
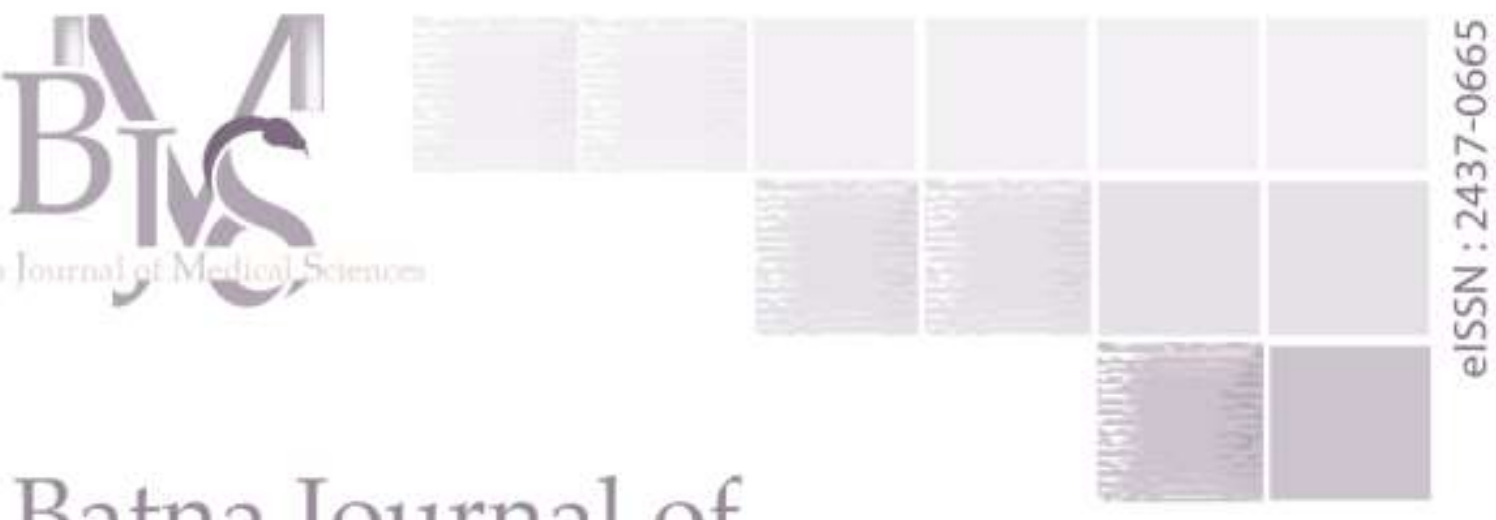

Batna Journal of

lVIedical Sciences
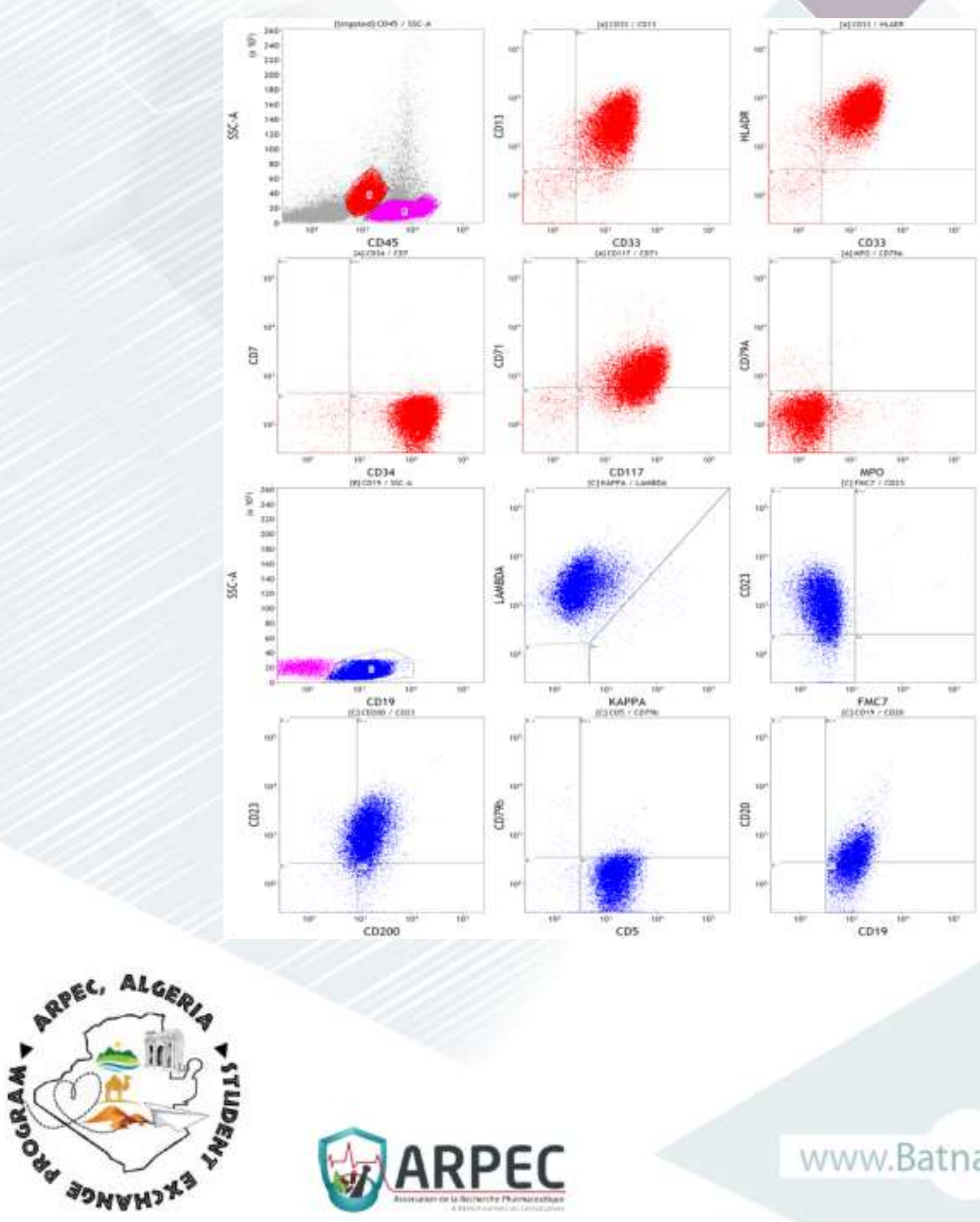

ARPEC 


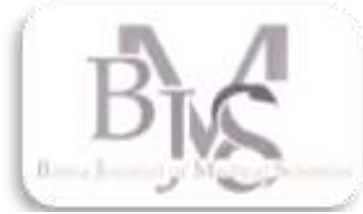

- $\bigcirc$

\title{
Batna Journal Of Medical Sciences
}

\author{
Organe officiel de l'association de la \\ Recherche Pharmaceutique
}

Volume 7, Numéro 2 (2020). Pages 71 - 209

\author{
TABLE DES MATIÈRES
}

\section{Articles originaux}

Anticancer Drug Design: Development of Cyclin-Dependent Kinase Inhibitors Using In Silico Techniques. Matmour D, Achachi N, Mérad $Y$, Toumi H. pages 71-73

Frequency of Peripheral Artery Disease in association with Coronary Artery Disease: a cross-sectional monocentric study in eastern Algeria. Merghit R, Ait Athmane M, Lakehal A. pages 74-78

Fibrillation auriculaire au cours du syndrome coronarien aigu avec élévation du segment ST. Zouzou H. pages 79 83

Audit de la prise en charge de la douleur à l'Hôpital Berdi F, Ifezouane J, Zhim I, Zakariya I, Lamsaouri J, Tadlaoui Y. pages 84-86

Effets des combinaisons glycémie 1 heure supérieure à 1,55 $\mathrm{g} / \mathrm{L}$ avec des profils glycémiques normaux après une HGPO chez des femmes obèses Guedjati MR, Boukebbal $M$, Gacem H, Ahmane A, Taibi AD, Hebboul G, Gasmi D, Lachekhab K, Djebabra A, Benadjrouda S. pages 87-91

Valeurs pronostiques de la Troponine T Hypersensible et du NT-proBNP chez les hémodialysés chroniques asymptomatiques. Médina Arab $M$, Zenati $A$, Labou N, Missoum M, Ainouz N, Bennoui M, Guechi Z. pages 92-96

Épidémiologie de la mortalité maternelle dans la Wilaya de Sétif, de 2014 à 2019 Abdoun M, Merabet N, Nouiouet N, Rezig F, Saoudi S. pages 97-101

Intérêt du jeûne dans l'exploration du bilan lipidique Nawal Habak, Dihia Bouaichi, Hanifa Abbache, Nadia OuldBessi, Ammar Chikouche. pages 102-104

Les uvéites de l'enfant « à propos de 62 cas » Kerrouche $\mathrm{K}$, Beldjilali S, Hafoudi M. pages 105-108

La mortalité maternelle dans la wilaya de Batna de 2014 à 2017 Khelalfa L, Oudjehih M, Ablous N, Bouzenita ZD, Toureche K, Benaldjia H, Beichi F, Boumaraf K. pages 109113

Épidémiologie des candidémies en réanimation médicale au CHU de Batna-Algérie Hamouda O. pages 114-116
Prescriptions d'anti-infectieux dans une pharmacie de Batna Keddad A, Oudjehih M. pages 117-121

Composition chimique, activité antimicrobienne et antioxydante de I'huile essentielle de Brocchia cinerea VIS. d'Algérie Ben-Moussa MT, Khelil K, Harkat $\mathrm{H}$, Lakehal S, Hadef $Y$. pages 122-128

La carence martiale chez l'enfant de moins de cinq ans à Batna Bouhdjila A. pages 129-133

Les données de la bactériologie en matière des BHRe au $\mathrm{CHU}$ de Batna Benmhidi M, Boukhalfa S, Benammar S, Makhloufi M, Lounis A, Khernane C. pages 134-136

Intérêt de la quantification de l'AgHBs chez le porteur chronique du virus de l'hépatite $B$ Benyahia $A$, Tebbal $S$, Chiboub B, Sadelaoud M, Bouncer H, Ameghchouche AH, Aouachria A. pages 137-141

\section{Mises au point}

Pandémie du déficit en vitamine $D$ et effets extra-osseux Abdellaoui S, Bengana B, Boukabous A, Lefkir-Tafiani S. pages 142-147

Détection précoce des désordres glycémiques par la glycémie une heure après une hyperglycémie provoquée par voie orale Guedjati MR. pages 148-150

Impact de l'irradiation solaire sur la santé Chader H, Gacem H. pages 151-158

Association asthme et diabète n'est pas fortuite Aissani $S$, Zitouni A. pages 159-161

Les thérapies comportementales et cognitives : une perspective islamique Benmebarek Z. pages 162-166

Méthémoglobinémie et surdosage à la Dapsone : Revue de la littérature. Zamoum R, Kaddour S. pages 167-170

Certificat de décès en Algérie : intérêt, circuit, formulaire et directives de rédaction Abdoun M. pages 171-175

Arthrite Juvénile Idiopathique. Avancées et défis Hadef $D$, Slimani S. pages 176-181
La "Mesure de l'Homme" : Historique de l'anthropométrie et de l'auxologie. Boutrid N, Rahmoune H. pages 182-185

\section{Cas cliniques}

Anévrisme de la crosse aortique distale englobant l'origine de l'artère sous Clavière gauche : à propos d'un cas Lakehal R, Bendjaballah S, Aimer F, Bouharagua R, Khacha K, Bouzid A. pages 186-188

Tumeur neuroendocrinienne de l'ovaire, une localisation inhabituelle: A propos d'un cas et revue de la littérature Benabdelhafidh Z. pages 189-191

Cas rare d'hémorragie digestive grave survenant sur diverticuloses jéjunales Chetibi A, Saidani M. pages 192193

Abcès cervical tardif sur plaie pénétrante par flèche : à propos d'une observation Seza JCK, Lame CA, Loum B, Bivahagumye L, Ngendakuriyo G. pages 194-196

Analyse moléculaire d'une famille algérienne de NEM2A au CPMC, Alger Chikouche A, Ould Bessi N, Habak N. pages 197-200

Un cas d'embolie pulmonaire après guérison chez un patient atteint de COVID-19 Benhocine Y. pages 201-203

Premier cas d'un Zona exceptionnel du creux poplité Righi $\mathrm{N}$ Mansouri OK, Lina B. pages 204-205

La Covid-19 atypique, à propos d'un cas Righi N, Debbabi M, Hamoud S, Sadelaoud M, Toumi W. pages 206-207

\section{Lettre à l'éditeur}

Coexistence of myeloblasts and B-CLL cells in peripheral blood Brahimi M, Ramdani H, Guenna M, Bouali Y, Bekadja MA. pages 208-209

\section{EDITORIAL OFFICE :}

Publication Director : Samy SLIMANI

Editor-in-Chief : Hocine GACEM

Executive Deputy Editor : Abdelhak ABABSA MOUAKI

Editorial Board : Amel AHMANE (Batna, Algérie), Ghania BELAALOUI (Batna, Algérie), Mohamed BRAHIMI (Oran, Algérie), Karima CHAABNA (Doha, Qatar), Adel GOURI (Annaba, Algérie), Assia HADDOUCHE (Blida, Algérie), Ihsane HMAMOUCHI (Rabat, Maroc), Noureddine IDRISS KHODJA (Montréal, Canada), Kawtar NASSAR (Casablanca, Maroc), Abdou Rajack NDIAYE (Dakar, Sénégal), Adlen NEZZAR (Batna, Algérie), Sidi EI Wafi OULD BABA (Nouakchott, Mauritanie). 


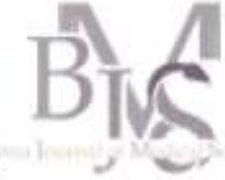

○

- 0

-

○

1. Therapeutic Chemistry Laboratory, Department of Pharmacy, University of Sidi Bel-Abbes, 22000, Algeria.

\section{Central Laboratory,}

University Hospital Center of Sidi Bel-Abbes, 22000, Algeria.

3. Research in Pharmaceutical Development Laboratory, Pharmacovigilance Department, University Hospital Center $1^{\text {st }}$ November, University of Oran, 31000, Algeria.

4. General Directorate of Scientific Research and Technological Development (DGRSDT), Algiers, Algeria.

Corresponding author : Derouicha MATMOUR drmatmour24@hotmail.fr

DOI : $\underline{\text { https://doi.org/10.48087/ }}$ BJMSoa.2020.7201

Received : June 03, 2020

Accepted : September 09, 2020

Published : November 09, 2020

This is an open access article distributed under the terms of the Creative Commons

Attribution International License (CC BY 4.0), which permits unrestricted use, distribution, and reproduction in any medium or format, provided the original author and journal are appropriately credited.

\section{Citation :}

Matmour D, Achachi N, Mérad Y, ToumI H. Anticancer Drug Design: Development of CyclinDependent Kinase Inhibitors Using In Silico Techniques. Batna J Med Sci 2020;7(2):71-3. https://doi.org/10.48087/BJMS oa.2020.7201

\title{
Anticancer Drug Design: Development of Cyclin-Dependent Kinase Inhibitors Using In Silico Techniques
}

\author{
Conception de médicaments anticancéreux: Développement in silico \\ d'inhibiteurs de kinases cycline dépendante
}

\author{
Derouicha Matmour ${ }^{1,2,3,4 *}$, Nawel Achachi ${ }^{3,4}$, Yassine Mérad ${ }^{2,4}$, Houari Toumi ${ }^{3,4}$
}

\begin{abstract}
In mammalian cells, proliferation is controlled by the cell cycle, where cyclin-dependent kinases regulate critical checkpoints. CDK4 is considered highly validated anticancer drug target due to its essential role regulating cell cycle progression at the G1 restriction point. Our objective is designing novel CDK4 inhibitors using Structure-Based Drug Design and Quantitative StructureActivity Relationship techniques. We used bioinformatics tools and biological databases. QSAR study of CDK4 inhibitors has given us an idea on the physicochemical features of studied compounds and their correlation with the $\mathrm{IC}_{50}$ activity. The docking study has helped to highlight the molecule key elements to refine in order to get a more potent compound of CDK4.The Molecule under the code 21366124 which has the low $\mathrm{IC}_{50}=3$ nmole shows the most binding affinity with score value of $\Delta G=-9,8$ $\mathrm{kcal} / \mathrm{mol}$. As prospects, it would be very interesting to synthesize this drug candidate and to test its inhibitory activity on cell culture of breast cancer
\end{abstract}

Keywords: CDK4, Inhibitors, QSAR, SBDD, Docking, Breast cancer.

\section{INTRODUCTION}

The strategy of Structure-Based Drug Design (SBDD) has accelerated many drug discovery projects and already yielded several promising anticancer leads acting towards different targets, including Cyclin-Dependent-Kinases (CDKs) [1].

The Quantitative Structure-Activity Relationship (QSAR) techniques have been widely used for predicting a broad spectrum of biological activities [2].

Protein kinases are key enzymes in regulatory signals of major physiological functions. Dysfunction of these proteins is often associated with diseases such as cancer, inflammatory or neuro-degenerative diseases. That's why protein kinases have become essential therapeutic targets [3].

Sustained proliferative capacity is a hallmark of cancer. In mammalian cells, proliferation is controlled by the cell cycle, where CDKs regulate critical checkpoints. CDK4 is considered highly validated anticancer drug target due to its essential role regulating cell cycle progression at the G1 restriction point [4].

\section{RÉSUMÉ}

Dans les cellules de mammifères, la prolifération est contrôlée par le cycle cellulaire, où les kinases dépendantes de la cycline régulent les points de contrôle critique. La CDK4 est considérée comme étant une cible thérapeutique anticancer hautement validée en raison de son rôle essentiel dans la régulation de la progression du cycle cellulaire au point de restriction G1. L'objectif de ce travail est de concevoir de nouveaux inhibiteurs CDK4 à l'aide de techniques de conception de médicaments basées sur la structure et sur l'activité biologique. Des outils de bio-informatique et des bases de données biologiques ont été utilisés. L'étude QSAR des inhibiteurs CDK4 a donné une idée sur les caractéristiques physicochimiques des composés étudiés et leur corrélation avec l'activité $\mathrm{IC}_{50}$. L'étude d'amarrage moléculaire a permet de mettre en évidence les éléments clés de la molécule dockée afin d'obtenir un inhibiteur CDK4 plus puissant. La molécule sous le code 21366124 qui a la plus faible IC 50 = 3 nmole, s'est avérée la plus affine avec un score d'énergie $\Delta G=-9,8 \mathrm{kcal} / \mathrm{mol}$. En perspectives, il serait très intéressant de synthétiser cette molécule candidate et de tester son effet inhibiteur sur une culture cellulaire de cancer du sein.

Mots-clés : CDK4, inhibiteurs, QSAR, SBDD, Docking, Cancer du sein.

In this study, our main objective was to identify new anticancer drug candidates, using the SBDD and QSAR techniques for designing novel CDK4 inhibitors.

\section{MATERIALS AND METHODS}

The present study combines ligand-based drug design and structure-based drug design approaches.

In one hand, 2D quantitative structure activity relationship (2D-QSAR) was used to generate models from 83 compounds belonging to CDK4 inhibitors and separated to training and test sets. Validation of the QSAR model was judged by root-mean-square error (RMSE) and the correlation factor $\left(\mathrm{R}^{2}\right)$ [5-7].

In the other hand, docking studies of six inhibitors were carried to determine their binding mode into the active site of CDK4 and to interpret the efficacy of molecules that's capable to inhibit CDK4 $[8,9]$.

We used software's of SBDD and QSAR wich are based on molecular modeling techniques and in silico virtual screening $[\mathbf{9}, \mathbf{1 0}]$. 


\section{RESULTS AND DISCUSSION}

\section{2D-QSAR Study}

2D-QSAR study was performed in order to find a mathematical correlation between the structure and physicochemical properties of CDK4 inhibitors and their inhibitory activity that was expressed as PIC $_{50}\left(-\log \mathrm{IC}_{50}\right)$.

The model I showed in Figure 1 exhibited a correlation between the experimentally observed and predicted values of CDK4 inhibitors. The resultant correlation regression analysis plot showed a linear relationship in model I with RMSE $=0.2458, \mathrm{R}=0.9345$ and $\mathrm{R}^{2}=0.8733$. No molecule was an outlier in the database using MOE's plotting applications in the model. We have also verified that the model was valid with $\mathrm{R} 2$ $=0.15$ and $\mathrm{Q} 2=-0.37$ (Figure 2).

The correlation coefficient between the experimental observed and predicted value of test set compounds of inhibitors was 0,70 .

\section{traintest2.M6 (PLS), Untitled}

YPred:Comp. 3](YVar Picas (M))/YVar(YVar PIC

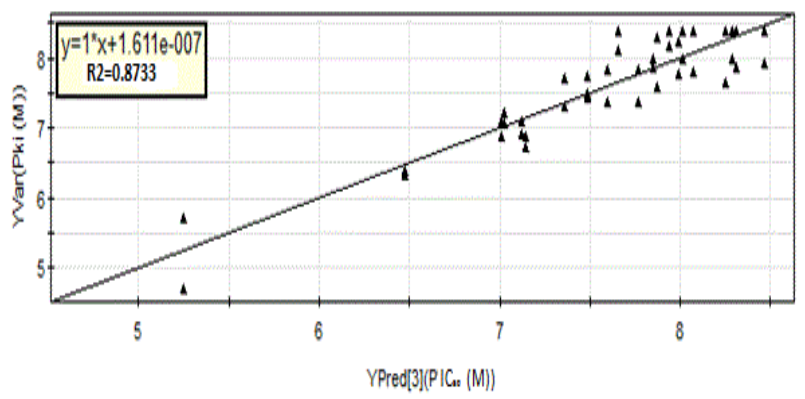

Figure 1. Linear correlation plots of 2D-QSAR.

traintest2.M6 (PLS): Validate Model

Pki (M) Intercepts: $R 2=(0.0,0.159), Q 2=(0.0,-0.374)$
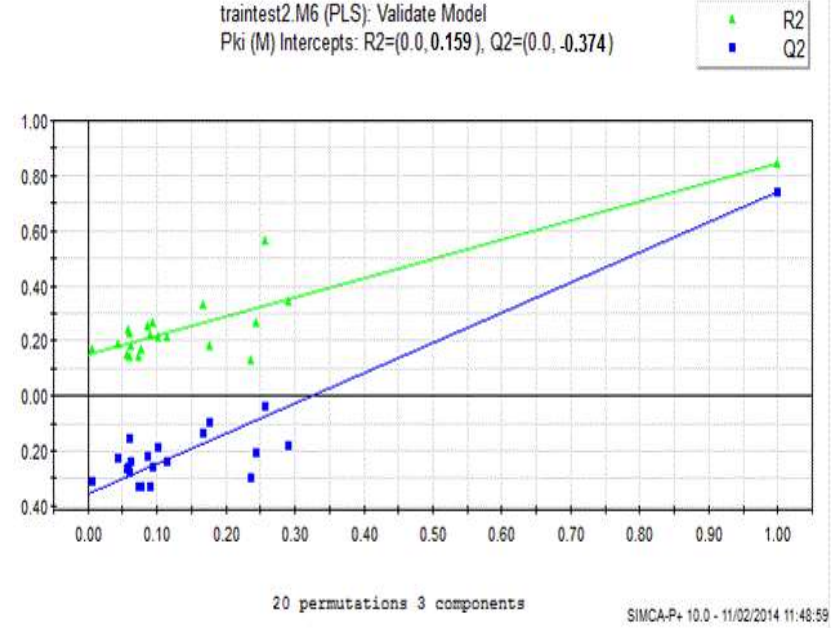

Figure 2. Model validation.

\section{Molecular Docking Study}

Figure 3 shows the 3D crystal structure of CDK4 extracted from PDB (Code: 4DHU) and Figure 4 shows the selected target site of CDK4, the magenta spheres represent where the molecular docking was focused.

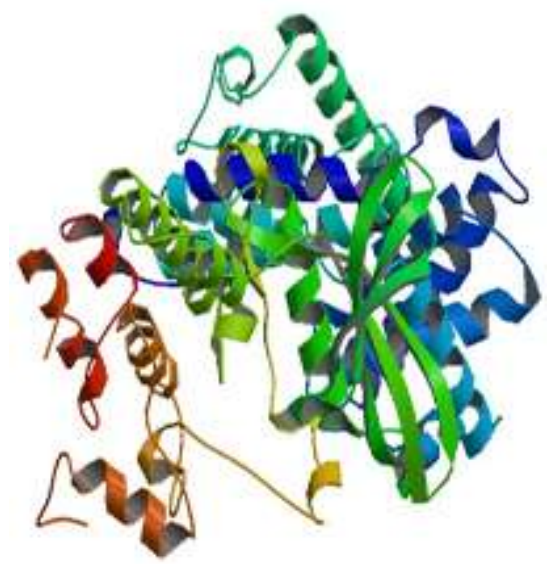

Figure 3. 3D crystal structure of CDK4.

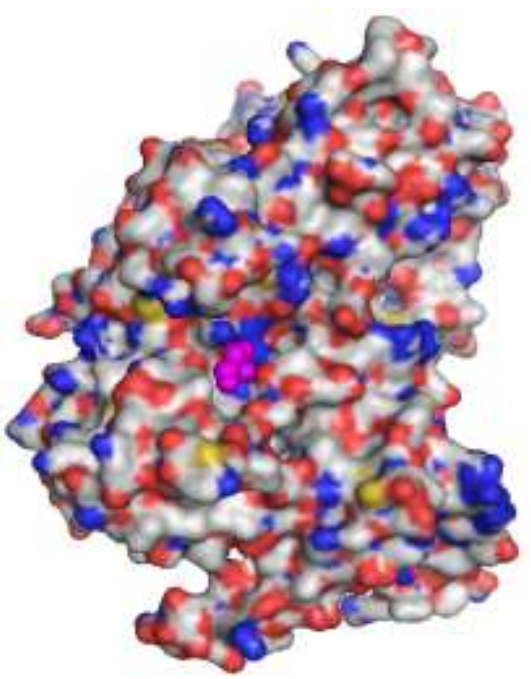

Figure 4. Selected target site of CDK4.

The Coumpond under the code 21366124 is successfully docked in the active site of CDK4. Its $\mathrm{IC}_{50}=3 \mathrm{nmole}$ and its binding energy $=-9.8 \mathrm{Kcal} / \mathrm{mol}$, therefore, it proved the most affine. It formed three hydrogen bonds with the residues: Arg136, Thr165 and Asp73 whose lengths: $3.04 \mathrm{~A}^{\circ}, 2.94 \mathrm{~A}^{\circ}$ and $2.92 \mathrm{~A}^{\circ}$. Three hydrophobic pockets formed and interaction piStacking between imidazole nucleus of ligand and pyrrolidine nucleus of L-proline amino acid (Figure 5).

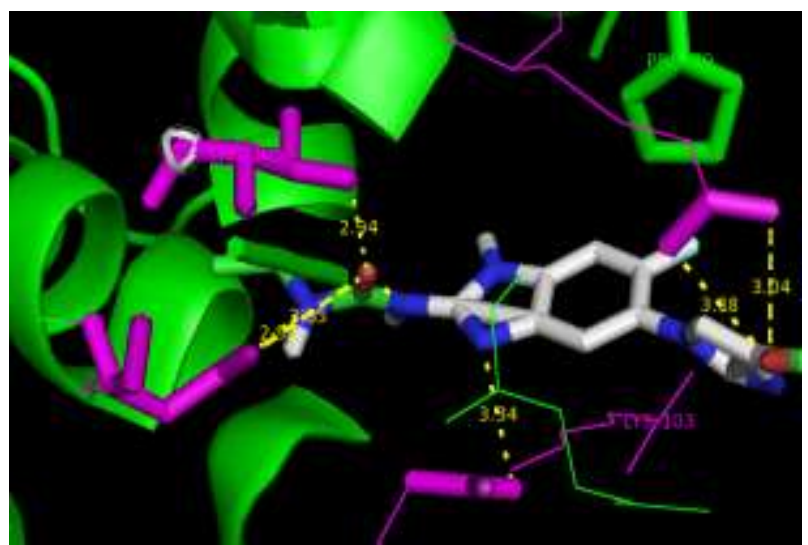

Figure 5. Interaction between CID 21366124 and CDK4. 


\section{CONCLUSION}

We have performed a structure-activity relationships study of CDK4 as potential therapeutic target. QSAR study of CDK4 inhibitors has given us an idea on the physicochemical features of studied compounds and their correlation with the $\mathrm{IC}_{50}$ activity. Furthermore, the docking study has helped to highlight the molecule key elements to refine in order to get a more potent compound of CDK4.

The molecule under the code 21366124 which has the low $\mathrm{IC}_{50}=3$ nmole shows the most binding affinity with score value of $\Delta \mathrm{G}=-9,8 \mathrm{kcal} / \mathrm{mol}$ and it presents the best interactions and energy score testifying the complex stability. As prospects, it would be very interesting to synthesize this drug candidate and to test its inhibitory activity on cell culture of breast cancer.

\section{CONFLICT OF INTEREST}

The authors declare that they have no conflict of interests.

\section{REFERENCES}

1. Cavasotto C, Orry, A. Ligand Docking and Structure-Based Virtual Screening in Drug Discovery. Current Topics in Medicinal Chemistry. 2007; 7: 1006-1014

2. Arkadiusz ZD, Tomasz A and Jorge G. Computational Methods in Developing Quantitative Structure-Activity Relation-ships (QSAR): A Review. Combinatorial Chemistry \& High Throughput Screening. 2006; 9: 213-228.
3. Sandrine Piguel, Chi Hung Nguyen, Delphine Martin, Tom Baladi and J. Aziz. Chimie des petites molécules pour le ciblage des protéines. Sondes de structures et sondes photoactivables pour les acides nucléiques et les kinases. Accessed December 09th 2018, Available at: https://studylibfr.com/doc/4594887/chimie-des-petitesmol\%C3\%A9cules-pour-le-ciblage-des-prot\%C3\%A9ines.

4. Concepción SM, Lawrence MG, María JL, Alfonso D. Cyclin dependent kinase (CDK) inhibitors as anticancer drugs. Bioorganic \& Medicinal Chemistry Letters. 2015; 25: 3420-3435.

5. Molecular Operating Environment (MOE). Integrated computer-aided molecular design platform for small molecule and biological therapeutics. Common platform for Chemists, Biologists and Crystallographers. Available at: https://www.chemcomp.com/Products.htm [Accessed $15^{\text {th }}$ July 2018].

6. CanSAR Database. Available at: https://cansarblack.icr.ac.uk/ [Accessed $15^{\text {th }}$ July 2018].

7. Gramatica P. Principles of QSAR Models Validation: Internal and External. QSAR \& Combinatorial Science. 2007; 26: 694-701.

8. Trott $\mathrm{O}$ and Olson AJ. AutoDock Vina: Improving the Speed and Accuracy of Docking with a New Scoring Function, Efficient Optimization, and Multithreading. Journal of Computational Chemistry. 2010; 31: 455-461.

9. Rohini R, Souda PR, Vishwanath R and al. Selective ATP competitive leads of CDK4: Discovery by 3D-QSAR pharmacophore mapping and molecular docking approach. Computational Biology and Chemistry. 2017;71: 224229.

10. Anupama Pandrangi. Molecular Docking and QSAR Studies on CDK4 Inhibitors Using In Silico Techniques. Journal of Pharmaceutical and Scientific Innovation. 2014;3(2):164-169.

This article was published in the "Batna Journal of Medical Sciences" BJMS, the official organ of the « Association pour la Recherche Pharmaceutique et l'Enrichissement des Connaissances - Batna »

The content of the Journal is "Open Access" and allows the reader to download and use the content for personal or educational purposes without requesting permission from the publisher/author.

Advantages of publishing in BJMS :

- Open access : once published, your article is available for free download

- Free submission: no submission fee, unlike most "Open Access" journals

- Possibility to publish in 3 languages : French, English, Arabic

- Quality of proofreading: geographically independent proofreaders/reviewers, respecting anonimity, to guarantee the neutrality and quality of the manuscripts.

For more information, contact BatnaJMS@gmail.com or log on to the journal's website: www.batnajms.net 

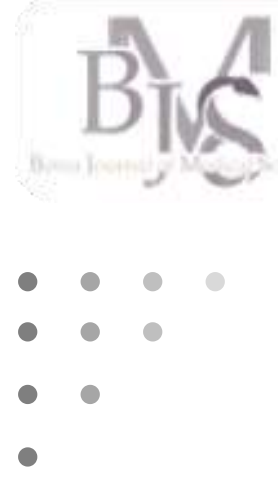

${ }^{1}$ Department of cardiology, University Hospital Center of Constantine, Algeria 25000

2Department of cardiology, University Hospital Center of Annaba, Algeria 23000

${ }^{3}$ Department of epidemiology University Hospital Center of Constantine, Algeria 25000

Correspondance à :

Rachid MERGHIT

mer18net@yahoo.fr

DOI :https://doi.org/10.48087/ BJMSoa.2020.7202

\section{Historique de l'article :}

Reçu le 02 juin 2020

Accepté le 22 août 2020

Publié le 09 novembre 2020

This is an open access article distributed under the terms of the Creative Commons Attribution International License (CC BY 4.0), which permits unrestricted use, distribution, and reproduction in any medium or format, provided the original author and journal are appropriately credited.

\section{Pour citer l'article :}

Merghit R, Ait Athmane M, Lakehal A. Frequency of Peripheral Artery Disease in association with coronary artery disease: a crosssectional monocentric study in eastern Algeria. Batna J Med Sci 2020;7(2):74-8. https://doi.org/10.48087/B IMSoa.2020.7202

Frequency of Peripheral Artery Disease in association with Coronary Artery Disease: a cross-sectional monocentric study in eastern Algeria

\author{
Fréquence de l'association artériopathie oblitérante des membres inférieurs \\ et coronaropathie : résultats d'une étude transversale monocentrique à l'est \\ algérien
}

\title{
Rachid Merghit ${ }^{1}$, Mouloud Ait Athmane ${ }^{2}$, Abdelhak Lakehal ${ }^{3}$
}

\section{RÉSUMÉ}

Introduction. Les coronariens ayant une atteinte vasculaire périphérique ont un pronostic cardiovasculaire plus sévère. Il paraît donc intéressant de dépister cette association, notamment en mesurant l'index de pression systolique qui est un moyen simple, non invasif, et non coûteux ayant un apport diagnostic important, afin de détecter des lésions silencieuses, mais menaçantes, et d'identifier un sous-groupe de coronariens à plus haut risque cardiovasculaire, nécessitant une prise en charge plus spécifique. Objectifs. Estimer la fréquence de L'artériopathie oblitérante des membres inférieurs (AOMI) chez les patients coronariens, recrutés en cardiologie dans les centres hospitalo-universitaires de la ville de Constantine et déterminer les facteurs de risques de cette association. Patients et méthodes. Notre étude est descriptive, transversale, mono centrique réalisée en unités des explorations cardiovasculaires de l'hôpital militaire régional universitaire de Constantine. Les sujets inclus avaient aux moins une lésion coronaire significative $\geq 50 \%$ sur une artère coronaire principale, pour chaque patient, une anamnèse orientée et un examen clinique cardiovasculaire ont précédé la mesure de L'index de pression systolique (IPS). L'AOMI était définie par un IPS inférieur à 0.90 sur une des quatre artères distales des membres inférieurs. Le traitement et l'exploitation des données ont fait appel au logiciel Epi Info Statistics V7. Résultats. 300 patients coronariens, âgés en moyen de 61 ans à prédominance masculine nette, ont été inclus. La fréquence de l'association AOMI et coronaropathie était de $34,7 \%$, asymptomatique dans $18 \%$ des cas. L'âge $\geq 65$ ans, le tabac, le diabète, l'HTA, la dyslipidémie, la sédentarité, l'accident vasculaire cérébral (AVC) et l'atteinte coronaire sévère étaient les facteurs indépendants de l'association. L'odds ratio ajusté (ORa) est à : 3,67 (âge $\geq 65$ ans), 4,10 (tabac), 3,48 (diabète), 3,30 (HTA), 2,32 (dyslipidémie), 2,14 (sédentarité), 6,40 (AVC) et 2,36 (atteinte coronaire sévère). Conclusion. L'IPS, en plus d'être un outil de dépistage précoce de l'AOMI, sa diffusion en pratique médicale aiderait à l'approche et l'affinement du risque vasculaire des patients coronariens.

Mots-clés : IPS, AOMI, coronaropathie.

\section{INTRODUCTION}

Atherosclerosis and its corollary atherothrombosis are widespread in both developed and developing countries [1]. International registries, recruiting patients with at least arterial disease, record between 16 and $28 \%$ of multisite arterial disease, of which about one third of these atheromatous patients are, in fact, asymptomatic and at very high cardiovascular risk [2]. Wanted by measurement of the ankle-brachial systolic pressure index (ABI) in coronary patients, the prevalence of

\begin{abstract}
Introduction. Patients with both Coronary Artery Disease (CAD) and Peripheral Artery Disease (PAD) have a worse cardiovascular prognosis. It therefore seems interesting to detect this association, in particular by measuring the ankle-brachial systolic pressure index $(\mathrm{ABI})$ which is a simple, inexpensive and non-invasive way with a significant diagnostic input that can detect silent but threatening lesions. It can also identify a subgroup of patients with coronary artery disease at a higher cardiovascular risk requiring a more specific management. Aims. Estimate the frequency of peripheral artery disease (PAD) in patients with Coronary Artery Disease CAD who were recruited at the department of cardiology at the university hospital of Constantine. This study also aims to detect the risk factors of this association. Patients and methods. Our study is descriptive, cross-sectional, single-centered in cardiovascular exploration units of the of the regional military university hospital of Constantine. The included subjects had at least one significant coronary lesion in a major coronary artery using radial Coronary angiography. Guided medical history and a cardiovascular clinical examination preceded the measurement of the $\mathrm{ABI}$ for each patient. PAD was defined by an ABI of less than 0.90 in one of the four distal arteries of the two lower limbs. Data was analyzed and processed by Epi-Info Statistics V7. Results. 300 coronary patients, an average age of 61 years, mostly males, took part in the study. The frequency of the combination PAD and coronary artery disease was $34.7 \%$ using the $\mathrm{ABI}$, asymptomatic in $18 \%$ of cases. independent factors of the association were : Age $\geq 65$ [aOR 3,67, P<0,0001], tobacco [aOR 4,10, P<0,002], diabetes [aOR 3,48, $\mathrm{P}<0,0001]$, AHT [aOR 3,30, $\mathrm{P}<0,0001$ ], dyslipidemia [aOR 2.32, $\mathrm{P}<0,009$ ], inactivity [aOR 2,14, $\mathrm{P}<0,015]$, stroke [aOR 6,4, $\mathrm{P}<0,015]$ and severe coronary impairment [aOR 2,36, $\mathrm{P}<0,015]$. Conclusion. $\mathrm{ABI}$, in addition to being an early detection tool for PAD, its dissemination in medical practice would help in the approach and refinement of vascular risk in coronary heart patients.
\end{abstract}

Keywords : ABI, PAD, CAD.

peripheral arterial disease (PAD) can reach more than $30 \%$ according to the age of the subjects and can even exceed $40 \%$ in hospitalized subjects [3].

\section{MATERIELS AND METHODS}

\section{Study population}

Our epidemiological study is descriptive, analytical and monocentric conducted on a sample of 300 consecutive coronary patients, at the regional military university hospital of Constantine, with at least one lesion of $50 \%$ in 
a major coronary artery, using radial coronary angiography regardless of their age and gender, excluding those who refused to participate in the study and patients with acute ischemia of the lower limbs.

\section{Study}

The patients received a collection of anthropometric measurements (weight, height, calculation of BMI), a collection of information (cardiovascular risk factors, cardiovascular and cerebral pathologies), a comprehensive clinical examination, a biological balance including complete lipid profile (HDLC ,Total cholesterol, triglyceride, LDL-C), fasting blood glucose, creatinine levels, calculated creatinine clearance according to the MDRD formula and HBA1C for diabetic patients. we measured the $\mathrm{ABI}$ in dorsal decubitus, the upper limbs undressed, in a patient relaxed for more than 10 minutes, by a pocket doppler (Sonotrax Vascular Lite, brand: EDAN), equipped with a probe of $8 \mathrm{MHz}$, with a sphygmomanometer (brand: Riester) . After obtaining a stable Doppler signal, the cuff was inflated by $20 \mathrm{~mm} \mathrm{Hg}$ beyond the signal removal pressure and then slowly deflated by $2 \mathrm{~mm} \mathrm{Hg}$ per second until an audible signal reappeared. The retained value was equivalent to systolic ankle blood pressure. The recorded arteries were classically, for each lower limb, the posterior tibial artery in the back-malleolar gutter, the Dorsalis pedis artery at the foot [4]. This procedure was performed twice on each limb; at each arm. The Doppler signal was picked up at the humeral or radial level, with the cuff positioned as when measuring the usual blood pressure (BP). We chose the most sensitive method to calculate the ABI. The ratio between the lowest levels of systolic pressure on the highest brachial systolic pressure of both arms. The ABI was calculated for each of the arteries of each limb [5]. The lowest index of the two lower limbs was the one considered, making the diagnosis of PAD if lower or equal 0.9 in the presence or absence of symptomatology.

If $\mathrm{ABI}$ ankle was limited to $0.91-0.99$, a hemodynamic treadmill walking test (Skinner Strandness test) with taking distal pressures at rest and after an effort was indicated in search of an subclinical PAD; the diagnosis was retained when the distal pressure dropped by at least $20 \%$ from the first minute after stopping the effort. In the case where the ABI ankle was strictly greater than 1.3 , a supplement of hemodynamic exploration was performed by measuring the systolic pressure index in toe (TBI) for each member using a photoplethysmograph. All of our coronary patients have benefited from a Trans thoracic cardiac ultrasound with measurement of the LV mass, overall left ventricular systolic ejection fraction using the Simpson Biplan method and evaluation of LV diastolic function by measuring LV filling pressures.

\section{Data analysis}

All patient examinations were performed by the same cardiologist (lead investigator), which were then initially recorded on a data sheet prepared for this purpose, later transferred to a database (EXCEL 2013 file) designed for the same purpose. Statistical analysis was performed using Epi Info Statistics V7. The results are presented with 95\% confidence intervals, in the form of average, median, standard deviation, and minimum and maximum values, for quantitative variables. In the form of percentages with their standard deviation for qualitative variables.

\section{RESULTS}

\section{Characteristics demographics of our sample}

Between June 2015 and March 2016, we collected 300 consecutive coronary patients. The average age of this population was $61.3 \pm 11.3$ years, extremes of age ranging from 23 to 85 years, and a median of 62 years, with a significant male predominance $(\mathrm{P}<0,0001)$. This population was relatively small (average BMI $27.9 \pm 4.7 \mathrm{~kg} / \mathrm{m} 2$, Average waist circumference $95.6 \pm 11.2 \mathrm{~cm}$ ). The majority of our coronaries had more than three cardiovascular risk factors (CVRF) (72.7\%). The predominant CVRF were arterial hypertension (AHT) (58.7\%), sedentarity (57.3\%), dyslipidemia (52.7\%), overweight (49\%), and diabetes (47.4\%). The least observed CVRF were active smoking (32.3\%), obesity (29.3\%), and Familial Coronaropathy (26.4\%). Diabetes was associated with AHT and dyslipidemia in $36 \%, 47.3 \%$ of cases respectively; triple association was observed in $37.7 \%$. Personal antecedents of cerebrovascular disease (ischemic stroke, hemorrhagic stroke), were observed in $2.7 \%$. The majority of our patients $(60.7 \%)$ went through coronary angiography for an acute coronary syndrome, the rest for stable ischemic heart disease, an increased left ventricular mass (LVM), was found in 54\%. The left ventricular ejection fraction (LVEF), using the Simpson biplane method, was retained in $83.34 \%$ and $54.3 \%$ of our patients had an alteration of LV diastolic function. $41.67 \%$ had Single vessel disease, $30.7 \%$ double vessel disease, $22 \%$ triple vessel disease.

\section{Peripheral arterial exploration}

As a result of this investigation, it was found that the frequency of Symptomatic PAD was $16 \%$, and the frequency of intermittent claudication of the lower limbs according to the Edinburgh questionnaire $11.67 \%$. The intake of the clinical examination was poor, finding a decrease or abolition of pulse $11 \%$ and pathological auscultation in $7.34 \%$. For 300 patients and on the basis of four ABI for each of them, we totaled 1200 $\mathrm{ABI}$ with an average $\mathrm{ABI}$ of $1.07 \pm 0.26$ on the right side and 1.08 \pm 0.24 on the left side. The ABI profile according to TASC II and AHA [6] was normal in $44.7 \%, \mathrm{ABI} \leq 0,9$ evoking PADS in $17.3 \%$. A dodgy $\mathrm{ABI}$ in $17 \%$, an $\mathrm{ABI}>1.3$ evoking arterial incompressibility in $21 \%$. After measuring the $A B I$ of stress in situations where the $\mathrm{ABI}$ is questionable $(0.9-\mathrm{ABI}-1)$, and measuring the systolic pressure index to the big toe in situations where the $\mathrm{ABI}$ was 1.3 , the definitive $\mathrm{ABI}$ profile of our population was in $57 \%$ in favor of the absence of PAD, a Media calcinosis observed in $21 \%$, in relation to an isolated mediacalcosis $(8.3 \%)$ or mixed impairment $(12.7 \%)$ and the presence of $\mathrm{PAD}$ in $34.7 \%$ for either an isolated PAD, diagnosed after measuring resting $\mathrm{ABI}$ in 52 patients $(17.3 \%)$, a PAD diagnosed after measuring stress $\mathrm{ABI}$ in questionable cases in 14 patients $(4.7 \%)$, or a mixed impairment ( calcose media and associated PAD ) in 38 patients (12.7\%). In total, ABI was pathological in 129 coronary scans, representing a frequency of $43 \%$ of the overall population. The frequency of the combination PAD and coronary artery disease, diagnosed using the $\mathrm{ABI}$ is $34.7 \% \pm 5,3,95 \%$ CI $(29.3 \%-40 \%)$. This population is therefore composed of 104 patients including 48 with symptomatic PAD and 56 with asymptomatic PAD.

\section{Analysis of the factors correlated with the occurrence of PAD in our coronary heart}

Multi-varied analysis, with a type of logistic regression, was carried out by the SPSS 22. The risk of PAD related to each factor, adjusted for all other factors, was represented by Odds Adjusted Ratio (ORa) estimated with its confidence interval at $95 \%$. The various parameters predisposing to the installation of PAD in our coronary artery have been identified and are in order of frequency: personal antecedents of brain events type ischemic stroke or transient ischemic attack (TIA) (ORa 6.40), smoking (ORa 4.10), age advanced age 65 (ORa 3.67), diabetes (3.48), AHT(ORa 3.30), severe coronary impairment (0Ra 2.36), aorto-coronary bypass surgery (ORa 3.20), and to a lesser degree dyslipidemia and inactivity (ORa 2.32, 2.14). 
However, some factors, which were found to be significant in the bi-varied analysis, no longer stood out in the multivariate analysis; these are: male sex, normal BMI, severe kidney failure, systolic LV dysfunction, diastolic LV dysfunction and increased LV mass (Table1 and 2).

Tableau 1. Comparative study between different coronary populations

\begin{tabular}{|c|c|c|c|c|c|c|}
\hline \multirow[t]{2}{*}{ Variables } & \multirow{2}{*}{$\begin{array}{l}\text { without PAD } \\
\quad(n=196)\end{array}$} & \multirow{2}{*}{$\begin{array}{l}\text { with PAD } \\
(n=104)\end{array}$} & \multirow[b]{2}{*}{$\mathbf{P}$} & \multicolumn{2}{|c|}{ with PAD } & \multirow[b]{2}{*}{$\mathbf{P}$} \\
\hline & & & & $\underset{(n=56)}{\operatorname{asymptomatic}}$ & $\begin{array}{c}\text { symptomatic } \\
(n=48)\end{array}$ & \\
\hline \multicolumn{7}{|l|}{ Sex $(n-\%)$} \\
\hline Men & $145(74)$ & $90(86,5)$ & & $50(89,2)$ & $40(83,3)$ & NSD \\
\hline Women & $51(26)$ & $14(13,5)$ & $N S D$ & $6(10,8)$ & $8(16,7)$ & \\
\hline \multicolumn{7}{|l|}{ Age } \\
\hline Average (years) & $58,14 \pm 11,25$ & $67,2 \pm 8,4$ & 0,0001 & $67,8 \pm 7$ & $66,9 \pm 8$ & $N S D$ \\
\hline \multicolumn{7}{|l|}{ range (years) } \\
\hline$\geq 65$ & $64(32,6)$ & $68(65,4)$ & 0,0001 & $36(64,2)$ & $32(66,7)$ & $N S D$ \\
\hline$<65$ & $132(67,4)$ & $36(34,6)$ & & $20(35,7)$ & $16(33,3)$ & \\
\hline \multicolumn{7}{|l|}{ Number of CVRF } \\
\hline$\geq 3 \mathrm{CVRF}$ & $124(63,3)$ & $94(90,4)$ & 0,0001 & $49(87,5)$ & $45(93,8)$ & $N S D$ \\
\hline$<3 \mathrm{CVRF}$ & $72(36,7)$ & $10(9,6)$ & & $7(12,5)$ & $3(6,52)$ & \\
\hline \multicolumn{7}{|l|}{ BMI (Kg/m2) } \\
\hline BMI average & $28,7 \pm 4,63$ & $26,3 \pm 43$ & 0,0001 & 26,08 & 26,71 & $N S D$ \\
\hline $\mathrm{BMI} \geq 30(\mathrm{n}-\%)$ & $78(39,8)$ & $10(9,6)$ & 0,0001 & & & \\
\hline $\mathrm{BMI}<30(\mathrm{n}-\%)$ & $118(60,2)$ & $94(90,4)$ & & & & \\
\hline \multicolumn{7}{|l|}{ Diabetes (n-\%) } \\
\hline Diabetic & $74(37,8)$ & $68(65,4)$ & 0,0001 & $40(71,4)$ & $28(58,4)$ & \\
\hline Non diabetic & $122(62,2)$ & $36(34,6)$ & & $16(28,6)$ & $20(41,7)$ & 0,003 \\
\hline Average duration (years) & $11,7 \pm 7,8$ & $11,03 \pm 9,11$ & $N S D$ & & & \\
\hline Diabetes encienty (years) $\geq 10$ & $43(58,1)$ & $35(51,5)$ & $N S D$ & & & \\
\hline Diabetes balance (n-\%) & $51(58,0)$ & $15(27,8)$ & 0,002 & & & \\
\hline Good balance & $26(29,5)$ & $27(50,0)$ & & & & \\
\hline \multicolumn{7}{|l|}{ Bad balance } \\
\hline \multicolumn{7}{|l|}{ Tabacco (n-\%) } \\
\hline Smoker & $108(55,1)$ & $78(75)$ & & $41(73,2)$ & $37(77,0)$ & $N S D$ \\
\hline Non-smoker & $88(44,9)$ & $26(25)$ & 0,002 & $15(26,8)$ & $11(22,9)$ & \\
\hline Amount of smoking toxicity & $28 \pm 17,80$ & $37,95 \pm 19,36$ & & & & \\
\hline \multicolumn{7}{|l|}{ HT (n-\%) } \\
\hline $\mathrm{HT}$ & $98(50,0)$ & $78(75,0)$ & 0,0001 & $41(73,2)$ & $37(77,0)$ & $N S D$ \\
\hline No HT & $98(50,0)$ & $26(25,0)$ & & $15(26,8)$ & $11(22,9)$ & \\
\hline Average duration (years) & $8,16 \pm$ & $8,18 \pm$ & $N S D$ & & & \\
\hline HT encienty (years) $\geq 10$ & 6,134 & 8,614 & $N S D$ & & & \\
\hline HT balance & $40(40,8)$ & $26(33,3)$ & & & & \\
\hline Good balance & $42(42,1)$ & $27(34,3)$ & 0,003 & & & \\
\hline Bad balance & $57(57,9)$ & $51(65,7)$ & & & & \\
\hline \multicolumn{7}{|l|}{ Dyslipidimia(n-\%) } \\
\hline Dyslipidimia (LDLc < & $101(51,5)$ & $74(71,2)$ & & $39(69,6)$ & $35(72,9)$ & \\
\hline $\begin{array}{l}0,7 \mathrm{~g} / \mathrm{l}) \\
\end{array}$ & & & 0,009 & & & NSD \\
\hline $\begin{array}{r}\text { No dyslipidemia } \\
(\mathrm{LDLc} \geq 0,7 \mathrm{~g} / \mathrm{l})\end{array}$ & $95(48,5)$ & $30(28,8)$ & & $17(30,4)$ & $13(27,0)$ & \\
\hline \multicolumn{7}{|l|}{ Familial Coronaropathy (n-\%) } \\
\hline Coronaropathy F & $51(26,0)$ & $28(26,9)$ & $N S D$ & $16(28,6)$ & $12(25)$ & $N S D$ \\
\hline No coronaropathy F & $145(74,0)$ & $76(73,1)$ & & $40(71,4)$ & $36(75)$ & \\
\hline Severe renal failure & $2(1,0)$ & $5(4,8)$ & $N S D$ & $3(5,4)$ & $2(4,1)$ & $N S D$ \\
\hline \multicolumn{7}{|l|}{ Sedentarity (n-\%) } \\
\hline (+)sedentarity & $102(52,0)$ & $70(67,3)$ & 0,015 & $47(66,1)$ & $33(71,3)$ & $N S D$ \\
\hline (-)sedentarity & $94(48,0)$ & $34(32,7)$ & & $19(33,9)$ & $15(28,7)$ & \\
\hline
\end{tabular}


(Table 1 continued)

\begin{tabular}{|c|c|c|c|c|c|c|}
\hline $\begin{array}{l}\text { Stroke (n-\%) } \\
\text { Stroke } \\
\text { No stroke }\end{array}$ & $\begin{array}{r}2(1,0) \\
194(99,0)\end{array}$ & $\begin{array}{r}6(5,8) \\
98(94,2)\end{array}$ & 0,0001 & $\begin{array}{r}4(7,1) \\
52(92,9)\end{array}$ & $\begin{array}{r}2(4,1) \\
46(95,9)\end{array}$ & $N S D$ \\
\hline $\begin{array}{l}\text { LVEF (n-\%) } \\
\text { LVEF altered } \\
\text { LVEF normal }\end{array}$ & $\begin{array}{r}18(9,2) \\
178(90,8)\end{array}$ & $\begin{array}{l}32(30,77) \\
72(69,23)\end{array}$ & $N S D$ & $\begin{array}{l}18(32,1) \\
38(67,9)\end{array}$ & $\begin{array}{l}14(29,1) \\
34(70,9)\end{array}$ & $N S D$ \\
\hline $\begin{array}{c}\text { L VFP (n-\%) } \\
\text { HIGH LVFP } \\
\text { Normal LVFP }\end{array}$ & $\begin{array}{r}95(48,5) \\
101(51,5)\end{array}$ & $\begin{array}{l}68(65,4) \\
36(34,6)\end{array}$ & $N S D$ & $\begin{array}{l}36(64,3) \\
20(35,7)\end{array}$ & $\begin{array}{l}32(66,7) \\
16(33,3)\end{array}$ & $N S D$ \\
\hline $\begin{array}{l}\text { LV mass (n-\%) } \\
\text { Normal LV mass } \\
\text { Increased LV mass }\end{array}$ & $\begin{array}{r}105(53,6) \\
91(46,4)\end{array}$ & $\begin{array}{l}33(31,7) \\
71(68,3)\end{array}$ & $N S D$ & $\begin{array}{l}18(32,1) \\
38(67,9)\end{array}$ & $\begin{array}{l}15(31,6) \\
33(68,4)\end{array}$ & $N S D$ \\
\hline $\begin{array}{l}\text { Severe coronary artery disease } \\
(n-\%)\end{array}$ & $19(9,70)$ & $64(61,53)$ & 0,015 & $32(57,1)$ & $32(66,7)$ & $N S D$ \\
\hline
\end{tabular}

NSD: no significant difference; HT: arterial hypertension; BMI: body mass Index; CVRF: cardiovascular risk factors

LVEF: left ventricular ejection fraction; LVM: left ventricular mass; LVFP: left ventricular filling pressures

Tableau 2. Factors related to the occurrence of the PAD

\begin{tabular}{|c|c|c|c|}
\hline Variables & $\begin{array}{l}\text { adjusted odds ratio } \\
\text { (aOR) }\end{array}$ & $95 \% \mathrm{CI}$ aOR & $\mathbf{P}$ \\
\hline Male & 0,53 & $0,172-1,654$ & 0,277 \\
\hline Age $\geq 65$ years & 3,67 & $1,975-6,851$ & 0,0001 \\
\hline Diabetes & 3,48 & $1,843-6,589$ & 0,0001 \\
\hline AHT & 3,30 & $1,703-6,406$ & 0,0001 \\
\hline Smoking & 4,10 & $1,674-10,069$ & 0,002 \\
\hline Dyslipidemia & 2,32 & $1,234-4,381$ & 0,009 \\
\hline Normal BMI & 0,12 & $0,051-0,293$ & 0,0001 \\
\hline $\begin{array}{l}\text { Severe Renal } \\
\text { failure }\end{array}$ & 0,17 & $0,027-1,089$ & 0,062 \\
\hline Sedentarity & 2,14 & $1,159-3,971$ & 0,015 \\
\hline Stroke & 6,40 & $1,60-14,20$ & 0,0001 \\
\hline Altered LVEF & 0,58 & $0,164-2,025$ & 0,390 \\
\hline Increased LVFP & 0,90 & $0,422-1,948$ & 0,801 \\
\hline Increased LVM & 0,36 & $0,164-2,025$ & 0,7 \\
\hline $\begin{array}{l}\text { Severe } \\
\text { coronary artery } \\
\text { disease }\end{array}$ & 2,36 & $1,159-3,822$ & 0,015 \\
\hline
\end{tabular}

\section{DISCUSSION}

Regardless of the stage of CAD, the presence of a PAD is a factor of bad prognosis and at least an aggravating factor [3]. The measure of $\mathrm{ABI}$ is the cornerstone of the lower arterial limb exploration strategy as an immediate complement to the clinical examination, not only to diagnose PAD in many cases, but also to help to identify a significant segment of the asymptomatic population, already at high cardiovascular risk. Studies show that there is no significant difference in risk of death and vascular events, at five years between symptomatic and asymptomatic patients [7]. The relative simplicity of this test and its high sensitivity seem to indicate this for the detection of PAD in pauci- or asymptomatic populations.
This led us to use it during our work to diagnose the PAD and then deduce the frequency of association with coronary artery disease especially since the contribution of questionnaires (Rose, Edinburgh etc.) and diagnostic clinical examination of PAD are reduced, as demonstrated by numerous epidemiological studies [8].

During our study; any symptomatic or asymptomatic patient with one of the following criteria was considered arteritic: an $\mathrm{ABI} \leq 0.9$ at rest, an $\mathrm{ABI} \leq 0.9$ after stress sensibilisation (positive Skinner Strandness test) or an ABI toe $\leq 0.7$ in a patient with an $\mathrm{ABI}>1.3$. Based on these conditions, the frequency of the combination of coronary artery disease and PAD was $34.7 \% \pm 5.3,95 \%$ CI $(29.3 \%$ - 40\%). Various studies objectively target a high frequency of PAD in coronary patients; however, the prevalence figures remain heterogeneous [3]; this is naturally due to the recruitment method of coronary patients (population selected versus population and therefore average age) and the PAD diagnostic method. Our data on the prevalence of PAD is consistent with the most recent literature, and even appear to be superior to other studies; several explanations for this difference are possible:

- The diagnosis of PAD, in our study, was based on interrogation, clinical examination and measurement of the resting $\mathrm{ABI}$ (possibly supplemented by a measure of the stress $\mathrm{ABI}$, in borderline cases, and measurement of the TBI, if mediacalcose.

- $\quad$ The ABI threshold used in our study to diagnose the PAD was an $\mathrm{ABI} \leq 0.9$, (0.9 included), which probably increased the number of detected patients with PAD compared to studies using an $\mathrm{ABI}<0.9$ as a criterion.

- The method of calculating ABI also influences the heterogeneity of PAD prevalence. Given the lack of consensus on the calculation of ABI. the use of the lowest $\mathrm{ABI}$, in our study, provides a better sensitivity to a slight decrease in specificity [9] and more identification of patients with high cardiovascular risk, and consequently a better estimate of the frequency of PAD in patients with Coronary disease.

- Also noteworthy is the high prevalence of cardiovascular risk factors found in our study. Indeed, we have noted a high prevalence of arterial hypertension (AHT), diabetes, 
tobacco, and dyslipidemia. These cardiovascular risk factors are associated with PAD in most of the conducted studies.

In our serie, the frequency of asymptomatic PAD diagnosed with $\mathrm{ABI}$ was higher than that of symptomatic intermittent claudication (IC) based PAD using the Edinburgh questionnaire, the ratio between the two frequencies was 2.97, in the literature. The prevalence of PAD determined by the ABI measurement is generally 2.5 higher than that determined by the CI [10-12]. This high frequency of asymptomatic PAD in our series can be explained by the fact that it is often underestimated in coronary scans. The various parameters predisposing to the installation of PAD in our coronary artery were personal antecedents of ischemic stroke, smoking, advanced age - 65 years, AHT, severe coronary impairment, dyslipidemia and sedentarity. However, some of the factors that were found to be significant in the bi-varied analysis no longer stood out in the multi-variance analysis such as sex or gender difference which disappeared after logistical regression and adjustment to confounding factors, such as age and CVRF mainly all-male tobacco in our study; a normal BMI was a rather protective factor, This seems to be related to the involvement of obesity in other risk factors of PAD such as hypertension, type II diabetes and dyslipidemia. This interaction may be responsible for decreasing the statistical power of the results [13].

LVM was significantly increased in the arteritic versus nonarteric group $(\mathrm{P}=0.0001)$; this finding is similar for LVFP $(\mathrm{P}=0.005)$, but these links disappear in multi-variance analysis. This loss of statistical power can be explained by the entanglement of several factors in the increase in LVM mainly AHT and diabetes, as well as for LVFP (AHT, diabetes, LVM, CAD). According to our analysis, LV dysfunction is significantly related to the occurrence of PAD in coronary patients in bivaried analysis $(\mathrm{P}=0.03)$. After a multi-variate analysis, this link disappears $(P=0.390)$, probably in relation to the decrease in the statistical power of this factor after adjusting to other factors essentially, the severe CAD often incriminated in the LV dysfunction.

\section{CONCLUSION}

Currently, the ABI is recognized by the majority of international recommendations as a rapid, low cost, noninvasive, and reproducible way to complete any clinical examination (any patient with at least one CVRF or in any subject with at least one atherothrombotic vascular disease). It must be available to any specialist practitioner or not, taking care of this type of patients and as part of a systematic vascular assessment, to establish or eliminate a PAD, symptomatic or asymptomatic.

\section{CONFLICT OF INTEREST}

The authors declare that they have no conflict of interests.

\section{REFERENCES}

1. Fowkes, F.G., et al., Comparison of global estimates of prevalence and risk factors for peripheral artery disease in 2000 and 2010: a systematic review and analysis. Lancet, 2013. 382(9901) : p. 1329-40.

2. Becker, F., Artériopathie oblitérante des membres inférieurs. Journal des Maladies Vasculaires, 2016. 41(2) : p. 96.

3. Kownator,S et al, Stratégie de la conduite des examens chez le patient polyvasculaire, in EMC - Angéiologie VL - IS - SP - YP -. 2016.

4. Hiatt, W.R., Medical treatment of peripheral arterial disease and claudication. N Engl J Med, 2001. 344(21): p. 1608-21.

5. Espinola-Klein, C., et al., Different calculations of ankle-brachial index and their impact on cardiovascular risk prediction. Circulation, 2008. 118(9): p. 961-7.

6. Haute Autorité de Santé (HAS). Recommandations pour la pratique clinique : Prise en charge de l'artériopathie chronique oblitérante athéroscléreuse des membres inférieurs (indications médicamenteuses, de revascularisation et de rééducation). 2006; Available from: https://www.has sante.fr/portail/upload/docs/application/pdf/AOMI_recos.pdf.

7. Golomb, B.A., T.T. Dang, and M.H. Criqui, Peripheral arterial disease: morbidity and mortality implications. Circulation, 2006. 114(7): p. 68899.

8. Yao, S.T., J.T. Hobbs, and W.T. Irvine, Ankle systolic pressure measurements in arterial disease affecting the lower extremities. Br J Surg, 1969. 56(9): p. 676-9.

9. Schroder, F., et al., A modified calculation of ankle-brachial pressure index is far more sensitive in the detection of peripheral arterial disease. J Vasc Surg, 2006. 44(3): p. 531-6.

10. Criqui, M.H., et al., The prevalence of peripheral arterial disease in a defined population. Circulation, 1985. 71(3): p. 510-5.

11. Hirsch, A.T., et al., Peripheral arterial disease detection, awareness, and treatment in primary care. JAMA, 2001. 286(11): p. 1317-24.

12. Schroll, M. and O. Munck, Estimation of peripheral arteriosclerotic disease by ankle blood pressure measurements in a population study of 60-year-old men and women., in J Chronic Dis. 1981. p. 261-269.

13. Nadjia, K.H., Prévalence de L'artériopathie oblitérante des membres inférieurs chez le coronarien Algérien. (Doctoral dissertation, Université Benyoucef Benkhedd, Alger), 2013.

Cet article a été publié dans le « Batna Journal of Medical Sciences » BJMS, l'organe officiel de « l'association de la Recherche Pharmaceutique - Batna»

Le contenu de la Revue est ouvert « Open Access » et permet au lecteur de télécharger, d'utiliser le contenu dans un but personnel ou d'enseignement, sans demander l'autorisation de l'éditeur/auteur.

Avantages à publier dans BJMS :

- Open access : une fois publié, votre article est disponible gratuitement au téléchargement

- Soumission gratuite : pas de frais de soumission, contrairement à la plupart des revues « Open Access »

- Possibilité de publier dans 3 langues : français, anglais, arabe

- Qualité de la relecture : des relecteurs/reviewers indépendants géographiquement, respectant l'anonymat, pour garantir la neutralité et la qualité des manuscrits.

Pour plus d'informations, contacter BatnaJMS@gmail.com ou connectez-vous sur le site de la revue : www.batnajms.net 
Fibrillation auriculaire au cours du syndrome coronarien aigu avec élévation du segment ST

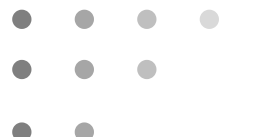

○

1 Faculté de Médecine,

Université de Batna 2 - Algérie

${ }^{2}$ Service de cardiologie, $\mathrm{CHU}$ Batna - Algérie

\section{Correspondance à :}

Hanane ZOUZOU

hanane zouzou@yahoo.fr

DOI : https://doi.org/10.48087/ BJMSoa.2020.7203

\section{Historique de l'article :}

Reçu le 02 juin 2020

Accepté le 04 septembre 2020

Publié le 09 novembre 2020

Il s'agit d'un article en libre accès distribué selon les termes de la licence Creative Commons Attribution International License (CC BY 4.0), qui autorise une utilisation, une distribution et une reproduction sans restriction sur tout support ou format, à condition que l'auteur original et la revue soient dûment crédités.

Pour citer l'article :

Zouzou H. Fibrillation auriculaire au cours du syndrome coronarien aigu avec élévation du segment ST. Batna J Med Sci 2020;7(2):79-83. https://doi.org/10.48087/ BJMSoa.2020.7203

\author{
Atrial fibrillation during acute coronary syndrome with ST elevation
}

Hanane Zouzou ${ }^{1,2}$

\section{RÉSUMÉ}

Introduction. La fibrillation auriculaire (FA) est le trouble du rythme supra ventriculaire le plus fréquent ; sa survenue dans le syndrome coronarien aigu (SCA) avec élévation du segment ST, complique la prise en charge et aggrave le pronostic, son incidence et ses facteurs prédictifs ont fait l'objet de plusieurs études internationales, cependant ses données épidémiologiques manquent en Algérie. Objectifs. L'objectif principal de notre étude est la détermination de la fréquence de la FA dans le SCA avec élévation du segment ST, alors que l'objectif secondaire est l'analyse des facteurs prédictifs et la mortalité associée à cette arythmie. Méthodes et matériels. Dans cette étude prospective, menée dans le service de cardiologie du CHU Hussein Dey (AlgerAlgérie), 467 patients présentant un syndrome coronarien aigu avec élévation du segment ST (87 femmes et 380 hommes) ont été colligés entre le 28 Février 2014 et le 16 Juillet 2015 ; l'enregistreur ECG Holter des 48 heures est placé à l'admission. Les tests ANNOVA ou H de Kruskal ont été utilisés pour la comparaison des variables quantitatives, le test $\chi 2$ ou le test exact de Fisher ont été utilisés pour les variables qualitatives, tous les tests ont été réalisés avec un risque de 1 ère espèce de $5 \%$. Résultats. La fréquence de la FA est de $6 \%$ (28 patients), IC95\% : [3.8\%-8.2\%], l'analyse multi variée a permis l'identification des facteurs prédictifs indépendants suivants: l'âge avancé et le territoire droit du SCA, Le risque de mortalité exprimé par le Hazard Ration (HR) est à 5 (IC95\% : 1.6-15.58), p : 0.004), les facteurs prédictifs de la mortalité sont l'échec de la thrombolyse, l'atteinte rénale, le territoire droit du syndrome coronarien aigu, et la fibrillation ventriculaire. Conclusion. La fibrillation auriculaire est une complication rythmique fréquente, durant le syndrome coronarien aigu avec sus décalage du segment ST, ses deux facteurs prédictifs selon notre étude sont l'âge et le territoire droit du syndrome coronarien aigu, sa survenue augmente le risque de la mortalité hospitalière, ce risque élevé est en parti expliqué par la stratégie thérapeutique.

Mots clés: syndrome coronarien aigu, fibrillation auriculaire, trouble du rythme, trouble de la conduction

\section{INTRODUCTION}

La fibrillation auriculaire est le trouble du rythme supra ventriculaire le plus fréquent ; sa survenue dans le syndrome coronarien aigu (SCA) avec élévation du segment ST, complique la prise en charge et aggrave le pronostic, le mécanisme physiopathologique de la fibrillation auriculaire est complexe et multifactoriel, l'ischémie de la paroi auriculaire ou l'étirement suite à l'augmentation de la pression intra auriculaire constituent le substratum pour l'installation de cette arythmie. L'inflammation, le système nerveux autonome, l'activation

\begin{abstract}
Background. Atrial fibrillation (AF) is the most frequent supraventricular rhythm disorder; its occurrence in acute coronary syndrome (ACS) with ST-segment elevation complicates management and worsens the prognosis, its incidence and predictive factors have been the subject of several international studies, however its epidemiological data is lacking in Algeria. Aims. The main objective of our study is the determination of the frequency of atrial fibrillation in acute coronary syndrome with elevated ST segment, the secondary objective was the analysis of predictive factors of this arrhythmia, and related mortality. Methods and materials. In this prospective study, conducted in the cardiology department of the Hussein Dey University Hospital (Algiers-Algeria), 467 patients with acute coronary syndrome with ST segment elevation (87 women and 380 men) were collected between February 28, 2014 and July 16, 2015; the 48hour Holter ECG recorder was placed at admission. The Kruskal ANNOVA or $\mathrm{H}$ tests were used for the comparison of quantitative variables, the $\chi 2$ test or the Fisher exact test were used for the qualitative variables, all tests were performed with a 1 st species risk of $5 \%$.

Conclusion. Atrial fibrillation is a common rhythmic complication, during acute coronary syndrome with elevated ST segment, its two predictors according to our study are the age and right territory of acute coronary syndrome, its occurrence increases the risk of hospital mortality, this high risk is partly explained by the therapeutic strategy. Results. The incidence of $\mathrm{AF}$ is $6 \%$ (28 patients), IC95\%: 3.8\%-8.2\%], multivariate analysis identified the following independent predictors: advanced age and right ACS territory, Hazard Ration (HR) risk of mortality is 5 (95\% CI: $1.6-15.58), \mathrm{p}: 0.004)$, Predictors of mortality are thrombolysis failure, renal impairment, right ACS territory, and ventricular fibrillation.
\end{abstract}

Keywords : acute coronary syndrome, atrial fibrillation, rhythm disorder, conduction disorder.

hormonale participent à son initiation et sa perpétuation, ainsi l'altération de la fonction ventriculaire gauche et l'augmentation des pressions de remplissage, l'inflammation du péricarde, l'hypoxie, l'hypokaliémie, les catécholamines, le dysfonctionnement sinusal, favorisent l'installation de ce trouble. [1]

La fibrillation auriculaire survient durant les 24 premières heures dans le syndrome coronarien aigu inférieur en rapport avec l'occlusion de la coronaire droite, et après 24 heures dans la localisation antérieure associée à la dysfonction ventriculaire gauche. 
Son incidence et ses facteurs prédictifs ont fait l'objet de plusieurs études internationales, cependant ses données épidémiologiques manquent en Algérie.

L'objectif principal de notre étude est la détermination de la fréquence des troubles du rythme et de la conduction dans le syndrome coronarien aigu avec élévation du segment ST, alors que l'objectif secondaire est l'analyse des facteurs prédictifs de ces arythmies et la mortalité associée, parmi ces troubles du rythme étudiés, figure la fibrillation auriculaire dont les résultats sont rapportés dans cet article.

\section{MATÉRIELS ET MÉTHODES}

Dans cette étude prospective, menée dans le service de cardiologie du CHU Hussein Dey (Alger-Algérie), 467 patients présentant un syndrome coronarien aigu avec élévation du segment ST (87 femmes et 380 hommes) ont été colligés entre le 28 Février 2014 et le 16 Juillet 2015.

L'enregistreur ECG Holter des 48 heures placé à l'admission. L'ECG de surface 17 dérivations à l'admission puis répété durant l'hospitalisation, l'échocardiographie doppler, la coronarographie et le bilan biologique sont des examens qui ont été pratiqués chez la majorité des patients.

Les troubles du rythme et de la conduction les plus importants ont été regroupés ensemble pour constituer le groupe des troubles du rythme et de la conduction. Les patients présentant le même type de trouble étaient regroupés ensemble, le nom attribué à chaque groupe est celui du trouble qui le caractérise; il existe des chevauchements entre les groupes, ainsi plusieurs troubles peuvent exister chez le même patient.

La constitution de chaque groupe du trouble du rythme implique la constitution du groupe opposé sans le trouble correspondant, ce dernier groupe sert pour l'étude comparative; chaque groupe constitué est donc décrit puis comparé au groupe opposé correspondant. Concernant la fibrillation auriculaire, les patients ayant présenté cette arythmie en phase aiguée, constitue le groupe de la fibrillation auriculaire, ce dernier est comparé au groupe sans fibrillation auriculaire.

L'analyse descriptive de l'échantillon a été estimée par la moyenne \pm l'écart type, ou le nombre et le pourcentage selon la variable, les tests ANNOVA ou H de Kruskal ont été utilisés pour la comparaison des variables quantitatives, le test $\chi 2$ ou le test exact de Fisher ont été utilisés pour les variables qualitatives, tous les tests ont été réalisés avec un risque de 1 ère espèce de $5 \%$.

\section{RÉSULTATS}

\section{Fréquence de la fibrillation auriculaire}

Un total de 467 patients présentant un syndrome coronarien aigu avec élévation du segment ST (87 femmes et 380 hommes) ont été colligés.

La fibrillation auriculaire a été enregistrée chez 35 patients, dont 7 sont connus et traités pour cette arythmie. La fibrillation auriculaire (FA) complication rythmique du syndrome coronarien aigu a été documentée chez 28 patients.

La fréquence de la fibrillation auriculaire est donc de $6 \%$ (28 patients), IC95\% : [3.8\%-8.2\%]. C'est le trouble du rythme supra ventriculaire le plus fréquent, sa fréquence de survenue n'est pas influencée par la réalisation de l'ECG Holter, car chez les 28 patients, l'ECG de surface a permis sa détection; chez 20 patients soit les $71.42 \%$ de ce groupe ont présenté ce trouble à l'arrivée.

\section{Facteurs de risque}

les facteurs de risque retrouvés dans ce groupe sont répartis comme suit : L'hypertension artérielle : 16 patients (57.14\%), Le diabète: 9 patients $(32.14 \%)$, Le tabac:11 patients (39.28\%), Les antécédents cardiovasculaires familiaux précoces : 1 patient (3.57\%), L'obésité : 7 patients (7.40\%), La dyslipidémie: 6 patients (21.42\%), La ménopause: 8 patientes (100\%), L'association HTA et diabète : 8 patients (28.57\%), L'HTA diabète et tabac : 1 patient (3.57\%), L'HTA diabète et ménopause :2 patientes $(7.14 \%)$.

La moyenne de l'index de la masse corporelle est de $25.37 \pm 3.56$

Le score de GRACE supérieur à 155 (risque élevé de mortalité hospitalière) : 17 patients (60.71\%), Le délai de consultation, avant la 6ème heure : 23 patients (82.14\%).

\section{Les caractéristiques cliniques}

3 patients (10.71\%) ont présenté un état de choc cardiogénique, 4 patients (14.28\%) ont présenté des signes d'insuffisance cardiaque gauche et 2 patients $(7.14 \%)$ ont présenté des signes d'insuffisance cardiaque droite. La moyenne de la tension artérielle systolique est de $118 \pm 51.27$ $\mathrm{mm} \mathrm{Hg}$ et la moyenne de la tension artérielle diastolique est de $70.89 \pm 27.08 \mathrm{~mm} \mathrm{Hg}$.

Le syndrome coronarien aigu antérieur étendu a été observé chez 7 patients, circonférentiel chez 2 patients, antérieur chez 3 patients, latéral haut chez 3 patients, antéro-septal chez 1 patient, inférobasal chez 11 patients, inférieur chez 3 patients et droit chez 7 patients, la moyenne de la fréquence cardiaque à l'admission est de $90.64 \pm 30.43 \mathrm{p} / \mathrm{min}$, de l'intervalle PR après réduction de la FA : $154.28 \pm 30.81 \mathrm{msec}$, de la durée du QRS : $71.42 \pm 16.71 \mathrm{msec}$, du sus décalage de $4.53 \pm 2.89 \mathrm{~mm}$, du sous décalage $1.78 \pm 1.75 \mathrm{~mm}$, de l'amplitude de l'onde $\mathrm{T}$ : $6.82 \pm 3.68 \mathrm{~mm}$ et du QT corrigé $425.16 \pm 56.72 \mathrm{msec}$, persistance du sus décalage chez 6 patients.

Les fibrinolytiques ont été administrés chez 24 patients soit $85.71 \%$,

La persistance de la douleur après thrombolyse a été observée chez 5 patients $(20.8 \%)$ et la persistance du sus décalage chez les 5 patients $(20.8 \%)$.

Concernant les patients qui ont présenté la FA durant l'hospitalisation malgré la thrombolyse, la persistance de la douleur et du sus décalage, a été observée chez 2 patients $(8.3 \%)$.

\section{Échocardiographie doppler}

Pratiquée chez 24 patients, la fraction ventriculaire gauche inférieure à $40 \%$ a été retrouvée chez 3 patients (12.5\%), l'hypertrophie ventriculaire gauche chez 8 patients $(28.57 \%)$, la moyenne de la surface de l'oreillette gauche est de $18.17 \pm 4.10 \mathrm{~cm}^{2}$, celle de l'oreillette droite $12.56 \pm 2.72 \mathrm{~cm}^{2}$, du diamètre diastolique du ventricule gauche : $52.79 \pm 6.69 \mathrm{~mm}$, $\mathrm{du}$ diamètre du ventricule droit : $25.60 \pm 3.48 \mathrm{~mm}$, de la pression artérielle pulmonaire systolique : $28.04 \pm 7.76 \mathrm{~mm} \mathrm{Hg}$, l'akinésie des parois chez 13 patients (54.16\%), et l'insuffisance mitrale significative chez 5 patients soit $17.85 \%$.

\section{Arythmies associées}

Le bloc de branche droit complet: 6 patients, le bloc de branche gauche : 2 patients, le dysfonctionnement du nœud sinusal : 2 patients, le bloc auriculo ventriculaire complet : un patient, la fibrillation ventriculaire : 7 patients, la tachycardie ventriculaire soutenue: 2 patients, les tachycardies ventriculaires non soutenues : 9 patients. 


\section{- $\quad$ ECG Holter (figure 1)}

Pratiqué chez 24 patients, en plus des troubles sus cités, la tachycardie auriculaire : un patient, les salves d'extrasystoles auriculaires: 11 patients, les salves d'extrasystoles ventriculaires: 13 patients, le rythme idioventriculaire accéléré: 6 patients, les extrasystoles ventriculaires polymorphes : un patient, et le phénomène $\mathrm{R}$ sur $\mathrm{T}: 2$ patients.

\section{- Biologie}

La créatininémie moyenne était de $12.55 \pm 4.15 \mathrm{mg} / \mathrm{l}$, la troponine $\mathrm{T}$ hs $\geq$ à $5 \mathrm{ng} / \mathrm{ml}: 9$ patients soit $39.13 \%$.

\section{Coronarographie}

Réalisée chez 19 patients, les lésions sévères ont été retrouvées chez 6 patients, la sténose serrée du tronc commun gauche chez 1 patient, de l'artère inter ventriculaire antérieure chez 9 patients, de l'artère circonflexe chez 5 patients, de l'artère coronaire droite chez 6 patients, les lésions bi tronculaires chez 6 patients, les lésions tri tronculaires chez 2 patients, le flux TIMI 0 chez 1 patient.

\section{- Mortalité}

4 patients sont décédés durant les 48 premières heures d'hospitalisation. Un patient décédé dans la 1ère heure de son hospitalisation, un patient à la 3ème heure, un patient à la 5 ème heure et un patient à la 11 ème heure

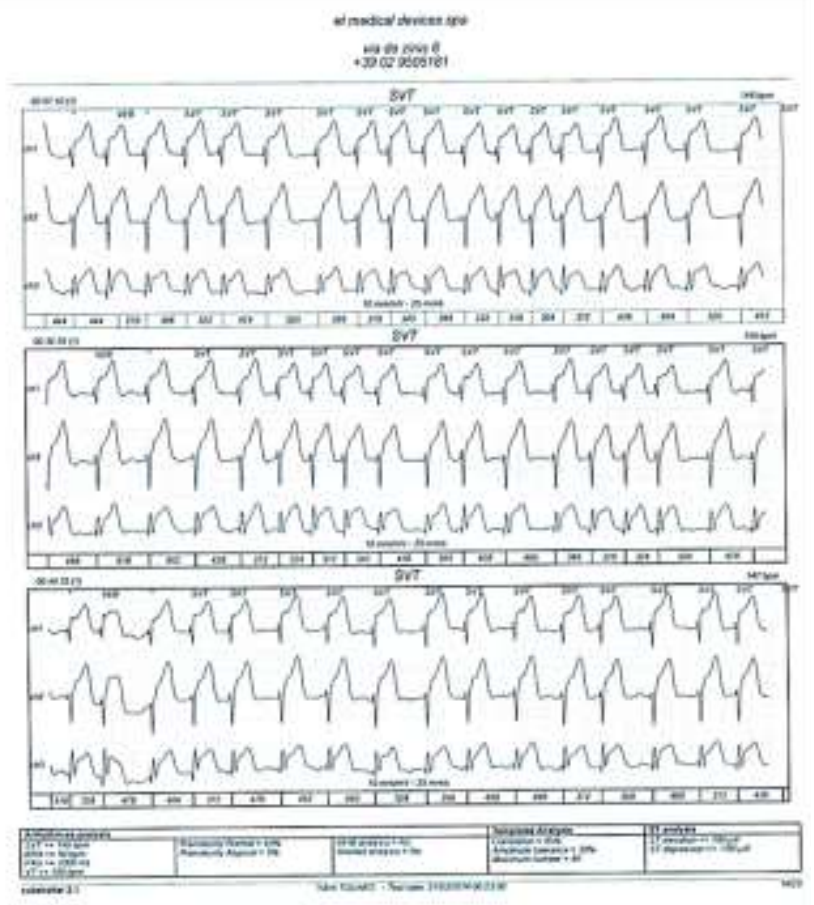

Figure 1 : FA durant le SCA avec élévation du segment ST

\section{Résultats après comparaison des deux groupes avec et} sans fibrillation auriculaire

\section{Les facteurs prédictifs}

Selon l'étude uni variée, les facteurs qui ont un lien statistiquement significatif avec la fibrillation auriculaire sont les suivants : L'âge supérieur ou égal à 68 ans, Le score de GRACE élevé supérieur ou égal à 155 , Le territoire droit du SCA, la surface de l'oreillette droite $\geq 12 \mathrm{~cm}^{2}$, l'insuffisance mitrale significative, la créatininémie élevée, La persistance de la douleur indépendamment de la thrombolyse, et l'échec de la thrombolyse.
Mais après analyse multi variée, les deux facteurs prédictifs indépendants de la fibrillation auriculaire retenus étaient l'âge $\geq 68$ ans et le territoire droit du syndrome coronarien aigu. (Figure 2)

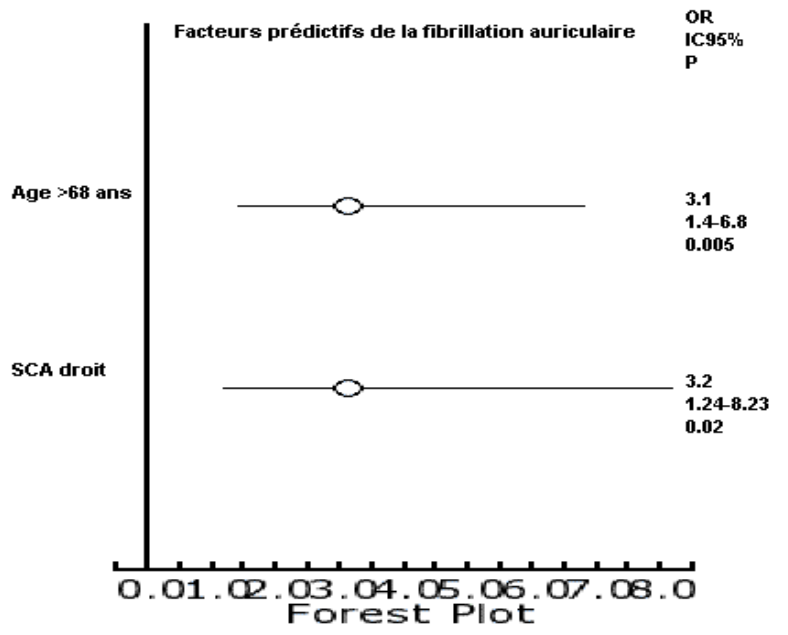

Figure 2 : Facteurs prédictifs de la fibrillation auriculaire

- L'âge avancé, supérieur ou égal à 68 ans, est donc fortement lié à la survenue de la fibrillation auriculaire dans le SCA, la FA est connue par sa fréquence élevée chez le sujet âgé, ceci se confirme dans notre étude, les modifications anatomiques et électro physiologiques liées à l'âge, constituent le substratum et favorisent l'initiation et la perpétuation de la FA en présence d'une gâchette en l'occurrence le syndrome coronarien aigu.

- Le territoire droit du syndrome coronarien aigu, entraine une nécrose de la paroi auriculaire droite.

La magnitude du lien entre la fibrillation auriculaire et ses facteurs prédictifs est faible, le coefficient V de Cramer ne dépasse pas 0.2 (Tableau 1).

Tableau 1 : magnitude du lien entre la fibrillation auriculaire et ses facteurs prédictifs.

\begin{tabular}{lcc} 
Facteurs prédictifs de la FA & Coefficient V de Cramer & $\mathbf{p}$ \\
\hline Age & 0.151 & 0.001 \\
\hline Syndrome coronarien droit & 0.141 & 0.002
\end{tabular}

\section{La mortalité}

Le risque relatif de décès précoce chez les patients avec fibrillation auriculaire était 5 fois plus élevé que le reste de la population (HR : 5), (Figure 3), le risque relatif de la fibrillation ventriculaire était 7 fois plus élevé (RR : 6.98).

Les facteurs prédictifs de la mortalité dans le groupe de la fibrillation auriculaire étaient :

- Le territoire droit du syndrome coronarien aigu (SCA) (OR : 9.9), favorise l'insuffisance cardiaque droite connue par son pronostic péjoratif.

- La créatininémie $\geq 17 \mathrm{mg} / \mathrm{l}(\mathrm{OR}:$ 8), favorise les perturbations métaboliques, électro physiologiques et hémodynamiques. 
- La persistance du sus décalage après la thrombolyse (OR : 34.6), augmente l'étendu de la nécrose, et les dégâts myocardiques

- La fibrillation ventriculaire (OR: 12) synonyme de la mort.

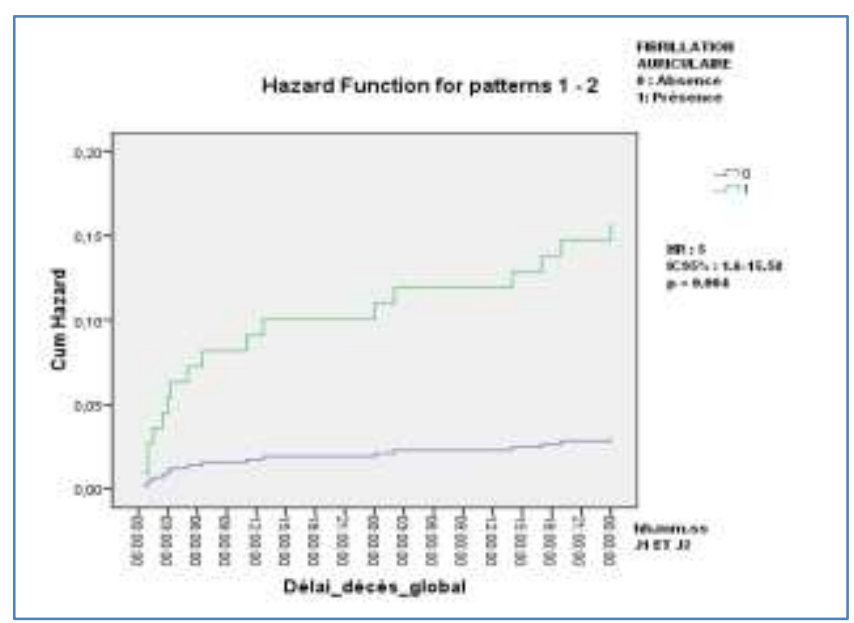

Figure 3. Courbe de la mortalité hospitalière (48H) dans le groupe de la FA vs groupe sans FA

Tous ces facteurs favorisent l'instabilité hémodynamique, les perturbations métaboliques et les modifications électro physiologiques à l'origine du décès.

La magnitude du lien faible ou modérée entre la mortalité et ses facteurs prédictifs n'est pas significative ( $p>0.05$ ) (Tableau 2).

Tableau 2 : Magnitude du lien entre la mortalité et ses facteurs prédictifs dans le groupe de la fibrillation.

\begin{tabular}{lcc}
\hline $\begin{array}{l}\text { Facteurs prédictifs de la } \\
\text { mortalité dans le groupe } \\
\text { de la FA }\end{array}$ & Coefficient V de Cramer & $\mathbf{p}$ \\
\hline SCA droit & 0.236 & 0.212 \\
\hline Créatininémie & 0.120 & 0.53 \\
\hline Persistance du sus décalage & 0.343 & 0.07 \\
\hline Fibrillation ventriculaire & 0.236 & 0.212 \\
\hline
\end{tabular}

\section{DISCUSSION}

La fréquence : La fréquence de la fibrillation auriculaire dans notre étude était de $6 \%$, presque la même que celle rapportée dans la littérature (5 à $7 \%$ ). [2-4] Cette similarité peut être expliquée par la survenu précoce de la majorité de ces troubles et donc indépendamment de la thérapeutique.

La moyenne d'âge dans notre population était de 60 ans,

facteur favorisant la survenue de cette arythmie fortement liée à l'âge avancé.

Les facteurs prédictifs : Dans une étude publiée en 2012 [5], les facteurs prédictifs retrouvés étaient le tabac, la pression artérielle basse, la fréquence cardiaque élevée, le score de GRACE élevé, et le taux élevé de la troponine, mais les 4 derniers facteurs sont liés entre eux et reflètent l'état hémodynamique précaire, exprimé par un score de GRACE élevé.

Selon le registre ARIAM publié en 2013 [6], parmi les facteurs prédictifs retenus de la fibrillation auriculaire (FA) : l'âge, le sexe féminin, les antécédents de la FA, la fréquence cardiaque élevée à l'admission, et l'insuffisance cardiaque, de même les deux derniers facteurs étaient liés et reflétaient l'état hémodynamique altéré.
Dans une étude publiée en 2014 [7], les facteurs prédictifs retrouvés par Galvão Braga et al étaient l'âge, l'hypertension artérielle, les antécédents de valvulopathie ou de pontage coronaire, l'infection respiratoire, l'insuffisance cardiaque, le taux élevé des Pro-BNP et de la créatininémie, la fraction d'éjection basse, le diamètre large de l'oreillette gauche ; après l'analyse multi variée, les facteurs prédictifs indépendants retenus étaient l'âge, le diamètre large de l'oreillette gauche et la fraction d'éjection $\leq 40 \%$.

Selon une étude publiée en 2011 [8], l'insuffisance rénale est un facteur prédictif de la fibrillation auriculaire.

Dans cette étude et selon l'analyse uni variée, les facteurs statistiquement liés à la fibrillation auriculaire étaient l'âge $\geq$ 65 ans, le score de GRACE élevé, la surface de l'oreillette droite $\geq 12 \mathrm{~cm}^{2}$, l'insuffisance mitrale, le territoire droit du SCA, la créatininémie $\geq 14 \mathrm{mg} / \mathrm{l}$, la persistance de la douleur et du sus décalage après thrombolyse.

Les facteurs prédictifs indépendants retenus après analyse multi variée, étaient l'âge et le territoire droit du SCA, cette exclusion des facteurs sus cités est essentiellement liée à leur dépendance, l'âge, la créatininémie élevée sont inclus dans le score de GRACE, la surface de l'oreillette droite liée au territoire droit.

La mortalité : Selon une étude publiée en 2000, l'incidence de la mortalité hospitalière dans le groupe avec fibrillation auriculaire est de $25.3 \%$ vs $16 \%$ dans le groupe sans fibrillation auriculaire, selon cette étude et après l'analyse multi variée, la fibrillation auriculaire reste un facteur prédictif indépendant de la mortalité avec un Odds Ratio de 1.21 [9].

Alors que l'incidence de la mortalité hospitalière varie entre $8.8 \%$ et $14.5 \%$ selon les études les plus récentes.

Selon Galvão Braga et al, l'incidence de la mortalité hospitalière est de $8.8 \%$ dans le SCA compliqué de fibrillation auriculaire, alors qu'elle de $4.1 \%$ dans le groupe sans fibrillation auriculaire ( $p<0.044$; OR 3.1). [7]

Selon le registre ARIAM publié en 2014, l'incidence de la mortalité est de $14 \%$ dans le SCA compliqué de FA alors qu'elle est de $5.2 \%$ chez les patients sans FA. (p<0.001, HR 1.7). [6]

La fibrillation auriculaire augmente de façon significative le risque de la fibrillation ventriculaire, de la mort subite, du choc cardiogénique et de l'insuffisance cardiaque, alors que l'augmentation du risque thromboembolique n'est pas significative. [6]

La fibrillation auriculaire même transitoire, augmente le risque de son installation et des accidents thromboemboliques à moyen et long terme. [10].

Dans une étude prospective publiée en 2015, l'incidence de la mortalité hospitalière dans le SCA compliqué de FA est de $12.2 \%$. [11].

Dans notre étude, le taux de mortalité est élevé $14.28 \%$ dans le groupe avec fibrillation auriculaire vs $2.9 \%$ dans le groupe sans FA (HR : 5, IC95\% [1.65-15.58]. p = 0.004), de même pour le risque de la survenue de la FV est très élevé OR : 6.9, IC95\% [2.6-18.3], $\mathrm{P}=0.000$.

Ce taux élevé de la mortalité hospitalière précoce est lié aux facteurs suivants : le territoire droit du SCA (OR : 9.9), la créatininémie $\geq 17 \mathrm{mg} / \mathrm{l}$ (OR : 8), la persistance du sus décalage après la thrombolyse (OR : 34.6$)$, et la fibrillation ventriculaire (OR : 12), et donc liée à la stratégie thérapeutique, surtout que dans notre étude, rare sont les patients qui ont bénéficié de l'angioplastie primaire ou de sauvetage 


\section{CONCLUSION}

La fibrillation auriculaire est une complication rythmique fréquente, durant le syndrome coronarien aigu avec sus décalage du segment ST, ses deux facteurs prédictifs selon notre étude sont l'âge et le territoire droit du syndrome coronarien aigu, sa survenue augmente le risque de la mortalité hospitalière, ce risque élevé est en parti expliqué par la stratégie thérapeutique.

Remerciement: un remerciement à tout le personnel qui a participé à la réalisation de ce travail.

Déclaration d'intérêts : les auteurs ne déclarent aucun conflit d'intérêt en rapport avec cet article.

\section{RÉFÉRENCES}

1. Wang, Y.-M. Yang, J. Zhu. Mechanisms of new-onset atrial fibrillation complicating acute coronary syndrome. Herz March 2015, Volume 40, Issue 1, pp 18-26.

2. Renato D. Lopes, Laine E. Elliott, Harvey D. White, Judith S. Hochman, Frans Van de Werf, Diego Ardissino, et al. Antithrombotic therapy and outcomes of patients with atrial fibrillation following primary percutaneous coronary intervention: results from the APEX-AMI trial. European Heart Journal (2009) 30, 2019-2028.

3. F Pizzetti, F M Turazza,M G Franzosi, S Barlera, A Ledda, A P Maggioni, et al. Incidence and prognostic significance of atrial fibrillation in acute myocardial infarction: the GISSI-3 data. Heart 2001;86:527-532 527.
4. Anna Lisowsk, Agnieszka Tycińska, Małgorzata Knapp, Piotr Lisowski, Włodzimierz. Musiał The incidence and prognostic significance of cardiac arrhythmias and conduction abnormalities in patients with acute coronary syndromes and renal dysfunction. Kardiol Pol 2011; 69, 12: 1242-1247.

5. McManus DD1, Huang W, Domakonda KV, Ward J, Saczysnki JS, Gore JM et al. Trends in atrial fibrillation in patients hospitalized with an acute coronary syndrome. Am J Med. 2012 Nov;125(11) :1076-84.

6. Almendro-Delia M, Valle-Caballero MJ, Garcia-Rubira JC, Muñoz-Calero B, Garcia-Alcantara A, Reina-Toral A et al. Study Group. Prognostic impact of atrial fibrillation in acute coronary syndromes: results from the ARIAM registry. Eur Heart J Acute Cardiovasc Care. 2014 Jun;3(2):141-8.

7. Carlos Galvão Braga, Vítor Ramos, Catarina Vieira, Juliana Martins, Sílvia Ribeiro, António Gaspar, et al. New-onset atrial fibrillation during acute coronary syndromes: Predictors and prognosis. Rev Port Cardiol. 2014;33 (5):281---287.

8. Anna Lisowska, Agnieszka Tycinska, Malgorzata Knapp, Piotr Lisowski, Wlodzimierz J, Musial. The incidence and prognostic significance of cardiac arrhythmias and conduction abnormalities in patients with acute coronary syndromes and renal dysfunction. Kardiol Pol 2011; 69, 12:1242-1247.

9. Saif S. Rathore, Alan K. Berger, Kevin P. Weinfurt, Kevin A. Schulman, William J. Oetgen, Bernard J. Gersh, et al, Acute Myocardial Infarction Complicated by Atrial Fibrillation in the Elderly Prevalence and Outcomes. Circulation. 2000;101:969-974.

10. Schmitt J, Duray G, Gersh BJ, Hohnloser SH. Atrial fibrillation in acute myocardial infarction: a systematic review of the incidence, clinical features and prognostic implications. Eur Heart J. 2009;30:1038-45.

11. Karim Stamboul, Marianne Zeller, Laurent Fauchier, Aurélie Gudjoncik, Philippe Buffet, Fabien Garnier, et al Prognosis of silent atrial fibrillation after acute myocardial infarction at 1-year follow-up. Heart 2015;101:864869.

Cet article a été publié dans le « Batna Journal of Medical Sciences » BJMS, l'organe officiel de « l'association de la Recherche Pharmaceutique - Batna»

Le contenu de la Revue est ouvert « Open Access » et permet au lecteur de télécharger, d'utiliser le contenu dans un but personnel ou d'enseignement, sans demander l'autorisation de l'éditeur/auteur.

Avantages à publier dans BJMS :

- Open access : une fois publié, votre article est disponible gratuitement au téléchargement

- Soumission gratuite : pas de frais de soumission, contrairement à la plupart des revues « Open Access »

- Possibilité de publier dans 3 langues : français, anglais, arabe

- Qualité de la relecture : des relecteurs/reviewers indépendants géographiquement, respectant l'anonymat, pour garantir

la neutralité et la qualité des manuscrits.

Pour plus d'informations, contacter BatnaJMS@gmail.com ou connectez-vous sur le site de la revue : www.batnajms.net 


\section{Bis}

○ $\bigcirc$

-

$+2$

○

${ }^{1}$ Pole pharmacie, HMIMV-Rabat

${ }^{2}$ Faculté de médecine et de pharmacie-Rabat-Maroc

\section{Correspondance à :}

Fadoua BERDI

berdifadoua@gmail.com

DOI : https://doi.org/10.48087/ BJMSoa.2020.7204

\section{Historique de l'article :}

Reçu le 06 juin 2020

Accepté le 11sepembre 2020

Publié le 09 novembre 2020

Il s'agit d'un article en libre accès distribué selon les termes de la licence Creative Commons Attribution International License (CC BY 4.0), qui autorise une utilisation, une distribution et une reproduction sans restriction sur tout support ou format, à condition que l'auteur original et la revue soient dûment crédités.

Pour citer l'article :

Berdi F, Ifezouane J, Zhim I, Zakariya I, Lamsaouri J, Tadlaoui Y. Audit de la prise en charge de la douleur à l'Hôpital. Batna Med Sci 2020;7(2):84-6. https://doi.org/10.48087/ BJMSoa.2020.7204

\title{
Audit de la prise en charge de la douleur à l'Hôpital
}

\author{
Audit of Pain Management at the Hospital
}

\author{
Fadoua Berdi ${ }^{1,2}$, Jihane Ifezouane ${ }^{1,2}$, Imane Zhim ${ }^{1,2}$, Imane Zakariya ${ }^{1,2}$, Jamal \\ Lamsaouri $^{1,2}$, Yasmina Tadlaoui ${ }^{1,2}$
}

\section{RÉSUMÉ}

Introduction. La douleur est un motif de consultation très fréquent, chaque syndrome algique justifie des modalités particulières de prise en charge thérapeutique, selon son origine, ses caractéristiques et le terrain. L'objectif de l'étude est d'évaluer la prise en charge de la douleur à l'hôpital afin de déceler d'éventuelles déficiences. Matériel et méthodes. Un questionnaire a été destiné aux médecins de tous les services de l'hôpital. Les principaux thèmes étaient : le statut du médecin prescripteur, les méthodes et le temps consacré pour l'évaluation de la douleur, les antalgiques prescrits ainsi que les voies d'administration, la douleur chez l'enfant et enfin l'objectif de la prise en charge de la douleur. Résultats. On a reçu 100 réponses, avec 22\% généralistes, $40 \%$ résidents et $17 \%$ des professeurs, dont $37 \%$ ont reçu une formation sur la douleur. $62 \%$ ont un protocole de prise en charge de la douleur dans leurs services. $81 \%$ prescrivent un antalgique dès l'expression de la douleur par les patients. La moyenne du temps, pour évaluer la douleur a été de 5,82 minutes avec un écart type de 4,92. $52 \%$ utilisent la méthode EVA, 4\% I'EN, 66\% évaluent la douleur selon les plaintes des patients, 35\% selon les réactions des patients, $23 \%$ selon les mouvements des patients, $20 \%$ selon l'expression faciale des patients. $98 \%$ prescrivent le paracétamol en première intention, $45 \%$ de la morphine, $72 \%$ des AINS, $30 \%$ du tramadol et $24 \%$ utilisent en plus de ces analgésiques, d'autres molécules tel que le néfopam, la codéine. Les obstacles rencontrés lors de la prise en charge de la douleur sont $27 \%$ d'ordre économique, $24 \%$ dû à la non adhésion des patients, 36\% la non disponibilité de certains antalgiques. $89 \%$ estiment que le niveau de prise en charge de la douleur dans le service est excellent, contre $56 \%$ avec un niveau moyen et $8 \%$ un niveau insuffisant. $83 \%$ affirment que la prise en charge de la douleur est obligatoire et $82 \%$ faisant partie d'une assurance qualité des soins. Conclusion. La prise en charge de la douleur est pluridisciplinaire, les professionnels de santé doivent avoir une formation sur la nécessité de prise en charge de la douleur qui entre dans le cadre de l'assurance qualité des soins et devient obligatoire pour la prise en charge du patient.

Mots clés : la douleur, antalgiques, douleur aigue, douleur chronique, qualité des soins.

\section{INTRODUCTION}

La douleur est un motif de consultation fréquent, elle est définie par l'international Association for Study of Pain comme "Une expérience sensorielle et émotionnelle désagréable en relation avec une lésion tissulaire réelle ou potentielle, ou décrite en termes d'une telle lésion. " [1]. Cette définition est valable pour toutes les douleurs, et reflète bien la complexité du phénomène douloureux. La douleur est responsable de $20 \%$ de toutes les consultations en externe et $12 \%$ du total des prescriptions médicales [1]. Elle constitue un des symptômes

\section{ABSTRACT}

Background. Pain is a very frequent reason for consultation, each pain syndrome justifies specific methods of therapeutic management, depending on its origin, characteristics and field. The objective of the study is to assess the management of pain in the hospital in order to detect possible impairments. Material and methods. A questionnaire was sent to doctors from all departments of the hospital. The main themes were: the statute of the prescribing doctor, the methods and the time devoted to the evaluation of pain, the analgesics prescribed as well as the administration routes, pain in children and finally the objective of taking in charge of pain. Results. We received 100 responses, with $22 \%$ general practitioners, $40 \%$ residents and $17 \%$ teachers, $37 \%$ of whom received pain training. $62 \%$ have a pain management protocol in their services. $81 \%$ prescribe pain relievers as soon as patients express pain. The mean time to assess pain was 5.82 minutes with a standard deviation of $4.92 .52 \%$ use the EVA method, $4 \%$ the EN, $66 \%$ assess pain based on patient complaints, $35 \%$ based on patient feedback, $23 \%$ based on patient movement, $20 \%$ based on patient facial expression. $98 \%$ prescribe paracetamol as first line, $45 \%$ of morphine, $72 \%$ of NSAIDs, $30 \%$ of tramadol and $24 \%$ use in addition to these analgesics, other molecules such as nefopam, codeine. The obstacles encountered during the management of pain are $27 \%$ economic, $24 \%$ due to the non-adherence of patients, $36 \%$ the unavailability of certain analgesics. $89 \%$ consider that the level of pain management in the service is excellent, compared to $56 \%$ with an average level and $8 \%$ with an insufficient level. $83 \%$ say that pain management is mandatory and $82 \%$ are part of quality assurance of care. Conclusion. Pain management is multidisciplinary, health professionals must have training in the need for pain management, which is part of quality assurance of care and becomes compulsory for patient management.

Keywords: pain, analgesics, acute pain, chronic pain, quality of care.

médicaux les plus débilitants, ce qui justifie qu'elle est actuellement considérée comme le cinquième signe vital. Elle est considérée comme un problème majeur au niveau individuel mais également aux niveaux social et économique [2].

La prise en charge de la douleur est pluridisciplinaire, elle fait partie de la qualité des soins. Le but est d'identifier l'intensité, la localisation et l'étiologie de la douleur afin d'instituer au plus vite un traitement adapté. La douleur aigue est immédiate et brève, se caractérise par un début et une fin. 
Elle est dû à un excès de nociception et disparait avec la guérison de la lésion causale, comme exemples les douleurs dentaires, fracture ou une brûlure. La douleur chronique est une douleur-maladie, qui se caractérise par la persistance d'une durée $>6$ mois, même après guérison de la cause. Elle est plus complexe, car elle entraîne des perturbations physiques et psychiques, notamment un ralentissement locomoteur et intellectuel, une humeur dépressive et des troubles du sommeil, comme exemples, les douleurs dû à un cancer et les douleurs rhumatismales [3].

La démarche de la prise en charge, diffère en fonction du type de la douleur (aigue ou chronique). Pour les douleurs aigues, on doit agir rapidement pour soulager les douleurs intenses. Pour les douleurs chroniques, une évaluation plus poussée et une prise en charge multidimensionnelle seraient nécessaires [4]. Pour une prise en charge correcte de la douleur, on doit principalement réaliser un interrogatoire avec le patient ou sa famille surtout pour les enfants, les sujets âgées ou en déficience cognitive [5]. Puis une évaluation chiffrée de l'intensité de la douleur, celle-ci doit être faite à intervalle régulier afin d'apprécier le soulagement des patients et adapter le traitement le cas échéant. Après l'interrogatoire et l'évaluation de la douleur, un examen clinique s'impose et puis une décision thérapeutique sans oublier la réévaluation $d u$ traitement [6]. L'objectif de notre étude était d'évaluer le niveau de la prise en charge de la douleur par les médecins au sein de notre hôpital.

\section{MATÉRIELS ET MÉTHODES}

Un questionnaire sur l'évaluation de la prise en charge de la douleur, a été établi et destiné aux médecins de l'hôpital de tous les services des soins. 150 questionnaires ont été distribués. Cette enquête comportait 15 questions, réparties en 4 thèmes :

- La première partie comportait les renseignements sur le prescripteur, le statut du médecin, sa spécialité et son éventuelle formation sur la prise en charge de la douleur.

- La deuxième partie a été consacrée aux modalités de la prise en charge de la douleur, notamment le protocole adopté, les méthodes d'évaluation de la douleur avant de prescrire des antalgiques, le temps consacré pour son évaluation, les antalgiques prescrits ainsi que les voies d'administration.

- La troisième partie a été consacrée à la prise en charge de la douleur chez l'enfant, avec les méthodes utilisées afin d'évaluer la douleur.

- La quatrième partie comportait la qualité de prise en charge de la douleur, les obstacles rencontrés et l'estimation de la qualité de la prise en charge de la douleur selon les médecins.

L'étude statistique des résultats obtenus a été réalisée par le logiciel SPSS version 23.

\section{RÉSULTATS}

Sur 150 questionnaires distribués au niveau de tous les services de l'hôpital, 100 ont répondu, et donc un taux de réponse de $67 \%$. Les participants ont été répartis, entre $22 \%$ des généralistes, $40 \%$ des résidents de différentes spécialités et $17 \%$ des professeurs. Parmi les 100 participants, $37 \%$ ont déjà reçu une formation sur la douleur.

Concernant le protocole de prise en charge de la douleur, $62 \%$ des médecins participants affirment en avoir un, contre $38 \%$ qui n'ont pas un protocole validé dans leurs services. Pour la décision thérapeutique, On a constaté que $81 \%$ prescrivent un traitement antalgique dès l'expression de la douleur par les patients. La moyenne du temps estimé par les médecins afin d'évaluer la douleur a été de 5,82 minutes avec un écart type de 4,92. Conçernant les méthodes d'évaluation adoptées, $52 \%$ utilisent la méthode EVA, 4\% I'EN, 66\% évaluent la douleur selon les plaintes des patients, $35 \%$ selon les réactions des patients, $23 \%$ selon les mouvements des patients, $20 \%$ selon l'expression faciale des patients.

Plusieurs antalgiques sont prescrits à l'hôpital, d'après les résultats obtenus, on a constaté que $98 \%$ prescrivent le paracétamol en première intention, $45 \%$ de la morphine, $72 \%$ des AINS, $30 \%$ du tramadol et $24 \%$ utilisent en plus de ces analgésiques, d'autres molécules tel que le néfopam, la codéine.

Les voies d'administration les plus utilisées étaient comme suit : $97 \%$ la voie orale qui est utilisé en première intention, $47 \%$ la voie intraveineuse, et $15 \%$ utilisent aussi d'autres voies, tel que la voie sous cutanée, médullaire et des patchs surtout pour les douleurs cancéreuses. La totalité des médecins participants confirment avoir réévalué la douleur chez leurs patients.

80 médecins qui ont répondu à l'évaluation de la douleur chez l'enfant. Parmi lesquels, 32\% l'évaluent en entretenant un dialogue avec les parents de l'enfant, $56 \%$ en observant l'enfant, $43 \%$ en maintenant un dialogue avec l'enfant lui-même, et seulement $15 \%$ des médecins participants évaluent la douleur chez l'enfant selon les tests recommandés.

Les obstacles que les médecins rencontrent sont d'ordre économique dans $27 \%$ des cas, $24 \%$ estiment que l'obstacle majeur est la non adhésion des patients, 36\% la non disponibilité des antalgiques. $89 \%$ estiment que le niveau de prise en charge de la douleur dans leur service est excellent, $56 \%$ estiment un niveau moyen et $8 \%$ un niveau insuffisant de prise en charge de la douleur. L'intérêt de la prise en charge de la douleur est rapporté par tous les médecins participants à l'étude. $83 \%$ trouvent que la prise en charge de la douleur est obligatoire, $82 \%$ l'estiment faisant partie d'une assurance qualité des soins, $70 \%$ voient qu'elle est importante pour la prise en charge du patient et $45 \%$ suggère que la prise en charge de la douleur améliore la qualité des soins.

\section{DISCUSSION}

Les résultats de notre étude ont mis en place des points positifs et des points négatifs. Parmi les points positifs, on note l'estimation de l'intérêt de la prise en charge de la douleur vu par les médecins, $83 \%$ sont conscients que la prise en charge de la douleur est obligatoire et fait partie de la qualité des soins. $70 \%$ ont montré une bonne prise de conscience de l'importance de la prise en charge de la douleur pour le patient. En effet, une étude similaire a été réalisée dans des hôpitaux libanais, a montré aussi que l'intérêt de la prise en charge de la douleur a été rapporté par tous les médecins interrogés et tous la considèrent comme une obligation et faisant partie de la qualité des soins [7].

Il est bien évident qu'une bonne prise en charge de la douleur commence d'abord par l'évaluation de celle-ci [8]. La HAS a mis en évidence des outils d'évaluation, parmi lesquels [9] : L'échelle visuelle analogique, l'échelle numérique et l'échelle verbale simple, qui ont été validées pour mesurer l'intensité de la douleur. Elles n'apprécient pas les autres dimensions de la douleur et ne permettent pas de préciser le diagnostic des mécanismes sous-jacents. 
L'utilisation en pratique clinique quotidienne des mesures de l'intensité de la douleur est utile pour mieux détecter les malades ayant besoin d'un traitement symptomatique et les malades avec des douleurs chroniques nécessitant une réévaluation périodique.

$90 \%$ des médecins participant à notre étude appliquent ces recommandations et utilisent les différents outils décrits, pour évaluer les douleurs chez les patients.

Il n'y a pas de correspondance consensuelle entre les différents outils d'évaluation et les antalgiques utilisés. La HAS a établi une correspondance entre les outils d'auto-évaluation et l'intensité de la douleur, seuils d'intervention thérapeutique en auto et hétéro-évaluation.

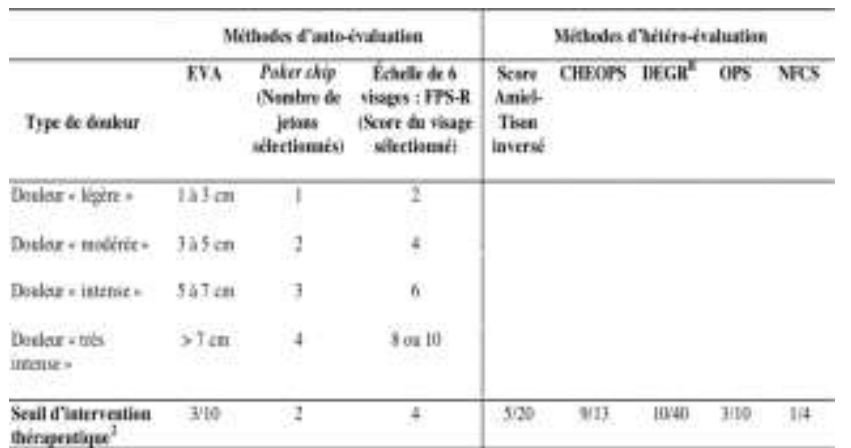

Figure 1. Correspondance entre outils d'évaluation et intervention thérapeutique [9].

Une étude traitant la douleur post opératoire, a montré que $100 \%$ des anesthésistes utilisent du paracétamol et $65 \%$ des AINS [10] La morphine est prescrite par $92 \%$ des anesthésistes par voie intraveineuse, par $8 \%$ per os, par $57 \%$ par voie souscutanée et par $65 \%$ des anesthésistes par voie médullaire. Des blocs nerveux périphériques sont réalisés par $60 \%$ des anesthésistes [11]. Les résultats de notre étude convergent vers ceux de la littérature.

En pédiatrie, il existe des recommandations de la HAS associant les niveaux de douleur au palier d'antalgiques à prescrire. Ces recommandations sont extrapolables à l'adulte. En effet, l'enfant perçoit la douleur comme tout le monde. Plusieurs travaux ont prouvé les effets délétères de l'absence de traitement ou de prévention de la douleur dans le développement neurosensoriel du bébé et dans son comportement futur face aux évènements de la vie et notamment face aux soins. L'enfant doit bénéficier d'une prise en charge de la douleur au même titre que tout individu quel que soit son âge et sa pathologie $[05,12]$.

Selon la HAS, les outils d'évaluation sont différents selon l'âge de l'enfant. Pour chaque classe d'âge, les mieux validés sont :

- Auto-évaluation : préconisée pour les enfants âgés de plus de 6 ans. Cette méthode a montré d'excellentes qualités métrologiques. Elle peut donc être utilisée en toute confiance, sous réserve d'explications adaptées au niveau de compréhension de l'enfant. Parmi les outils d'auto-évaluation, l'échelle visuelle analogique (EVA) qui est considérée comme l'outil de référence dans cette classe d'âge.

- EVA associée à un autre outil, tels que les jetons ou échelle de 6 visages: est préconisé chez les enfants de 4 à 6 ans. Si les scores obtenus par les 2 outils sont divergents, ils doivent être considérés comme non valides, c'est-à-dire non informatifs. Dès lors, seule l'hétéro-évaluation est possible.
- Hétéroévaluation: pour les enfants moins de 4 ans. L'évaluation de la douleur est plus complexe car elle repose principalement sur l'observation de son comportement.

Dans notre étude, Tous les médecins répondant à la question consacrée à la prise en charge de la douleur ont confirmé la nécessité de traiter la douleur chez un enfant.

Parmi les points négatifs relevées d'après notre étude, seulement $30 \%$ des médecins participant à l'enquête ont déjà suivi une formation sur la prise en charge de la douleur, et donc la notion de prise en charge de la douleur reste encore insuffisamment perçue par de nombreux médecins. Aussi, l'obstacle le plus rapporté par les médecins lors de notre enquête était la non disponibilité de certaines classes thérapeutiques des antalgiques, ceci peut être expliqué par le système d'approvisionnement de l'hôpital en produits de santé soumis au code des marchés publics et au budget limité alloué à la pharmacie.

\section{CONCLUSION}

Le point fort qui ressort de cette enquête est la prise de conscience de la prise en charge de la douleur au niveau des services. Les progrès qu'il faudrait accomplir sont la nécessité d'informer les professionnels de santé et d'amener l'établissement de santé à s'engager dans un programme de prise en charge institutionnelle de la douleur et d'avoir un comité de douleur à l'hôpital.

Déclaration d'intérêts: les auteurs ne déclarent aucun conflit d'intérêt en rapport avec cet article.

\section{RÉFÉRENCES}

1. Alford DP, et al. Update in pain medicine. J. Gen Intern Med 2008; 23(6):841-5.

2. Stewart WF et al. Lost productive time and cost due to common pain conditions in the US workforce. JAMA 2003; 290(18):2443-54.

3. Galinski M, Beaune S, Lapostolle F, Adnet F. Prise en charge de la douleur aiguë en urgence. EMC - Médecine d'urgence. 2015 ;10(3) (http://www.sciencedirect.com/science/article/pii/S19595182(15)59017-X)

4. Peter P. Pham, Michael P. Gaspar, Patrick M. Kane. Medical Management for Pain. In : De Terri M. Skirven, A. Lee Osterman, Jane Fedorczyk, Pete C. Amadio, Sheri Felder, Eon K Shin. Rehabilitation of the Hand and Upper Extremity, E-Book. Elsevier Health Sciences ; 14 janv. 2020. P1350-1353.

5. Prise en charge de la douleur aigue et chronique chez l'enfant. Recommandations de bonnes pratiques. ANSM. Juin 2009.

6. McGrath B el al. Thirty percent of patients have moderate to severe pain 24 hours after ambulatory surgery: a survey of 5703 patients. Can Journal Anaesthesie. 2004; 51:886-891.

7. Madi-Jebaran S et al. Enquête nationale sur la prise en charge de la douleur aiguë postopératoire dans les hôpitaux libanais. Annales Françaises d'Anesthésie et de Réanimation. 2009 ; 28(5):496-500.

8. Waljee JF et al. The use of opioid analgesics following common upper extremity surgical procedures: a national, population-based study. Plast Reconstr Surg. 2016;137(2):355e-364e.

9. HAS. Prise en charge de la douleur. Mis en ligne le 26 juin 2017

10. Apfelbaum JL et al. Postoperative pain experience: results from a national survey suggest postoperative pain continues to be undermanaged. Anesthesie Analg. 2003;97:534-540.

11. Rodgers J, Cunningham K, Fitzgerald K, Finnerty E. Opioid consumption following outpatient upper extremity surgery. Journal Hand Surg. 2012;37(4):645-650.

12. Annequin D, Ngo J. Évaluation et prise en charge de la douleur chez le nouveau-né, l'enfant et l'adolescent. EMC - Traité de Médecine Akos. 2018 ;13(4). 


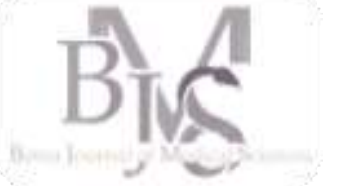

- $\bigcirc$

-

-

Service de physiologie clinique et explorations fonctionnelles métaboliques et nutrition $\mathrm{CHU}$ Benflis Touhami de Batna Algérie

Faculté de Médecine, Université de Batna 2 - Algérie

\section{Correspondance à :}

Mohamed Ridha Guedjati

m.guedjati@univ-batna2.dz

DOI : https://doi.org/10.48087/ BJMSoa.2020.7205

Historique de l'article :

Reçu le 11 juin 2020

Accepté le 10 septembre 2020

Publié le 09 novembre 2020

Il s'agit d'un article en libre accès distribué selon les termes de la licence Creative Commons Attribution International License (CC BY 4.0), qui autorise une utilisation, une distribution et une reproduction sans restriction sur tout support ou format, à condition que l'auteur original et la revue soient dûment crédités.

\section{Pour citer l'article :}

Guedjati MR, Boukebbal M, Gacem $\mathrm{H}$, et al. Effets des combinaisons glycémie 1 heure supérieure à 1,55 g/L avec des profils glycémiques normaux après une HGPO chez des femmes obèses.

Batna J Med Sci 2020;7 (2):87-91.

https://doi.org/10.48087/BI MSoa.2020.7205

Effets des combinaisons glycémie 1 heure supérieure à 1,55 g/L avec des profils glycémiques normaux après une HGPO chez des femmes obèses

\author{
Effects of 1 hour postload plasma glucose greater than $1.55 \mathrm{~g} / \mathrm{L}$ \\ combinations with normal glycemia profiles after an OGTT in obese \\ women \\ Mohamed Ridha Guedjati, Maamar Boukebbal, Hocine Gacem, Amel Ahmane, \\ Adeila Dallal Taibi, Ghania Hebboul, Dhikra Gasmi, Khaoula Lachekhab, Asma \\ Djebabra, Samira Benadjrouda
}

\section{RÉSUMÉ}

Introduction. Des études ont fait émerger l'existence de risque de prédiabète chez les patients qui ont une combinaison, profil glycémique normal et une glycémie à 1 heure supérieure à $1,55 \mathrm{~g} / \mathrm{L}$. Nous avons étudié les effets des combinaisons glycémie 1 heure (Gly $1 \mathrm{~h}$ ) après une hyperglycémie provoquée par voie orale HGPO avec un profil glycémique normal. Méthode. Une HGPO standardisée a été réalisée chez 100 femmes obèses. 46 avaient un profil glycémique normal. Une $\mathrm{GAJ}<1,00 \mathrm{~g} / \mathrm{L}$ et une glycémie à 2 heures, Gly $2 \mathrm{~h}<1,40 \mathrm{~g} / \mathrm{L}$. Elles étaient classées en deux groupes. Le groupe 1 (G1) ayant une GAJ $<1,00$ $\mathrm{g} / \mathrm{L}$, subdivisé en (G1a) ayant une Gly $1 \mathrm{~h}<1,55 \mathrm{~g} / \mathrm{L}$ et G1b ayant une Gly $1 \mathrm{~h} \geq$ à $1,55 \mathrm{~g} / \mathrm{L}$. Le groupe 2 (G2) avec une Gly $2 \mathrm{~h}<1,40 \mathrm{~g} / \mathrm{L}$, subdivisé en (G2a) ayant une Gly $1 \mathrm{~h}<1,55 \mathrm{~g} / \mathrm{L}$ et (G2b) ayant une Gly $1 \mathrm{~h} \geq 1,55$ $\mathrm{g} / \mathrm{L}$. Les tests de sensibilité - spécificité et l'Odds ratio ont été calculés à la recherche d'éventuelles corrélations. Résultats. Une corrélation très significative existait entre la Gly $1 \mathrm{~h} \geq 1,55 \mathrm{~g} / \mathrm{L}$ chez les sous-groupes G1b ( $\mathrm{n}=11, p=0,0001)$ et $\mathrm{G} 2 \mathrm{~b}(\mathrm{n}=11$, $p=0,0007$ ) et la valeur de $1,55 \mathrm{~g} / \mathrm{L}$. Cette valeur est hautement spécifique (spécificité 0,79 ) et moins sensible (sensibilité $0,64-0,54$ ). L'Odds ratio était de $[\mathrm{G} 1 \mathrm{~b}=5,85$ et $\mathrm{G} 2 \mathrm{~b}=3,41]$. Conclusion. La Gly $1 \mathrm{~h}$ à la valeur seuil de $1,55 \mathrm{~g} / \mathrm{L}$ peut mieux mettre en évidence des profils glycémiques à risque cardiométabolique.

Mots Clés. Glycémie à 1 heure, HGPO, tolérance normale au glucose, glycémie à jeun.

\section{INTRODUCTION}

Il est connu que les personnes qui ont une glycémie à jeun (GAJ) élevée et / ou une intolérance au glucose (ITG) présentent un risque accru de diabète de type 2 et de maladies cardiovasculaires (MCV). Le test d'hyperglycémie provoquée par voie orale (HGPO) est la méthode de diagnostic des différentes situations pathologiques [1]. Cependant, de nombreuses études longitudinales ont montré que certains individus qui développent un diabète de type 2 avaient une tolérance normale au glucose (TNG) au départ [2]. Par conséquent, il est essentiel d'identifier parmi les personnes qui ont un profil glycémique normal ceux qui peuvent encourir un risque cardiométabolique accru. Cette identification doit être précoce pour établir des programmes d'intervention précoce. Très récemment, un seuil de $1,55 \mathrm{~g} / \mathrm{L}$ de la glycémie après 1 heure (GLY $1 \mathrm{~h}$ ) après

\section{ABSTRACT}

Introduction. Studies have shown the existence of a risk of prediabetes in patients who have a combination, normal profile and one-hour glycemia level higher than $1.55 \mathrm{~g} / \mathrm{L}$. We studied the effects of 1-hour (1-h) post-load plasma glucose (PG) combinations with a normal profile after an oral glucose tolerance test OGTT. Method. A standardized OGTT was performed in 100 obese women. 46 had a normal profile. Fasting Glucose (FG) $<1.00 \mathrm{~g} / \mathrm{L}$ and 2 hours post-load plasma glucose (PG) 2 -hPG $<1.40$ $\mathrm{g} / \mathrm{L}$. They were classified into two groups. Group 1 (G1) having a FG $<1.00 \mathrm{~g} / \mathrm{L}$, subdivided into (G1a) having a $1-\mathrm{h} P \mathrm{PG}<1.55 \mathrm{~g} / \mathrm{L}$ and G1b having a $1-\mathrm{h} \mathrm{PG} \geq$ 1.55 g/L. Group 2 (G2) with a $2-\mathrm{h} P G<1.40 \mathrm{~g} / \mathrm{L}$, subdivided into (G2a) having a 1-h PG $<1.55 \mathrm{~g} / \mathrm{L}$ and (G2b) having a 1-h PG $\geq 1.55 \mathrm{~g} / \mathrm{L}$. The sensitivity specificity tests and the Odds ratio were calculated in search of possible correlations. Results. A very significant correlation existed between $1-\mathrm{h} \geq 1.55 \mathrm{~g} / \mathrm{L}$ in the subgroups G1b ( $\mathrm{n}=11, p=0.0001)$ and $\mathrm{G} 2 \mathrm{~b}$ $(\mathrm{n}=11, p=0.0007)$ and the value of $1.55 \mathrm{~g} / \mathrm{L}$. This value is highly specific (specificity 0.79 ) and less sensitive (sensitivity G1b=0.64 and G2b $=0.54$ ). The Odds ratio was $[\mathrm{G} 1 \mathrm{~b}=5.85$ and $\mathrm{G} 2 \mathrm{~b}=3.41]$. Conclusion. The 1 hour (1-h) post-load plasma glucose at the threshold value of $1.55 \mathrm{~g} / \mathrm{L}$ can be proposed for performing the cardiometabolic risk in normal profiles.

Key words: 1 hour glucose, OGTT, normal glucose tolerance, fasting glucose

une HGPO, permet d'identifier les sujets avec une tolérance normale du glucose (TNG) et à haut risque de diabète de type 2 futur [3-5].

Comme il a été prouvé, chez cette même catégorie (TNG), qu'une Gly $1 \mathrm{~h} \geqslant 1,55 \mathrm{~g} / \mathrm{L}$ est fortement associée à des profils de risque cardiométaboliques élevés tels que la dyslipidémie [6], l’hyperuricémie [7] et les lésions infracliniques des organes cibles, y compris l'athérosclérose carotidienne précoce [8], l'hypertrophie ventriculaire gauche [9] et la stéatose hépatique non alcoolique [10].

De plus, il a été démontré qu'un niveau élevé de la Gly $1 \mathrm{~h} \geqslant 1,55 \mathrm{~g} / \mathrm{L}$ est associé à un risque accru de MCV et de mortalité. De même, une Gly $1 \mathrm{~h} \geqslant 1,55 \mathrm{~g} / \mathrm{L}$ est plus prédictif de la morbidité cardiovasculaire que la glycémie 2 heures (Gly 2 h) après une HGPO [11-14]. Dans l'étude de Reykjavik, un niveau élevé de la Gly $1 \mathrm{~h}$ était associé à un risque plus élevé de maladie coronarienne chez les personnes non diabétiques [15]. 


\section{Objectif}

Étudier les effets des combinaisons entre la glycémie $1 \mathrm{~h}$ $\geq 1,55 \mathrm{~g} / \mathrm{L}$ après un test d'hyperglycémie provoquée par voie orale (HGPO) avec la glycémie à T0 (GAJ) inférieure à 1,00 $\mathrm{g} / \mathrm{L}$ et la glycémie à 120 minutes (Gly $2 \mathrm{~h}$ ) inférieure à 1,40 $\mathrm{g} / \mathrm{L}$ chez des femmes obèses.

\section{MATÉRIELS ET MÉTHODES}

\section{1- Sujets d'étude et conception}

Les patientes sont prises en charge pour leur obésité (IMC $\geq$ $30 \mathrm{Kg} / \mathrm{m}^{2}$ ). Une HGPO a été indiquée chez chacune d'elles dans le cadre de cette prise en charge ceci selon les critères de l'American Diabetes Association (ADA) [16]. Elles ont été recrutées de façon prospective, de janvier à décembre 2018, afin de subir un test d'HGPO standardisé de 2 heures selon les critères OMS. Le profil glycémique normal a été défini sur la base des critères de l'ADA [16]; Une glycémie à jeun (GAJ) $<1,00 \mathrm{~g} / \mathrm{L}$, une glycémie après 2 heures (Gly $2 \mathrm{~h}$ ) $<1,40 \mathrm{~g} / \mathrm{L}$. Les critères d'exclusion étaient les suivants : un IMC $<30$ $\mathrm{Kg} / \mathrm{m}^{2}$, un diabète sucré, une grossesse, un traumatisme récent, un cancer, une cardiomyopathie, une cardiopathie rhumatismale, une insuffisance cardiaque sévère, une maladie pulmonaire chronique, une maladie hépatique ou rénale, une maladie immunitaire, une maladie gastrointestinale chronique, une pancréatite chronique, des antécédents de maladie maligne ou des antécédents de traitements altérant le métabolisme du glucose.

Le seuil de la Gly $1 \mathrm{~h}$ a été considérée à la valeur de 1,55 g/L. Les patientes incluses $(n=100)$ ont été divisées en deux groupes pour l'analyse des données après avoir donné leur consentement éclairé. Le premier groupe (G1) concernait les femmes ayant une glycémie à T0 de l'HGPO (GAJ < 1,00 g/L). Ce groupe a été subdivisé en deux sous-groupes, $(G 1 a)$ dont les sujets ont une Gly $1 \mathrm{~h}<1,55 \mathrm{~g} / \mathrm{L}$ et $(G 1 b)$ dont les sujets ont une Gly $1 \mathrm{~h} \geq 1,55 \mathrm{~g} / \mathrm{L}$. Le deuxième groupe (G2) qui concernait les femmes ayant, à T120 de l'HGPO, une Gly $2 \mathrm{~h}$ $<1,40 \mathrm{~g} / \mathrm{L}$. De même, ce groupe a été subdivisé en deux sousgroupes, $(G 2 a)$ dont les sujets ont une Gly $1 \mathrm{~h}<1,55 \mathrm{~g} / \mathrm{L}$ et (G2b) dont les sujets ont une Gly $1 \mathrm{~h} \geq 1,55 \mathrm{~g} / \mathrm{L}$.

\section{2- Tests de laboratoire}

Les données du laboratoire ont été obtenues à partir d'échantillons de sang veineux prélevés lors d'une hospitalisation de jour. Les prélèvements ont été effectués chez des sujets à jeun d'au moins 12 heures et au repos. Une HGPO de $75 \mathrm{~g}$ a été réalisée avec un échantillonnage de 0,60 , 120 et 180 min pour la glycémie [16].

\section{3- L'analyse statistique}

Les variables continues ont été exprimées en tant que valeurs moyennes et écart-type pour les données normalement distribuées. Les variables catégorielles ont été exprimées en fréquences. Pour les comparaisons statistiques, un test $t$ de Student a été utilisé pour comparer les valeurs de la Gly $1 \mathrm{~h}$ à la valeur fixe de $1,55 \mathrm{~g} / \mathrm{L}$. Le test du chi carré a été utilisé pour les variables catégorielles. Dans cette analyse statistique il a été réalisé une évaluation, de la valeur prédictive d'un test diagnostic. L'intensité de liaison entre deux variables a été vérifiée par le coefficient de Youle. De même il a été réalisé une évaluation, du risque relatif, de l'Odds ratio avec des intervalles de confiance (IC à $95 \%$ ). Le $p$-value a été considérée comme statistiquement significatif à une valeur $<0,05$. Toutes les analyses ont été effectuées à l'aide du logiciel SPSS version 20.0 pour Windows.

\section{RÉSULTATS}

\section{1- Les caractéristiques de base}

Les sujets de l'étude étaient composés de 100 femmes dont l'âge moyen était de 44,13 $\pm 12,15$ ans et toutes obèses avec un IMC moyen de 36,84 $\pm 6,06$ (tableau1).

Tableau 1. Caractéristiques générales de la population d'étude $(n=100)$

\begin{tabular}{lcc}
\hline & Moyenne & Écart-type \\
\hline Age (ans) & 44,13 & 12,15 \\
\hline Poids (Kg) & 91,85 & 16,59 \\
\hline Taille (cm) & 157,77 & 6,12 \\
\hline IMC (Kg/m2) & 36,84 & 6,06 \\
\hline Masse grasse (Kg) & 41,16 & 8,84 \\
\hline Masse maigre (Kg) & 50,77 & 4,83 \\
\hline Graisse viscérale & 10,66 & 3,02 \\
\hline
\end{tabular}

La valeur seuil de la Gly $1 \mathrm{~h}$ à 1,55 g/L pendant l'HGPO a été utilisée pour diviser les sujets en 4 sous-groupes. Les sousgroupes G1a et G2a représentent (35\%) chacun, les sousgroupes $\mathrm{G} 1 \mathrm{~b}$ et $\mathrm{G} 2 \mathrm{~b}$ représentent $(11 \%)$ chacun. Les caractéristiques de base des patientes des différents groupes sont présentées dans le (tableau 2).

Tableau 2. Combinaison Gly 1h-GlyT0 et Gly $1 \mathrm{~h}$-Gly 120

Gly $1<1,55 \mathrm{~g} /$

Gly $1 \geq 1,55 \mathrm{~g} / \mathrm{L}$

$\begin{array}{llll}\text { Nombre } & \text { Test } t \text { de Student } & \text { Nombre } & \text { Test } t \text { de Student }\end{array}$

HGPO (g/L)

\begin{tabular}{|c|c|c|c|c|c|c|}
\hline Gly T0 & $<1$ & 35 (G1a) & NS & 11 (G1b) & $P=0.0001$ & 46 (G1) \\
\hline$(1,02 \pm 0,15)$ & $\geq 1$ & 19 & NS & 35 & NS & 54 \\
\hline$(1,33 \pm 0,36)$ & $\geq 1,40$ & 26 & NS & 28 & NS & 54 \\
\hline
\end{tabular}




\section{2- Les caractéristiques spécifiques}

Un lien, statistiquement très significatif, a été démontré si on rapportait les Gly $1 \mathrm{~h} \geqslant 1,55 \mathrm{~g} / \mathrm{L}$ des patientes des sousgroupes [G1b $(p=0,0001)$ et $\mathrm{G} 2 \mathrm{~b}(p=0,0007)]$ à la valeur fixe de 1,55. Â l'inverse, aucun lien n'a pu être démontré chez les autres sous-groupes avec une Gly $1 \mathrm{~h}<1,55 \mathrm{~g} / \mathrm{L}$ (tableau 2).

L'évaluation de cette valeur prédictive semble être mieux corrélée à l'éventuel désordre glycémique chez le groupe G1b $\left(\mathrm{Khi}^{2}=16,73, p \leq 0,001\right)$ que chez le groupe G2b $\left(\mathrm{Khi}^{2}=8,15\right.$ $p \leq 0,01)$. Quel que soit le sous-groupe, la valeur prédictive de 1,55 est statistiquement significative.
La valeur de 1,55 est hautement spécifique aussi bien chez G1b et G2b avec une spécificité de 79,09. Cependant cette valeur est moins sensible chez le sous-groupe G2b (sensibilité $=54,85$ ) que chez le sous-groupe G2a (sensibilité $=64,81$ ). De même cette valeur est hautement prédictive chez les deux sous-groupes. La VPP (valeur prédictive positive) est respectivement de 76,09 pour G1b et 71,79 pour G2b. La VPN (valeur prédictive négative) était de 64,81 pour G1b et de 51,38 pour G2b. De plus une forte liaison a été trouvée entre la valeur prédictive et l'éventuelle existence d'un désordre métabolique type prédiabète. Le cœfficient $\mathrm{Q}$ de Youle était de 0,71 pour G1b et de 0,55 pour G2b (tableau 3).

Tableau 3. Performance de la Gly $1 \mathrm{~h}$ : Tests de sensibilité - spécificité et valeurs prédictives

\begin{tabular}{|c|c|c|c|}
\hline & Groupe 1 GLY TO & Groupe 2 GLY T120 & Interprétation \\
\hline$\%$ de sensibilité & 64,81 & 51,85 & +/- sensible \\
\hline$\%$ de spécificité & 76,09 & 76,09 & Hautement spécifique \\
\hline$\%$ valeur prédictive positive & 76,09 & 71,79 & Hautement prédictive \\
\hline$\%$ valeur prédictive négative & 64,81 & 51,38 & Moyennement prédictive \\
\hline Taux de la maladie chez l'ensemble de la population & 54 & 54 & \\
\hline Taux de la positivité du signe testé chez la population & 46 & 39 & \\
\hline Indice de Youden & 0,41 & 0,28 & Modérément efficace \\
\hline $\mathrm{Khi}^{2}$ & $\begin{array}{c}16,73 \\
(p \leq 0,001)\end{array}$ & $\begin{array}{c}8,15 \\
(p \leq 0,01)\end{array}$ & Liaison significative \\
\hline Coefficient de Q de Yule & 0,71 & 0,55 & $\begin{array}{l}\text { Lien très fort ou fort entre la } \\
\text { valeur prédictive et la survenu } \\
\text { du prédiabète }\end{array}$ \\
\hline
\end{tabular}

L'évaluation de l'indicateur de risque et la liaison entre facteur d'exposition et le désordre a ramené une prévalence chez la population d'étude de $54 \%$ avec une borne inférieure de l'indice de confiance (IC $95 \%$ ) de 44,23 et une borne supérieure de 63,77. Dans cette analyse (tableau 4), le rapport des cotes (Odds ratio) était significatif chez les deux groupes (G1b: Odds ratio $=5,85$ vs $G 2 b$ : Odds ratio=3,41). L'indice de confiance (IC à 95\%) avait comme borne inférieure 2,44 pour G1b vs 1,41 pour G2b; la borne supérieure était de 14,16 pour G1 vs 7,99 pour G2b). Le risque relatif (RR) était de 2,16 (G1b) et de 1,68 (G2b). L'indice de confiance (IC à $95 \%$ ) avait comme borne inférieure de 44,23 et celle supérieure de 63,77.

Tableau 4. Analyse multivariée par régression logistique Risque relatif et Odds ratio

\begin{tabular}{lcc}
\hline Écart réduit & $\begin{array}{c}\text { Groupe 1 } \\
\text { GLY T0 }\end{array}$ & $\begin{array}{c}\text { Groupe 2 } \\
\text { GLY T120 }\end{array}$ \\
\hline Prévalence & $\begin{array}{c}4,09 \\
(p \leq 0,0001)\end{array}$ & $\begin{array}{c}2,85 \\
(p \leq 0,01)\end{array}$ \\
\hline IC à 95\% (borne inférieure) & 54 & 54 \\
\hline IC à 95 \% (borne supérieure) & 44,23 & 44,23 \\
\hline Risque attribuable (RA) \% & 63,77 & 63,77 \\
\hline Risque relatif (RR) & 40,9 & 29,17 \\
\hline Odds Ratio (OR) & $2,16^{*}$ & $1,68^{*}$ \\
\hline IC selon Woolf (borne inférieure) & $5,85^{*}$ & $3,43^{*}$ \\
\hline IC selon Woolf (borne supérieure) & 2,44 & 14,45 \\
\hline $\begin{array}{l}\text { IC selon OR Miettinen } \\
\text { (borne inférieure) }\end{array}$ & 2,51 & 8,13 \\
\hline $\begin{array}{l}\text { IC selon OR Miettinen } \\
\text { (borne supérieure) }\end{array}$ & 13,47 \\
\hline $\begin{array}{l}* \\
\text { malaélation significative entre la variable Gly } 1 \mathrm{~h} \geq 1,55 \mathrm{~g} / \mathrm{L} \text { et la survenue de la }\end{array}$ & & \\
\hline
\end{tabular}

\section{DISCUSSION}

Cette étude de cas témoins, menée chez des patientes obèses à risque cardiométabolique avec profil glycémique normal, a montré que des taux élevés de Gly $1 \mathrm{~h}$ après un test d'HGPO, étaient associés à un risque élevé de développer des désordres glycémiques. Les analyses menées chez des sous-groupes avec une GAJ (T0 de l'HGPO) ou bien de la Gly 2h (T120 de l'HGPO) ont montré que la valeur seuil de 1,55 g/L de la Gly $1 \mathrm{~h}$ est fortement liée au risque de développer ces désordres. De plus en plus de preuves ont montré que des sujets ayant une TNG sont à haut risque non seulement de diabète de type 2 mais également de MCV, car le diabète de type 2 et les MCV peuvent avoir des origines métaboliques communes [1718]. Abdul-Ghani et al ont démontré qu'une Gly1 $\mathrm{h} \geqslant 1,55$ $\mathrm{g} / \mathrm{L}$ suite à une HGPO est un meilleur paramètre prédictif du futur diabète de type 2 que la GAJ ou la Gly 2 h chez les sujets sans désordres glycémiques [3-5].

La combinaison d'une Gly $1 \mathrm{~h} \geqslant 1,55 \mathrm{~g} / \mathrm{L}$ à une GAJ normale dans notre étude semble être fortement liée (spécificité de $79 \%$, sensibilité de 64,81\%). Dans une étude Indienne, cette combinaison était proche (spécificité de 66\%, sensibilité de $64 \%$ ) pour une combinaison de la Gly $1 \mathrm{~h}$ $\geqslant 1,53 \mathrm{~g} / \mathrm{L}$ et la GAJ normale [19]. L'étude récente de Peddinti et al avait conclu à une sensibilité de $77 \%$ et une spécificité de $69 \%$ [20]. De plus, il a été démontré que les sujets avec TNG ayant une Gly $1 \mathrm{~h} \geqslant 1,55 \mathrm{~g} / \mathrm{L}$ présentent des désordres métaboliques et des lésions infracliniques des organes cibles, similaires aux individus ayant une intolérance au glucose (IG), eux-mêmes sont considérés à 
haut risque à la fois pour le diabète de type 2 et les MCV. Il convient de noter que des études de population à grande échelle ont suggéré qu'un niveau élevé de la Gly $1 \mathrm{~h}$ est fortement associé à un risque élevé de mortalité cardiovasculaire chez les personnes sans diabète au départ lors d'un suivi à long terme [11,14].

Abdul-Ghani et al [21] avait trouvé pour des combinaisons graduelles de la GAJ - Gly $1 \mathrm{~h}(\mathrm{GAJ}<0,9 \mathrm{~g} / \mathrm{L}-$ Gly $1 \mathrm{~h}>1,50 \mathrm{~g} / \mathrm{L}$; $0,90 \mathrm{~g} / \mathrm{L}<\mathrm{GAJ}<1,00 \mathrm{~g} / \mathrm{L}-$ Gly $1 \mathrm{~h}>1,50 \mathrm{~g} / \mathrm{L} ; \mathrm{GAJ}>1,00 \mathrm{~g} / \mathrm{L}-$ Gly $1 \mathrm{~h}>1,50 \mathrm{~g} / \mathrm{L}$ ) que l'OR (Odds Ratio) avec un IC $95 \%$ était dans l'ordre $[\mathrm{OR}=7,1(3,3-17,0)$; $\mathrm{OR}=11,3(5,0-25,8)$; $\mathrm{OR}=17(7,5-41,9)]$. Dans notre étude la combinaison $\mathrm{GAJ}<1,0$ $\mathrm{g} / \mathrm{L}$ - Gly $1 \mathrm{~h} \geq 1,55 \mathrm{~g} / \mathrm{L}$, a ramené un OR IC 95\% de 5,85 [2,44 - 14,16]. Il semblerait que la combinaison GAJ-Gly $1 \mathrm{~h}$ est mieux corrélée $\left(\mathrm{Khi}^{2}=16,73, p \leq 0,001\right)$ que celle TNG-Gly $1 \mathrm{~h}$ $\left(\mathrm{Khi}^{2}=8,15 p \leq 0,01\right)$. De même, Bergman [22] pour une combinaison TNG-Gly1h $\geq 1,55 \mathrm{~g} / \mathrm{L}$ avait démontré un OR IC $95 \%$ de $4,35[2,50-7,73]$, pour la meme combinaison dans notre étude OR IC $95 \%$ était de 3,41[1,41-7,99]. Les résultats présentés ici sont cohérents avec des observations précédentes qui montrent bien que les sujets NGT avec une Gly1 $\mathrm{h} \geqslant 1,55 \mathrm{~g} / \mathrm{L}$ présentaient une situation intermédiaire d'intolérance au glucose caractérisée par une résistance à l'insuline et un dysfonctionnement des cellules $\beta$ [23,24]. Il est proposé que la Gly $1 \mathrm{~h} \geqslant 1,55 \mathrm{~g} / \mathrm{L}$ puisse représenter une forme d'état prédiabétique et une pétition a été publiée dans ce sens [25]. Il a été observé chez des sujets avec une Gly $1 \mathrm{~h}$ $\geqslant 1,55 \mathrm{~g} / \mathrm{L}$ qu'ils présentaient un HOMA-IR plus élevé et des niveaux d'ISI plus bas, indiquant un niveau plus élevé d'une résistance à l'insuline [26]. Les auteurs suggèrent que la résistance à l'insuline est responsable de l'association d'une $\mathrm{Gly} 1 \mathrm{~h} \geqslant 1,55 \mathrm{~g} / \mathrm{L}$ aux maladies coronariennes. Le mécanisme par lequel des taux élevés de la Gly $1 \mathrm{~h}$ sont associés à une maladie coronarienne n'est pas défini [26]. Une étude plus approfondie qui traitera la combinaison de la Gly1h $\geqslant 1,55$ $\mathrm{g} / \mathrm{L}$ à des aspects comme la résistance à l'insuline, la maladie coronarienne et les facteurs inflammatoires, est plus que souhaitable. De ce fait, il a été observé des niveaux accrus de hs-CRP, de globules blancs et de neutrophiles chez des sujets avec une Gly1h $\geqslant 1,55 \mathrm{~g} / \mathrm{L}$. Il semblerait que ces signes infracliniques sont liés aux processus inflammations [27]. Cependant, les mécanismes potentiels doivent être mieux explorés par d'autres investigations.

Plusieurs limites doivent être considérées dans cette étude. Tout d'abord, une unique HGPO utilisée pour mesurer les seuils des Glycémies à une heure, ne peut être, en elle seule, totalement concluante. Il a été rapporté que les niveaux de la Gly $1 \mathrm{~h}$ après l'HGPO ont une variabilité intra et interindividuelle et parfois des valeurs faussement élevées $[28,29]$ ce qui peut induire une certaine imprécision dans la classification des sujets cela même peut affecter les résultats. Des études antérieures ont suggéré que les indices qui utilisent plusieurs points temporels d'une HGPO étaient plus reproductibles [29]. Des comparaisons de la reproductibilité entre la Gly 1 h, la Gly 2 h et d'autres indices dérivés de l'HGPO devraient être effectuées dans l'avenir. Deuxièmement, les résultats sont observés chez des femmes et des résultats différents pourraient être obtenus chez des hommes. Troisièmement, la conception de l'étude n'élimine pas les relations causales et potentielles entre les niveaux de Gly $1 \mathrm{~h}$ et les autres facteurs (Poids, IMC, composition corporelle en masse grasse, sédentarité, alimentation...) Enfin, il s'agit d'une étude à faible échantillon, des études multicentriques longitudinales dans une population à grande échelle sont nécessaires. Par conséquent, elles permettront un suivi pronostic à long terme des sujets, chez qui il y a association Gly $1 \mathrm{~h} \geqslant 1,55 \mathrm{~g} / \mathrm{L}$ et GAJ normale ou encore ceux qui ont une TNG combinée à une Gly $1 \mathrm{~h} \geqslant 1,55$ g/L.

\section{CONCLUSION}

Cette étude a montré des liens étroits entre des niveaux élevés de Gly $1 \mathrm{~h}$ et des valeurs normales de la glycémie à jeun ou celles d'une Gly $2 \mathrm{~h}$ suite à une HGPO standardisée. Les résultats sont pertinents à la lumière de précédentes observations qui mettent l'accent sur le rôle de la Gly $1 \mathrm{~h}$ dans l'identification précoce des personnes ayant une GAJ et/ou une TNG normales comme sujets à haut risque cardiométabolique. Étant donné que l'atteinte des organes cibles peut être infraclinique, il est suggéré de prêter attention particulière à la glycémie à $1 \mathrm{~h}$ après un test d'HGPO. La valeur $1,55 \mathrm{~g} / \mathrm{L}$ peut être proposée afin que les individus, avec GAJ normale et/ou qui ont une TNG, puissent bénéficier d'une prise en charge précoce, y compris sur le mode de vie tel que l'exercice physique ou encore l'alimentation.

Déclaration d'intérêts : les auteurs ne déclarent aucun conflit d'intérêt en rapport avec cet article.

\section{RÉFÉRENCES}

1. Nathan D, Davidson M, DeFronzo R, Heine R, Henry R, Pratley R et al. Impaired Fasting Glucose and Impaired Glucose Tolerance: Implications for care. Diabetes Care. 2007;30(3):753-759.

2. Unwin N, Shaw J, Zimmet P, Alberti K. Impaired glucose tolerance and impaired fasting glycaemia: the current status on definition and intervention. Diabetic Medicine. 2002;19(9):708-723.

3. Abdul-Ghani M, Abdul-Ghani T, Ali N, DeFronzo R. One-Hour Plasma Glucose Concentration and the Metabolic Syndrome Identify Subjects at High Risk for Future Type 2 Diabetes. Diabetes Care. 2008;31(8):1650 1655.

4. Abdul-Ghani M, Lyssenko V, Tuomi T, DeFronzo R, Groop L. Fasting Versus Postload Plasma Glucose Concentration and the Risk for Future Type 2 Diabetes: Results from the Botnia Study. Diabetes Care. 2008;32(2):281-286

5. Abdul-Ghani M, Williams K, DeFronzo R, Stern M. What Is the Best Predictor of Future Type 2 Diabetes?. Diabetes Care. 2007;30(6):15441548.

6. Shimodaira M, Niwa T, Nakajima K, Kobayashi M, Hanyu N, Nakayama T Correlation between serum lipids and 1-hour postload plasma glucose levels in normoglycemic individuals. Journal of Clinical Lipidology. 2014;8(2):217-222.

7. Perticone F, Sciacqua A, Perticone M, Arturi F, Scarpino P, Quero M et al. Serum Uric Acid and 1-h Postload Glucose in Essential Hypertension. Diabetes Care. 2011;35(1):153-157.

8. Succurro E, Marini M, Arturi F, Grembiale A, Lugarà M, Andreozzi F et al. Elevated one-hour post-load plasma glucose levels identifies subjects with normal glucose tolerance but early carotid atherosclerosis. Atherosclerosis. 2009;207(1):245-249.

9. Sciacqua A, Miceli S, Carullo G, Greco L, Succurro E, Arturi F et al. OneHour Postload Plasma Glucose Levels and Left Ventricular Mass in Hypertensive Patients. Diabetes Care. 2011;34(6):1406-1411.

10. Sesti G, Hribal M, Fiorentino T, Sciacqua A, Perticone F. Elevated $1 \mathrm{~h}$ postload plasma glucose levels identify adults with normal glucose tolerance but increased risk of non-alcoholic fatty liver disease. BM] Open Diabetes Research \& Care. 2014;2(1):e000016.

11. Vaccaro O, Ruth K, Stamler J. Relationship of Postload Plasma Glucose to Mortality With 19-Yr Follow-Up. Comparison of one versus two plasma glucose measurements in the Chicago Peoples Gas Company Study. Diabetes Care. 1992;15(10):1328-1334.

12. Orencia A, Daviglus M, Dyer A, Walsh M, Greenland P, Stamler J. OneHour Postload Plasma Glucose and Risks of Fatal Coronary Heart Disease and Stroke among Nondiabetic Men and Women: The Chicago Heart Association Detection Project in Industry (CHA) Study. Journal of Clinical Epidemiology. 1997;50(12):1369-1376. 
13. Bergman M, Chetrit A, Roth J, Dankner R. One-hour post-load plasma glucose level during the OGTT predicts mortality: observations from the Israel Study of Glucose Intolerance, Obesity and Hypertension. Diabetic Medicine. 2016;33(8):1060-1066.

14. Bardini G, Dicembrini I, Cresci B, Rotella C. Inflammation Markers and Metabolic Characteristics of Subjects With 1-h Plasma Glucose Levels. Diabetes Care. 2009;33(2):411-413.

15. Manco M, Panunzi S, Macfarlane D, Golay A, Melander O, Konrad T et al. One-Hour Plasma Glucose Identifies Insulin Resistance and -Cell Dysfunction in Individuals With Normal Glucose Tolerance: Crosssectional data from the Relationship between Insulin Sensitivity and Cardiovascular Risk (RISC) study. Diabetes Care. 2010;33(9):20902097.

16. Bergman M, Manco M, Sesti G, Dankner R, Pareek M, Jagannathan R et al. Petition to replace current OGTT criteria for diagnosing prediabetes with the 1-hour post-load plasma glucose $\geq 155 \mathrm{mg} / \mathrm{dl}(8.6 \mathrm{mmol} / \mathrm{L})$. Diabetes Research and Clinical Practice. 2018;146:18-33.

17. Cao L, Wang P, Luan H, Chen H, Luo C, Zhu D et al. Elevated 1-h postload plasma glucose levels identify coronary heart disease patients with greater severity of coronary artery lesions and higher risk of 1-year re-admission. Diabetes and Vascular Disease Research. 2020;17(1):147916411989697.

18. Ikonomidis I, Stamatelopoulos K, Lekakis J, Vamvakou G, Kremastinos D. Inflammatory and non-invasive vascular markers: The multimarker approach for risk stratification in coronary artery disease. Atherosclerosis. 2008;199(1):3-11.

19. Rushforth N, Bennett P, Steinberg A, Miller M. Comparison of the Value of the Two- and One-hour Glucose Levels of the Oral GTT in the Diagnosis of Diabetes in Pima Indians. Diabetes. 1975;24(6):538-546.

20. Utzschneider K, Prigeon R, Tong J, Gerchman F, Carr D, Zraika S et al. Within-subject variability of measures of beta cell function derived from a 2 h OGTT: implications for research studies. Diabetologia. 2007;50(12):2516-2525.
21. Bergman M, Chetrit A, Roth J, Dankner R. One-hour post-load plasma glucose level during the OGTT predicts mortality: observations from the Israel Study of Glucose Intolerance, Obesity and Hypertension. Diabetic Medicine. 2016;33(8):1060-1066.

22. Pareek M, Bhatt D, Nielsen M, Jagannathan R, Eriksson K, Nilsson P et al. Enhanced Predictive Capability of a 1-Hour Oral Glucose Tolerance Test: A Prospective Population-Based Cohort Study. Diabetes Care. 2017;41(1):171-177.

23. Sarwar N, Aspelund T, Eiriksdottir G, Gobin R, Seshasai S, Forouhi N et al. Markers of Dysglycaemia and Risk of Coronary Heart Disease in People without Diabetes: Reykjavik Prospective Study and Systematic Review. PLoS Medicine. 2010;7(5):e1000278.

24. Classification and Diagnosis of Diabetes: Standards of Medical Care in Diabetes-2020. Diabetes Care. 2019;43(Supplement 1):S14-S31.

25. Gensini G. A more meaningful scoring system for determining the severity of coronary heart disease. The American Journal of Cardiology. 1983;51(3):606.

26. Coutinho M, Gerstein H, Wang Y, Yusuf S. The relationship between glucose and incident cardiovascular events. A metaregression analysis of published data from 20 studies of 95,783 individuals followed for 12.4 years. Diabetes Care. 1999;22(2):233-240.

27. Kuang L, Huang Z, Hong Z, Chen A, Li Y. Predictability of 1-h postload plasma glucose concentration: A 10-year retrospective cohort study. Journal of Diabetes Investigation. 2015;6(6):647-654.

28. Peddinti G, Bergman M, Tuomi T, Groop L. 1-Hour Post-OGTT Glucose Improves the Early Prediction of Type 2 Diabetes by Clinical and Metabolic Markers. The Journal of Clinical Endocrinology \& Metabolism. 2018;104(4):1131-1140.

29. Abdul-Ghani M, Stern M, Lyssenko V, Tuomi T, Groop L, DeFronzo R. Minimal Contribution of Fasting Hyperglycemia to the Incidence of Type 2 Diabetes in Subjects With Normal 2-h Plasma Glucose. Diabetes Care. 2009;33(3):557-561.

Cet article a été publié dans le « Batna Journal of Medical Sciences » BJMS, l’organe officiel de « l'association de la Recherche Pharmaceutique - Batna»

Le contenu de la Revue est ouvert « Open Access » et permet au lecteur de télécharger, d’utiliser le contenu dans un but personnel ou d'enseignement, sans demander l'autorisation de l'éditeur/auteur.

Avantages à publier dans BJMS :

- Open access : une fois publié, votre article est disponible gratuitement au téléchargement

- Soumission gratuite : pas de frais de soumission, contrairement à la plupart des revues « Open Access »

- Possibilité de publier dans 3 langues : français, anglais, arabe

- Qualité de la relecture : des relecteurs/reviewers indépendants géographiquement, respectant l'anonymat, pour garantir la neutralité et la qualité des manuscrits.

Pour plus d'informations, contacter BatnaJMS@gmail.com ou connectez-vous sur le site de la revue : www.batnajms.net 


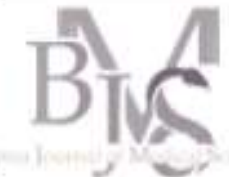

○ $\bigcirc$

-

-

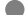

${ }^{1}$ Unité de Biochimie. LBC. CHU Hussein Dey, Alger - Algérie

${ }^{2}$ Laboratoire central de biologie. CHU Bab el Oued, Alger - Algérie

${ }^{3}$ Service nephrologie. Hopital Kolea, Tipasa - Algérie

${ }^{4}$ Clinique de néphrologie hémodialyse Dialamine - Algérie

5Unité d'hémodialyse Dar El Beida. CHU Rouiba, université Alger1 - Algérie

${ }^{6}$ Service néphrologie. CHU Hussein Dey, Alger - Algérie

${ }^{7}$ Laboratoire hormonologie. EHS CPMC, Alger - Algérie

${ }^{8}$ Faculté de médecine d'Alger, université Alger1 - Algérie

\section{Correspondance à :}

Médina ARAB

biochmed@hotmail.com

DOI :https://doi.org/10.48087/ BJMSoa.2020.7206

\section{Historique de l'article :}

Reçu le 15 juin 2020

Accepté le 15 septembre 2020

Publié le 09 novembre 2020

Il s'agit d'un article en libre accès distribué selon les termes de la licence Creative Commons Attribution International License (CC BY 4.0), qui autorise une utilisation, une distribution et une reproduction sans restriction sur tout support ou format, à condition que l'auteur original et la revue soient dûment crédités.

Pour citer l'article :

Arab M, Zenati A, Labou N, Missoum M, Ainouz N, Bennoui M, Guechi Z. Valeurs pronostiques de la Troponine T Hypersensible et du NT-proBNP chez les hémodialysés chroniques asymptomatiques. Batna J Med Sci 2020;7(2):92-6 https://doi.org/10.48087/ BJMSoa.2020.7206

\title{
Valeurs pronostiques de la Troponine T Hypersensible et du NT- proBNP chez les hémodialysés chroniques asymptomatiques
}

\author{
Pronostic value of Troponine T High sensitive and NT-proBNP in \\ asymptomatics chronic heamodialysis
}

\author{
Médina Arab ${ }^{1,7,8}$, Akila Zenati ${ }^{2,8}$, Nadia Labou $^{3}$, Mohamed Missoum ${ }^{4}$, Nouba \\ Ainouz $^{5,8}$, Mohamed Bennoui ${ }^{6,8}$, Zehor Guechi ${ }^{1,8}$
}

\section{RÉSUMÉ}

Introduction. L'hémodialyse permet aujourd'hui la survie de plus d'un million et demi de patients dans le monde, avec une qualité de vie de plus en plus acceptable. Le risque de mortalité est 20 fois plus élevé chez les patients en hémodialyse chronique par rapport à la population générale d'âge égal, incriminant les maladies cardiovasculaires comme premières causes de mortalité. Objectif. Étudier de la variation des marqueurs cardiaques TnThs et NTproBNP chez une population d'hémodialysée chronique et leurs rôles pronostiques dans la survenue la morbi-mortalité cardiovasculaire. Patients et méthodes. Il s'agit d'une étude de cohorte prospective observationnelle et longitudinale réalisée sur 145sujets urémiques chroniques asymptomatiques traités par hémodialyse suivi pendant 02 ans. Résultats. 93,8\% des patients ont des taux de TnThs et $100 \%$ ont des taux de NTproBNP supérieurs aux valeurs de référence. La mortalité globale cumulée est de $11,7 \%(\mathrm{n}=17)$ avec une moyenne de survie de 22,57 mois. L'analyse univariée retrouve une valeur pronostique pour la TnThs et NT-proBNP avec respectivement des HR brut de 4,99 et 5,008. Cependant, l'analyse multivariée ne retrouve pas ces marqueurs comme des facteurs prédictifs indépendant de mortalité. Conclusion. La TnThs et NT-proBNP sont de mauvais marqueurs pronostiques non indépendants de mortalité globale.

Mots clés: hémodialysés chroniques, marqueur pronostique, mortalité globale, NT-proBNP, TnThs.

\section{INTRODUCTION}

L'hémodialyse est une technique non physiologique car elle fait appel à des liquides de dialyse dont la biocompatibilité n'est pas parfaite, à des membranes de dialyse moins performantes que le filtre glomérulaire. De plus c'est une technique d'épuration intermittente contrairement aux reins qui travaillent continuellement et en temps réel [1].

Cependant, malgré son apport au confort et à la survie des patients qui y sont soumis, le risque de mortalité est 20 fois plus élevé dans cette population par rapport à la population générale d'âge égal. Les maladies cardiovasculaires sont considérées comme première cause de mortalité [2] représentées par les maladies coronaires à $40 \%$ et l'hypertrophie ventriculaire $75 \%$ [3].

\section{ABSTRACT}

Introduction. Now hemodialysis allows the survival of more than one and a half million patients worldwide, with an increasingly acceptable quality of life.The risk of death is 20 times higher in patients undergoing chronic hemodialysis, compared to the general population of equal age, incriminating cardiovascular diseases as the leading causes of death. Aim. Study of the variation of cardiac markers TnThs and NT-proBNP in a population of chronic hemodialysis and their prognostic roles in the occurrence of cardiovascular morbidity and mortality. Patients and methods. This is a prospective, observational and longitudinal cohort study performed on 145 asymptomatic chronic uremic subjects treated with hemodialysis followed for 2 years. Results. $93.8 \%$ of patients have TnTh levels and $100 \%$ who have NT-proBNP levels higher than the reference values. The overall cumulative mortality is $11.7 \%(n=17)$ with a mean survival of 22.57 months. The univariate analysis found a prognostic value for TnThs and NT-proBNP with respectively grossHR 4.99 and 5.008. However, multivariate analysis does not find these markers as predictors independent of mortality. Conclusion. TnThs and NT-proBNP are poor non-independent prognostic markers of overall mortality.

Keywords: chronic dialysis patients, prognostic marker, overall mortality, NT-proBNP, TnThs.

L'augmentation de l'incidence des événements cardiovasculaires dans cette population peut s'expliquer d'une part, par la présence de facteurs de risque dits classiques: âge, hypertension, diabète, tabagisme, la dyslipidémie et d'autre part, par des facteurs de risque dits non classiques liés à l'insuffisance rénale tels que la rétention hydrosodée, l'anémie et l'hyperparathyroïdie [4,5].

Le terme de Syndrome cardio-rénal est utilisé pour décrire la coexistence de dysfonction cardiaque et rénale où l'altération aigue ou chronique d'un organe peut induire une altération aigue ou chronique de l'autre organe [6]. Selon Ronco et al (2008) [7], il existe une altération systolique et/ou diastolique du myocarde chez les insuffisants rénaux chroniques. C'est ce qui a été décrit comme syndrome cardio-rénal de type 4. 
En médecine d'urgence, le recours à l'utilisation des marqueurs cardiaques n'est plus à démontrer.

Les avancées technologiques avec le développement des techniques d'immunodosages a rendu possible le dosage de nouveaux marqueurs tels que les troponines dans le diagnostic du syndrome coronarien aigu et le NT- pro BNP et la Galectine 3 dans l'insuffisance cardiaque aigue $[8,9]$. L'objectif de cette étude est de déterminer la valeur pronostique de la TnThs et du NT-proBNP chez des patients urémiques chroniques traités par hémodialyse.

\section{PATIENTS ET MÉTHODES}

Il s'agit d'une étude de cohorte observationnelle, longitudinale et pronostique s'étalant sur une période de de 35 mois.

L'étude avait porté sur des patients en insuffisance rénale chronique stade terminal traités par hémodialyse de suppléance, utilisant générateur de marque FRESENIUS 4008S, une membrane de dialyse de type Helixone. Le recrutement a été effectué au niveau de 4 centres d'hémodialyse :

Unité d'hémodialyse du CHU Hussein Dey

Unité d'hémodialyse de l'hôpital de Koléa

Unité d'hémodialyse de Dar el Beida

Une clinique privée.

Cette étude avait débuté en avril 2014 et tous les patients ont bénéficié d'un suivi pendant 2 ans.

\section{Critères d'inclusion}

Patients en dialyse ambulatoire âgés de plus de 18 ans et hémodialysés chroniques depuis plus de 6 mois.

\section{Critères d'exclusion}

Patients ayant eu des événements cardiovasculaires en l'occurrence, le syndrome coronarien aigu, pontage coronarien, artériopathie des membres inférieurs, amputation.

\section{Programme de travail}

Des prélèvements sanguins ont été effectués sur tube EDTA et tube hépariné au milieu de la semaine, avant la séance de dialyse pour un bilan biochimique: urée, créatinine, CRP ultrasensible, triglycérides, cholestérol total, HDLc, LDLc, calcium, phosphore, PAL, Albumine, la $250 \mathrm{H}$ vitamine D et la PTH, hémoglobine avec dosage des marqueurs cardiaques: troponine Ths et NT-proBNP.

Après ce bilan biologique, l'attention particulière a été focalisée sur une éventuelle apparition d'événements cardiovasculaires (Infarctus du myocarde, angine de poitrine, insuffisance cardiaque, accident vasculaire cérébral, angioplastie coronarienne avec ou sans pose de stent) et de décès toute cause confondue pendant 24 mois.

Le dosage de la TnThs et du NT-proBNP ont été réalisés sur Cobas e411Roche.

\section{Analyse statistique}

La saisie et l'analyse des données a été faite sur le logiciel SPSS 22.0.

\section{RÉSULTATS}

\section{Caractéristiques générales des patients}

145 patients urémiques chroniques ont été traités par hémodialyse cliniquement asymptomatiques. L'Age médian était de 50 ans avec des extrêmes de 18 et 87ans. Une prédominance masculine a été observée dans notre population avec un sexratio à 1,23. L'IMC moyen était de $22,25 \pm 4,09 \mathrm{~kg} / \mathrm{m}^{2}$ avec un minimum de $14 \mathrm{Kg} / \mathrm{m}^{2}$ et un maximum de $35 \mathrm{Kg} / \mathrm{m}^{2} .64 \%$ des patients étaient hypertendus et $19 \%$ diabétiques (Tableau1).

Tableau 1 : caractéristiques clinico-biologiques des hémodialysés chroniques asymptomatiques

\begin{tabular}{|c|c|c|}
\hline Paramètres & Patients (n) & $\begin{array}{l}\text { Valeurs } \\
\text { usuelles }\end{array}$ \\
\hline HTA (\%) & 64 (n 93) & \\
\hline Diabète (\%) & 19 (n 27) & \\
\hline Tabagiques (\%) & $9($ n 13) & \\
\hline IMC $\left(\mathrm{Kg} / \mathrm{m}^{2}\right)$ & $22,25 \pm 4,09(n 131)$ & $18,5-25$ \\
\hline Urée g/l & $1,14 \pm 0,28 n 145$ & $0,15-0,45$ \\
\hline Créatinine $\mathrm{mg} / \mathrm{l}$ & $89,61 \pm 26,71$ n 145 & $4-12$ \\
\hline Acide urique $\mathrm{mg} / \mathrm{l}$ & $55,36 \pm 12,47$ n 145 & $20-70$ \\
\hline Triglycérides g/l & $1,36[0,85-1,72]$ n 145 & $0,5-1,5$ \\
\hline Cholestérol total g/l & $1,56 \pm 0,35 \mathrm{n} 145$ & $1,5-2,00$ \\
\hline HDLc g/l & $0,36[0,31-0,44]$ n 145 & $>0,40$ \\
\hline LDLc g/l & $0,83[0,65-0,99]$ n 139 & $<1,30$ \\
\hline Non HDLc g/l & $1,18 \pm 0,36$ n 145 & $<1,60$ \\
\hline CRPhs mg/l & $3,70[2,00-6,40]$ n145 & $<1$ \\
\hline Albumine $\mathrm{g} / \mathrm{I}$ & $41,63[39,74-44,19]$ n 145 & $35-50$ \\
\hline Calcium mg/l & $87[81-94]$ n 145 & 84-105 \\
\hline Phosphore mg/l & $48[37-57]$ n144 & $25-48$ \\
\hline $25 \mathrm{OH}$ vitamine $\mathrm{D}$ totale $\mathrm{ng} / \mathrm{ml}$ & $18,68[12,42-39,23]$ n 144 & $>30$ \\
\hline PTH pg/ml & $198[78,29-574]$ n 145 & $15-65$ \\
\hline PAL UI/I & $113[75,9-201]$ n144 & $<140$ \\
\hline Hémoglobine g/dl & $9,77 \pm 1,84$ n 137 & $12-16$ \\
\hline TnThs $\mu \mathrm{g} / \mathrm{l}$ & $0,046[0,033-0,069]$ n 145 & $<0,014$ \\
\hline NT-proBNP pg/ml & $8132[2946-27996]$ n 144 & $<125$ \\
\hline
\end{tabular}

\section{Caractéristiques clinico-biologiques}

Les principales étiologies à l'origine de l'insuffisance rénale chronique terminale étaient: la néphropathie diabétique $(17 \%)$, la néphro-angiosclérose $(12 \%)$ et la polykystose rénale (9\%). Cependant, chez 38\% de nos patients, l'étiologie de l'insuffisance rénale chronique reste indéterminée. 93,8\% des patients urémiques avaient un taux TnThs supérieurs au 99eme percentile dont $35,17 \%$ avaient des taux se situant entre $0,05 \mu \mathrm{g} / \mathrm{l}$ et $0,1 \mu \mathrm{g} / \mathrm{l}$ et $10,34 \%$ supérieur à $0,1 \mu \mathrm{g} / \mathrm{l}$. Quant au NT-proBNP, $100 \%$ des patients présentaient des taux au seuil de $125 \mathrm{pg} / \mathrm{ml}$, les taux de NT-proBNP étaient augmentés de 4 à 280 fois la valeur seuil.

Les taux de troponine TnThs ont été subdivisés en tertile, groupe $1<0,032 \mathrm{ug} / \mathrm{l}$, groupe 2 représentant les taux de $0,033 \mu \mathrm{g} / \mathrm{l}-0,071 \mu \mathrm{g} / \mathrm{l}$ et groupe 3 représentant les taux supérieurs à $0,071 \mu \mathrm{g} / \mathrm{l}$.

Les taux de NT-proBNP ont été subdivisés en quartile: groupe 1 représentant les taux inférieurs à $2847 \mathrm{pg} / \mathrm{ml}$, groupe $2: 2848-8132$, groupe $3: 8133-29971$ et le groupe 4 $>29971 \mathrm{pg} / \mathrm{ml}$.

Le tableau 2 résume les caractéristiques clinico-biologiques selon les groupes de NT-proBNP qui montre des différences significatives entre le NT-proBNP, taux d'hémoglobine, PTH, PAL, Triglycérides, cholestérol total, non HDLc et HTA.

Le tableau 3 résume les caractéristiques clinico-biologiques selon les groupes de TnTs qui montre des différences significatives entre $250 \mathrm{H}$ vitamine D et le NT-proBNP. 
Pendant la durée de suivi qui était de deux ans, 06 patients ont été perdus de vue, un seul cas avait eu un accident vasculaire cérébral et 17 décès ont été enregistrés durant cette période.

La mortalité cumulée au cours de la première année était de $5,5 \%(8 / 145)$ et cours de la deuxième année le taux est passé à
6,4\% (9/139). La prévalence de la mortalité sur deux ans était de $11,7 \%$. Le taux de mortalité était de 5,31 pour 1000 patients mois. Les causes de décès enregistrés étaient : 11 par arrêt cardiaque; 01Suite à un carcinome ovarien; 01 Post syndrome infectieux; 01post AVC, 03 étaient de causes inconnues.

Tableau 2. Caractéristiques clinico-biologique selon le groupe de NT-proBNP chez les hémodialysés chroniques asymptomatiques

\begin{tabular}{|c|c|c|c|c|c|}
\hline NTproBNPpg/ml & $<2847$ & $2848-8132$ & 8133-29971 & $>29971$ & $\mathbf{P}$ \\
\hline Age ans & $50,33 \pm 13,93$ & $49,40 \pm 15,09$ & $50,13 \pm 15,50$ & $54,21 \pm 16,70$ & 0,53 \\
\hline Sexe H & $22 / 36$ & $20 / 36$ & $21 / 36$ & $17 / 37$ & 0,578 \\
\hline Durée de dialyse mois & $111,52 \pm 96,35$ & $109,61 \pm 80,32$ & $118,48 \pm 100,99$ & $111,08 \pm 80,43$ & 0,97 \\
\hline Diabète & $5 / 36$ & $7 / 36$ & $6 / 36$ & $9 / 37$ & 0,698 \\
\hline HTA & $18 / 36$ & $16 / 36$ & $8 / 36$ & $10 / 37$ & 0,038 \\
\hline Tabac & $2 / 36$ & $4 / 36$ & $5 / 31$ & $2 / 37$ & 0,501 \\
\hline Urée g/l & $1,17 \pm 0,33$ & $1,13 \pm 0,25$ & $1,11 \pm 0,25$ & $1,16 \pm 0,31$ & 0,800 \\
\hline Créatinine mg/l & $93,22 \pm 25,75$ & $94,78 \pm 21,65$ & $89,94 \pm 24,74$ & $83,01 \pm 22,18$ & 0,20 \\
\hline Acide urique $\mathrm{mg} / \mathrm{l}$ & $58,66 \pm 15,80$ & $56,63 \pm 11,07$ & $51,63 \pm 11,08$ & $54,54 \pm 10,69$ & 0,098 \\
\hline Albumine $\mathrm{g} / \mathrm{l}$ & $40,92 \pm 5,13$ & $40,30 \pm 6,47$ & $41,34 \pm 5,15$ & $39,93 \pm 4,59$ & 0,655 \\
\hline CRPus $\mathrm{mg} / \mathrm{l}$ & $5,40 \pm 4,90$ & $4,90 \pm 5,61$ & $5,58 \pm 5,55$ & $8,00 \pm 7,72$ & 0,13 \\
\hline Triglycérides g/l & $1,89 \pm 0,87$ & $1,71 \pm 0,84$ & $1,50 \pm 0,18$ & $1,21 \pm 0,53$ & 0,006 \\
\hline HDLc g/I & $0,37 \pm 0,10$ & $0,39 \pm 0,11$ & $0,38 \pm 0,11$ & $0,37 \pm 0,09$ & 0,504 \\
\hline LDLc g/l & $0,96 \pm 0,28$ & $0,83 \pm 0,27$ & $0,85 \pm 0,27$ & $0,81 \pm 0,26$ & 0,12 \\
\hline Non HDLc g/l & $1,33 \pm 0,34$ & $1,19 \pm 0,36$ & $1,16 \pm 0,37$ & $1,07 \pm 0,34$ & 0,026 \\
\hline Calcium mg/l & $87,50 \pm 8,72$ & $85,17 \pm 10,10$ & $90,33 \pm 8,18$ & $86,14 \pm 8,72$ & 0,075 \\
\hline Phosphore mg/l & $48,76 \pm 17,42$ & $52,17 \pm 17,44$ & $47,89 \pm 17,34$ & $46,19 \pm 14,60$ & 0,484 \\
\hline PAL UI/I & $208,01 \pm 214,29$ & $230,24 \pm 303,39$ & $107,94 \pm 54,89$ & $135,15 \pm 88,43$ & 0,023 \\
\hline PTH pg/ml & $571,55 \pm 607,89$ & $514,62 \pm 563,98$ & $298,34 \pm 442,44$ & $322,45 \pm 322,17$ & 0,045 \\
\hline 25 OH VITAMINE D ng/ml & $26,81 \pm 17,97$ & $27,98 \pm 20,62$ & $21,21 \pm 13,31$ & $23,62 \pm 20,28$ & 0,376 \\
\hline Hémoglobine g/dl & $10,26 \pm 1,87$ & $10,21 \pm 1,52$ & $9,27 \pm 1,81$ & $9,31 \pm 1,94$ & 0,026 \\
\hline $\mathrm{IMC} \mathrm{kg} / \mathrm{m}^{2}$ & $23,41 \pm 4,09$ & $22,53 \pm 3,55$ & $22,27 \pm 3,75$ & $20,90 \pm 4,20$ & 0,088 \\
\hline
\end{tabular}

Tableau3 : caractéristiques clinico-biologiques selon le groupe de TnThs chez les hémodialysés chroniques asymptomatiques

\begin{tabular}{|c|c|c|c|c|}
\hline TnTHSug/l & $<0,032$ & $0,033-0,071$ & $>0,071$ & p \\
\hline Age ans & $45,32 \pm 11,60$ & $50,63 \pm 15,44$ & $57,90 \pm 16,13$ & 0,002 \\
\hline Sexe $\mathrm{H}$ & $22 / 38$ & $37 / 71$ & $21 / 36$ & 0,728 \\
\hline $\begin{array}{l}\text { Durée de } \\
\text { dialyse mois }\end{array}$ & $98,86 \pm 79,59$ & $126,14 \pm 94,72$ & $100,14 \pm 84,72$ & 0,19 \\
\hline Diabete & $5 / 38$ & $9 / 71$ & $13 / 36$ & 0,008 \\
\hline HTA & $19 / 38$ & $48 / 71$ & $10 / 36$ & 0,096 \\
\hline TABAC & $4 / 38$ & $8 / 63$ & $1 / 35$ & 0,323 \\
\hline Urée g/l & $1,15 \pm 0,28$ & $1,18 \pm 0,27$ & $1,07 \pm 0,27$ & 0,31 \\
\hline $\begin{array}{l}\text { Créatinine } \\
\mathrm{mg} / \mathrm{l}\end{array}$ & $96,93 \pm 26,18$ & $90,92 \pm 27,39$ & $84,40 \pm 21,17$ & 0,09 \\
\hline $\begin{array}{l}\text { Acide urique } \\
\mathrm{mg} / \mathrm{l}\end{array}$ & $55,86 \pm 13,29$ & $55,23 \pm 12,63$ & $55,08 \pm 11,58$ & 0,95 \\
\hline Albumine g/l & $40,61 \pm 4,56$ & $41,70 \pm 5,39$ & $40,34 \pm 4,27$ & 0,49 \\
\hline CRPus mg/l & $3,80 \pm 3,14$ & $5,94 \pm 6,14$ & $8,44 \pm 7,51$ & 0,004 \\
\hline $\begin{array}{l}\text { Cholestérol } \\
\text { total g/l }\end{array}$ & $1,61 \pm 0,32$ & $1,50 \pm 0,32$ & $1,48 \pm 0,35$ & 0,27 \\
\hline $\begin{array}{l}\text { Triglycérides } \\
\text { g/l }\end{array}$ & $1,66 \pm 0,65$ & $1,38 \pm 0,68$ & $1,39 \pm 0,69$ & 0,33 \\
\hline HDLc g/l & $0,38 \pm 0,12$ & $0,38 \pm 0,10$ & $0,35 \pm 0,10$ & 0,33 \\
\hline LDLc g/l & $0,90 \pm 0,28$ & $0,83 \pm 0,25$ & $0,83 \pm 0,28$ & 0,23 \\
\hline Non HDLc g/l & $1,22 \pm 0,31$ & $1,11 \pm 0,32$ & $1,11 \pm 0,38$ & 0,42 \\
\hline Calcium mg/l & $85 \pm 8,24$ & $87,54 \pm 9,70$ & $87,50 \pm 6,73$ & 0,24 \\
\hline $\begin{array}{l}\text { Phosphore } \\
\mathrm{mg} / \mathrm{l}\end{array}$ & $51,94 \pm 18,60$ & $48,27 \pm 16,47$ & $45,38 \pm 12,35$ & 0,38 \\
\hline PAL UI/I & $210,37 \pm 200,55$ & $173 \pm 231,59$ & $146,53 \pm 104,29$ & 0,39 \\
\hline
\end{tabular}

(Suite Tableau 3)

\begin{tabular}{lllll} 
PTH pg/ml & $479,75 \pm 494,71$ & $437,45 \pm 530,69$ & $251,51 \pm 279,68$ & 0,18 \\
\hline $\begin{array}{l}\text { 25 OH } \\
\text { VITAMINE D } \\
\text { ng/ml }\end{array}$ & $31,69 \pm 18,56$ & $27,03 \pm 20,45$ & $19,61 \pm 13,57$ & 0,014 \\
\hline $\begin{array}{l}\text { Hémoglobine } \\
\text { g/dl }\end{array}$ & $10,48 \pm 1,74$ & $9,42 \pm 1,84$ & $9,68 \pm 1,99$ & 0,056 \\
\hline IMC kg/m ${ }^{2}$ & $22,70 \pm 3,88$ & $21,18 \pm 3,97$ & $22,87 \pm 4,95$ & 0,071 \\
\hline $\begin{array}{l}\text { NT } \\
\text { proBNPpg/ml }\end{array}$ & $7703 \pm 10070$ & $14063 \pm 12039$ & $21584 \pm 13644$ & 0,000 \\
\hline
\end{tabular}

Le tableau 4 montre que les hémodialysés décédés étaient plus âgés, avec des taux moyens d'albumine à $38 \mathrm{~g} / \mathrm{l}$, des taux élevés en TnThs et en NT-proBNP ainsi qu'une insuffisance en $250 \mathrm{H}$ vitamine D totale.

La survie moyenne globale de notre cohorte était de 22,57 mois avec un IC95\% de $(21,86-23,28)$ mois.

Le risque proportionnel de COX univarié de la TnThs et du NTproBNP avaient une forte valeur pronostique pour des taux de TnThs $>0,071 \mu \mathrm{g} / \mathrm{l}$ avec un HR univarié de 4,99 (IC95\% 1,0523,52 ) avec une p value de 0,042 et pour des valeurs de NTproBNP supérieures à $29970 \mathrm{pg} / \mathrm{ml}$ avec un HR univarié de 5,008 (IC95\% 1,08-23,18) avec P 0,039. 
Tableau 4. Caractéristiques clinico-biologiques entre les hémodialysés vivants et les hémodialysés décédés

\begin{tabular}{llll}
\hline Paramètres & Hémodialysés vivants & Hémodialysés décédés & P Value \\
\hline Age (ans) & $49[38-55](122)$ & $76[56-79](17)$ & $0,000^{*}$ \\
Durée de dialyse(mois) & $105[47,5-157,5](122)$ & $60[18-152](17)$ & 0,123 \\
Sexe (H)\% & $54(66 / 122)$ & $52(9 / 17)$ & 0,929 \\
IMC & $22,15 \pm 4,08(112)$ & $22,27 \pm 4,12(14)$ & 0,762 \\
HTA \% & $68(84 / 122)$ & $35(6 / 17)$ & $0,007^{*}$ \\
Diabète \% & $14,7(18 / 122)$ & $23(4 / 17)$ & 0,353 \\
Tabac \% & $9,8(12 / 122)$ & $5,88(1 / 17)$ & 0,600 \\
Albumine(g/l) & $42,11[38,8-44,15](122)$ & $38[34-39,8](17)$ & $0,000^{*}$ \\
Créatinine(mg/l) & $91,53 \pm 27,18(122)$ & $75,10 \pm 22,30(17)$ & $0,012^{*}$ \\
Urée (g/l) & $1,16 \pm 0,27(122)$ & $1,05 \pm 0,37(17)$ & 0,344 \\
CRP US (mg/l) & $3,53(0,06-21)(122)$ & $6,93(0,27-21)(17)$ & 0,208 \\
Cholestérol total(g/l) & $1,58 \pm 0,35(122)$ & $1,42 \pm 0,34(17)$ & 0,078 \\
Triglycerides (g/l) & $1,41[0,94-1,83](122)$ & $1,15[1,01-1,25](17)$ & 0,158 \\
HDLc(g/l) & $0,36[0,30-0,43](122)$ & $0,35[0,31-0,46](17)$ & 0,787 \\
LDL (g/l) & $0,85[0,68-1,04](118)$ & $0,70[0,55-0,99](17)$ & 0,094 \\
Chol T/HDLc & $4,45 \pm 1,53(122)$ & $4,15 \pm 1,86(17)$ & 0,250 \\
Non HDLc (g/l) & $1,19 \pm 0,36(122)$ & $1,04 \pm 0,37(17)$ & 0,093 \\
Calcium (mg/l) & $86[82-93](122)$ & $87[85-91](17)$ & 0,514 \\
Phosphore (mg/l) & $48[38-58](122)$ & $43[35,5-51](17)$ & 0,340 \\
PAL (U/l) & $116[77,5-176](122)$ & $101[74-182](17)$ & 0,739 \\
PTH pg/ml & $230[87,2-550,5](122)$ & $160[70,31-495](17)$ & 0,979 \\
250H vitaminaDtotale & $20,81[12,34-36,7](121)$ & $14,90[8,61-18,35](17)$ & $0,026^{*}$ \\
TnThsug/l & $0,045[0,030-0,066](122)$ & $0,065[0,041-0,122](17)$ & $0,010^{*}$ \\
NT proBNPpg/ml & $7702[2826-29766](121)$ & $35000[5934-35000](17)$ & $0,024^{*}$ \\
Hémoglobine (g/dl) & $9,74 \pm 1,84(115)$ & $9,85 \pm 2,02(17)$ & 0,762
\end{tabular}

(n) : nombre de patients vivants ou décédés, * : valeurs de $p<0,05$

Après ajustement à l'âge, albumine, LDLc et l'hypertension, les HR ajustés sont devenus non significatifs.

Cependant, Le taux d'albumine inférieur à $40 \mathrm{~g} / \mathrm{l}$ avait présenté un facteur pronostic indépendant avec un facteur de 3,97 suivi de l'âge avec un facteur de 1,078. Quant à l'hypertension, elle présentait moins de risque de mortalité (cf tableau5)

Tableau 5. Risque proportionnel de Cox de la TnThs et NT-proBNP dans la mortalité globale

\begin{tabular}{|c|c|c|c|c|}
\hline Paramètre & $\begin{array}{l}\text { HR non ajusté } \\
\text { (IC95\%) }\end{array}$ & $\mathbf{P}$ & $\begin{array}{l}\text { HR ajusté } \\
\text { (IC95\%) }\end{array}$ & $\mathbf{P}$ \\
\hline \multicolumn{5}{|l|}{ TnTHS sug/l } \\
\hline TnTHS $<0,032$ & Référent & 0,058 & Référent & \\
\hline TnTHSO 0,033- & $1,94(0,40-$ & 0,406 & $1,77(0,32-$ & 0,508 \\
\hline 0,071 & $9,35)$ & 0,042 & $9,60)$ & 0,366 \\
\hline TnTHS $>0,071$ & $\begin{array}{l}4,99(1,05- \\
23,52)\end{array}$ & & $\begin{array}{l}2,27(0,38- \\
13,53)\end{array}$ & \\
\hline \multicolumn{5}{|l|}{ NT-proBNP pg/ml } \\
\hline NTproBNP $<2847$ & Référent & 0,051 & Référent & \\
\hline NTproBNP 2848- & $2,03(0,37-$ & 0,413 & $2,01(0,35-$ & 0,426 \\
\hline 8132 & $11,10)$ & 0,986 & 11.36) & 0,677 \\
\hline $\begin{array}{l}\text { NTproBNP 8133- } \\
29971\end{array}$ & $\begin{array}{l}0,98(0,13- \\
6,97)\end{array}$ & 0,039 & $\begin{array}{l}1,55(0,194- \\
12.48)\end{array}$ & 0,148 \\
\hline NTproBNP>29971 & $\begin{array}{l}5,008(1,08- \\
23,18)\end{array}$ & & $\begin{array}{l}3,39(0,64- \\
17.70)\end{array}$ & \\
\hline
\end{tabular}

\section{DISCUSSION}

L'IRCT est un enjeu majeur de santé publique du fait de l'élévation de son incidence. Dans le monde, le profil épidémiologique varie d'un pays à un autre. Dans les pays développés, elle touche les sujets âgés. L'HTA et le diabète sont les principales étiologies de l'IRCT. En effet, en France les patients prévalents en dialyse sont en majorité des hommes $60 \%$ avec un sexratio de 1,$1 ; 64 \%$ des patients en dialyse ont 65 ans et plus et $40 \%$ ont 75 ans et plus. La néphropathie hypertensive et vasculaire représente $26 \%$ des causes de l'IRCT suivi de la néphropathie diabétique $22 \%$ et $16 \%$ sont d'origines indéterminées [10].

L'Algérie compte parmi les pays en voie de développement. Dans notre cohorte, le profil épidémiologique se rapproche des études des pays voisins [11,12].
Les taux élevés de troponines TnThs rendent l'interprétation difficile dans le contexte de l'urgence chez ces patients. Les résultats obtenus concordent avec l'étude de Wolley et al (2013) [13] qui retrouve une augmentation de TnThs chez 98\% de la population d'hémodialysés.

La cause de l'élévation chronique de la troponine fait l'objet de plusieurs études. Cette augmentation serait probablement multifactorielle : endommagement du myocarde par toxicité urémique, ischémie macrovasculaire ou microvasculaire et la diminution de la clairance rénale [14]. Ajoutés à ces mécanismes, d'autres facteurs pourraient expliquer l'augmentation de la troponine tels: les lésions oxydatives, les changements hémodynamiques liés à la dialyse et à l'anémie sans oublier l'augmentation du processus inflammatoire [15].

Au cours de cette présente étude le taux de la troponine Ths est influencé par l'âge et le diabète, plusieurs autres études ont abouti au même constat $[13,16]$. L'association constatée au cours de notre analyse entre la CRPus et la troponine Ths, a aussi été démontrée par l'étude de Filippi [17] et Stolear [18] mettant en évidence la prévalence élevée de l'état inflammatoire et du stress oxydant chez les hémodialysés indépendamment de toute infection ou néoplasiequi résulterait de l'insuffisance rénale chronique elle-même. Les taux élevés retrouvés du NT-proBNP dans notre cohorte, sont en accord avec l'étude Fahim 2015[19] qui retrouve une augmentation de $93 \%$ dans la population dialysée. Cette nette augmentation de NT-proBNP reflète le statut hydrique, la fonction rénale résiduelle, le schéma de dialyse et les pathologies cardiaques présentes telles que les dysfonctions ventriculaires gauches et ischémie infraclinique dans la population dialysée [19].

L'étude Satyan et al [20] a retrouvé des résultats similaires quant à la variation de NT-proBNP par rapport l'IMC et la pression systolique. En effet, la production des peptides natriurétiques est conséquente à l'augmentation de la pression artérielle et à la surcharge volémique chez les hémodialysés mais aussi chez les patients avec perte musculaire sévère et cachectiques secondaire à la perte de protéine commune à la population. 
Selon l'étude de Ikeda 2016 le NT-proBNP peut être un marqueur indépendant de mal nutrition chez les hémodialysés indépendamment du statut hydrique, l'inflammation chronique et la dysfonction cardiaque [21].

Nous avons noté une prévalence de mortalité de 11,7\% avec une durée de survie moyenne de 22,57 mois. Dans l'étude Fouda au Cameroun (2017), la survie des patients dialysés est plus faible avec une moyenne de 8 mois. Les patients décèdent au cours des 3 premiers après initiation de la dialyse. Ce fait, s'explique par la présence de plusieurs facteurs: le transfert tardif, le manque d'adhérence au traitement, ruptures du matériel de dialyse et les comorbidités [22].

Il faut reconnaitre que depuis 2010, il n'y a plus de zone d'insécurité sanitaire en matière d'hémodialyse en Algérie. Depuis 2004, les caisses de sécurité sociale ont conventionné un grand nombre de centres privés d'hémodialyse.

En ajustant la troponine T HS à l'âge, l'albumine, l'HTA et le taux de LDL, le HR diminue de 2,27 (0,38-13,53) et devient non significatif. Ce qui revient à dire que la troponine Ths n'est pas un facteur pronostique indépendant de la mortalité globale.

La méta-analyse de Li al 2015[23] qui a porté sur l'étude de la prédiction de la troponine cardiaque dans la mortalité globale et cardiovasculaire chez les insuffisants rénaux chroniques, a retrouvé une hétérogénéité dans les populations et les sousgroupes étudiés. En effet les_études avec des échantillons de faibles tailles montrent une augmentation significative de la mortalité globale avec un HR 3,20 (2,65-3,87) comparativement aux_études avec des échantillons de tailles élevées où le HR est de 1,54(0,88-2,70).

Le HR non ajusté du NT-pro-BNP retrouvé est de 5,00. Cependant, après ajustement à l'âge, l'albumine, LDL, HTA le HR diminue de $3,39(0,64-17,00)$ et devient non significatif. Comparativement à l'étude de Satyan2007 [20] réalisée sur 150 hémodialysés chroniques suivi pendant deux ans, retrouve que les quartiles $3(3276-7880 \mathrm{pg} / \mathrm{ml})$ et quartiles 4 $(7880-35000 \mathrm{pg} / \mathrm{ml})$ sont associés à la mortalité globale avec des HR non ajusté respectifs de 4,78(1,58-14,51) et $4,08(1,31-14,40)$; après ajustement à l'hémoglobine, tabac, PAS, PAD et marqueur de la dysfonction ventriculaire systolique, le NT-proBNP devient un facteur indépendant de mortalité.

Cette discordance des résultats peut s'expliquer par le nombre de décès rencontré au cours de notre étude où 17 patients sont décédés sur 145 contre 44 sur 150 pour l'étude de Satyan [20] durant la période de deux ans.

\section{CONCLUSION}

On peut dire que la TnThs et le NT-proBNP semblent être des marqueurs de mauvais pronostiques mais non indépendant de la mortalité.

Déclaration d'intérêts: les auteurs ne déclarent aucun conflit d'intérêt en rapport avec cet article.

\section{RÉFÉRENCES}

1. Jungers $P$, Man NK, Joly $D$, Legendre $C$. L'insuffisance rénale chronique : prévention et traitement. Lavoisier ; 2011. 337 p.

2. Le Feuvre C. Maladie coronaire chez I'hémodialysé. Lettre du cardiologue. .2000; N³30:20-26.
3. Foley RN, Parfrey PS, Sarnak MJ. Epidemiology of cardiovascular disease in chronic renal disease Am J Kidney dis. 1998; 32 : S112- 9

4. Madore F. Facteurs de risque vasculaire et insuffisance rénale. médecine/sciences. 2004;20(12):1100-3.

5. Meier P, Saudan P, Burnier M, Martin P-Y. Comorbidité et facteurs de risque cardiovasculaire liés à l'insuffisance rénale chronique. Revue medicale suisse $2003, \mathrm{~N}^{\circ} 2426$

6. Ahmed MS, Wong CF, Pai P. Cardiorenal syndrome - a new classification and current evidence on its management. Clin Nephrol. 2010;74(4):245-57.

7. Ronco C, Haapio M, House AA et al. Cardiorenal syndrome. J Am Coll Cardiol. 2008; 52:1527-1539.

8. HAS. Les marqueurs cardiaques dans la maladie coronarienne et l'insuffisance cardiaque en médecine ambulatoire. Juillet 2010

9. Bonnefoy E, Gabbarz E, Bugugnani M.J et al. Les marqueurs cardiaques. Cahier de formation biologie médicale. 2002; $\mathrm{N}^{\circ} 27$.

10. Registre français des traitements de suppléance de l'insuffisance rénale chronique REIN. Rapport annuel 2015.https://www.agencebiomedecine.fr/IMG/pdf/rapport_rein_2015.pdf

11. Zouari L, Omri S, Turki S, Maâlej M et al. Qualité de vie des insuffisants rénaux chroniques hémodialysés: a propos de 71 cas. La tunisie médicale 2016 ; 94 (1) : 40-45

12. Asserraji $\mathrm{M}$, Maoujoud $\mathrm{O}$, Belarbi $\mathrm{M}$, Oualim Z. Profil epidémiologique de l'insuffisance rénale terminale à l'hôpital militaire rabat, maroc. $\begin{array}{llll}\text { Panafriacn medical journal. 2015; 20:439. } & \end{array}$ doi:10.11604/pamj.2015.20.439.3352

13. Wolley M, Stewart R, Curry E, Davidson J et al. Variation in and prognostic importance of troponin $\mathrm{T}$ measured using a high-sensitivity assay in clinically stable haemodialysis patients. Clin Kidney J. 2013; 6: 402-409 doi: 10.1093/ckj/sfs122

14. Dikow R, Hardt SE. The uremic myocardium and ischemic tolerance : a world of difference. Circulation 2012;125(10):1215-6

15. Parikh RH, Seliger SL, deFilippi $\mathrm{CR}$. Use and interpretation of high sensitivity cardiac troponins in patients with chronic kidney disease with and without acute myocardial infarction. Clin Biochem. 2015;48(4-5):247-53.

16. Artunc F, Mueller C, Breidthardt T, Twerenbold R, Peter A, Thamer C, et al. Sensitive Troponins - Which Suits Better for Hemodialysis Patients? Associated Factors and Prediction of Mortality. PLOS ONE. 2012;7(10):e47610.

17. deFilippi C, Wasserman S, Rosanio S, Tiblier E, Sperger H, Tocchi M, et al. Cardiac Troponin T and C-Reactive Protein for Predicting Prognosis, Coronary Atherosclerosis, and Cardiomyopathy in Patients Undergoing Long-term Hemodialysis. JAMA. 2003;290(3):353-9.

18. Stolear JC, Georges B, Shita A, Verbeelen D. The predictive value of cardiac troponin $\mathrm{T}$ measurements in subjects on regular haemodialysis. Nephrol Dial Transplant. 1999;14(8):1961-7.

19. Fahim MA, Hayen A, Horvath AR, Dimeski G, Coburn A, Johnson DW, et al. N-Terminal Pro-B-Type Natriuretic Peptide Variability in Stable Dialysis Patients. Clin J Am Soc Nephrol CJASN. 2015;10(4):620-9.

20. Satyan S, Light RP, Agarwal R. Relationships of N-terminal pro-Bnatriuretic peptide and cardiac troponin $T$ to left ventricular mass and function and mortality in asymptomatic hemodialysis patients. Am J Kidney Dis Off J Natl Kidney Found. 2007;50(6):1009-19.

21. Ikeda M, Honda H, Takahashi K, Shishido K, Shibata T. N-Terminal Pro-BType Natriuretic Peptide as a Biomarker for Loss of Muscle Mass in Prevalent Hemodialysis Patients. PloS One. 2016;11(11):e0166804.

22. Fouda H, Ashuntantang G, Kaze F, Halle M-P. La survie en hémodialyse chronique au Cameroun. Pan Afr Med J [Internet]. 24 févr 2017 [cité 9 mars 2019];26. Disponible sur: https://www.ncbi.nlm.nih.gov/pmc/articles/PMC5409996/

23. Li W-J, Chen X-M, Nie X-Y, Zhang J, Cheng $Y-J$, Lin X-X, et al. Cardiac troponin and $\mathrm{C}$-reactive protein for predicting all-cause and cardiovascular mortality in patients with chronic kidney disease: A metaanalysis. Clinics. 2015;70(4):301-11. 


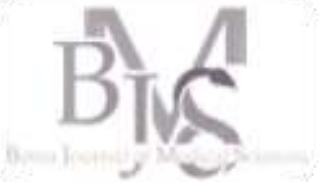

\section{Épidémiologie de la mortalité maternelle dans la Wilaya de Sétif, de 2014 à 2019}

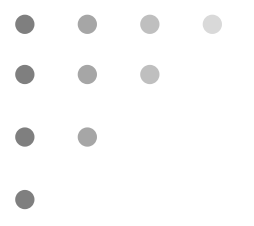

${ }^{1}$ Service épidémiologie et médecine préventive CHU de Sétif

2Direction de la Santé et la Population de Sétif

\section{Correspondance à :}

Meriem ABDOUN

drabdounmeriem19@gmail.com

DOI :https://doi.org/10.48087/ BJMSoa.2020.7207

Historique de l'article :

Reçu le 15 juin 2020

Accepté le 11 septembre 2020

Publié le 09 novembre 2020

Il s'agit d'un article en libre accès distribué selon les termes de la licence Creative Commons Attribution International License (CC BY 4.0), qui autorise une utilisation, une distribution et une reproduction sans restriction sur tout support ou format, à condition que l'auteur original et la revue soient dûment crédités.

Pour citer l'article :

Abdoun M, Merabet N, Nouiouet N, Rezig F, Saoudi S. Épidémiologie de la mortalité maternelle dans la Wilaya de Sétif, de 2014 à 2019. Batna J Med Sci 2020;7(2):97-101. https://doi.org/10.48087/BI MSoa.2020.7207

\section{Epidemiology of maternal mortality in the Wilaya of Sétif, from 2014 to 2019}

Meriem Abdoun ${ }^{1}$, Nadia Merabet ${ }^{1}$, Nesrine Nouiouet ${ }^{1}$, Fouzi Rezig ${ }^{2}$, Souad Saoudi ${ }^{2}$.

\section{RÉSUMÉ}

Objectif. L'objectif de notre étude est de décrire le profil épidémiologique des décès maternels dans la Wilaya de Sétif durant les années 2014-2019. Matériels et méthodes. Les définitions utilisées sont celles de l'OMS L'analyse porte sur les données observées à partir des statistiques des déclarations obligatoires des décès maternels par la mise en place d'un Audit National des Décès Maternels depuis 2014. Il s'agit d'étude descriptive des décès maternels survenus dans la wilaya de Sétif, durant les années 2014-2019. Résultats. 141 décès maternels ont eu lieu entre 2014 et 2019. L'âge moyen des femmes décédées était de 32 ans (écart-type 5,7 ans). La tranche d'âge 25-29 ans est la plus touchée (33,3\%). 79\% des décès maternels ont eu lieu dans un établissement hospitalier public, 20\% dans une clinique privée et seulement $1 \%$ à domicile. La première cause des décès maternels est due aux hémorragies obstétricales avec un taux de 37,6\%, suivie par les complications de l'hypertension artérielle (22\%) et l'embolie amniotique (15,6\%). Les infections sont impliquées dans $5 \%$ des décès. Conclusion. De nombreuses améliorations sont encore possibles, d'une part pour connaître la cause exacte conduisant à ce drame, d'autre part, pour optimiser les soins notamment en matière d'hémorragie obstétricale et de complications hypertensives étant donné qu'elles demeurent les premières causes de décès.

Mots clés : mortalité maternelle, indicateur de soins obstétricaux, causes de décès.

\section{INTRODUCTION}

La mortalité maternelle est un indicateur de la qualité des soins obstétricaux. Elle constitue un indicateur important de développement sanitaire d'un pays. En Algérie, La mortalité maternelle reste préoccupante et pose un problème de santé publique majeur. L'Organisation Mondiale de la Santé (OMS) a estimé que chaque année dans le monde, au moins un demi-million de femmes meurent à cause de la grossesse, de l'avortement ou des suites de couches [1]

En souscrivant aux Objectifs du Millénaire pour le Développement (OMD), l'Algérie s'est engagée à lutter contre la mortalité maternelle et d'atteindre un Rapport de Mortalité Maternelle (RMM) de 50 pour $100 \quad 000$ naissances vivantes (NV) [2]. En 1999, l'enquête conduite par l'Institut National de Santé Publique (INSP) a trouvé un RMM à 117 pour 100000 NV. En 2014, le RMM est passé à 60.3 pour $100000 \mathrm{NV}$. La progression lente de

\section{ABSTRACT}

Objective. The objective of our study is to describe the epidemiological profile of maternal deaths in the Wilaya of Sétif during the years 2014-2019. Materials and methods. The definitions used are those of the WHO. The analysis focuses on the data observed from the statistics of mandatory reporting of maternal deaths through the implementation of a National Audit of Maternal Deaths since 2014. This is a descriptive study of maternal deaths occurring in the wilaya of Sétif during the years 2014-2019. Results. 141 maternal deaths occurred between 2014 and 2019. The average age of the women who died was 32 years (standard deviation 5.7 years). The 25-29 age group was the most affected (33.3\%). $79 \%$ of maternal deaths occurred in a public hospital, $20 \%$ in a private clinic and only $1 \%$ at home. The first cause of maternal death is due to obstetric haemorrhage with a rate of $37.6 \%$, followed by complications of high blood pressure $(22 \%)$ and amniotic embolism (15.6\%). Infections are involved in $5 \%$ of deaths. Conclusion. Many improvements are still possible, on the one hand to know the exact cause leading to this tragedy, and on the other hand to optimise care, particularly in terms of obstetric haemorrhage and hypertensive complications, as these remain the leading causes of death.

Key words : maternal mortality, obstetric care indicator, causes of death.

la réduction de la mortalité maternelle a été à l'origine de l'initiative du plan national de réduction de la mortalité maternelle 20152019 [3].

L'OMS défini la mort maternelle comme «tout décès d'une femme survenu au cours de la grossesse ou dans un délai de 42 jours après sa terminaison, quelles que soient la durée ou la localisation, pour une cause quelconque déterminée ou aggravée par la grossesse, ou les soins qu'elle a motivés, mais ni accidentelle, ni fortuite» [4].

Ces morts maternelles se répartissent en deux groupes : les décès par cause obstétricale directe " résultent de complications obstétricales (grossesse, travail et suites de couches), d'interventions, d'omissions, d'un traitement incorrect ou d'un enchaînement d'événements résultant de l'un quelconque des facteurs ci-dessus » et les décès par cause obstétricale indirecte " résultent d'une maladie préexistante ou d'une affection apparue au cours de la grossesse sans qu'elle soit due à des 
causes obstétricales directes, mais qui a été aggravée par les effets physiologiques de la grossesse » [4].

\section{Objectif}

L'objectif de notre étude est de décrire le profil épidémiologique des décès maternels dans la wilaya de Sétif durant les années 2014-2019.

\section{MATÉRIELS ET MÉTHODES}

\section{Type d'étude}

Nous avons mené une étude descriptive des décès maternels survenus dans la wilaya de Sétif, durant les années 2014 2019.

\section{Population de l'étude}

Était inclus dans l'étude tout décès d'une femme qui répond à la définition de la mortalité maternelle de l'OMS dans la wilaya de Sétif.

Était exclue toute mort maternelle tardive qui est le décès d'une femme résultant de causes obstétricales directes ou indirectes survenue dans un délais de plus de 42 jours.

\section{Source des données}

Les données ont été recueillies par un audit des décès maternels qui a été mis en place, en Algérie, par arrêté ministériel $\mathrm{N}^{\circ} 89$ du 4 juillet 2013 instituant la déclaration obligatoire de tout décès maternel qu'ils surviennent dans une structure de santé (publique, privé) ou à domicile.

\section{RÉSULTATS}

\section{Fréquence et évolution}

141 décès maternels ont été colligés durant la période d'étude. Ces décès se répartissent en fonction des années selon le Tableau 1. On a enregistré 23,5 décès annuels en moyenne avec un maximum de décès enregistré au cours de l’année 2018 (31 décès).
Ces chiffres représentent un rapport moyen de mortalité maternelle de 44,2 décès pour 100000 NV. L'évolution annuelle du RMM depuis 2014 à 2019 montre une augmentation significative jusqu'à l'année 2018 (respectivement 36,3 en 2014 contre 58,1 pour $100000 \mathrm{NV}$ en 2018). C'est lors de cette année qu'on a enregistré un maximum de décès, suivi d'une diminution importante au cours de l'année 2019 (26,3 pour 100000 NV).

La proportion de décès maternels parmi les décès de femmes en âge de procréer est représentée dans le Tableau 1.

Le poids de ces décès parmi le total des décès de femmes en âge de procréer (15 à 49 ans) était de 7,8\%.

\section{Age}

L'âge moyen des femmes décédées de mort maternelle était de 32 ans avec un écart type de 5,7 ans. Dans notre étude, la parturiente la plus jeune avait 17 ans, alors que la plus âgée avait 45 ans. L'évolution de l'âge moyen par année montre une augmentation allant de 31,1 ans à 33 ans mais non significative $(p>0,05)$ (Figure 1$)$.

Figure 1 : évolution de l'âge moyen des décès maternels, Sétif 20142019

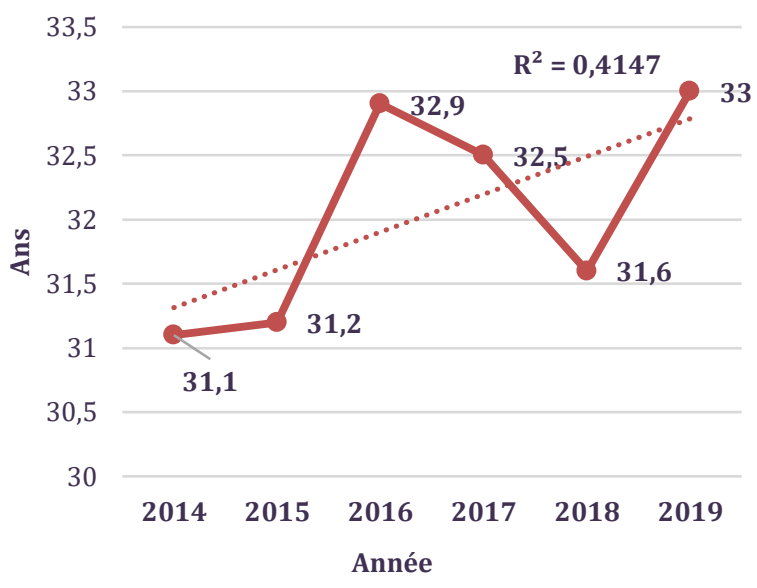

Tableau 1 : Effectif annuel des décès maternels, taux de mortalité maternelle pour 100000 naissances vivantes, poids de ces décès parmi le total des décès de femmes en âge de procréer (15 à 49 ans) en \%, Sétif 2014-2019

\begin{tabular}{|c|c|c|c|c|c|}
\hline Année & $\begin{array}{c}\text { Décès } \\
\text { maternels }\end{array}$ & $\begin{array}{l}\text { Naissances } \\
\text { vivantes }\end{array}$ & $\begin{array}{l}\text { Ratio Mortalité } \\
\text { Maternel }\end{array}$ & $\begin{array}{l}\text { Décès féminin 15- } \\
\text { 49ans }\end{array}$ & $\begin{array}{l}\text { Part des décès maternels sur } \\
\text { l'ensemble des décès** }\end{array}$ \\
\hline 2014 & 19 & 52318 & 36,3 & 280 & $6,8 \%$ \\
\hline 2015 & 26 & 52635 & 49,4 & 327 & $7,9 \%$ \\
\hline 2016 & 25 & 53815 & 46,4 & 350 & $7,1 \%$ \\
\hline 2017 & 26 & 53534 & 48,6 & 268 & $9,7 \%$ \\
\hline 2018 & 31 & 53338 & 58,1 & 294 & $10,5 \%$ \\
\hline 2019 & 14 & 53250 & 26,3 & 276 & $5,1 \%$ \\
\hline Total & 141 & 318890 & 44,2 & 1795 & $7,8 \%$ \\
\hline
\end{tabular}

* taux pour 100000 vivantes

** Part que représentent les décès maternels sur le total des causes de la mortalité féminine entre 15 et 49 ans 
Près d'un tiers $(33,3 \%)$ des décès maternels sont survenus chez des femmes âgées de 25-29 ans. Il faut noter que 7,1\% des femmes décédées avaient moins de 24 ans (Tableau 2).

Tableau 2. Répartition des décès maternels par groupes d'âges, Sétif 2014-2019

\begin{tabular}{lcc}
\hline $\begin{array}{l}\text { Groupes d'âge } \\
\text { (ans) }\end{array}$ & Décès maternels & Pourcentage \\
\hline$<20$ & 02 & $1,4 \%$ \\
\hline $20-24$ & 08 & $5,7 \%$ \\
\hline $25-29$ & 47 & $33,3 \%$ \\
\hline $30-34$ & 31 & $22 \%$ \\
\hline $35-39$ & 34 & $24,1 \%$ \\
\hline $40-44$ & 18 & $12,8 \%$ \\
\hline$\geq 45$ & 01 & $0,7 \%$ \\
\hline Tous âges & 141 & $100 \%$ \\
\hline
\end{tabular}

Au cours des deux périodes étudiées 2014-2016 et 2017-2019, la fréquence de la mortalité maternelle a augmenté pour les 25-39 ans. Elle a notablement et significativement diminué pour les 20-24 ans et les plus de 44 ans (Tableau 3).

Tableau 3. Répartition des décès maternels par groupes d'âges et par période, Sétif 2014-2016 et 2017-2019

\begin{tabular}{lcccc}
\hline $\begin{array}{l}\text { Période } \\
\text { (année) }\end{array}$ & \multicolumn{2}{c}{ 2014-2016 } & \multicolumn{2}{c}{ 2017-2019 } \\
\hline $\begin{array}{l}\text { Groupes } \\
\text { d'âge (ans) }\end{array}$ & Effectifs & $\%$ & Effectifs & $\%$ \\
\hline$<20$ & 02 & $2,8 \%$ & 00 & $0 \%$ \\
\hline $20-24$ & 05 & $7,2 \%$ & 03 & $4,2 \%$ \\
\hline $25-29$ & 23 & $32,8 \%$ & 24 & $33,8 \%$ \\
\hline $30-34$ & 14 & $20 \%$ & 17 & $23,9 \%$ \\
\hline $35-39$ & 15 & $21,5 \%$ & 19 & $26,8 \%$ \\
\hline $40-44$ & 10 & $14,3 \%$ & 08 & $11,3 \%$ \\
\hline$\geq 45$ & 01 & $1,4 \%$ & 00 & $0 \%$ \\
\hline Tous âges & $\mathbf{7 0}$ & $\mathbf{1 0 0} \%$ & $\mathbf{7 1}$ & $\mathbf{1 0 0} \%$ \\
\hline *chaque période comporte trois années & & &
\end{tabular}

\section{Wilaya de résidence}

Entre 2014-2019, 83\% des défuntes résidaient dans la wilaya de Sétif et $17 \%$ hors wilaya avec respectivement 7,1\% wilaya de Bordj Bou Arreridj, 6,4\% de M'sila et 3,5\% autres wilaya (Tableau 4). Il faut souligner que les femmes ne décèdent pas systématiquement là où elles résident.

Tableau 4. Répartition des décès maternels par Wilaya de résidence, 2014-2019

\begin{tabular}{lcc}
\hline Wilaya & Effectif & $\%$ \\
\hline Sétif & 117 & $83 \%$ \\
\hline Bordj Bou Arreridj & 10 & $7,1 \%$ \\
\hline M'sila & 09 & $6,4 \%$ \\
\hline Autres & 05 & $3,5 \%$ \\
\hline Total & $\mathbf{1 4 1}$ & $\mathbf{1 0 0 \%}$ \\
\hline
\end{tabular}

\section{Lieu du décès}

$79 \%$ des décès maternels ont eu lieu dans un établissement hospitalier public, $20 \%$ dans une clinique privée et seulement $1 \%$ à domicile.

\section{Voie d'accouchement}

Parmi les 125 femmes décédées pendant et après l'accouchement, plus de la moitié avaient accouché par voie basse et $44 \%$ avaient subi une césarienne.

\section{La parité}

La parité constitue un facteur étiologique important dans la mortalité maternelle. Dans notre étude la parité moyenne était de 1,48 $\pm 1,7$ avec des extrêmes allant de 0 à 10 pares. Nous avons classé les femmes décédées en 5 groupes : nullipares ( 0 accouchement), primipares ( 1 accouchement), paucipares ( 2 à 3 accouchements), multipares (4 à 6 accouchements) et grandes multipares (plus de 7 accouchements). La répartition de la parité des femmes décédées a montré que presque les deux tiers étaient des primipares et des paucipares. La distribution est présentée dans le Tableau 5.

Tableau 5. Répartition des décès maternels selon la parité, le moment du décès, Sétif 2014-2019

\begin{tabular}{lcc}
\hline & Effectifs & Pourcentages \\
\hline Parité & & \\
\hline Nullipares & 03 & $2,1 \%$ \\
\hline Primipares & 47 & $33,3 \%$ \\
\hline Paucipares & 48 & $34,1 \%$ \\
\hline Multipares & 35 & $24,8 \%$ \\
\hline Grandes multipares & 08 & $5,7 \%$ \\
\hline Total & $\mathbf{1 4 1}$ & $\mathbf{1 0 0 \%}$ \\
\hline Moment du décès & 10 & $7,1 \%$ \\
\hline $\begin{array}{l}\text { Au cours de } \\
\text { l'avortement }\end{array}$ & & \\
\hline $\begin{array}{l}\text { Au cours de la } \\
\text { grossesse* }\end{array}$ & 06 & $4,2 \%$ \\
\hline
\end{tabular}

\section{Pendant et après}

accouchement

$\begin{array}{lll}\leq 24 \text { heures } & 82 & 58,1 \% \\ >1 \mathrm{j} \leq 42 \mathrm{j} & 43 & 30,5 \%\end{array}$

\begin{tabular}{lcc}
\hline Total & 141 & $100 \%$ \\
\hline "Décès des mères n'ayant pas accouché &
\end{tabular}

"Décès des mères n'ayant pas accouché

\section{Moment du décès}

La période du post-partum à 42 jours, concentre $88,6 \%$ des décès maternels dont la moitié en post partum immédiat. $7,1 \%$ des décès maternels sont survenus au cours d'un avortement et $4,2 \%$ des femmes sont décédées enceintes (décès des mères n'ayant pas accouché) (Tableau 5). 


\section{Causes de décès}

Le Tableau 6 représente la distribution des causes de décès maternels entre. Les causes directes des décès maternels étaient les plus fréquentes (125 décès soit 88,7\%). L'hémorragie était la première cause de décès, responsable de 37,6 \% des décès maternels. La deuxième cause est représentée par les complications de l'hypertension artérielle retrouvée dans $22 \%$ des décès. Les embolies amniotiques se placent en troisième position $(15,6 \%)$. Il faut noter que toutes les embolies amniotiques n'ont pas été anatomiquement prouvées. Viennent ensuite les infections (5\%), les maladies thrombo-emboliques $(4,2 \%)$. On a noté également deux décès maternels dus aux exagérations des signes sympathiques.

Chez 16 patientes $(11,3 \%)$, le décès était lié à une cause obstétricale indirecte. Parmi ces décès, trois sont dus à des pathologies de l'appareil circulatoire, même effectif pour les décès par cancers, on a enregistré un décès du à un lupus.

Par ailleurs, chez quatre patientes le décès était inexpliqué. Malheureusement l'autopsie n'a pas été réalisée dans aucun de ces cas.

Tableau 6. Répartition des décès maternels selon la cause, Sétif 2014-2019

\begin{tabular}{lll}
\hline Causes & Effectifs & Pourcentages \\
\end{tabular}

Causes directes

\begin{tabular}{ccc}
\hline $\begin{array}{l}\text { Hémorragies } \\
\text { obstétricales }\end{array}$ & 53 & $37,6 \%$ \\
\hline $\begin{array}{l}\text { Hémorragies non } \\
\text { précisée }\end{array}$ & 26 & $18,4 \%$ \\
Rupture utérine & 19 & $13,5 \%$ \\
CIVD & 06 & $4,2 \%$ \\
Placenta prævia & 02 & $1,4 \%$ \\
\hline Complications & 31 & $22 \%$ \\
\hline d'hypertension & 26 & $18,4 \%$ \\
\hline Éclampsie & 05 & $3,5 \%$ \\
\hline HELLP syndrome & 22 & $15,6 \%$ \\
\hline Embolies amniotique & 07 & $5 \%$ \\
\hline Infection & 06 & $4,2 \%$ \\
\hline Thrombo-embolies & 04 & $2,8 \%$ \\
\hline $\begin{array}{l}\text { Embolies } \\
\text { pulmonaires }\end{array}$ & 02 & $1,4 \%$ \\
\hline $\begin{array}{l}\text { Thrombose veineuse } \\
\text { cébrale }\end{array}$ & 02 & $1,4 \%$ \\
\hline $\begin{array}{l}\text { Exagération des signes } \\
\text { sympathiques }\end{array}$ & 04 & $2,8 \%$ \\
\hline Inconnues & $\mathbf{1 2 5}$ & $\mathbf{8 8 , 7 \%}$ \\
\hline Total causes directes & & \\
\hline
\end{tabular}

Cause indirecte

\begin{tabular}{lcc}
\hline $\begin{array}{l}\text { Pathologies de l'appareil } \\
\text { circulatoire }\end{array}$ & 03 & $2,1 \%$ \\
Cancers & 03 & $2,1 \%$ \\
Lupus & 01 & $0,7 \%$ \\
\multicolumn{1}{c}{ Autres } & 09 & 6,4 \\
\hline Total cause indirecte & $\mathbf{1 6}$ & $\mathbf{1 1 , 3 \%}$ \\
& & \\
\hline Total toutes causes & $\mathbf{1 4 1}$ & $\mathbf{1 0 0 \%}$ \\
\hline
\end{tabular}

\section{DISCUSSION}

La présente étude a été basée essentiellement sur les données issues de l'audit des décès maternels de la wilaya de Sétif entre 2014 et 2019.

Le rapport de mortalité maternelle global dans la wilaya de Sétif est de 44,2 pour 100000 NV. L'évolution au fil des années a montré que le RMM le plus élevé été enregistré en 2018 soit 58,1 pour 100.000 NV suivi d'une diminution importante au cours de l'année 2019 (26,3 pour 100000 NV). Ceci peut s'expliquer par l'application de la loi n 18-11 du 2 juillet 2018 relative à la santé, obligeant dans son article 73 tous les professionnels de la santé à déclarer la femme enceinte et l'inscrire dès le troisième trimestre de grossesse, selon son choix, auprès d'une maternité publique ou privée. Nous constatons par ailleurs que ce RMM est inférieur à la moyenne nationale qui est de 60,3 pour $100000 \mathrm{NV}$ [5]. Notre RMM représente un cinquième $(1 / 5)$ du chiffre moyen dans les pays en voie de développement qui est de 230 pour $100000 \mathrm{NV}$ [6]. Il est plus élevé par rapport au pays développés [7]. D’une manière plus générale le RMM retrouvé dans notre étude nous place en position intermédiaire entre les pays développés et les pays en voie de développement.

L'âge des femmes est un facteur non contesté de risque de mort maternelle. Dans la littérature médicale, l'âge de la mère est reconnu comme l'un des facteurs les plus liés à la mortalité maternelle. En effet, selon l'OMS [8,9], les extrêmes d'âge présentent des risques accrus de décès. Contrairement, à notre étude, près d'un tiers $(33,3 \%)$ des décès maternels sont survenus chez des femmes âgées de 25 à 29 ans avec une moyenne d'âge de 32 ans $\pm 5,7$ ans. L'étude réalisée au niveau national par l'INSP en 2001 retrouve un âge moyen de 33 ans. Avec un maximum de décès entre 40 et 44 ans. En France, Entre 2010 et 2012, près de $30 \%$ des décès maternels sont survenus chez des femmes âgées de 35 à 39 ans avec un âge moyen de de 33,7 ans [10].

La parité constitue un facteur étiologique important dans la mortalité maternelle. L'OMS rappelle que les grandes multipares présentent un risque augmenté de mortalité et de morbidité maternelle. Inversement, dans notre étude on constate que presque les deux tiers des décès maternels sont des primipares et des paucipares. Alors que l'INSP en 2001a retrouvé un taux de $23,1 \%$ des décès chez les grandes multipares.

Parmi les 141 décès colligés, le décès maternel est survenu en post partum dans $88 \%$ des cas dont $58,1 \%$ dans les 24 premières heures et $30,5 \%$ dans les 42 jours ayant suivi l'accouchement. La revue de la littérature révèle de façon quasi-universelle le rôle prédominant de la période du post partum dans la survenue de décès maternels et la nécessité d'augmenter la durée de la surveillance pendant cette période avec renforcement de la surveillance des 24 premières heures après l'accouchement.

L'étude des décès maternels selon les étiologies est extrêmement importante, car elle nous permet d'élaborer des stratégies et des conduites à tenir pour les réduire dans la mesure du possible. Au cours de la période étudiée, les causes obstétricales directes $(88,7 \%)$ dominent largement les étiologies de décès. $80,2 \%$ de ces décès maternels sont attribués à quatre causes directes: les hémorragies obstétricales, les complications de l'hypertension artérielle, les embolies amniotiques et les infections.

Dans notre série, les hémorragies occupaient la première place et sont retrouvées dans $37,6 \%$ des décès maternels. 
Dans le dernier rapport du comité national d'experts sur la mortalité maternelle, elles constitueraient également la première cause de mortalité dans le monde et représentent $25 \%$ des causes de morts obstétricales directes [11]. La détermination d'un groupe de femmes à risque d'hémorragie doit faire partie de la prévention primaire et doit avoir comme objectif le dépistage des situations prévisibles d'accidents hémorragiques.

Les maladies hypertensives étaient à l'origine de 31 cas de décès maternels soit un taux de $22 \%$, occupant ainsi la seconde place derrière les hémorragies. On considère qu'au niveau mondial, les complications hypertensives sont responsables annuellement de $27 \%$ décès maternels, en particulier dans les pays en voie de développement [11]. Les rapports français et anglais estiment que $90 \%$ des décès liés aux complications hypertensives sont évitables et qu'elles sont dus à l'absence de surveillance durant la grossesse [12].

Les embolies amniotiques se placent en troisième position $(15,6 \%)$. Leur fréquence est difficile à chiffrer car le seul examen de certitude est histologique. Il faut noter que toutes les embolies amniotiques dans notre étude n'ont pas été anatomiquement prouvées.

Depuis l'avènement des antibiotiques, les décès maternels suite à une infection n'apparaissent presque plus dans les statistiques des pays développés. Malheureusement cette redoutable complication demeure parmi les premières causes de décès maternels dans les pays en développement et est responsable de $11 \%$ des décès maternels [11]. Dans notre étude, le taux était de $5 \%$.

\section{CONCLUSION}

Au terme de la présente étude, nous pouvons conclure que la mortalité maternelle reste relativement élevée en Algérie malgré les avancées en termes de législation et de programmes de réduction de la mortalité maternelle. Tous nos efforts doivent tendre à la faire diminuer, et ceci en recherchant la cause exacte des décès maternels.

De ce fait, l'adoption des nouvelles recommandations de l'OMS concernant d'abord la nouvelle classification qui comprend en effet, non plus deux (causes directes et causes indirectes), mais trois groupes de décès maternels incluant un nouveau groupe : les complications imprévues de la prise en charge. Ce troisième groupe permet de mieux cerner les causes iatrogènes. Ensuite, l'intégration d'une nouvelle entité dans les statistiques appelée: maternal near miss. Cette entité est définie comme toute femme ayant frôlé la mort mais ayant survécu suite à une complication survenue au cours de la grossesse, de l'accouchement ou dans les 42 jours qui ont suivi sa terminaison. Le but étant d'évaluer la qualité de soins fournis aux femmes enceintes [13].
Enfin, la création d'un comité d'experts sur la mortalité maternelle avec sa mise en place au niveau de chaque wilaya. Il sera composé d'experts gynécologues-obstétriciens, anesthésistes, épidémiologistes et sages-femmes. Il aura pour objectif l'expertise des décès maternels en évitables ou non évitables dans une perspective de prévention.

Déclaration d'intérêts: les auteurs ne déclarent aucun conflit d'intérêt en rapport avec cet article.

\section{REFERENCES}

1. Mortalité maternelle, bilan statistique. 2008 Sept;(7):1-2.

2. OMS, UNICEF, UNFPA, Groupe de la Banque mondiale et Division de la population des Nations Unies. Tendances de la mortalité maternelle : 19902015. Estimations de l'OMS, de I'UNICEF, de I'UNFPA, du Groupe de la Banque mondiale et de la Division de la population des Nations Unies. Genève 2015 :1-3.

3. Ministère de la Santé de la Population et de la Réforme Hospitalière d'Algérie. Direction Générale de la Prévention et de la Promotion de la Santé. PLAN NATIONAL DE REDUCTION DE LA MORTALITE MATERNELLE 20152019:13-14.

4. Classification statistique internationale des maladies et des problèmes de santé connexes. Dixième Révision. Genève, Organisation mondiale de la Santé.2008;2:179-180.

5. Abrouk $S$ et al : Caractéristiques des décès maternels au cours du 2ème semestre 2014. Institut national de Santé Publique, Direction Générale de la Prévention et de la Promotion de la Santé.2015;(19).

6. Organisation Mondiale de la Santé. Mortalité Maternelle. Aide-mémoire. 2016 Nov;(348).

7. Maternal Mortality in 2000 : Estimates Developed by WHO, UNICEF and UNFPA. World Health Organization. Genève. 2004;:28-29.

8. Ban Ki-moon. Objectifs du Millénaire pour le développement, Rapport. Nations unies New York. 2015;:75.

9. OMS, Sous-estimation de la mortalité maternelle. Communiqué OMS Genève. 1996 Fév 5;:1-2.

10. Marianne $P$, Fabien $B$, Marie-Hélène $B C$. Epidémiologie de la mortalité maternelle en France, de 1996 à 2002 : fréquence, facteurs et causes. BEH. 2006 Déc 12;(50) :392-395.

11. Say L, Chou D, Gemmill A, Tunçalp Ö, Moller AB, Daniels JD, et al. Global Causes of Maternal Death: A WHO Systematic Analysis Lancet Global Health. 2014;2(6):323-333.

12.André Benbassa et al. CNEMM : La mortalité maternelle en France : considérations épidémiologiques et cliniques (1999 - 2001) et recommandations. BEH. 2006 Déc 12; (50):396-399.

13. Jerbi M. La mortalité maternelle en question. Journal de Gynécologie Obstétrique et Biologie de la Reproduction. 2010;(39):173-174.

Cet article a été publié dans le « Batna Journal of Medical Sciences » BJMS, l’organe officiel de « l'association de la Recherche Pharmaceutique - Batna»

Le contenu de la Revue est ouvert «Open Access » et permet au lecteur de télécharger, d'utiliser le contenu dans un but personnel ou d'enseignement, sans demander l'autorisation de l'éditeur/auteur.

Avantages à publier dans BJMS :

- Open access : une fois publié, votre article est disponible gratuitement au téléchargement

- Soumission gratuite : pas de frais de soumission, contrairement à la plupart des revues « Open Access »

- Possibilité de publier dans 3 langues : français, anglais, arabe

Qualité de la relecture : des relecteurs/reviewers indépendants géographiquement, respectant l'anonymat, pour garantir

la neutralité et la qualité des manuscrits.

Pour plus d'informations, contacter BatnaJMS@gmail.com ou connectez-vous sur le site de la revue : www.batnajms.net 


\section{Bis}

- $\bigcirc$

- 0

P

○

${ }^{1}$ Laboratoire de biochimie. Faculté de médecine Benyoucef Benkheda Alger-1 - Algérie

2EHS CPMC, Alger - Algérie

\section{Correspondance à :}

Nawal HABAK

nawal.bioch@gmail.com

DOI :https://doi.org/10.48087/ BJMSoa.2020.7208

\section{Historique de l'article :}

Reçu le 18 juin 2020

Accepté le 16 septembre 2020

Publié le 09 novembre 2020

Il s'agit d'un article en libre accès distribué selon les termes de la licence Creative Commons Attribution International License (CC BY 4.0), qui autorise une utilisation, une distribution et une reproduction sans restriction sur tout support ou format, à condition que l'auteur original et la revue soient dûment crédités.

\section{Pour citer l'article :}

Habak N, Bouaichi D, Abbache $\mathrm{H}$, et al. Intérêt du jeûne dans l'exploration du bilan lipidique. Batna J Med Sci 2020;7(2):102-4. https://doi.org/10.48087/B] MSoa.2020.7208

\title{
Intérêt du jeûne dans l'exploration du bilan lipidique
}

\author{
Interest of fasting in the exploration of the lipid balance
}

\author{
Nawal Habak, Dihia Bouaichi, Hanifa Abbache, Nadia OuldBessi, Ammar \\ Chikouche.
}

\section{RÉSUMÉ}

Introduction. Le bilan lipidique correspond à un ensemble d'analyses permettant de mettre en évidence des anomalies du métabolisme des lipides et d'optimiser la prise en charge diététique et/ou le besoin thérapeutique pour le suivi, la surveillance et l'évaluation du risque athérogène. L'objectif de cette étude est d'évaluer la place du jeûne dans les recommandations pré analytiques du dosage des paramètres lipidiques. Matériels et Méthodes. Notre étude prospective a été réalisée sur 27 prélèvements sanguins sur tube hépariné, des patients en deux temps : à jeun et non à jeun après la prise d'un repas consistant. Le dosage des deux paramètres : Triglycérides et le Cholestérol total, ont été réalisé par la méthode colorimétrique enzymatique. Résultats. Nos résultats ont été classés en deux groupes pour chaque paramètre. L'étude statistique a été réalisée sur le logiciel Graph Pad Prism7. La comparaison des deux groupes TG par l'application du test non paramétrique de Wilcoxon retrouve $\mathrm{p}<0,0001$, ce qui signifie la présence d'une différence significative entre les deux groupes. La comparaison des deux groupes Chol par l'application du test paramétrique test $\mathrm{T}$ retrouve $\mathrm{p}<0,0001$, ce qui signifie la présence d'une différence significative entre les deux groupes. Conclusion. À travers cette étude, bien qu'elle soit réalisée sur un faible échantillonnage, nous permet de souligner l'importance du jeûne dans l'analyse des paramètres du bilan lipidique, ce qui concorde avec plusieurs travaux de la littérature qui ont permis d'instaurer le jeûne dans les recommandations lors de l'exploration du métabolisme lipidique.

Mots clés : cholestérol, triglycérides, jeûne.

\section{INTRODUCTION}

Les lipides sont des macromolécules ayant une propriété commune d'être relativement insolubles dans l'eau et solubles dans les solvants organiques apolaires (1).

Ces substances hydrophobes sont véhiculées dans le sang sous forme de structures micellaires solubles, résultant de l'association de lipides et de protéines (Apolipoprotéines), appelés les lipoprotéines, exception faite pour les acides gras non estérifiés transportées par l'albumine $(2,3)$.

L'étude des lipides est dominée par un groupe de pathologies hétérogènes appelé dyslipoprotéinémies. La plupart de ces dyslipémies sont athérogènes avec formation de la plaque d'athérome d'où l'intérêt de les dépister précocement par des examens biologiques simples, pour une prise en charge diététique et/ou thérapeutique adaptée (4-6).

\section{ABSTRACT}

Introduction. A lipid profil corresponds to a set of analyses to identify abnormalities in lipid metabolism to optimize dietary management and/or therapeuticand evaluation of atherogenic risk. The aim of this study is to evaluate the place of the fastingin the pre-analytical recommendations of the dosage of lipid parameters. Materials and Methods. Our prospective study was carried out on 27 blood samples of patients over two stages: fasting and not fasting after taking a consist snack. The dosage of the two parameters TG and Chol were achieved by the enzymatic color method. Results. Our results were categorized into two groups for each parameter: The statistical study was conducted on Graph Pad Prism7 software. The comparison of the two TG groups by the application of the Wilcoxon nonparametric test found $\mathrm{p}<0.0001$, which means significant difference between the two groups. The comparison of the two Chol groups by the application of the T-test parametric test is found in the two groupsp $<0.0001$, which means significant difference between the two groups. Conclusion. Through this study, although it is carried out on a low sample, allows us to underline the importance of fasting in the analysis of lipid profil which is steady with several works in the literature that have allowed fasting in recommendations when exploring lipid metabolism.

Keywords : cholesterol, triglycerides, fasting.

Le bilan lipidique correspond à un ensemble d'analyses permettant de mettre en évidence des anomalies du métabolisme des lipides et d'optimiser la prise en charge, le suivi, la surveillance et l'évaluation $\mathrm{du}$ risque athérogène (7).

Ce bilan regroupe le dosage du cholestérol total (Chol), HDL cholestérol (high-density lipoprotein), LDL cholestérol (Low Density Lipoprotein), les triglycérides (TG) et les apolipoprotéines (8).

Il est recommandé d'effectuer l'analyse après un jeûne de $12 \mathrm{~h}$, situation difficile à supporter particulièrement chez les enfants, les personnes âgées et les diabétiques.

L'objectif de notre travail est d'évaluer l'impact du jeûne sur le bilan lipidique en comparant deux paramètres: le cholestérol et les Triglycérides à jeun et non à jeun. 


\section{MATÉRIELS ET MÉTHODES}

Il s'agit d'une étude prospective dans laquelle nous avons inclus des patients ne présentant aucune anomalie connue du bilan lipidique, absence de diabète ou d'anomalie thyroïdienne ; et ils n'ont déclaré aucune prise thérapeutique pouvant affecter les paramètres lipidiques.

Les patients consentis ont été prélevés sur deux temps à jeun et non à jeun après 2 heure de la prise d'un repas consistant.

Les échantillons de sang ont été prélevés sur tube hépariné, dans la même journée à jeun puis non à jeun (après 2 heure de temps de la prise d'un petit déjeuner consistant) chez tous les patients.

Le dosage du cholestérol et des triglycérides a été réalisé par les méthodes enzymatiques colorimétriques (Trinder) pour les deux temps de prélèvement.

L'étude statistique a été réalisée sur le logiciel Graph Pad Prism7.

\section{RÉSULTATS ET DISCUSSION}

Nous avons colligé 27 patients, dont $44.44 \%$ hommes (12 patients) et $55.55 \%$ femmes (15 patientes) avec un sex-ratio de 0,8 . Les critères d'inclusion et le consentement des patients ont influencé le nombre d'échantillon recueilli.

Nos résultats ont été classés en deux groupes pour les deux paramètres :

- Triglycérides : pour le groupe 1 à jeun : la médiane était à $1.46 \mathrm{~g} / \mathrm{l}$ et pour le groupe 2 non à jeun : la médiane était à $1.69 \mathrm{~g} / \mathrm{l}$. (Tableau.1)

Tableau 2 . Taux des TG à jeun et non à jeun.

\begin{tabular}{|c|c|c|c|}
\hline Patients & À jeun & Non à jeun & $\begin{array}{c}\text { Variation } \\
\text { TG non à jeun - TG à jeun } \\
\end{array}$ \\
\hline 1 & 0,759 & 0,831 & 0.072 \\
\hline 2 & 0,99 & 1,136 & 0.146 \\
\hline 3 & 1,515 & 1,822 & 0.307 \\
\hline 4 & 4,669 & 4,83 & 0.161 \\
\hline 5 & 1,94 & 1,984 & 0.044 \\
\hline 6 & 1,706 & 1,782 & 0.076 \\
\hline 7 & 1,524 & 1,812 & 0.288 \\
\hline 8 & 1,667 & 1,97 & 0.303 \\
\hline 9 & 0,822 & 0,833 & 0.011 \\
\hline 10 & 1,09 & 1,197 & 0.107 \\
\hline 11 & 1,334 & 1,475 & 0.141 \\
\hline 12 & 0,564 & 0,569 & 0.005 \\
\hline 13 & 0,596 & 0,68 & 0.084 \\
\hline 14 & 1,049 & 1,063 & 0.014 \\
\hline 15 & 1,119 & 1,26 & 0.141 \\
\hline 16 & 0,573 & 0,7 & 0.127 \\
\hline 17 & 1,466 & 1,694 & 0.228 \\
\hline 18 & 3,601 & 3,956 & 0.355 \\
\hline 19 & 1,953 & 1,972 & 0.019 \\
\hline 20 & 2,494 & 2,611 & 0.117 \\
\hline 21 & 1,162 & 1,253 & 0.091 \\
\hline 22 & 1,99 & 2,124 & 0.134 \\
\hline 23 & 1,433 & 1,498 & 0.065 \\
\hline 24 & 0,97 & 0,998 & 0.028 \\
\hline 25 & 4,394 & 4,58 & 0.186 \\
\hline 26 & 2,511 & 2,726 & 0.215 \\
\hline 27 & 2,33 & 2,45 & 0.12 \\
\hline
\end{tabular}

- Cholestérol : pour le groupe 1 à jeun : la médiane était de 1.61 et pour le groupe 2 : non à jeun la médiane était de1.78 g/l. (Tableau. 2).
Tableau 1. Taux du cholestérol à jeun et non à jeun.

\begin{tabular}{|c|c|c|c|}
\hline Patients & À jeun & Non à jeun & $\begin{array}{c}\text { Variation } \\
\text { Non à jeun - À jeun }\end{array}$ \\
\hline 1 & 2,571 & 1,787 & $-0,784$ \\
\hline 2 & 1,617 & 1,784 & 0,167 \\
\hline 3 & 0,874 & 0,84 & $-0,034$ \\
\hline 4 & 2,083 & 2,178 & 0,095 \\
\hline 5 & 1,607 & 1,662 & 0,055 \\
\hline 6 & 1,335 & 1,357 & 0,022 \\
\hline 7 & 1,731 & 1,795 & 0,064 \\
\hline 8 & 1,068 & 1,109 & 0,041 \\
\hline 9 & 1,452 & 1,446 & $-0,006$ \\
\hline 10 & 1,607 & 1,705 & 0,098 \\
\hline 11 & 1,403 & 1,34 & $-0,063$ \\
\hline 12 & 1,088 & 1,126 & 0,038 \\
\hline 13 & 2,387 & 2,482 & 0,095 \\
\hline 14 & 1,244 & 1,171 & $-0,073$ \\
\hline 15 & 1,862 & 1,988 & 0,126 \\
\hline 16 & 1,29 & 1,315 & 0,025 \\
\hline 17 & 2,012 & 2,032 & 0,02 \\
\hline 18 & 2,671 & 2,586 & $-0,085$ \\
\hline 19 & 2,498 & 2,518 & 0,02 \\
\hline 20 & 3,019 & 3,117 & 0,098 \\
\hline 21 & 2,396 & 2,448 & 0,052 \\
\hline 22 & 2,512 & 2,68 & 0,168 \\
\hline 23 & 2,476 & 2,534 & 0,058 \\
\hline 24 & 1,314 & 1,325 & 0,011 \\
\hline 25 & 3,311 & 3,384 & 0,073 \\
\hline 26 & 1,44 & 1,49 & 0,05 \\
\hline 27 & 1,48 & 2,122 & 0,642 \\
\hline
\end{tabular}

Nous avons procédé à une comparaison de deux variables quantitatives appariées. La vérification de la normalité a été réalisée par le test de Pearson qui a retrouvé une distribution qui ne suit pas la loi normale pour les triglycérides $(\mathrm{p}=0.0015 ; \mathrm{p}=0.0029)$, contrairement au cholestérol $(\mathrm{p}=0$. $48, \mathrm{p}=0.34$ )

La comparaison des deux groupes cholestérol (à jeun, non à jeun) par l'application du test paramétrique test $\mathrm{T}$ retrouve $(\mathrm{p}<0,0001)$, ce qui signifie présence d'une différence significative entre les deux groupes (Figure 1).

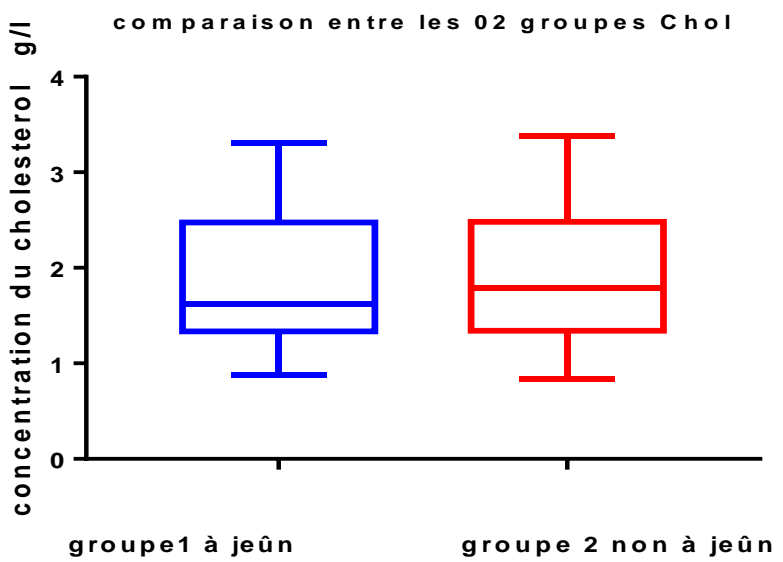

Figure 1. Comparaison du taux de cholestérol entre les deux groupes à jeun et non à jeun 
La comparaison des deux groupes triglycérides (à jeun, non à jeun) par l'application du test non paramétrique de Wilcoxon retrouve $\mathrm{p}<0,0001$, ce qui signifie présence d'une différence significative entre les deux groupes (Figure 2).

\section{Test de Wilcoxon comparaison entre les $\mathbf{0 2}$ groupes: dosage des triglycérides}

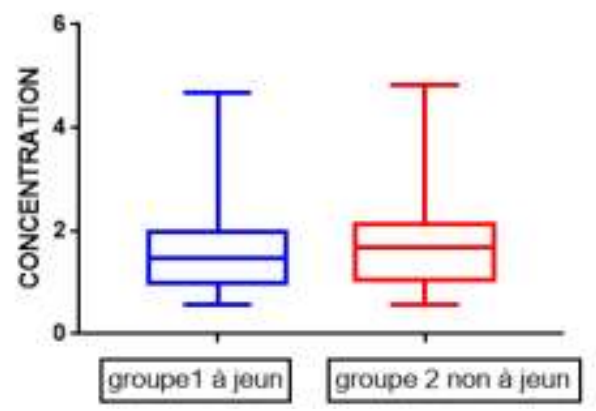

Figure 1. Comparaison du taux des triglycérides entre les deux groupes à jeun et non à jeun

Plusieurs travaux se sont intéressés à évaluer l'intérêt de cette recommandation pré-analytique de jeûne au moment du prélèvement pour le dosage du bilan lipidique.

L'étude récente de Nordestgrad et al ainsi que celle de Bensal et al ont montré que le jeûne n'a aucune influence sur le dosage des triglycérides et du cholestérol total, HDL cholestérol, LDL cholestérol $(9,10)$.

Cependant, d'autres auteurs ont souligné que les équations d'évaluation du risque cardiovasculaire ont été élaborées sur des sujets à jeun depuis $12 \mathrm{~h}$ (11).

Du point de vue de la physiologie post prandiale, l'étude réalisée en 2017 par Couderc et ala mis l'accentsur la présence des échanges et des transferts delipides entre les lipoprotéines, ainsi après un repas normal, la concentration en LDL cholestérol est abaissée (en moyenne de 0,2mmol/L ou $0,08 \mathrm{~g} / \mathrm{L}$, et la présence de chylomicrons entraîneune erreur par défaut lors du calcul du LDL-cholestérol par l'équation de Friedewald (12).

L'équipe de Nordestgrad en 2016 ont suggéré l'obligation du jeûne dans certaines situations cliniques,surtout chez les personnes avec des dyslipidémies primitives; alors que dans d'autres situations, un bilan non à jeun peut être toléré, en particulier en cas d'hospitalisation pour un syndrome coronaire aigu ou chez l'enfant (9).

\section{CONCLUSION}

Par ce modeste travail et malgré le nombre faible d'échantillon inclus, nos résultats retrouvent une différence significative des concentrations pour les 2 paramètres cholestérol et triglycérides entre les 2 groupes à jeun et non à jeun; ce qui rejoignent les recommandons du dosage après un jeûne de $12 \mathrm{~h}$.

La surveillance des paramètres lipidiques permet de prévenir les maladies cardiovasculaires. Soit pour une prévention primaire, chez les personnes n'ayant jamais eux d'accidents cardiovasculaires, ou une prévention secondaire, chez les personnes ayant déjà fait un accident cardiovasculaire.
La mise en évidence précoce d'une dyslipidémie, permet d'instaurer des mesures diététiques et/ou thérapeutiques; ainsi il est actuellement retenu que le jeune de $12 \mathrm{~h}$ est recommandé dans l'exploration du bilan lipidique, exception faite dans certaines circonstances (enfants, personnes âgées, ...)

DECLARATION D'INTERETS : les auteurs ne déclarent aucun conflit d'intérêt en rapport avec cet article.

\section{RÉFÉRENCES}

1. Armand $M$, Pasquier $B$, Borel $P$, Andre $M$, Senft $M$, Peyrot $J$, et al. Emulsion et absorption des lipides: importance des proprietes physicochimiques. OCL-Oleagineux-Corps Gras-Lipides. 1997;4(3):178-84.

2. Hassan N, Jean-Marie B. Actualités sur le métabolisme des lipoprotéines plasmatiques. Médecine thérapeutique / Endocrinologie. 2002;4(4):194 8.

3. Gautier T, Masson D, Lagrost L. Métabolisme des lipoprotéines de haute densité (HDL). Archives of Cardiovascular Diseases Supplements. 2011;3(4):267-72.

4. Serraj K, Hamaz S, Alaoui H, Bachir H, Andres E. Les dyslipidémies: grands principes et approche simplifiée. Médecine thérapeutique. 2016;22(5):307-11

5. Rodondi N, Waeber G, editors. Dyslipidémies: comment utiliser les nouvelles recommandations au cabinet médical? Forum Médical Suisse; 2018: EMH Media.

6. Miller M, Stone NJ, Ballantyne C, Bittner V, Criqui MH, Ginsberg HN, et al. Triglycerides and cardiovascular disease: a scientific statement from the American Heart Association. Circulation. 2011;123(20):2292-333.

7. Linton MF, Yancey PG, Davies SS, Jerome WG, Linton EF, Song WL, et al. The role of lipids and lipoproteins in atherosclerosis. Endotext [Internet] MDText. com, Inc.; 2019.

8. Lima-Oliveira G, Volanski W, Lippi G, Picheth G, Guidi GC. Pre-analytical phase management: a review of the procedures from patient preparation to laboratory analysis. Scandinavian Journal of Clinical and Laboratory Investigation. 2017;77(3):153-63.

9. Nordestgaard BG, Langsted A, Mora S, Kolovou G, Baum H, Bruckert E, et al. Fasting is not routinely required for determination of a lipid profile: clinical and laboratory implications including flagging at desirable concentration cut-points-a joint consensus statement from the European Atherosclerosis Society and European Federation of Clinical Chemistry and Laboratory Medicine. European Heart Journal. 2016;37(25):1944-58.

10. Bansal S, Buring JE, Rifai N, Mora S, Sacks FM, Ridker PM. Fasting compared with nonfasting triglycerides and risk of cardiovascular events in women. Jama. 2007;298(3):309-16.

11. Langsted A NB. Nonfasting lipids, lipoproteins, and apolipoproteinsin individuals with and without diabetes: 58434 individuals from the CopenhagenGeneral Population Study. Clin Chem. 2011;57:482-489.

12. Couderc R, Antar M, Bonnefont-Rousselot $\mathrm{D}$, Paul J-L, Therond P, editors. Le bilan lipidique en 2017. Annales de biologie clinique; 2017. 


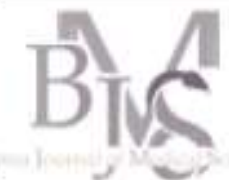

- $\bigcirc$

- 1

Service d'ophtalmologie pédiatrique, Établissement Hospitalier Spécialisé (EHS) « Canastel », Oran - Algérie.

\section{Correspondance à :}

Kheira KERROUCHE

ophtalmo kerrouche@yahoo.fr

DOI :https://doi.org/10.48087/ BJMSoa.2020.7209

\section{Historique de l'article :}

Reçu le 23 juin 2020

Accepté le 15 septembre 2020

Publié le 09 novembre 2020

Il s'agit d'un article en libre accès distribué selon les termes de la licence Creative Commons Attribution International License (CC BY 4.0), qui autorise une utilisation, une distribution et une reproduction sans restriction sur tout support ou format, à condition que l'auteur original et la revue soient dûment crédités.

\section{Pour citer l'article :}

Kerrouche K, Beldjilali S, Hafoudi M. Les uveites de l'enfant « à propos de 62 cas ». Batna J Med Sci 2020;7(2):105-8. https://doi.org/10.48087/ B]MSoa.2020.7209

\title{
Les uvéites de l'enfant «à propos de 62 cas »
}

\author{
Children's uveitis "about 62 cases
}

\author{
Kheira Kerrouche, Said Beldjilali, Mabrouk Hafoudi
}

\section{RÉSUMÉ}

Objectif. Était de décrire les caractéristiques épidémiologiques, cliniques, étiologiques et évolutives des uvéites pédiatriques reçus au service d'ophtalmologie EHS Canastel Oran et de souligner leur impact sur le pronostic fonctionnel. Matériel et méthodes. Il s'agit d'une étude descriptive, rétrospective sur une période de 09 ans, du 1er janvier 2011 au 31 décembre 2019. Elle regroupe tous les cas d'uvéites ayant nécessité l'hospitalisation. Tous les enfants ont bénéficié d'un bilan ophtalmologique complet, d'un bilan étiologique et d'un suivi régulier allant de 06 mois à 6 ans. Résultats. Au total 62 cas d'uvéites ont été recensés, avec une moyenne de 7 cas/an. L'âge moyen des patients est de 10 ans avec un sex-ratio 1,5. La cause inflammatoire était prépondérante dominée par l'arthrite juvénile idiopathique et l'HLA B27, 37\% des cas étaient idiopathiques, alors que l'étiologie infectieuse n'était présente que dans $27,5 \%$ cas. L'atteinte était bilatérale dans $60 \%$ des cas, avec une prédominance des localisations antérieure et intermédiaire (62\%). En plus du traitement spécifique antibiotique et antiviral dans les formes infectieuses l'ensemble des patients ont reçu une corticothérapie par voie locale et générale adaptée à chaque étiologie. Les complications oculaires ont été retrouvées dans $43,5 \%$ des cas, dominées par l'hypertonie dans $30 \%$, la cataracte dans $13 \%$. L'acuité visuelle finale était inférieure à $1 / 10$ dans $25 \%$, chez 6 cas au niveau des deux yeux. Conclusion. Les caractéristiques des uvéites pédiatriques diffèrent d'une étude à une autre, cependant certaines particularités retrouvées dans notre étude sont habituellement décrites dans la plupart des séries à savoir, la fréquence des formes idiopathiques et celle liée à l'arthrite chronique juvénile ainsi que des complications potentiellement cécitantes qui sont secondaires à l'inflammation et au traitement.

Mots clés : uvéite, enfant, étiologie, complication.

\section{INTRODUCTION}

L'uvéite de l'enfant est une affection souvent grave, heureusement rare ne représentant que 3 à $10 \%$ de l'ensemble des uvéites [1,2]. Sa gravité réside dans sa présentation souvent insidieuse "silencieuse » l'enfant exprime peu les troubles fonctionnelles conduisant fréquemment à sa découverte au stade de complications [3].

La démarche diagnostique étiologique devant une uvéite pédiatrique doit être bien codifiée et urgente. Elle est guidée par un interrogatoire précis, un examen ophtalmologique et général bien conduits qui orientent et canalisent le bilan para clinique. Quant au traitement, il est d'abord étiologique, il fait appel souvent à la corticothérapie locale et générale avec ses

\section{ABSTRACT}

Aim. Was to describe the epidemiological, clinical, etiological and evolutionary characteristics of pediatric uveitis received at the Ophthalmology Department of Canastel Hospital Oran and to highlight their impact on functional prognosis. Methods. This is a descriptive study, retrospective over a period of 09 years, from January 1, 2011 to December 31, 2019. It includes all cases of uveitis requiring hospitalization. All the children received a complete ophthalmological check-up, an etiological assessment and regular follow-up from 06 months to 6 years. Results. A total of 62 cases of uveitis were recorded, with an average of 7 cases/year. The average age of the patients is 10 years with a sex ratio of 1.5. The inflammatory cause was dominated by juvenile idiopathic arthritis and HLA B27, 37\% of cases were idiopathic, while the infectious etiology was present in only $27.5 \%$ of cases. Affection was bilateral in $60 \%$ of cases, with a predominance of anterior and intermediate localizations (62\%). In addition to specific antibiotic and antiviral treatment for the infectious forms, all patients received local and general corticosteroid therapy adapted to each etiology. Ocular complications were found in $43.5 \%$ of cases, dominated by hypertonia in $30 \%$, cataract in $13 \%$. Final visual acuity was less than $1 / 10$ in $25 \%$, in 6 cases in both eyes. Conclusion. The characteristics of pediatric uveitis differ from one study to another, however certain particularities found in our study are usually described in most series, namely, the frequency of idiopathic forms and that related to chronic juvenile arthritis as well as potentially blinding complications that are secondary to inflammation and treatment.

Key words : uveitis, child, etiology, complication.

nombreux effets secondaires sur un organisme en pleine croissance.

\section{Objectif}

Notre objectif était de décrire le profil épidémiologique et clinique des patients présentant une uvéite, hospitalisés dans notre service.

\section{PATIENTS ET MÉTHODES}

Nous avons effectué une étude descriptive, rétrospective sur une période de 09 ans, du 1er janvier 2011 au 31 décembre 2019.

Elle regroupe tous les cas d'uvéites ayant nécessité l'hospitalisation au service d'ophtalmologie pédiatrique (EHS Canastel d'Oran) durant cette période. Tous les patients ont bénéficié d'un bilan ophtalmologique complet comprenant : 
Un interrogatoire minutieux des parents et de l'enfant (si son âge le permet) à la recherche des antécédents récents ou anciens d'atteinte infectieuse ou inflammatoire systémique sans oublier de consulter son carnet de santé.

Un examen ophtalmologique complet bilatéral et symétrique débutant par l'évaluation de l'acuité visuelle de loin et de près de chaque œil après une bonne correction optique, un examen du segment antérieur et du fond œil avec une prise du tonus oculaire.

Des explorations para cliniques ophtalmologiques orientées en fonction de la forme anatomique de l'uvéite et de la transparence des milieux: une angiographie fluorosceinique, une tomographie en cohérence optique (OCT), une échographie oculaire en mode B.

Un bilan étiologique de base comprenant : une formule de numération sanguine (FNS), une C réactive protéine (CRP), une vitesse de sédimentation (VS), une électrophorèse des protéines, des sérologies (de la syphilis, la toxoplasmose, la toxocarose), une intradermoréaction à la tuberculine, un télethorax et un examen clinique pédiatrique.

Le complément du bilan étiologique est orienté par l'anamnèse et les données de l'examen clinique. Les bilans spécifiques qui ont été demandé dans notre série sont les anticorps antinucléaires, le facteur rhumatoïde, l'inhibiteur de l'enzyme de conversion, calcémie la calciurie, l'étude cytologique et biochimique du LCR, l'IRM cérébrale et médullaire, le quantiféron, la biopsie des glandes salivaires accessoires, l'audiogramme et le typage HLAB27.

Avant de commencer le traitement une évaluation de la glycémie de la fonction rénale et un ionogramme est demandé.

Le traitement est d'abord symptomatique local faisant appels aux collyres dilatateurs (mydriaticum ou atropine) et les anti-inflammatoires locaux (collyre, sous conjonctivale et sous ténonienne) associés selon les cas à des collyres hypotonisants. Le traitement général est adapté à l'étiologie retrouvée. Dans l'uvéite infectieuse l'utilisation d'une antibiothérapie associée secondairement à la corticothérapie systémique alors que cette dernière est systématique dans les autres formes d'uvéites.

Le suivi est régulier et à long terme, le rythme est adapté à la forme de l'uvéite à son caractère chronique et ou récurrent.

\section{RÉSULTATS}

Sur une période de 09 ans, allant de 2011 à 2019, nous avons hospitalisé et pris en charge dans notre service d'ophtalmologie pédiatrique de l'EHS de Canastel, 62 cas d'uvéite avec une moyenne annuelle de 7 cas par an.

La moyenne la plus élevée était de 11 cas enregistrés durant l'année 2019. Nous avons noté aussi que $64 \%$ des cas ont été enregistrés en fin d'automne et durant l'hiver.

L'âge moyen des patients était de 10 ans (allant de 4 à 16 ans), Quarante pourcent des patients avaient un âge entre 9 et 12 ans. La sex-ratio est de 1,5.

L'acuité visuelle à l'admission variée entre voir bougée la main et $4 / 10$.
Les données cliniques ont montré que l'atteinte était bilatérale dans $62 \%$ des cas. L'uvéite antérieure représentait $32 \%$, l'intermédiaire $30,6 \%$ tandis que la panuvéite représentait $22,5 \%$ selon le tableau ${ }^{\circ} 1$.

La forme non granulomateuse et synéchiante représentait $78 \%$.

Tableau 1. Répartition suivant le type anatomique des uvéites

\begin{tabular}{ll}
\hline Type anatomique & $\mathrm{N}(\%)$ \\
\hline Uvéite antérieure & $20(32)$ \\
\hline Uvéite intermédiaire & $19(30,6)$ \\
\hline Panuvéite & $14(22,5)$ \\
\hline Uvéite postérieure & $9(0,14)$ \\
\hline
\end{tabular}

Le bilan étiologique est revenu négatif chez 23 cas (37\%) concluant en une forme idiopathique alors que l'étiologie infectieuse a représenté 27,5\% (17 cas) dominée par la toxoplasmose et l'herpès. La cause inflammatoire était présente dans 35,5\% (22 cas) dominée par l'arthrite chronique (AJI) et la spondylarthropathie. La figure $\mathrm{n}^{\circ} 1$ résume la répartition des différentes étiologies de l'uvéite de notre série.
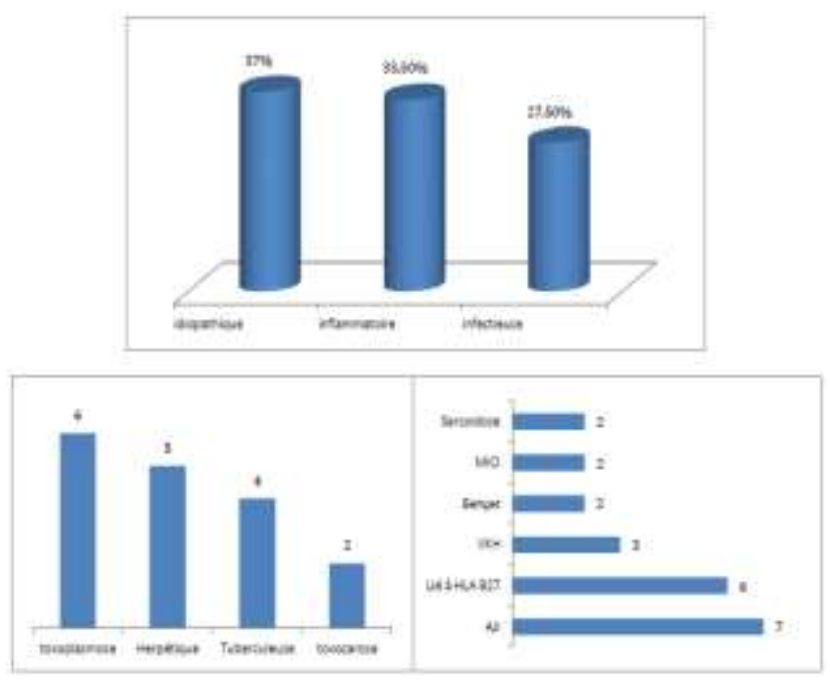

Uvéites infectieuses

Uvéites inflammatoires AJl : arthrite juvénile idiopathique. VKH : maladie de Vogt Koyanagi Harada, $\mathrm{MICl}$ : maladies inflammatoires chroniques intestinales.

Figure 1. Répartition des différentes étiologies de l’uvéite

Quarante trois pourcent de l'ensemble des patients soit 27 cas ont présenté des complications à l'admission et au cours de leur suivi dont neuf patients d'entre eux ont présenté plus de deux complications c'est-à-dire une cataracte associée à une hypertonie et un œdème maculaire.

Trente pourcents de l'ensemble des patients soit 19 cas ont eu une hypertonie oculaire dont 09 cas aucours d'une uvéite antérieure, 18\% (11 cas) ont présenté une cataracte, 11\% (7 cas) ont été compliqués d'un œdème maculaire.

Le tableau $\mathrm{n}^{\circ} 2$ détaille la répartition des complications dans les différents types anatomiques d'uvéite (U): 
Tableau 2. Complications oculaires dans les différents types anatomiques d'uvéite

\begin{tabular}{llllll}
\hline Type d'uvéite & U. antérieure & U. intermédiaire & Panuvéite & U. postérieure & Total \\
& N (\%) & N (\%) & N (\%) & N (\%) & N (\%)
\end{tabular}

Complications

\begin{tabular}{llllll}
\hline Hypertonie oculaire & $9(14)$ & $5(8)$ & $5(8)$ & 0 & $19(30)$ \\
Cataracte & $6(32)$ & $3(5)$ & $2(3,2)$ & 0 & $11(18)$ \\
OEdème maculaire & 0 & $5(8)$ & $1(1,6)$ & $1(1,6)$ & $7(11)$ \\
Décollement de rétine & 0 & 0 & $1(1,6)$ & 0 & $1(1,6)$ \\
Kératite en bandelette & $3(5)$ & 0 & 0 & 0 & $3(5)$ \\
\hline
\end{tabular}

L'évolution de l'acuité visuelle sous traitement a montré qu'un quart des patients ont une acuité visuelle finale inférieure à $1 / 10$ ème.

La figure $\mathrm{n}^{\circ} 2$ montre la répartition des acuités visuelles finales.

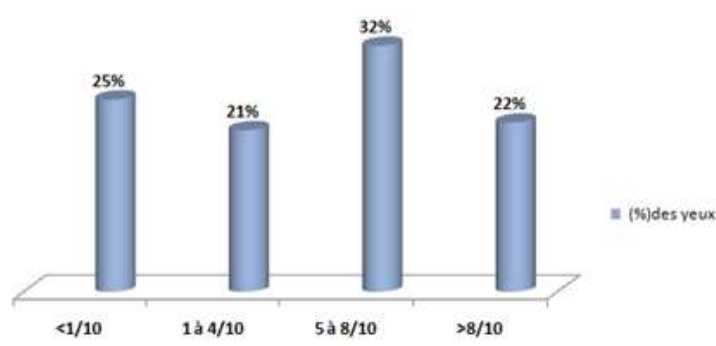

Figure 2. Répartition des acuités visuelles finales

\section{DISCUSSION}

Bien que les mécanismes pathogéniques de l'inflammation soient identiques aux atteintes observées chez l'adulte, l'uvéite de l'enfant se distingue par plusieurs particularités :

Sur le plan épidémiologique par sa rareté, sa fréquence ne dépasse pas les $10 \%$ de l'ensemble des uvéites [1,2].

L'âge moyen au diagnostic diffère suivant les séries et suivant le type anatomique mais aussi selon l'étiologie, il est compris entre six et dix ans dans $40 \%$ des cas [4]. L'atteinte touche aussi bien les filles que les garçons, cependant une prédominance féminine est notée dans certaines étiologies spécifiques telle que l'arthrite juvénile idiopathique [5]. Dans la littérature consultée, aucune notion de recrudescence saisonnière de l'uvéite chez l'enfant n'a été signalée.

Nos résultats rejoignent ces données épidémiologiques, sauf que dans notre série nous avons noté plutôt une nette prédominance masculine ainsi qu'une augmentation du nombre de cas en hiver.

Sur le plan clinique, l'uvéite pédiatrique se caractérise par la pauvreté des signes d'appels fonctionnels, l'enfant manifeste rarement la symptomatologie initiale de l'inflammation surtout dans les atteintes intermédiaires et postérieures et même dans certaines formes antérieures sans rougeur oculaire telle que l'AJI ; ce qui engendre un important retard de diagnostic.
L'uvéite est souvent bilatérale chez l'enfant [3]. La forme anatomique antérieure est prépondérante, elle représente 30-50\%, suivi par l'uvéite postérieure $20-30 \%$ [3-5]. Nous rejoignant ces données sur la fréquence de l'atteinte bilatérale, et antérieure de l'uvéite cependant dans notre série, l'uvéite intermédiaire vient en 2 ème position avec 30,6\%.

Chez l'enfant, il existe certaines étiologies qui lui sont spécifiques et qu'il faut connaitre, telles que: l'arthrite juvénile idiopathique, la toxocarose, le Cinca syndrome et la maladie de Kawasaki [5]. Ces deux dernières pathologies sont cependant rares.

Les principales étiologies des uvéites pédiatriques rapportées dans les grandes séries de la littérature sont par ordre de fréquence les uvéites idiopathiques 28,8\%, suivies des uvéites dans le cadre de l'arthrite juvénile idiopathique $20,9 \%[3,6]$

Dans notre série l'uvéite idiopathique représentait 37\%, suivie de l'uvéite inflammatoire dans $35,5 \%$ et dans cette forme c'est l'AJI qui est l'étiologie la plus fréquente avec 32\%.

Les uvéites dans le cadre de l'AJI de notre série ont été toutes antérieures, inaugurales de la pathologie chez deux cas, découvertes au stade de complications sévères bilatérales chez trois patientes. Ces chiffres se rapprochent des données de la littérature.

Pour nos uvéites infectieuses, la toxoplasmose représente $35 \%$, sa présentation anatomique était postérieure et unilatérale pour tous les cas, tandis que l'uvéite herpétique qui vient en seconde position avec $29 \%$ des cas, sa manifestation était variée dans notre série. Elle s'est présentée sous la forme d'une kérato-uvéite antérieur granulomateuse hypertensive unilatérale chez trois cas, sous la forme d'une rétinite nécrosante chez un cas et sous la forme d'une panuvéite chez le dernier cas. Quant aux deux cas de toxocarose, ils ont touché uniquement le segment postérieur, ces résultats sont similaires aux données de la littérature [7].

Parmi les étiologies inflammatoires en dehors de l'AJI, nous avons enregistré une série variée de pathologies à savoir : six cas de spondylarthropathie à manifestation antérieure aigue bruyante, trois cas de Vogt Koyanagi Harada sous forme de panuvéite bilatérale à prédominance postérieure typique avec atteinte cutanée et neurologique. Deux cas de sarcoïdose oculaire sous forme d'une uvéite antérieure granulomateuse associée à une vascularite confirmée par la biopsie des glandes salivaires accessoires et la positivité de l'enzyme de conversion de l'angiotensine. Tandis que pour les deux cas de la maladie de Behçet, le diagnostic était typique chez le 1 er cas 
par la présence de l'aphtose buccale et la panuvéite à hypopion, alors que le 2ème cas s'est présenté avec une vascularite occlusive bilatérale veineuse qui s'est complétée secondairement par l'aphtose buccale.

Chez les deux cas de la maladie de Crohn le diagnostic a été confirmé par la biopsie de la muqueuse intestinale.

Cependant notre série n'a comportée aucun cas de Cinca syndrome, ni de maladie de Kawasaki.

Concernant le traitement, la totalité de nos patients ont bénéficié d'une corticothérapie locale et générale instaurée en urgence précédée d'un traitement spécifique dans les formes infectieuses. Seulement deux cas ont bénéficié de l'indication des immunosuppresseurs prescrites et suivi par l'interniste, il s'agit d'un cas de maladie de Behçet et un cas d'AJI.

Les complications de l'inflammation sont fréquentes, très nombreuses et handicapantes chez l'enfant d'autant plus qu'elles sont déjà présentes au diagnostic. Chaque type anatomique et étiologique d'uvéite s'individualise par une complication, à titre d'exemple, les uvéites antérieures de l'AJI se compliquent de cataracte, d'hypertonie et de kératite en bandelette, tandis que les uvéites intermédiaires se compliquent fréquemment de cataracte et surtout d'œdème maculaire $[8,9,10]$. Dans notre série $43 \%$ des patients ont présenté une ou plusieurs complications de l'uvéite auxquelles s'ajoutent celles liées au traitement cortisonique (cataracte et glaucome cortisonique). Ce chiffre se rapproche des données de la littérature $[2,11]$.

Ces différentes complications ont retenti sur le pronostic visuel de nos patients avec des acuités visuelles finales inférieures à $1 / 10$ (considérée comme cécité légale) chez $25 \%$ d'entre eux. L'étude faite par Chebil A et al sur 49 cas, $18 \%$ ont gardé une acuité visuelle inférieure à $1 / 10$ [6], alors que l'étude de Laghmari $M$ et al sur 20 cas d'uvéites pédiatriques a rapporté que $28 \%$ de leurs patients ont conservé une acuité visuelle inférieure à 1/10 [12].

\section{CONCLUSION}

L'uvéite pédiatrique reste une affection grave, de diagnostic clinique facile, mais étiologique souvent négatif nécessitant la multiplication des examens para cliniques. Son évolution est habituellement insidieuse et chronique émaillée de complications souvent amblyogènes malgré le traitement cortisonique qui occupe une place importante et incontournable. Elle nécessite de ce fait une prise en charge bien codifiée et une collaboration multidisciplinaire.
Déclaration d'intérêts: les auteurs ne déclarent aucun conflit d'intérêt en rapport avec cet article.

\section{RÉFÉRENCES}

1. Clarke LA, Guex-Crosiera Y, Hofer M. Epidemiology of uveitis in children over a 10-year period. Clin Exp Rheumatol 2013; 31(4): 633-7.

2. Guex-Crosiera Y, Vaudaux.J, Hofer M. Prise en charge d'une uvéite pédiatrique. Paediatrica Vol. 26 No. 5; 2015.

3. Smith JA, Mackensen F, Sen HN, et al. Epidemiology and course of disease in childhood uveitis. Ophthalmology 2009; 116(8): 1544-51. 51.e1

4. Brézin A. Les uvéites. Rapport de la société française d’ophtalmologie Paris : Masson (2010).

5. Poulin M, Mortemousque B. Etiologies de l'uvéite chez l'enfant et bilan étiologique. Réflexions ophtalmologiques; $\mathrm{N}^{\circ}$ 171-Tome 19- janvier 2014, pp 15-18.

6. Chebil A et al. Étude épidémiologique des uvéites de l'enfant à propos de 49 cas. JFO janvier 2012 ; Volume 35, numéro 1 pages 30-34.

7. Guex-Crosiera $Y$ et al. L'uvéite non infectieuse de l'enfant Recommandations des groupes de travail de l'Uvéite et de Rhumatologie pédiatrique Forum Med Suisse 2012; 12(38):739-741.

8. Rosenberg KD, Feuer WJ, Davis JL. Ocular complications of pediatric uveitis. Ophthalmology 2004; 111: 2299-306.

9. Ducos de Lahitte $\mathrm{G}$ et al. Maculopathy in uveitis o juvenile idiopathic arthritis: an optical coherence tomography study. $\mathrm{Br} J$ Ophthalmol 2008; 92: 64-9.

10. Bonnet C, Monnet D. Uvéites non infectieuses. Urgence en ophtalmologie. Elsevier; Masson Rapport de la SFO 2018; chap.5.2 ; pp 380-400.

11. Anesi SD, Foster CS. Importance of recognizing and preventing blindness from juvenile idiopathic arthritis- associated uveitis. Arthritis Care Res (Hoboken) 2012; 64(5): 653-7.

12. Laghmari $\mathrm{M}$ et al. L'uvéite de l'enfant à propos de 20 cas. JFO volume 26, N6 juin 2003 pp.609-613.

\footnotetext{
Cet article a été publié dans le «Batna Journal of Medical Sciences» BJMS, l'organe officiel de "l'association de la Recherche Pharmaceutique - Batna»

Le contenu de la Revue est ouvert « Open Access » et permet au lecteur de télécharger, d'utiliser le contenu dans un but personnel ou d'enseignement, sans demander l'autorisation de l'éditeur/auteur.

Avantages à publier dans BJMS :

- Open access : une fois publié, votre article est disponible gratuitement au téléchargement

- Soumission gratuite : pas de frais de soumission, contrairement à la plupart des revues « Open Access »

- Possibilité de publier dans 3 langues : français, anglais, arabe

- Qualité de la relecture : des relecteurs/reviewers indépendants géographiquement, respectant l'anonymat, pour garantir la neutralité et la qualité des manuscrits.

Pour plus d'informations, contacter BatnaJMS@gmail.com ou connectez-vous sur le site de la revue : www.batnajms.net
} 


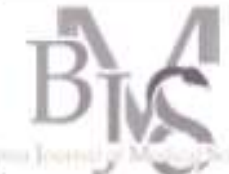

- $\bigcirc$

-

-

○

Service d'épidémiologie et de médecine préventive $\mathrm{CHU}$ de Batna -Algérie.

\section{Correspondance à :}

Messaouda OUDJEHIH messa18@yahoo.fr

DOI :https://doi.org/10.48087/ BJMSoa.2020.7210

\section{Historique de l'article :}

Reçu le 01 juillet 2020

Accepté le 25 septembre 2020

Publié le 09 novembre 2020

Il s'agit d'un article en libre accès distribué selon les termes de la licence Creative Commons Attribution International License (CC BY 4.0), qui autorise une utilisation, une distribution et une reproduction sans restriction sur tout support ou format, à condition que l'auteur original et la revue soient dûment crédités.

\section{Pour citer l'article :}

Khelalfa L, Oudjehih M, Ablous $\mathrm{N}$, et al. La mortalité maternelle dans la wilaya de Batna de 2014 à 2017. Batna J Med Sci 2020;7(2):109-13. https://doi.org/10.48087/BJMS oa.2020.7210

\title{
La mortalité maternelle dans la wilaya de Batna de 2014 à 2017
}

\author{
Maternal mortality in Batna from 2014 to 2017
}

\author{
Loubna Khelalfa, Messaouda Oudjehih, Nacira Ablous, Zina Dounia Bouzenita, \\ Khadidja Toureche, Hanane Benaldjia, Fayçal Beichi, Khawla Boumaraf.
}

\section{RÉSUMÉ}

Introduction. Le taux de mortalité maternelle est un indicateur de la prise en charge de la femme enceinte. Les objectifs de l'étude étaient de calculer le taux de mortalité maternelle dans la Wilaya de Batna et dresser un profil des caractéristiques épidémiologiques et cliniques des cas du 1er janvier 2014 au 31 décembre 2017. Matériels et méthodes. Étude descriptive rétrospective qui avait porté sur les décès maternels parvenus à la direction de la santé de la wilaya. Les variables de l'étude étaient basées sur celles du formulaire de déclaration obligatoire de décès maternel. Résultats. Durant la période d'étude 55 décès maternels avaient été déclarés pour 136482 naissances vivantes (NV), soit un taux de mortalité maternelle (TMM) global de 40,3 décès maternels / 100000 NV. La daïra de Batna avait enregistré le taux le plus élevé avec $(12,7 \%)$, L'âge moyen des femmes était de 32 ans +/- 0,7 ans avec des extrêmes allant de 21à 43 ans. Le secteur public avait enregistré la quasi-totalité des décès (98\%) dont près de la moitié $(49,1 \%)$ étaient survenus dans le post-partum immédiat, causées en premier lieu par les hémorragies de la délivrance avec un pourcentage de 39\%, suivies par les septicémies puerpérales et les complications de l'HTA dans 20\% et $15 \%$ des cas respectivement. Conclusion. Le taux de mortalité maternelle à Batna est inférieur au taux national, ceci pourrait s'expliquer par la prise en charge satisfaisante des parturientes de la wilaya ; sous réserve de prendre en considération la part des décès survenus à domicile et ceux non déclarés difficiles à chiffrer.

Mots clé : mortalité maternelle, déclaration obligatoire, Batna, Algérie.

\section{INTRODUCTION}

Le décès maternel est défini comme étant « tout décès d'une femme, survenu au cours de la grossesse ou dans un délai de 42 jours après sa terminaison, quelle qu'en soit la durée ou le site de la grossesse, pour une cause liée a ou aggravée par la grossesse ou les soins qu'elle a motivé, hormis toute cause accidentelle ou fortuite » (1). Le taux de mortalité maternelle est un indicateur de la qualité du suivi obstétrical et de la prise en charge des parturientes (2).

Depuis les années 1940, la mortalité maternelle dans les pays développés est devenue de plus en plus rare, descendant en 2012 au-dessous du seuil de 5 décès maternels pour 100.000 naissances vivantes (NV) dans certains pays européens. En effet, le taux de mortalité maternelle en 2015, était de 12 pour 100000 $\mathrm{NV}$ dans les pays développés, alors qu'il était de 239 pour $100000 \mathrm{NV}$ dans les pays en

\section{ABSTRACT}

Introduction. The maternal mortality rate is an indicator of the care provided for pregnant women. The objectives of the study were to calculate the maternal mortality rate in the Wilaya of Batna and to draw up a profile of the epidemiological and clinical characteristics of cases from January 1, 2014 to December 31, 2017. Materials and methods. Retrospective descriptive study which had focused on maternal deaths that had reached the health department of the wilaya. The variables of the study were based on those of the mandatory declaration form of maternal death. Results. During the study period, 55 maternal deaths were reported for 136,482 live births, representing an overall maternal mortality rate (MMR) of 40.3 maternal deaths/100,000 live births. The daïra of Batna recorded the highest rate $(12.7 \%)$. The average age of women was 32 years +/- 0.7 years, with extremes ranging from 21 to 43 years. The public sector had recorded almost all deaths $(98 \%)$, of which almost half $(49.1 \%)$ occurred in the immediate postpartum period, caused primarily by hemorrhage during delivery (39\%), followed by puerperal sepsis and complications of hypertension in $20 \%$ and $15 \%$ of cases respectively. Conclusion. The maternal mortality rate in Batna is lower than the national rate, this could be explained by the Satisfactory care of parturients in the wilaya ; subject to taking into consideration the share of deaths occurring at home and those not declared difficult to quantify.

Key words : maternal mortality, compulsory declaration, Batna, Algeria. développement et à revenu faible et dont la plupart des décès auraient pu être évités $[3,4]$.

En 2015, l'objectif 5 du Millénaire pour le développement (OMD5) qui visait à réduire la mortalité maternelle de $75 \%$ dans le monde en rendant universel l'accès à la médecine procréative n'a pas été atteint. Le rapport de l'OMD5 avait rapporté que seuls $51 \%$ des pays possédaient des données sur les causes de la mortalité maternelle (5).

En Algérie un plan quinquennal national 20152019, de réduction de la mortalité maternelle a été initié par le ministère de la santé de la population et de la réforme hospitalière (MSPRH) (6). Par ailleurs un audit des décès maternels a été mis en place, par arrêté ministériel $\mathrm{N}^{\circ} 89$ du 4 juillet 2013 instituant la déclaration obligatoire de tout décès maternel (7). 
Les objectifs de l'étude étaient de calculer, le taux de mortalité maternelle de 2014 à 2017 et de dresser un profil des caractéristiques épidémiologiques et cliniques de la mortalité maternelle durant ces quatre années.

\section{MATÉRIELS ET MÉTHODES}

Il s'agissait d'une étude descriptive rétrospective, sur la période allant du 1er janvier 2014 au 31 décembre 2017. Elle avait porté sur les données des décès maternels parvenus à l'unité de la population de la direction de la santé de la wilaya de Batna.

Étaient inclus dans l'étude l'ensemble des décès maternels répondant à la définition du décès maternel survenus dans les établissements de santé, des différentes communes de la wilaya et déclarés à la direction de la santé et la population (DSP) de Batna. Les décès maternels survenus à domicile n'ayant pas fait l'objet d'une déclaration étaient exclus de l'étude.

Les variables prises en compte dans l'étude étaient celles qui figuraient sur le formulaire de déclaration obligatoire de décès maternel conçu par le ministère de la santé et de la population, réparties en quatre items :

Les informations relatives aux femmes décédées : âge et lieu de résidence, lieu du décès qu'il soit à domicile, établissement de santé public ou privé, présence d'une évacuation.

Le moment de décès qu'il soit pendant la grossesse, pendant le travail et l'accouchement, dans le post-partum immédiat, dans les 42 jours suivant un avortement ou un accouchement.

La cause du décès ; hémorragie de la délivrance, complications dues à l'hypertension artérielle (HTA) gravidique, septicémie puerpérale, rupture utérine ou autres hémorragies.

Données relatives à la déclaration : date et lieu de déclaration et nom du médecin déclarant.

Les données recueillies à partir du registre des décès maternels de la DSP avaient été confirmées et complétées à partir du registre de la mortalité hospitalière du service d'épidémiologie et de médecine préventive ainsi que celui du bureau des entrées du CHU de Batna.

Les taux de mortalité maternelle étaient exprimés par le rapport entre le nombre des femmes décédées à la suite des conséquences obstétricales directes et indirectes et le nombre des naissances vivantes déclarées au service de démographie de la DSP de la wilaya de Batna.

\section{Analyses statistiques des données}

Le logiciel Microsoft Office Excel 2013 a été utilisé pour la réalisation de l'analyse des données. Les variables étaient de type qualitatif et quantitatif ; des effectifs et des pourcentages ont été calculés et comparés selon le test khi ${ }^{2}$ de Fisher en raison de la taille réduite des effectifs, avec un risque d'erreur de première espèce $\alpha$ à $5 \%$.

\section{Aspects éthiques}

La confidentialité des données et l'anonymat des femmes ainsi que l'identité du médecin déclarant ont été respectés.

\section{RÉSULTATS}

Durant la période allant du 1 janvier 2014 au 31 décembre 2017, la DSP avait déclaré 55 décès maternels sur le territoire de la wilaya de Batna pour 136482 (NV, soit un taux de mortalité maternelle (TMM) global de 40,3 DM / 100000 NV.
La daïra de Batna avait enregistré le pourcentage le plus élevé des décès maternels $(12,7 \%)$, suivi par la daïra de Barika $(10,9 \%)$, les daïras de N'gaous et Merouana avec (09\%).

L'âge moyen des femmes décédées était de 32 ans +/- 0,7 ans avec des extrêmes allant de 21 ans à 43 ans. La tranche d'âge la plus touchée était celle des 30 à 34 ans avec 38\% des cas.

Le taux le plus élevé dans la wilaya de Batna, était enregistré en 2017 avec 50,99 DM pour $100.000 \mathrm{NV}$, alors qu'il était de 28,98 pour 100.000 NV en 2016 (tableau 1).

Tableau 1. Caractéristiques des décès maternels

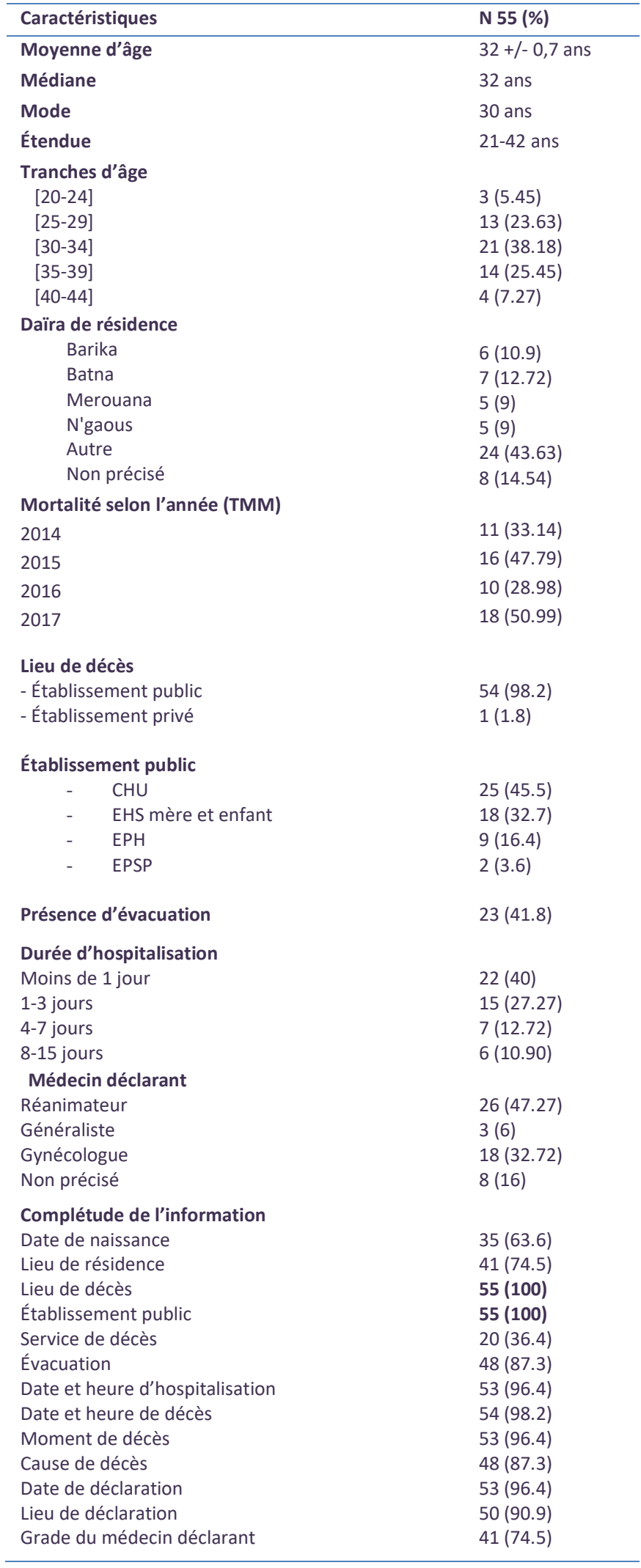


Le secteur public était pourvoyeur de la quasi-totalité des décès (98\%), parmi eux (46\%) étaient survenus au CHU de Batna et (33 \%) à l'EHS mère et enfant de la ville de Batna. Les évacuations vers ces deux structures représentaient $(40 \%)$ de l'ensemble des décès. Les structures hospitalières périphériques avaient compté (21\%) des décès.

Près de la moitié (44\%) des décès étaient survenus dans les premières 24 heures suivant l'admission dans une structure de santé avec des extrêmes allant d'une heure à 15 jours et une durée moyenne d'hospitalisation de 02 jours (tableau 1).

Les décès étaient déclarés, respectivement par un médecin réanimateur ou un gynécologue dans (47\%) et (33\%) des cas. Le délai de déclaration du décès qui ne devrait pas excéder les 48 heures n'était respecté que dans (62\%) des cas.

Le formulaire de déclaration obligatoire de décès maternel n'était pas complètement renseigné par les médecins déclarants; en effet, seule la wilaya de décès, le type d'établissement où a eu lieu le décès étaient notifiés sur l'ensemble des fiches de déclaration, alors que le moment de décès n'était notifié que dans $96.4 \%$ et la cause de décès dans 87.3\% des cas (tableau 1).

Près de la moitié des décès $(49,1 \%)$ étaient survenus dans le post-partum immédiat, suivis par ceux survenus dans les 42 jours suivant l'accouchement dans un cas sur cinq.

La cause de décès n'était pas mentionnée sur trois supports de déclaration obligatoire de décès maternels. Les hémorragies de la délivrance occupaient le $1^{\mathrm{er}}$ rang des causes de décès avec un pourcentage de 39\%, suivies par les septicémies puerpérales et les complications de l'HTA dans $20 \%$ et $15 \%$ des cas respectivement. Les causes de décès n'étaient pas statistiquement associées à une tranche d'âge particulière. (Tableau 2).

\section{DISCUSSION}

Dans la wilaya de Batna et conformément à la définition de l'organisation mondiale de la santé et sur une période de quatre années, 55 DM étaient identifiés pour 136482 NV, soit un TMM global de 40,3 DM / 100000 NV. L'évolution au fil des années avait montré que le taux le plus élevé était enregistré en 2017 soit 50,99 DM pour $100.000 \mathrm{NV}$, alors qu'il était de 28,98 pour $100.000 \mathrm{NV}$ en 2016. S'agit-il d'une augmentation réelle du taux des décès, d'une amélioration des déclarations des décès ou d'une fluctuation des taux de mortalité maternelle en raison de la rareté du phénomène. Par ailleurs l'étude n'avait pris en considération que les décès maternels déclarés ; la proportion des décès à domicile et les décès non déclarés reste difficile à estimer, Cependant ce taux pourrait être faible vu que les accouchements à domicile en Algérie sont devenus de plus en plus rares (8).

En dépit de cette limite, l'étude avait permis de dresser un profil des décès maternels dans la wilaya de Batna. Ce taux était inférieur à la moyenne nationale qui était de 60,3 pour $100000 \mathrm{NV}$ en 2014. Ceci pourrait s'expliquer par la couverture sanitaire satisfaisante par les établissements de santé dispensant une assistance d'un agent de santé qualifié lors de l'accouchement et de soins post-partum pour la prise en charge des parturientes notamment les maternités rurales et les centres de protection maternelle et infantile (PMI) et suivi par les gynécologues du secteur privé sur l'ensemble du territoire de la wilaya de Batna où les femmes devraient bénéficier d'au moins quatre consultations anténatales.

Ce TMM montrait que nous étions en position intermédiaire entre les pays en voie de développement et les pays développés (9).

Tableau 2. Répartition selon les taux standardisés et les circonstances des décès

\begin{tabular}{|c|c|c|c|c|c|c|c|}
\hline & \multicolumn{5}{|c|}{ Tranche d'âge } & \multirow{2}{*}{$\begin{array}{c}\text { Total N=55 } \\
(\%) \\
\end{array}$} & \multirow{2}{*}{$\begin{array}{l}\mathbf{P}^{*} \\
/\end{array}$} \\
\hline & {$[20-24]$} & [25-29] & [30-34] & [35-39] & {$[40-44]$} & & \\
\hline \multicolumn{8}{|l|}{ Taux de mortalité maternelle pour } \\
\hline $100000 \mathrm{NV}$ & 2.19 & 9.52 & 15.38 & 10.25 & 3.66 & 40.3 & / \\
\hline \multicolumn{8}{|l|}{ Moment de décès } \\
\hline Grossesse & & 2 & 1 & 0 & 0 & $4(7.27)$ & \\
\hline Le travail et l'accouchement & & 1 & 5 & 1 & 0 & $7(12.72)$ & 0.45 \\
\hline Post-partum immédiat & & 7 & 10 & 9 & 2 & $28(50.9)$ & \\
\hline 42 jours suivant un avortement & & 2 & 1 & 1 & 1 & $5(9.09)$ & \\
\hline 42 jours suivant un accouchement & & 1 & 4 & 3 & 1 & $11(20)$ & \\
\hline \multicolumn{8}{|l|}{ Nature des causes directes } \\
\hline Hémorragie de la délivrance & 0 & 2 & 8 & 6 & 0 & $16(29.09)$ & \multirow{5}{*}{0.24} \\
\hline Complication liée à une HTA gravidique & 1 & 1 & 2 & 0 & 1 & $5(9.09)$ & \\
\hline Septicémies puerpérales & 0 & 3 & 1 & 2 & 1 & $7(12.72)$ & \\
\hline Autres causes & 1 & 5 & 11 & 5 & 2 & $24(43.63)$ & \\
\hline Non précisé & 1 & 1 & 1 & 0 & 0 & $3(5.45)$ & \\
\hline
\end{tabular}

*Khi ${ }^{2}$ calculé est un $\mathrm{khi}^{2}$ de Fisher 
Les pays à contexte socioculturel similaire, tels que le Maroc et la Tunisie avaient enregistré respectivement 121 et 44.8 décès pour 100000 naissances vivantes $[9,10]$.

Le décès maternel commençait à progresser à partir de 25 ans et la tranche d'âge la plus touchée était celle des 30 à 34 ans avec $38 \%$ des cas, ceci pourrait s'expliquer par l'augmentation de l'âge au mariage $[11,12]$. Alors que dans les pays en développement, le risque de mortalité maternelle était plus élevé chez les adolescentes de moins de 15 ans (14). En France, 50\% des décès concernaient des femmes entre 30 et 39 ans (15) .

Le moment du décès était représenté par le post-partum dans $69.1 \%$ des cas, avec 49,1\% dans les 24 heures suivant l'accouchement et $20 \%$ dans les 42 jours suivant l'accouchement, alors que $16 \%$ survenaient pendant le travail. La revue de la littérature révèle de façon quasiuniverselle le rôle prédominant de la période du post-partum dans la survenue du DM (3).

Ces résultats rejoignent ceux de l'étude réalisée par l'INSP en 2015 et qui avait montré que 77,92\% des DM survenaient après l'accouchement, 9,7\% pendant le travail et $9 \%$ pendant la grossesse (8) .

Dans notre étude, la majorité des décès maternels (74\%) étaient dus à des causes obstétricales directes, dont les causes hémorragiques (39\%), les septicémies (20\%) suivies par les complications secondaires à l'HTA (15\%). Alors que selon l'OMS, les trois quarts de l'ensemble des décès maternels sont représentées par l'hémorragie sévère, les infections, l'hypertension durant la grossesse et l'avortement pratiqué dans de mauvaises conditions de sécurité. Le même constat était observé dans les études occidentales $[15,16]$.

$\mathrm{Au}$ Maroc, en 2014, l'enquête confidentielle sur les décès maternels avait trouvé des résultats similaires. En effet, les causes hémorragiques représentaient la 1ère cause de décès (35\% des cas), les complications liées à l'HTA gravidique à $20 \%$ des cas et les complications septiques à $15 \%$ des cas (10).

En Tunisie, selon Tej et al. et en 2015, les décès maternels étaient en rapport avec une cause directe dans la grande majorité des cas $(81 \%$ des cas $)$, avec les causes hémorragiques dans $35 \%$ des cas et les complications de la pré-éclampsie étaient incriminées dans 19,35\% (11). Il ressort de ces résultats et selon les orientations des différents programmes que l'amélioration des soins avant la conception, au cours de la grossesse et autour de l'accouchement pourraient améliorer le taux de mortalité maternelle.

La déclaration des décès était assurée par un médecin spécialiste dans $80 \%$ des cas (33\% gynécologue et $47 \%$ médecin réanimateur).

Le respect du délai de déclaration réglementé était retrouvé dans $62 \%$ des cas. Parmi les $34 \%$ chez qui les délais n'étaient pas respectés, certains décès n'étaient déclarés qu'au bout de trois mois dans $08 \%$ des cas.

L'OMS estime à 78\% les soins qualifiés à l'accouchement en Afrique du nord et moyen orient.

Le nombre élevé de cas des décès survenus dans la Wilaya de Batna nous incite à mener des études plus larges et à exploiter les audits réalisés autour du décès maternel pour mieux appréhender cet aspect de mortalité, évitable pour la plupart des cas et survenant chez une population jeune.

Par ailleurs les soins obstétricaux et néonatals d'urgence pourrait jouer un rôle important dans la réduction de la mortalité maternelle différents niveaux de prévention dans l'offre des soins de qualité avant, pendant la grossesse, lors de l'accouchement et dans les suites de couches pourraient améliorer le taux de mortalité maternelle (18).

En conclusion en Algérie, le TMM constitue un indicateur clé de la santé publique et la surveillance de la mortalité maternelle est un reflet de la qualité du système de soins. Le MSPRH, en se basant sur les recommandations des organismes de santé mondiale (19) avait mis en place un ensemble de décisions visant à réduire la mortalité maternelle; le Plan National de la Réduction Accélérée de la Mortalité Maternelle (PNRAMM) 2015-2019 visait la protection de la santé de la mère et de l'enfant et la réduction des inégalités à l'accès aux soins de qualité pour les parturientes (20), L'audit des décès maternels a été mis en place, par arrêté ministériel $N^{\circ} 89$ du 4 juillet 2013 instituant la déclaration obligatoire de tout décès maternel et s'appuie sur un ensemble de décisions du Ministère de la santé. En outre, et depuis son installation officielle en 2015, le Comité d'experts chargé de l'audit des décès maternels (CECADAM), était en charge de la supervision des audits.

L'audit est un instrument utile pour le suivi et l'évaluation ; il s'agit d'un dossier standardisé, son renseignement permet l'analyse des causes de décès des facteurs de risques et des pratiques professionnelles En dépit de la lourdeur de la réalisation de l'audit; ces avantages permettent d'identifier les secteurs où la qualité des soins prodigués devraient ou pourraient être améliorée et permet d'organiser la mise en œuvre des changements nécessaires pour répondre aux standards de soins définis (21) .

Déclaration d'intérêts: les auteurs ne déclarent aucun conflit d'intérêt en rapport avec cet article.

\section{RÉFÉRENCES}

1. World Health Organization Report on the World Health Organization. Working Group on the Classification of Maternal Deaths and Severe Maternal Morbidities. Geneva; 2009.

2. Zhang W-H, Alexander S. Objectifs pour un développement durable-pour l'analyse et pour la planification en santé. Revue de médecine périnatale 2017;9(1):3-6.

3. The un maternal mortality estimation inter-agency group.A global, regional, and national levels and trends in maternal mortality between 1990 and 2015, with scenario-based projections to 2030: a systematic analysis L. LANCET. 2016; 387 (10017): 462-7.

4. Alkema L, Chou D, Hogan D, Zhang S, Moller AB, Gemmill A. Trends in maternal mortality: 2000 to 2017: estimates by WHO, UNICEF. Lancet. 2016 387 (10017): 462-74.

5. Nations unies, New York, 2015. Objectifs du Millénaire pour le développement : rapport 2015.

6. Ministere de la santé, de la population et de la reforme hospitaliere. Direction de la prévention de la promotion de la santé, Plan national de réduction de la mortalité maternelle 2015-2019, Algérie.

7. République Algérienne Démocratique et Populaire. Ministère de la Santé, de La Population et de la Réforme Hospitalière. Arrêté N89 du 04 Juillet 2013 Instituant La déclaration obligatoire de décès maternel.

8. Institut National de Santé Publique, Algérie. Caractéristiques des décès maternels au cours du 2ème semestre 2014. Algérie, Mai 2015.

9. Centers for Disease Control and Prevention. Pregnancy-related mortality in the United States. Available at www.cdc.gov/reproductivehealth/maternalinfanthealth/pmss.html. Retrieved June 23, 2017. 


\section{ARTICLE ORIGINAL}

10. Royaume du Maroc, Ministere de la santé, Plan d'action 2012-2016. Enquête confidentielle sur les décès maternels de 2015 dans les six régions prioritaires au Maroc. Rapport de synthèse.

11. Tej Dellagi R, Bougatef S, Ben Salah F, Ben Mansour N, Gzara A, Gritli I, Ben Romdhane $\mathrm{H}$, Rachdi MT. l'enquête nationale tunisienne sur la mortalité maternelle de 2010 : a propos des données de tunis. La tunisie Medicale. 2014;92 (08): 560-566.

12. République Algérienne Démocratique et Populaire. Ministère de I'Industrie et des Mines. Agence Nationale de Développement de I'Investissement. Démographie algérienne 2018.

13. République algérienne démocratique et populaire. Office national des statistiques. Démographie algérienne 2015.n 740.

14. Patton GC, Coffey C, Sawyer SM, Viner RM, Haller DM, Bose K, et al. Global patterns of mortality in young people: a systematic analysis of population health data.. Lancet. 2009;374:881-892.

15. Saucedoa M, Deneux-Tharaux Bouvier C. Épidémiologie de la mortalité maternelle en France, 2007-2009.Journal de Gynécologie Obstétrique et Biologie de la Reproduction. 42(7);2013:613-627.
16. Dumont A. Réduire la mortalité maternelle dans les pays en développement: quelles sont les interventions efficaces? Revue de médecine périnatale 2017;9(1):7-14.

17. Say L, Chou D, Gemmill A, Tunçalp Ö, Moller AB, Daniels JD, et al. Global Causes of Maternal Death: A WHO Systematic Analysis. Lancet Global Health. 2014;2(6): e323-e333.

18. Organisation Mondiale de la Santé, la stratégie mondiale pour la santé de la femme, de l'enfant et de l'adolescent (2016-2030) Survivre, s'épanouir, transformer,2015.

19. Stratégie mondiale pour la santé de la femme, de l'enfant et de I'adolescent (2016-2030).

20. République Algérienne Démocratique et Populaire, ministère de La Santé, de La Population Et de La Reforme Hospitalière, direction Générale de La Prévention et de La Promotion de la Santé Plan National de Réduction de La Mortalité Maternelle 2015 - 2019.

21. De Brouwere V, ZinnenV, Delvaux T. Conduire des Revues des cas de Décès Maternels (RDM). Guide et outils pour les professionnels de la santé. Londres,Fédération Internationale de Gynécologie et d’Obstétrique, FIGOLOGIC. 2012

Cet article a été publié dans le « Batna Journal of Medical Sciences » BJMS, l’organe officiel de « l'association de la Recherche Pharmaceutique - Batna»

Le contenu de la Revue est ouvert « Open Access » et permet au lecteur de télécharger, d’utiliser le contenu dans un but personnel ou d'enseignement, sans demander l'autorisation de l'éditeur/auteur.

Avantages à publier dans BJMS :

- Open access : une fois publié, votre article est disponible gratuitement au téléchargement

- Soumission gratuite : pas de frais de soumission, contrairement à la plupart des revues « Open Access »

Possibilité de publier dans 3 langues : français, anglais, arabe

- Qualité de la relecture : des relecteurs/reviewers indépendants géographiquement, respectant l'anonymat, pour garantir la neutralité et la qualité des manuscrits.

Pour plus d'informations, contacter BatnaJMS@gmail.com ou connectez-vous sur le site de la revue : www.batnajms.net 


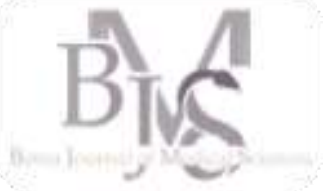

\title{
Épidémiologie des candidémies en réanimation médicale au $\mathrm{CHU}$ de Batna-Algérie
}

\author{
Epidemiology of candidemia in the intensive care unit at the university \\ hospital Batna-Algeria.
}

\author{
Ouanassa Hamouda
}

Service de Parasitologie Mycologie, CHU Batna - Algérie

\section{Correspondance à :}

Ouanassa HAMOUDA

hamoudaouanassa@yahoo.fr

DOI :https://doi.org/10.48087/ B]MSoa.2020.7211

\section{Historique de l'article :}

Reçu le 01 juillet 2020

Accepté le 26 septembre 2020

Publié le 09 novembre 2020

Il s'agit d'un article en libre accès distribué selon les termes de la licence Creative Commons Attribution International License (CC BY 4.0), qui autorise une utilisation, une distribution et une reproduction sans restriction sur tout support ou format, à condition que l'auteur original et la revue soient dûment crédités.

\section{Pour citer l'article :}

Hamouda O. Épidémiologie des candidémies en réanimation médicale au CHU de Batna-Algérie. Batna J Med Sci 2020;7(2):114-16. https://doi.org/10.48087/B IMCna 20207311

\section{RÉSUMÉ}

Introduction. Les candidémies sont des affections graves responsables d'une mortalité élevée malgré le progrès thérapeutique. Patients et méthodes. Il s'agit d'une étude prospective descriptive réalisée au niveau du service de la réanimation médicale au CHU de BATNA pendant trois ans. Pour chaque patient des prélèvements des sites périphériques ont été réalisés afin de calculer l'index de colonisation de Pittet. L'identification des espèces isolées a été réalisée avec le milieu CHROMagar Candida et Api Candida Biomérieux. Résultats. Un total de 28 cas de candidémies correspond á 28 hémocultures positives a été diagnostiqué concernant 28 patients. Le sexe ratio était de1,8. L'incidence globale était de 2,17 cas pour 1000 admissions. Les principaux motifs d'hospitalisation étaient le choc septique, la détresse respiratoire et la pathologie neurologique. La présence d'une colonisation ( $\geq 2$ sites), une antibiothérapie á large spectre, d'un cathéter intra vasculaire étaient les facteurs de risque les plus retrouvés. Sur l'ensemble des souches isolées Candida parapsilosis était l'espèce majoritairement isolée. La mortalité était importante $71 \%$ ce qui souligne la gravité de la maladie.

Mots clé: Candidémie, Hémoculture, Candida spp, réanimation médicale, Index de colonisation.

\section{INTRODUCTION}

Les candidémies sont définies par la présence prouvée d'une levure de genre Candida dans une (ou plusieurs) hémoculture. Ce sont des infections graves responsables d'une mortalité élevée relativement fréquentes en réanimation [1].

Candida est une levure opportuniste qui n'exerce son pouvoir pathogène qu'en présence de facteurs favorisants, c'est le genre le plus fréquemment retrouvé au cours des infections fongiques. Les candidémies sont classées en quatrième place des infections hématogènes, mais leur prévalence varie selon la population étudiée [2-4].

Les objectifs de notre étude étaient :

$\checkmark$ Étudier l'épidémiologie des candidémies dans le service de réanimation médicale au CHU de BATNA.

$\checkmark \quad$ Analyser les facteurs de risque.

$\checkmark$ Montrer l'intérêt de l'index de colonisation dans la survenue des candidémies.

$\checkmark \quad$ Évaluer le taux de mortalité.

\section{ABSTRACT}

Introduction. Candidemia are serious conditions responsible for high mortality despite therapeutic progress. Patients and methods. This is a prospective descriptive study carried out in the medical intensive care unit of the UHC of BATNA for three years. For each patient, samples from peripheral sites were taken to calculate the Pittet colonization index. The identification of the isolated species was carried out with the CHROMagar Candida and Api Candida Biomérieux medium. Results. A total of 28 cases of candidemia corresponding to 28 positive blood cultures were diagnosed in 28 patients. The sex ratio was 1,8 . The overall incidence was 2.17 cases per 1000 admissions. The main reasons for hospitalization were septic shock, respiratory distress and neurological pathology. The presence of colonization ( $\geq 2$ sites), broad spectrum antibiotic therapy, and an intravascular catheter were the most common risk factor. Out of all the strains isolated Candida parapsilosis was the predominantly isolated species. Mortality was significant at $71 \%$, which underlines the seriousness of the disease.

Key words : Candidemia, Candida spp, medical intensive care unit, Blood culture, Colonization index.

\section{PATIENTS ET MÉTHODES}

\section{Protocole d'étude}

Il s'agit d'une étude prospective descriptive réalisée sur une période de trois ans (Janvier 2016 au Décembre 2018) chez les patients du service de réanimation médicale du Centre Hospitalier Universitaire de BATNA ALGERIE.

Ont été inclus dans notre étude tous les patients ayant au moins une hémoculture positive á Candida spp, d'âge $\geq 16$ ans et hospitalisés plus de 48 heures au niveau du service de la réanimation médicale.

Ont été exclus de notre étude les patients ayant des prélèvements profonds positifs à levures autres que Candida spp.

Durant la période de l'étude, l'index de colonisation (IC) de Pittet a été calculé pour chaque patient á partir des prélèvements d'au moins de cinq sites périphériques (nasal, auriculaire, buccal, anal ou rectal et urinaire). Chaque échantillon a été ensemencé sur un milieu chromogène (CHROMagar CANDIDA) incubé á $37^{\circ} \mathrm{C}$ pendant 48 heures, ce qui a 
permis la détection spécifique des quatre espèces de Candida en donnant des colonies de couleurs différentes. L'identification des colonies blanches a été réalisée avec la galerie Api CANDIDA Biomérieux, et la lecture a été effectuée après 48 heures.

L'hémoculture a été réalisée sur des flacons fongiques contenant le milieu Sabouraud Chloramphénicol avec ou sans actidione au même moment que les prélèvements périphériques et á chaque fois en présence d'un syndrome infectieux.

Une infection prouvée est confirmée en présence d'une candidémie.

\section{RÉSULTATS}

\section{Caractéristiques des patients}

Durant la période d'étude, 1288 patients ont été hospitalises en réanimation médicale dont 28 avaient une candidémie soit une incidence cumulée de 2,17 pour 1000 admissions.

L'âge moyen était de 48,13 ans avec des extrêmes de 16 et 83 ans. Le sexe ratio $\mathrm{H} / \mathrm{F}$ était de 1,8.

Les motifs d'hospitalisation les plus fréquents étaient : le choc septique dans $31 \%$ des cas, la détresse respiratoire et la pathologie neurologique dans $15 \%$ des cas, l'accident vasculaire cérébral dans $12 \%$ des cas, le coma dans $11 \%$ des cas, la péritonite et la chirurgie abdominale dans $8 \%$ des cas (Figure 1)

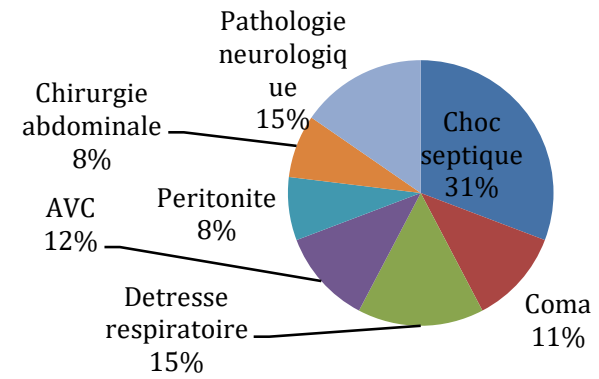

Figure 1. Répartition des patients selon le motif d'hospitalisation.

Tous les patients avaient au moins un ou plusieurs facteurs de risque avec une prédominance de la colonisation $\geq 2$ sites, l'antibiothérapie á large spectre et le séjour $\geq 7$ jours et la présence d'un cathéter veineux central (voir tableau 1).

Tableau1 : Principaux facteurs de risque.

\begin{tabular}{lcc}
\hline Facteur de risque & $\begin{array}{c}\text { Nombre de } \\
\text { patients }\end{array}$ & Fréquence \\
\hline Age $\geq 60$ & 11 & $39,28 \%$ \\
Séjour $\geq 7$ jours & 25 & $89,28 \%$ \\
$\begin{array}{l}\text { Cathéter veineux central } \\
\text { (CVC) }\end{array}$ & 21 & $75 \%$ \\
Dialyse & 3 & $10,71 \%$ \\
$\begin{array}{l}\text { Antibiothérapie à large } \\
\text { spectre } \\
\text { Chirurgie récente }\end{array}$ & 26 & $92,85 \%$ \\
Chirurgie digestive récente & 6 & $21,42 \%$ \\
Colonisation $\geq 2$ sites & 6 & $21,42 \%$ \\
Ventilation mécanique & 28 & $100 \%$ \\
Sonde vésicale & 7 & $25 \%$ \\
\hline
\end{tabular}

Au total, 28 index de colonisation (IC) ont été calculés.

En fonction de la valeur seuil de positivité (IC $\geq 0,5$ ), les patients se sont répartis en deux groupes :

Groupe 1 : 6 patients $(21,42 \%)$ ont été faiblement ou modérément colonisés (IC $<0,5)$.

Groupe 2 : 22 patients $(78,57 \%)$ ont été fortement colonisés (IC $\geq 0,5$ ).

\section{Résultats mycologiques}

Dans notre étude, 28 séries de prélèvements ont été réalisées (urine, ano-rectal, buccal, nasal, auriculaire, urinaire), soit 140 prélèvements et 28 hémocultures étaient positives á Candida. Dans l'ensemble des cultures positives les espèces non albicans étaient prédominantes par apport á l'espèce Candida albicans

Dans les prélèvements superficielle $C$ glabrata était l'espèce non albicans la plus fréquemment isolée (Fig2) et dans les hémocultures l'espèce $C$ parapsilosis était l'espèce la plus isolée (Fig3).

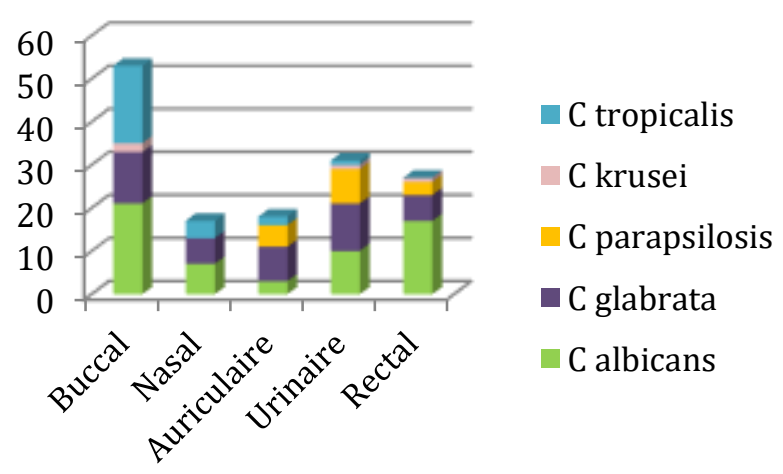

Figure 2. Distribution des espèces Candida dans les différents prélèvements superficiels.

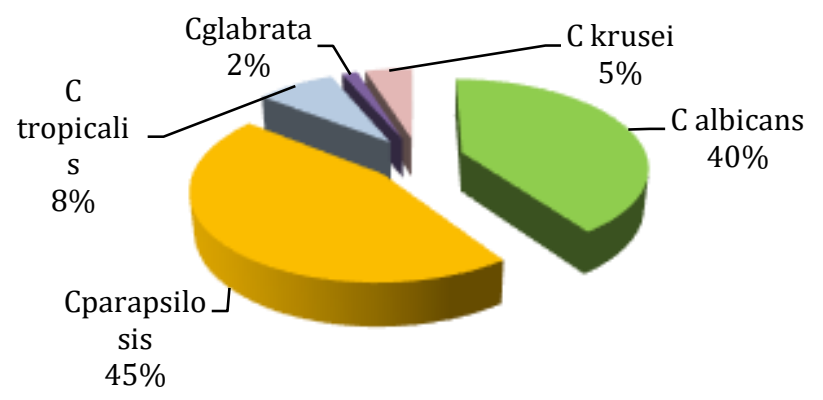

Figure 3. Distribution des espèces Candida dans les hémocultures

\section{Évolution :}

La mortalité dans notre étude était significative $71 \%$ ce qui souligne la gravité de cette affection.

\section{DISCUSSION}

Cette étude prospective descriptive avait comme objectif principal de mettre un état des lieux sur l'épidémiologie des candidémies au service de la réanimation médicale au CHU de BATNA.

Dans notre étude l'âge moyen était de 48,31 ans. Selon plusieurs études ; les âges les plus exposés au risque de candidémie, se situent bien souvent entre 50 et 65 ans [5-7]. 
Concernant la répartition selon le sexe, on note une prédominance du sexe masculin, avec un sexe ratio $\mathrm{H} / \mathrm{F}=1,8$, ce qui est conforme avec toutes les données publiées jusqu'à présent $[6,7]$.

Dans notre étude, l'incidence moyenne des candidemies était de 2,17 pour 1000 admissions, ce taux est proche de celui retrouvé dans plusieurs études : Nolla-Salas et al (1997) avec un taux de 2 /1000 admissions [8], Charles et al (2003) avec un taux de 2,1 / 1000 admissions [9] et Colombot al (2006) avec un taux de 2,49/1000 admissions [10].

Cette incidence est considérée élevée et peut être expliquée par la multiplication des facteurs de risque des candidémies dans notre hôpital.

Les candidémies étaient associée dans notre étude á plusieurs facteurs de risque comme le séjour prolonger, la colonisation $\geq 0,5$, l'antibiothérapie á large spectre et la présence du CVC.

Dans notre étude on a retrouvé la prédominance des espèces non albicans par apport à l'espèce Candida albicans ce qui est conforme avec plusieurs études [11-13].

L'espèce majoritairement isolée des hémocultures était $C$ parapsilosis, un résultat conforme á plusieurs études ayant retrouvées une prédominance de cette espèce $[7,14-16]$.

Candida parapsilosis est une espèce liée étroitement à l'utilisation des CVC. Ainsi la mise en place d'un cathéter veineux central entraine un traumatisme adventitiel au niveau de la porte d'entrée á l'origine d'un thrombus qui sera colonisé secondairement par les Candida parapsilosis saprophyte á l'état normal de la peau et par conséquence des embolies fongiques disséminées [17].

L'utilisation d'antibiotique à large spectre favorise l'émergence du C parapsilosis [18]. (92,85\%) de nos patients avaient reçu des antibiotiques à large spectre (Vancomycine, Imipenème, Ertapenème, Ciprolon, Amikacine).

Dans notre étude la proportion de la mortalité était de (71\%), elle est considérée élevée si on la compare avec celles retrouvées dans plusieurs études [5,7]. Ce qui souligne la gravité de cette affection.

\section{CONCLUSION}

Les Candidémies restent des affections sévères et en constante augmentation, en raison de l'augmentation de la population á risque. Elles occupent une place grandissante au sein des infections nosocomiales. Leur pronostic reste très mauvais non seulement du fait de la gravité des pathologies sous-jacentes qui constituent un facteur de risque des candidémies, mais aussi vue la gravité de l'infection même responsable d'une mortalité élevée.

Déclaration d'intérêts: les auteurs ne déclarent aucun conflit d'intérêt en rapport avec cet article.

\section{RÉFÉRENCES}

17. Ascioglu S, Rex JH, de Pauw B, Bennett JE, Bille J, Crokaert F, et al. Defining opportunistic invasive fungal infections in immunocompromised patients with cancer and hematopoietic stem cell transplants: an international consensus. Clin Infect Dis 2002; 34: 7-14.

18. Wisplinghoff $H$, Bischoff $T$, Tallent SM, Seifert $H$, Wenzel RP, Edmond MB Nosocomial bloodstream infections in US hospitals: Analysis of 24,179 cases from a prospective nationwide surveillance study. Clin Infect Dis 2004; 39: 309-17.
1. Marchetti O, Bille J, Fluckiger U, Eggimann P, Ruef C, Garbino J, et al. Epidemiology of candidemia in Swiss tertiary care hospitals: Secular trends, 1991-2000. Clin Infect Dis 2004; 38:311-320. Clin Infect Dis 2004; 38: 311-20. 6 .

2. Pfaller MA, Diekema DJ. Epidemiology of invasive candidiasis: a persistent public health problem. Clin Microbiol Rev 2007; 20: 133-63.

3. De Waele JJ, Vogelaers D, Blot S. Fungal infections in patients with severe acute pancreatitis and the use of prophylactic therapy. Clin Infect Dis. 2003; 37: 208-13.

4. Horn DL, Neofytos D, Anaissie E, Fishman J, Steinbach WJ, Olyaei AJ, et a . Epidemiology and Outcomes of Candidemia in 2019 Patients: Data from the Prospective Antifungal Therapy Alliance Registry Clinical Infectious Diseases 2009; 48: 1695-703.

5. Bassetti M, Merelli M, Righi E, Diaz-Martin A, Rosello A M, Luzzati R, et al.Epidemiology, Species Distribution, Antifungal Susceptibility, and Outcome of Candidemia across Five Sites in Italy and Spain Journal of Clinical Microbiology p. 4167-4172 December 2013.

6. Tadec L, Talarmin J-P, Gastinne $T$, Bretonnière $C$, Miegeville M, Le Pape $P$, et al. Epidemiology, risk factor, species distribution, antifungal resistance and outcome of Candidemia at a single French hospital : a 7-year study. Mycoses. 2016 May; 59(5) : 296-303.

7. Nolla-Salas J, Sitges-Serra A, León-Gil C, Martínez-Gonzàlez J, LeónRegidor MA, Ibàñez-Lucía $P$, et al. Candidemia in non-neutropenic critically ill patients : analysis of prognostic factors and assessment of systemic antifungal therapy. Study Group of Fungal Infection in the ICU. Intensive Care Med. 1997 Jan; 23(1) : 23-30.

8. Charles PE, Doise JM; Quenot JP. Medical and surgical patients difference of outcome between candidemia in critically ill patients. Intensive care med 2003; 29: 2162-9.

9. Montravers P, Gauzit R, Muller C, Marmuse JP, Fichelle A, Desmonts JM Emergence of antibiotic-resistant bacteria in cases of peritonitis after intraabdominal surgery affects the efficacy of empirical antimicrobial therapy. Clin Infect Dis 1996; 23 : 486-94.

10. Ortiz Ruiz G, Osorio J, Valderrama S, Alvarez D, Elias Diaz R, Calderon J, et al. Risk factors for candidemia in nonneutropenic critical patients in Colombia. Medicina Intensiva. 2016; 40 : 139-144.

11. Montagna MT, Caggiano G, Lovero G. Epidemiology of invasive fungal infections in the intensive care unit : Results of a multicenter Italian survey (AURORA Project) Infection. 2013; 41(3) : 645-653.

12. Gupta Priyanka, Prateek Shashank, Chatterjee1 Biswaroop, Kotwal1 Arti, Singh Amit K et Mittal1 Garima. Prevalence of Candidemia in ICU in a Tertiary Care Hospital in North India Int. J. Curr. Microbiol. App. Sci (2015) 4(6) : 566-575.

13. Pappas PG, Rex JH, Lee J, Hamill RJ, Larsen RA, Powderly W, et al. A Prospective Observational Study of Candidemia : Epidemiology, Therapy, and Influences on Mortality in Hospitalized Adult and Pediatric Patients. Clin Infect Dis. 2003; 37 : 634-43.

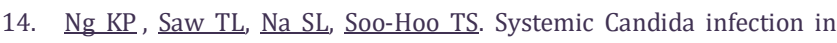
University hospital 1997-1999: the distribution of Candida biotypes and antifungal susceptibility patterns. Mycopathologia. 2001; 149(3) :141-6.

15. Medrano DI, Brilhante RS, Cordeiro Rde A, Rocha MF, Rabenhorst SH Sidrim J. Candidemia in a Brazilian hospital : the importance of Candida parapsilosis. Rev Inst Med Trop Sao Paulo. 2006 Jan-Feb; 48(1) : 17-20.

16. Aubertine CL, Rivera M, Rohan SM. Comparative study of the new colorimetric VITEK 2 yeast identification card versus the older fluorometric card and of CHROMagar Candida as a source medium with the new card. J Clin Microbiol 2006; 44(1) : 227-8. 

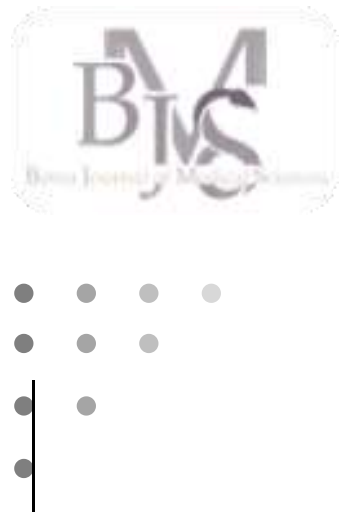

${ }^{1}$ Pharmacien libéral - Batna 05000, Algérie

${ }^{2}$ Journaliste médical - Batna

${ }^{3} \mathrm{CHU}$ de Batna

4Faculté de médecine, Université de Batna 2

${ }^{5}$ Laboratoire LGRIAS, Université de Batna 2, Algérie

\section{Correspondance à :}

Abdellatif KEDDAD

apimc@yahoo.fr

DOI :https://doi.org/10.48087/ BJMSoa.2020.7212

\section{Historique de l'article :}

Reçu le 03 juin 2020

Accepté le 03 octobre 2020

Publié le 09 novembre 2020

Il s'agit d'un article en libre accès distribué selon les termes de la licence Creative Commons Attribution International License (CC BY 4.0), qui autorise une utilisation, une distribution et une reproduction sans restriction sur tout support ou format, à condition que l'auteur original et la revue soient dûment crédités.

\section{Pour citer l'article :}

Keddad A, Oudjehih M, Prescriptions d'antiinfectieux dans une pharmacie de Batna. Batna J Med Sci 2020;7(2):117-121. https://doi.org/10.480 87/BJMSoa.2020.7212

\title{
Prescriptions d'anti-infectieux dans une pharmacie de Batna
}

\author{
Prescriptions of antimicrobials in a community pharmacy in Batna
}

\author{
Abdellatif Keddad ${ }^{1,2}$, Messaouda Oudjehih ${ }^{3,4,5}$
}

\section{RÉSUMÉ}

Les maladies infectieuses représentaient en 2007, en Algérie $4,58 \%$ du motif des consultations. La prévalence des patients hospitalisés traités par anti infectieux (AI) dans le pays était de $45,5 \%$ en 2015 . L'objectif de cette étude était la description des prescriptions contenant des anti infectieux (AI), sur la base d'une analyse rétrospective des ordonnances remboursables par la sécurité sociale, reçues au niveau d'une officine pharmaceutique privée de la ville de Batna (Algérie) sur une période d'une année allant de juillet 2013 à juin 2014. L'étude a montré qu'environ une ordonnance sur trois contenait au moins une ligne d'AI et que ceux-ci avaient représenté $13,1 \%$ de l'ensemble des médicaments prescrits toutes classes pharmaco thérapeutiques confondues. Si les plus gros prescripteurs en volume ont été les médecins généralistes $(51,4 \%$ des ordonnances), les AI avaient occupé chez les chirurgiens dentistes 1 ligne sur 2. Le profil de ces prescriptions dans le temps a mis en évidence 2 pics maximaux d'Al systémiques en saison froide à savoir mars et octobre. C'est la famille des pénicillines qui occupe avec 32,6\% des lignes d'AI, le haut du tableau des produits les plus prescrits dans cette classe.

Mots clés. Anti-infectieux, ordonnance, officine

\section{INTRODUCTION}

Les maladies infectieuses représentaient en 2007 en Algérie 4,58\% du motif des consultations [1]. La prévalence des patients hospitalisés traités par anti infectieux (AI) dans le pays était de $45,5 \%$ en 2015 soit 2 à 3 fois plus élevée que celle observée dans les CHU/CHR français en 2012 [2] alors que le Centre National de Pharmacovigilance rapportait en 2011 un taux de 54,1\% de prescriptions d'AI [3].

En 2006 en Tunisie, les maladies infectieuses et parasitaires étaient à l'origine de 2,9\% des décès [4]. La résistance bactérienne aux antimicrobiens due aux bactéries multi résistantes (BMR), est selon l'Organisation Mondiale de la Santé (OMS) de plus en plus préoccupante [5]. Il s'agit d'un phénomène mondial pour lequel l'organisation a tiré le signal d'alarme face au risque sanitaire potentiel qu'il fait courir aux populations. Les stratégies élaborées pour prévenir et maitriser les résistances, se basent sur le suivi de la

\section{ABSTRACT}

In 2007, infectious diseases in Algeria accounted for $4.58 \%$ of the reason for consultations. The prevalence of hospitalized patients treated with antimicrobials was $45.5 \%$ in the country in 2015 . The aim of this study is to quantify and describe the prescriptions containing antimicrobials, on the basis of the exploitation of a retrospective analysis of prescriptions reimbursed by social insurance, archives in a community pharmacy in the city of Batna (Algeria) for one year, from July 2013 to June 2014, with the aim of better targeting strategies for the proper use of antimicrobials. The study has shown that around 1 in 3 prescriptions contains at least one line of AI drugs accounted for $13.1 \%$ of all the drugs prescribed. If the largest prescribers by volume were general practitioners $(51.4 \%)$ of prescriptions for all medical specialities combined), in dental surgeon, AI occupied 1 line out of 2 of their prescriptions. Two maximum AI prescription peaks have been highlighted int the cold season, namely February and October. The penicillin family occupies $32.6 \%$ of the AI lines at the top of the table.

Keywords. Antimicrobials, prescription, community pharmacy

consommation des anti-infectieux (AI) [6] et sur la collecte de données de santé issues de la sécurité sociale [7 ], alors que des études font le lien entre le niveau d'utilisation des antibiotiques et la fréquence de la résistance [8].

En Algérie, c'est en 2002 qu'a été créé le réseau national de surveillance de la résistance aux antibiotiques. La constitution de données de santé permet d'une part d'affiner la prise de décision, et d'autre part elle constitue un élément d'évaluation.

Dans notre pays, en secteur libéral, nous n'avons pas trouvé de publications rapportant les niveaux de consommation des AI.

L'objectif de l'étude était de dresser le profil des prescriptions d'AI inscrits dans la nomenclature nationale (NN), chez les assurés sociaux pour les ordonnances reçues dans une officine de Batna durant une année ; de juillet 2013 à juin 2014. 


\section{MATÉRIELS ET MÉTHODES}

Il s'agit d'une étude descriptive rétrospective et exhaustive basée sur l'analyse des ordonnances remboursables par la sécurité sociale reçues dans le cadre du tiers payant et archivées au niveau d'une officine pharmaceutique privée de la ville de Batna sur une durée d'une année de juillet 2013 à juin 2014.

Ont été incluses dans l'étude, toutes les ordonnances reçues en une année, dans le cadre du conventionnement tiers payant (système CHIFA) avec l'organisme de sécurité sociale des salariés (CNAS). Par ailleurs, le Logiciel de Gestion de l'Officine (LGO), a permis l'exploitation des données en matière de consommations des médicaments en valeur et en volume selon les différentes classes thérapeutiques.

\section{Critères d'exclusion}

Ont été exclues les ordonnances présentées en dehors du cadre du tiers payant.

Les ordonnances présentées dans le cadre du tiers payant avec l'organisme des assurés sociaux non salariés CASNOS.

Les prescriptions présentées sur papier libre et en automédication.

Les informations recueillies portaient d'une part sur l'identification du prescripteur (spécialité, localité) et du patient (âge, sexe), et d'autre part, les produits pharmaceutiques appartenant à la classe des $\mathrm{AI}$ de la NN, qui ont été prescrits. Leur référent (princeps) est relevé sur la base Thériaque (c) qui est une base d'information sur les médicaments, la dénomination commune internationale (DCI) de la nomenclature nationale (NN) des médicaments du ministère de la santé, la famille $\mathrm{AI}$, la classification Anatomique Thérapeutique Chimique (ATC) le standard international à 7 positions. L'objectif du système ATC est l'uniformisation des codifications des médicaments, et partant de là, la production de statistiques permettant les comparaisons entre différents secteurs de la santé publiques ou privés.

Après avoir saisi les données sur une feuille Excel du logiciel propriétaire Microsoft Office, une sélection utilisant les filtres en fonction des données recherchées a été utilisée en combinaison avec l'option des tableaux croisés dynamiques pour produire les résultats présentés.

\section{Traitement des données}

Le matériel recueilli (les prescriptions) a servi, d'une part à collecter les informations en lien avec l'identification du prescripteur et du patient, et d'autre part, l'enregistrement de tout AI prescrit, ainsi que son dosage et sa forme galénique.

La base initiale était composée d'une feuille Excel comportant 24 colonnes compartimentées ainsi : 3 pour référencer le matériel archivé, 1 pour la date de la prescription, 7 pour l'identification du prescripteur, 2 pour les données relatives au patient, 3 affectées aux médicaments prescrits et 4 pour les coûts et durée du traitement. A ces données, ont été ajoutées pour cette étude, 6 colonnes spécifiques aux AI prescrits ainsi distribuées : (1) le nom de la spécialité, (2) son référent sur la base Thériaque (C), (3) la DCI figurant sur la $\mathrm{NN}$, (4) la famille, (5) la classification ATC à 7 positions, (6) la classification ATC à 3 positions. Une analyse statistique descriptive a été menée décrivant la fréquence et le contexte des prescriptions des AI.

Nous avons respecté les aspects éthiques relatifs à l'anonymat des prescripteurs et des patients.

\section{RÉSULTATS}

\section{Population}

Dans le cadre du conventionnement avec la caisse de sécurité sociale des salariés CNAS, un total de 3143 ordonnances d'assurés sociaux reçues dans l'officine entre juillet 2013 et juin 2014 ont fait l'objet de notre étude.

Dans notre officine, le chiffre d'affaires (CA) des ordonnances servies dans le cadre du tiers payant a représenté $86,5 \%$ des ventes de médicaments. Les AI ont occupé, sur les 24 classes pharmaco thérapeutiques de la NN 7,3\% en valeur et $11,6 \%$ en volume arrivant en $4 \mathrm{e}$ position, derrière les classes (1) métabolisme diabète, (2) cardiologie angiologie, (3) antalgiques.

Les prescriptions de médecine générale, avaient occupé $38,6 \%$ de l'ensemble de notre échantillon, tandis que la chirurgie dentaire en occupait $2.6 \%$. En tout, 22 spécialités médicales ont été identifiées avec une provenance géographique distribuée sur 9 wilayas, dont 97,4\% pour la seule wilaya de Batna, siège de l'officine. Le sex-ratio était de 0,7 avec des âges extrêmes allant de 2 mois à 90 ans.

Nos données ont mis en avant que 36,7\% des prescriptions $(n=1152)$ contenaient au moins 1 AI soit environ une ordonnance sur trois. Le nombre total de lignes toutes classes pharmaco thérapeutiques confondues a été de 11 671, parmi lesquelles 1527 lignes d'AI soit une fréquence de 13,1\%. Sur les 1012 lignes produites par le secteur privé 11,9\% ont été des $\mathrm{AI}$, tandis que pour les 515 lignes du secteur public on retrouve $16,2 \%$ de lignes d'AI.

Deux groupes d'âge ont été les cibles principales des AI systémiques, celui des $0-10$ ans avec $13,3 \%(n=163)$ et celui des $50-60$ ans avec $22,2 \%(n=271)$ (figure 1$)$.

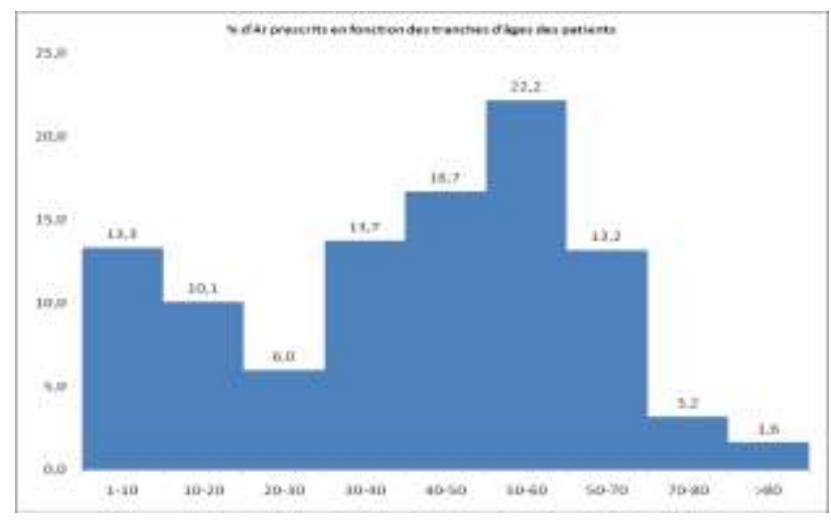

Figure 1. Distribution des AI en fonction de l'âge des patients

\section{Fréquences de prescriptions d'AI au sein d'une même spécialité médicale}

$\mathrm{Au}$ sein de la chirurgie dentaire 53,3\% des lignes prescrites ont été des $\mathrm{AI}$, puis vient l'ophtalmologie où l'on a retrouvé $(30,1 \%)$ des lignes, suivent l'urologie (29\%), la pédiatrie $(27,2 \%)$ et l'ORL $26,8 \%$.

Si $88,7 \%$ des ordonnances ne comportaient qu'un $(n=840)$ à deux $(n=515)$ AI, la valeur maximale de 5 AI toutes formes confondues a été relevée dans $1,6 \%$ des cas $(n=25)$. Il s'agissait dans ce cas d'associations d'AI systémiques avec des AI locaux. 


\section{Familles, DCI et classes ATC d'AI prescrits}

Ont été recensées dans l'étude, 16 familles d'AI (tableau 1). Celle des pénicillines arrivait en tête de liste des fréquences avec $32,7 \%(n=499)$ des produits prescrits.

Elle a été suivie par les macrolides avec 16,6\% (n=253). Ces 16 familles étaient composées de 59 dénominations communes internationales (DCI). Parmi celles-ci, en haut du tableau, l'amoxicilline a été la plus prescrite et retrouvée dans $28,6 \%$ des ordonnances $(16,8 \%$ seule et $11,6 \%$ associée à l'acide clavulanique).

Tableau 1. Fréquence de prescription des AI par famille

\begin{tabular}{lcc}
\hline Familles & $\mathbf{n}$ & $\mathbf{\%}$ \\
\hline PENICILLINE & 499 & 32,7 \\
MACROLIDES & 253 & 16,6 \\
ANTIFONGIQUES & 145 & 9,5 \\
CEPHALOSPORINES & 140 & 9,2 \\
ANTIBIOTIQUES LOCAUX & 101 & 6,6 \\
AMINOSIDES & 84 & 5,5 \\
IMIDAZOLES & 81 & 5,3 \\
QUINOLONES & 50 & 3,3 \\
CYCLINES & 35 & 2,3 \\
FUSIDATE & 33 & 2,2 \\
ANTI INFECTIEUX URINAIRES & 30 & 2,0 \\
SULFAMIDES & 23 & 1,5 \\
ANTI INFECTIEUX LOCAUX & 17 & 1,1 \\
ANTISEPTIQUES & 15 & 1,0 \\
ANTI HELMINTIQUES & 14 & 0,9 \\
ANTIVIRAUX & 7 & 0,5 \\
TOTAL & $\mathbf{1 5 2 7}$ & $\mathbf{1 0 0 , 0}$ \\
\hline
\end{tabular}

À noter un rapport inversé où les macrolides ont été les plus prescrits au niveau de deux spécialités médicales : la pédiatrie où ils ont représenté $43,6 \%$ des AI systémiques vs $23,6 \%$ pour les pénicillines, et la pneumologie avec $41,8 \%$ de macrolides vs 32,8\% de pénicillines. En explorant le type d'AI par classe ATC prescrits (tableau 2), il est apparu que la classe des AI systémiques (antibactériens J01 et antimycosiques J02), totalisait 69\% des lignes $(n=1054)$. Le second groupe (ophtalmologie S01 otologie S02) arrivant loin derrière avec $8,9 \%(n=136)$ réparti entre l'ophtalmologie $(n=102)$ et l'otologie $(n=34)$.

\section{Prescription en fonction de la période}

$\mathrm{Au}$ cours de l'année, l'évolution des prescriptions des AI systémiques (classe ATC J01 et J02) (graphique 2), avait montré 2 pics. Le premier au mois de mars avec $12 \%(n=126)$ des prescriptions, le second en octobre avec $11,7 \%(n=123)$ des prescriptions.

Les 4 derniers mois (de septembre à décembre) et les 4 premiers mois (janvier à avril), avaient totalisé respectivement $40 \%(n=422)$ et $41,2 \%(n=434)$ des lignes de prescriptions des AI systémiques. Le mois de juillet ayant présenté la plus basse fréquence. Pour les AI toutes formes galéniques (systémiques et non systémiques), un profil similaire avait été obtenu.
Tableau2. Distribution des prescriptions des AI par classe ATC dans notre échantillon

\begin{tabular}{|c|c|c|c|}
\hline Classes ATC retrouvées & Ref & $\mathbf{N}$ & $\%$ \\
\hline J01 Antibactériens (usage systémique) & $\mathrm{J} 01$ & 1023 & 67,0 \\
\hline S01 Ophtalmologie & S01 & 102 & 6,7 \\
\hline $\begin{array}{l}\text { D01 Antimycotiques à usage } \\
\text { dermatologique }\end{array}$ & D01 & 91 & 6,0 \\
\hline P01 Antiprotozoaires & P01 & 78 & 5,1 \\
\hline $\begin{array}{l}\text { G01 Anti-infectieux et antiseptiques à } \\
\text { usage gynécologique }\end{array}$ & G01 & 43 & 2,8 \\
\hline R01 Médicaments pour le nez & R01 & 43 & 2,8 \\
\hline S02 Otologie & S02 & 34 & 2,2 \\
\hline J02 Antimycotiques (usage systémique) & $\mathrm{J} 02$ & 31 & 2,0 \\
\hline $\begin{array}{l}\text { D06 Antibiotiques et agents } \\
\text { chimiothérapeutiques à usage } \\
\text { dermatologique }\end{array}$ & D06 & 26 & 1,7 \\
\hline $\begin{array}{l}\text { A07 Antidiarrhéiques, anti- } \\
\text { inflammatoires intestinaux/agents anti- } \\
\text { infectieux }\end{array}$ & A07 & 16 & 1,0 \\
\hline $\begin{array}{l}\text { D07 Préparations dermatologiques à base } \\
\text { de corticostéroïdes }\end{array}$ & D07 & 16 & 1,0 \\
\hline P02 Anthelmintiques & P02 & 14 & 0,9 \\
\hline A01 Préparations stomatologiques & $\mathrm{A} 01$ & 9 & 0,6 \\
\hline D10 Anti-acnéiques & D10 & 1 & 0,1 \\
\hline Total & & 1527 & 100,0 \\
\hline
\end{tabular}

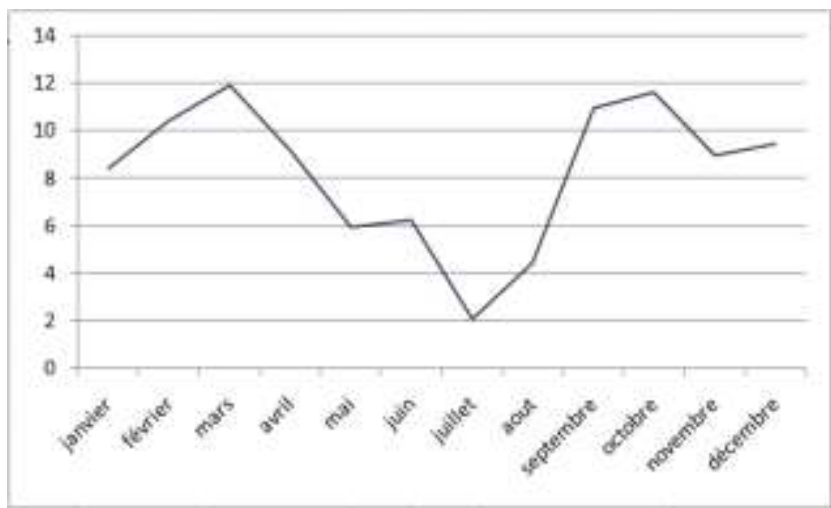

Figure 2. Evolution du \% des prescriptions d'AI systémiques au cours de l'année

\section{DISCUSSION}

Sur la base des 3143 ordonnances analysées, 32,7\% de prescriptions contenaient au moins 1 AI comparés aux 41,2\% de prescriptions d'antibiotiques retrouvées en Inde au cours de la même période et pendant la même durée d'étude [9]. Cette classe pharmaco thérapeutique revêt une importance certaine pour la santé publique. En termes de consommation de médicaments, les données fournies par le LGO de l'officine, rapportaient une part de $86,5 \%$ du CA pour les ordonnances servies dans le cadre du tiers payant. Sur les 24 classes pharmaco-thérapeutiques les AI occupaient 7,3\% des ventes en valeur et $11,6 \%$ en volume (unités ventes). Cette classe se situait en $4 \mathrm{e}$ position, après respectivement les classes métabolisme diabète, puis cardiologie angiologie, puis les antalgiques antispasmodiques. 
Il s'agit à notre connaissance de la première étude menée dans la ville de Batna pour dresser le profil de la prescription des AI dans une officine donnée. Nos données ne portent que sur une officine pharmaceutique privée d'une ville moyenne algérienne, elles ne prétendent à aucune représentation géographique. Celles-ci portent uniquement sur les ordonnances d'assurés sociaux atteints d'une maladie chronique ou non ainsi que leurs ayants droits, reçues entre juillet 2013 et juillet 2014 dans le cadre du conventionnement avec la CNAS (caisse de sécurité sociale des salariés). Il faut noter qu'au cours de notre étude, des ruptures avaient été enregistrées. Elles ont concerné en particulier 1 aminoside (gentamicine), 1 polymixine (colistine). Ces ruptures de médicaments, certaines chroniques (colistine) d'autres aigues, pourraient biaiser les résultats.

En dépit de ces limites, notre étude semble rejoindre les résultats de l'enquête nationale santé Tahina 2007 [1], qui avait rapporté une morbidité due aux maladies infectieuses et parasitaires de $4,68 \%$ chez les femmes et 5,51\% chez les hommes liée à une forte consommation d'antibiotiques. Si les résultats ont identifié la médecine générale comme étant le plus gros prescripteur d'AI en volume, avec 51,4\% de l'ensemble des ordonnances, c'est la chirurgie dentaire qui est la spécialité où l'on en prescrit le plus (1 ligne sur 2).

Il faut rappeler toutefois qu'en dentisterie, les prescriptions sont soumises à une liste limitative comportant les AI, les antalgiques et les anti-inflammatoires. Au Brésil, des orthodontistes avaient déclaré avoir prescrit des antibiotiques dans des situations où ceux-ci n'étaient pas justifiés [10]. Cela suggère, dans les plans de prévention, une évaluation des mesures d'hygiène mises en place dans les cabinets dentaires. A cela s'ajoute l'évaluation de la pertinence de la prescription des AI dans la pratique dentaire qui a priori ne nécessite pas systématiquement une couverture antibiotique des patients. Une probable actualisation peut être envisagée, en vue de revoir les schémas thérapeutiques et ainsi réduire la fréquence de prescription de ces médicaments, comme rapporté par une étude qui montre que $89,4 \%$ des antibio prophylaxies en chirurgie dentaire, n'étaient pas justifiées [11].

Nous retrouvons deux tranches d'âges les 0-10 ans (13,3\%) et les 50-60 ans (22,2\%) qui sont les plus exposées aux prescriptions d'AI. Tandis qu'une étude néerlandaise fait apparaitre un taux de prescription supérieur chez les adolescents vs enfant de 0 à 11 ans [12].

Les résultats que nous avons obtenus, ont fait apparaitre en tête des prescriptions, la famille des pénicillines avec 32,6\% des anti-infectieux prescrits (tableau 1). Cette famille est apparue en tête des prescriptions d'antibiotiques avec $60,8 \%$ dans le secteur officinal en Tunisie en 2013 [13], ainsi qu'en France avec $62,9 \%$ en 2015[14]. Ces résultats sont en cohérence avec l'ensemble des travaux qui rapportent le lien entre la fréquence de prescription des AI et la résistance aux anti-microbiens (RAM). Dans cette famille, l'amoxicilline a été la DCI la plus prescrite avec 16,9\% des lignes de prescriptions d'AI. Cette DCI couvrait en 2010 selon le ministère de la santé, 24,22 \% de parts de marché avec une valeur de 1.293.812.146 DA (tableau 2).

Par cette prescription dominante de l'amoxicilline, notre étude confirme les résultats obtenus en lien avec le pourcentage d'Escherichia coli résistant à cet antibiotique [15].
Cette résistance a été la plus élevée avec l'amoxicilline/ampicilline avec une moyenne de $79,65 \%$ en milieu hospitalier et $72,81 \%$ pour les patients externes.

La mise en évidence de deux pics de prescriptions maximales au cours de la saison froide, permet d'identifier les périodes où le renforcement des stratégies d'usage rationnel des antibiotiques est le plus optimal, dont le Programme National de lutte contre les maladies diarrhéiques et infections respiratoires aigües PNLMDIRA. Cette période est naturellement propice aux infections des voies respiratoires, et nous rapproche des prélèvements des voies respiratoires basses, qui ont mis évidence $23,74 \%$ de bactéries multirésistantes (BMR) [15].

Dans un contexte, où les éléments chiffrés sur le sujet sont relativement pauvres dans notre pays, ces résultats peuvent constituer un point de départ, obtenu à un temps $\mathrm{T}$-1, et ainsi permettre d'évaluer l'impact de stratégies mises en place à T0 en comparant les résultats obtenus après leur mise en place à $\mathrm{T}+1$. De ce point de vue, les données disponibles au niveau de la sécurité sociale constituent un véritable Big data de grande valeur, par le fait qu'elles sont régulièrement alimentées par l'ensemble des officines conventionnées du pays.

\section{CONCLUSION}

Nos résultats pourraient bien converger avec le fait que l'exposition massive d'une population à un $\mathrm{AI}$ contribue à la sélection de souches résistantes [16].

La mise en place de référentiel pour l'usage rationnel des AI est éclairée par les résultats obtenus, à l'image du modèle du Conseil du Médicament du Québec élaboré en 2007 [17] contribuant à une stricte prescription des antibiotiques aux seules situations cliniques où une efficacité a été démontrée [18].

L'accès pour les chercheurs ou les académiciens aux bases de données existantes, dont la BIG DATA de la sécurité sociale en particulier sur le suivi de la consommation des AI, contribuerait à l'élaboration d'outils d'aide à la dispensation au niveau des officines et en milieu hospitalier. Ces données contribueraient par ailleurs à l'élaboration de protocole précis d'usage rationnel des $\mathrm{AI}$ tant pour les prescripteurs, que pour les dispensateurs. Le suivi régulier de la consommation des antibiotiques en santé humaine et animale, est l'objectif 11 du plan stratégique de lutte contre les résistances aux antimicrobiens, élaboré par nos voisins tunisiens [19].

Déclaration d'intérêts: les auteurs ne déclarent aucun conflit d'intérêt en rapport avec cet article.

\section{RÉFÉRENCES}

1. Institut National de Santé Publique Enquête Nationale Santé Tahina 2007 p43. p45.

2. Atif M L, Boubechou N, Beddek M, Bezzaoucha A, Evolution sur 3 années de la prévalence des traitements antibiotiques dans un $\mathrm{CHU}$ en Algérie $\mathrm{CHU}$ Blida. 2016 Médecine et Maladies Infectieuses 46(4):36-37.

3.Centre National de Pharmacovigilance. http://www.sante.dz/jms2011/khris_cnpm.pdf (consulté le 25 décembre 2017).

4. Turki I. Etat de santé des Tunisiens en 2030.Mémoire master professionnel de prospective appliquée. Université virtuelle de Tunis ; juin 2008, p11.

5. Organisation Mondiale de la Santé. Journée mondiale de la santé https://www.who.int/world-health-day/2011/fr/ (consulté le28 mars 2018). 
6. Randriatsarafara F M, Ralamboson J, Rakotoarivelo R et al. Consommation d'antibiotiques au Centre Hospitalier Universitaire d'Antananarivo : prévalence et défis stratégiques. Santé Publique, 2015/2 (Vol. 27), p. 249-255.

7. Guide pour une méthode de calcul de la consommation des antibiotiques dans les établissements de santé et en ville - Travaux du comité national de suivi du plan pour préserver l'efficacité des ATB 2006 (p16).

8. Boyer A. Maitrise de la résistance bactérienne : réflexion sur la phase empirique de l'antibiothérapie en réanimation. Thèse INSERM U657, Bordeaux 2, 2012 p43.

9. Farooqui $\mathrm{H} \mathrm{H}$, Mehta A, Selvaraj S. Outpatient antibiotic prescription rate and pattern in the private sector in India: evidence from medical audit data. 2013-2014 PLOS ONE 2019;

10. M R Bolfoni, Fg Pappen et Al. Antibiotic prescription for endodontic infection: a survey of Brazilian endodontists. - International Endodontic Journal. 2018;51(2):148-156.

11. Freyder C. Evaluation des pratiques de prescription d'antibioprophylaxie en chirurgie dentaire chez les patients adultes au CHU de Nice - Thèse 2017 Faculté de chirurgie dentaire de Nice (p32).

12. Ivanovska V, Hek K, Mantel K, et al. Age specific antibiotic prescribing and adherence to guidelines in pediatric patients in primary - Pediatr Infect Dis J. 2018 Mar;37(3):218-223.
13. Toumi S., Miled S., Fradi I. Etat des lieux sur la consommation nationale des antibiotiques en Tunisie 2011 -2013 ministère de la santé, direction de la pharmacie.

14. Cavalié P, Hider-Mlynarz et al. Evolution de la consommation des antibiotiques en France entre 2000 et 2015 - Rapport ANSM p18.

15. Rahal $\mathrm{k}$ et al. Réseau Algérien de surveillance de la Résistance des Bactéries aux Antibiotiques (AARN), 17ème rapport d'évaluation 2016, édition 2018 p117.

16. Guillemot D. Les liens consommation des antibiotiques/résistance bactérienne. Revue Médicale Suisse 2000 vol 420793.

17. Institut National d'excellence en Santé et en services sociaux du Quebec. (Consulté le 01 avril 2018) https://www.inesss.qc.ca/fileadmin/doc/INESSS/Outils/GUO/InfectResp Feui $\underline{\text { lle-suivi-Interactive.pdf }}$

18. Recommandations de bonnes pratiques dans la prescription des antibiotiques en pratique buccodentaire- Agence Française de sécurité sanitaire des produits de santé, juillet 2011 p8.

https://www.ansm.sante.fr/var/ansm_site/storage/original/application/753c 041773b2cebeab1ec25bdba06d33.pdf Consulté le 10 aout 2020

19. Plan d'action national de lutte contre la résistance aux antimicrobiens en Tunisie 2019 -2023. Ministère de la santé (p24).

Cet article a été publié dans le « Batna Journal of Medical Sciences » BJMS, l’organe officiel de « l'association de la Recherche Pharmaceutique - Batna»

Le contenu de la Revue est ouvert « Open Access » et permet au lecteur de télécharger, d'utiliser le contenu dans un but personnel ou d'enseignement, sans demander l'autorisation de l'éditeur/auteur.

Avantages à publier dans BJMS :

- Open access : une fois publié, votre article est disponible gratuitement au téléchargement

- Soumission gratuite : pas de frais de soumission, contrairement à la plupart des revues « Open Access »

- Possibilité de publier dans 3 langues : français, anglais, arabe

- Qualité de la relecture : des relecteurs/reviewers indépendants géographiquement, respectant l'anonymat, pour garantir la neutralité et la qualité des manuscrits.

Pour plus d'informations, contacter BatnaJMS@gmail.com ou connectez-vous sur le site de la revue : www.batnajms.net 


\section{Bis}

- $\bigcirc$

-

O

○

${ }^{1}$ Laboratoire de pharmacognosie, département de pharmacie, faculté de médecine de Batna, Algérie

${ }^{2}$ Laboratoire de microbiologie, établissement hospitalier public de Biskra, Algérie

${ }^{3}$ Laboratoire Physio-Toxicologie, Pathologie cellulaire moléculaire-Biomolécules

${ }^{4}$ Département de PharmacieFaculté de Médecine Université de Batna

${ }^{5}$ Laboratoire de chimie analytique, département de pharmacie, faculté de médecine d'Annaba, Algérie

${ }^{6}$ Laboratoire pour le développement et le contrôle des préparations pharmaceutiques hospitalières.

${ }^{7}$ Département de pharmacie de la faculté de médecine de l'université Badji MokhtarAnnaba.

\section{Correspondance à :}

Mohammed Tahar BEN-MOUSSA taherpgnosie@yahoo.fr

DOI :https://doi.org/10.48087/BI MSoa.2020.7213

\section{Historique de l'article :}

Reçu le 02 juin 2020

Accepté le 06 septembre 2020

Publié le 09 novembre 2020

Il s'agit d'un article en libre accès distribué selon les termes de la licence Creative Commons

Attribution International License (CC BY 4.0), qui autorise une

utilisation, une distribution et une reproduction sans restriction sur tout support ou format, à condition que l'auteur original et la revue soient dûment crédités.

\section{Pour citer l'article :}

Ben Moussa M.T, Khelil K, Hassina $\mathrm{H}$, et al. Composition chimique, activité antimicrobienne et antioxydante de l'huile essentielle de Brocchia cinerea VIS. d'Algérie. Batna J Med Sci 2020;7(2):122-8. https://doi.org/10.48087/BJMS oa.2020.7213

Composition chimique, activité antimicrobienne et antioxydante de l'huile essentielle de Brocchia cinerea VIS. d'Algérie

\author{
Chemical composition, antimicrobial and antioxidant activity of the essential \\ oil of Brocchia cinerea VIS. from Algeria
}

\title{
Mohammed Tahar Ben-Moussa ${ }^{1}$, Khaled Khelil ${ }^{2}$, Hassina Harkat ${ }^{3,4}$, Samia Lakehal ${ }^{5}$, Youcef Hadef ${ }^{6,7}$
}

\section{RÉSUMÉ}

Ce travail vise l'étude de la composition chimique, de l'activité antioxydante et antimicrobienne de l'huile essentielle (HE) de Brocchia cinerea d'Algérie vis-à-vis de trois bactéries, d'un champignon. L'analyse chromatographique (CG/SM) a montré que l'HE de Brocchia cinerea est caractérisée par la présence du Beta.-Thujone (46,80), 1-Methyl-2-(1'-methylethenyl)-3'ethenylcyclopropylmethanol $(14,59)$ et du 1,8-Cineole (12,63), limonen-10-ol (9,47), accompagnés d'autres composés à des teneurs relativement faibles : 1(7),3,8-0Menthatriene (3,45), (-)-Camphor $(2,11)$. L'activité antioxydante déterminée par le test DPPH a montré que l'HE présente un faible potentiel antioxydant avec l'IC 50 $=6652 \mu \mathrm{g} / \mathrm{ml}$ et IC $50=140.53$ par FRAP, en comparaison à d'autres espèces de la famille des Asteraceae. Aux concentrations étudiées, l'essence a manifesté une forte activité antibactérienne et antifongique. Cette bioactivité est due principalement à la richesse de cette essence en Beta.-Thujone connu pour son efficacité contre les agents microbiens.

Mots clés : Brocchia cinerea, Huile essentielle, Activité antimicrobienne, Activité antioxydante, Composition chimique.

\begin{abstract}
This work aims to study the chemical composition, antioxidant and antimicrobial activity of the essential oil (EO) of Brocchia cinerea from Algeria against three bacteria and one fungus. Chromatographic analyses (GC/MS) have shown that the essential oil of Brocchia cinerea is characterized by the presence of Beta-. Thujone (46.80), 1-Methyl-2-(1'-methylethenyl)-3'ethenylcyclopropylmethanol (14.59) and 1,8-Cineole (12.63), limonen-10-ol (9.47), accompanied by other compounds at relatively low levels: 1(7),3,8-oMenthatriene (3.45), (-)-Camphor (2.11). The antioxidant activity determined by the DPPH test showed that EO has a low antioxidant potential with IC $50=6652 \mu \mathrm{g} / \mathrm{ml}$ and IC $50=140.53$ per FRAP, compared to other species of the Asteraceae family. At the concentrations studied, the essence showed strong antibacterial and antifungal activity. This bioactivity is mainly due to the richness of this species in Beta-Thujone known for its effectiveness against microbial agents.
\end{abstract}

Keywords : Brocchia cinerea, Essential oil, Antimicrobial activity, Antioxidant activity, Chemical composition.

\section{Abréviations}

HE : huile essentielle

$\mathbf{m L}$ : millilitre

Kg : Kilogramme

CPG : chromatographie phase gazeuse

SM : spectrométrie de masse

${ }^{\circ} \mathrm{C}$ : degré Celsius

g : gramme

L : litre

ATCC : American Type Culture Collection

$\mathrm{MH}:$ Mueller-Hinton

$\mu \mathrm{L}$ : microlitre

CFU : Unité Formant Colonie

$\mathbf{h}$ : heure
CMI : concentration minimale inhibitrice

CMB : concentration minimale bactéricide

PDB : Potato dextrose broth

PDA : Potato dextrose Agarose

DPPH : 1,1-diphénylpicrylhydrazyl

FRAP : Ferric reducing-antioxidant power

mM : milli mole

AA : activité antioxydante,

Abs : absorbance

$\mathbf{n m}$ : nanomètre

$\mathrm{FeCl}_{3}$ : Chlorure ferrique

UV-VIS : ultraviolet - visible

IC50 : inhibition concentration 50

\section{INTRODUCTION}

Les plantes ont été la ressource de base pour la plupart des besoins de l'homme depuis l'époque des aborigènes. Récemment, l'intérêt pour les plantes médicinales s'est accru en raison de l'augmentation de l'utilisation des plantes médicinales et de leurs effets thérapeutiques $(1,2)$.

Les plantes étant une grande source de métabolites secondaires bioactifs, elles jouent un rôle vital dans le domaine du développement des médicaments (HE) (3-5).
Ces métabolites sont des produits à forte valeur ajoutée, utilisées non seulement dans les industries pharmaceutiques mais aussi cosmétiques et agroalimentaires. La famille des Asteraceae englobe de nombreuses espèces et variétés, et la composition chimique des HE de certaines d'entre elles a été étudiée depuis longtemps (6).

Aussi, les huiles de plusieurs espèces de la famille des Asteraceae sont investiguées pour leurs propriétés antibactériennes et antifongiques et également pour leur activité antioxydante (6-8). 
En Algérie, la famille des Asteraceae renferme 408 espèces répartis en 109 genres (9). Brocchia cinerea (Delile) Vis. est une plante annuelle herbacée laineuse de $5-15 \mathrm{~cm}$ complètement, tomenteuses. Les feuilles et les tiges vert blanchâtre sont recouvertes de poils minuscules épais. Ses tiges sont dressées ou diffuse, ses fleurs toutes en tubes et ses feuilles sont d'épaisseur divisée à une partie supérieure avec deux ou trois segments, en la tige de la branche supérieure il y a des inflorescences jaunes. Cette plante se développe dans des conditions désertiques (végétales xérophiles) et favorise les sols de sable limoneux $(10,11)$. Brocchia cinerea est une plante xérophyte ; il prospère dans des conditions désertiques avec une pluviométrie annuelle moyenne de $100 \mathrm{~mm}$. La plante préfère les sols sablo-limoneux, et on la trouve généralement sur des lits d'oueds non salins sur des sols sableux graveleux (12). Géographiquement, il est largement distribué en Afrique du Nord, en particulier dans les régions sahariennes de l'Algérie et du Maroc, la région de la mer Rouge, le Sinaï, la dépression de Qattara et le Mali (13).

Traditionnellement, Brocchia cinerea (Delile) Vis est largement utilisé pour traiter plusieurs maladies comme les coliques, la toux, la diarrhée et les troubles digestifs. La plante est généralement appliquée sous forme de décoction, de macération, de perfusion et d'inhalation (14-16). En plus de ses utilisations médicinales, les nomades utilisent la plante comme additif de thé pour améliorer le goût du thé. Il est également utilisé pour filtrer le beurre de chèvre, en raison de ses bonnes propriétés de conservation (17).

En Algérie, peu de travaux ont été consacrés à l'étude du profil chimique de l'HE de Brocchia cinerea. À notre connaissance, l'activité antibactérienne, antifongique et antioxydante de cette essence n'a fait l'objet d'aucune étude auparavant. Dans ce contexte s'inscrit le présent travail dont l'objectif essentiel consiste à étudier l'activité antimicrobienne, antioxydante et la composition chimique de l'HE de Brocchia cinerea d'Algérie.

\section{MATÉRIELS ET MÉTHODES}

\section{Matériel végétal}

Les échantillons de la partie aérienne (tiges, feuilles et fleurs) de Brocchia cinerea ont été récoltés au mois de Mars dans la région de M'lili Biskra d'Algérie.

\section{Mode d'obtention des HE}

Les huiles essentielles sont extraites par hydro-distillation des parties aériennes de cette espèce pendant 3 heures de temps à l'aide d'un appareil d'extraction normalisée par la pharmacopée européenne (18). L'opération est répétée plusieurs fois pour chaque échantillon de la matière végétale sèche. Le rendement en huile essentielle est déterminé en $\mathrm{mL} / \mathrm{Kg}$ de matière sèche. L'huile essentielle est ensuite stockée à $4^{\circ} \mathrm{C}$ à l'abri de la lumière.

\section{Appareillage et protocole expérimentale de l'analyse des huiles essentielles par CPG/SM}

L'analyse chromatographique des huiles essentielles a été réalisé par chromatographie en phase gazeuse couplée à un spectromètre de masse de type Clarus 600 D MS (Perkin Elmer USA). La colonne capillaire utilisée est de type RESTEK $\mathrm{Rtx}^{\circledR}$-5MS de 30 mètres de longueur, $0,25 \mathrm{~mm}$ de diamètre interne épaisseur du film $0.25 \mu \mathrm{m}$, la phase stationnaire. Les injections ont été faites en mode splitless. L'hélium a été employé comme gaz vecteur à un débit de $1 \mathrm{~mL} / \mathrm{min}$. Les températures de l'injecteur et de la ligne de transfert ont été portées à $250^{\circ} \mathrm{C}$. La température initiale a été fixée à $60^{\circ} \mathrm{C}$ et maintenue pendant 1 minute, puis augmentée de $3{ }^{\circ} \mathrm{C} / \mathrm{min}$ jusqu'à $200{ }^{\circ} \mathrm{C}$ et on maintient en isotherme pendant 13 minutes.
L'acquisition est faite en impact électronique à $70 \mathrm{eV}$, avec une source à $250{ }^{\circ} \mathrm{C}$ en mode Scan (de 40 jusqu'à 600). L'identification des composés ont été réalisé par comparaison des spectres de masse avec ceux donnés par les librairies WILEY et NIST.

Les huiles essentielles ont été diluées dans de l'éthanol absolu à une concentration de $1 \mathrm{~g} / \mathrm{L}$.

La teneur en pourcentage des constituants des huiles essentielles est déterminée par la méthode de normalisation interne.

\section{Micro-organismes étudiés}

L'activité antibactérienne et antifongique a été évaluée sur différents microorganismes :

- bactéries : les trois souches bactériennes (Escherichia coli, Pseudomonas aeruginosa et Staphylococcus aureus) choisies au cours de cette étude sont de type ATCC.

Elles ont été entretenues par repiquage sur gélose (Sigma) nutritive favorable à leur croissance pendant 24 heures, à l'obscurité et à $37^{\circ} \mathrm{C}$;

- moisissures : Candida albicans, a été choisies pour sa fréquence élevée dans les infections digestive et gynécologique. Elle est cultivée sur le milieu nutritif PDA (Potato Dextrose Agar) 48 heures, à $37^{\circ} \mathrm{C}$ et a l'obscurité.

\section{Activité antibactérienne}

La préparation d'inoculum bactérien est habituellement effectuée après plusieurs étapes. Initialement, les échantillons maintenus congelés ou réfrigérés doivent être activés dans un milieu $\mathrm{MH}$ liquide. Après 6 à 8 heures à $35^{\circ} \mathrm{C}$, une aliquote est transférée dans un milieu de $\mathrm{MH}$. Après $24 \mathrm{~h}$ à $35^{\circ} \mathrm{C}$, les colonies de culture axénique peuvent être mises en suspension dans $5 \mathrm{~mL}$ de solution saline stérile $(8,5 \mathrm{~g} / \mathrm{L}$ de $\mathrm{NaCl}$ ) et mesuré à l'aide d'un densitomètre 0,5 McFarland (correspondant à $1-2$ x 108 UFC / mL) (19). Le milieu de culture est constitué de Muller Hinton liquide avec 0.5\% tween 80 .

La technique est généralement réalisée dans des plaques en U avec 96 puits et présente des variations par rapport à la méthode originale décrite par Eloff (20). $20 \mu \mathrm{L}$ de l'huile essentielle est ajouté dans le premier puits qui contient 170 $\mu \mathrm{L}$ de bouillon Mueller-Hinton (tween $80: 0.5 \%$ ) les autres puits contient déjà $95 \mu \mathrm{l}$ Mueller-Hinton (tween 80 : 0.5\%) Après homogénéisation du premier puits $95 \mu \mathrm{l}$ du mélange du premier puits est transféré au deuxième puits et ainsi de suite, les $95 \mu \mathrm{l}$ du dernier puits sont éliminé.

À la fin $5 \mu \mathrm{L}$ d'une suspension bactérienne de 3,5 × $10^{7} \mathrm{CFU} /$ $\mathrm{mL}$ sont ajoutés à chaque puits. Les résultats sont lus après une période d'incubation de $24 / 35^{\circ} \mathrm{C}$.

La valeur CMI est la concentration la plus faible du produit naturel qui inhibe visuellement la croissance microbienne. A partir des tubes sans croissance visible, $10 \mu \mathrm{L}$ de solution sont retirés et étalés sur gélose Mueller-Hinton et incubés pendant encore $24 \mathrm{~h} / 35^{\circ} \mathrm{C}$ pour déterminer la concentration bactéricide minimale (CMB). L'absence d'unités formant des colonies (ou une croissance inférieure à $0,1 \%$ de l'inoculum initial) indique que les huiles essentielles sont bactéricides. Le pourcentage d'inhibition de la croissance bactérienne par des huiles essentielles des deux plantes peut également être calculé en utilisant un spectrophotomètre en comparaison avec des puits témoins positifs (milieu de culture sans extraits et exempt des microorganismes) et des puits témoins négatifs (antibiotique plus microorganisme). 


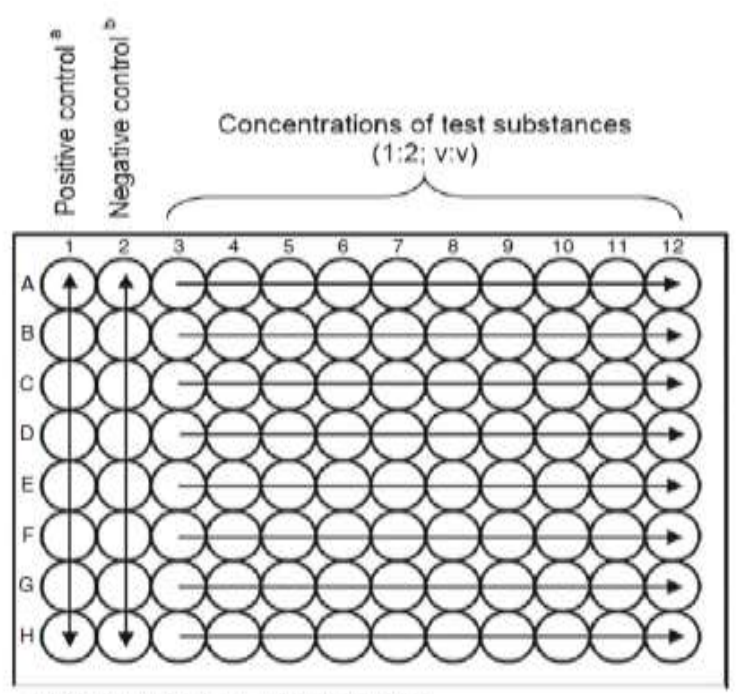

Microorganism + culture media;

${ }^{b}$ Microorganism + culture media + antibiotic.

Figure 1. Microplaque utilisée dans l'activité antibactérienne et antifongique

\section{Activité antifongique}

\section{Préparation du milieu de culture}

L'infusion de pomme de terre se prépare en faisant bouillir dans l'eau $200 \mathrm{~g}$ de pommes de terre tranchées (lavées mais non pelées) pendant 30 minutes en laissant décanter le bouillon obtenu et en le filtrant à travers un coton. Ensuite en ajoutant de l'eau distillée pour un volume final d'un litre. Puis $20 \mathrm{~g}$ de dextrose et autant d'agar-agar en poudre y sont dissous avant une stérilisation par autoclave à $100 \mathrm{kPa}$ pendant 15 minutes.

\section{Potato dextrose broth}

Un milieu de culture similaire est préparé de manière identique au PDA, mais en omettant l'agar-agar et en ajoutant $5 \mathrm{ml}$ de tween 80 pour un litre.

Une souche pathogène de Candida albicans est repiquée sur le milieu de culture (PDA + tween 80 à $0.5 \%)$ et incubée à $37^{\circ} \mathrm{C}$ pendant $48 \mathrm{~h}$.

À l'aide de l'eau physiologique, une solution de Candida albicans est préparée avec une densité $0.5 \mathrm{McF}$ arland à l'aide d'un densitomètre et laissée reposer pendant 3 minutes.

La suspension fongique est diluée à $1 / 10$ dans le PDB.

Les puits du plateau de micro-titrage sont remplis avec $85 \mu \mathrm{l}$ de PDB sauf le premier puits $170 \mu \mathrm{l}$ de PDB et $20 \mu \mathrm{l}$ de l'huile essentielle, $95 \mu \mathrm{l}$ du mélange du premier puits est transféré au deuxième puits ainsi de suite, les $95 \mu \mathrm{l}$ du dernier puits doivent être éliminé et $5 \mu \mathrm{l}$ de la suspension fongique sont ajouté à chaque puits.

Les puits témoins de croissance contiennent $5 \mu \mathrm{L}$ de suspension fongique et $95 \mu \mathrm{L}$ de PDB.

Un agent antifongique est ajouté à la suspension fongique pour un contrôle positif et le milieu de culture avec la suspension fongique comme contrôle négatif.

Le bac est incubé sans agitation à $35^{\circ} \mathrm{C}$ jusqu'à $70 \mathrm{~h}$, en vérifiant la croissance visible dans les puits après 24 et $48 \mathrm{~h}$. Le produit naturel démontrant une activité antifongique empêchera toute croissance discernable de Candida albicans (21).

\section{Activité antioxydante}

a) Activité de piégeage des radicaux DPPH

Le pouvoir antioxydant de l'HE et des extraits méthanoliques des deux plantes a été testé par la méthode qui utilise le DPPH (1,1-diphénylpicrylhydrazyl) comme un radical libre relativement stable. $(22,23)$

Dans ce test, le DPPH de couleur violette se réduit en un composé jaune, le diphénylpicrylhydrazine, dont l'intensité de la couleur est inversement proportionnelle à la capacité réductrice des antioxydants présents dans le milieu. $(24,25)$ La réaction est réalisée dans un volume total de $2.5 \mathrm{ml}$ contenant $2 \mathrm{ml}$ de DPPH à $0,1 \mathrm{mM}$ solubilisé dans le méthanol. Les échantillons de l'HE et des extrais ont été préparés par dissolution dans le méthanol absolu, ces solutions dites solutions mères subiront ensuite des dilutions pour obtenir les concentrations finales (1, 26-28).

L'acide ascorbique est utilisé comme un antioxydant de référence, une solution mère de $0.05 \mathrm{mg} / \mathrm{ml}$ est préparés, cette solution subira des dilutions pour obtenir des concentrations finales de 1 à $10 \mu \mathrm{g} / \mathrm{mL}$.

Les échantillons sont ensuite laissés à l'obscurité pendant 60 minutes, et la décoloration par rapport au témoin négatif contenant uniquement la solution du DPPH est mesurée à $515 \mathrm{~nm}$. L'activité antioxydante est estimée selon l'équation suivante :

$\mathrm{AA}=([$ Abscontrol-Abstest $] /$ Abscontrol $) \times 100$

$\mathrm{AA}$ : activité antioxydante, Abs : absorbance à $515 \mathrm{~nm}$.(29).

\section{b) FRAP}

Le pouvoir réducteur du fer $(\mathrm{Fe} 3+)$ dans les extraits est déterminé selon la méthode décrite par OYAIZU (1986) (BOUGANDOURA, 2013) $(30,31)$. La méthode de la réduction du fer est basée sur la réduction de fer ferrique en sel de fer (bleu de Prusse) par les antioxydants qui donnent la couleur bleue selon la figure 2 .

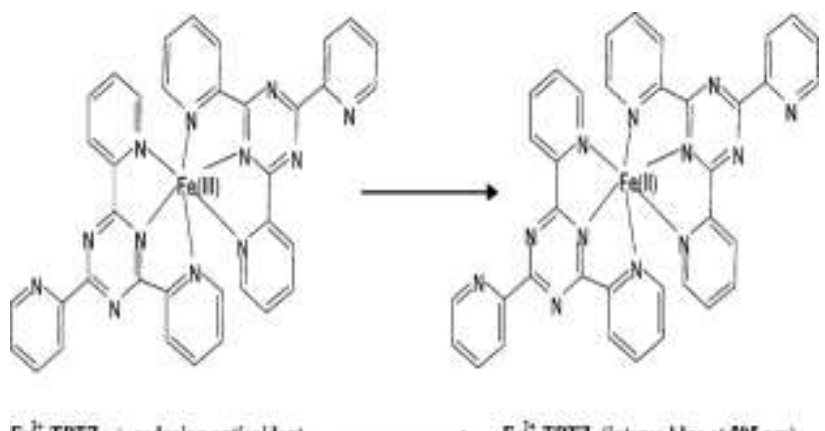

$\mathrm{Fe}^{+}$-TPTZ + redecing antioxidant

\section{$\mathrm{Fe}^{\mathrm{I}}$-TPTZ (intense blue at $595 \mathrm{~nm}$ )}

Figure 2. Schéma sur la réaction de test FRAP (Ferric reducing antioxidant power)

TPTZ : ferric2,4,6-tripyridyl-s-triazine.

$\mathrm{Fe}^{2+}$ : Ions ferreux.

$\mathrm{Fe}^{3+}$ : Ions ferriques

Un millilitre de l'extrait et des huiles essentielles à différentes concentrations est mélangé avec $0,5 \mathrm{~mL}$ d'une solution tampon phosphate $0,2 \mathrm{M}(\mathrm{pH} 6,6)$ et $0,5 \mathrm{ml}$ d'une solution de ferricyanure de potassium $\mathrm{K}_{3} \mathrm{Fe}(\mathrm{CN})_{6}$ à $1 \%$.

L'ensemble est incubé au bain-marie à $50^{\circ} \mathrm{C}$ pendant $20 \mathrm{~min}$ et ensuite laissé refroidir, $0.5 \mathrm{ml}$ d'acide trichloracétique à $10 \%$ sont ajoutés pour stopper la réaction. Les tubes sont centrifugés à $3000 \mathrm{tr} / \mathrm{min}$ pendant $10 \mathrm{~min}$. Un aliquote $(0,5 \mathrm{~mL})$ de surnageant est combinée avec $0,5 \mathrm{ml}$ d'eau distillée et $0,1 \mathrm{~mL}$ d'une solution aqueuse de $\mathrm{FeCl}_{3}$ (Chlorure ferrique) à $0,1 \%$. 
La lecture de l'absorbance du milieu réactionnel se fait à 700 $\mathrm{nm}$ contre un blanc semblablement préparé, en remplaçant l'extrait par de l'eau distillée qui permet de calibrer l'appareil (spectrophotomètre UV-VIS).

Le contrôle positif est représenté par un standard d'un antioxydant ; l'acide ascorbique dont l'absorbance a été mesuré dans les mêmes conditions que les échantillons. Une augmentation de l'absorbance correspond à l'augmentation de l'activité antioxydante (32).

Le pouvoir réducteur de fer est exprimé par IC50 qui correspond à la concentration de l'échantillon donnant une absorbance de 0.5 .

\section{Analyse statistique}

Les méthodes classiques de statistiques ont été utilisées pour calculer les moyennes et les écarts-types. Toutes les mesures ont été reproduites trois fois, et les données sont présentées sous forme de moyenne \pm écart-type.

Les analyses de la variance ont été réalisées par l'Anova avec le logiciel «SPSS ». Une probabilité de $p$ inférieur à 0.05 a été adoptée comme critère de différences significatives.

\section{RÉSULTATS ET DISCUSSION}

\section{Rendement et composition chimique}

Les rendements moyens en HE de Brocchia cinerea ont été exprimés en millilitre par rapport à $1 \mathrm{Kg}$ de matière sèche de la partie aérienne de la plante. Ce taux d'environ 9 est supérieur à celui obtenu par Mebarka \& al (2015) et Chouikh \& al $(2015)(33,34)$.

Les constituants majoritaires (Tableau 1 et figure 3) Beta. Thujone $\quad(46,80), \quad 1$-Methyl-2-(1'-methylethenyl) -3'ethenylcyclopropylmethanol $(14,59)$ et du 1,8-Cineole $(12,63)$, limonen-10-ol $(9,47)$, accompagnés d'autres composés à des teneurs relativement faibles : 1(7),3, 8-oMenthatriene $(3,45),(-)$-Camphor $(2,11)$.

Cette huile diffère par sa composition de celle extraite des feuilles de la même espèce cultivée à Ouargla par Bouziane et al,dont les constituants majoritaires sont Thujone (47.72 $\%)$; camphor (10.54\%); santolinatriene $(8.00 \%)$; eucalyptol (6.37\%); acétate (4.17\%), terpinen-4-ol (2.77\%) (Bouziane $\mathrm{M}$ et all, 2013)(17).

Chouikh et al a trouvé les composés suivants 3-Carène (30.99\%); thujone $(21.73 \%)$; santolina triene (18.58\%); camphor (6.21\%); eucalyptol (2.79\%); 7'Oxaspiro[cyclopropane- ,4'tricyclo[3.3.1.0 $(6,8)]$ nonan-2'one] (2.98\%); terpinen-4-ol (3.64\%); $\rho$-menth-1-en-8-ol (3.01\%); transpinocarveol (1.28\%)(34).

Au Bechar, Les composés majoritaires trouvés par Djellouni sont (E)-citral (24.01\%); cislimonene epoxide (18.26\%); thymol methylether (15.04\%); carvacrol (15.03\%); transcarveol (13.79\%); carvone (3.06\%); transpiperitol $(2.54 \%)(35)$.

Ces résultats indiquent que nous sommes en Algérie en présence de trois chémotypes de Brocchia cinerea : le chémotype à Beta. -Thujone, chémotype 3-Carène et le chémotype à (E)-citral.
Tableau 1. Composition chimique de l'huile essentielle extraite de Brocchia cinerea récoltées à M'lili Biskra (Algérie).

\begin{tabular}{|c|c|c|c|}
\hline & Composants & TR & 8 \\
\hline 01 & $\begin{array}{l}\text { 1-Methyl-2-(1'-methylethenyl)-3'- } \\
\text { ethenylcyclopropylmethanol }\end{array}$ & 5,56 & 14,59 \\
\hline 02 & l-Phellandrene & 6,08 & 0,53 \\
\hline 03 & DELTA.3-Carene & 6,45 & 0,29 \\
\hline 04 & .gamma.-Terpinene & 6,76 & 0,18 \\
\hline 05 & Camphene & 6,7 & 0,10 \\
\hline 06 & Sabinene & 7,4 & 1,21 \\
\hline 07 & 2-Beta.-Pinene & 7,54 & 0,14 \\
\hline 08 & 3-(Cyclopentylmethyl)-1,2-pentadiene & 8,62 & 0,05 \\
\hline 09 & alpha.-Caryophyllene & 8,72 & 0,37 \\
\hline 10 & $\begin{array}{l}\text { 3-[2',4'-Dimethylphenyl]-2,2- } \\
\text { dimethylpropanal }\end{array}$ & 9,04 & 1,09 \\
\hline 11 & 1-Allyltricyclo[4.1.0(2,7)] heptane & 9,23 & 0,62 \\
\hline 12 & 1,8-Cineole & 9,39 & 12,63 \\
\hline 13 & Z-thujenol & 10,02 & 0,16 \\
\hline 14 & 1-Phellandrene & 10,28 & 0,72 \\
\hline 15 & gamma.-Terpinene & 10,71 & 0,35 \\
\hline 16 & Isoamyl-2-Methyl Butyrate & 11,93 & 0,63 \\
\hline 17 & .alpha.-Thujone & 12,07 & 0,07 \\
\hline 18 & Trans Caryophyllene & 12,17 & 0,17 \\
\hline 19 & Beta.-Thujone & 12,61 & 46,80 \\
\hline 20 & Sabinol & 12,74 & 0,11 \\
\hline 21 & 1-Menthene & 13,17 & 0,06 \\
\hline 22 & 1-Allyltricyclo[4.1.0(2,7)] heptane & 13,63 & 0,15 \\
\hline 23 & $(-)$-Camphor & 13,75 & 2,11 \\
\hline 24 & 1(7),3,8-o-Menthatriene & 14,4 & 3,45 \\
\hline 25 & $\begin{array}{l}\text { 1-formyl-1-(2- } \\
\text { phenylethyl)cyclohexane }\end{array}$ & 14,7 & 0,18 \\
\hline 26 & $\begin{array}{l}\text { 1-Isopropyl-4-methyl-1,4- } \\
\text { cyclohexadiene }\end{array}$ & 15,09 & 1,66 \\
\hline 27 & Myrtenol & 15,44 & 0,06 \\
\hline 28 & $\begin{array}{l}\text { Cyclohexène, 1-methyl-4-(1- } \\
\text { methylethenyl }\end{array}$ & 15,65 & 0,81 \\
\hline 29 & (3Z,6Z) -Dodeca-3,6-dien-1-ol & 16,28 & 0,04 \\
\hline 30 & Cis-3-Hexenyl. Alpha. Methylbutyrate & 17,41 & 0,41 \\
\hline 31 & Cis-10-Pinanemethanol & 18,44 & 0,05 \\
\hline 32 & Limonen-10-ol & 19,26 & 9,47 \\
\hline 33 & $\begin{array}{l}\text { Cyclo Pyran } 1.3 \text { Dione 4.4a.5.6 } \\
\text { Tetrahydro 4.7 Dimethyl }\end{array}$ & 19,69 & 0,3 \\
\hline 34 & Lavandulyl Acetate & 19,9 & 0,3 \\
\hline 35 & Thymol & 20,37 & 0,05 \\
\hline 36 & cis-Jasmone & 24,44 & 0,09 \\
\hline 37 & 1(7),4, 8-o-Menthatriene & 28,12 & 0,06 \\
\hline 38 & gamma. -Curcumen-12-ol & 46,02 & 0,02 \\
\hline 39 & $(+,-)$-E-Nuciferol & 46,08 & 0,04 \\
\hline 40 & aromadendrene 2 & 46,24 & 0,01 \\
\hline 41 & Italicen-5-ol & 46,41 & 0,02 \\
\hline \multicolumn{2}{|c|}{ Total } & & 100,00 \\
\hline
\end{tabular}




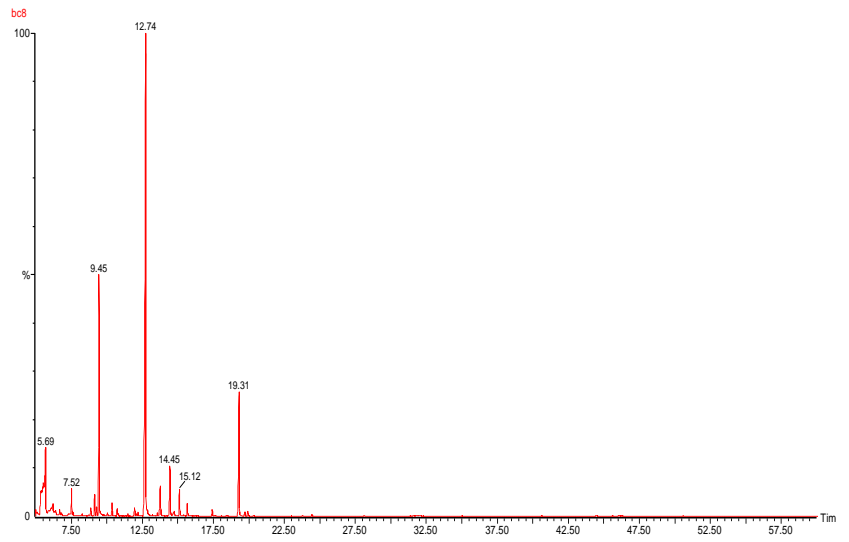

Figure 3. Chromatogramme de l'huile essentielle extraite de Brocchia cinerea récoltées à M’lili Biskra (Algérie).

Le Tableau 2 montre les résultats de l'activité antibactérienne et antifongique de l'HE de Brocchia cinerea. On note que cette essence a exercé une forte activité antibactérienne. La CMI est de $20.92 \mu \mathrm{g} / \mathrm{mL}$ pour Escherichia coli et Pseudomenas aeruginosa, alors que Staphylococcus aureus étaient plus sensibles avec une CMI de 02,61 $\mu \mathrm{g} / \mathrm{mL}$.

À l'égard de Candida albicans, l'essence de Brocchia cinerea a également montré une forte bioactivité, avec une CMI de 10,82 $\mu \mathrm{g} / \mathrm{mL}$. L'activité antifongique de l'essence de Brocchia cinerea est comparable au Fluconazole ou la CMI $\leq 8$.

Aligianis et al. (36) ont proposé une classification de l'activité antimicrobienne des produits végétaux, basée sur les résultats de la CMI, comme suit:

Inhibiteurs puissants - CMI inférieure à $500 \mu \mathrm{g} / \mathrm{ml}$;

Inhibiteurs modérés - CMI entre 600 et $1500 \mu \mathrm{g} / \mathrm{ml}$;

Inhibiteurs faibles - CMI supérieure à $1600 \mu \mathrm{g} / \mathrm{ml}$.

L'huile essentielle de cette plante est bactéricide parce que $\mathrm{CMB} / \mathrm{CMI}<$ à 32 et aussi fongicide parce que $\mathrm{CMF} / \mathrm{CMI}<$ à 32 .

Tableau 2. Activité antimicrobienne et antifongique de l'huile essentielle de Brocchia cinerea.

\begin{tabular}{lcccc}
\hline & $\begin{array}{c}\text { CMI } \\
(\mu \mathrm{g} / \mathbf{m L})\end{array}$ & $\begin{array}{c}\text { CMB } \\
(\mu \mathrm{g} / \mathbf{m L})\end{array}$ & CMB/CMI & $\begin{array}{l}\text { Nature de } \\
\text { l'activité }\end{array}$ \\
\hline $\begin{array}{l}\text { Escherchia coli } \\
\text { Pseudomenas }\end{array}$ & 20,92 & 20,92 & 1 & Bactéricide \\
$\begin{array}{l}\text { aeruginosa } \\
\begin{array}{l}\text { Staphylococcus } \\
\text { aureus }\end{array}\end{array}$ & 02,61 & 41,85 & 2 & Bactéricide \\
$\begin{array}{l}\text { Candida } \\
\text { albicans }\end{array}$ & 10,82 & 10,82 & 16 & Bactéricide \\
\hline
\end{tabular}

Cette huile essentielle a montré une meilleure activité contre les bactéries Gram-positives que les bactéries Gram-négatives car la grande majorité des extraits de plantes sont plus actifs contre les bactéries à Gram positif. La plus grande résistance des bactéries à Gram négatif peut être expliquée par le fait que la membrane externe des bactéries à Gram négatif présente une arille à de nombreuses substances, y compris les antibiotiques (37) et que l'espace périplasmique contient des enzymes capables de dégrader des molécules étrangères (38). De plus, les bactéries Gram-négatives ont des pompes à efflux qui réduisent les taux cellulaires d'antibiotiques (39).
Les résultats obtenus ont révélé les propriétés antibactériennes in vitro potentielles des huiles essentielles des deux espèces étudiées, indiquant l'importance des études liées à leur application dans le traitement antibiotique des maladies infectieuses.

\section{Activité antioxydante DPPH}

\section{Activité antioxydante des HE}

La surproduction des radicaux libres dans l'organisme et le déficit du système de défense endogène sont une composante importante dans la physiopathologie de plusieurs affections (maladies cardiovasculaires, neurologiques et processus néoplasiques).

Actuellement, la recherche vise à renforcer ces défenses endogènes par des substances naturelles issues de plantes, qui sont douées des propriétés antiradicalaires. L'intérêt croissant des effets bénéfiques de l'antioxydant sur la santé a mené au développement d'un grand nombre de tests pour déterminer les capacités antioxydantes des extraits naturels. Deux méthodes ont été employées : piégeage du radical libre DPPH (capacité antiradicalaire) et chélation et la réduction de l'ion ferrique (pouvoir réducteur).

Tableau 3. Activité antioxydante de l'huile essentielle extraite de Brocchia cinerea récoltées à M’lili Biskra (Algérie).

\begin{tabular}{lll}
\hline & $\begin{array}{l}\text { Huile essentielle } \\
(\mu \mathrm{g} / \mathrm{mL})\end{array}$ & $\begin{array}{l}\text { Acide Ascorbique } \\
(\mu \mathrm{g} / \mathrm{mL})\end{array}$ \\
\hline DPPH & $11794,55 \pm 159,53$ & $3,9 \pm 0,4$ \\
FRAP & $143,92 \pm 2,28$ & $2,41 \pm 0,1$ \\
\hline
\end{tabular}

L'activité antioxydante de l'essence de Brocchia cinerea a été évaluée par deux technique DPPH et FRAP.

La concentration qui fournit 50\% d'inhibition (IC 50) calculée à partir de la courbe de la Figure 4 et présentée dans le Tableau 3 est de l'ordre de 11794,55 \pm 159,53 $\mu \mathrm{g} / \mathrm{ml}$. Le pouvoir antioxydant de l'acide ascorbique est de $(3,9 \pm 0,4$ $\mu \mathrm{g} / \mathrm{ml})$. Avec cette valeur $(11794,55 \pm 159,53 \mu \mathrm{g} / \mathrm{ml})$, l'activité antioxydante de l'HE de Brocchia cinerea est beaucoup plus que celle de T. vulgaris $(450,11 \pm 5,23 \mu \mathrm{g} / \mathrm{ml})$, Ch. nobile $\quad(602,73 \pm 4,8 \mu \mathrm{g} / \mathrm{ml}), \quad$ Ziz. clinopodioides $(1238,82 \pm 9,3 \mu \mathrm{g} / \mathrm{ml})$, Cu. cyminum $(1255,52 \pm 8,92 \mu \mathrm{g} / \mathrm{mL})$ et Zinc. officinale (5595,06 $\pm 8,24 \mu \mathrm{g} / \mathrm{mL}$ ) (40).

Le résultat de l'activité réductrice de fer est de $143,92 \pm 2,28 \mu \mathrm{g} / \mathrm{mL}$ pour l'huile essentielle de Brocchia cinerea et de $2,41 \pm 0,1 \mu \mathrm{g} / \mathrm{ml}$ pour l'acide ascorbique.
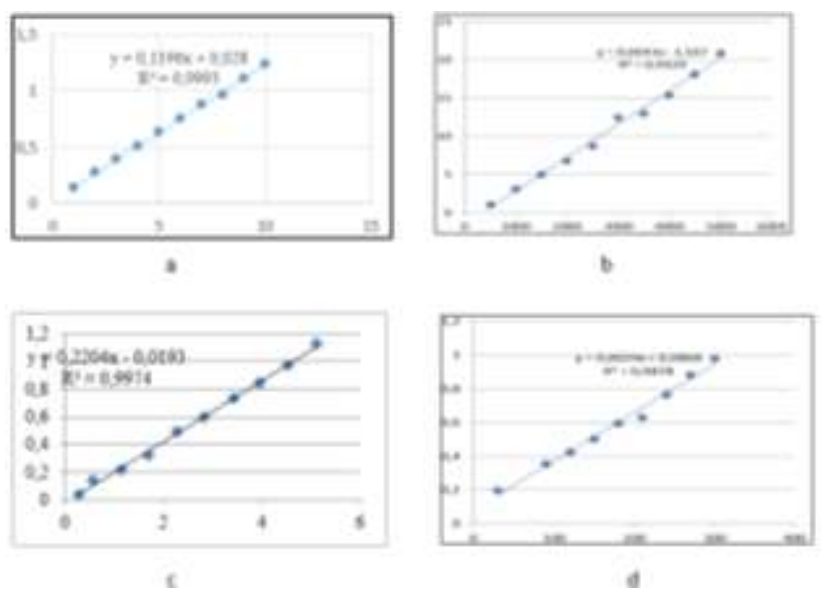

Figure 4 Courbes d'étalonnage pour la détermination de l'activité antioxydante.

$$
\begin{array}{ll}
\text { a-acide ascorbique par DPPH } & \text { b-HE par DPPH } \\
\text { c-acide ascorbique par FRAP } & \text { d-HE par FRAP }
\end{array}
$$




\section{CONCLUSION}

Dans ce travail, nous avons caractérisé la composition chimique de l'essence de Brocchia cinerea des régions arides sud-est d'Algérie. L'identification des constituants a été réalisée en se fondant sur leur spectre de masse obtenu par chromatographie en phase gazeuse couplée à la spectrométrie de masse. L'analyse chromatographique a permis d'identifier 41 constituants avec une codominance de Beta-Thujone $(46,80), \quad$ 1-Methyl-2-(1'-methylethenyl)-3'ethenylcyclopropylmethanol (14,59) et du 1,8-Cineole $(12,63)$, limonen-10-ol $(9,47)$. L'efficacité antimicrobienne des HE de Brocchia cinerea d'Algérie a été démontrée vis-à-vis de trois bactéries ATCC et un champignon.

Notre intérêt s'est orienté aussi vers l'activité antioxydante de l'essence de Brocchia cinerea dans le cadre de la recherche de nouveaux antioxydants d'origine naturelle, afin d'épargner l'utilisation des antioxydants synthétiques dont certains d'entre eux peuvent être toxiques ou carcinogènes. Les résultats que nous avons obtenus confirment que l'HE de cette espèce possède un pouvoir antioxydant très faible.

Cette importante activité antimicrobienne est due principalement à la richesse de cette essence en Beta.Thujone. Ce composé cétonique est très connu pour ses propriétés contre les agents microbiens.

L'ensemble de ses résultats laisse entrevoir des perspectives de la recherche de formulation à base des essences de Brocchia cinerea à la place de certains conservateurs de synthèse dans le domaine de l'industrie agroalimentaire, pharmaceutique et cosmétique.

Déclaration d'intérêts: les auteurs ne déclarent aucun conflit d'intérêt en rapport avec cet article.

\section{RÉFÉRENCES}

1. Amarti F, El Ajjouri M, Ghanmi M, Satrani B, Aafi A, Farah A, et al. Composition chimique, activité antimicrobiennne et antioxydante de l'huile essentielle de Thymus zygis du Maroc. Phytothérapie. 2011;9(3):149.

2. Silpa P, Roopa K, Thomas TD. Production of plant secondary metabolites: Current status and future prospects. Biotechnological Approaches for Medicinal and Aromatic Plants: Springer; 2018. p. 3-25.

3. Koumaglo KH, Dotse K, Bettini F, Bayle J-C. Composition chimique de l'huile essentielle de Chromolaena odorata (L) King et Robinson (Asteraceae) du Togo: Effets de séchage et du site de récolte. J Soc Ouest-Afr Chem. 2009;28:11-6.

4. Paolini J. Caractérisation des huiles essentielles par CPG/Ir, CPG/SM (IE et IC) et RMN du carbone-13 de Cistus albidus et de deux Asteraceae endémiques de Corse: Eupatorium cannabinum subsp. corsicum et Doronicum corsicum 2005.

5. Jose S, Thomas TD. Comparative phytochemical and anti-bacterial studies of two indigenous medicinal plants Curcuma caesia Roxb. and Curcuma aeruginosa Roxb. International Journal of Green Pharmacy (IJGP). 2014;8(1).

6. Juteau F, Masotti V, Bessiere JM, Dherbomez M, Viano J. Antibacterial and antioxidant activities of Artemisia annua essential oil. Fitoterapia. 2002;73(6):532-5.

7. Kordali S, Kotan R, Mavi A, Cakir A, Ala A, Yildirim A. Determination of the chemical composition and antioxidant activity of the essential oil of Artemisia dracunculus and of the antifungal and antibacterial activities of Turkish Artemisia absinthium, A. dracunculus, Artemisia santonicum, and Artemisia spicigera essential oils. Journal of agricultural and food chemistry. 2005;53(24):9452-8.

8. Benabadji $\mathrm{N}$, Bouazza $\mathrm{M}$. Contribution à une étude bioclimatique de la steppe à Artemisia herba-alba Asso. dans l'Oranie (Algérie occidentale). Science et changements planétaires/Sécheresse. 2000;11(2):117-23.
9. Chatelain C, Medjahdi B, Benhouhou SS. eFlore du Maghreb, une flore électronique basée sur la Nouvelle flore d'Algérie de P. Quézel et S. Santa. Ecologia mediterranea: Revue internationale d'écologie méditerranéenne= International Journal of Mediterranean Ecology. 2018;44(2):131-6.

10. Quézel P, Santa S. Nouvelle flore de l'Algérie et des régions désertiques méridionales. 1963.

11. Benchelah A-C, Bouziane H, Maka M. Fleurs du Sahara, arbres et arbustes, voyage au coeur de leurs usages avec les Touaregs du Tassili. Phytothérapie. 2004;2(6):191-7.

12. Boulos L. Medicinal plants of North Africa. Medicinal plants of North Africa. 1983.

13. Ozenda P. Flora and vegetation of the Sahara: CNRS; 1991.

14. Bellakhdar J. La pharmacopée marocaine traditionnelle. 1997.

15. Beloued A. Plantes médicinales d'Algérie: Offices des publications universitaires; 2005.

16. Djellouli M, Moussaoui A, Benmehdi H, Ziane L, Belabbes A, Badraoui M, et al. Ethnopharmacological studyand phytochemical screening of three plants (Asteraceae family) from the region of South West Algeria. Asian journal of natural \& applied sciences. 2013;2:59-65.

17. Bouziane M, Hadj-mahammed M. Chemical Composition of the Essential oil of Brocchia cinerea Grown in South Eastern of Algeria. Asian Journal of Chemistry. 2013;25(7):3917.

18. Européenne P. Ge édition (2008). Conseil de l’Europe, Strasbourg, France.

19. Institute CaLS. standardization of sensitivity tests with antimicrobials by disc diffusion. Guideline 2003;M2-A8(CLSI, Wayne, PA).

20. Eloff JN. A sensitive and quick microplate method to determine the minimal inhibitory concentration of plant extracts for bacteria. Planta medica. 1998;64(08):711-3.

21. Hayhoe EJ, Palombo EA. Screening for antibacterial, antifungal, and anti quorum sensing activity. Metabolomics Tools for Natural Product Discovery: Springer; 2013. p. 219-25.

22. Blois MS. Antioxidant determinations by the use of a stable free radical. Nature. 1958;181(4617):1199.

23. Sharma OP, Bhat TK. DPPH antioxidant assay revisited. Food chemistry. 2009;113(4):1202-5.

24. Kiers CT, De Boer J, Olthof R, Spek A. The crystal structure of a 2, 2diphenyl-1-picrylhydrazyl (DPPH) modification. Acta Crystallographica Section B: Structural Crystallography and Crystal Chemistry. 1976;32(8):2297-305.

25. Sanchez-Moreno C. Methods used to evaluate the free radical scavenging activity in foods and biological systems. Food science and technology international. 2002;8(3):121-37.

26. Huang C-Y, Wu S-J, Yang W-N, Kuan A-W, Chen C-Y. Antioxidant activities of crude extracts of fucoidan extracted from Sargassum glaucescens by a compressional-puffing-hydrothermal extraction process. Food chemistry. 2016;197:1121-9.

27. El Youbi AEH, Bousta D, Jamoussi B, Greche H, El Mansouri L, Benjilali J, et al. Activités antioxydante, apoptotique et antiproliférative de Tetraena gaetula (Emb. \& Maire) Beier \& Thulin et de Berberis hispanica Boiss. \& Reut. originaires du Maroc. Phytothérapie. 2012;10(3):151-60.

28. Dehpour AA, Ebrahimzadeh MA, Fazel NS, Mohammad NS. Antioxidant activity of the methanol extract of Ferula assafoetida and its essential oil composition. Grasas y aceites. 2009;60(4):405-12.

29. Fatima KT, Azed KM, Nacira A, Cherifa B. Antioxydant activity and preventive possibility of Algerian medicinal plant Matricaria pubescens on hepatic toxicity. Analele Stiintifice ale Universitatii" Al I Cuza" din Iasi. 2016;62(1):148.

30. Oyaizu M. Antiangiogenic properties of natural polyphenols from red wine and green tea. Jap J Nut. 1986;44:307-15.

31. Bougandoura N, Bendimerad N. Evaluation de l'activité antioxydante des extraits aqueux et méthanolique de Satureja calamintha ssp. Nepeta (L.) Briq. Nature \& Technology. 2013(9):14.

32. Ghaisas M, Navghare V, Takawale A, Zope V, Deshpande A. In-vitro antioxidant activity of Tectona grandis Linn. Pharmacologyonline. 2008;3:296-305. 


\section{ARTICLE ORIGINAL}

33. Mebarka B, HADJ-Mahammed M. CARACTERISATION PHYTOCHIMIQUE DE DEUX EXTRAITS DE Brocchia cinerea Vis.(ASTERACEAE) PAR GC-MS: ACTIVITE ANTI-CLOSTRIDIUM. 2015.

34. Atef C, Boualem M, Cherif MM, Youcef $H$, Azzedine C. Chemical composition and antimicrobial activity of essential oils in Xerophytic plant Cotula cinerea Del (Asteraceae) during two stages of development: flowering and fruiting. 2015.

35. Djellouli M, Benmehdi H, Mammeri S, Moussaoui A, Ziane L, Hamidi N. Chemical constituents in the essential oil of the endemic plant Cotula cinerea (Del.) from the southwest of Algeria. Asian Pacific Journal of Tropical Biomedicine. 2015;5(10):870-3.

36. Aligiannis N, Kalpoutzakis E, Mitaku S, Chinou IB. Composition and antimicrobial activity of the essential oils of two Origanum species. Journal of agricultural and food chemistry. 2001;49(9):4168-70.
37. Palombo EA, Semple SJ. Antibacterial activity of traditional Australian medicinal plants. Journal of ethnopharmacology. 2001;77(2-3):151-7.

38. Duffy CF, Power RF. Antioxidant and antimicrobial properties of some Chinese plant extracts. International journal of antimicrobial agents. 2001;17(6):527-9.

39. Köhler $T$, Pechère J-C, Plesiat $P$. Bacterial antibiotic efflux systems of medical importance. Cellular and Molecular Life Sciences CMLS. 1999;56(910):771-8.

40. Sharifzadeh A, Javan AJ, Shokri H, Abbaszadeh S, Keykhosravy K. Evaluation of antioxidant and antifungal properties of the traditional plants against foodborne fungal pathogens. Journal de Mycologie Múdicale. 2016;26(1):e11-e7.

Cet article a été publié dans le « Batna Journal of Medical Sciences » BJMS, l'organe officiel de « l'association de la Recherche Pharmaceutique - Batna»

Le contenu de la Revue est ouvert « Open Access » et permet au lecteur de télécharger, d'utiliser le contenu dans un but personnel ou d'enseignement, sans demander l'autorisation de l'éditeur/auteur.

Avantages à publier dans BJMS :

- Open access : une fois publié, votre article est disponible gratuitement au téléchargement

- Soumission gratuite : pas de frais de soumission, contrairement à la plupart des revues « Open Access »

- Possibilité de publier dans 3 langues : français, anglais, arabe

- Qualité de la relecture : des relecteurs/reviewers indépendants géographiquement, respectant l'anonymat, pour garantir la neutralité et la qualité des manuscrits.

Pour plus d'informations, contacter BatnaJMS@gmail.com ou connectez-vous sur le site de la revue : www.batnajms.net 


\section{$B S^{2}$}

- $\bigcirc$

-

P

○

Faculté de Médecine, Université de Batna 2 - Algérie

Service de pédiatrie EHS mère et enfant Meriem Bouattoura,

Batna -Algérie

\section{Correspondance à :}

Abderrachid BOUHDJILA

\section{Bouhdjilarachid@yahoo.fr}

DOI :https://doi.org/10.48087/ BJMSoa.2020.7214

Historique de l'article :

Reçu le 23 juin 2020

Accepté le 29 septembre 2020

Publié le 09 novembre 2020

Il s'agit d'un article en libre accès distribué selon les termes de la licence Creative Commons Attribution International License (CC BY 4.0), qui autorise une utilisation, une distribution et une reproduction sans restriction sur tout support ou format, à condition que l'auteur original et la revue soient dûment crédités.

\section{Pour citer l'article :}

Bouhdjila A. La carence martiale chez l'enfant de moins de cinq ans à Batna. Batna J Med Sci 2020;7(2):129-133. https://doi.org/10.48087/BJMS oa.2020.7214

\title{
La carence martiale chez l'enfant de moins de cinq ans à Batna
}

\author{
Martial deficiency in children under five years of age in Batna
}

\author{
Abderrachid Bouhdjila
}

\section{RÉSUMÉ}

Introduction. La carence martiale touche les groupes des populations à risque (femmes enceintes et enfants en bas âge) aussi bien dans les pays développés que ceux en développement. En Algérie l'ampleur du problème n'est pas bien connue, car les études sont rares et les outils d'évaluation, des indicateurs biochimiques peu fiables. Objectifs. Déterminer la prévalence de la carence martiale chez l'enfant de zéro à cinq ans dans la ville de Batna, étudier ses répercussions sur le développement physique et psychomoteur de l'enfant et évaluer le traitement 1 et les mesures de prévention adéquates. Matériels et méthodes. Sur un échantillon de 354 enfants de zéro à cinq ans, dans la ville de Batna, nous avons mesuré la ferritine sérique, l'hémoglobine, les récepteurs de la transferrine, nous avons analysé les signes cliniques de la carence et évalué la croissance et le développement psychomoteur des enfants. Les enfants carencés ont reçu un traitement à base de fer. Des contrôles ont été effectués à la fin du traitement martial. Résultats. La prévalence de la carence martiale est variable en fonction de la tranche d'âge, elle est de $41 \%$ chez le nouveau-né dont $25 \%$ sont en état de carence avancée (anémie par carence martiale) de 1à 6 mois elle est de $40,5 \%$ chez le nourrisson de 7 à 12 mois elle est de $51,9 \%$; Chez l'enfant de $13-60$ mois elle est de $42,3 \%$ et $29,3 \%$ sont en carence sévère (anémie par carence martiale). Les facteurs de risque retrouvés sont : la non supplémentation durant la grossesse, la mauvaise diversification alimentaire des nourrissons, le faible apport en fer alimentaire, chez les enfants carencés nous avons observé des anomalies du développement psychomoteur et de l'IMC. Conclusion. La carence martiale est fréquente chez les enfants de 0 à 5 ans à Batna. La supplémentation en fer des femmes enceintes, la bonne diversification alimentaire, un régime alimentaire équilibré et riche en fer permettraient de prévenir cette carence.

Mots clés : carence martiale, anémie, apport en fer alimentaire, supplémentation, prévention.

\section{INTRODUCTION}

En 2007 l'OMS a publié un rapport, sur la situation nutritionnelle de la population mondiale et révèle $47 \%$ des enfants d'âge préscolaire, à travers le monde, souffrent d'anémie ; dans $50 \%$ des cas, l'anémie est due à une carence en fer [1,2] Beaucoup d'entre eux n'atteignent pas leurs pleines performances neurocognitives du fait des répercussions de cette carence en fer sur leur développement.

En Algérie aucune étude nationale sur le statut martial de la population de moins de cinq ans n'a été réalisée et seulement quelques études locales, utilisant des paramètres différents, peu fiables pour évaluer le profil martial.

\begin{abstract}
Introduction. Martial deficiency affects at-risk population groups (pregnant women and young children) in both developed and developing countries. In Algeria, the extent of the problem is not well known, as studies are rare and evaluation tools, biochemical indicators unreliable. Aims. To determine the prevalence of martial deficiency in children aged 0 to 5 years in the city of Batna, to study its impact on the physical and psychomotor development of the child and to evaluate the treatment 1 and adequate prevention measures. Materials and methods. In a sample of 354 children from 0 to 5 years old in the city of Batna, we measured serum ferritin, hemoglobin, transferrin receptors, analyzed clinical signs of deficiency and assessed the growth and psychomotor development of children. The deficient children received iron therapy. Controls were carried out at the end of the martial treatment. Results. The prevalence of martial deficiency is variable according to age group, it is $41 \%$ in newborns of which $25 \%$ are in an advanced state of deficiency (martial deficiency anemia) from 1 to 6 months, it is $40.5 \%$ in infants from 7 to 12 months, it is $51.9 \%$; in children from $13-60$ months, it is $42.3 \%$ and $29.3 \%$ are in severe deficiency (martial deficiency anemia). The risk factors found are: non-supplementation during pregnancy, poor dietary diversification of infants, low dietary iron intake, in deficient children we observed abnormalities in psychomotor development and BMI. Conclusion. Martial deficiency is frequent in children from 0 to 5 years old in Batna. Iron supplementation of pregnant women, good dietary diversification, a balanced diet rich in iron would prevent this deficiency.
\end{abstract}

Key words: martial deficiency, anemia, dietary iron intake, supplementation, prevention.

\section{Objectifs}

Déterminer la prévalence de la carence martiale chez les enfants de la naissance a cinq ans dans la ville de Batna.

Identifier les facteurs de risque de la carence martiale chez cette population.

\section{MATÉRIELS ET MÉTHODES}

Étude descriptive prospective sur douze mois du 1 janvier au 31decembre 2012.

\section{Contexte de l'étude : la ville de Batna}

La ville de Batna est une commune de la wilaya de Batna, dont elle est le chef-lieu, située à $435 \mathrm{~km}$ au sud-est d'Alger elle est la $5^{5}$ plus 


\section{Population et recrutement}

Nous avons tiré au sort 354 enfants, dont l'âge varie de quelques heures à 60 mois. Nous avons étudié le dossier obstétrical des 51 mamans des nouveau nés.

\section{Critères de non inclusion}

Les nouveaux nés prématurés (nés avant 37 semaines de gestation),

Les nouveaux nés malades,

Les enfants présentant des hémorragies digestives,

Les enfants ayant reçus dans les mois passés ou recevant au moment de l'enquête des médicaments contenant du fer,

Les enfants ayant une pathologie inflammatoire,

Les enfants issus de familles connues pour une anémie constitutionnelle.

\section{Les paramètres biologiques étudiés sont :}

Une formule de numération sanguine (FNS) est réalisée sur automate, pour évaluer l'hémoglobine $(\mathrm{Hb})$; Un taux de C réactive protéine CRP ; Un taux de ferritine sérique (Fs) Les récepteurs sériques de la transferrine (RsTF).

Les valeurs biologiques retenues pour définir la carence martiale et l'anémie par carence martiale sont regroupées dans le tableau 1.

Tableau 1. Diagnostic biologique de la carence martiale et de l'anémie par carence martiale

\begin{tabular}{clccc}
\hline \multicolumn{1}{c}{ Statut martial } & $\begin{array}{c}\text { Fs } \\
(\mu \mathrm{g} / \mathrm{l})\end{array}$ & $\begin{array}{c}\mathrm{RsTF} \\
(\mathrm{g} / \mathrm{l})\end{array}$ & $\begin{array}{c}\mathrm{Hb} \\
(\mathrm{g} / \mathrm{dl})\end{array}$ \\
Femme au & Carence martiale & $<35$ & $>1,9$ & $>10$ \\
3éme TG* & $\begin{array}{c}\text { Anémie/ carence } \\
\text { martiale }\end{array}$ & $<35$ & $>3$ & $<10$ \\
& Carence martiale & $<35$ & $>6$ & \\
Nouveau-né & $\begin{array}{l}\text { Anémie/ carence } \\
\text { martiale }\end{array}$ & $<35$ & $>6$ & $<13,5$ \\
& $\begin{array}{l}\text { Carence martiale } \\
\text { 1-12mois }\end{array}$ & $<12$ & $>6$ & \\
& $\begin{array}{l}\text { Anémie/ carence } \\
\text { martiale }\end{array}$ & $<10$ & $>6$ & $<10$ \\
& $\begin{array}{l}\text { Carence martiale } \\
\text { 13-60mois }\end{array}$ & $<12$ & $>3$ & \\
& $\begin{array}{l}\text { Anémie/ carence } \\
\text { martiale }\end{array}$ & $<10$ & $>3$ & $<11$ \\
& & & & \\
\hline
\end{tabular}

*TG : trimestre de grossesse, FS ferritine sérique exprimé en microgramme par litre de sang ; RsTF récepteurs solubles de la transferrine exprimée en gramme par litre de sang, $\mathrm{Hb}$ hémoglobine exprimé en gramme par litre de sang

En fonction de ces résultats les enfants sont classés en 3 groupes :

- Absence de carence en fer

- Carence en fer

- Anémie par carence en fer

Nous avons étudié, les facteurs de risque de la carence en fer en analysant le questionnaire formulé pour l'étude :

- Le profil martial de la mère (bilan effectué lors de l'hospitalisation pour accouchement et comportant: Taux de ferritine, d'hémoglobine, récepteurs solubles de la transferrine

- La supplémentation en fer durant la grossesse

- Le niveau socioéconomique du ménage

- Le nombre de grossesses et le délai entre chaque grossesse

- Le niveau intellectuel et la fonction de la mère
- Le mode d'allaitement

- L'âge de diversification

- Les habitudes alimentaires (la prise des viandes, poisson et œufs), céréales, les légumes secs, légumes frais et fruits, type de lait consommé).

Pour les enfants présentant une carence martiale ou une anémie quel que soit le degré de sévérité, un traitement à base de fer médicinal sous forme de gouttes ou de sirop de fer ferreux a été prescrit.

\section{Analyse statistique}

Les variables qualitatives sont exprimées en pourcentage avec intervalle de confiance à $95 \%$.

Les variables quantitatives sont exprimées en moyennes \pm écarts type ou en DS.

Pour la comparaison de deux variables quantitatives nous avons utilisé le test de chi2 (Pearson ou Fisher), pour le degré de signification du test, $p$ est significatif s'il $\leq 0,05$ (5\%).

Nous avons utilisé le logiciel EPI info, version 3. 5.4, et le logiciel SPSS version 22.

\section{RÉSULTATS}

\section{Caractéristiques sociodémographiques}

Répartition en fonction des tranches d'âge (voir tableau 2)

51 nouveaux nés et 303 enfants âgés de 1 à 60 mois ont été retenus dans l'étude. La moyenne d'âge de la population était de 22,1 mois avec une légère prédominance masculine et un Sex-ratio égal à 1.2 .

Tableau 2 Répartition de la population de 1à 60 mois selon les tranches d'âge

\begin{tabular}{lll}
\hline Age (mois) & Fréquence (n) & Pourcentage (\%) \\
$1-6$ & 43 & 14,2 \\
$7-12$ & 52 & 17,2 \\
$13-24$ & 58 & 19,2 \\
$25-36$ & 54 & 17,8 \\
$37-48$ & 42 & 13,8 \\
$49-60$ & 54 & 17,8 \\
Total & 303 & 100 \\
\hline
\end{tabular}

Profil martial de la population de 0 à 60 mois de la population étudiée

Le taux moyen d'hémoglobine était estimé à $11,34 \mathrm{~g} / \mathrm{dl}$ $\pm 1,67$ avec des extrêmes de 6,75 et 19,66 .

La ferritinémie moyenne était de 24,07 $\pm 22,7$ avec des extrêmes de 3 et $175 \mathrm{mcg} / \mathrm{l}$.

Pour les récepteurs solubles de la transferrine, la moyenne était de 3,18 $\pm 1,99$ avec des valeurs extrêmes de 0,12 et 11,12.

Parmi cette population $43,9 \%$ avaient un taux de ferritinémie inferieur a $12 \mathrm{mcg} / \mathrm{l}$ donc en état de carence martiale et 29,3\% un taux d'hémoglobine étaient au stade d'anémie avec un taux d'hémoglobine inferieur à $11 \mathrm{~g} / \mathrm{dl}$ pour les enfants des plus de 28jours et inferieurs à $13,5 \mathrm{~g} / \mathrm{dl}$ pour les enfants de zéro à 28jours. 


\section{Profil martial par tranches d'âge}

\section{Caractéristiques sociodémographiques des mères de nouveau-nés}

Nous avons distingué deux groupes parmi les 51 mamans de nouveau-nés; un groupe (30) des nouveaux nés supplémentés en fer durant la grossesse et l'autre (21) non supplémenté (tableau 3).

Tableau 3. Caractéristiques sociodémographiques des mères des nouveaux nés (n51)

\begin{tabular}{llll}
\hline Mères & $\begin{array}{l}\text { Supplémentées } \\
\text { (n30) }\end{array}$ & $\begin{array}{l}\text { Non } \\
\text { supplémentées (n } \\
21)\end{array}$ & $\mathrm{p}$ \\
$\begin{array}{l}\text { Age moyen } \\
\text { (année) }\end{array}$ & $26 \pm 7,3$ & $28,3 \pm 8,6$ & DNS \\
Parité & $2,3 \pm 0,3$ & $3,6 \pm 1,4$ & $<0,001$ \\
$\begin{array}{l}\text { Espace } \\
\text { intergénésique } \\
\text { (année) }\end{array}$ & $3,1 \pm 02$ & $2 \pm 04$ & 0,01 \\
$\begin{array}{l}\text { Revenu familial } \\
\text { (DA) }\end{array}$ & $40.000 \pm 1423$ & $22276 \pm 1235$ & $<0,001$ \\
\hline
\end{tabular}

DA dinar algérien

\section{Profil martial de la naissance à 1 année}

$41 \%$ des nouveau-nés avaient une ferritinémie inferieur à 35 microgramme/l donc en état de carence martiale et $25 \%$ avaient un taux d'hémoglobine inférieur à $13,5 \mathrm{~g} / \mathrm{dl}$ donc en état d'anémie par carence martiale.

Quand on analyse le profil martial néonatal et le statut maternel on retrouve que $\mathbf{8 5 , 7 \%}$ des nouveaux nés de mères non supplémentées en fer avaient une carence martiale et $61,9 \%$ avaient une anémie (tableau 4). 35,2\% des mamans des nouveaux nés étaient en carence martiale et $15,6 \%$ avaient une anémie. $41 \%$ des mères n'étaient pas supplémentées et Parmi celles non supplémentées, 85,7\% avaient une carence en fer et 38,2\% une anémie

Tableau 4. Ferritinémie néonatale et maternelle selon la supplémentation maternelle en fer

\begin{tabular}{llllc}
\hline Ferritine $(\mathrm{n}=51)$ & Mère & $\begin{array}{l}\text { Nouveau- } \\
\text { né }\end{array}$ & $\begin{array}{l}\text { Fréquence } \\
(\%)\end{array}$ & $\mathrm{p}$ \\
$\begin{array}{l}\text { Supplémentées } \\
\text { (30) }\end{array}$ & $45,3 \pm 2,3$ & & $\begin{array}{l}30 / 30 \\
(100)\end{array}$ & $<0.001$ \\
Non & $19,4 \pm$ & 20,2 & $18 / 21$ & \\
supplémentées & 1,3 & $\pm 3,6$ & $(85,7)$ & \\
$(21)$ & & & & \\
\hline
\end{tabular}

Pour les nourrissons de 1 mois à 12 mois, $39,5 \%$ avaient une carence et $37,2 \%$ avaient une anémie parmi les nourrissons de 1à 6moiset 51,9\% des nourrissons de 7-12mois avaient une carence en fer et 21,2\% avaient une anémie (tableau 5).

Tableau 5. Profil martial des enfants âgés de 1 à 12 mois

\begin{tabular}{lcllll}
\hline $\begin{array}{l}\text { Variable } \\
\text { biologique }\end{array}$ & \multicolumn{2}{c}{$1-6$ mois $(\mathrm{n}=43)$} & \multicolumn{2}{c}{$7-12$ mois $(\mathrm{n}=52)$} & $\mathrm{p}$ \\
& Population & $\begin{array}{l}\text { Groupe } \\
\text { carencé }\end{array}$ & Population & $\begin{array}{l}\text { Groupe } \\
\text { carencé }\end{array}$ & \\
$\mathrm{Hb}(\mathrm{g} / \mathrm{dl})$ & $11,3 \pm 1,21$ & 9,55 & $11,1 \pm 0,1$ & $9,2 \pm 0,2$ & $<0,001$ \\
& & $37,2 \%$ & & $21.2 \%$ & \\
Fs (ùg/l) & $22,1 \pm 0,2$ & 9,2 & $21,2 \pm 0,2$ & $8,2 \pm 0,2$ & \\
& & $39,5 \%$ & & $51.9 \%$ & \\
RsTF & $4,7 \pm 1,2$ & 8,83 & $3,3 \pm 0,16$ & $7,8 \pm 0,8$ & \\
(mg/l & & $39,5 \%$ & & $51.9 \%$ &
\end{tabular}

\section{Carence martiale et apports alimentaires en fer}

Après l'âge de 6mois (âge de la diversification) Seulement $23,4 \%$ recevaient des protéines animales (viandes, poulet, poisson) ;80\% reçoivent des céréales maison (non enrichies en fer) et $58 \%$ reçoivent du lait non enrichi en fer (lait pasteurisé reconstitué).

\section{Carence martiale et revenu familial}

Chez les nourrissons de 7à12mois L'apport alimentaire en fer est positivement lié au revenu du ménage. Il est de $4,8 \pm 1,2 \mathrm{mg} /$ par jour quand le revenu du père est $50 \%$ audessus du salaire minimum ( $p<0,001)$ (tableau 3 )

De même cet apport est augmenté $4,8 \pm 1,2 \mathrm{mg} / \mathrm{j}$ lorsque la mère a un revenu équivalent au SMIG (18000 dinars algérien) par rapport à un revenu plus faible $(\mathrm{p}<0,001)$.

Pendant les premiers 6mois, le fer est apporté uniquement par le lait quel que soit le type. 41,8\% des nouveaux nés avaient été allaité au sein exclusivement

\section{Profil martial des 13-60mois}

La valeur moyenne de la ferritine chez les 208 enfants de 13à 60 mois était de 23,79 $\mu \mathrm{g} / \mathrm{l}$ chez 88 enfants soit 42,3\%, elle est de $10,3 \pm 0,3 \mu \mathrm{g} /$ donc il y'a carence martiale.

La carence martiale était plus fréquente entre 13 mois et 24 mois (50\%).

La valeur moyenne de l'hémoglobine chez les 208 enfants de 13 à 60 mois était de $11,42 \mathrm{~g} / \mathrm{dl}$, chez $29,3 \%$ il y'avait une anémie (l'hémoglobine était inférieure à $10,4 \pm 0,6 \mathrm{~g} / \mathrm{dl}$ ).

$50 \%$ des enfants âgés de 13 à 24 mois avaient une carence et $43,1 \%$ avaient une anémie

Durant la deuxième moitié de la première année de vie, le fer est apporté par les aliments de diversification et le lait. La diversification alimentaire était tardive, après $6 \mathrm{mois}$ chez $72,8 \%$ des nourrissons, Les protéines animales n'ont été introduites que chez $23,4 \%$ des nourrissons et le lait pasteurisé non enrichi en fer est consommé par près de $59 \%$ des enfants (Figure 1); La consommation journalière de fer était faible : 4,8mg.

La consommation en fer héminique été régulière chez 21,7 \% de nos enfants

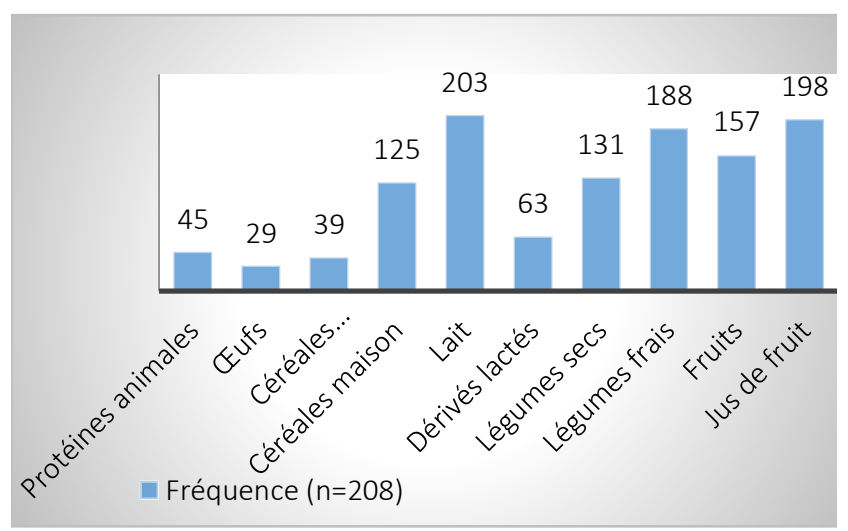

Figure 1. Apports alimentaires chez les enfants de 1360 mois

\section{DISCUSSION}

$47 \%$ des enfants d'âge préscolaire à travers le monde, souffrent d'anémie; dans $50 \%$ des cas, l'anémie est due à une carence en fer [3]. Pour évaluer l'ampleur du problème dans 
Batna, nous avons analysé le profil martial ainsi les facteurs de risque de carence martiale chez 354 enfants âgés de 0à60mois. Dans cette population 43,9\% avaient une carence martiale et $29,3 \%$ une anémie.

Ces taux sont inferieurs a ceux retrouvé dans les autres études algériennes [4, 5] cela serait dû à la différence des groupes étudiés et peut être normes des paramètres biologiques utilisés, ces taux sont assez voisins de ceux au Maroc [6] et en Tunisie [7] cependant ils sont plus élevés qu'en Europe, la prévalence de la carence martiale à l'âge d'1 an été évaluée dans 11 pays différents à la même époque à $7,2 \%$ (0 à $33 \%$ ), associée à une anémie chez 2,3\% (0 à $12 \%$ ) [3] et nettement inférieurs aux taux africains [8].

L'étude des facteurs de risque de carence martiale chez le nouveau-né a révélé que $35,2 \%$ des mamans des nouveaux nés étaient en carence martiale et $15,6 \%$ avaient une anémie, ces taux sont inférieurs à ceux retrouvé par Ghorashi en IRAN [9] et Demmouche à l'ouest de l'Algérie [10], 41\% des mères n'étaient pas supplémentées et Parmi celles non supplémentées, $85,7 \%$ avaient une carence en fer et 38,2\% une anémie. Les nouveaux nés carencés étaient tous issus de mères non supplémentées.

L'espace intergénésique des mères était en moyenne de $3,2 \pm 0,2$ ans, un espace inter génésique inférieur à 2 ans constitue un facteur de risque de carence martiale chez les mamans $(p<0,001)$. Ce facteur de risque est retrouvé dans l'étude de Benhassine à Alger [4] Smahi à Tlemcen [5], Demmouche à Sidi bel Abbes [11] et Ghorachi en en Iran [9].

Pendant les premiers 6mois, le fer est apporté uniquement par le lait quel que soit le type : 39,5\% avaient une carence martiale et $37,2 \%$ avaient une anémie. Dans notre étude le taux de ferritinémie des nourrissons de 1-6 mois suit celui de leurs mères: 35,2\% des mères des enfants de 1 à 6 mois étaient carencés en fer et $39,5 \%$ de leurs bébés l'étaient également $(\mathrm{p}<0,001)$

Durant la deuxième moitié de la première année de vie, le fer est apporté par les aliments de diversification et le lait; $51,9 \%$ des nourrissons âgés de 7 à 12 mois avaient un taux de ferritine de $8,2 \pm 0,2 \mu \mathrm{g} / \mathrm{l}$ donc carencés et $21,2 \%$ avaient une anémie.

41,8\% avaient été allaité au sein exclusivement, vs un taux national de 7\% [12]. Pour Pizarro, l'allaitement exclusif au sein jusqu'à 9 mois a exposé $36 \%$ à une carence martiale [13]

La diversification alimentaire était tardive, après 6 mois chez $72,8 \%$ des nourrissons, Les protéines animales n'ont été introduites que chez $23,4 \%$ des nourrissons et le lait pasteurisé non enrichi en fer est consommé par près de $59 \%$ des enfants.

La consommation journalière de fer était faible : 4,8mg, loins des apports recommandés $(8 \mathrm{mg} / \mathrm{j})$. On Remarque, alors que la diversification alimentaire a été mal gérée par les mamans ce qui a exposé les nourrissons à la carence martiale.

Bramhagen (Suède) [14] où dans une population âgée de 12 mois, $27 \%$ avaient des apports en fer inferieur aux normes recommandées pour la population scandinave (8mg/j) 10,3\% des nourrissons avaient une carence en fer et $2,3 \%$ avaient une anémie.

Chez les 208 enfants âgés de 13 à 60 mois, 42,3\% avaient une carence.et $29,3 \%$ une anémie.

Si l'on regarde la tranche d'âge où la carence est la plus élevée, $50 \%$ des enfants âgés de 13 à 24 mois avaient une carence et $43,1 \%$ avaient une anémie, ceci serait dû à une mauvaise gestion de la diversification alimentaire.
Pour Benhassine [4] ,47 \% d'enfants entre 13 et 24mois avaient une carence martiale sévère Les facteurs de risque liés à la carence dans ce groupe de population étaient la faible quantité de fer consommée ; le faible revenu de la mère, le faible niveau d'instruction de la mère, et le faible revenu du père $(p<0,001)$.

Au Maroc, $45 \%$ des enfants âgés de 6 mois à 5 ans ont une carence en fer [6].

Ferrara et al (Italie2014) [15] avaient retrouvé sur une cohorte de 1250 enfants âgés de 8 à 36mois sur les 3 décennies de 1980 à 2000 ; que 14,3\% avaient une carence martiale et $6 \%$ avaient une anémie par carence martiale ; les facteurs de risque liés à cette carence étaient le faible revenu économique, la diversification alimentaire tardive après l'âge de 6 mois, l'introduction du lait de vache avant l'âge d'un an, et le manque de supplémentation en fer.

La consommation en fer héminique n'a été régulière que chez $21,7 \%$ de nos enfants, Cette faible tendance à la consommation de fer héminique est rapportée dans l'étude de Smahi [5] et les autres études où on remarque que la faible biodisponibilité du fer apporté par le régime, basé sur la consommation de légumes et de céréales était la cause de l'anémie par carence martiale au Maroc [6] et dans d'autres pays $[1,3,8]$.

\section{CONCLUSION}

La carence en fer est un problème majeur de santé publique chez l'enfant de la naissance à cinq ans à Batna avec 43. 9\% carence martiale et $29,3 \%$ ont une anémie par carence martiale. C'est au cours de la première année de vie que le risque est grand, ceci est dû à la mauvaise gestion de la diversification.

Les facteurs de risque de la carence martiale relevés dans notre étude sont :

-Non supplémentation des femmes enceintes

-Parité supérieure à 3 et espace inter génésique inferieur à 2ans

-Mauvaise diversification alimentaire

-Faible revenu familial

-Régime alimentaire pauvre en fer

Déclaration d'intérêts: les auteurs ne déclarent aucun conflit d'intérêt en rapport avec cet article.

\section{RÉFÉRENCES}

1. Lynch $S$.Why nutritional iron deficiency persists as a worldwide problem. J Nutr.2011,141: S763-76.

2. Mclean E, Egli I, Cogswell M, Benoist B et Wojdyla D. Prévalence mondiale de l'anémie chez les enfants d'âge préscolaire les femmes enceintes et les femmes en âge de procreer. In: Badham $\mathrm{j}$, Zimmermann $M$, Kraemer $k$. Le guide de l'anémie nutritionnelle. Zurich:Sight and life press. 2007 p1 1-12.

3. Dupont . Prévalence de la carence martiale. Archives de Pédiatrie 2017;24:5S45-5S48.

4. Benhassine F. Les anemies nutritionnelles en milieu rural algerien chez,le nourrisson de 3mois à 36 mois, comparaison avec le milieu urbain [thése de DESM ,medecine].Université d'Alger; faculté de medecine ; 1997.

5. Smahi MC. Prevalence et facteurs de risque de la carence martiale chez les nourrissons de la commune de Tlemcen [thése de DESM, medecine].université de Tlemcen ; faculté de medecine ; 2011. 


\section{ARTICLE ORIGINAL}

6. El HamdouchiA, El Kari K, Rjimati L, El Haloui N, El MzibriL M, Aguenaou $\mathrm{H}$ et Mokhtar N. Impact de l'enrichissement de la farine en ferélémentaire sur la prévalence de l'anémie chez les enfants en âge préscolaire au Maroc. EMHJ. 2010, 16 (11) :1148-1152.

7. Les anémies en tunisie : causes et mesures d'intervention. Institut national de nutrition et de technologie alimentaire .2002. Ministère de la santé publique. Tunis, $156 \mathrm{p}$.

8. Sanou R, Turgeon-O'Brien H, Desrosiers T. Prévalence et déterminantsnon alimentaires de l'anémie et de la carence en fer chez des orphelins et enfants vulnérables d'âge préscolaire auBurkina Faso. Nutr Clin Metabol 2008;2210-19.

9. EmamGhorashi F, Heidari T. Iron status of babies born to iron deficient anaemic mothers in an iranian hospital la revue de santé de la méditerranée orientale, 2004, 10, (6).

10. Demmouche, A., Moulessehoul Prévalence de l'anémie ferriprive au cours de la grossesse dans la wilaya de Sidi Bel Abbes (ouest de l'Algérie) Antropo .2010, 21 Suppl: S39-48.
11. Demmouche F. Effect of iron supplementation among pregnant women at mother-and-baby clinic of sidi bel abbès, west algeria cahiers d'études et de recherches francophones / santé . JanvierFévrier-Mars 2004, 14, (1): 21-9.

12. Suivi de la situation des enfants et des femmes Enquête nationale à indicateurs multiples Rapport principal MICS 3 ALGERIE .2006 .MSPRH/UNICEF 2006.

13. Pizarro F, Lozoff B. Prevention of iron-deficiency anemia: comparison of high- and low-iron formulas in term healthy infants after six months of life. J Pediatr 1998;132: 635-640.

14. Bramhagen AC. Factors influencing iron nutrition among one-year-old healthy childrenin. Sweden.Journal of Clinical Nursing.2011, 20:18871894.

15. Ferrara M, Bertocco F, Ferrara D, Incarnato L, Capozzi L.Iron deficiency screening in the first three years of life: A three-decadelong retrospective case study. Hematology 2014. 19. (4):239-243

Cet article a été publié dans le « Batna Journal of Medical Sciences » BJMS, l'organe officiel de « l'association de la Recherche Pharmaceutique - Batna»

Le contenu de la Revue est ouvert « Open Access » et permet au lecteur de télécharger, d'utiliser le contenu dans un but personnel ou d'enseignement, sans demander l'autorisation de l'éditeur/auteur.

Avantages à publier dans BJMS :

- Open access : une fois publié, votre article est disponible gratuitement au téléchargement

- Soumission gratuite : pas de frais de soumission, contrairement à la plupart des revues « Open Access »

- Possibilité de publier dans 3 langues : français, anglais, arabe

- Qualité de la relecture : des relecteurs/reviewers indépendants géographiquement, respectant l'anonymat, pour garantir la neutralité et la qualité des manuscrits.

Pour plus d'informations, contacter BatnaJMS@gmail.com ou connectez-vous sur le site de la revue : www.batnajms.net 


\section{Bis}

- $\bigcirc$

-

○

○

Laboratoire de bactériologie. CHU de Batna.

Faculté de médecine de Batna

\section{Correspondance à :}

Messaoud BENMHIDI

mahdi05mess@gmail.com

DOI :https://doi.org/10.48087/ BJMSoa.2020.7215

\section{Historique de l'article :}

Reçu le 13 Septembre 2020

Accepté le 25 Octobre 2020

Publié le 09 novembre 2020

Il s'agit d'un article en libre accès distribué selon les termes de la licence Creative Commons Attribution International License (CC BY 4.0), qui autorise une utilisation, une distribution et une reproduction sans restriction sur tout support ou format, à condition que l'auteur original et la revue soient dûment crédités.

\section{Pour citer l'article :}

Benmhidi M. Les donnée de la bactériologie en matière de BHRe au CHU de Batna . Batna J Med Sci 2020;7(2):134-6. https://doi.org/10.48087/BJM Soa.2020.7215

\title{
Les données de la bactériologie en matière des BHRe au CHU de Batna
}

\author{
emerging highly resistant bacteria data at the University Hospital of Batna
}

\author{
Messaoud Benmhidi, Sana Boukhalfa, Sonia Benammar, Meriem Makhloufi, \\ Asma Lounis, Chahinez Khernane
}

\section{RÉSUMÉ}

Les Bactéries multi résistantes posent un problème de santé publique, Thérapeutique, de Pronostic et de Prise en charge. Actuellement, la diffusion de carbapénèmases constitue le problème clinique le plus important en matière de résistance aux antibiotiques chez les Gram négatifs, en particulier chez les entérobactéries et le risque de transmission entre patients et élevé. Cette étude a pour objectifs de donner une répartition des BHRe isolées par service et par prélèvements et de sensibiliser aux risques de l'antibiorésistance

Il s'agit d'une étude rétrospective avisée descriptive des BHRe isolées des prélèvements pathologiques provenant des différents services du CHU de Batna.

Notre enquête a porté sur une année. Tous les prélèvements ont été traités au niveau du laboratoire de bactériologie par un examen microscopique, une culture sur milieux gélosés spécifiques et un antibiogramme selon les normes CLSI. Ces examens ont abouti à un taux de BHRe de 2,20\% pour les EPC et 0,5\% pour les ERV.

Seulement deux espèces sont considérées comme BHRe et qui sont Enterococcus faecium résistants aux glycopeptides quelques soit le mécanisme de résistance (ERV) et Entérobactéries résistantes aux carbapénèmes par production de carbapénèmases (EPC).

Mots clés : antibiotiques, BHRe, ERV, EPC, CLSI

\section{INTRODUCTION}

L'émergence de la résistance aux antibiotiques (ATB) est un enjeu majeur de santé publique à l'échelle internationale [1].

L'augmentation de la résistance aux antibiotiques est préoccupante chez les bacilles à Gram Négatifs tel que Pseudomonas, Acinetobacter baumanii et les entérobactéries qui sont les pathogènes importants chez l'homme.

La résistance aux (C3G) est apparue en 1980 avec l'émergence d'enzymes appelée bêtalactamases à spectre élargi (BLSE) qui sont résistantes à toutes les bêtalactamines même aux céphalosporines de troisième génération C3G, à l'exception des cephamycines puis en 2000, l'émergence rapide d'autres BLSE de type CTX-M.

L'émergence des EBLSE depuis le début des années 1990 a favorisé la consommation des carbapénèmes.

\section{ABSTRACT}

Multi-resistant bacteria pose a problem of public health, treatment, prognosis and management. Diffusion of carbapenemases is the most important clinical problem in antibiotic resistance among gram negative, especially in enterobacteria and the risqué of transmission between patientsis high. The objectives of this study are ti give a division of the BHRe isolated by service and by sample and to raise awareness of the risks of antibiotic resistance.

It is a descriptive retrospective study of BHRe isolated from pathological sample coming from various services of the Batna University Hospital.

Our investigation focused on one year. All the samples are processed in the bacteriology laboratory by a microscopic examination, culture on specific agar media and antibiogram according to CLSI standards. These examinations resulted in a BHRe rate of $2.20 \%$ for the $\mathrm{EPC}$, and $0.5 \%$ for VRE.

Only two species are considered as BHRe which are; Entrococcus faecium resistant to glycopeptides regardeless of the resistance mechanism (ERV), and Enterobacteriaceae resistant to carbapenems by production of carbapenemares (EPC).

Key words: antibiotics, BHRe, ERV, EPC, CLSI

Leur utilisation semble compromise avec l'émergence de bactéries devenues résistantes aux carbapénèmes (BHRe).

La résistance aux carbapénèmes chez ces espèces est liée à l'association des mécanismes de résistance suivants : surexpression des BLSE surtout chez les entérobactéries du groupe qui sont porteurs de céphalosporinases avec pompe à efflux, imperméabilité ou l'action des carbapénèmases [2].

Les gènes codant pour les carbapénèmases sont portés par les plasmides associés aux résistances à d'autres Antibiotiques [3].

Par définition, les bactéries hautement résistantes émergentes (BHRe) sont des commensales du tube digestif, résistantes à de nombreux antibiotiques et ayant des mécanismes de résistance transférables entre bactéries et ayant diffusé que sur des modes sporadiques ou épidémiques limités [4,5]. 
Parmi ces espèces, Enterococcus faecium résistants aux glycopeptides (ERV), quel que soit le mécanisme de résistance, et Entérobactéries résistantes aux carbapénèmes par production de carbapénèmases (EPC) [4]. Une antibiothérapie inappropriée due à des BHRe est constamment associée à une augmentation de la mortalité hospitalière $[6,7]$.

Les Bactéries multi résistantes posent un problème de santé publique, Thérapeutique, de Pronostic et de Prise en charge. Actuellement, la diffusion de carbapénèmases constitue le problème clinique le plus important en matière de résistance aux antibiotiques chez les Gram négatifs, en particulier chez les entérobactéries et le risque de transmission entre patients et élevé.

Cette étude a pour objectif de donner une répartition des BHRe isolées par service et par prélèvements et de sensibiliser aux risques de l'antibio-résistance

\section{MATERIELS ET METHODES}

Il s'agit d'une étude rétrospective à visée descriptive des BHRe étudiée sur une période d'une année en 2017 au niveau du laboratoire de bactériologie du CHU de Batna.

Les différents prélèvements étudiés sont des pus, des hémocultures, des urines, des LCR et des dispositifs invasifs. Ces prélèvements sont munis d'une fiche de renseignement, ils sont issus des différents services du CHU de Batna.

Les bactéries isolées provenaient des prélèvements réalisés chez tous les patients admis au CHU ainsi que des prélèvements externes, celles isolées des prélèvements répétitifs et chez le Personnel soignant ont été exclues.

Chaque prélèvement a été soumis à une étude microscopique à l'êtas frais avec des colorations de Gram

L'ensemencement a été fait sur des milieux géloses (milieu Hectoen, milieu au sang cuit et milieux sur gélose nutritive) avec une incubation de $24 \mathrm{~h}$ suivie d'identification biochimique par Galerie API et d'antibiogramme par la méthode de diffusion selon CLSI : Test de Hodge modifié

Pour l'identification moléculaire du mécanisme de résistance, un CIM test par la méthode d'inactivation des carbapénémases, ainsi qu'une PCR ont été réalisés. La PCR a été réalisée dans un mélange réactionnel de l'extrait d'ADN, de la Taq polymérase, des amorces et des quatre désoxyribonucléosides triphosphates (dNTP) en excès dans une solution tampon.

Les données ont été collectées sur la base du logiciel Whonet 5.6.

En plus de l'identification des espèces, les paramètres déterminés ont été, essentiellement, la fréquence, la répartition par service de provenance et les résistances associés à d'autres antibiotiques.

\section{RÉSULTATS}

Dans cette étude, nous avons pu isoler parmi les prélèvements reçus $23 \mathrm{EPC}$ et des $2 \mathrm{ERV}$. Les résultats des examens bactériologiques révèlent un total de 1044 entérobactéries dont 23 sont des EPC, soit un pourcentage de $2.20 \%$ (Figure.1)

La répartition des EPC par service de provenance est donnée par la figure 2 qui démontre une prédominance des EPC dans le service des brulés avec un taux de 39\% suivi de l'hématologie, la néphrologie, la chirurgie et la réanimation avec un taux de 13\% chacun; le service des urgences et le prélèvement externes occupent la dernière place avec un taux de $4 \%$ chacun.

Concernant les espèces porteuses de carbapénémases, Klebsiella pneumoniae vient en tête avec un pourcentage de $56 \%$, suivie d'Escherichia Coli avec 22\% puis Enterobacter sp et Proteus sp ; 7\% chacun.

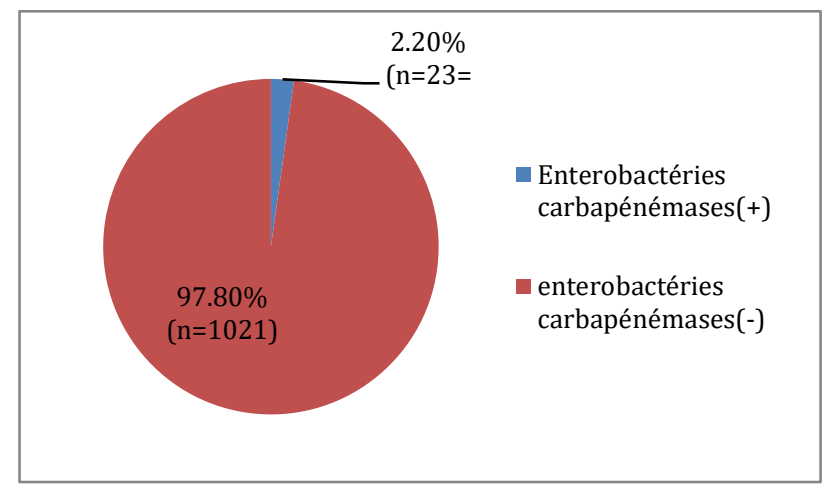

Figure 1. Fréquence des EPC.

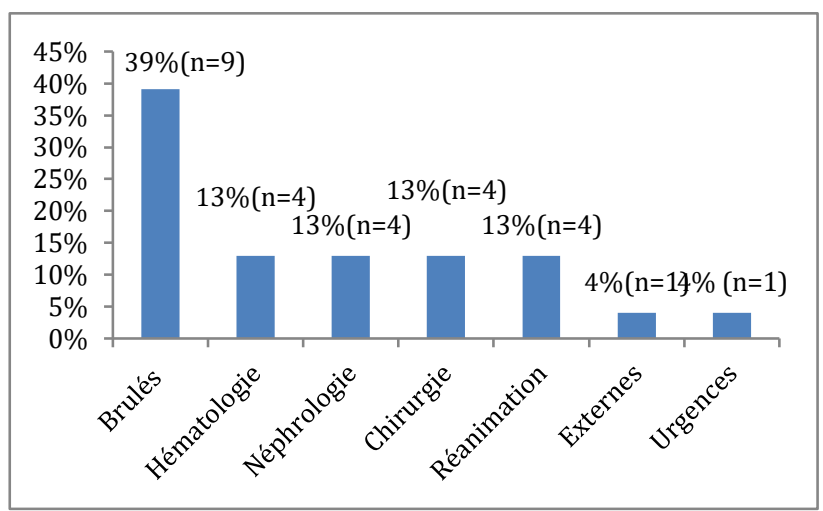

Figure 2. Prédominance des EPC par services.

Toutes les EPC isolées étaient, également, résistantes à l'ampicilline et à la céfazoline, $98 \%$ étaient résistante aussi à l'amoxicilline/acide clavulanique et $85 \%$ au cefotaxime. Les résistances associées concernaient également la gentamicine $(70 \%)$ et l'imipénème $(70 \%)$ et à un moindre degré la ciprofloxacine (35\%) et la fosfomycine (5\%) (figure 3).

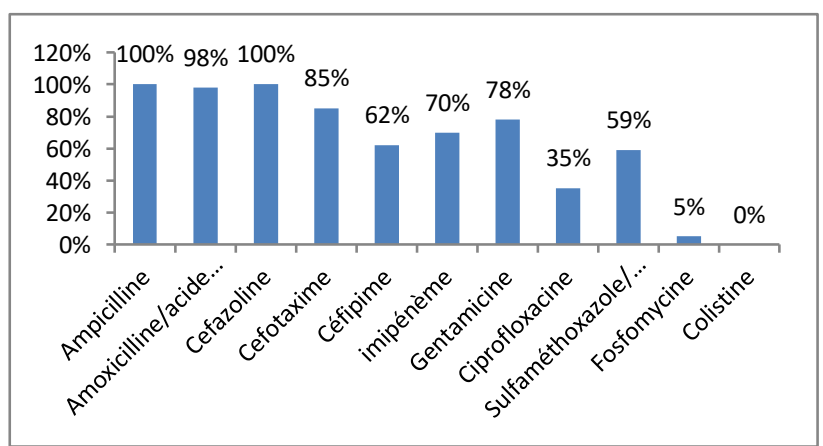

Figure 3. Résistances associées des EPC à d'autres antibiotiques.

Sur un total de 400 coccis gram positif, deux se sont révélés ERV soit un pourcentage de $0.5 \%$. Il s'agit d'Enterococcus faecium qui a été retrouvé au niveau des services de réanimation et d'hématologie. 
Les bactéries isolées étaient à la fois résistantes à l'ampicilline, gentamicine, rifampicine, lévofloxacine, vancomycine, teicoplanine et tétracyclines (figure 4)

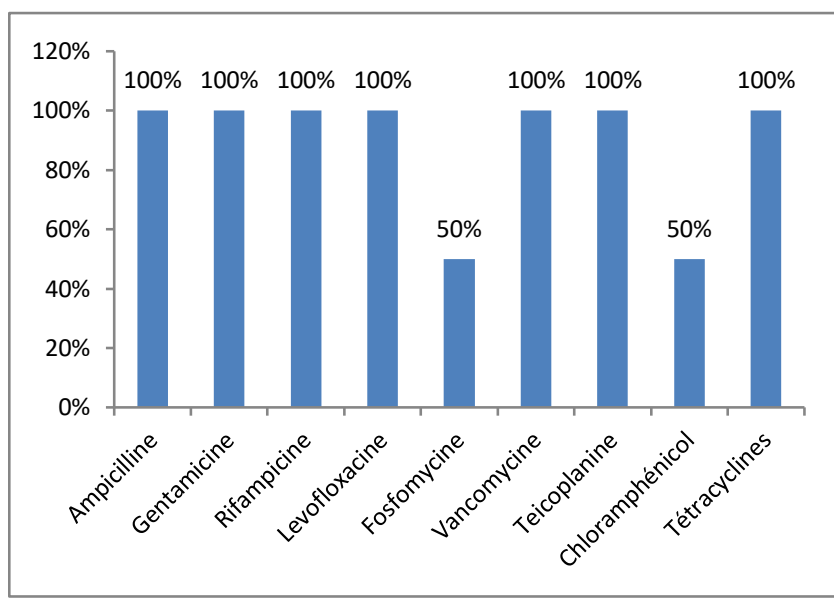

Figure 4. Résistances associées des ERV à d'autres antibiotiques

\section{DISCUSSION}

Le microbiologiste était en charge de l'identification de la BHRe, culture sur géloses spécifiques des EPC ou ERV et identification moléculaire du mécanisme de résistance. Les méthodes de diagnostic rapide permettent un premier rendu $\mathrm{du}$ résultat en quelques heures [8]. 2,20\% des entérobactéries sont porteuses de carbapénèmases [9], L'espèce dominante est Klebsiella Pneumoniae avec une fréquence de $56 \%$ après Escherichia coli de $22 \%$.

En France, Klebsiella pneumoniae est impliquée dans $59 \%$ des épisodes et Escherichia coli dans $34 \%$ des épisodes [10] Selon les données de ce réseau, la proportion de souches résistantes aux carbapénèmes était stable et < $1 \%$ en 2013 en France pour K. pneumoniae et pour E. coli. En comparaison, cette proportion pour $K$. pneumoniae était en forte augmentation en Grèce (59\%) et en Italie (34 \%) où ces souches sont devenues endémiques et fréquemment responsables d'infections invasives [10].

Cette résistance a été confirmée par des tests complémentaires et par la PCR qui a révélé la présence du gène OXA-48 pour les entérobactéries. L'OXA-48 épargne les C3G, les résultats montrent $62 \%$ de résistance au C3G ce qui signifie la présence d'une BLSE portée par une CTX M-15. Les mécanismes OXA-48 sont les plus fréquents et sont retrouvés dans $78 \%$ des épisodes en France, plus rarement NDM et KPC [10].

La sensibilité à la colistine est variable, certaines souches sont naturellement résistantes. La CMI des souches normalement sensibles à la colistine a été testée, elle était de l'ordre de 0,5 avec une faible résistance à la fosfomycine $5 \%$ et une résistance de $35 \%$ à la ciprofloxacine et $59 \%$ de résistance au sulfamethoxazole triméthoprime.

Les EPC sont fréquents au niveau des brulés avec une fréquence de $39 \%$ par rapport à d'autres services comme le service d'Hématologie et de néphrologie de 13\%; un protocole de surveillance a été installé en conséquence.

Parmi les coccis à Gram positif, on a retrouvé $0,5 \%$ en Réanimation médicale et en Hématologie. Entérocoques Faecium multi résistants (ERV) [11] avec une sensibilité de $50 \%$ à la fosfomycine et au Chloramphenicol.

\section{CONCLUSION}

La maîtrise de la diffusion de ces germes (dépistage précoce, maintien des précautions complémentaires contact, signalisation, dépistage éventuel des sujets contacts et suivi du portage) ainsi qu'un usage raisonné de l'antibiothérapie afin de limiter la pression de sélection sont les principaux leviers d'action à la disposition du clinicien. Des souches de bactéries hautement résistantes (BMR ou BHR) sont décrites de manière croissante à travers le monde alors, qu'en même temps, très peu de nouvelles molécules antibiotiques sont développées et disponibles. De nouvelles thérapeutiques, de nouveaux tests de diagnostic plus rapides et des connaissances plus approfondies sur la dynamique bactérienne (interactions résistance/virulence) sont tous aussi nécessaires. L'évolution des Bacilles à Gram négatif d'une multi résistance à une résistance à tous les antibiotiques représente La menace du future ce qui nécessite la préservation des carbapénèmes et des Glycopeptides.

Déclaration d'intérêts: les auteurs ne déclarent aucun conflit d'intérêt en rapport avec cet article.

\section{RÉFÉRENCES}

1. WHO. Antimicrobial resistance: global report on surveillance. 2014; Available from: http://www.who.int/drugresistance/ documents/surveillancereport/en/.

2. Bonnin RA, Nordmann P, Poirel L, Screening and deciphering antibiotic resistance in Acinetobacter Baumanii :a state of the art ,Exp Revanti In fTher 2013;11:571-583

3. Nordmann P. Carbapénèmase producing Enterobacteriaceae:overiew of a major public health challenge. Med Mal Infect 2014;44:51-56

4. Magiorakos, A.P., et al. Multidrug-resistant, extensively drug-resistant and pandrug-resistantbacteria: an international expert proposal for interim standard definitions for acquired resistance. ClinMicrobiol Infect, 2012;18 (3):268-81.

5. Haut Conseil de la Faculté Publique, Recommandations pour la prévention de la transmission croisée des "Bactéries Hautement Résistantes aux antibiotiques émergentes » (BHRe). 2013

6. Kollef, M.H., et al.Inadequate antimicrobial treatment of infections: a risk factor for hospital mortality among critically ill patients. Chest 1999;115(2):462-74.

7. Shorr, A.F., et al. Inappropriate antibiotic therapy in Gram-negative sepsis increases hospital length of stay. Crit Care Med, 2011;39(1):46-51.

8. Nordmann, P. and L. Poirel. Strategies for identification of carbapenemase-producing Enterobacteriaceae. J Antimicrob Chemother, 2013;68(3):487-9.

9. Nordmann $P$, Poirel $L$, Doertet $L$ Rapid detection of carbapénèmasesproducing Enterobacteriaecae.Emerg Infect Dis 2012;18:1503-1507

10. Santé publique France. Episodes impliquant des entérobactéries productrices de carbapénèmas es en France. Situation épidémiologique du 4 septembre 2015 [En ligne]. France;2015 [Cité le 25 octobre 2020]. Disponible: https://www.santepubliquefrance.fr/maladies-et-

traumatismes/infections-associees-aux-soins-et-resistance-auxantibiotiques/infections-associees-aux-soins/documents/bulletinnational/episodes-impliquant-des-enterobacteries-productrices-decarbapenemases-en-france.-situation-epidemiologique-du-4-septembre2015

11. Benammar S, Pantel A, Aujoulat F, et al. First molecular characterization of related cases of healthcare-associated infections involving multidrugresistant Enterococcus faecium vanA in Algeria. Infect Drug Resist. 2018;11:1483-1490. Published 2018 Sep 17. doi:10.2147/IDR.S164487 


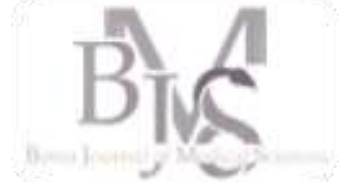

- $\bigcirc$

- $\bigcirc$

P

○

${ }^{1}$ Faculté de médecine,

Université de Batna 2 - Algérie

2 Faculté de médecine, université de Ouargla - Algérie

${ }^{3}$ Laboratoire d'analyse médicales M. Sadlaoud - Algérie

${ }^{4}$ Faculté de médecine, Université de Batna 2 - Algérie

${ }^{5}$ EPH Batna - Algérie

${ }^{6}$ Faculté de médecine, Université de Béchar - Algérie

Correspondance à :

Anis BENYAHIA

anis.benyahia@univ-batna2.dz

DOI :https://doi.org/10.48087/ BJMSoa.2020.7216

Historique de l'article :

Reçu le 4 septembre 2020

Accepté le 2 novembre 2020

Publié le 09 novembre 2020

Il s'agit d'un article en libre accès distribué selon les termes de la licence Creative Commons Attribution International License (CC BY 4.0), qui autorise une utilisation, une distribution et une reproduction sans restriction sur tout support ou format, à condition que l'auteur original et la revue soient dûment crédités.

\section{Pour citer l'article :}

Benyahia A, Tebbal S, Chiboub B. Intérêt de la quantification de l'Ag HBs chez le porteur chronique du virus de l'hépatite B. Batna J Med Sci 2020;7(2):137-41 https://doi.org/10.48087/BIM $\underline{\text { Soa.2020.7216 }}$

\title{
Intérêt de la quantification de l'AgHBs chez le porteur chronique du virus
} de l'hépatite $B$

\author{
Interest of the quantification of HBsAg in the chronic carrier of the hepatitis \\ $B$ virus
}

\author{
Anis Benyahia ${ }^{1}$, Soraya Tebbal ${ }^{1}$, Bouthayna Chiboub ${ }^{2}$, Mourad Sadelaoud ${ }^{3}$, \\ Hocine Bouncer ${ }^{4}$, Amira Housna Ameghchouche ${ }^{5}$, Assia Aouachria 6
}

\section{RÉSUMÉ}

La quantification de l'AgHBs a montré un intérêt dans la classification du patient dans les différentes phases de l'infection chronique B. Pour évaluer l'impact de celle-ci, nous avons mené une étude prospective chez les porteurs chroniques du VHB, sur une période de 5 ans. Nous avons colligé 210 patients $(114$ hommes; 96 femmes), avec un âge moyen de 43 ans ( 18 - 80 ans), une cytolyse dans $12 \%$ des cas $(25 / 210)$ et $90 \%$ des patients AgHBe négatif (189/210). L'ADN-VHB moyen est de 3,25 $\pm 2 \mathrm{Log} \mathrm{UI} / \mathrm{ml}$. Une fibrose modérée à sévère (F2, F3, F4) est noté dans $21,42 \%(45 / 210)$. Le taux moyen de l'AgHBs est de 2,84 $\pm 1,1 \mathrm{Log} \mathrm{UI} / \mathrm{ml}$, il est de 4,36 $\mathrm{LogUI} / \mathrm{ml}$ chez le sujet jeune versus $3 \mathrm{LogUI} / \mathrm{ml}$ pour le sujet âgé. Nous avons noté une corrélation entre le taux de l'AgHBs et celui de l'ADN VHB ( $\mathrm{r}=0,40, \mathrm{p}<0,001)$. Ce taux est plus élevé pour l'AgHBe positif $(3,81 \mathrm{LogUI} / \mathrm{ml}$ vs 2,77 LogUI/ml). Les taux moyens d'AgHBs sont de 4,$33 ; 3,13 ; 2,87$ et $3,19 \operatorname{logUI} / \mathrm{ml}$ aux phases $1,2,3$ et 4 respectivement. Entre phase 1 et phase 3, il est de 3,6 \pm $1,5$ vs $2,07 \pm 1(p<0,001)$. Entre phase active ( 2 et 4$)$ et phase inactive (3), il est de $3,35 \pm 0,7$ vs $2,07 \pm 1$ $(p<0,00001)$. Pour un taux d'ADN VHB $<2000 \mathrm{UI} / \mathrm{ml}$ et d'AgHBs $<1000 \mathrm{UI} / \mathrm{ml}$, la proportion de porteurs inactifs est de 43\% (90/210) contre 59\% (124/210) par la définition usuelle (ADN VHB $(<2000 \mathrm{UI} / \mathrm{ml})$.

Mots-clés: hépatite chronique B; quantification de l'AgHBs ; portage inactif.

\section{INTRODUCTION}

L'hépatite $B$ pose un problème mondial de santé publique. Le nombre de porteurs chroniques est estimé à 257 millions [1]. L'Algérie, d'endémicité intermédiaire, a une séroprévalence du portage chronique de l'AgHBs de 2,15\% [2].

Depuis sa découverte en 1965 par Blumberg [3], la détection qualitative de l'AgHBs est utilisée à des fins diagnostiques. Sa clairance, qui est rare, est possible soit spontanément ou après traitement [4].

L'intérêt pour la quantification de l'AgHBs repose sur l'étude princeps [5], montrant une association positive avec l'ADNccc (covalently closed circular). La disponibilité récente de tests quantitatifs standardisés a relancé la recherche autour de ce marqueur [6].

Si la charge virale renseigne sur le nombre de virions circulants, la quantification de l'AgHBs

\section{ABSTRACT}

The quantification of HBsAg has shown an interest in the classification of the patient in the different phases of chronic B infection. To evaluate the impact of this, we conducted a prospective study in chronic HBV carriers over a period of 5 years. We collected 210 patients (114 men; 96 women), with a mean age of 43 years (18 - 80 years), cytolysis in $12 \%$ of cases $(25 / 210)$ and $90 \%$ of HBeAg-negative patients (189/210). Mean HVB-DNA is $3.25 \pm 2 \mathrm{Log} \mathrm{IU} / \mathrm{ml}$. Moderate to severe fibrosis (F2, F3, F4) is noted in $21.42 \%(45 / 210)$. The mean HBsAg level is $2.84 \pm 1.1 \mathrm{Log} \mathrm{IU} / \mathrm{ml}$, it is $4.36 \mathrm{Log} \mathrm{IU} / \mathrm{ml}$ in the young subject versus $3 \mathrm{Log} \mathrm{IU} / \mathrm{ml}$ for the elderly subject. We noted a correlation between HBsAg and HBV-DNA levels $(\mathrm{r}=0.40, \mathrm{p}<0.001)$. This rate is higher for positive $\mathrm{HBeAg}$ (3.81 LogUI/ml vs. $2.77 \mathrm{LogUI} / \mathrm{ml}$ ). Average HBsAg levels are $4.33 ; 3.13 ; 2.87$ and $3.19 \operatorname{logUI} / \mathrm{ml}$ in phases $1,2,3$ and 4 respectively. Between phase 1 and phase 3 it is 3.6 \pm 1.5 vs. $2.07 \pm 1(\mathrm{p}<0.001)$. Between active phase ( 2 and 4) and inactive phase (3) it is $3.35 \pm 0.7$ vs $2.07 \pm 1$ ) $(\mathrm{p}<0.00001)$. For HBV DNA $<2000 \mathrm{IU} / \mathrm{ml}$ and HBsAg $<$ $1000 \mathrm{IU} / \mathrm{ml}$, the proportion of inactive carriers is $43 \%$ $(90 / 210)$ versus $59 \%(124 / 210)$ by the usual definition (HBV DNA $(<2000 \mathrm{IU} / \mathrm{ml})$.

Keywords: chronic hepatitis B, quantification of HBsAg, inactive carrier.

mesure la fraction de virions complets et celle, plus importante, de virions vides. Celle-ci permet de mieux caractériser le statut du patient et en particulier du porteur inactif.

Elle permet également de prédire la réponse au traitement, la durée optimale de ce traitement ainsi que la perte éventuelle de l'antigène HBs [1].

Nous proposons l'évaluation de l'impact de la quantification de l'AgHBs sur l'histoire naturelle de l'hépatite chronique $\mathrm{B}$ chez les patients suivis pour portage chronique du VHB.

\section{PATIENTS ET MÉTHODES}

Il s'agit d'une étude descriptive, prospective, monocentrique, menée au service d'infectiologie de l'établissement public hospitalier de Batna, incluant des patients porteurs chroniques du VHB sur période entre allant de septembre 2013 à mars 2018. 
Deux cent dix patients porteurs chroniques de l'AgHBs, âgés de plus de 17 ans, ont été inclus dans l'étude pour lesquelles des quantifications de l'AgHBs ont été réalisées.

Ont été exclus de l'étude les patients coinfectés par le virus de l'hépatite $\mathrm{C}$ et le virus d'immunodéficience humaine, les perdus de vue et ceux dont les données étaient incomplètes.

Les données épidémiologiques, cliniques, le statut qualitatif de l'AgHBs, l'AgHBe et la charge virale du VHB ont été recueillies à l'inclusion.

Tous les patients ont été classés en fonction des différentes phases de l'infection chronique par le VHB dont les critères de diagnostic étaient basés sur les lignes directrices de pratique clinique de l'Association européenne pour l'étude du foie (EASL 2017) [7].

\section{Quantification de l'AgHBs}

Les taux de l'AgHBs dans les échantillons de sérum ont été quantifiés à l'aide du test Elecsys II utilisant des anticorps monoclonaux et polyclonaux permettant une amélioration de la reconnaissance de l'AgHBs et des mutants AgHBs.

La linéarité de la technique Elecsys II s'étend de 0,05 à 52.000 $\mathrm{UI} / \mathrm{ml}$ avec une dilution automatique intégrée. L'analyse de ce test a été pratiquée dans le laboratoire d'analyse médicale du Dr Sadlaoud Batna.

\section{Quantification de l'ADN-VHB}

L'ADN-VHB était quantifié par la technique d'amplification PCR en temps réel (COBAS AmpliPrep / COBAS TaqMan) avec un seuil de détection à $20 \mathrm{UI} / \mathrm{ml}$. Le titrage de l'ADN-VHB a été pratiqué à l'institut Pasteur d'Alger.

Le dosage des paramètres biochimiques et sérologiques était réalisé par des tests standardisés. Le statut du patient était défini au cours du suivi, conformément aux recommandations EASL 2012.

\section{Analyse statistique}

La saisie et l'analyse des données est faite sur le logiciel Epi Info 7. Les variables quantitatives sont présentées en valeurs moyennes ou médianes accompagnées de leur indice de dispersion.

Des tests de Chi2, analyses de variance, tests de Student en séries non appariées, ou le coefficient de corrélation $\mathrm{R}$ de Spearman ont été utilisés afin de rechercher des liens possibles entre l'AgHBs et les caractéristiques des patients incluant des données épidémiologiques, des paramètres virologiques, des paramètres cliniques, des paramètres biochimiques et histologiques. Dans toutes les analyses, une valeur seuil de $p<0,05$ a été considérée comme statistiquement significative.

\section{RÉSULTATS}

\section{Caractéristiques générales des patients}

Deux cents dix patients porteurs chroniques du VHB, naïfs de tout traitement antiviral, ont bénéficié d'une quantification de l'AgHBs, 114 hommes et 96 femmes.

La moyenne d'âge était de $43 \pm 13$ ans (18 - 80 ans). La classe d'âge la plus représentée était celle des 25 - 34 ans soit $28 \%$ (60/210) (figure 1). L'IMC moyen était de 27,6, la cytolyse a été noté dans $12 \%$ des cas $(25 / 210)$ et $90 \%$ des patients (189/210) étaient AgHBe négatif (tableau 1).

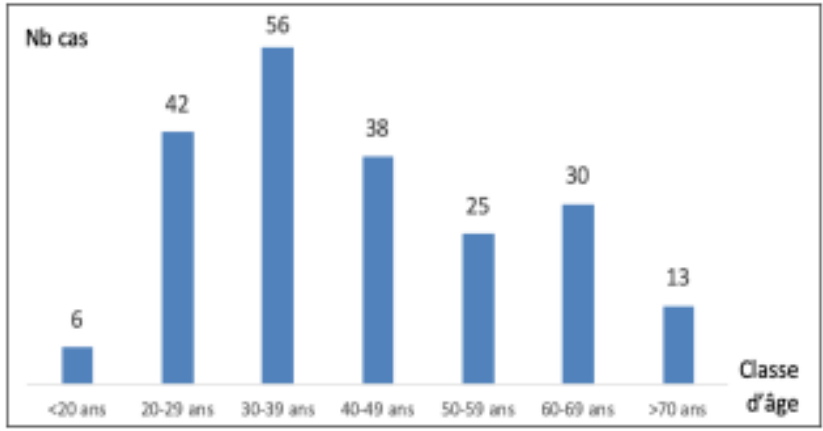

Figure 1. Répartition des patients par classe d'âge.

La répartition montre que $46,2 \%$ des patients $(97 / 210)$ ont un taux inférieur à $1000 \mathrm{UI} / \mathrm{ml}$; un taux de 23,3\% (49/210) pour un AgHBs compris entre 1000 et $3500 \mathrm{UI} / \mathrm{ml}$ et un taux élevé dans 31,5\% des cas (64/210) (tableau 2). Le taux d'AgHBs moyen est de $4965 \pm 858 \mathrm{UI} / \mathrm{ml}(2,84 \pm 1,1$ $\operatorname{LogUI} / \mathrm{ml})$.

Tableau 1. Caractéristiques générales des patients à l'inclusion $(n=210)$.

\begin{tabular}{cc}
\hline \multicolumn{1}{c}{ Caractéristiques } & Valeurs \\
\hline Age (année) & $43(18-80$ ans) \\
\hline Hommes (nb, \%) & $114(54.2)$ \\
\hline ALAT normale $(\mathrm{nb}, \%)$ & $185(88 \%)$ \\
\hline AgHBe positif (nb, ml) & $21(10 \%)$ \\
\hline AgHBs (UI/ml, LogUI/ml) & $2,84 \pm 1,1 ; 4965 \pm 858$ \\
\hline ADN VHB moyen (LogUI/ml) & $3,25 \pm 2$ \\
\hline Statut du patient (n, \%) & \\
- Phase1 & $12(5.71)$ \\
- Phase2 & $9(4.28)$ \\
- Phase3 & $124(59)$ \\
- Phase4 & $65(31)$ \\
& \\
- F0-F1 & $165(78,6)$ \\
- F2 & $27(12,9)$ \\
- F3 & $8(3,8)$ \\
- F4 & $10(4,8)$ \\
\hline
\end{tabular}

La figure 2 montre le déclin du taux d'AgHBs suivant les classes d'âge. Le titre de l'AgHBs le plus élevé est observé chez le sujet jeune de moins de 20 ans avec une moyenne de $22.913 \mathrm{UI} / \mathrm{ml}$, par la suite cette dernière diminue avec l'âge. Les taux d'AgHBs les plus bas sont observés dans la classe d'âge $>70$ ans (taux moyen : $1.134 \mathrm{UI} / \mathrm{ml}$ ) et les classes d'âge $45-49$ ans et $60-64$ ans (1290 et $1198 \mathrm{UI} / \mathrm{ml})$.

Tableau 2. Distribution des taux de l'AgHBs ( $\mathrm{n}=210)$

\begin{tabular}{cccc}
\hline $\begin{array}{c}\text { Taux AgHBs } \\
(\mathbf{U I} / \mathbf{m l})\end{array}$ & $\begin{array}{c}\text { Nombre } \\
\text { de cas }\end{array}$ & $\mathbf{\%}$ & $\begin{array}{c}\text { Sous total } \\
\text { (nb, \%) }\end{array}$ \\
\hline$<0,5$ & 5 & 2,38 & \\
$<10$ & 13 & 6,19 & 97 \\
$10-99$ & 22 & 10,47 & $(46 \%)$ \\
$100-499$ & 36 & 17,14 & \\
$500-1000$ & 21 & 10 & 49 \\
$1000-2000$ & 30 & 14,28 & $(23 \%)$ \\
$2000-3500$ & 19 & 9 & 64 \\
$3500-10000$ & 35 & 16,67 & $(31 \%)$ \\
$>10000$ & 29 & 13,8 & \\
\hline Total & 210 & 100 &
\end{tabular}




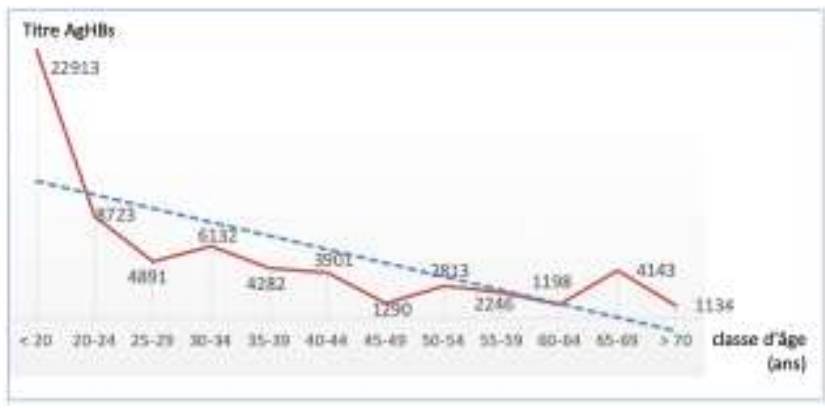

Figure 2. Répartition des taux moyens de l'AgHBs (UI/ml) par tranche d'âge

La charge virale moyenne du VHB est de 3,25 $\pm 2 \mathrm{Log} \mathrm{UI} / \mathrm{ml}$. La cytolyse est observée chez $12 \%(25 / 210)$ des patients. Quarante-cinq patients $(21,42 \%)$ ont une fibrose modérée à sévère (F2, F3, F4) (tableau 1).

Dans le volet diagnostique, selon la classification usuelle, nous avons recensé $59 \%$ des patients porteurs inactifs $(124 / 210), 35,2 \%(74 / 210)$ des patients avec hépatite active et $12(5,7 \%)(12 / 210)$ de patients immunotolérants.

\section{Corrélation AgHBs et ADN VHB}

La figure 3 représente la distribution des couples de valeurs AgHBs/ADN VHB en log $\mathrm{UI} / \mathrm{ml}$ et met en évidence une corrélation entre le taux d'AgHBs et la charge virale B. En effet, le taux d'AgHBs augmente avec l'élévation de la charge virale, il y'a une différence statistique significative $(p<0,001)$.

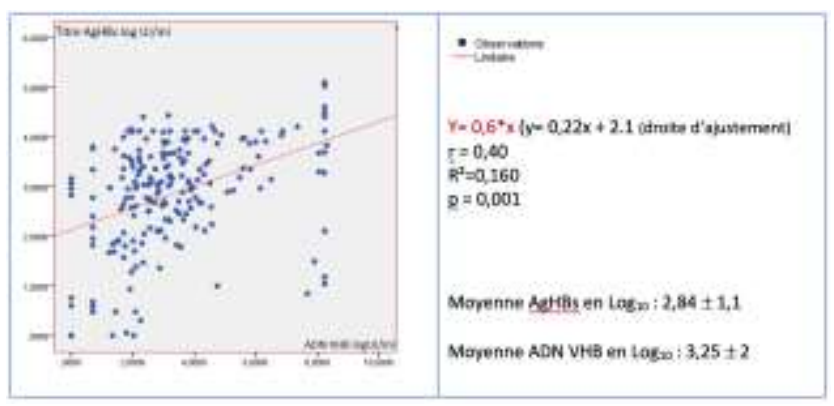

Figure 3. Corrélation AgHBs et ADN VHB ( $\mathrm{n}=210)$

\section{Quantification de l'antigène HBs et histoire naturelle de l'infection à VHB}

Le diagnostic des différentes phases de l'histoire naturelle du VHB selon les niveaux de l'ADN-VHB, de l'ALAT et de l'évaluation de la fibrose hépatique (tableau 3) retrouve $59 \%$ $(124 / 210)$ de porteurs inactifs (phase 3). Cependant, dans notre étude, en utilisant la quantification de l'AgHBs, l'analyse de cette classe d'effectif ne retrouve que $43 \%$ des patients $(90 / 210)$ qui présentent un taux d'AgHBs $<1000 \mathrm{UI} / \mathrm{ml}$ et par conséquent classés "vrais" porteurs inactifs. La fréquence pour cette catégorie de population où l'AgHBs $>1000$ est de $27 \%(34 / 124)$. Il s'agit probablement de faux porteurs inactifs. Cette population est alors 'reclassée' en phase 4 .
Tableau 3. Classification diagnostique par taux d'AgHBs $(n=210)$

\begin{tabular}{lllll}
\hline \multirow{2}{*}{ Phases } & \multicolumn{2}{l}{ Dg ADN-VHB } & Dg QHBs \\
\cline { 2 - 5 } & Effectif & $\%$ & Effectif & $\%$ \\
\hline Infection AgHBe (+) [phase 1] & 12 & 5,71 & 12 & 6 \\
Hépatite AgHBe (+) [phase 2] & 9 & 4,28 & 9 & 4 \\
Infection AgHBe (-) [phase 3] & 124 & 59 & 90 & 43 \\
Hépatite AgHBe (-) [phase 4] & 65 & 31 & 99 & 47 \\
\hline Total & 210 & 100 & 210 & 100 \\
\hline $\begin{array}{l}\text { Dg ADN-VHB : diagnostic par } \\
\text { quantification AgHBs }\end{array}$ & ADNB; Dg QHBs : diagnostic par
\end{tabular}

\section{Taux d'AgHBs selon le statut du patient}

Répartition selon le statut de l'AgHBe

Le taux moyen de l'AgHBs est significativement plus élevé chez les 21 patients AgHBe positifs en comparaison aux 189 patients AgHBe négatifs ( $\mathrm{p}<0,001)$. Il est respectivement de $3,81 \pm 1,3 \log \mathrm{UI} / \mathrm{ml}$ versus $2,77 \pm 1,06 \mathrm{log} \mathrm{UI} / \mathrm{ml}$. La charge virale VHB est également différente : 7,4 $\pm 1,34 \operatorname{logUI} / \mathrm{ml}$ pour les patients (AgHBe positif) versus 2,78 $\pm 1,5 \mathrm{log} \mathrm{UI} / \mathrm{ml}$ pour les patients (AgHBe négatif). La différence statistique est significative $(\mathrm{p}<0,000001)$.

Répartition selon le statut: porteur inactif (phase 3) vs hépatite active (phases 2 et 4) vs immunotolérant (phase 1)

La moyenne du taux de l'AgHBs des patients de phase 1 est de $3,6 \pm 1,5 \operatorname{logUI} / \mathrm{ml}$ et celle des patients des phases 2 et 4 est de 3,34 $\pm 0,7 \log U I / m l$. Statistiquement, la différence n'est pas significative ( $\mathrm{p}=\mathrm{NS}$ ).

Entre phase 1 et phase $3(3,6 \pm 1,5$ vs $2,07 \pm 1)$, la différence statistique est significative $(p<0,001)$. Entre phases 2 et 4 et phase $3(3,35 \pm 0,7$ vs $2,07 \pm 1)$, la différence est significative $(p<0,00001)$. La différence entre les 3 groupes était significative $(\mathrm{p}<0,0001)$ (tableau 4$)$.

Tableau 4. Taux moyen de l'AgHBs et de l'ADN VHB selon les phases diagnostiques $(\mathrm{n}=210)$

\begin{tabular}{llll}
\hline Phases & Effectif & $\begin{array}{c}\text { AgHBs moyen } \\
\text { (LogUI/ml) }\end{array}$ & $\begin{array}{l}\text { ADN VHB } \\
\text { moyen } \\
\text { (LogUI/ml) }\end{array}$ \\
\hline Phase 1 (IT) & 12 & $3,59 \pm 1,48$ & $7,85 \pm 0,62$ \\
Phases 2 et 4 (HA) & 108 & $3,34 \pm 09$ & $3,83 \pm 1,68$ \\
Phase 3 (PI) & 90 & $2,07 \pm 1,03$ & $1,84 \pm 0,97$ \\
\hline Total & 210 & $2,83 \pm 1,10$ & $3,24 \pm 2,03$ \\
\hline
\end{tabular}

La charge virale diffère selon les phases : phase 1, phases 2 et 4 et phase 3 (tableau 4). La charge virale moyenne chez les patients en phase 1 est de 7,85 $\pm 0,6 \log \mathrm{UI} / \mathrm{ml}$ et chez les patients en phases 2 et 4 de 3,83 $\pm 1,7 \operatorname{logUI} / \mathrm{ml}$. La différence statistique est significative $(p<0,000001)$. Entre phase 1 et phase $3(7,85$ vs $1,84 \pm 1 \log )$, la différence est significative $(\mathrm{p}<0,00001)$. Et entre phases 2 et 4 et phase $3(3,83$ vs 1,84 $\log )$. La différence est significative $(\mathrm{Z}=9,933 ;(\mathrm{p}<0,00001)$. Statistiquement, il existe une différence significative entre les différentes phases ou statuts. 


\section{Stades de fibrose selon les phases diagnostiques}

La fibrose hépatique ( $\geq$ F2) est retrouvée dans $21 \%$ des cas $(45 / 210) ; 27 / 45$ cas $(60 \%)$ en $\mathrm{F} 2 ; 8 / 45$ cas $(18 \%)$ en $\mathrm{F} 3$ et $10 / 45$ cas $(22 \%)$ en $\mathrm{F} 4$. Il est à noter que 8 patients classés porteurs inactifs (selon la combinaison : ADN $<2000 \mathrm{UI} / \mathrm{ml}$ et AgHBs $<1000 \mathrm{UI} / \mathrm{ml}$ ) présentent une fibrose modérée (F2) (tableau 5).

Tableau 5 : Répartition de la fibrose selon les phases diagnostiques $(\mathrm{n}=210)$

\begin{tabular}{llllll}
\hline Statut & F0-F1 & F2 & F3 & F4 & Total \\
\hline Infection AgHBe+ & 12 & 0 & 0 & 0 & 12 \\
Hépatite AgHBe+ & 0 & 4 & 3 & 2 & 9 \\
Infection AgHBe - & 82 & 8 & 0 & 0 & 90 \\
Hépatite AgHBe - & 71 & 15 & 5 & 8 & 99 \\
\hline \multirow{2}{*}{ Total } & 165 & 27 & 8 & 10 & \multirow{2}{*}{210} \\
\hline
\end{tabular}

\section{DISCUSSION}

L'antigène de surface de l'hépatite $\mathrm{B}$ (AgHBs) a été le premier marqueur sérologique découvert pour l'infection par le VHB et a été utilisé comme marqueur classique pour le diagnostic de l'infection par le VHB [3].

Certaines études ont montré que le titre d'AgHBs circulant refléterait la quantité d'ADNccc présent dans le foie [6-8-9]. Il est considéré comme un marqueur substitutif de l'ADNccc.

Depuis le développement d'outils standardisés, de nombreuses études portant sur la quantification de l'AgHBs ont été réalisées pour montrer son intérêt éventuel dans le suivi de l'histoire naturelle et la prédiction de la réponse au traitement dans l'hépatite chronique B [10].

Il a été montré que son titre varie de façon importante au cours de l'évolution de l'hépatite chronique B, qu'il est inversement corrélé au contrôle immunitaire, indiquant que le titre de l'AgHBs reflète l'interaction complexe entre virus et système immunitaire fournissant ainsi des informations complémentaires à la charge virale VHB [5-11].

Notre étude confirme la corrélation positive entre les taux d'AgHBs et l'ADN VHB. Ce lien est maintenant bien établi, bien qu'il soit plus fort chez les patients AgHBe positif (durant la phase d'immunotolérance) que chez les patients AgHBe négatif [11-12].

La quantification de l'AgHBs montre des taux significativement plus élevés chez les patients AgHBe positifs que chez les patients AgHBe négatifs. Cette différence a été retrouvée dans différentes études aussi bien européennes qu'asiatiques $[11,13,14]$.

Notre étude, a montré une différence significative des titres sériques d'AgHBs entre les quatre phases de l'histoire naturelle du VHB. Ces résultats sont en accord avec ceux des études précédentes [11-15-16]. De nombreuses études confirment l'importance clinique de la quantification de l'AgHBs, pour suivre l'histoire naturelle de l'hépatite chronique B. Le titre d'AgHBs varie significativement lors des différentes phases de l'infection chronique, avec les titres les plus élevés à la phase d'immunotolérance et les plus faibles à la phase de portage inactif. Ce titre refléterait le contrôle immunologique sur l'infection. Combiné à la quantification de l'ADN viral, il permettrait d'identifier les vrais porteurs inactifs.
Dans notre étude, en tenant compte de la combinaison quantification de l'AgHBs et de l'ADN VHB, la proportion du portage inactif du VHB passe de $59 \%$ à $42,85 \%$, et la fréquence des patients reclassés de la phase 3 en phase 4 est à un taux de $27,41 \%$.

La difficulté chez les patients porteurs chroniques du VHB est de déterminer à quelle phase de l'infection ils se situent pour décider ou non de l'initiation d'un traitement. Il est difficile, sur la seule normalité des transaminases et de l'ADN VHB < $2000 \mathrm{UI} / \mathrm{ml}$ de différencier les patients ayant une infection chronique VHB AgHBe négatif (porteurs inactif) de ceux ayant une hépatite chronique AgHBe négatif [17], en raison notamment des fluctuations de l'ADN VHB d'une part et de la période de rémission transitoire d'une hépatite active d'autre part.

Après un an de suivi, avec une détermination trimestrielle des transaminases et de l'ADN VHB, près de deux tiers des patients initialement identifiés comme des infections chroniques à VHB AgHBe négatif étaient confirmés. Le reste des patients présentaient une hépatite chronique AgHBe négatif, avec une fluctuation de la charge virale et des transaminases nécessitant un traitement antiviral $[13,18,19]$.

Par ailleurs, un certain nombre de patients avec des transaminases normales de façon répétée, présentent des fluctuations de l'ADN VHB entre 2000 et $20000 \mathrm{UI} / \mathrm{ml}$ tout en restant d'authentiques infections chroniques à VHB AgHBe négatif [20].

Plusieurs études, essentiellement européennes, ont évalué l'intérêt du titrage de l'AgHBs pour le diagnostic de l'infection chronique à VHB AgHBe négatif. Dans une première étude [13], chez des patients de génotype D, avec un suivi mensuel des transaminases et de la virémie pendant un an, la combinaison d'un AgHBs $<1000 \mathrm{UI} / \mathrm{ml}$ et d'un ADN-VHB < $2000 \mathrm{UI} / \mathrm{ml}$ avait une performance diagnostique de $94,3 \%$ et une valeur prédictive positive de 87,3\% sur une seule détermination pour identifier les patients ayant une infection chronique à VHB AgHBe négatif. Des résultats comparables ont été observés dans une étude française [21].

De même, chez des patients de génotype $B$ et $C$ dans la cohorte REVEAL, cette combinaison avait une performance diagnostique de $78 \%$ avec une valeur prédictive positive de $83 \%$ [22]. Ces différences peuvent être liées d'une part à des génotypes du VHB différents et d'autre part à des différences dans le suivi et aussi dans la fréquence de la surveillance.

Une grande étude rétrospective dans 8 centres de référence repartis dans le monde portant sur 292 patients AgHBe négatifs avec initialement des transaminases normales et un ADN VHB $<20000 \mathrm{UI} / \mathrm{ml}$, suivis sur une période médiane de 8 ans, a étudié la valeur prédictive du titrage de l'AgHBs [23]. La combinaison d'un AgHBs < $1000 \mathrm{UI} / \mathrm{ml}$ et d'un ADN VHB < $2000 \mathrm{UI} / \mathrm{ml}$ identifiait les infections chroniques à VHB AgHBe négatif pendant toute la période de suivi avec une spécificité de $88 \%$, une valeur prédictive positive de $92 \%$ et une performance diagnostique de $71 \%$. La meilleure combinaison en termes de spécificité et de valeur prédictive positive dans cette étude était l'association d'un AgHBs $<100$ $\mathrm{UI} / \mathrm{ml}$ et d'un ADN VHB $<2000 \mathrm{UI} / \mathrm{ml}$ avec une spécificité de $98 \%$ et une valeur prédictive de $97 \%$.

Par ailleurs, cette étude identifiait d'une part des vrais «porteurs inactifs » avec un AgHBs < 100 UI /ml, un ADN VHB $\leq 2000 \mathrm{UI} / \mathrm{ml}$, des transaminases normales, et des patients en «rémission VHB » avec un AgHBs entre 100 et $1000 \mathrm{UI} / \mathrm{ml}$, un ADN VHB < $2000 \mathrm{UI} / \mathrm{ml}$ et des transaminases normales et d'autre part des porteurs actifs avec un AgHBs > $1000 \mathrm{UI} / \mathrm{ml}$ ou un ADN VHB > $2000 \mathrm{UI} / \mathrm{ml}$. Ainsi, une révision 
des définitions en incluant le niveau de l'AgHBs pourrait faciliter la gestion des patients atteints d'hépatite chronique B AgHBe négatif [23].

Au-delà du titrage de l'AgHBs, plusieurs études récentes ont étudié l'intérêt du titrage de l'antigène du core (AgHBcr) pour identifier les infections chronique à $\mathrm{VHB}$ AgHBe négatif. Le titrage de l'AgHBcr semble plus discriminant que le titrage de l'AgHBs pour identifier les patients ayant une infection chronique AgHBe négatif chez les patients avec un ADN VHB $<2000 \mathrm{UI} / \mathrm{ml}$ quel que soit le génotype VHB alors que le titrage de l'AgHBs était utile uniquement chez les patients de génotype $\mathrm{D}[24]$.

\section{CONCLUSION}

La quantification de l'AgHBs représente un outil de suivi virologique des hépatites chroniques $B$. En association avec la quantification de l'ADN-VHB sérique, la pertinence clinique de la quantification de l'AgHBs repose sur un rôle prédictif démontré récemment dans de nombreuses études. La quantification de l'AgHBs est devenue quasi-systématique mais son utilisation en pratique clinique et l'interprétation de son taux ne sont pas définies. En effet, aucune recommandation n'a été faite quant à son application en pratique courante, excepté son dosage précoce au cours du traitement par interféron, conseillé par l'EASL 2012.

L'objectif de notre étude était d'évaluer l'impact de son utilisation en vie réelle sur une cohorte prospective de patients atteints d'hépatite chronique B et suivis au service d'infectiologie à l'EPH de Batna.

Notre étude vient de confirmer le rôle de la quantification de l'AgHBs pour différencier une infection chronique VHB AgHBe négatif (porteurs inactifs) des patients avec hépatite active AgHBe négatif. Ainsi, l'inclusion du niveau de l'AgHBs pourrait faciliter la gestion des patients atteints d'hépatite chronique B AgHBe négatif.

Déclaration d'intérêts: les auteurs ne déclarent aucun conflit d'intérêt en rapport avec cet article.

\section{RÉFÉRENCES}

1. Guidelines for the Prevention, Care and Treatment of Persons with Chronic Hepatitis B Infection. Geneva: World Health Organization; 2015 Mar. ISBN-13: 978-92-4-154905-9.

2. Tebbal $\mathrm{S}$, Bouguermouh $\mathrm{A}$, Belabbes $\mathrm{H}$ et al. Enquête nationale séroépidémiologique de l'AgHBs en Algérie. (IPA, INSP, OMS 1998); Communication orale. Alger 1998.

3. Blumberg BS, Sutnick AI, London WT. Hepatitis and leukemia: their relation to Australia antigen. Bull N Y Acad Med. 1968;44(December (12)):1566-1586.]]

4. EASL. 2012. J Hepatol Clinical Practice Guidelines: management of chronic hepatitis B;J Hepatol 2012;mar 20.].

5. Werle-Lapostolle B, Bowden S, Locarnini S, et al. Persistence of cccDNA during the natural history of chronic hepatitis $B$ and decline during adefovir dipivoxil therapy. Gastroenterology $2004 ; 126$ : 1750 8.]
6. Deguchi M, Yamashita N, Kagita M, et al. Quantitation of hepatitis B surface antigen by an automated chemiluminescent microparticle immunoassay. J Virol Methods 2004;115:217-22.].

7. European Association for the Study of the Liver. Electronic addresse, European Association for the Study of the L. EASL 2017 Clinical Practice Guidelines on the management of hepatitis B virus infection. J Hepatol 2017;67:370-98.

8. Chen $\mathrm{C}-\mathrm{H}$, Lee $\mathrm{C}-\mathrm{M}$, Wang J-H, Tung H-D, et al. Correlation of quantitative assay of hepatitis B surface antigen and HBVDNA levels in asymptomatic hepatitis B virus carriers. Eur J GastroenterolHepatol. 2004;16(November (11)):1213-1218.

9. Rodella A, Galli C, Terlenghi L, Perandin Fet al N. Quantitative analysis of $\mathrm{HBsAg}$, IgM anti-HBc and anti-HBc avidity inacute and chronic hepatitis B. J ClinVirol Off Publ Pan Am SocClinVirol. 2006;37(November (3)):206212.

10. Chan $\mathrm{HL}$, Wong VW, Tse AM, Tse $\mathrm{CH}$, et al. Serum hepatitis B surface antigen quantitation can reflect hepatitis $\mathrm{B}$ virus in the liver and predict treatment response. Clin Gastroenterol Hepatol 2007;5:1462-8.].

11. Jaroszewicz J, Calle Serrano B, Wursthorn K, et al. Hepatitis B surface antigen [HBsAg] levels in the natural history of hepatitis B virus [HBV]infection : a European perspective. J Hepatol $2010 ; 52$ : 514-22.].

12. Zeng L-Y, Lian J-S, Chen J-Y, Jia $\mathrm{H}-\mathrm{Y}$, et al. Hepatitis B surface antigen levels during natural history of chronic hepatitis $\mathrm{B}$ : a Chinese perspective study. World J Gastroenterol WJG. 21 juill 2014;20(27):9178-84.

13. Brunetto $M R$, Oliveri $F$, Colombatto $P$, et al. Hepatitis $B$ surface antigen serum levels help to distinguish active from inactive hepatitis B virus genotype D carriers. Gastroenterology 2010;139:483-90.

14. Nguyen $\mathrm{T}$, Thompson AJ, Bowden $\mathrm{S}$, et al. Hepatitis B surface antigen levels during the natural history of chronic hepatitis $B$ : a perspectiveon Asia. J Hepatol 2010;52:508-13.

15. Janssen HLA, Sonneveld MJ, Brunetto MR. Quantification of serum hepatitis B surface antigen: is it useful for the management of chronic hepatitis B? Gut. 5 janv 2012;61(5):641-5.

16. Martinot-Peignoux $M$, Lapalus $M$, Asselah $T$, Marcellin $P$. The role of $\mathrm{HBsAg}$ quantification for monitoring natural history and treatment outcome. Liver Int Off J Int Assoc Study Liver. févr 2013;33 Suppl 1:125-32.).

17. Invernizzi $F$, Vigano $M$, Grossi $G$, Lampertico $P$. The prognosis and management of inactive HBV carriers. Liver Int 2016;36 Suppl 1: 100-4.3.

18. Manno M, Camma C, Schepis F, Bassi F, et al. Natural history of chronic HBV carriers in northern Italy: morbidity and mortality after 30 years. Gastroenterology 2004;127:756-63.

19. Martinot-Peignoux $M$, Boyer $N$, Colombat $M$, Akremi $R$, et al. Serum hepatitis B virus DNA levels and liver histology in inactive HBsAg carriers. J Hepatol 2002;36:543-6.

20. Zacharakis GH, Koskinas J, Kotsiou S, Papoutselis M, et al. Natural history of chronic HBV infection: a cohort study with up to 12 years follow-up in North Greece (part of the Interreg I-II/ EC-project). J Med Virol 2005;77:173-9.

21. Martinot-Peignoux M, Lapalus M, Laouenan C, Lada O, et al. Prediction of disease reactivation in asymptomatic hepatitis $\mathrm{B}$ e antigen-negative chronic hepatitis $B$ patients using baseline serum measurements of HBsAg and HBV-DNA .J Clin Virol 2013;58:401-7.

22. Liu J, Yang $\mathrm{HI}$, Lee $\mathrm{MH}$, Jen $\mathrm{CL}$, et al. Serum Levels of Hepatitis B Surface Antigen and DNA Can Predict Inactive Carriers With Low Risk of Disease Progression. Hepatology 2016;64: 381-9.

23. BrouwerWP, Chan HL, BrunettoMR, Martinot- Peignoux M, et al. Repeated Measurements of Hepatitis B Surface Antigen Identify Carriers of Inactive HBV During Long-term Follow-up. Clin Gastroenterol Hepatol 2016;14:1481-9 e1485.].

24. Riveiro-Barciela $M$, Bes $M$, Rodriguez-Frias $F$, Tabernero $D$, et al. Serum hepatitis B core-related antigen is more accurate than hepatitis $B$ surface antigen to identify inactive carriers, regardless of hepatitis $B$ virus genotype. Clin Microbiol Infect 2017. 


\section{BIs}

- $\bigcirc$

-

P

○

Service de Rhumatologie. CHU Beni Messous, Alger - Algérie

\section{Correspondance à :}

Selma ABDELLAOUI

sante76@hotmail.fr

DOI :https://doi.org/10.48087/ BJMSra.2020.7217

\section{Historique de l'article :}

Reçu le 3 Juin 2020

Accepté le 15 Septembre 2020

Publié le 09 novembre 2020

Il s'agit d'un article en libre accès distribué selon les termes de la licence Creative Commons Attribution International License (CC BY 4.0), qui autorise une utilisation, une distribution et une reproduction sans restriction sur tout support ou format, à condition que l'auteur original et la revue soient dûment crédités.

\title{
Pandémie du déficit en vitamine $D$ et effets extra-osseux
}

\author{
Vitamin D deficiency pandemic and extra bone effects
}

\author{
Selma Abdellaoui, Bilal Bengana, Abdenour Boukabous, Salima Lefkir-Tafiani
}

\section{RÉSUMÉ}

Le phénomène de déficit en vitamine $\mathrm{D}$ a pris une grande ampleur aujourd'hui, ce déficit connaît un effet de « pandémie» mondiale, il n'épargne aucune frontière ni catégorie d'âge. Les connaissances de la physiologie de cette vitamine ont progressé de manière considérable, faisant passer sa conception d'une hormone purement osseuse à une hormone ayant un rôle sur la santé globale. En effet, son double rôle d'immunomodulateur et de contrôle de la prolifération cellulaire est possible grâce à son métabolite actif secrété de façon autocrine par certains tissus, et la répartition ubiquitaire de ses récepteurs. En plus de cette description d'effets pléiotropes à la fois dans des modèles cellulaires, expérimentales et cliniques, de plus en plus d'études épidémiologiques ont montré l'importance de la carence en vitamine $\mathrm{D}$ dans la population générale, adulte, comme pédiatrique. Sur le plan diagnostic, la mesure du taux de la 25-hydroxy-vitamine D (25-OHD) est une méthode très fiable pour évaluer les réserves. D'autre part, beaucoup de facteurs de risque ont été mis en évidence et des populations à risque ont été identifiées. En ce qui concerne la supplémentation, il a été récemment suggéré que la stabilité de la concentration de la 25-OHD ne peut être obtenue lors de la supplémentation intermittente que si l'espacement entre les prise est inférieur à 3 mois (de l'ordre de 1 mois) . Quelques travaux récents montrent, que l'utilisation de doses journalières modérées de vitamine D plutôt que de fortes doses administrées de manière intermittente serait à privilégier.

Mots clés : Déficit en vitamine D, Effet extra-osseux, supplémentation en vitamine D.

\section{ABSTRACT}

The phenomenon of vitamin D deficiency has become widespread today, this deficit is experiencing a global "pandemic" effect, it spares no border or age category. Knowledge of the physiology of this vitamin has advanced considerably, shifting its conception from a purely bone hormone to one that has a role in overall health. Indeed, its dual role of immunomodulator and control of cell proliferation is possible thanks to its active metabolite secreted in an autocrine way by certain tissues, and the ubiquitous distribution of its receptors.

In addition to this description of pleiotropic effects in both cellular, experimental and clinical models, more and more epidemiological studies have shown the importance of vitamin D deficiency in the general adult population as well as in pediatrics. From a diagnostic standpoint, measuring the level of 25-hydroxy-vitamin D (25-OHD) is a very reliable method for evaluating reserves. On the other hand, many risk factors have been identified and at risk populations have been identified. With regard to supplementation, it has recently been suggested that the stability of the concentration of 25OHD can only be obtained during intermittent supplementation if the spacing between doses is less than 3 months (of the order 1 month). Some recent studies show that the use of moderate daily doses of vitamin D rather than large doses administered intermittently should be preferred.

Keywords: Vitamin D deficiency, , extra bone effect of vitamin D, vitamin D supplementation.

\section{Pour citer l'article :}

Abdellaoui S. Pandémie du déficit en vitamine $D$ et effets extra-osseux. Batna J Med Sci 2020;7(2):142-7. https://doi.org/10.48087/B JMSra.2020.7217

\section{INTRODUCTION}

Longtemps considérée comme une hormone essentiellement utile pour réguler le métabolisme phosphocalcique et la minéralisation osseuse, la vitamine D suscite depuis vingt ans, un intérêt grandissant. Dans un contexte de "pandémie» de déficit en vitamine $\mathrm{D}$ dans la population générale [1],

La progression des connaissances fondamentales et cliniques sur l'influence pluritissulaire de ce stéroïde est vertigineuse et tous les grands secteurs de la médecine sont concernés : le tissu osseux et le risque de fracture, le muscle et le risque de chute, le système nerveux central (SNC) et les fonctions cognitives. Le psychisme, l'immunité et l'autoimmunité, le risque de cancer mais aussi celui d'infection et d'accident cardiovasculaire [2].

Sur la plan diagnostic biologique, la plupart des experts internationaux s'accordent pour définir des seuils minimaux de concentration sérique limite de $25-\mathrm{OHD}$ à $20 \mathrm{ng} / \mathrm{mL}(50 \mathrm{nmol} / \mathrm{L})$ pour définir le déficit en vitamine $\mathrm{D}$ et une limite de $30 \mathrm{ng} / \mathrm{mL}(75 \mathrm{nmol} / \mathrm{L})$ pour définir l'insuffisance en vitamines D chez l'adulte [3]. Chez l'enfant, les consensus sont moins clairs, mais on considère qu'une concentration sérique minimale de $20 \mathrm{ng} / \mathrm{mL}$ est nécessaire [4].

\section{Vitamine D et physiologie osseuse}

La biosynthèse de cette hormone liposoluble commence au niveau cutané sous l'effet du rayonnement ultraviolet UVB (290 à 315nm) [5], 
continue au niveau hépatique par l'hydroxylation en 25 et se termine au niveau rénal par l'hydroxylation en 1 . La vitamine D existe sous deux formes principales : la forme de stokage (25-OHD ou calcidiol) et la forme active (1-25-OH2D ou calcitriol). La 1-25-OH2D est une hormone stéroïde, elle agit au niveau cellulaire avec une liaison initiale cytoplasmique au récepteur de la vitamine $\mathrm{D}$ (VDR) qui appartient à la superfamille des récepteurs nucléaires, et qui va ensuite se transloquer dans la cellule et se fixer au VDRE (vitamin D responsive element) et ainsi déclencher l'expression ou la répression des gènes cibles contrôlés par la vitamine D [6]. De ce fait, la découverte de dérivés de la vitamine $D$, dont le $1,250 H D$ qui constitue sa forme active agissant sur l'homéostasie phosphocalcique et le métabolisme osseux et, surtout, la découverte de VDR, a donné à la vitamine D un statut de pro-hormone. Le rôle de la vitamine D dans la régulation de l'homéostasie phosphocalcique est bien connu.

une minéralisation osseuse optimale est assurée par le maintien d'un état de normocalcémie suite à l'absorption intestinal du calcium et du phosphore. Au niveau intestinal, la vitamine D stimule l'absorption de calcium et phosphore, permettant ainsi de maintenir un état de normocalcémie nécessaire pour une minéralisation osseuse adéquate. Au niveau rénal, elle stimule la réabsorption tubulaire du calcium et inhibe la synthèse de la parathormone (PTH), hormone hypercalcémiante et phosphaturiante. À l'inverse, la PTH stimule l'expression de $1 \alpha$-hydroxylase, pour stimuler la conversion de 25-OH en 1-25-OH2D. De son côté le FGF 23 (fibroblast growth factor 23), une hormone phosphaturiante, qui inhibe la synthèse de $1 \alpha$-hydroxylase (induisant une diminution de la 1-25-OH2D) avec un rétrocontrôle positif de la 1-25-OH2D sur la synthèse du FGF23 par l'ostéocyte [7].

$\mathrm{Au}$ niveau osseux, la 1-25-OH2D n'a pas d'effet direct sur la minéralisation, mais elle agit via le maintien d'une calcémie et phosphorémie efficaces [8]. Elle a une action sur les ostéoclastes en stimulant le précurseurs myélomonocytaires en lignée ostéoclastique, et une action sur les ostéoblastes en stimulant la sécrétion de protéines sériques telles que l'ostéocalcine, l'ostéoprotégérine et le RANK-ligand (receptor activator of nuclear factor kappa-B ligand). En effet, l'état du squelette à l'âge adulte est déterminé par la qualité de la croissance et de la minéralisation osseuse pendant l'enfance : C'est au cours des 20 premières années de vie que se constitue $90 \%$ de la masse osseuse définitive. De plus, 25\% du capital osseux final est acquis pendant les 2 ans de l'adolescence, c'est dire l'importance de l'optimisation des facteurs de l'environnement (nutrition, activité physique, facteurs hormonaux) durant cette phase critique d'acquisition de la masse osseuse. Au niveau ostéoblastique, elle stimule la sécrétion de nombreuses protéines spécifiques (par exemple l'ostéocalcine, l'ostéoprotégérine et RANKligand [receptor activator of nuclear factor kappa-B ligand]), alors qu'au niveau ostéoclastique elle stimule la différenciation des précurseurs myélomonocytaires en lignées ostéoclastiques. La qualité de la croissance et de la minéralisation osseuses pendant l'enfance joue un rôle important sur l'état squelettique à l'âge adulte ; en effet, $90 \%$ de la masse osseuse définitive se constitue au cours des 20 premières années de vie. De nombreux facteurs, notamment génétiques et environnementaux (par exemple la nutrition, l'activité physique et les facteurs hormonaux), sont impliqués dans la constitution du capital osseux, mais une des phases critiques de l'acquisition de la masse osseuse est représentée par l'adolescence, $25 \%$ du capital osseux final étant en effet acquis sur deux ans, c'est dire l'importance de l'optimisation des facteurs environnementaux lors de cette période [9]. Par ailleurs, durant la période périnatale divers facteurs joueraient un rôle capital comme les facteurs maternels (tabagisme, déficit en vitamine D) ou foetaux (retard de croissance) Ainsi, la minéralisation osseuse semblerait être «programmée » pendant cette période.

\section{Vitamine D et pédiatrie}

De nombreux travaux ont rapporté le déficit en vitamine D dans la population pédiatrique.

Une étude lyonnaise monocentrique prospective en néphrologie pédiatrique avait montré qu'une grande majorité d'enfants étaient carencés en vitamine $D$, indépendamment de la fonction rénale [10]. Une étude québécoise a montré que plus de $90 \%$ des enfants et des adolescents sains avaient des concentrations circulantes en vitamine D suboptimales et les déficits sévères en vitamine $\mathrm{D}$ étaient plus fréquents chez les adolescents les plus âgés [11]. Enfin, une étude européenne, dans neuf pays différents, a retrouvé des concentrations suboptimales chez $81 \%$ des adolescents avec une augmentation de la vitamine $\mathrm{D}$ avec l'âge et une diminution de cette hormone avec l'augmentation de l'indice de masse corporelle [12].

\section{Effets extra-osseux de la vitamine D}

La vitamine $D$, qui n'est pas stricto sensu une vitamine, puisque sa synthèse est possible à partir du déhydrocholestérol $[13,14]$. De plus, son rôle extra-osseux, indépendant du métabolisme calcique serait suggéré par l'action de la vitamine $D$ sur 500 gènes ainsi que par l'expression de son récepteur spécifique dans de nombreux cas [15].

\section{Chez l'enfant}

Lors de la grossesse, le rôle de la vitamine D a été mis en évidence, avec des études cliniques montrant une association entre déficit en $25-\mathrm{OH}$ vitamine $\mathrm{D}$ chez la femme enceinte et augmentation de l'incidence des complications maternelles (prééclampsie sévère, cholestase gravidique, diabète gestationnel par exemple), fœetales (petit poids de naissance, prématurité) et néonatales (infections pulmonaires au cours des premiers mois de vie notamment) [16]. De plus, des nourrissons par ailleurs en bonne santé ont plus de risques d'avoir une bronchiolite à VRS (virus respiratoire syncytial) au cours de la première année de vie s'ils étaient déficitaires en 25-OHD au cordon [17].

Les nouveau-nés prématurés de faible poids de naissance sont des sujets particulièrement à risque de carence en vitamine $\mathrm{D}$ et de rachitisme du prématuré. Il existe une relation directe entre la concentration de $25-\mathrm{OH}$ vitamine D au cordon et le statut vitaminique maternel [18]. Il a été suggéré dans l'étude nord-américaine Nurses' Health Study II qu'une supplémentation en vitamine D pendant l'adolescence pourrait limiter le risque de développer des tumeurs bénignes du sein à l'âge adulte [19] ; en Ontario, le temps passé à l'extérieur pendant l'adolescence était significativement associé à une diminution du risque de cancer du sein à l'âge adulte dans une étude cas témoins de 6571 femmes, sans pour autant que le lien entre exposition solaire et vitamine D ne puisse être formellement démontré [20]. En ce qui concerne la dysimmunité, Banwell et al. ont montré, d'une manière semblable à ce qui est observé chez les adultes, qu'un déficit en vitamine D est un facteur de risque de survenue de sclérose en plaques dans la population pédiatrique canadienne [21]. Une analyse secondaire de la Third National Health and Nutrition Examination Survey a montré une association inverse entre concentrations circulantes de $25-0 \mathrm{H}$ vitamine $\mathrm{D}$ et survenue récente d'une infection des voies aériennes supérieures [22].

Majak et al. ont montré chez l'enfant et l'adolescent asthmatiques qu'une supplémentation en vitamine $\mathrm{D}$ native peut permettre de diminuer la fréquence des exacerbations 
d'asthme [23]. Une étude de 3182 adolescents issus de la cohorte britannique ALSPAC que la vitamine D pourrait être un facteur protecteur contre le développement des psychoses [24], sachant que d'autres études ont aussi établi un lien entre concentrations basses en $25-\mathrm{OH}$ vitamine $\mathrm{D}$ et dépression [25]. Chez l'adulte, un déficit en vitamine D est un facteur de risque de survenue de diabète de type 1 [26] et du fait que la vitamine $\mathrm{D}$ participe à la régulation du système immunitaire, il a été postulé qu'elle pouvait être utilisée dans la prévention et le traitement du diabète de type1 (mais les essais de supplémentation se sont avérés inefficaces), de cancer [27], de maladies inflammatoires ou dysimmunitaires (sclérose en plaques [28], psoriasis [29], polyarthrite rhumatoïde [30], lupus érythémateux [31]) et d'infection (tuberculose, épisodes oto-rhino-laryngologiques hivernaux) [32].

\section{Chez l'adulte :}

Peu importe le domaine considéré, il est clair que la concentration sérique de vitamine $\mathrm{D}$ constitue un puissant biomarqueur de l'état de santé d'un patient de plus de 50ans. Les concentrations basses de la 25-OHD sont associées avec un excès de mortalité et de nombreuses pathologies en particulier le syndrome métabolique, l'obésité, les cancers, le risque d'infection, le risque de maladie auto-immune, le risque de chute et de fracture et peut être une part du déclin cognitif des sujets âgés. Certes dépendante de l'ethnie et de l'âge, cette concentration sérique en vitamine D est aussi le reflet de l'Indice de masse corporelle (IMC), de l'activité physique et du mode de vie en général. Il est donc possible que la haute prévalence de l'insuffisance en vitamine D, observée dans tous les pays, soit en partie expliquée par un mode de vie, urbain, suralimenté et sédentaire, mode de vie qui en lui-même favorise la survenue de ces pathologies. Les infections grippales [33], les autres infections virales des voies aériennes supérieures ou les infections bactériennes (notamment mycobacterium tuberculosis) pourraient être favorisée par une insuffisance en vitamine D [34-36]. Une étude finlandaise a montré que la supplémentation en vitamine $\mathrm{D}$ au cours de la première année de vie était associée à une diminution du risque de $78 \%$ de développement du diabète de type1 [37].

Dans le modèle murin de lupus érythémateux systémique, l'administration de 1-25-0H2D prévient le développement des lésions cutanées et réduit la protéinurie [38]. Les données humaines sont plus contradictoires dans cette maladie notamment en raison de multiples facteurs confondants (glucocorticoïdes, tabagisme, IMC, photosensibilité, écran solaire). Dans une autre étude de patients atteints de polyarthrite rhumatoïde (PR), les patients avec les critères de PR avaient un taux de 25-OHD et de1-25OH2D plus bas que les témoins. Ce taux était corrélé à la sévérité de la PR (DAS28, CRP, HAQ) et est prédictif du HAQ (Health assessment questionnaire) à un an et une augmentation de $10 \mathrm{ng} / \mathrm{mL}$ était associée à une diminution du score DAS28 de 0,30 et de $25 \%$ du taux de la CRP (C-reactive protein) [39]. Le risque de cancer colorectal était diminué de $50 \%$ chez les patients avec une concentration sérique de 25 OHD supérieure à $82,5 \mathrm{nmol} / \mathrm{L}$ par rapport aux patients avec une concentration inférieure à $30 \mathrm{nmol} / \mathrm{L}$ [40]. Une autre étude a montré que le risque de cancer du sein serait réduit de $50 \%$ chez les patientes dont la concentration de 25-OHD était supérieure à $130 \mathrm{nmol} / \mathrm{L}$ [41] par contre le risque de cancer de prostate n'était pas influencé par le taux de vitamine D [42]. Au cours de l'étude WHI [43], l'apport quotidien de $500 \mathrm{UI}$ de vitamine $\mathrm{D}$ a été réalisée pendant sept ans, un effet sur la taille du cancer du sein a été observé mais sans impact sur l'incidence. Lappe et al. [44] ont publié des résultats très favorables à la vitamine D (réduction de $60 \%$ du risque de cancer à quatre ans), avec des doses plus adaptées (1000UI/j). Goldner et al., ont rapporté que chez des sujets obèses, la fréquence des carences est de $61 \%$ et celle des insuffisances de $90 \%$ contre respectivement 12 et $32 \%$ chez des témoins appariés pour l'âge, le sexe, l'origine ethnique et l'exposition solaire [45] et en définissant l'hyperparathyoïdie par un taux de PTH supérieure à70ng/L, $49 \%$ des sujets obèses étaient en hyperparathyroïdie, contre $2 \%$ pour les sujets témoins. De plus, après by-pass gastrique (BPG) l'absorption orale de vitamine D serait diminuée de $42 \%$ [46]. La perte de poids serait en cause au même titre que la carence en vitamine D [47]. De nombreuses études ont également retrouvé un lien entre la carence en vitamine D et le risque de survenue d'un diabète et d'un accident cardiovasculaire [48]. Il est donc possible que les concentrations basses de vitamine $\mathrm{D}$, soit témoin d'un mode de vie très sédentaire. Chez les séniors, la prévalence d'hypovitaminose D a été estimée entre 40 et $50 \%$ chez les sujets âgés de plus de 65 ans non-chuteurs, mais atteint plus de $70 \%$ chez les chuteurs $[49,50]$. Il a également été observé, dans une population de sujets âgés institutionnalisés, que les chuteurs avaient des concentrations sériques de vitamine D significativement plus basses que les non-chuteurs [51]. Une observation a fait suggérer que l'effet positif de la vitamine D sur la puissance musculaire pourrait ne pas être lié à la force musculaire, mais à la vitesse de contraction musculaire [52]. Enfin, on connaît l'implication du stress oxydatif et des phénomènes inflammatoires, dégénératifs et vasculaire au cours de l'histoire naturelle des pathologies démentielles de la personne âgée [53], des études sont en faveur d'un lien entre vitamine D et cognition chez la personne âgée [54].

\section{Supplémentation en vitamine D}

En regard des effets de la vitamine $\mathrm{D}$, des conséquences de l'insuffisance ou de la carence, des mesures préventives, sans qu'il y ait de consensus quant aux modalités de cette prévention ont été prises aussi bien chez l'enfant que chez l'adulte [55-58].

Un taux sérique de 250HD compris entre 20 et $60 \mathrm{ng} / \mathrm{mL}$ chez la population générale et entre 30 et $60 \mathrm{ng} / \mathrm{mL}$ au cours d'une ostéoporose, d'une insuffisance rénale chronique ou d'une malabsoption constitue l'objectif principal d'une supplémentation optimale.

\section{Chez l'enfant}

Le Comité de nutrition de la société française de pédiatrie a publié en 2012 des recommandations de supplémentation chez l'enfant et l'adolescent en fonction de l'âge, de la présence ou non de certains facteurs de risque bien identifiés (Tableau 1) [59]. La prescription de vitamine D reste une prescription médicamenteuse, et il existe des situations où la vitamine $\mathrm{D}$ est contre-indiquée, notamment dans certaines pathologies génétiques empêchant la dégradation de la vitamine $\mathrm{D}$ active (mutation de la 24 -hydroxylase). Il convient donc de vérifier avant toute prescription de vitamine $\mathrm{D}$ l'absence de contre-indications, et notamment un antécédent d'hypercalcémie, de lithiase ou de néphrocalcinose, ce qui nécessiterait un bilan complémentaire.

\section{Chez l'adulte :}

Des travaux récents suggèrent que l'utilisation de doses journalières modérées de vitamine $\mathrm{D}$ plutôt que de fortes doses administrées de manière intermittente serait à privilégier chez les sujets chuteurs [60-62].

La prescription d'une prise quotidienne de vitamine $D$ est 
par ailleurs plus difficile, sans forme galénique bien adaptée du moins en Algérie où la seule forme galénique disponible est la 200000UI. Par ailleurs, l'administration journalière ou intermittente de vitamine $\mathrm{D}$ semble, à doses équivalentes, induire la même réduction de la sécrétion de PTH, ce qui est important pour l'efficacité osseuse de la vitamine D. De ce fait, le Groupe de Recherche et d'Information sur l'Ostéoporose GRIO [63] recommande de garder une dose intermittente tout en choisissant les posologies les moins élevées parmi celles disponibles et les intervalles les plus courts possibles. Ainsi, si l'on vise une concentration $>30$ $\mathrm{ng} / \mathrm{mL}$, on pourra proposer 50000 UI par semaine pendant 8 semaines chez ceux dont la concentration initiale de 25 OHD est $<20 \mathrm{ng} / \mathrm{mL}$, ou 50000 UI par semaine pendant 4 semaines chez ceux dont la concentration de 25-0HD est entre 20 et $30 \mathrm{ng} / \mathrm{mL}$. Ce traitement d'attaque sera suivi par un traitement d'entretien de $50000 \mathrm{UI} /$ mois, la 25-OHD sera dosée à 6 mois afin d'ajuster la posologie (en sachant que l'élévation de la concentration n'est pas identique entre les individus) [64]. En Algérie, afin de tenter de rester en adéquation avec les recommandations du GRIO, la dose de 200000UI/mois pendant 3 mois puis relais 200000UI à 3 mois paraît raisonnable. Dans l'éventualité d'une disposition future de formes pharmaceutiques de vitamine $\mathrm{D}$ adaptées à une administration journalière (1000 à 1500 $\mathrm{UI} / \mathrm{j}$ ), il serait possible, chez les patients observants, de prescrire 3000 à $5000 \mathrm{UI} / \mathrm{j}$ pendant 3 mois chez ceux ayant une concentration $<20 \mathrm{ng} / \mathrm{mL}$ suivies par un traitement au long cours par 1000 à $3000 \mathrm{UI} / \mathrm{j}$.

Tableau 1. Recommandations 2012 de supplémentation en vitamine D chez l'enfant et l'adolescent selon le Comité de la nutrition de la Société française de pédiatrie [59].

\begin{tabular}{lll}
\hline & $\begin{array}{l}\text { Pas de facteurs de } \\
\text { risque }\end{array}$ & $\begin{array}{l}\text { Avec facteurs de } \\
\text { risque }\end{array}$ \\
\hline Femme enceinte & $\begin{array}{l}\text { Dose de charge } \\
\text { unique de } 80000 \text { à }\end{array}$ \\
& $\begin{array}{l}100000 \text { UI au début } \\
\text { du 7ème mois de } \\
\text { grossesse }\end{array}$
\end{tabular}

\begin{tabular}{|c|c|}
\hline Nourrisson allaité & 1000 à $1200 \mathrm{UI} / \mathrm{J}$ \\
\hline $\begin{array}{l}\text { Enfant }<18 \text { mois } \\
\text { recevant un lait } \\
\text { enrichi en } \\
\text { vitamine D }\end{array}$ & 600à $800 \mathrm{UI} / \mathrm{j}$ \\
\hline
\end{tabular}

\begin{tabular}{|c|c|c|}
\hline $\begin{array}{l}\text { Enfant }<18 \text { mois } \\
\text { recevant un lait } \\
\text { non enrichi en } \\
\text { vitamine D }\end{array}$ & 1000 à $1200 \mathrm{UI} / \mathrm{j}$ & \\
\hline $\begin{array}{l}\text { Enfant de } 18 \text { mois } \\
\text { à } 5 \text { ans }\end{array}$ & $\begin{array}{l}2 \text { doses de charges } \\
\text { de } 80000 \text { à } 100000 \\
\text { UI en hiver } \\
\text { (novembre et } \\
\text { février) }\end{array}$ & $\begin{array}{l}\text { Dose de charge } \\
\text { trimestrielle }\end{array}$ \\
\hline $\begin{array}{l}\text { Enfant de } 5 \text { à } \\
\text { 10ans }\end{array}$ & $\begin{array}{l}\text { Pas de } \\
\text { supplémentation }\end{array}$ & $\begin{array}{l}2 \text { doses de } \\
\text { charges de } 80000 \\
\text { à } 100000 \text { UI en } \\
\text { hiver (novembre } \\
\text { et février) }\end{array}$ \\
\hline $\begin{array}{l}\text { Adolescent de } 10 \text { à } \\
15 \text { ans }\end{array}$ & $\begin{array}{l}2 \text { doses de charges } \\
\text { de } 80000 \text { à } 100000 \\
\text { UI en hiver } \\
\text { (novembre et } \\
\text { février) }\end{array}$ & $\begin{array}{l}\text { Dose de charge } \\
\text { trimestrielle }\end{array}$ \\
\hline
\end{tabular}

\section{CONCLUSION}

Du fait des changements d'habitudes de vie (obésité, vie à l'intérieur, photoprotection), le déficit en vitamine D native est de plus en plus fréquent dans la population générale alors que la vitamine $\mathrm{D}$ a des effets bénéfiques squelettiques et phosphocalciques (stimulation de l'absorption intestinale de calcium et de phosphore notamment) et extra osseux (effets anti-infectieux, anti-inflammatoires, antihypertenseurs, etc.). Afin d'optimiser le pic de masse osseuse, il faudrait appliquer les recommandations de supplémentation en vitamine $\mathrm{D}$ des enfants de 0 à 18 ans, et savoir identifier les situations à risque de carence en vitamine D et/ou en calcium, tout en promouvant une activité physique adéquate. À ce titre, les apports calciques chez l'enfant nécessitent d'être régulièrement stimulés car une proportion non négligeable d'enfants et d'adolescents n'est pas dans les cibles d'apports calciques recommandés. Puis ces recommandations seront poursuivies à l'âge adulte. Ainsi, étant donné que la forme galénique journalière n'est pas disponible, l'administration intermittente est à encourager selon les recommandations du GRIO de 2011. Autrement dit, l'idéal serait de préconiser la dose la plus faible parmi les posologies disponibles, séparée par des intervalles d'administration courts. De nouveaux essais thérapeutiques randomisés de grande ampleur et prenant en compte la concentration initiale de vitamine $\mathrm{D}$, les cofacteurs classiques sont indispensables afin de mieux évaluer l'impact extraosseux d'un traitement par la vitamine D impliqué dans diverses pathologies.

Déclaration d'intérêts: les auteurs ne déclarent aucun conflit d'intérêt en rapport avec cet article.

\section{RÉFÉRENCES}

1. Sempos CT, Durazo- Arvizu RA, Dawson-Hughes B, Yetley EA, Looker AC Schleicher RL. Is there a reverse J-shaped association between 25hydroxyvitamin D and all-cause mortality? Results from th U.S. nationally representative NHANES. J Clin Endocrinol Metab 2013;98:3001-9.

2. Adams JS, Hewison M. Update in vitamin D. J Clin Endocrinol Metab 2010;95:471-8.

3. Priemel M, von Domarus C, Klatte TO, Kessler S, Schlie J, Meier. Bone mineralization defects and vitamin $\mathrm{D}$ deficiency histomorphometric analysis of iliac crest bone biopsies and circulating 25-hydrovitamin D in 675 patients. J Bone Miner Res 2010; 25:305-12.

4. Misra M, Pacaud D, Petryk A, Collett- Solberg PF, Kappy M. Drug and Therapeutics Committee of the Lawson wilkins Petritric Endocrine Society Vitamin $D$ deficiency in children and its management: review of current knowledge and recommendations. Pediatrics 2008 ; 122:398417.

5. Garabédian M. La vitamine D. Traité des maladies métaboliques osseuses de I'adulte. Paris: Médecine Sciences Flammarion;2008.p.89105.

6. Bacchetta J, Ranchin B, Dubourg L, Cochat P. Vitamine D : un acteur majeur en santé? Arch Pediatr 2010;17:1687-95.

7. Bacchetta J, Cocha P, Salusky I B. FGF 23 et Klotho: Les nouveaux incontournables du métabolisme phosphocacique. Arch Pediatr 2011; 18:686-95.

8. Lieben L, Masuyama R, Torrekens S, Van Looveren R, Schrooten J, Baatsen P. Normocalcemia is maintained in mice under conditions of calcium malabsorption by vitamin D-induced inhibition of bone mineralization. J Clin Invest 2012;122:1803-15.

9. Bachetta. J, Vitamine $D$ en pédiatrie. Journal de pédiatrie et de puériculture 2019 ; 6 : 310-2. Doi : 10.1016/j.jpp.2019.09.004

10. Bacchetta J, Dubourg L, Harambat J, Ranchin B, Abou-Jaoude P, Arnaud $S$. The influence of glomerular filtration rate and age on fibroblast growth factor 23 serum levels in pediatric chronic kidney disease. J Clin Endocrinol Metab 2010;95:1741-8.

11. Mark S. Vitamin D status and recommendations to improve vitamin D status in Canadian youth. Appl Physiol Nutr Metab 2010;35:718. 
12. González-Gross M, Valtuena J, Breidenassel C, Moreno LA, Ferrari M, Kersting M. Vitamin D status among adolescents in Europe: the Healthy Lifestyle in Europe by Nutrition in Adolescence study. $\mathrm{Br} J$ Nutr 2012;107:755-64.

13. Belderbos ME, Houben ML, Wilbrink B, Lentjes E, Bloemen EM, Kimpen JLL. Cord blood vitamin D deficiency is associated with respiratory syncytial virus bronchiolitis. Pediatrics 2011;127:e1513-20.

14. Briot K, Audran M, Cortet B, et al. Vitamin D: skeletal and extra skeletal effects; recommendations for good practice. Presse Med 2009;38(1):4354.

15. Schlinger J.L, Monnier. L. The history of vitaminD, a hundred-year-old hormone looming less large than initially and transiently expected. Med of metab dis 2019;13(4): 375-83.

16. Holick MF. Vitamin D deficiency. N Engl J Med 2007;357(3):266-81.

17. Dror DK. Vitamin D status during pregnancy: maternal, fetal, and postnatal outcomes. Curr Opin Obstet Gynecol 2011;23:422-6.

18. Burris HH, Van Marter $L$, McElrath TF, Tabatabai P, Litonjua AA, Weiss ST. Vitamin D status among preterm and full-term infants at birth. Pediatr Res 2014;75:75-80.

19. Su X, Colditz GA, Collins LC, Baer HJ, Sampson LA, Willett WC. Adolescent intakes of vitamin $D$ and calcium and incidence of proliferative benign breast disease. Breast Cancer Res Treat 2012;134:78391.

20. Anderson LN, Cotterchio M, Kirsh VA, Knight JA. Ultraviolet sun-light exposure during adolescence and adulthood and breast cancer risk: a population-based case-control study among Ontario women. Am J Epidemiol 2011;174:293-304.

21. Banwell B, Bar-Or A, Arnold DL, Sadovnick D, Narayanan S, McGowan M Clinical, environmental, and genetic determinants of multiple sclerosis in children with acute demyelination: a prospective national cohort study. Lancet Neurol 2011;10:436-45.

22. Ginde AA, Mansbach JM, Camargo CA. Association between serum 25hydroxyvitamin $D$ level and upper respiratory tract infection in the Third National Health and Nutrition Examination Survey. Arch Intern Med 2009;169:384-90.

23. Majak P, Olszowiec-Chlebna M, Smejda K, Stelmach I. Vitamin D supplementation in children may prevent asthma exacerbation triggered by acute respiratory infection. J Allergy Clin Immunol 2011;127:1294-6.

24. Tolppanen A-M, Sayers A, Fraser WD, Lewis G, Zammit S, McGrath J. Serum 25-hydroxyvitamin D3 and D2 and non-clinical psychotic experiences in childhood. PloS One 2012;7:e41575.

25. Kjærgaard M, Waterloo K, Wang CEA, Almås B, Figenschau Y, Hutchinson MS. Effect of vitamin D supplement on depression scores in people with low levels of serum 25-hydroxyvitamin D: nested case-control study and randomised clinical trial. Br J Psychiatry 2012;201:360-8.

26. Hyppönen $E$, Läärä $E$, Reunanen $A$, Järvelin $M R$, Virtanen $S M$. Intake of vitamin D and risk of type 1 diabetes: a birth-cohort study. Lancet 2001;358:1500-3.

27. Peterlik M, Grant WB, Cross HS. Calcium, vitamin D and cancer. Anticancer Res 2009;29:3687-98

28. Hayes CE. Vitamin D: a natural inhibitor of multiple sclerosis. Proc Nutr Soc 2000;59:531-5.

29. Nancy A-L, Yehuda S. Prediction and prevention of autoimmune skin disorders. Arch Dermatol Res 2009;301:57-64.

30. Merlino LA, Curtis J, Mikuls TR, Cerhan JR, Criswell LA, SaagKG. Vitamin $D$ intake is inversely associated with rheumatoid arthritis: results from the lowa Women's Health Study. Arthritis Rheum 2004;50:72-7.

31. Kamen D, Aranow C. Vitamin D in systemic lupus erythematous. Curr Opin Rheumatol 2008;20:532-7.

32. Liu PT, Stenger S, Li H, Wenzel L, Tan BH, Krutzik SR. Toll-like receptor triggering of a vitamin D-mediated human antimicrobial response. Science 2006;311:1770-3.
33. Goldstein MR, Mascitelli L,Pezzetta F. Pandemic influenza $\mathrm{A}(\mathrm{H} 1 \mathrm{~N} 1)$ :mandatory vitamin D supplementation? Med Hypotheses 2010;74:756.

34. Yamshchikov AV, Desai NS, Blumberg HM et al.Vitamin D for treatment and prevention of infectious diseases: a systematic review of randomized controlled trials. Endocr Pract 2009;15:438-49.

35. Wejse C, GomesVF, Rabna P et al. Vitamin D a supplementary treatment for tuberculosis: a double-blind, randomized, placebo-controlled trial. Am J Respir Crit Care Med 2009; 179:843-50.

36. Bartley J. Vitamin D, innate immunity and upper respiratory tract infection. J Laryngol Otol 2010;124:465-9.

37. Hypponen E, Laara E, Reunanen A, et al. Intake of vitamin D and risk of type 1 diabetes: a birth-cohort study. Lancet 2001;358:1500-3.

38. Lemire JM, Ince A, Takashima M.1,25-Dihydroxyvitamin D3 attenuates the expression of experimental murine lupus of MRL/Imice. Autoimmunity 1992;12:143-8.

39. Patel S, Farragher T, Berry J, et al. Association between serum vitamin D metabolite levels and disease activity in patients with early inflammatory polyarthritis. Arthritis Rheum 2007; 56:2143-9.

40. Gorham ED, Garland CF, Garland FC et al. Optimal vitamin D status for colorectal cancer prevention: a quantitative meta-analysis. Am J Prev Med 2007:32:210-6.

41. Garland CF, Gorham ED, Mohr SB et al. Vitamin D and prevention of breast cancer: pooled analysis. J Steroid Biochem Mol Biol2007;103:70811.

42. IARC.Vitamin D and Cancer. IARC Working Group Reports. Lyon, France: International Agency for Research on Cancer;2008.

43. Chlebowski RT, Johnson KC, Kooperberg C, et al. Calcium plus vitamin D supplementation and the risk of breast cancer. J Natl Cancer Inst 2008:100:1581-91.

44. Lappe JM, Travers-Gustafson D, Davies KM, et al. Vitamin D and calcium supplementation reduces cancer risk: results of a randomized trial. Am J Clin Nutr 2007;85:1586-91.

45. Goldner WS, Stoner JA,Thompson J, et al. Prevalence of vitamin D insufficiency and deficiency in morbidly obese patients: a comparison with non-obese controls. Obes Surg 2008;18:145-50.

46. Flores L, Osaba MJ, Andreu A et al. Calcium and vitamin D supplementation after gastric by pass should be individualized to improve or avoid hyperparathyroidism. Obes Surg 2010; 20:738-43.

47. Balsa JA, Botella-Carretero Jl, Peromingo R et al. Chronic increase of bone turnover markers after biliopancreatic diversion is related to secondary hyperparathyroidism and weight loss. Relation with bone mineral density. Obes Surg 2010;20:468-73.

48. Parker J, Hashmi O, Dutton D, et al. Levels of vitamin D and cardiometabolic disorders: systematic review and meta-analysis. Maturitas 2010;65:225-36.

49. Gloth MF, Tobin JD. Vitamin D deficiency in older people. J Am Geriatr Soc $1995 ; 43: 822-8$.

50. Thomas MK, Lloyd-Jones MD, Thadhadi RI, et al. Hypovitaminosis D in medical inpatients. N Engl J Med 1998;338:777-83.

51. Stein MS, Wark JD, Scherer SC et al. Falls relate to vitamin D and parathyroid hormone in an Australian nursing home and hostel. J Am Geriatr Soc 1999;47:1195-201.

52. Annweiler $\mathrm{C}$, Bridenbaugh $\mathrm{S}$, Schott $\mathrm{AM}$ et al. Vitamin $\mathrm{D}$ and muscle function: new prospects? Biofactors 2009;35:3-4.

53. Annweiler C, Schott AM, Berrut G, et al. Vitamin D and ageing: neurological issues. Neuropsychobiology 2010 ; 62(3) 139-50.

54. Annweiler C, Schott AM, Berrut G, et al. Vitamine D et cognition, du soleil pour le crépuscule de l'esprit. Ann Gerontol 2009;2:239-42. 
55. Holick MF. Vitamin D deficiency. N Engl J Med 2007;357(3):266-81.

56. Bischoff-Ferrari HA, Giovannucci E, Willett WC, et al. Estimation of optimal serum concentrations of 25-hydroxy-vitamin D for multiple health outcomes. Am J Clin Nutr 2006;84(1):18-28.

57. Tang BM, Eslick GD, Nowson C et al. Use of calcium or calcium in combination with vitamin $\mathrm{D}$ supplementation to prevent fractures and bone loss in people aged 50 years and older: a meta-analysis. Lancet 2007;370(9588):657-66.

58. Cashman KD, Hill TR, Lucey AJ et al. Estimation of the dietary requirement for vitamin $D$ in healthy adults. Am J Clin Nutr 2008;88(6):1535-42.

59. Vidailhet M, Mallet E, Bocquet A, Bresson J-L, Briend A, Chouraqui J-P. Vitamin D still a topical matter in children and adolescents. A position paper by the Committee on Nutrition of the French Society of Pediatrics. Arch Petriatr $2012 ; 19: 316-28$.

60. Sanders KM, Stuart AL, Williamson EJ et al. Annual high-dose oral vitamin $D$ and falls and fractures in older women: a randomized controlled trial. J.A.M.A 2010;303:1815-22.
61. Smith H, Anderson F, Raphael $P$, et al. Effect of annual intramuscular vitamin $D$ on fracture risk in elderly men and women-A populationbased, randomized, double-blind, placebo-controlled trial. Rheumatology 2007;46:1852-7.

62. Välimäki VV, Löyttyniemi E, Pekkarinen T et al. How well are the optimal serum $250 H D$ concentrations reached in high-dose intermittent vitamin D therapy? A placebo-controlled study on comparison between 100000 IU and 200000 IU of oral D3 every 3 months in elderly women. Clin Endocrinol (Oxf) 2016;84:837-44.

63. Benhamou CL, Souberbielle JC, Cortet B, et al. La vitamine D chez I'adulte : recommandations du GRIO. Presse Med 2011; 40:673-82.

64. Autier P, Gandini S, Mullie P. A Systematic Review: Influence of Vitamin D Supplementation on Serum 25-Hydroxyvitamin D Concentration. J Clin Endocrinol Metab 2012; 97:2606-13.

Cet article a été publié dans le « Batna Journal of Medical Sciences » BJMS, l'organe officiel de « l'association de la Recherche Pharmaceutique - Batna»

Le contenu de la Revue est ouvert « Open Access » et permet au lecteur de télécharger, d'utiliser le contenu dans un but personnel ou d'enseignement, sans demander l'autorisation de l'éditeur/auteur.

Avantages à publier dans BJMS :

- Open access : une fois publié, votre article est disponible gratuitement au téléchargement

- Soumission gratuite : pas de frais de soumission, contrairement à la plupart des revues « Open Access »

- Possibilité de publier dans 3 langues : français, anglais, arabe

- Qualité de la relecture : des relecteurs/reviewers indépendants géographiquement, respectant l'anonymat, pour garantir la neutralité et la qualité des manuscrits.

Pour plus d'informations, contacter BatnaJMS@gmail.com ou connectez-vous sur le site de la revue : www.batnajms.net 


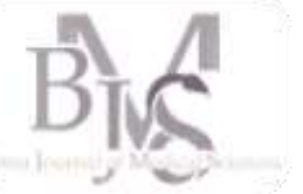

\title{
Détection précoce des désordres glycémiques par la glycémie une heure après une hyperglycémie provoquée par voie orale
}

\author{
Early detection of glycemia disorders using one-hour post load plasma \\ glucose after an oral glucose tolerance test
}

Mohamed Redha Guedjati

Service de physiologie clinique et explorations fonctionnelles métaboliques et nutrition CHU de Batna

\section{Correspondance à :}

Mohamed Redha GUEDJATI m.guedjati@univ-batna2.dz DOI :https://doi.org/10.48087/ B]MSra.2020.7218

Historique de l'article :

Reçu le 3 juin 2020

Accepté le 15 Septembre 2020

Publié le 09 novembre 2020

Il s'agit d'un article en libre accès distribué selon les termes de la licence Creative Commons Attribution International License (CC BY 4.0), qui autorise une utilisation, une distribution et une reproduction sans restriction sur tout support ou format, à condition que l'auteur original et la revue soient dûment crédités.

\section{Pour citer l'article :}

Guedjati MR. Détection précoce des désordres glycémiques par la glycémie une heure après une hyperglycémie provoquée par voie orale. Batna J Med Sci 2020;7:148-50. https://doi.org/10.48087/B] MSra.2020.7218

\section{RÉSUMÉ}

L'hyperglycémie provoquée par voie orale (HGPO) est indiquée et effectuée chez les patients pour lesquels on suspecte des anomalies du métabolisme du glucose. Les critères de diagnostic du diabète sont assez codifiés. Cependant l'état prédiabétique est associé à une perturbation moins prononcée de la glycémie à jeun et/ou de celle après 2 heures. Pour réaliser une HGPO, il faut mesurer la glycémie avant l'administration de $75 \mathrm{~g}$ glucose et deux heures après. Des prélèvements à 30,60 et 90 minutes sont souvent réalisés en plus des deux prélèvements des temps obligatoires. Depuis quelques années, la mesure de la glycémie 60 minutes après l'administration de $75 \mathrm{~g}$ de glucose, est devenue une pratique de plus en plus courante. Cette pratique semble être plus fiable en post HGPO chez des sujets dont le profil glycémique normal et qui ont une glycémie à 60 minutes supérieure ou égale à la valeur de 1,55 g/L.

Mots-clés : Prédiabète ; Diabète sucré ; Glycémie à 60 minutes ; HGPO.

\section{INTRODUCTION}

Les critères diagnostiques du diabète sont connus depuis de longues années. Une glycémie supérieure ou égale à 2,00 g/l (11,1 mmol/l) à tout moment, permet de diagnostiquer un diabète [1].

En 2003, la limite inférieure de la glycémie à jeun (GAJ) permettant d'identifier l'hyperglycémie modérée à jeun (HMJ) de l'anglais Impaired Fasting Glucose (IFG) a été fixée à la valeur de 1,00 g/L (5,6 mmol/l) [2] De même, le diagnostic de l'intolérance au glucose (IG) se définit à partir d'une glycémie à 2 heures ( $\mathrm{T} 120$ minute) $\geq$ à 1,40 g/L. À des valeurs inférieures on parle de tolérance normale au glucose (TNG) de l'anglais Normal Glucose Tolerance (NGT) [2]. Ces deux valeurs sont toujours d'actualité et surtout elles permettent de mettre en évidence un état transitoire avant la survenue du diabète, appelé l'état prédiabétique [3].

Le test de base qui détermine les désordres du métabolisme du glucose est le test d'hyperglycémie provoquée par voie orale (HGPO) [1]. Pour le réaliser, il faut mesurer la glycémie avant l'administration de 75 g de glucose et deux heures après. D'autres prélèvements aux temps intermédiaires (T30, T60 et T90) sont souvent réalisés en plus des

\begin{abstract}
Oral glucose tolerance test (OGTT) is a test that is indicated and performed in patients with suspected abnormalities in glucose metabolism. The criteria for diagnosing diabetes are fairly codified. Pre-diabetic status is associated with or a less pronounced disturbance of Fasting glucose and/or at 2 hours glucose tolerance. To achieve OGTT, the plasma glucose must be measured before administering $75 \mathrm{~g}$ glucose and two hours after. Blood samples at 30,60 and 90 minutes are often taken in addition to the two obligatory samples times. Recently, measuring plasma glucose 60 minutes after the administration of glucose has become an increasingly common practice. This practice seems to be more reliable in post OGTT in subjects who's normal glycemia profile and who have plasma glucose level at 60 minutes greater than or equal to the value of $1.55 \mathrm{~g} / \mathrm{L}$.
\end{abstract}

Keywords: Pre-diabetes; Diabetes mellitus; Plasma glucose at 60 minutes; OGTT.

deux prélèvements obligatoires. La glycémie est évaluée 30 minutes après l'administration de glucose lorsqu'on suspecte une hypoglycémie réactive.

Le test peut être prolongé à 180 minutes.

L'HGPO test diagnostic des désordres métaboliques transitoires

L'HGPO est effectuée si on suspecte des troubles du métabolisme du glucose chez des patients qui n'ont pas de diabète diagnostiqué. Elle permet surtout de diagnostiquer la phase métaboliquement transitoire et asymptomatique des troubles connus comme un état prédiabétique. Cet état regroupe deux situations.

La première s'appelle l'hyperglycémie modérée à jeun (HMJ) de l'anglais Impaired fasting gluose (IFG). La glycémie doit être, dans ce cas entre 1,00 et 1,25 g/L $(5,6-6,9 \mathrm{mmol} / \mathrm{L})$. La deuxième situation se voit quand la glycémie 120 minutes est située entre 1,40 et $1,99 \mathrm{~g} / \mathrm{L}$ (7,8 - 11,1 mmol/L). Elle s'appelle l'intolérance au glucose (IG) de l'anglais Impaired glucose tolerance $(I G T)[1,2]$.

Il existe une certaine inadéquation des critères de diagnostics (tableau 1) ADA OMS et IEC [4]. Cette inadéquation questionne de nombreux praticiens dans la prise de décision diagnostique. 
Tableau 1. Critères diagnostics du prédiabète

\begin{tabular}{lccc}
\hline & OMS* & $\mathrm{ADA}^{* *}$ & ICE*** \\
\hline $\begin{array}{l}\text { Hyperglycémie } \\
\text { modérée à }\end{array}$ & $1,10-125 \mathrm{~g} / \mathrm{L}$ & $1,00-25 \mathrm{~g} / \mathrm{L}$ & - \\
jeun (HMJ) & $6,1-6,9 \mathrm{mmol} / \mathrm{L}$ & $5,6-6,9 \mathrm{mmol} / \mathrm{L}$ & \\
& & & \\
Intolérance au & $1,40-1,99 \mathrm{~g} / \mathrm{L}$ & $1,40-1,99 \mathrm{~g} / \mathrm{L}$ & - \\
glucose (IG) & $7,8-11,0 \mathrm{mmol} / \mathrm{L}$ & $7,-11,0 \mathrm{mmol} / \mathrm{L}$ & \\
HbA1c $\%$ & & $5,7-6,4 \%$ & $6,0-6,4 \%$ \\
\hline
\end{tabular}

* OMS: Organisation Mondiale de la Sante, ADA**: American Diabetes Association, ICE*** : International Expert Committee

Depuis quelques années la mesure de la glycémie 60 minutes après l'administration de $75 \mathrm{~g}$ de glucose interpelle les chercheurs $[5,6,7]$. Elle est devenue une pratique de plus en plus courante. Elle permet une meilleure prédiction du développement du diabète type 2 [8]. Selon la durée de suivi (en moyenne 7 à 8 ans), des taux élevés de la glycémie à 60 minutes sont statistiquement mieux prédictifs que la glycémie à 120 minutes [7-8]. Paddock et al proposent la glycémie 60 minutes après un test d'HGPO comme une alternative afin d'identifier des individus présentant un risque élevé de diabète de type 2 [9]. Le diagnostic du diabète type 2 par la glycémie 120 minutes était justifié par une approximation du niveau de cette glycémie liée à une distribution de la prévalence des atteintes vasculaires notamment les rétinopathies. En 1979 the National Diabetes Data Group (NDDG) avait recommandé des mesures des glycémies intermédiaires à 30, 60 et 90 minutes chez des sujets avec une (IG) et qui ont une glycémie 120 minutes entre $1,40 \mathrm{~g} / \mathrm{L}$ et $1,99 \mathrm{~g} / \mathrm{L}$.

\section{La glycémie à 60 minutes : valeurs seuils prédictives}

La pratique du dosage de la glycémie à 60 minutes semble être de plus en plus fiable en post HGPO chez des sujets avec un profil glycémique normal et qui ont une glycémie à 60 minutes supérieure ou égale à la valeur de 1,55 g/L [6-8]. En fait c'est la valeur de la glycémie à 60 minutes qui suscitait plus d'intérêt du seuil à partir duquel, on estime qu'il y a un risque cardiométabolique [10] ; Dans une étude menée chez des jeunes de race blanche ayant une tolérance normale au glucose de l'anglais Normal Glucose Tolerance (NGT) qui étaient en surpoids et/ou obèses, la valeur de glycémie à 60 minute $\geq 1,325 \mathrm{~g} / \mathrm{L}$ a pu identifier une diminution de la sensibilité et de la sécrétion d'insuline avec tendance à l'accentuation de leur profil cardiométabolique [11].

Lors de l'évaluation du risque des troubles métaboliques, des auteurs ont identifié une accentuation de ce risque si le taux de la glycémie à 1 heure est $\geq 1,548 \mathrm{~g} / \mathrm{L}(\geq 8,6 \mathrm{mmol} / \mathrm{l})$ $[12,13]$. Des études rétrospectives, ont établi comme valeur seuil de 1,584 g/L (8,85 mmol/l) 1 heure après une HGPO comme limite pour encourir un risque de syndrome métabolique [13]. La valeur de 1,55 g/L semble être la mieux étudiée concernant le risque de développement du diabète type 2. Les travaux d'Abdul-Ghani [5, 6, 7] sur la Gly 60 ont montré une courbe ROC inférieure de la Gly 120 par rapport à celle de la Gly $60(0,79$ vs 0,84$)$. La présence d'une Gly $60 \geq$ $1,55 \mathrm{~g} / \mathrm{L}$ associée à un état de HMJ ou IG, est mieux prédictive que quand il y a une HMJ ou une IG. De même la courbe ROC de l'hémoglobine glyquée (HbA1c) se trouve au-dessous de celle de la Gly 60 (0,73 vs 0,84$)$. L'HbA1c est moins corrélée à la sensibilité de l'insuline que la Gly 60 à la valeur de 1,55 g/L [14].

$\mathrm{Si}$ la valeur seuil semble moins préoccupante que l'introduction de la Gly 60 comme moyen de diagnostics des désordres métaboliques du glucose, nombreux sont ceux qui ont adopté et défendu la valeur limite de 1,55g/L [14,15].

Les risques liés aux taux élevés de la glycémie à 60 minutes.

Il a été fait état de l'utilité d'exploiter une concentration plasmatique de glucose à 60 minutes pour la prédiction du diabète de type 2 et des événements cardiovasculaires [16]. De même, il a été démontré que la glycémie à 60 minutes $\geq 1,55 \mathrm{~g} / \mathrm{L}$ avec une tolérance normale au glucose (TNG), est associée à un profil lipidique athérogène similaire à celui observé chez les personnes qui ont une intolérance au glucose (IG) et surtout similaire au profil lipidique des personnes nouvellement diagnostiquées comme diabète de type $2[17,18]$. Des auteurs ont également montré que la viscosité sanguine augmente avec l'augmentation de la glycémie 60 minutes [19].

Il a été suggéré que le sous-groupe avec tolérance normale au glucose (TNG) et ayant une glycémie à 60 minutes $\geq 1,55 \mathrm{~g} / \mathrm{L}$ ont une viscosité sanguine comparable à celle observée chez les sujets avec (HMJ) et / ou avec (IG). Une glycémie à 60 minutes $\geq 1,55 \mathrm{~g} / \mathrm{L}$ est fortement associée à un dysfonctionnement des cellules $\beta$ et une diminution de la sensibilité à l'insuline [13]. De ce fait, il a été observé des niveaux élevés de hs-CRP, de globules blancs et de neutrophiles chez des sujets avec une Gly1h $\geqslant 1,55 \mathrm{~g} / \mathrm{L}$.

Il semblerait que ces signes infracliniques sont liés aux processus inflammatoires $[8,13]$. Le risque d'atteinte rénale a été constaté chez des sujets qui ont une glycémie 60 minutes de loin supérieure à 1,55 g/L [20]. Le risque coronarien est l'un des risques les plus redoutés en cas de désordres du métabolisme du glucose.

La résistance à l'insuline est responsable, et de manière peu précise, de l'association d'une Gly $60 \geqslant 1,55 \mathrm{~g} / \mathrm{L}$ aux maladies coronariennes [21].

Aspects physiopathologiques en appui à la Gly 60 minutes.

Trois phases distinguent le passe d'une NTG au diabète type 2 [14]

La première phase se caractérise par une diminution de la sensibilité à l'insuline au niveau du muscle squelettique et des adipocytes. Les cellules $\beta$ du pancréas augmentent leur sécrétion d'insuline.

La deuxième phase se caractérise par un maintien de la sécrétion des cellules $\beta$ du pancréas, mais la masse de ces cellules commence à diminuer entrainant une altération irréversible de leur réaction au glucose.

La troisième phase au cours de laquelle les cellules $\beta$ énormément détériorées, ne peuvent plus maintenir l'homéostasie du glucose, le désordre devient patent. Il peut s'agir essentiellement de diabète.

À la lumière de cette évolution et au fur et à mesure que la Gly 120 minutes augmente, la sensibilité des cellules $\beta$ au glucose diminue bien que la sécrétion d'insuline puisse être maintenu, il y a dépendance de la réponse des cellules $\beta$ au stimulus du glucose. Cette réponse tend à masquer un désordre potentiellement installé.

En cas de TNG et Gly $60 \geq 1,55 \mathrm{~g} / \mathrm{L}$ on constate une diminution de la sensibilité des cellules $\beta$ au glucose avec maintien d'une sécrétion résiduelle d'insuline ce qui explique le caractère TNG et d'où réponse précoce du pancréas qui peut se traduire par une Gly $60 \geq 1,55 \mathrm{~g} / \mathrm{L}$ (figure 1 ). 


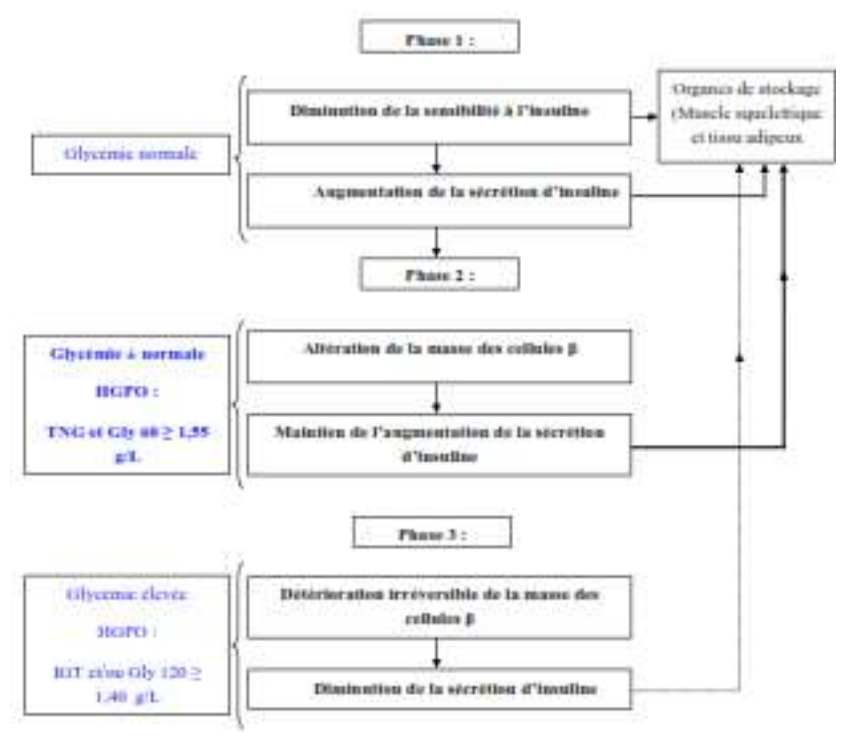

Figure1. Intérêt de la Gly 60 pour le diagnostic précoce désordres glycémiques en cas de TNG

\section{CONCLUSION}

Les approches actuelles pour le diagnostic du prédiabète paraissent sous optimales. Il est proposé que la glycémie à 60 minutes après une HGPO puisse être utilisée pour détecter précocement un état prédiabétique. Plusieurs travaux considèrent que la valeur seuil de 1,55 g/L est fortement prédictive de l'apparition des désordres de la régulation hormonale du glucose chez des individus considérés comme ayant une TNG. Ce type d'individus a une fonction des cellules $\beta$ détériorée avec une diminution de leur sensibilité au glucose et surtout un maintien d'une sécrétion stable d'insuline. Un diagnostic précoce de cette catégorie d'individus par une glycémie à 60 minutes, offre une opportunité d'une prise en charge par un changement du mode de vie. Les modifications précoces des habitudes alimentaires et l'instauration d'une activité physique améliorent la réponse résiduelle des cellules $\beta$. Ces mesures de diagnostic et de prise en charge précocement entreprises offrent les possibilités de stopper, ou à la limite, de retarder l'évolution vers un diabète.

\section{Encadré. Points essentiels}

- L'HGPO reste un test de grande utilité dans le diagnostic des désordres du métabolisme du glucose notamment l'état prédiabétique.

- La glycémie à 60 minutes après un test d'HGPO, trouve une place primordiale dans la détection précoce des désordres du métabolisme du glucose chez des individus avec TNG.

- La valeur 1,55 g/L est, à priori, le seuil qui prête une meilleure prédiction de l'atteinte précoce des organes; d'ailleurs, mieux que la glycémie à 120 minutes voire même de l'HbA1c.

- Une détection, aussi précoce qu'elle soit, par une glycémie à 60 minutes $\geq 1,55 \mathrm{~g} / \mathrm{L}$ des désordres glycémiques, permet l'anticipation de la prise en charge par des modifications de mode de vie. Action efficace sur l'activité résiduelle des cellules $\beta$.

Déclaration d'intérêts: les auteurs ne déclarent aucun conflit d'intérêt en rapport avec cet article.

\section{RÉFÉRENCES}

1. American Diabetes Association. Classification and Diagnosis of Diabetes: Standards of Medical Care in Diabetes-2020. Diabetes Care. 2019;43(Supplement 1):S14-S31.

2. Genuth $S$, Alberti KG, Bennett $P$ et al. Report of the Expert Committee on the Diagnosis and Classification of Diabetes Mellitus. Diabetes Care. 2003;26(Supplement 1):S5-S20.

3. Otto-Buczkowska E, Dryżałowski M. The utility of serum glucose measurement at 1 hour of the oral glucose tolerance test. Clinical Diabetology. 2016;5(4):127-130.

4. Makaroff $L$. The need for international consensus on prediabetes. The Lancet Diabetes \& Endocrinology. 2017;5(1):5-7.

5. Abdul-Ghani $M$, Abdul-Ghani $T$, Ali $N$ et al. One-Hour Plasma Glucose Concentration and the Metabolic Syndrome Identify Subjects at High Risk for Future Type 2 Diabetes. Diabetes Care. 2008;31(8):1650-1655.

6. Abdul-Ghani M, Lyssenko V, Tuomi T et al. Fasting Versus Postload Plasma Glucose Concentration and the Risk for Future Type 2 Diabetes: Results from the Botnia Study. Diabetes Care. 2008;32(2):281-286.

7. Abdul-Ghani M, Stern M, Lyssenko V et al. Minimal Contribution of Fasting Hyperglycemia to the Incidence of Type 2 Diabetes in Subjects With Normal 2-h Plasma Glucose. Diabetes Care. 2009;33(3):557-561.

8. Oh T, Min S, Ahn C et al. Normal Glucose Tolerance with a High 1-Hour Postload Plasma Glucose Level Exhibits Decreased $\beta$-Cell Function Similar to Impaired Glucose Tolerance. Diabetes \& Metabolism Journal. 2015;39(2):147.

9. Paddock $\mathrm{E}$, Hohenadel $\mathrm{M}$, Piaggi $\mathrm{P}$ et al. One-hour and two-hour postload plasma glucose concentrations are comparable predictors of type 2 diabetes mellitus in Southwestern Native Americans. Diabetologia. 2017;60(9):1704-1711.

10. Oh T, Lim S, Kim K et al. One-hour postload plasma glucose concentration in people with normal glucose homeostasis predicts future diabetes mellitus: a 12-year community-based cohort study. Clinical Endocrinology. 2016;86(4):513-519.

11. Marcovecchio M, Bagordo M, Marisi E et al. One-hour post-load plasma glucose levels associated with decreased insulin sensitivity and secretion and early makers of cardiometabolic risk. Journal of Endocrinological Investigation. 2017;40(7):771-778.

12. Su J, Chen T, Xu F et al. Glycemic variability in normal glucose regulation subjects with elevated 1-h postload plasma glucose levels. Endocrine. 2013;46(2):241-248.

13. Priya $M$, Amutha $A$, Pramodkumar $T$ et al. $\beta$-Cell Function and Insulin Sensitivity in Normal Glucose-Tolerant Subjects Stratified by 1-Hour Plasma Glucose Values. Diabetes Technology \& Therapeutics. 2016;18(1):29-33.

14. Bergman M, Manco M, Sesti G et al. Petition to replace current OGTT criteria for diagnosing prediabetes with the 1-hour post-load plasma glucose $\geq 155 \mathrm{mg} / \mathrm{dl}$ ( $8.6 \mathrm{mmol} / \mathrm{L})$. Diabetes Research and Clinical Practice. 2018;146:18-33.

15. Bergman $M$, Jagannathan $R$, Buysschaert $M$ et al. Lessons learned from the 1-hour post-load glucose level during OGTT: Current screening recommendations for dysglycaemia should be revised. Diabetes/Metabolism Research and Reviews. 2018;34(5):e2992.

16. Bardini G, Dicembrini I, Cresci B et al. Inflammation Markers and Metabolic Characteristics of Subjects With 1-h Plasma Glucose Levels. Diabetes Care. 2009;33(2):411-413.

17. Andreozzi F, Mannino G, Perticone M et al. Elevated 1-h post-load plasma glucose levels in subjects with normal glucose tolerance are associated with a pro-atherogenic lipid profile. Atherosclerosis. 2017;256:15-20.

18. Fiorentino T, Marini M, Andreozzi $F$ et al. One-Hour Postload Hyperglycemia Is a Stronger Predictor of Type 2 Diabetes Than Impaired Fasting Glucose. The Journal of Clinical Endocrinology \& Metabolism. 2015;100(10):3744-3751.

19. Marini M, Fiorentino T, Andreozzi $\mathrm{F}$ et al. Elevated 1-h post-challenge plasma glucose levels in subjects with normal glucose tolerance or impaired glucose tolerance are associated with whole blood viscosity. Acta Diabetologica. 2017;54(8):775-784.

20. Succurro E, Arturi F, Lugarà $M$ et al. One-Hour Postload Plasma Glucose Levels Are Associated with Kidney Dysfunction. Clinical Journal of the American Society of Nephrology. 2010;5(11):1922-1927.

21. Cao L, Wang $\mathrm{P}$, Luan $\mathrm{H}$ et al. Elevated 1 -h postload plasma glucose levels identify coronary heart disease patients with greater severity of coronary artery lesions and higher risk of 1-year re-admission. Diabetes and Vascular Disease Research. 2020;17(1):147916411989697. 


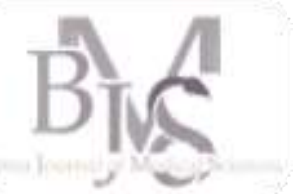

\title{
Impact de l'irradiation solaire sur la santé
}

\author{
Impact of solar radiation on health
}

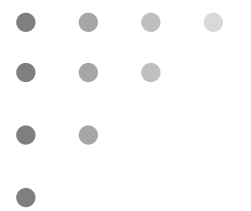

${ }^{1}$ Laboratoire de PharmacologieDépartement de PharmacieFaculté de Médecine-Université Alger 1- Algérie

\section{${ }^{2}$ Service de}

pharmacotoxicologie-Agence Nationale des produits

pharmaceutiques, Alger- Algérie

${ }^{3}$ Laboratoire de PharmacologieDépartement de Pharmacie-

Faculté de Médecine-Université Batna 2- Algérie

\section{Correspondance à :}

Henni CHADER

hennichader@hotmail.fr

DOI : $\underline{\text { https://doi.org/10.48087/ }}$ BJMSra.2020.7219

\section{Historique de l'article :}

Reçu le 18 juin 2020

Accepté le 14 Août 2020

Publié le 09 novembre 2020

Il s'agit d'un article en libre accès distribué selon les termes de la licence Creative Commons Attribution International License (CC BY 4.0), qui autorise une utilisation, une distribution et une reproduction sans restriction sur tout support ou format, à condition que l'auteur original et la revue soient dûment crédités.

\section{Pour citer l'article :}

Chader H et Gacem H. Impact de l'irradiation solaire sur la santé. Batna J Med Sci 2020;7(2):151-8. https://doi.org/10.48087/B IMSra.2020.7219

\section{RÉSUMÉ}

L'exposition aux irradiations solaires n'est pas sans risque sur la santé bien que l'homme lui attribue des effets bénéfiques pour l'organisme et une source d'embellissement pour les adeptes du bronzage. Des irradiations électromagnétiques du soleil, les rayons ultraviolets (UV) sont les plus dangereux pour l'organisme malgré qu'ils soient derrière la transformation du 7-déshydrocholestérol en cholécalciférol (D3), une vitamine impliquée grandement dans le métabolisme phosphocalcique. Les innombrables effets délétères que provoque le rayonnement solaire peuvent être d'apparition précoce, retardée ou à long terme ; ils peuvent être bénins comme l'effet coup de soleil ou graves à l'image du cancer de la peau. La réactivité du revêtement cutané vis-à-vis des rayons UV solaires varie avec le phototype qui se trouve sous l'influence de facteur génétique conditionnant la qualité et la quantité de la mélanine synthétisée par les mélanocytes.

Mots clés: peau, soleil, rayons ultraviolets, stress oxydatif, photodermatoses, photosensibilisation.

\section{INTRODUCTION}

Le soleil est une méga centrale thermonucléaire qui produit un rayonnement composé d'une suite infinie et continue de radiations électromagnétiques qui irradient dans toutes les directions de l'espace. L'ensemble de ces radiations constituent le spectre solaire. Seule les rayons visibles, ultraviolets (UV) et infrarouges (IR) arrivent sur la surface terrestre, le reste est absorbé par la couche d'ozone et par l'atmosphère [1].

Le rayonnement visible est constitué de radiations colorées (violet, indigo, bleu, vert, jaune, orange, rouge) qui associées entre elles, font la lumière du jour. La limite inférieure du rayonnement visible se situe à $400 \mathrm{~nm}$, la limite supérieure à $800 \mathrm{~nm}$. Les rayons infrarouges (IR) ont une longueur d'onde comprise entre 800 et $1400 \mathrm{~nm}[1]$.

Les irradiations des domaines infrarouge et visible ne semblaient pas être néfastes pour l'organisme mais certaines études ciblant les effets de la lumière visible ou IR sur la peau ont conclu que ces 2 types de rayonnement solaire peuvent également contribuer au photovieillissement prématuré de la peau par la formation d'espèces réactives de l'oxygène (ERO) $[2,3]$.

Environ 5\% du rayonnement solaire est émis

\section{ABSTRACT}

Exposure to solar radiations is not free of health risks despite the fact that humans attribute beneficial effects to it for the organism and considered as a source of embellishment for tanning enthusiasts. From the sun's electromagnetic radiation, ultraviolet (UV) rays are the most dangerous for the body despite the fact that they are behind the transformation of 7-dehydrocholesterol into cholecalciferol (D3), a vitamin greatly involved in phosphocalcic metabolism. The innumerable harmful effects caused by solar radiation can be early, delayed or long-term; they can be as mild as sunburn or as severe as skin cancer. The reactivity of the skin covering to solar UV rays varies with the phototype, which is under the influence of genetic factors conditioning the quality and quantity of melanin synthesized by melanocytes.

Key words : skin, sun, ultraviolet rays, oxydative stress, photo dermatoses, photosensitization.

sous forme d'UV dont la bande comprise entre 100 et $400 \mathrm{~nm}$ est divisée en trois parties selon la longueur d'onde : les rayons ultraviolets $\mathrm{C}$ (UVC) de 100 à $280 \mathrm{~nm}$; les rayons ultraviolets $B$ (UVB) de 280 à $315 \mathrm{~nm}$ et les rayons ultraviolets A (UVA) de 315 à $400 \mathrm{~nm}$; Les $95 \%$ restants comprennent la lumière visible (50 \%) et les infrarouges (45\%) [4].

Les UVC n'atteignent pas le sol car ils sont totalement absorbés par la couche d'ozone, seuls les UVA et les UVB irradient la surface de la terre où ils représentent respectivement $95 \%$ et $5 \%$ du rayonnement solaire en ultraviolet atteignant la terre [1] (Figure 1).

\section{GENERALITES SUR LE RAYONNEMENT SOLAIRE}

\section{I.1. Diffusion des rayons ultraviolets à travers la peau}

La plus grande partie $(70 \%)$ des rayons UVB sont arrêtés par la couche cornée, $20 \%$ arrivent à atteindre le corps muqueux de l'épiderme, et $10 \%$ le derme superficiel. La majorité des rayons UVA traversent la couche cornée mais seuls 20 à $30 \%$ atteignent le derme profond. Les rayonnements visibles et infrarouge traversent l'épiderme, le derme et parviennent jusqu'à l'hypoderme [5]. Plus la longueur d'onde d'une irradiation est longue, plus elle pénètre profondément la peau [5]. 


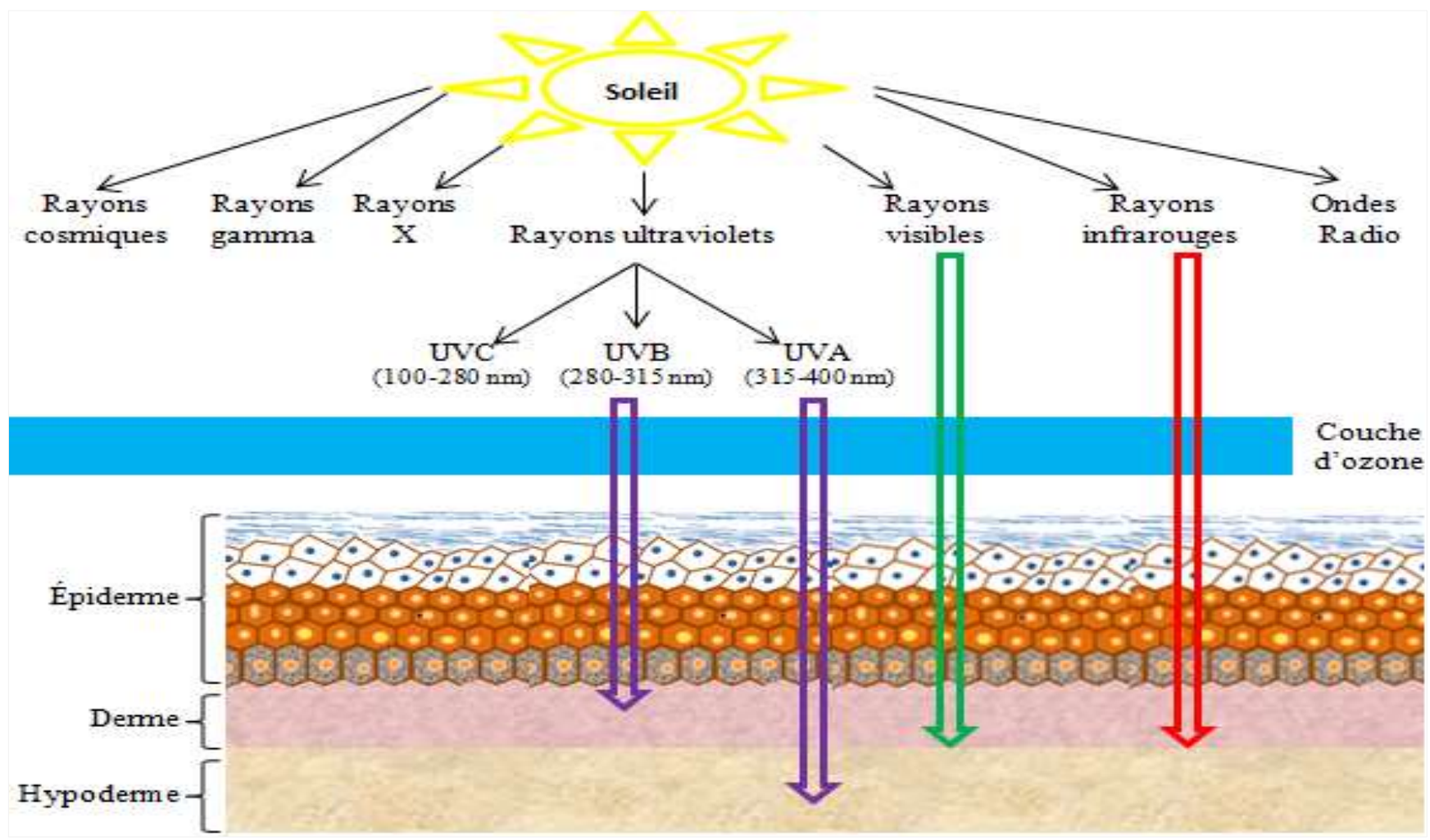

Figure 1. Spectre des radiations électromagnétiques du soleil et diffusion à travers la peau (D’après Césarini M. J-P et Robert P, 1985) [5].

\section{I.2. Action des rayons ultraviolets sur la matière vivante}

Les radiations solaires, par le biais des rayons UV, sont responsables des réactions photochimiques au niveau cutané. À l'échelle cellulaire il existe des molécules appelées chromophores capables d'absorber les UV grâce à leurs doubles liaisons contenues dans leurs structures [6-8]. Les UV interagissent avec les chromophores par deux mécanismes photo-réactionnels.

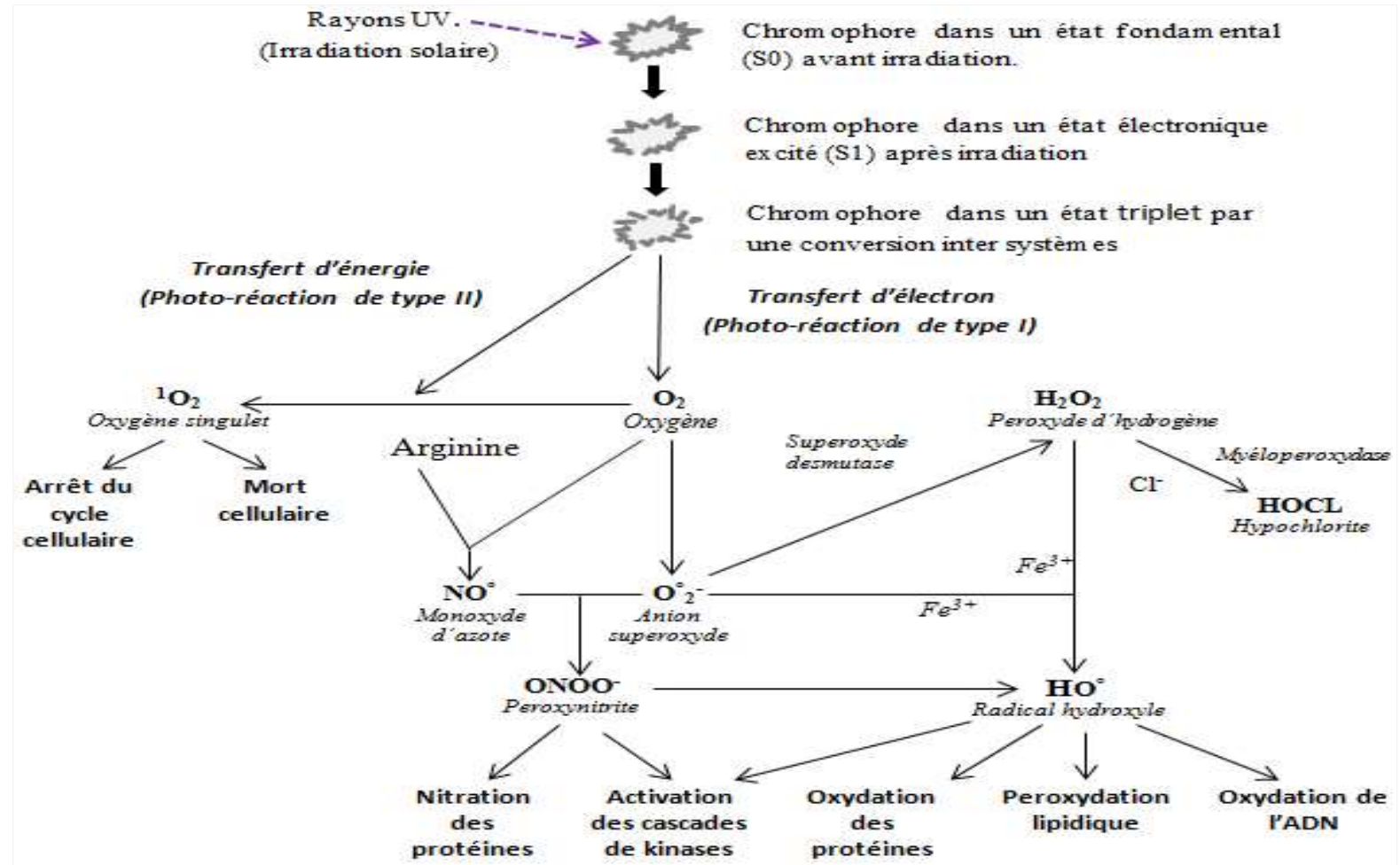

Dans la photo-réaction de type I, les chromophores qui en absorbant les rayons UV vont passer d'un état fondamental à un état électronique excité puis vers un état triplet par une conversion inter-systèmes. Devenu ion, le chromophore va transférer son électron en excès vers une molécule d'oxygène moléculaire pour donner naissance au radical superoxyde $\mathrm{O}^{\circ}{ }_{2}$ qui va à son tour réagir pour former d'autres espèces réactives selon le schéma illustré dans la figure 2 . 
Devenu ion, le chromophore va transférer son électron en excès vers une molécule d'oxygène moléculaire pour donner naissance au radical superoxyde $0^{\circ}{ }_{2}$ qui va à son tour réagir pour former d'autres espèces réactives selon le schéma illustré dans la figure 2 .

La photo-réaction de type II est enclenchée par le chromophore excité qui par un transfert d'énergie vers l'oxygène moléculaire, ce dernier est excité et entre dans son premier état singulet $0^{\circ} 1_{2}$. L'ensemble des radicaux ainsi produits par ces deux réactions avec l'oxygène moléculaire constitue une famille appelée espèces réactives d'oxygènes (ERO) ; les dommages engendrés par l'accumulation des ERO dans la cellule sont qualifiés de stress oxydant $[9,10]$.

\section{I.3. Dommages macromoléculaires causés par les espèces} réactives d'oxygène (ERO)

\section{I.3.1. Altérations des protéines}

En oxydant les protéines, les ERO générées par les UVA comme les UV B entrainent des modifications structurales et fonctionnelles. Les protéines contenant les acides aminés aromatiques sont les plus modifiées et les enzymes contenant ce type de protéines voient leurs activités biologiques disparaitre une fois oxydées. Les modifications structurales post oxydatives sont représentées par l'altération des groupements thiols, formation de ponts disulfures et accentuation du caractère hydrophobe qui augmente l'agrégation des protéines et les rend plus résistantes à la protéolyse physiologique [1]. Les protéines oxydées constituent les marqueurs du stress oxydant qu'on retrouve $\mathrm{au}$ cours du vieillissement, maladies inflammatoires et cancers [12-16].

\section{I.3.2. Altérations de l'ADN}

Les attaques contre l'ADN sont causées à la fois par les rayons UVA et UVB; ces derniers agissent directement sur l'ADN alors que les UVA le font indirectement par le biais des ERO générées plus particulièrement le radical hydroxyle $\mathrm{HO}^{\circ}$ [17]. Les altérations ainsi induites peuvent se traduire par des modifications de bases azotées, destruction de la liaison entre la base et le désoxyribose générant un site abasique, cassure de brin et enfin création de pontage avec des protéines. Tous ces dommages peuvent entrainer un arrêt de division cellulaire par blocage des mécanismes de réplication, un arrêt de la synthèse protéique par blocage des mécanismes de transcription-traduction et même une mort cellulaire [16-20]. Pour réparer ces altérations, la cellule fait appel à un processus de réparation-excision lequel n'est pas dénué d'erreurs, des mutations peuvent alors apparaitre dans le cas où il y'a accident de réparation de l'ADN responsable de la modification du patrimoine génétique cellulaire $[8,12,13,21$, 22].

\section{I.3.3. Altérations des lipides (Peroxydation lipidique)}

Les acides gras polyinsaturés (AGPI.) sont la cible privilégiée de l'attaque radicalaire essentiellement du radical hydroxyle. Cette peroxydation se déroule en 3 étapes [23] :

- L'initiation qui consiste en l'arrachement d'un atome d'hydrogène d'un groupement méthylène (-CH2-) adjacent à deux doubles liaisons pour former un radical diène conjugué $(\mathrm{R} \bullet)$

- La propagation qui débute lorsqu'une molécule d'oxygène moléculaire attaque le radical acide gras $(\mathrm{R})$ pour former un radical peroxyle (R-000) capable d'arracher un atome d'hydrogène à une autre molécule d'acide gras adjacente et se transforme en hydroperoxyde créant ainsi une réaction en chaîne.
- La terminaison dans laquelle les hydroperoxydes peuvent être neutralisés par le glutathion peroxydase ou continuer à se décomposer en produits secondaires tel que le malondialdéhyde (MDA) qui est doté d'une réactivité très importante et peut agir avec les protéines et l'ADN.

La peroxydation lipidique où l'oxydation des lipides par les ERO entraine une diminution de la fluidité et la résistance membranaire, l'augmentation de la perméabilité au calcium et l'altération des récepteurs membranaires [16-24].

\section{I.3.4. Altérations des polysaccharides}

Les ERO oxydent essentiellement le glucose et les protéoglycanes de la substance fondamentale de la matrice extracellulaire du derme [25].

\section{EFFETS DES RADIATIONS SOLAIRES SUR LA PEAU}

L'agressivité des radiations solaires au niveau cutané dépend de la durée d'exposition, de l'intensité du rayonnement solaire, du taux de réflexion par le sol. Les effets néfastes observés au niveau de la peau après exposition aux rayons solaires sont nombreux: coups de soleil, vieillissement cutané, cancers cutanés, réactions de photosensibilisation. Les effets induits par une exposition solaire de l'organisme peuvent être bénéfiques ou délétères; d'apparition précoce, retardée ou à long terme [5, 26] (Figure 3).

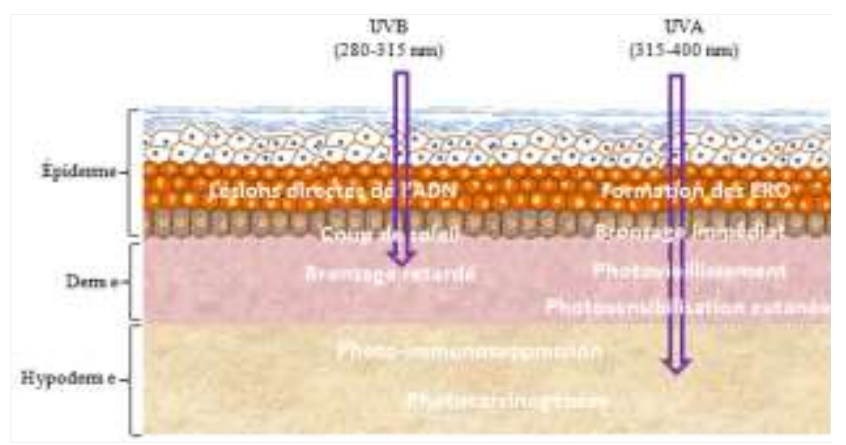

Figure 3. Schéma illustrant les effets induits par l'irradiation ultraviolette au niveau cutané (D’après $M$. Wlaschek et collaborateurs, 2001) [26].

\section{II.1. Effets précoces}

Dans la plupart des cas, les effets précoces sont induits par les rayons UVB qui d'une part, provoquent la dégradation cellulaire par perméabilisation de la membrane lysosomiale et relargage d'enzymes protéolytiques qui dégradent les constituants de la cellule, et d'autre part, bloquent de manière réversible la synthèse d'ADN et d'ARN. Les effets immédiats observés regroupent la synthèse de la vitamine $\mathrm{D}$ dans l'épiderme, la vasodilatation dermique qui se traduit par une rougeur de la peau (érythème) suivie d'une élévation de la température cutanée et d'une sensation de chaleur, une légère coloration de la peau qui dure quelques heures (phénomène de Meirowski) et apparaissant au bout de 30 minutes d'exposition solaire où les UVA dont l'implication est largement démontrée provoquent un réarrangement et une accélération de la maturation des mélanines noires contenues dans les kératinocytes [5-26].

\section{II.2. Effets retardés}

\section{II.2.1. L'érythème actinique}

L'effet du coup de soleil causé par les rayons UVB est une réaction inflammatoire violente qui se traduit par un érythème important. 
Les membranes et les noyaux des cellules épidermiques sont lésés, et les mécanismes de réparation cellulaire sont enclenchés [27]. Vingt minutes d'exposition à midi en période d'été sont suffisantes pour enclencher le mécanisme de la réaction inflammatoire. Le délai d'apparition de l'érythème est d'environ deux à six heures après l'exposition solaire. Le maximum d'intensité est atteint au bout de douze à vingtquatre heures [5-26].

Sur le plan histologique, l'exposition solaire provoque un épaississement de l'épiderme, connue sous l'appellation d'hyperplasie épidermique, et en particulier la couche cornée d'où l'appellation hyperkératose. Cet épaississement est dû à l'action des UVA sur les cellules basales qui commencent à se diviser plus activement à partir du 3ème jour de l'exposition solaire. L'épaississement de la couche cornée a pour effet de diminuer la pénétration des rayons UV dans la peau ainsi que leurs effets délétères [5-26].

\section{II.2.2. Bronzage ou pigmentation retardée}

L'augmentation du pigment mélanique dans l'épiderme est responsable du bronzage qui est la coloration cuivrée de la peau qui débute deux jours après l'exposition au soleil. Ce délai correspond au temps nécessaire aux mélanocytes, après activation par les UVB, pour synthétiser le stock supplémentaire de mélanine qui sera distribuée aux kératinocytes voisins [28-29].

La pigmentation est maximale au bout d'une vingtaine de jours et disparait progressivement en l'absence d'une nouvelle exposition [30]. L'intensité du bronzage est étroitement liée au phototype du sujet (Tableau 1) car la qualité de la mélanine produite est génétiquement programmée et n'est pas modifiée par l'irradiation UV ; plus le phototype est sombre, plus le sujet bronze [5-26].

Tableau 1. Classification des érythèmes solaires (D'après $M$. Déparois, 2014) [27].

\begin{tabular}{|c|c|c|c|c|}
\hline $\begin{array}{l}\text { Classification } \\
\text { des érythèmes }\end{array}$ & $1^{\text {er }}$ degré & $2^{\text {e degré }}$ & $3^{\text {e }}$ degré & $4^{\mathrm{e}}$ degré \\
\hline Couleur & Rosée & Rouge vif & Violine & Cyanique \\
\hline $\begin{array}{l}\text { Autres signes } \\
\text { cutanés }\end{array}$ & Aucun & Aucun & OEdème & $\begin{array}{l}\text { Bulles ou } \\
\text { phlyctènes }\end{array}$ \\
\hline $\begin{array}{l}\text { Signes } \\
\text { généraux }\end{array}$ & Aucun & Aucun & Aucun & $\begin{array}{l}\text { Fièvre: } \\
39-40^{\circ} \mathrm{C} \\
\text { Malaise } \\
\text { général }\end{array}$ \\
\hline $\begin{array}{l}\text { Délai } \\
\text { d'apparition } \\
\text { (heure) }\end{array}$ & $6-24$ & $2-12$ & & $6-12$ \\
\hline Durée (jour) & $1-2$ & $2-3$ & 4 & $4-6$ \\
\hline Desquamation & $\begin{array}{l}\text { Fine et } \\
\text { rapide }\end{array}$ & $\begin{array}{l}\text { Fine et } \\
\text { rapide }\end{array}$ & En lambeaux & $\begin{array}{l}\text { En } \\
\text { lambeaux }\end{array}$ \\
\hline $\begin{array}{l}\text { Pigmentation } \\
\text { mélanique } \\
\text { de la peau }\end{array}$ & Aucune & $\begin{array}{l}\text { Plus ou } \\
\text { moins } \\
\text { importante }\end{array}$ & $\begin{array}{l}\text { Coloration } \\
\text { importante }\end{array}$ & Aucune \\
\hline
\end{tabular}

\section{II.2.3. La photo-immunosuppression}

Les UVB et à un degré moindre les UVA ont un effet immunosuppresseur réversible qui apparait quelques heures après une exposition solaire. Le rétablissement de la fonction immunitaire se fait au bout de trois semaines.

La dépression immunitaire photo-induite entraine une diminution des réactions d'hypersensibilité de contact où les lymphocytes T sont grandement impliqués [27, 31, 32].
II.3. Effets apparaissant à long terme après exposition solaire réitérée

II.3.1. Le vieillissement cutané photo-induit où héliodermie

L'exposition chronique des régions découvertes de la peau aux rayons solaires entraine un vieillissement cutané prématuré dont les altérations spécifiques sont différentes de celles liées à l'âge. Les rayonnements en cause sont les UVA qui pénètrent davantage dans la peau et produisent des altérations plus profondes que celles des UVB. L'héliodermie où vieillissement actinique est sous l'influence de l'ensoleillement cumulé et des facteurs génétiques tels que le phototype et la prédisposition familiale [27].

Sur le plan clinique, l'héliodermie se caractérise par l'épaississement de la peau du visage, du dos, des mains et de l'avant-bras qui avec le temps elle devient jaunâtre et sèche avec apparition de rides qui se creusent, de télangiectasies et d'une pigmentation irrégulière. Sur le plan biochimique, les rayons UVB, entrainent la senescence des fibroblastes dermiques qui se traduit par une diminution de la synthèse du collagène et une dégradation d'élastine [33] ; les rayons UVA agissent sur les fibroblastes dermiques en augmentant à la fois l'expression du gène apoptotique et la synthèse des métalloprotéinases (mmp-1, mmp-9) et du TNF- $\alpha$ tout en augmentant la production du pro-collagène $1 \alpha 1$ [34]. À un stade avancé de l'héliodermie, l'épiderme s'amincit, le nombre de mélanocytes et de cellules de Langerhans diminue [35].

\section{II.3.2. Carcinogénèse}

Les expositions solaires cumulées depuis le premier jour de la vie sont responsables de l'apparition des épithéliomas. Les mélanomes malins sont initiés par les expositions solaire de courte durée mais intenses et répétées [5-26]. Dans le mécanisme du développement du mélanome, les rayons ultraviolets jouent un grand rôle dans l'initiation tumorale en entrainant la mutation du gène de l'apoptose P53 de la cellule mélanocytaire directement par dégradation de l'ADN par les UVB ou indirectement par le biais des ERO générées par les UVA [17]. Les UVA comme les UVB sont également impliqués dans les autres stades de la cancérogénèse [13-36].

\section{II.4. Les dermatoses liées à l'exposition solaire}

\section{II.4.1. Les dermatoses photo-aggravées}

C'est un ensemble de pathologies génétiques ou acquises dont les réactions cutanées déjà importantes sont exacerbées par l'exposition aux rayons solaires. On retrouve: la dermatose bulleuse, la télangiectasie, le lupus érythémateux, l'hématodermie, l'herpès et l'acné [5].

\section{II.4.2. Les photodermatoses par déficience du système de protection}

Les sujets albinos et les malades atteints de vitiligo sont très sensibles aux irradiations solaires à cause de l'absence de protection liée à une anomalie de la synthèse de la mélanine. Le xeroderma pigmentosum, syndrome xérodermoïde et l'érythème prolongé sont des dermatoses caractérisées par une déficience des systèmes de réparation de l'ADN [5].

\section{II.4.3. Les photodermatoses par anomalie métabolique}

Les porphyries et la pellagre sont la conséquence d'un dérèglement métabolique. Les porphyries sont la résultante de la formation au niveau sanguin d'un métabolite photodynamisant (porphyrines et/ou de leurs précurseurs) suite à un déficit de l'une des huit enzymes de la chaine métabolique de l'hème $[5,37,38]$. 
La pellagre, quant à elle, est due à l'accumulation dans la peau d'un produit photoactif [39] secondaire à une anomalie métabolique du tryptophane responsable d'un déficit en vitamine PP (nicotinamide) donnant naissance à des accidents de photosensibilisation [37].

\section{II.4.4. Les photodermatoses idiopathiques}

Ce sont des dermatoses qui se manifestent par un état d'hypersensibilité dès la première exposition solaire. Sous cette appellation, on retrouve: la lucite, les érythèmes polymorphes, l'urticaire solaire, le prurit solaire et l'hydroa vacciniforme [5-27].

\section{II.4.4.1. La lucite}

D'après Césarini et Robert, le terme de lucite recouvre trois affections distinctes : lucite estivale bénigne, lucite polymorphe et la lucite hivernale bénigne [5-27].

\section{A. La lucite estivale bénigne}

Elle touche surtout la femme (80\%) [40] après la première exposition solaire brutale ou prolongée au début des vacances d'été [41] ou lors d'une exposition d'un week-end ensoleillé [40]. Tout en épargnant le visage, elle se manifeste par des démangeaisons pouvant empêcher le sommeil [41], de très petites cloques ou de petites taches rouges souvent confondues avec l'urticaire.

Elle disparait progressivement en une à deux semaines mais récidive lors de nouvelles expositions au soleil. Les récidives sont le plus souvent plus graves le plus souvent $[40,41]$.

\section{B. La lucite polymorphe}

C'est la photodermatose à médiation immunologique la plus courante dans le monde avec une prévalence pouvant atteindre 10 à $20 \%$ en Europe occidentale et aux États-Unis [42-44]. Elle affecte le plus souvent les jeunes femmes avec une incidence 4 fois plus que l'homme, de tous types de peau, dans la deuxième à la troisième décennie de la vie $[42,45]$, Elle débute $30 \mathrm{mn}$ à 1-3 jours après la première exposition solaire du printemps et disparait généralement en 7 à 10 jours [44].

Les lésions se présentent sous forme de papules, vésicules, papulovésicules, plaques ou nodules non cicatriciels, érythémateux et peu prurigineux [44]. Ces lésions disparaissent en quelques jours en évitant l'exposition au soleil.

\section{La lucite hivernale bénigne}

C'est une éruption de plaques rouges-violacées gonflées qui démangent et apparaissent sur le front, les tempes, les pommettes et les oreilles. Elle touche les sujets jeunes, le plus souvent fillette ou jeune fille de moins de 15 ans, apparaissant après une exposition brutale au soleil à des altitudes dépassant les 1300 mètres [40]. Elle touche essentiellement les personnes atteintes de lucite estivale bénigne [37, 39] laissant penser qu'elle est uniquement l'expression de la LEB suite à une exposition brutale à la lumière intense d'un soleil réfléchi sur la neige [46].

\section{II.4.4.2. Autres photodermatoses idiopathiques}

Qu'il s'agisse d'urticaire solaire à réponse immédiate (1 à 5 mn après l'exposition) [47, 48], d"hydroa vacciniforme, de prurigo actinique, tous les deux débutant dans l'enfance (avant 10 ans) et disparaissant à l'adolescence $[49,50]$ tout en laissant des cicatrices [51] ou de la dermatite actinique chronique à début eczématiforme et pouvant aller jusqu'à l'érythrodermie [52]; ils ont deux particularités communes : une survenue rare et des manifestations graves.

\section{II.5. Les dermatoses de photosensibilisation}

La photosensibilisation cutanée est l'ensemble des phénomènes pathologiques liés à la rencontre au niveau de la peau d'une substance photoréactive avec une radiation lumineuse à une ou plusieurs longueurs d'ondes [53-54]. De nombreuses molécules exogènes qui par des mécanismes distincts peuvent provoquer une dermite phototoxique ou une dermite photo-allergique qui sont des dermatoses de photosensibilisation [53-54].

\section{II.5.1. Dermite phototoxique}

La dermite phototoxique où photo-irritation est une réaction toxique aigue se manifestant sans aucune prédisposition particulière chez tous les individus sous forme d'une brûlure solaire exagérée circonscrite aux parties découvertes de l'organisme et apparaissant après exposition concomitante de la peau à la lumière et certains produits chimiques photosensibles appliqués localement ou administrés par voie systémique [53-55] (Tableau 2).

La dermite phototoxique reproduit cliniquement et histologiquement l'image d'une brûlure solaire : érythème plus ou moins œdémateux au stade initial, bulles à un stade plus avancé [26]. Ces manifestations apparaissent uniquement au niveau des zones irradiées, les régions sousorbitaires, sous-nasales, sous-mentonnières sont épargnées [55]. Des sensations immédiates de brûlure ou de piqûre caractérisent certaines réactions de photosensibilisation par certains agents chimiques $[53,55,56]$.

L'érythème disparait en quelques jours à quelques semaines mais parfois il peut laisser de légères séquelles pigmentaires $[53,55,56]$. Histologiquement, une nécrose cellulaire plus ou moins importante pouvant toucher tout l'épiderme est observée [56].

\section{II.5.1.1. Mécanisme}

Le point de départ de la dermite phototoxique est la génération des ERO par les molécules phototoxiques sous l'action des UVA [57-59]. Dans la quête de rétablissement de leur neutralité électrique, les ERO ainsi générés vont oxyder les molécules des membranes cytoplasmique et lysosomiale des kératinocytes épidermiques induisant ainsi la perte de l'intégrité membranaire et le relargage de Tumor Necrosis Factor $\alpha($ TNF $\alpha)$ et d'interleukines $1 \alpha, 1 \beta$ (IL-1 $\alpha$ et $\beta$ ) qui sont des cytokines pro-inflammatoires dotées d'un pouvoir d'activation de la cascade immunologique non antigène dépendante en stimulant la libération d'autres cytokines qui recrutent et activent directement les lymphocytes $\mathrm{T}$ acteurs majeures de la réaction inflammatoire $[65,70]$.

Tableau 2. Substances phototoxiques (D’après A. Prusinowski, 2018) [61].

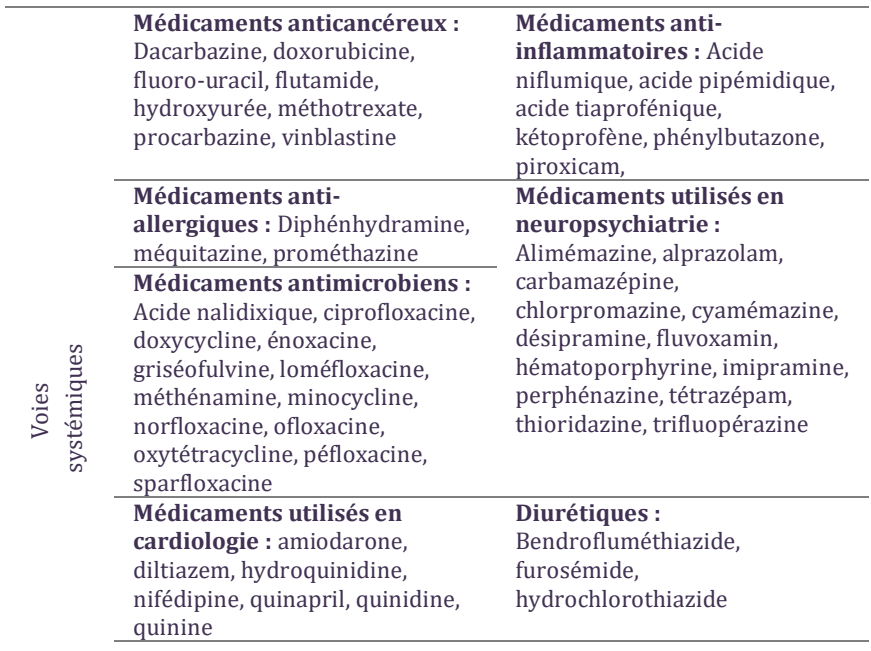


(Suite Tableau 2)

\begin{tabular}{ll}
\hline $\begin{array}{l}\text { Médicaments utilisés en } \\
\text { nutrition : Cyclamate de sodium, } \\
\text { fénofibrate, simvastatine }\end{array}$ & $\begin{array}{l}\text { Médicaments utilisés en } \\
\text { dermatologie : Acide para- } \\
\text { aminobenzoïque, } \\
\text { hydroxychloroquine, } \\
\text { isotrétinoïne, méthoxypsoralène }\end{array}$ \\
\hline $\begin{array}{l}\text { Médicaments topiques : } \\
\text { Chlorprométhazine, }\end{array}$ & $\begin{array}{l}\text { Antiseptiques : Citronnelles, } \\
\text { bithionol, hexamidine, } \\
\text { triclocarban, triclosan }\end{array}$ \\
diphénhydramine, isothipendyl, & \\
\hline \multirow{2}{*}{$\begin{array}{l}\text { kétoprofène, oxytétracycline, } \\
\text { piroxicam, prométhazine, } \\
\text { sulfadiazine }\end{array}$} & \\
\hline
\end{tabular}

\section{II.5.2. Dermite photo-allergique}

La dermite photo-allergique est une réaction d'hypersensibilité retardée à médiation cellulaire qui met en jeu le système immunitaire et apparait que chez quelques individus prédisposés après exposition concomitante aux rayons solaires et les produits photosensibilisants appliqués sur la peau ou administrés par voie systémique (Tableau 3). Cliniquement, la dermite photo-allergique se manifeste par des lésions de type eczéma (érythème œdémateux, vésiculeux ou bulleux) et peut prendre un aspect urticarien, lichénifié ou lupique dans certains cas.

Contrairement à la dermite phototoxique, l'éruption cutanée peut s'étendre aux zones couvertes, persister pendant des mois ou des années et ne régresser que lentement après élimination de la molécule photosensibilisante par l'organisme. La photosensibilisation rémanente est caractérisée par des récidives apparaissant à chaque exposition solaire malgré l'arrêt du traitement avec la substance photo-allergisante $[8,60,62]$. Sur le plan histologique, la dermite photo-allergique montre un infiltrat lymphohistiocytaire périvasculaire avec modification épidermique de types exocytose et spongiose [63].

\section{II.5.2.1. Mécanisme}

Le mécanisme de la dermite photo-allergique correspond à celui de la réaction d'hypersensibilité retardée dont la première phase se déroule au niveau des couches vivantes de l'épiderme et corresponds à la transformation en haptène de la molécule photosensibilisante sous l'action des rayons UVA ou UVB [57, 60,62,64]. En se fixant à une protéine de la couche granuleuse épidermique qu'il dénature, l'haptène devient un antigène complet ; considéré comme corps étranger, cet antigène est porté par les cellules de Langerhans qui migrent dans le derme pour le présenter aux lymphocytes
$\mathrm{T}$ naïfs et les activer in situ mais également au niveau du ganglion lymphatique qui draine le territoire cutané où la substance photosensibilisante est présente.

Au niveau du ganglion lymphatique les lymphocytes T activés se transforment en une population de lymphoblastes. Ces derniers après un certain nombre de divisions cellulaires, redeviennent de petits lymphocytes $\mathrm{T}$ appartenant à plusieurs sous- populations : les lymphocytes T mémoires, les lymphocytes $\mathrm{T}$ effecteurs et les lymphocytes $\mathrm{T}$ suppresseurs. Les lymphocytes T mémoire de l'hypersensibilité retardée sont sensibilisés et sont porteurs de récepteurs de membrane spécifiques pour l'allergène en cause.

Dans un organisme déjà sensibilisé où circulent les lymphocytes $\mathrm{T}$ à mémoire, la réintroduction de l'haptène provoque, en 24 à 48 heures, des signes cliniques d'une dermite de contact allergique. Le retard observé dans la survenue de la réponse de l'organisme à l'haptène s'explique par le délai nécessaire à la formation de l'haptène en allergène et sa présentation par les cellules de Langerhans aux lymphocytes T à mémoire sensibilisés [57, 60, 62, 64].

Tableau 3. Exemples de substances photo-allergisantes systémiques (D’après A. Prusinowski, 2018) [61].

\begin{tabular}{|c|c|c|}
\hline 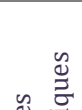 & $\begin{array}{l}\text { Médicaments } \\
\text { antimicrobiens : } \\
\text { sulfonamides }\end{array}$ & $\begin{array}{l}\text { Médicaments anti- } \\
\text { allergiques : Chlorpromazine, } \\
\text { prométhazine, thioridazine }\end{array}$ \\
\hline$>\frac{\bar{d}}{\infty}$ & $\begin{array}{l}\text { Médicaments utilisés en } \\
\text { cardiologie : Quinidine, } \\
\text { thiazides (diurétiques) }\end{array}$ & $\begin{array}{l}\text { Médicaments } \\
\text { hypoglycémiants: } \\
\text { Sulfonylurées }\end{array}$ \\
\hline
\end{tabular}

\section{PHOTOTYPES CUTANES}

En 1975, Thomas Bernard Fitzpatrick proposa une classification des phototypes cutanés (Tableau 4) en se basant sur de la sensibilité de la peau aux rayons solaires, le potentiel à développer un érythème actinique, le potentiel de bronzage, la couleur de la peau (carnation), les taches de rousseurs (éphélide) et la couleur des cheveux. Cette classification a été adoptée plus tard par la FDA Américaine. La sensibilité de l'individu à développer un érythème actinique (coup de soleil) dépend du type et de la quantité de mélanine contenue dans les mélanocytes épidermiques (Tableau 5). Ce dernier critère permet de classer les individus en caucasoïdes, mongoloïdes et négroïdes. Les sujets albinos sont dénués de pigmentation mélanique [29].

Tableau 4. Phototypes cutanés selon Thomas Bernard Fitzpatrick (1975) [65]

\begin{tabular}{|c|c|c|c|c|}
\hline Phototype & Classification selon le type et la quantité de mélanine & Éphélide & Carnation & Coup de soleil (Bronzage) \\
\hline $\mathbf{0}$ & Cheveux blancs & 0 & Albinos (sensible) & $\begin{array}{l}\text { Brûle très facilement } \\
\text { (Ne bronze jamais) }\end{array}$ \\
\hline I & Caucasoïdes à mélanosomes porteurs de phaeomélanine (cheveux roux) & +++ & Laiteuse (sensible) & $\begin{array}{l}\text { Brûle très facilement } \\
\text { (Ne bronze jamais) }\end{array}$ \\
\hline II & Caucasoïdes à mélanosomes porteurs d'eumélanine (cheveux blonds) & ++ & Claire (sensible) & $\begin{array}{l}\text { Brûle très facilement } \\
\text { (Bronze très peux) }\end{array}$ \\
\hline IIIA & Caucasoïdes à mélanosomes porteurs d'eumélanine (cheveux chatins) & + & Claire (normale) & $\begin{array}{l}\text { Brûle modérément } \\
\text { (Bronze graduellement) }\end{array}$ \\
\hline IV & Mongoloïdes (cheveux brun clair) & 0 & Mate (insensible) & $\begin{array}{l}\text { Brûle très peu } \\
\text { (Bronze toujours bien) }\end{array}$ \\
\hline $\mathbf{V}$ & Mongoloïdes (cheveux brun foncé) & 0 & Mate & $\begin{array}{l}\text { Brûle rarement } \\
\text { (Bronze abondamment) }\end{array}$ \\
\hline VI & Négroïdes (cheveux noirs) & 0 & Noire (insensible) & $\begin{array}{l}\text { Ne brûle jamais } \\
\text { (Fortement pigmenté) }\end{array}$ \\
\hline
\end{tabular}


Tableau 5. Effets du soleil sur les peaux claires (D’après M. Déparois, 2014) [27].

\begin{tabular}{lll}
\hline Temps d'exposition & $\begin{array}{l}\text { Rayonnements } \\
\text { solaires }\end{array}$ & Effets engendrés \\
\hline \multirow{3}{*}{ Minutes } & UVB & Synthèse vitamine D \\
\cline { 2 - 3 } & IR & Chaleur/rougeur/sueur \\
\cline { 2 - 3 } & UVA & $\begin{array}{l}\text { Pigmentation } \\
\text { immédiate }\end{array}$ \\
\hline \multirow{3}{*}{ Heures } & UVB & Coup de soleil \\
\cline { 2 - 3 } & UVA & Lucite \\
\hline \multirow{3}{*}{ Jours } & UVB/UVA & Photosensibilisation \\
\cline { 2 - 3 } & UVB & Bronzage \\
\hline Années & UVA & $\begin{array}{l}\text { Épaississement } \\
\text { épidermique }\end{array}$ \\
& UVB-UVA & Cancers cutanés \\
\cline { 2 - 3 } & UVB-UVA & Vieillissement cutané \\
\hline
\end{tabular}

\section{CONCLUSION}

La toxicité des rayons ultraviolets du soleil a été signalée depuis plusieurs décennies et vérifiée par de nombreux travaux scientifiques. Chacun d'entre nous doit connaitre son phototype afin d'anticiper sur les complications pouvant être induites par l'exposition solaire, et c'est en évitant ou en réduisant les expositions et/ou en utilisant efficacement les moyens de la photoprotection exogène.

Les processus de carcinogenèse et de photovieillissement induits par les UV à long terme peuvent être contrebalancés ou atténués par une alimentation riche en polyphénols et en vitamines A, C, E qui sont des antioxydants qui par leur pouvoir scavenger renforcent la photoprotection endogène. L'utilisation de produits cosmétiques photosensibilisants doit être évitée à plus haut point.

En ce qui concerne les médicaments phototoxiques et photoallergisants, dans le cas où leur prescription est incontournable, le médecin et le pharmacien doivent informer le malade de l'obligation d'éviter de s'exposer au soleil et d'utiliser les écrans ou filtres solaires pendant toute la durée du traitement.

Déclaration d'intérêts: les auteurs ne déclarent aucun conflit d'intérêt en rapport avec cet article.

\section{RÉFÉRENCES}

1 Lean J. The sun's variable radiation and its relevance for earth. Annu. Rev. Astron. Astrophys. 1997, 35, 33-67.

2 Zastrow L, Groth N, Klein F, Kockott D, Lademann J, Renneberg R, Ferrero L. The Missing Link - Light-Induced (280-1,600 nm) Free Radical Formation in Human Skin. Skin Pharmacol Physiol 2009;22:31-44. doi: $10.1159 / 000188083$

3. Frank Liebel, Simarna Kaur, Eduardo Ruvolo, Nikiforos Kollias, Michael D. Southall, Irradiation of Skin with Visible Light Induces Reactive Oxygen Species and Matrix-Degrading Enzymes, Journal of Investigative Dermatology, Volume 132, Issue 7, 2012, Pages 1901-1907, https://doi.org/10.1038/jid.2011.476.

4. Svobodova A, Walterova D, Vostalova J (2006) Ultraviolet light induced alteration to the skin. Biomed Pap Med Fac Univ Palacky Olomouc Czech Repub 150: 25-38.

5. Césarini JP et Robert P. Rayons ultraviolets. In Robert P. et col. Dermopharmacologie clinique. Paris : édition Maloine, 1985, 223-230.

6. Giusti AM., Raimondi M., Ravagnan G., Sapora O. and Parasassi T. Human cell membrane oxidative damage induced by single and fractionated doses of ionizing radiation: a fluorescence spectroscopy study. International Journal of Radiation Biology 1998, 74(5), 595-605.

7. Schmitz S, Garbe C, Jimbow K, Wulff A, Daniels H, Eberle J. and Orfanos $\mathrm{CE}$. Photodynamic action of ultraviolet A: induction of hydroperoxides: recent results. Cancer Res. 1995, 139, 43-55.
8. Robertson DG, Epling GA, Kiely JS, Bailey DL and Song B. Mechanistic studies of the phototoxic potential of PD117596, a quinolone antibacterial compound. Toxicology and Applied Pharmacology 1991, 111(2), 221-232.

9. Widel M, Krzywon A, Gajda K, Skonieczna M, Rzeszowska-Wolny J. Induction of bystander effects by UVA, UVB, and UVC radiation in human fibroblasts and the implication of reactive oxygen species. Free Radical Biology and Medicine, 2014, 68, 278-287.

10. Takahashi H., Suzuki Y., Miyauchi Y., Yoshio Hashimoto y., Ishida AY. and lizuka H. Roxithromycin decreases ultraviolet B irradiation-induced reactive oxygen intermediates production and apoptosis of kératinocytes. Journal of Dermatological Science 2004, 34(1), 25-33.

11 Huang W., Bianco A., Brigante M., Mailhot G. UVA-UVB activation of hydrogen peroxide and persulfate for advanced oxidation processes: Efficiency, mechanism and effect of various water constituents. Journal of Hazardous Materials, 2018, 347, 279-287.

12. Brem R., Guven M., Karran P. Oxidatively-generated damage to DNA and proteins mediated by photosensitized UVA. Free Radical Biology and Medicine, 2017, 107, 101-109.

13. Carrara I-M., Melo G-P.,Bernardes S-S, Neto F-S, Zambelli Ramalho LN.,Marinello P-C., Luiz R-C., Cecchini R., Alessandra Lourenco CecchiniA-L. Looking beyond the skin: Cutaneous and systemic oxidative stress in UVB induced squamous cell carcinoma in hairless mice. Journal of Photochemistry \& Photobiology, B: Biology,2019,195,17-26.

14. Pincemail J, Heusele C, Bonté F, Limet R, Defraigne J-O. Stress oxydant, antioxydants nutritionnels et vieillissement. Actualité Médicale Internationale. Métabolismes - Hormones - Nutrition. 2001, 5(4), 158164.

15. Favier A. Le stress oxydant : Intérêt conceptuel et expérimental dans la compréhension des mécanismes des maladies et potentiel thérapeutique. Annales Pharmaceutiques Françaises. 2006, 64(6), 390-396.

16. Darr D. and Fridovich I. Free radicals in cutaneous biology. J. Invest Dermatol. 1994, 102, 671-675.

17. Noonan F-P., De Fabo E-C. UVB and UVA Initiate Different Pathways to p53-Dependent Apoptosis in Melanocytes. Journal of Investigative Dermatology, 2009, 129(7), 1608-1610.

18. Mc Michael M-A. "Oxidative stress, antioxidants, and assessment of oxidative stress in dogs and cats." Journal of Veterinary Medical Associatiation. 2007, 231(5), 714-720.

19. Grandjean, D. Comprendre le stress oxydatif cellulaire chez le chien. Le Nouveau Praticien Véterinaire. 2005, 5(22), 11-16.

20. Godar DE. Preprogrammed and programmed cell death mechanisms of apoptosis: UV-induced immediate and delayed apoptosis. Photochem Photobiol 1996, 63(6), 825-830.

21. Marrot L. and Agapakis-Causse C. Differences in the photogenotoxic potential of two fluoroquinolones as shown in diploid yeast strain (Saccharomyces cerevisae) and supercoiled DNA. Mutation Research 2000, 468(1), 1- 9.

22 Hunting DJ, Gowans BJ, Brasseur N. and Van Lier JE. DNA damage and repair following treatment of $\mathrm{V}-79$ cells with sulfonated phthalocyanines. Photochemistry and Photobiology 1987, 45(6), 769-773.

23. Ogura R, Sugiyama M, Nishii J and Haramaki N. Mechanism of lipid radical formation following exposure of epidermal homogenate to ultraviolet light. J. Invest. Dermatol. 1991, 97(6), 1044-1047.

24. Philipsa N., Smith J., Keller T. and Gonzalez S. Predominant effects of Polypodium leucotomos on membrane integrity, lipid peroxidation, and expression of elastin and matrixmetalloproteinase-1 in ultraviolet radiation exposed fibroblasts, and kératinocytes. Journal of Dermatological Science 2003, 32(1), 1-9.

25. Lenzi F. Contribution à l'étude du stress oxydant cellulaire chez le chien de traineau en course de sprint. Thèse de Doctorat en Médecine Vétérinaire. Campus vétérinaire de Lyon.2011.

26. Wlaschek M, Tantcheva-Poor I, Naderi L, Ma W, Schneider LA, Wolf ZR, et al. Solar UV irradiation and dermal photoaging. Journal of Photochemistry and Photobiology B: Biology. 2001;63(1-3):41-51.

27. Déparois M. Les effets des rayonnements UV sur la peau : les conseils du pharmacien d'officine. Thèse de Doctorat en Pharmacie. Faculté de pharmacie et de médecine Rouan. 2014 
27. Van Schanke A., Jongsma M-J., Bisschop R., Van Venrooij Gemma M. C. A L., Rebel H. and De Gruijl F-R. Single UVB Overexposure Stimulates Melanocyte Proliferation in Murine Skin, in Contrast to Fractionated or UVA-1 Exposure. J Invest Dermatol, 2005, 124(1), 241-247.

28. Giacomoni P-U. Open questions in photobiology III. Melanin and photoprotection. Journal of Photochemistry and Photobiology B: Biology 1995, 29(1), 87-89.

29. Choi W., Miyamura Y., Wolber R., Smuda C., Reinhold W.,Liu H., Ludger Kolbe L. and Hearing V-J. Regulation of Human Skin Pigmentation in situ by Repetitive UV Exposure: Molecular Characterization of Responses to UVA and/or UVB. Journal of Investigative Dermatology, 2010, 130(6), 1685-1696.

30. Poon T-SC., Barnetson R-St-C., and Halliday G-M. Sunlight-Induced Immunosuppression in Humans Is Initially Because of UVB, Then UVA, Followed by Interactive Effects. J Invest Dermatol, 2005;125(4):840 -846.

31 Morison W-L. Effects of ultraviolet radiation on the immune system in humans, Photochem. Photobiol., 1989, 50(4), 515-524.

32 Cavinato M. and Dürr P-J. Molecular mechanisms of UVB-induced senescence of dermal fibroblasts and its relevance for photoaging of the human skin. Experimental Gerontology, 2017, 94, 78-82.

33. Xiaofeng Wang, Hui Hong, Jianping Wu. Hen collagen hydrolysate alleviates UVA-induced damage in human dermal fibroblasts. Journal of Functional Foods, 2019, 63,1-10.

34. Krutmann J. Ultraviolet A radiation-induced biological effects in human skin: relevance for photoaging and photodermatoses. Journal of Dermatological Science 2000, 23 (Suppl.1), S22-S26.

35. De Gruijl FR., Sterenborg HJ. and Forbes PD. The wavelength dependence of skin cancer induction by ultraviolet irradiation of albino hairless mice. Cancer Research. 1993, 53(1), 53-60.

36. Ablard P. Photodermatoses. Photoprotection. EMC - Pédiatrie - Maladies infectieuses $2001 ;[4-115-\mathrm{A}-10]$

37. Schmutz JL. Porphyries. Ann Dermatol Vénéréol. 2007 ;134(5-2) : 73-80. https://doi.org/10.1016/S0151-9638(07)89252-0.

38. Beani J-C. Photodermatoses. EMC, Dermatologie. 2014, [98-785-A-10]. Doi : 10.1016/S0246-0319(14)66854-0

39. Jeanmougin $\mathrm{M}$, Dutartre $\mathrm{H}$, Lorette $\mathrm{G}$.Lucites idiopathiques de l'enfant. Annales de Dermatologie et de Vénéréologie. 2007 ;134(5-2) :38-44. https://doi.org/10.1016/S0151-9638(07)89246-5.

40. Jeanmougin $\mathrm{M}$, Peyron J-L, Thomas $\mathrm{P}$, et al. Lucite estivale bénigne : prévention par un topique associant des filtres anti-UVA et des antioxydants. Annales de Dermatologie et de Vénéréologie 2006 ;133(5):425-428. DOI: 10.1016/S0151-9638(06)70932-2

41 Artz, CE, Farmer, CM \& Lim, HW Polymorphous Light Eruption: une revue. Curr Derm Rep 2019;8:110-116 https://doi.org/10.1007/s13671019-0264-y

42. Hönigsmann, H. (2008), Polymorphous light eruption. Photodermatology, Photoimmunology \& Photomedicine, 24: 155-161. doi:10.1111/j.16000781.2008.00343.x

43. Isedeh P, Lim HW. Polymorphous light eruption presenting as pinhead papular eruption on the face. J Drugs Dermatol. 2013 Nov;12(11):1285-6. PMID: 24196338

65. Wolf P, Byrne SN, Gruber-Wackernagel A. New insights into the mechanisms of polymorphic light eruption: resistance to ultraviolet radiation-induced immune suppression as an aetiological factor. Exp Dermatol. 2009 Apr;18(4):350- 6. doi: 10.1111/j.16000625.2009.00859.x. PMID: 19348001.
45. Hammadi H. Les photodermatoses: démarche diagnostique. El Hakim 2020;27(5):93-8. Disponible: https://elhakim.net/images/PEDIATRIE/Les-photodermatoses.pdf

46. Bourrain JL, Amblard P. Les photoallergies. Revue Française d'Allergologie et d'Immunologie Clinique 1997;37(5):661-667. https://doi.org/10.1016/S0335-7457(97)80064-0.

47. Alora MB, Taylor CR. Solar urticaria: case report and phototesting with lasers. J Am Acad Dermatol. 1998 Feb;38(2 Pt 2):341-3. doi: 10.1016/s0190-9622(98)70579-1. PMID: 9486712

48. BARBAROTS, PLANTIN P. Dermatite atopique photo-aggravée. Ann Dermatol Venereol 2007;1227(5502):3-93, ISSN 0151-9638, http://dx.doi.org/AD-05-2007-134-5-C2-0151-9638-101019-200703029

49. Beani J.C. Les photoallergies graves. Rev.Fr.Allergol 2008;738(4):301-374. http://dx.doi.org/10.1016/i.allerg.2008.02.016

50. Beani J.C. Les photosensibilisations graves. Ann Dermatol Venereol. 2008;1518(1):1-98. http://dx.doi.org/10.1016/i.annder.2008.10.024

51 Adamski H. Actualités des photodermatoses. réalités Thérapeutiques en Dermato-Vénérologie. 2018 ; 277:46-9. Disponible : https://jird.info/wpcontent/uploads/2019/02/06-1.pdf

52 Hinton A-N., Goldminz A-M. Feeling the burn phototoxicity and photoallergy. Dermatologic clinics. 2014, 32(3), 277-290.

53. Beijersbergen van Henegouwen GMJ. Systemic phototoxicity of drugs and other xenobiotics, J. Photochem. Photobiol. B: Biol. 1991, 10(3), 183210.

54. Yamamoto O. and Tokura Y. Photocontact dermatitis and chloracne: two major occupational and environmental skin diseases induced by different actions of halogenated chemicals. Journal of Dermatological Science 2003, 32(2), 85-94.

55. Jeanmougin M. Photosensibilité (phototoxicité et photo-allergie). In Robert P. et al. Dermopharmacologie clinique. Paris : édition Maloine, $1985,223-230$

56. Robert S. Dawe, Sally H. Ibbotson. Drug-Induced Photosensitivity. Dermatologic clinics. 2014, 32(3), 363-368.

57. Umezawa N., Arakane K., Ryu A., Mashiko S., Hirobe M. and Nagano T. Participation of reactive oxygen species in phototoxicity induced by quinolone antibacterial agents. Archives of Biochemistry and Biophysics 1997, 342(2), 275-281.

58. Wagai N. and Tawara K. Possible direct role of reactive oxygens in the cause of cutaneous phototoxicity induced by five quinolones in mice. Archives of Toxicology 1992, 66(6), 392-397.

59. Elma D., Amanda K. Introduction to photobiology. Dermatologic clinics. 2014, 32(3), 255-266.

60. Prusinowski A. Les effets médicaments photosensibilisants : Conseils à l'officine. Thèse de Doctorat en Pharmacie. Faculté de pharmacie de Lille. 2018.

61 Craig A., Cather M., Hui Xu. Photoimmunology. Dermatologic clinics. 2014, 32(3), 277-290.

62 Emmett Edwar A. Evaluation of the photosensitive patient. Dermatologic clinics. 1986, 4(2), 195-202.

63. Yoshiki Tokura. Immune responses to photohaptens: implications for the mechanisms of photosensitivity to exogenous agents. Journal of Dermatological Science. 2000, 23(Supplement1), S6-S7.

64. Fitzpatrick T. Soleil et peau. J Med Esthet. 1975; 2: 33-34.

Cet article a été publié dans le « Batna Journal of Medical Sciences » BJMS, l'organe officiel de « l'association de la Recherche Pharmaceutique - Batna»

Le contenu de la Revue est ouvert « Open Access » et permet au lecteur de télécharger, d'utiliser le contenu dans un but personnel ou d'enseignement, sans demander l'autorisation de l'éditeur/auteur.

Avantages à publier dans BJMS :

- Open access : une fois publié, votre article est disponible gratuitement au téléchargement

Soumission gratuite : pas de frais de soumission, contrairement à la plupart des revues « Open Access »

Possibilité de publier dans 3 langues : français, anglais, arabe

- Qualité de la relecture : des relecteurs/reviewers indépendants géographiquement, respectant l'anonymat, pour garantir la neutralité et la qualité des manuscrits.

Pour plus d'informations, contacter BatnaJMS@gmail.com ou connectez-vous sur le site de la revue : www.batnajms.net 


\section{Bis}

- $\bigcirc$

-

P

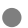

Service de pneumologie, Hôpital Mohammed Seghir El Nekkache, Université d'Alger, Algérie

\section{Correspondance à :}

Samia AISSANI

drs.aissani@hotmail.fr

DOI :https://doi.org/10.48087/ BJMSra.2020.7220

\section{Historique de l'article :}

Reçu le 26 juin 2020

Accepté le 24 septembre 2020

Publié le 09 novembre 2020

Il s'agit d'un article en libre accès distribué selon les termes de la licence Creative Commons Attribution International License (CC BY 4.0), qui autorise une utilisation, une distribution et une reproduction sans restriction sur tout support ou format, à condition que l'auteur original et la revue soient dûment crédités.

Pour citer l'article :

Aissani S, Zitouni A

Association asthme et diabète n'est pas fortuite. Batna J Med Sci 2020;7(2):159-61. https://doi.org/10.48087/B] MSra.2020.7220

\title{
Association asthme et diabète n'est pas fortuite
}

\author{
Asthma and diabetes association is not accidental
}

Samia Aissani, Ali Zitouni

\section{RÉSUMÉ}

L'association asthme et diabète a suscité de nombreuses interrogations du fait de l'augmentation simultanée de la prévalence de ces deux pathologies. Plusieurs facteurs peuvent favoriser la survenue du diabète chez les asthmatiques. La prescription pendant longtemps de fortes doses de corticoïdes associée ou pas à des corticoïdes par voie générale augmentent le risque de diabète particulièrement chez les non contrôlés. Ces corticoïdes sont souvent associés aux béta 2 mimétiques qui peuvent eux aussi entrainer des hyperglycémies. Le risque de diabète chez les asthmatiques se multiplie après 40 ans et lorsqu'il existe une notion de diabète familial. Parmi les facteurs du non contrôle de l'asthme, l'obésité et l'exposition au tabac qui sont des facteurs favorisant l'insulino-résistance responsable de la survenue du diabète chez les asthmatiques. À contrario, l'asthme peut apparaitre plusieurs années après le diabète. La recherche actuelle a montré que le poumon diabétique est le site de changements microangiopathiques tout comme le cœur et le rein. Des modifications pulmonaires induites par le diabète augmentent l'inflammation bronchique et le risque de maladies obstructives comme l'asthme.

Mots clés : asthme, diabète, obésité, tabac, corticoïdes, béta2 mimétiques, microangiopathies

\section{INTRODUCTION}

L'asthme et le diabète représentent deux comorbidités en constante croissance dans le monde. Ce constat n'est pas récent puisque Einar Helander a déjà signalé en 1958, le risque élevé de diabète chez les asthmatiques (1).

Par la suite une étude rétrospective d'une cohorte de 2392 asthmatiques a été faite entre 1964 et 1983 et a retrouvé un taux d'incidence du diabète de 1384 pour 100.000 habitants chez les asthmatiques (2). Chez la femme le risque est aussi élevé. C'est ce qui a été rapporté dans un travail fait chez 38570 femmes asthmatiques ou bronchopathes chroniques, âgées de plus 45 ans. Après 12 mois d'observation, $6.4 \%$ des femmes ont développé un diabète type $2(3)$.

\section{FACTEURS FAVORISANTS LE DIABETE CHEZ LES ASTHMATIQUES}

Plusieurs facteurs peuvent favoriser la survenue du diabète chez les asthmatiques. Il peut s'agir de facteurs liés aux médicaments antiasthmatiques ou au terrain prédisposant (figure1)

\section{ABSTRACT}

The association asthma and diabetes has raised many questions because of the simultaneous increase in the prevalence of these two pathologies. Several factors can favor the occurrence of diabetes in asthmatics. The prescription for long periods of high doses of corticosteroids combined or not with corticosteroids by general route increases the risk of diabetes especially in uncontrolled cases. These corticosteroids are often given with beta 2 mimetics which can also lead to hyperglycemia. The risk of diabetes in asthma increases after 40 years and when there is a notion of familial diabetes. Among the factors of non-control of asthma, obesity and exposure to tobacco which also factors promoting insulin resistance and therefore inflammation responsible for the occurrence of diabetes in asthmatics. Conversely, asthma can appear several years after diabetes. Current research has shown that the diabetic lung is the site of microangiopathic changes just like the heart and the kidney. Diabetes-induced lung changes increase bronchial inflammation and the risk of obstructive diseases like asthma.

Keywords: asthma, diabetes, obesity, tobacco, corticoids, beta 2 mimetics, microangiopathies

\section{Les médicaments antiasthmatiques} diabétogènes

$>\quad$ Les corticoïdes au long cours par voie générale entrainent une insulinorésistance et multiplient ainsi le risque de diabète par 2 ou 3 . L'intolérance au glucose et la diminution de la sensibilité à l'insuline sont fréquentes et peuvent induire un diabète de type 2 ou aggraver un diabète préexistant (4).

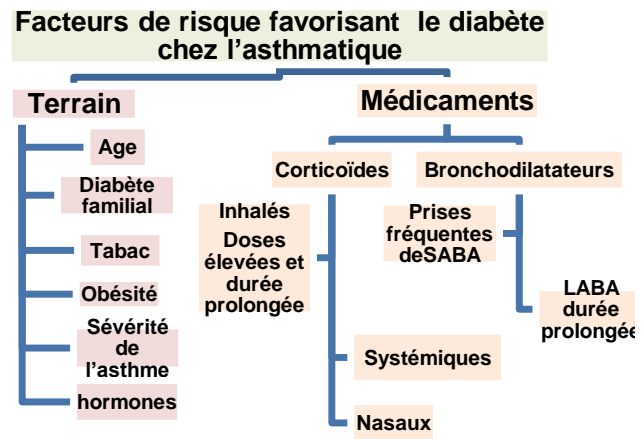

Figure 1. Facteurs de risque de diabète chez l'asthmatique. SABA : bronchodilatateur de courte durée d'action. LABA : bronchodilatateur de longue durée d'action. 
2. Le terrain

$>\quad$ Age

Le risque de diabète surtout de type 2 augmente avec l'âge (8). L'âge moyen de survenue du diabète de type 2 dépasse 40 ans (9) ; Ce qui fait recommander la recherche du diabète chez les quadragénaires en particulier les asthmatiques.

\section{Diabète familial}

Les personnes dont un parent est atteint de diabète de type 2 , sont prédisposées au diabète en raison de l'existence de plusieurs facteurs communs: environnementaux, nutritionnels et génétiques (10).

\section{$>\quad$ Exposition régulière au tabac}

Le tabagisme actif ou passif augmente les risques de développer un diabète de type 2. Cet effet est en partie lié à l'augmentation du risque de syndrome métabolique surtout chez les fumeurs (11).

La nicotine semble également exercer un effet toxique direct au niveau du pancréas et des récepteurs à l'insuline et induit une hyperglycémie et une résistance à l'insuline (12). De plus, le tabagisme induit une inflammation chronique qui peut également contribuer au développement du diabète (13).

Tout comme le risque de diabète, le tabac favorise l'hyperréactivité bronchique et donc risque de survenue de l'asthme et du non contrôle de l'asthme (14). On sait à l'heure actuelle que l'exposition au tabac augmente le risque de développer un asthme chez l'enfant.

Chez l'adulte, les résultats restent controversés sur l'effet du développement de l'asthme. Ce qui est certain ce qu'il entraine un non contrôle de l'asthme (15) par augmentation de la corticorésistance (16).

Au Final, le tabagisme augmente les risques de développer les deux comorbidités l'asthme et le diabète.

\section{Obésité}

L'asthme et l'obésité ont considérablement progressé durant les deux dernières décennies dans la plupart des pays du Monde. Il ne pourrait pas s'agir d'une simple coïncidence liée aux modifications de notre mode de vie puisqu'un certain nombre de données objectives épidémiologiques, cliniques et physiopathologiques plaident pour un lien réel entre les deux affections.

L'obésité est identifiée comme facteur de risque d'un nombre non négligeable de maladies chroniques dont l'asthme et le diabète (figure 2). Elle représente un des facteurs de sévérité et du non contrôle de l'asthme $(17,18)$. La sévérité de l'asthme chez les obèses impose des doses élevées de corticoïdes inhalées avec parfois plusieurs cures de corticoïdes par voie générales et par an (19) pouvant majorer plus l'obésité et augmenter le risque de diabète (figure 1).

\section{$>\quad$ Les hormones}

La particularité chez la femme obèse réside dans la double influence de l'obésité sur l'inflammation bronchique. D’une part, elle stimule l'action des adipocytes et par conséquent libération de nombreux médiateurs de l'inflammation (20).

D'autre part, elle peut augmenter les taux d'estradiol par transformation androgénique au niveau du tissu adipeux (21). Des taux élevés d'œstrogènes sont connus pour avoir un effet pro-inflammatoire sur la bronche des asthmatiques et par conséquent un non contrôle de l'asthme. (22).

A noter que l'obésite et l'hypereostrogénie sont deux facteurs de risque de l'insulinorésistance et par conséquent du diabete (23).

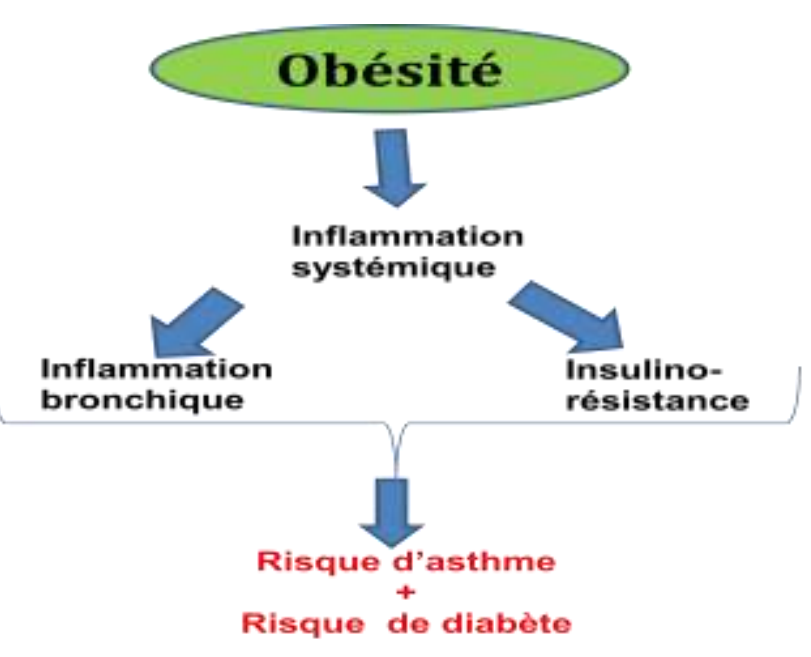

Figure 2. Effets de l'obésité sur l'asthme et le diabète.

\section{FACTEURS FAVORISANT LA SURVENUE DE L'ASTHME CHEZ LES DIABETIQUES}

L'asthme se caractérise par une réaction inflammatoire chronique médiée par les cellules T helper type 2 (Th2). Au cours du diabète de type 1 , les cellules Th1 et Th 2 s'opposent réciproquement les uns aux autres et l'équilibre entre leurs réponses fournit une explication possible (24) ; Ceci est aussi impliqué dans la physiopathologie de l'asthme notamment chez les diabétiques (25).

En plus de la lipotoxicité fréquemment retrouvée chez les diabétiques surtout de type 2 , la recherche actuelle a montré que le poumon diabétique est le site de changements microangiopathiques tout comme le cœur et le rein (26). Tout récemment, on a démontré l'effet néfaste de l'hyperglycémie sur le poumon.

L'insuline inhibe la prolifération des cellules T helper de type 1 au profit des $\mathrm{T}$ helper type 2 et favorise la survie des mastocytes, la dégranulation et la libération d'histamine. Elle active les macrophages inflammatoires pulmonaires et prolifère et contracte les cellules des muscles lisses des voies respiratoires. Elle augmente le dépôt de la matrice extracellulaire dans les poumons et favorise la fibrose. Au final, à défaut de l'insuline, le fonctionnement du poumon sera altéré et deviendra le site de changements microangiopathiques. La conséquence des modifications pulmonaires induites par le diabète est l'augmentation du risque des maladies respiratoires obstructives comme l'asthme, la BPCO (bronchopneumopathie chronique obstructive), des maladies inflammatoires du poumon comme les pneumopathies interstitielles et la fibrose, sans oublier le risque accru d'infections respiratoires (27).

\section{CONCLUSION}

L'association asthme et diabète est un phénotype particulier. Cette catégorie de patients asthmatiques et diabétiques nécessite une attention particulière car quel que soit le mécanisme, les soins sont le plus souvent complexe et couteux.

Afin de diminuer le risque de diabète chez les asthmatiques, il faut toujours justifier la prescription des corticoïdes quel que soit la présentation clinique, rechercher les signes de diabète chez les asthmatiques surtout après plusieurs années de corticothérapie même inhalée. Il est indispensable aussi de prendre en charge l'obésité qui un facteur de risque commun pour l'asthme et le diabète. Par ailleurs, des études sont nécessaires afin d'apprécier le risque de l'asthme chez les diabétiques. 


\section{POINTS IMPORTANTS}

Les médicaments antiasthmatiques peuvent augmenter le risque de diabète. Donc à rechercher systématiquement après plusieurs années de prescription

L'âge, le diabète familial, le tabagisme passif ou actif, l'obésité et les œstrogènes chez la femme augmentent l'insulinorésistance et par conséquent le diabète.

Le risque d'asthme chez les diabétiques est élevé en raison du déficit en insuline dont l'effet est anti-inflammatoire sur la muqueuse bronchique.

Déclaration d'intérêts: les auteurs ne déclarent aucun conflit d'intérêt en rapport avec cet article.

\section{RÉFÉRENCES}

1. Helander E. Asthma and diabetes. Acta Med Scand (1958) 162:165-74. doi:10.1111/j.0954-6820.1958.tb01762.

2. Juhn Y.J, Asthma may be linked to increased incidence of diabetes, heart disease. Annual Meeting of the American Academy of Allergy, Asthma \& Immunology; San Francisco, March, 2011; 18-22.

3. Song Y, Klevak A, Manson JE, Buring JE, Liu S. Asthma, chronic obstructive pulmonary disease, and type 2 diabetes in the Women's Health Study. Diabetes Res Clin Pract. 2010 Dec;90(3):365-71. doi: 10.1016/j.diabres.2010.09.010. Epub 2010 Oct 6. PMID: 20926152; PMCID: PMC2993844.

4. Pasieka AM, Rafacho A. Impact of glucocorticoid excess on glucose tolerance: clinical and preclinical evidence. Metabolites. 2016; 6:24.

5. Lipworth BJ. Systemic adverse effects of inhaled corticosteroid therapy. A systemic review and meta-analysis. Arch Intern Med. 1999;159:941-55.

6. Gulliford $\mathrm{MC}$ et col. Risk of diabetes associated with prescribed glucocorticoids in a large population. Diabetes Care. 2006, 29: 27282729.

7. Nanda A, Baptist AP, Divekar R, Parikh N, Seggev JS, Yusin JS, Nyenhuis SM. Asthma in the older adult. J Asthma. 2019; 18:1-12.

8. Saeedi P, Petersohn I, Salpea P, Malanda B, Karuranga S, Unwin N, et al. IDF Diabetes Atlas Committee. Global and regional diabetes prevalence estimates for 2019 and projections for 2030 and 2045: Results from the International Diabetes Federation Diabetes Atlas, $9^{\text {th }}$ edition. Diabetes Res Clin Pract. 2019 Nov ;157:107843. doi: 10.1016/j.diabres.2019.107843. Epub 2019 Sep 10. PMID : 31518657.

9. Bendinelli B, on behalf of the InterAct Consortium. Association between dietary meat consumption and incident type 2 diabetes: the EPIC-InterAct study. Diabetologia 2012; 56: 47e59.

10. Prudente S, Ludovico O, Trischitta V. Familial diabetes of adulthood: A bin of ignorance that needs to be addressed, Nutrition, Metabolism \& Cardiovascular Diseases. 2017; 27: 1053-1059
11. Kan Sun, Jianmin Liu, Guang Ning. Active Smoking and Risk of Metabolic Syndrome: A Meta-Analysis of Prospective Studies, PLOS ONE. 2012:7,10, 47791.

12. Facchini FS, Hollenbeck $C B$, Jeppesen J, et al. Insulin resistance and cigarette smoking. Lancet 1992;339: 1128-30.

13. Burke A, Fitzgerald GA. Oxidative stress and smoking-induced vascular injury. Prog Cardiovasc Dis 2003; 46:79-90

14. Prieto L, Gutiérrez V, Uixera S, Bertó JM.Effect of Cigarette Smoking on Airway Responsiveness to Adenosine 5'-Monophosphate in Subjects With Allergic Rhinitis, Chest. 2003 ;123(4):993-7.

15. Peiffer $\mathrm{G}$, Underner $\mathrm{M}$, Perriot J. Les effets respiratoires du tabagisme. Rev Pneumol Clin. 2018 ; 74(3) : 133-144.

16. Girodet PO. Quelle est l'efficacité des corticoïdes chez l'asthmatique qui fume. Rev Mal Respir 2008;25:185-92.

17. Saint-Pierre P, Bourdin A, Chanez P, Daures JP, Godard P. Are overweight asthmatics more difficult to control? Allergy Eur J Allergy Clin Immunol. 2006;61(1):79-84

18. Hersoug LG, Linneberg A. The link between the epidemics of obesity and allergic diseases: Does obesity induce decreased immune tolerance? Allergy Eur J Allergy Clin Immunol. 2007;62(10):1205-13.

19. Boulet L-P et Lessard A. Le rôle de l'obésité dans le développement de I'asthme : Évaluation et traitement de l'asthmatique obèse. Le clinicien. 2007: 5e symposium international de Québec sur la prévention/réadaptation cardiorespiratoire :83-88

20. Sismanopoulos N, Delivanis D, Mavrommati D, Hatziagelaki E, Conti P. Do mast cells link obesity and asthma? Eur J allergy Clin Immunol. 2013;68(19):8-15.

21. Zeitoun KM, Bulun SE. Aromatase: A key molecule in the pathophysiology of endometriosis and a therapeutic target. Fertil Steril. 1999;72(6):961-9.

22. Aissani S. Asthme et statut hormonal chez la femme [Internet]. Alger; 2017. Available from: Thèse de doctorat en sciences médicales.

23. Van Den Berge M, Heijink HI, Van Oosterhout AJM, Postma DS. The role of female sex hormones in the development and severity of allergic and non-allergic asthma. Clin Exp Allergy. 2009;39(10):1477-81.

24. Cardwell CR, Shields MD, Carson DJ, et al. A meta-analysis of the association between childhood type 1 diabetes and atopic disease. Diabetes Care. 2003;26:2568-2574.

25. Hsiao YT, Cheng WC, Liao WC, et al. Type 1 Diabetes and Increased Risk of Subsequent Asthma: A Nationwide Population-Based Cohort Study. Medicine (Baltimore). 2015;94(36):e1466. doi:10.1097/MD.0000000000001466

26. Popov D. Is lung a target of diabetic injury? the novel pro and cons evidences Proc. Rom. Acad., Series B, 2013, 15(2): 99-104.

27. Kolahian S, Leiss V, Nürnberg B. Diabetic lung disease: fact or fiction? Reviews in Endocrine and Metabolic Disorders. 2019; 20:303-319.

Cet article a été publié dans le « Batna Journal of Medical Sciences » BJMS, l'organe officiel de « l'association de la Recherche Pharmaceutique - Batna»

Le contenu de la Revue est ouvert « Open Access » et permet au lecteur de télécharger, d'utiliser le contenu dans un but personnel ou d'enseignement, sans demander l'autorisation de l'éditeur/auteur.

Avantages à publier dans BJMS :

- Open access : une fois publié, votre article est disponible gratuitement au téléchargement

- Soumission gratuite : pas de frais de soumission, contrairement à la plupart des revues « Open Access »

- Possibilité de publier dans 3 langues : français, anglais, arabe

- Qualité de la relecture : des relecteurs/reviewers indépendants géographiquement, respectant l'anonymat, pour garantir la neutralité et la qualité des manuscrits.

Pour plus d'informations, contacter BatnaJMS@gmail.com ou connectez-vous sur le site de la revue : www.batnajms.net 


\section{$\mathrm{B}$.}

- $\bigcirc$

-

P

Psychiatre libéral

Cité Larbi Benredjem

43000 Mila Algérie

Correspondance à :

Zoubie BENMEBAREK

zoubirbenmeb@gmail.com

DOI :https://doi.org/10.48087/ BJMSra.2020.7221

\section{Historique de l'article :}

Reçu le 28 juin 2020

Accepté le 24 septembre 2020

Publié le 09 novembre 2020

Il s'agit d'un article en libre accès distribué selon les termes de la licence Creative Commons Attribution International License (CC BY 4.0), qui autorise une utilisation, une distribution et une reproduction sans restriction sur tout support ou format, à condition que l'auteur original et la revue soient dûment crédités.

Pour citer l'article :

Benmebarek Z. Les thérapies comportementales et cognitives : une perspective islamique. Batna J Med Sci 2020;7(2):162-6. https://doi.org/10.48087/B JMSra.2020.7221

\title{
Les thérapies comportementales et cognitives : une perspective islamique
}

\author{
Cognitive behavioural therapy : an Islamic perspective
}

\author{
Zoubir Benmebarek
}

\section{RÉSUMÉ}

Depuis leur introduction en pratique clinique dans les années 70 , les thérapies comportementales et cognitives (TCC) ont connu un succès inégalé vu leur caractère empirique et leur efficacité dans le traitement de nombreux troubles psychiatriques. Ceci a encouragé leur adoption par la majorité des praticiens à travers le monde entier. Issues de la psychologie expérimentale, les TCC ont été développées essentiellement en occident et les principales études publiées concernaient des patients venant de cette région du monde. Pour appliquer les TCC à des patients d'autres cultures, il a toujours été nécessaire d'apporter des modifications soit dans leurs principes théoriques soit dans leurs techniques afin de les rendre compatibles aux autres contextes socioculturels et religieux. Dans le monde musulman, bien que globalement acceptées, les TCC ont fait l'objet d'évaluations et de critiques quant à leur harmonie avec les valeurs de l'Islam. Une littérature scientifique de plus en plus abondante, surtout en langue anglaise, aborde cette thématique bien qu'elle reste, à ce stade, essentiellement limitée à l'aspect théorique. Ce courant est quasi inexistant dans les pays francophones et le but de cette brève revue est de relever les points saillants des études sur les TCC vues d'une perspective islamique.

Mots-clés: Thérapie comportementale et cognitive, culture, religion, Islam.

\section{INTRODUCTION}

Les psychothérapies sont un outil thérapeutique important dans la prise en charge des troubles psychiatriques, pratiquées seules ou associées à une chimiothérapie médicamenteuse.

Les thérapies comportementales et cognitives (TCC) sont des psychothérapies reconnues parmi les six grandes familles d'aide psychologique. Leurs indications spécifiques, leur déroulement sur une période limitée de quelques mois et leur grande efficacité ont contribué à leur large développement [1].

Elles sont actuellement les seules qui se soient développées en étroite collaboration avec des recherches expérimentales. Dès leur introduction elles ont gagné la réputation de scientificité au point que certains affirment qu'ils ont réalisé pour la psychothérapie ce que Claude Bernard a fait pour la médecine [2].

La nature et le degré de ces ajustements est diversement apprécié; des professionnels de santé mentale de confession musulmane formulent assez souvent des critiques concernant les fondements théoriques et certaines applications des TCC.

\begin{abstract}
Since the 70s, Cognitive behavioural therapy (CBT) has become the most widely evidence-based practiced psychotherapy encompassing a large array of psychiatric disorders. It has spread across all countries and has been adopted by most physicians around the world. CBT was, essentially, conceptualized by and for westerners and most scientific data dealing with the subject stems from North America or Europe. It has always needed adjustment or modification, in its theoretical framework or in its techniques, to fit in other cultures, societies and religions outside the West. Muslim mental health professionals have highlighted that CBT, although generally well accepted, needs to be reviewed in some of its tenets to accommodate Muslim patients. Growing English- speaking scientific researches have been published in the last years dealing mainly with theoretical aspects; empiric research still lags behind. There is a dearth in French- speaking scientific publications about such a subject. This mini review is an attempt to bring this topic to the fore and to sketch the prominent findings and features of CBT from an Islamic perspective.
\end{abstract}

Keywords: Cognitive behavioural therapy, culture, religion, Islam.

Le travail de théorisation et de réalisation d'études empiriques sur des patients musulmans est de plus en plus prolifique au vu des publications scientifiques disponibles. Ceci est à même d'aboutir à l'adaptation de ces techniques au contexte culturel musulman et d'en faire bénéficier le maximum de patients $[3,4]$.

\section{DEFINITION - HISTORIQUE- PRINCIPES THEORIQUES ET TECHNIQUES}

Les thérapies comportementales et cognitives représentent l'application scientifique de la psychologie expérimentale. Elles mettent l'accent sur l'utilisation d'une méthodologie expérimentale afin de comprendre et de modifier les troubles psychologiques [5].

Leur origine remonte à la fin des années 1920 par la naissance de la psychologie de l'apprentissage. Elles connaissent un essor à partir des années 50-60 avec extension de la psychologie comportementale (théorie de l'apprentissage social, conditionnement opérant...L puis intégration de la psychologie cognitive (traitement de l'information). Elles se répandent comme psychothérapie dès les années 1970 d'abord pour la dépression puis pour d'autres troubles psychiatriques $[1,2]$. 
Elles se répandent comme psychothérapie dès les années 1970 d'abord pour la dépression puis pour d'autres troubles psychiatriques $[1,2]$.

Elles trouvent leur indication dans un large éventail de troubles psychiques allant des troubles anxieux et thymiques aux troubles psychotiques et des troubles d'adaptation aux troubles de la personnalité [6].

Plusieurs rapports institutionnels sur l'efficacité des psychothérapies ont donné un grade A (efficacité démontrée) dans de nombreuses indications, en particulier le rapport de l'Institut national de la santé et de la recherche médicale (Inserm) français de 2004 [7].

Leur base théorique repose sur 03 points essentiels :

L'activité cognitive affecte le comportement.

L'activité cognitive peut être étudiée, évaluée et modifiée.

Le changement du comportement est possible via le changement des cognitions. [8]

Les techniques se résument aux techniques d'exposition (désensibilisation systématique, exposition in vivo, prévention de la réponse, arrêt de la pensée, immersion...) et aux techniques d'apprentissage social (jeu de rôle, apprentissage vicariant ou modeling...) et les méthodes cognitives (pensées automatiques, distorsions cognitives, postulats et schémas...) [7,9].

La mise en pratique répond à un ensemble de principes: la conceptualisation $\mathrm{du}$ trouble en terme cognitif et comportemental, l'éducation, la collaboration et la participation active de la part du patient. La thérapie est limitée dans le temps et orientée vers un but défini, se focalise sur le présent, les sessions sont structurées, les techniques variées et personnalisées selon chaque patient [6]

\section{TCC ET CULTURE OCCIDENTALE}

Le principe du naturalisme séculier infiltre toute la culture occidentale politique, économique et s'étend aux sciences humaines. Parfois il exprime non pas une séparation entre le profane et le sacré mais une position antireligieuse. La distanciation de la psychologie par rapport à la religion est un fait établi, souvent indiscutable allant parfois jusqu' à l'affirmation que l'implication de la religion dans la psychologie est contreproductive et préjudiciable $[10,11]$.

Quand on évoque le terme psychologie tout court (ceci est aussi valable pour les psychothérapies y compris les TCC) cela présuppose implicitement une psychologie universelle alors qu'en réalité elle renvoie à la psychologie occidentale aux fondements théoriques séculiers et naturalistes et qui puise ses influences dans la culture gréco-romaine et dans la tradition judéo-chrétienne. Et la majorité des études scientifiques publiées ayant trait à la pratique de ces thérapies concernent des sujets venant de cette sphère culturelle que ce soit les chercheurs qui mènent ces études ou les patients objets de ces études [3].

En Amérique les recherches concernent essentiellement l'américain de la classe moyenne, de descendance européenne, hétérosexuel et de confession judéo chrétienne [12]. Certains ont fait le lien entre les TCC surtout sa variante la thérapie émotivo-rationnelle d'Ellis et le judaïsme arguant que les techniques de ces thérapies ont emprunté à cette religion beaucoup de ses préceptes et de ses idées fondamentales ( une compréhension honnête et sincère de soi est l'élément clé pour l'amélioration des compétences personnelles, l'intellect est capable de diriger les émotions, le comportement extérieur influence les pensées et les émotions, importance de l'autonomie, le bonheur et le malheur sont déterminés par l'homme lui-même....) [13].

L'hégémonie de ces conceptions est de plus en plus critiquée $\mathrm{au}$ vu de leurs limites de rendre compte de toute la complexité du psychisme humain dans sa diversité culturelle $[11,14]$. Il est impossible de rassembler tous les êtres humains sous une seule théorie explicative car même le rationnel et l'irrationnel ne sont pas évidents et consensuels pour tout le monde et sont infiltrés de valeurs culturelles et les indicateurs de santé mentale et de maladie ne sont pas universels; ce qui peut promouvoir le bien-être dans une société peut ne pas l'être dans une autre [15,16].

Il y a un besoin d'adapter la TCC aux diverses conditions sociales, ethniques, culturelles et religieuses et de tenir compte de ces différences dans la conceptualisation théorique et pratique afin d'améliorer la prise en charge, d'optimiser les résultats positifs et permettre à travers des approches créatives d'atteindre et de faire bénéficier un large éventail de population de patients [17].

\section{PSYCHOLOGIE, TCC ET ISLAM}

L'islam est la deuxième grande religion dans le monde avec 1.8 milliard de fidèles et deviendra la première religion monothéiste au monde en 2060 [18].

Il est à la fois culte et culture, spirituel et temporal. Son code de conduite s'étend de l'infime détail de la vie humaine aux questions majeures existentielles de la vie et fournit des réponses autant au pourquoi qu'au comment des choses.

Le Prophète Mohammed (QSSL) représente pour le musulman l'homme parfait qui a atteint le plus haut degré de soumission à Allah et de conformité aux préceptes de l'Islam. Il est l'exemple à suivre dans le chemin de la spiritualité et le modèle à imiter dans l'application des prescriptions religieuses.

La psychologie islamique est une approche indigène visant l'étude et la compréhension de la psychologie humaine instruite par l'enseignement et les connaissances tirés du Coran et de la Sunnah (tradition prophétique) [14,19]. Elle prend racine dans le paradigme ontologique qui est exprimé par la tradition islamique plutôt que dans les paradigmes occidentaux.

Les psychothérapies notamment les TCC découlent et suivent ce modèle quant à leur théorisation et leur pratique en intégrant les principes islamiques ou en adoptant les principes des TCC qui ne sont pas en conflits avec les préceptes de l'Islam [19].

Peu de praticiens ont été formés ou possèdent les connaissances requises pour aborder la TCC à partir d'une perspective islamique. Les praticiens sont en général diplômés des écoles laïques et naturalistes qui ne reconnaissent pas ou ne donnent pas de l'importance aux croyances religieuses.

Ceux qui sont intéressés par l'incorporation des conceptions islamiques dans les psychothérapies et dans les TCC le feront par un effort personnel mais ceci a ses limites et reste un travail de rapiéçage plutôt qu'une application méthodique d'une approche intégrative scientifiquement établie [19].

\section{TCC ET ISLAM : POINTS DE CONVERGENCES ET DE DIVERGENCES}

Ces dernières années ont connu une abondance de publications scientifiques établissant la différence entre une TCC occidentale et une TCC islamique. Le talon d'Achille de ces études réside dans leur aspect théorique et spéculatif se basant sur des arguments théologiques et la pauvreté des études empiriques scientifiquement validées qui pourraient 


\section{Points de divergences :}

Parmi les points de divergences théoriques Beshai et al. [3] relèvent :

Constructivisme versus réalisme: pour la TCC occidentale la réalité objective n'existe pas; elle est le fruit d'une construction idéique de l'individu tandis qu'en Islam la réalité objective existe bel et bien; elle reste cependant inaccessible aux sens dans sa globalité. Seul le Coran est le repère tangible et palpable de la réalité objective. La portée pratique de cette différence se traduit par exemple dans la discussion des postulats fondamentaux ou obligations morales en TCC (should / should not). Ces derniers sont généralement l'objet d'intervention thérapeutique en vue de leur modification ou leur réinterprétation alors qu'en Islam ces postulats ou obligations ne sont pas considérées comme pathologiques tant qu'ils sont compatibles avec les principes de l'Islam (respect des parents, abstention de l'alcool, chasteté...).

Empirisme versus science : En TCC occidentale le thérapeute et le patient rassemblent les preuves (empirisme collaboratif concernant une cognition dysfonctionnelle par exemple) et tirent des conclusions à postériori ( sans aucun préalable défini) alors qu'en Islam la science et l'empirisme sont considérés comme une extension de la théologie car provenant de la même source (Allah) et devraient être compatibles avec les principes de l'Islam qui sont déterminés à priori (notion de cognitions dysfonctionnelles et la façon de les modifier doivent répondre à cet impératif).

Origine de la détresse des individus : En TCC occidentale les individus sont considérés comme responsables de leurs malheurs (schémas cognitifs dysfonctionnels appris dès l'enfance) alors qu'en Islam le bien et le mal viennent ou sont permis par Allah (la maladie est alors une épreuve ou un châtiment).

Modification des comportements et des émotions : En TCC occidentale le changement comportemental fait suite aux modifications cognitives (relation causale) alors qu'en Islam le changement comportemental ne suit pas automatiquement les modifications cognitives sauf si Allah le veut.

Liberté et autocontrôle : en TCC occidentale les individus sont libres et ont un contrôle sur leurs actes alors qu'en Islam les individus ne sont pas totalement libres; des influences métaphysiques peuvent interférer avec leur liberté.

Les droits individuels: en TCC occidentale l'intérêt personnel prime sur l'intérêt collectif alors qu'en Islam c'est l'intérêt collectif qui prévaut.

Jafari [20] cite aussi d'autres éléments de divergence dans l'approche psychothérapique et qui sont aussi valables pour les TCC :

Perspective matérialiste versus perspective holistique: en occident les critères de succès sont basés sur les faits purement matériels (supériorité, santé physique, réussite sociale et professionnelle, bénéfices matériels) tandis que I'Islam encourage ses adhérents à donner importance autant aux réalisations matérielles qu'au développement spirituel et moral qui sont enchevêtrés et interdépendants.

Rationalisation de la culpabilité versus la repentance: dans la culture occidentale les comportements non désirés qui provoquent le sentiment de culpabilité sont surmontés par la rationalisation qui facilitera la réhabilitation; le psychothérapeute fera part de compréhension, soutien et empathie inconditionnée envers son patient. Alors qu'en Islam l'approche de la culpabilité se fera plutôt par la repentance qui aiderait le patient à revoir et modifier ce qui est considéré comme comportement indésirable voire un péché.

Autres points de dissonances relevés par différents auteurs [15, 21-24] :

Le style de communication explicite et direct, la notion d'épanouissement personnel, la question du genre, l'égalité entre hommes et femmes et leurs rôles respectifs, le locus de contrôle interne, l'entrainement aux habiletés sociales et l'assertivité tels préconisés par les TCC posent des difficultés dans la thérapie si elles sont prises sans considération contextuelle.

L'Islam tend à promouvoir l'interdépendance, l'épanouissement de la communauté, encourage un style de communication implicite qui prend en considération l'opinion des autres, le locus de contrôle est dirigé vers la famille, la communauté et à Allah ; le rôle de l'homme et de la femme et la notion d'égalité sont perçus différemment, la voie pour le bien- être est de se tourner vers Allah ce qui procure la satisfaction et le bonheur. Avoir une bonne opinion et positive des autres, une vision optimiste de l'avenir surtout une confiance infaillible envers Allah sont des éléments importants pour la santé mentale du point de vue islamique.

Les exercices à faire et les tâches à accomplir entre les sessions de TCC sont parfois difficiles à appliquer ; le patient musulman n'est pas toujours habitué à ce genre de coopération active et d'implication dans le processus psychothérapique et s'attend souvent à jouer un rôle passif et a besoin d'être dirigé. Enfin la relation entre effort et résultat n'est pas rigidement causale. Bien que l'Islam encourage et stimule l'effort et la persévérance il n'en fait pas du résultat une conséquence directe de l'effort fourni ; celui-ci reste soumis à la volonté divine.

\section{Points de convergence}

Ce n'est pas le processus de restructuration cognitive luimême qui est en conflit avec les principes islamiques mais le système de valeurs véhiculées par cette restructuration [4]. Et ce n'est pas toutes les valeurs car on retrouve certains aspects de la philosophie de base du comportementalisme qui ont des points de convergence avec les principes de l'islam dans la façon de changer un comportement [25]. La souplesse et à la malléabilité des TCC, l'aspect actif, pratique, directif et orienté vers un but des exercices des thérapies comportementales sont des éléments aisés à comprendre et à adopter par le musulman car en harmonie avec ses concepts religieux [4, $15,22]$.

L'importance donnée à la raison et à la logique, le caractère empirique et scientifique, la discussion et l'échange, et l'aspect psycho éducatif et instructif, l'insistance sur l'ici et le maintenant, le refus de puiser en problèmes personnels et intimes sont des points qui facilitent l'acceptation de la TCC $[4,22,25,26]$.

La notion de contrat thérapeutique, l'exposition, la désensibilisation, la relaxation et l'apprentissage social par imitation ou modeling sont facilement acceptés par les musulmans pour qui, par exemple, suivre un modèle comme le père, une figure religieuse et surtout le Prophète Mohammed a une signification importante et primordiale [26]. 


\section{CONSIDERATIONS PRATIQUES}

Avant d'exposer certains éléments pratiques des principes des TCC dans le contexte islamique il est utile de rappeler certains principes directeurs qui doivent être pris en considération quand on aborde ces techniques :

Pas mal de patients musulmans sont suspicieux face à la psychologie occidentale séculière qu'ils considèrent non adaptée à leur situation. Ceci pourrait expliquer, en plus de la croyance religieuse, le recours systématique et en premier lieu à la Rokia où le discours narratif s'inscrit et puise dans le modèle explicatif de la maladie partagé et accepté par la société.

L'islam est un élément central dans la vie des musulmans et le thérapeute doit y prêter attention car il affecte leurs vies à des niveaux multiples et différents [16].

L'islam est multidimensionnel et il faut demander ce qu'il veut dire pour chaque patient ; il est compris et appliqué différemment; éviter une vision simpliste et une surgénéralisation.

Savoir tirer les éléments positifs d'adaptation puisés dans la religion [16].

Les luttes psychiques religieuses islamiques peuvent nuire au patient, il faut savoir s'enquérir de ces crises de conscience, les normaliser et leur trouver des solutions satisfaisantes ; au besoin se faire aider par un imam [16].

La stigmatisation associée aux problèmes de santé mentale est répandue parmi les musulmans; essayer régulièrement d'instruire la population et favoriser la prise de conscience au sujet des troubles mentaux [16].

Expliquer les mécanismes et la logique des TCC en se référant à la tradition islamique aiderait les patients à devenir familier avec cette technique [25].

La thérapie n'est pas le lieu de changement de la culture ; celle-ci a sa propre logique elle est le fruit d'une expérience collective et de sagesse cumulée; s'attaquer aux croyances du patient peut lui nuire; c'est est une cause perdue d'avance et favorise l'arrêt du suivi [26].

\section{Quelques particularités pratiques d'une TCC islamique}

Appliquée de façon méthodique, étudiée et personnalisée, une TCC qui s'inspire des principes de l'Islam apportera des bénéfices supplémentaires au patient tels: meilleure observance thérapeutique, rétablissement rapide, moins de rechute et réduction des inégalités et disparités thérapeutiques [21].

On citera de manière non exhaustive [4,25, 27-31] :

- Comprendre la réalité temporale de ce Monde.

- Se concentrer sur l'Au-delà.

- Prendre conscience du sens de toute détresse ou affliction.

- $\quad$ Faire confiance et recourir à Allah (Tawakol).

- Se concentrer sur les bienfaits d'Allah.

- Se remémorer Allah (Dhikr) et lire le Coran.

- Imprécations (Du’aa).

- La prière : une forme de méditation de pleine conscience et une forme de relaxation; faciliterait le travail sur les pensées automatiques (métacognition).

- Le sens de repentance et de pardon (pour les patients déprimés qui ressentent de la culpabilité).
- $\quad$ Contemplation (Tafakur) : pensée profonde et prolongée partant de la création vers le Créateur.

- Thérapie opposée en imagination (tirée d'Al Ghazali) [31].

- Comparaison sociale (tirée de la théorie de comparaison sociale) : en Islam elle se fera avec ceux qui sont d'un rang inférieur et non avec ceux d'en haut, cette méthode cognitive est utile dans la dépression.

- L'effort, la persévérance la réussite sont encouragés mais leur résultat sera attribué à la volonté d'Allah.

- Compassion : avoir un schéma cognitif basé sur la compassion envers toute la création.

- Le code de conduite coranique favorise une bonne morale et un comportement pro social.

- Le pouvoir de la suggestion à son influence sur la modification du comportement.

- Gestion et contrôle de la colère dont l'approche islamique est originale et diffère de la manière occidentale.

- L'activation comportementale, technique qui encourage l'augmentation progressive de l'activité insistant sur celles qui procurent un sens de réussite et/ou de plaisir, est un concept qui est en harmonie avec les préceptes de l'islam qui encourage tout effort utile et bénéfique pour soi ou pour les autres indépendamment de ses résultats matériels aboutis ou non.

- Encourager les interactions sociales.

- Autres idées cognitives: prendre conscience des difficultés et des épreuves vécues par les Prophètes et les Saints; croire en la bonté et la bénéficience d'Allah ; préférer le volonté d'Allah à sa propre volonté; miséricorde pour le souffrant, aspect temporaire des difficultés humaines, tous les péchés sont pardonnables ; les bonnes actions et la repentance effacent les mauvaises actions ; nul n'est parfait, tout le monde peut se tromper ; la valeur des êtres est en rapport avec leur foi et le degré de croyance, remercier et reconnaitre les bienfaits d'Allah ; immortalité de l'âme.

\section{CONCLUSION}

Les TCC ont une efficacité indéniable et un bénéfice prouvé dans la prise en charge de divers troubles psychiatriques. Ces réussites ont permis leur dissémination rapide à travers le monde et leur adoption comme traitement psychothérapique de choix souvent de première intention.

L'application des TCC sur des populations et cultures autres que celles d'origine occidentale a mis en avant des problèmes de compatibilité culturelle.

Ces divergences ne sont pas insurmontables mais nécessitent un travail de théorisation et de pratique pour palier à ces dissonances. Si la théorisation a fait des avancées remarquables il n'en est pas de même pour les études pratiques sur les patients qui restent bien loin des espérances et même loin des capacités matérielles et humaines du monde musulman.

Il est plus que nécessaire d'encourager, à une échelle organisée et systématisée, la bonne pratique des TCC fondée sur les valeurs de l'Islam. Ceci profiterait au malade et permettrait même la réduction de la stigmatisation de la maladie, son acceptation et la réduction de la réticence qu'ont les patients à initier une psychothérapie qui ne répondrait pas à leurs croyances et à leurs valeurs. 
Déclaration d'intérêts : l'auteur ne déclare aucun conflit d'intérêt en rapport avec cet article.

\section{REFERENCES}

1. Mirabel-Sarron C. Les thérapies comportementales et cognitives : bases théoriques et indications. Ann Méd-Psychol 2011 ; 169 (6): 398402 .

2. Legéron $\mathrm{P}$, Van Rillaer J. Approche théorique des thérapies comportementales et cognitives chez l'adulte. EMC - Psychiatrie. 1999: 1-6 (37-820-A-40).

3. Beshai S, Clark CM, Dobson KS. Conceptual and pragmatic considerations in the use of cognitive-behavioral therapy with Muslim clients. Cogn Ther Res. 2013; 37(1): 197-206.

4. Hamdan A. Cognitive restructuring: An Islamic perspective. J Muslim Ment Health. 2008; 3(1): 99-116.

5. Cottraux J. Les thérapies comportementales et cognitives. 2ème tirage. Paris: Masson; 1992.

6. Beck J S. Cognitive behavior therapy: basics and beyond. 2nd Ed. New York: Guilford Press; 2011.

7. Cottraux J. Thérapies cognitives. EMC - Psychiatrie. 2016; 13:1-11 (37-820-A-50).

8. Dobson KS, Dozois DJA. Historical and philosophical bases of the cognitive-behavioral therapies. In: Dobson KS, editor. Handbook of cognitive- behavioral therapies. Third Edition. New York: The Guilford Press; 2010: 3-38.

9. Mirabel-Sarron C, Vera L. Techniques de thérapies comportementales. EMC - Psychiatrie. 2014 ; 30: 1-14 (37-820-A-45).

10. Main R. Secular and religious: the intrinsic doubleness of analytical psychology and the hegemony of naturalism in the social sciences. J Anal Psychol. 2013; 58(3): 366-386.

11. Reber JS. Secular Psychology: What's the Problem? J Psychol Theol. 2006; 34(3): 193-204.

12. Pantalone DW, Iwamasa GY, Martell CR. Cognitive-behavioral therapy with diverse populations. In: Dobson KS, editor. Handbook of cognitive- behavioral therapies. Third Edition. New York: The Guilford Press; 2010: 445-464.

13. Pies R. The Judaic foundations of rational-emotive behavioural therapy. Ment Health Relig Cult. 2011; 14(5): 459-472.

14. Utz A. Psychology from the Islamic perspective. English edition 1. Riyadh: International Islamic Publishing House; 2011.

15. Amer MM, Jalal B. Individual psychotherapy/counseling: psychodynamic, cognitive-behavioral, and humanistic-experiential models. In: Ahmed S, Amer MM, editors. Counseling Muslims: handbook of mental health issues and interventions. New York: Routledge; 2012: 87-117.
16. Abu Raiya H, Pargament KI. Religiously integrated psychotherapy with Muslim clients: From research to practice. Prof Psychol Res Pract. 2010; 41(2): 181-188.

17. Hays PA. Introduction: developing culturally responsive cognitivebehavioral therapies. In: Hays PA, Iwamasa GY, editors. Culturally responsive cognitive-behavioral therapy: assessment, practice, and supervision. First Edition. Washington DC: American Psychological Association; 2006: 3-19.

18. Lipka M, Hackett C. Why Muslims are the world's fastest-growing religious group [Internet]. Pew Research Center. [cité 20 juin 2020]. Disponible sur: https://www.pewresearch.org/facttank/2017/04/06/why-muslims-are-the-worlds-fastest-growingreligious-group/

19. Rothman A. An Islamic theoretical orientation to psychotherapy. In: Al-Karam CY, editor. Islamically integrated psychotherapy: uniting faith and professional. TEMPLETON Press; 2018: 25-56.

20. Jafari M. Counseling values and objectives: A comparison of western and Islamic perspectives. Am J Islam Soc Sci. 1993; 10(3): 326-339.

21. Husain A, Hodge DR. Islamically modified cognitive behavioral therapy: Enhancing outcomes by increasing the cultural congruence of cognitive behavioral therapy self-statements. Int Soc Work. 2016; 59(3): 393-405.

22. Hodge DR, Nadir A. Moving toward culturally competent practice with Muslims: modifying cognitive therapy with Islamic tenets. Soc Work. 2008; 53(1): 31- 41.

23. Williams V. Working with muslims in counselling: identifying sensitive issues and conflicting philosophy. Int J Adv Couns. 2005; 27(1): 125-130.

24. Fakhr El-Islam M. Arab culture and mental health care. Transcult Psychiatry. 2008; 45(4): 671-682.

25. Thomas J, Ashraf S. Exploring the Islamic tradition for resonance and dissonance with cognitive therapy for depression. Ment Health Relig Cult. 2011; 14(2): 183-190.

26. Dwairy MA. Counseling and psychotherapy with Arabs and Muslims: a culturally sensitive approach. New York: Teachers College Press; 2006.

27. Badri M. Contemplation: an Islamic psychospiritual study. The International Institute of Islamic Thought; 2000.

28. Lodi F. The HEART Method: Healthy Emotions Anchored in Rasool Allah's Teachings Cognitive therapy using prophet Mohammed as a psycho-spiritual exemplar. In: Al-Karam CY, editor. Islamically integrated psychotherapy: uniting faith and professional. TEMPLETON Press; 2018: 76-102.

29. Shahsavarani AM et al. Anger Management and Control in Social and Behavioral Sciences: A systematic review of literature on biopyschosocial model. Int J Med Rev. 3(1) ; 2016: 355-364.

30. Vasegh S. Cognitive therapy of religious depressed patients: common concepts between Christianity and Islam. J Cogn Psychother. 2011; 25(3):177-188

31. Yaacob NRN. Cognitive Therapy Approach from Islamic Psychospiritual Conception. Procedia - Soc Behav Sci. 2013; 97: 182-187.

\footnotetext{
Cet article a été publié dans le « Batna Journal of Medical Sciences » BJMS, l'organe officiel de « l'association de la Recherche Pharmaceutique - Batna»

Le contenu de la Revue est ouvert « Open Access » et permet au lecteur de télécharger, d'utiliser le contenu dans un but personnel ou d'enseignement, sans demander l'autorisation de l'éditeur/auteur.

Avantages à publier dans BJMS :

- Open access : une fois publié, votre article est disponible gratuitement au téléchargement

Soumission gratuite : pas de frais de soumission, contrairement à la plupart des revues « Open Access »

Possibilité de publier dans 3 langues : français, anglais, arabe

- Qualité de la relecture : des relecteurs/reviewers indépendants géographiquement, respectant l'anonymat, pour garantir la neutralité et la qualité des manuscrits.

Pour plus d'informations, contacter BatnaJMS@gmail.com ou connectez-vous sur le site de la revue : www.batnajms.net
} 


\section{$\mathrm{B}$}

- $\bigcirc$

-

○

Service de Toxicologie du CHU de BEO

Département de Pharmacie, Faculté de Médecine d'AlgerZiania, Ben-Aknoun

\section{Correspondance à :}

Radia ZAMOUM

radiazamoum@outlook.fr

DOI :https://doi.org/10.48087/ BJMSra.2020.7222

\section{Historique de l'article :}

Reçu le 31 Juillet 2020

Accepté le 29 septembre 2020

Publié le 09 novembre 2020

Il s'agit d'un article en libre accès distribué selon les termes de la licence Creative Commons Attribution International License (CC BY 4.0), qui autorise une utilisation, une distribution et une reproduction sans restriction sur tout support ou format, à condition que l'auteur original et la revue soient dûment crédités.

Pour citer l'article :

Zamoum R et Kaddour S. Méthémoglobinémie et surdosage à la Dapsone : Revue de la littérature. Batna J Med Sci 2020;7(2):167-70. https://doi.org/10.48087/B JMSra.2020.7222

\title{
Méthémoglobinémie et surdosage à la Dapsone : Revue de la littérature
}

\author{
Methemoglobinemia and Dapsone overdose : A Literature review
}

\author{
Radia Zamoum, Salma Kaddour
}

\section{RÉSUMÉ}

La Dapsone est une molécule à propriétés antibiotiques et immunomodulatrices. Son utilisation en médecine humaine est connue pour le traitement de la lèpre, de certaines dermatoses, du lupus bulleux et d'autres maladies. Cependant, son effet hématoxique, même à dose thérapeutique est avéré comme la survenue de l'hémolyse, d'une anémie hémolytique et de l'élévation du taux de la méthémoglobinémie. Cette dernière est d'autant plus importante que la Dapsonémie est élevée, d'où l'intérêt de déterminer le taux sanguin de la méthémoglobine chez les intoxiqués ou lors du traitement par la Dapsone. Le tableau clinique d'une intoxication par la Dapsone associe une cyanose, une dyspnée, des troubles neurologiques et cardiaques. L'arrêt du traitement permet la régression des symptômes et un retour au taux normal de la méthémoglobinémie. Le traitement repose sur la vidange gastrique ou le charbon actif, l'assistance respiratoire et le traitement spécifique (Bleu de méthylène). Plusieurs cas cliniques d'intoxication par la Dapsone sont décrits dans la littérature.

Mots clés: Dapsone, Méthémoglobinémie, Surdosage, Bleu de méthylène.

\section{INTRODUCTION}

La Dapsone, utilisée dès les années 1940 contre la lèpre, était destinée initialement à la teinture industrielle [1]. C'est une molécule appartenant à la famille des sulfones, connue pour ses effets antibiotiques et immunomodulateurs. Ainsi, la Dapsone est prescrite en dermatologie pour le traitement des dermatoses inflammatoires mais aussi comme traitement prophylactique chez les immunodéprimés. [2]

La Dapsone subit une biotransformation hépatique à l'origine de la formation de dérivés acétylés et de l'hydroxylamine. [2-4] Cette dernière est suspectée d'être responsable de sa toxicité hématologique.

L'effet hématotoxique persistant est observé en raison de la forte variabilité de sa demi-vie biologique. [3] Ces variations sont de 14 à 83 heures avec une demi-vie moyenne de 20 heures. [3]

La toxicité de la Dapsone peut être expliquée par des effets pharmacologiques, constants et dose-dépendants et des effets idiosyncrasiques, imprévisibles et de mécanisme mal connu. [2] L'hématotoxicité de la Dapsone associe une hémolyse, un effet méthémoglobinisant et une cyanose. [4]

\begin{abstract}
Dapsone is a molecule with antibiotic and immunomodulatory properties. Its use in human medicine is known for the treatment of leprosy, certain skin conditions, lupus bullosa and other diseases. However, its haematoxic effect, even at therapeutic doses, has been shown to result in the occurrence of hemolysis, hemolytic anemia and an increase in the level of methemoglobinemia. The latter is all the more important as the Dapsonemia is high, hence the importance of determining the blood level of methemoglobin in addicts or during treatment with Dapsone. The clinical picture of intoxication by Dapsone combines cyanosis, dyspnea, neurological and cardiac disorders. Stopping treatment allows symptoms to decrease and methemoglobinemia to return to normal levels. Treatment is based on gastric emptying or activated charcoal, respiratory assistance and specific treatment (methylene blue). Several clinical cases of Dapsone poisoning are described in the literature.
\end{abstract}

Keywords: Dapsone, Methemoglobinemia, Overdose, Methylene blue

\section{Dapsone et méthémoglobine}

Diverses étiologies peuvent être incriminées dans la production anormale de la méthémoglobine. Les causes génétiques, alimentaires, idiopathiques et toxicologiques sont les plus importantes. [1] La méthémoglobine résulte de l'oxydation du fer ferreux $\left(\mathrm{Fe}^{2+}\right)$ en fer ferrique $\left(\mathrm{Fe}^{3+}\right)$ au niveau de l'hémoglobine. Ainsi, l'hémoglobine devient une forme non fonctionnelle, incapable de transporter l'oxygène, indispensable au fonctionnement cellulaire. $[1,2]$

Plusieurs systèmes de réduction endogène existent, pour maintenir la MetHb à l'état réduit. Chez les individus normaux, seulement $1 \%$ environ de l'hémoglobine totale est sous forme de MetHb, à un moment donné. [5] Le système du cytochrome-b5 - MetHb réductase est le principal système de réduction de la MetHb et représente environ 99\% de la réduction quotidienne de la MetHb. [1] Une voie accessoire existe, induite par un transporteur d'électrons comme le bleu de méthylène, c'est la voie NADP-dépendante utilisant la MetHb réductase II. [6] L'acide ascorbique et le glutathion représentent des voies secondaires de réduction de 10 à $15 \%$ de MetHb physiologique. [1, 6] 
D'autres agents endogènes réducteurs de la MetHb existent. Ils comprennent la flavine réduite, la tétrahydroptérine, la cystéamine et la cystéine réduite sur les molécules de protéines. [1] Le taux de transformation de la MetHb en hémoglobine chez les individus normaux est d'environ 15\% par heure. [7]

La cause la plus fréquente de méthémoglobinémie, est l'ingestion ou l'exposition de la peau ou des muqueuses à des agents oxydants. Certains d'entre eux oxydent l'hémoglobine directement pour former de la méthémoglobine ; d'autres le font indirectement en réduisant l'oxygène libre en radicaux libres $\left(\mathrm{O}^{2}\right.$.), qui à leur tour oxydent l'hémoglobine. [8]

La Dapsone est un médicament responsable de la formation de la MetHb, dont le mécanisme repose sur sa biotransformation en un métabolite oxydant, l'hydroxylamine. Cette N-hydroxylation expliquerait la toxicité hématologique de la Dapsone. [2] D’où la nécessité de surveiller les taux de MetHb au cours du traitement par la Dapsone.

La Dapsone peut provoquer une méthémoglobinémie même à doses thérapeutiques. Contrairement à d'autres causes de méthémoglobinémie acquise, la Dapsone provoque souvent une méthémoglobinémie chronique de bas grade. [9]

Une corrélation entre les taux de méthémoglobinémie et le tableau clinique observé existe. Le Tableau 1 donne les symptômes en fonction des taux de méthémoglobinémie. Les taux physiologiques de méthémoglobinémie varient de 0,5 à $0,8 \%$ chez l'adulte et sont de 1,5\% chez le nouveau-né. [6]

Tableau 1: Corrélation des symptômes avec les taux de méthémoglobinémie. [6]

\begin{tabular}{|c|c|}
\hline Taux & Symptômes \\
\hline$>15-20 \%$ & Cyanose perceptible, coloration du sang \\
\hline$>20-45 \%$ & $\begin{array}{l}\text { Dyspnée, asthénie, vertige, céphalée, polypnée, } \\
\text { tachycardie }\end{array}$ \\
\hline$>45-50 \%$ & $\begin{array}{l}\text { Dépression du système nerveux central : lésions } \\
\text { neurologiques, troubles de la conscience, dépression } \\
\text { respiratoire }\end{array}$ \\
\hline$>55-70 \%$ & $\begin{array}{l}\text { Coma, convulsions, insuffisance cardiaque et troubles } \\
\text { du rythme }\end{array}$ \\
\hline$>70 \%$ & Décès possible \\
\hline
\end{tabular}

\section{Dosage de la méthémoglobine}

En raison de la diminution du taux de la MetHb de 10 à 20\%, toutes les 8 heures, son dosage doit être effectué rapidement après le prélèvement. Parmi les méthodes utilisées, la technique spectrophotométrique ou à l'aide d'un cooxymètre. Elle est basée sur les propriétés spectrales de la MetHb, par la mesure simultanée des absorbances à 6 longueurs d'ondes différentes. Ce qui permet le calcul de la concentration de MetHb après correction des interférences spectrales. $[10,11]$

L'identification et le dosage spécifique de la MetHb par spectrophotométrie peut se faire selon la méthode d'Evelyn et Malloy [6, 12,13] ou selon la méthode de Kaplan [6] Malgré la rapidité d'analyse répondant à l'urgence, que peut offrir le co-oxymètre, particulièrement en milieu hospitalier, la méthode spectrophotométrique d'Evelyn et Malloy demeure la méthode de référence. [14] De plus, le co-oxymètre ne permet pas la distinction de la MetHb, du bleu de méthylène administré comme antidote. [13]
Le prélèvement est réalisé sur un tube hépariné. Le fluorure de sodium est à proscrire car il est responsable de la formation du fluorométhémoglobine. Le sang total prélevé, doit être rapidement dilué (au vingtième) dans du tampon $(\mathrm{pH}$ 6,5) pour éviter de fausser le résultat. En effet, la MetHb est un pigment instable qui peut disparaitre par sa réduction en hémoglobine dans un sang non hémolysé ou se former, spontanément dans le sang recueilli. Les conditions de conservation optimales sont réunies par la congélation du prélèvement en tube bouché, jusqu'au moment de l'analyse. Dans le cas où le prélèvement est conservé à $+4^{\circ} \mathrm{C}$, sa dilution avec du soluté $\mathrm{NaCl}(7 \%)$ permet son analyse dans les deux à trois jours ou dans les $24 \mathrm{~h}$ s'il est conservé à température ambiante. [12]

\section{Cas d'intoxication décrits dans la littérature}

Ngo T-T et Truong J dans leur travail publié en 2007 [15], décrivent la relation de cause à effet qui existe entre le traitement par la Dapsone en prévention de la pneumonie, chez deux enfants cancéreux et la formation de la méthémoglobine. Une saturation en oxygène à $87 \% \mathrm{chez}$ le premier patient $(2,5$ ans $)$, accompagnée d'une méthémoglobinémie à $7,4 \%$, a été constatée. L'arrêt de l'administration de la Dapsone chez cet enfant, a permis le retour à des valeurs normales de méthémoglobinémie (Deux jours plus tard) et une saturation en oxygène à 94-95\%. Les mêmes constatations ont été données dans cet article, en ce qui concerne le deuxième enfant (11 ans), où la méthémoglobinémie était à $19,2 \%$ initialement et s'est normalisée au bout de quatre jours. Dans les deux cas, il s'agit d'une méthémoglobinémie symptomatique où la cyanose, une désaturation en oxygène et une coloration bleutée des muqueuses, ont été observées. [15]

Une étude rétrospective brésilienne [16], s'est intéressée aux cas d'intoxication par la Dapsone, chez 274 patients avec un âge variant de 1 mois à 50 ans. Les dosages dans le sang, de la méthémoglobine et de l'agent causal, ont révélé qu'il existe une corrélation significative $(n=144, r=0.32, p<0.05)$ entre la méthémoglobinémie et la Dapsonémie. Cependant, aucune corrélation n'a été observée entre la méthémoglobinémie ou le taux sanguin de la Dapsone et le temps écoulé depuis la prise de la Dapsone. [16]

Dans cette même étude [16], la cyanose, les vomissements, la confusion mentale, la tachycardie et la dyspnée, étaient les symptômes cliniques les plus cités. Une relation entre le nombre de comprimés ingérés (rapporté par le patient ou un parent) et les symptômes observés chez 120 patients intoxiqués, a été constatée. Un taux de $30 \%$ de méthémoglobine sanguine a été observé chez 154 patients tandis que 65 sujets, avaient $40 \%$ de méthémoglobinémie.

Un autre cas d'intoxication par la Dapsone aux USA, a été rapporté en 2005. [21] En effet, un patient de 18 ans, traité par plusieurs médicaments dont la Dapsone, était admis pour une laparotomie avec fermeture d'une fistule du tube G. Ses antécédents médicaux comprenaient une greffe de l'intestin grêle, 16 mois avant cette admission pour un syndrome de l'intestin court, avec une insuffisance nutritionnelle parentérale totale. À l'issue de cette opération chirurgicale, une dyspnée a été constatée, sans changement des signes vitaux. La saturation en oxygène $\left(\mathrm{SaO}_{2}\right)$ est restée à $92 \%$. Un dosage de la méthémoglobinémie a été effectué, en raison de la faible saturation et une pression artérielle élevée en oxygène $\left(\mathrm{PaO}_{2}\right)$. Ainsi, la méthémoglobinémie à $11,6 \%$ avec une $\mathrm{SaO}_{2}$ à $89 \%$ ont été observées. De ce fait, la Dapsone a été arrêtée, ce qui a permis la diminution du taux de méthémoglobine à 5,9\% au premier jour postopératoire et à $2,2 \%$ au deuxième jour. Le rétablissement s'est poursuivi sans incident. [17] 
L'imputabilité de la Dapsone dans la formation de la MetHb, a été avancée dans une autre publication suisse, faite en 2018 par El Gedailya A et al. [3] Il s'agit d'une patiente de 17 ans ayant présenté une coloration bleue des muqueuses, des vertiges et une fatigue importante. La prise de la Dapsone (300) pour le traitement de son acné, chez cette jeune fille, était à l'origine d'un taux initial élevé de MetHb $(27,9 \%)$. Cinq jours après, la patiente n'était plus cyanosée. [3]

Un autre cas d'intoxication par la Dapsone a été décrit [18] chez un enfant de 7 ans où le dosage de la méthémoglobinémie a révélé un taux de 35\%. En effet, L'enfant a été admis aux urgences pédiatriques, dans un tableau clinique, associant une cyanose péribuccale et acrocyanoses, un épisode de vomissements avec désaturation périphérique. Les causes infectieuse et cardiaque de cyanose ont été éliminées. Compte tenu de la possibilité d'empoisonnement, l'anamnèse a été menée et a révélé la prise de la Dapsone. La Dapsone a été prescrite à la maman, comme traitement prophylactique de la pneumonie à Pneumocystis jiroveci. Après la première perfusion au bleu de méthylène, la cyanose a diminué et une saturation périphérique de $93 \%$ a été obtenue, avec une $\mathrm{SaO}_{2}$ à $96 \%$ et une méthémoglobinémie à $12,5 \%$. Deux nouvelles infusions de bleu de méthylène ont été faites et une valeur de méthémoglobinémie de $8,2 \%$ a été obtenue. Quarante-huit heures après l'admission, la cyanose s'est à nouveau accentuée, avec une désaturation périphérique marquée. Un nouveau dosage de méthémoglobinémie a été réalisé avec une valeur de 14,7\%. Après 7 jours d'hospitalisation, la fille a évolué favorablement, sans cyanose ni désaturation. Le taux de méthémoglobinémie était à 1,2\%. [18]

Une femme de 23 ans, a été admise 2,5 heures après avoir ingéré 2,2 g de Dapsone. [19] Elle a développé une forte méthémoglobinémie $(39,9 \%)$ et a montré des signes de toxicité (instabilité hémodynamique et altération de l'état mental) malgré l'administration du charbon activé à plusieurs reprises, du bleu de méthylène, des vasopresseurs et l'intubation endotrachéale. Une hémofiltration veinoveineuse continue (CVVH) a ensuite été initiée suivie d'une hémodialyse intermittente avec hémoperfusion (IHD-HP). L"IHD et la CVVH ont permis respectivement de soustraire 95,3 mg et 67,8 mg de Dapsone, en 3,8 heures. Aucun rebond n'est survenu après l'arrêt du traitement épurateur. La patiente a été extubé 3,5 jours après et est sorti sans séquelles après 7 jours. [19]

\section{Prise en charge de l'intoxication par la Dapsone}

Devant toute intoxication médicamenteuse par ingestion, l'identification et l'arrêt du traitement en cause est le premier réflexe à adopter $[15,13]$, suivi du traitement évacuateur (Lavage gastrique) si le délai le permet (<1heure). [16] Le traitement symptomatique du surdosage à la Dapsone est celui de l'intoxication par les agents méthémoglobinisants. Il englobe l'oxygénothérapie normo ou hyperbare avec contrôle des voies aériennes, les benzodiazépines en cas de convulsions et le remplissage vasculaire pour contrecarrer l'hypotension. [16]

Le bleu de méthylène est le traitement de choix pour la prise en charge de la méthémoglobinémie acquise dépassant les $20 \%$ de MetHb, chez les intoxiqués symptomatiques (5), ou les $30 \%$ de MetHb chez les patients asymptomatiques. [15, 13] Il est administré par voie intraveineuse à une dose de 1 à $2 \mathrm{mg} / \mathrm{kg}$ pendant cinq minutes. [15, 13] L'effet maximal du bleu de méthylène se produit rapidement dans les 30 minutes. Si la réponse est insuffisante, des doses supplémentaires de bleu de méthylène peuvent être administrées après 1 heure. [13]
Selon une étude publiée en 2008, par Prasad R et al, l'administration continue du bleu de méthylène dès le début du traitement permet une réduction considérable des taux de MetHb dans le sang et devrait être préférée à la thérapie intermittente. [20]

L'administration du bleu de méthylène trouve son intérêt chez les immunodéprimés même à dose thérapeutique de Dapsone. En effet, il a été rapporté qu'une méthémoglobinémie à 9,2\% induite par la Dapsone a été observée, chez un sujet immunodéprimé de 53 ans [21], le traitement par cet antidote a permis son rétablissement et la disparition des symptômes (Dyspnée pendant 3 jours associée à de la fièvre, des frissons, des douleurs thoraciques pleurétiques et de la fatigue).

En raison de la longue demi-vie des médicaments méthémoglobinisants dont la Dapsone et de la courte demivie du bleu de méthylène, un effet rebond des taux de MetHb peut se produire jusqu'à 12 heures après l'administration de bleu de méthylène. De ce fait, une surveillance répétée des taux de MetHb avec la méthode d'Evelyn-Malloy est indiquée. [22]

Par ailleurs, cette thérapeutique peut servir aussi d'outil diagnostique, lorsque le dosage sanguin de la méthémoglobine n'est pas réalisable. C'est le cas publié en 2019, concernant une intoxication par la Dapsone chez une jeune fille de 22ans, où l'étiologie a été déterminée sur la base de l'anamnèse et des perturbations biotoxicologiques dues à la Dapsone, même si le taux de la méthémoglobinémie n'a pas été évalué. [13] Il est important de noter que le bleu de méthylène est un méthémoglobinisant en soi. [15, 16] En effet, des doses élevées (7 mg / kg) peuvent entraîner une hémolyse et une augmentation paradoxale des taux de MetHb allant jusqu'à $10 \%$. Le bleu de méthylène peut également augmenter le risque d'hémolyse induite par la Dapsone. [13]

L'acide ascorbique (Vitamine C) peut être aussi utilisé pour réduire les niveaux de méthémoglobinémie. $[2,8,13]$ L'exsanguino-transfusion peut s'avérer nécessaire si les patients ne répondent pas bien au bleu de méthylène et que les taux de méthémoglobinémie sont élevés $(>60 \%)$ où le pronostic vital est mis en jeu. $[13,16]$

Un test simple et rapide peut être fait et qui consiste à appliquer une goutte de sang sur du papier filtre pour évaluer son changement de couleur après une exposition à l'air. Le sang riche en MetHb reste de couleur brun chocolat. [13]

\section{CONCLUSION}

Le diagnostic d'une méthémoglobinémie est difficile à établir, en raison de ses symptômes non spécifiques. [13] En cas de surdosage, l'arrêt de la Dapsone peut améliorer l'état clinique de l'intoxiqué et permet la réduction progressive des taux de méthémoglobinémie. [15] Des taux sériques de méthémoglobine d'au moins $15 \%$ sont généralement responsables de cyanoses centrale et périphérique. [9] Le bleu de méthylène demeure un traitement antidotal spécifique à l'intoxication par la Dapsone. Son rôle est la reconversion de la méthémoglobine en hémoglobine indispensable au transport de l'oxygène. Il est important de faire une surveillance biologique et clinique avant d'instaurer le traitement par la Dapsone. La déficience en enzyme G6PD, l'hypoglycémie, l'insuffisance rénale ou hépatique ainsi que l'alcool aggravent l'intoxication par cette molécule. [6]

Déclaration d'intérêts: les auteurs ne déclarent aucun conflit d'intérêt en rapport avec cet article. 


\section{RÉFÉRENCES}

1. Wright RO, Lewander WJ, Woolf AD. Methemoglobinemia: Etiology, pharmacology, and clinical management. Ann Emerg Med November 1999; 34:646-656.

2. Farhi $D$, Bégon $E$, Wolkenstein $P$, Chosidow O. Dapsone (Disulone ${ }^{\circledR}$ ) en dermatologie. EMC - Dermatologie 2005:1-11 [Article 98-911-A-10].

3. El Gedaily A, Weiler S. Méthémoglobinémie sous dapsone. Actualités des centres régionaux de pharmacovigilance et de Tox Info Suisse. FORUM MÉDICAL SUISSE 2018;18(44):896-898. DOI: 10.4414/fms.2018.03401

4. Hanuschk D, Kozyreff A, Tafzi N, Tennstedt D et al. Perte brutale de vision consécutive à une méthémoglobinémie induite par la dapsone. Toxicologie Analytique et Clinique, December 2014 ;26(4) : 225-226. DOI ; 10.1016/i.toxac.2014.09.035

5. Jaffe ER, Hultquist DE. Cytochrome b5 reductase deficiency and enzymopenic hereditary methemoglobinemia, in Scriver CR, Beaudet AL, Sly WS, et al (eds): The Metabolic and Molecular Basis of Inherited Disease, ed 7. New York: McGraw-Hill, 1995:2267-2280.

6. Menu E.et Mehring M. Toxicologie Prépa-Pharma. Ed.1 De Boeck Supérieur s.a, Belgique ; 2015. 78-79. ISBN : 978-20152-8041-9393-5

7. Finch CA. Treatment of intracellular methemoglobinemia. Bull N Engl Med Ctr. 1947;6:241-245.

8. Skold A., Cosco D. L., Klein R. Methemoglobinemia: Pathogenesis, Diagnosis, and Management. Southern Medical Journal. November 2011; 104(11):757-761. DOI: 10.1097/SMJ.0b013e318232139f

9. Burke P, Jahangir K, Kolber MR. Dapsone-induced methemoglobinemia: case of the blue lady. Can Family Physician. 2013; 59:958-61.

10. Cannon DJ. Methemoglobin. Therapeutic Drug Monitoring (AACC) 1991; 13:3-4.

11. Faivre-Fiorina $B$, Caron $A$, Labrude $P$, Vigneron $C$. Les hémoglobines érythrocytaires, plasmatiques et substitutives face aux agents oxydants et réducteurs physiologiques. Ann Biol Clin 1998; 56:545-556.
12. Burgat-Sacaze V, Brun P. Godfrain J.C. Conditions du dosage de la méthémoglobine en toxicologie vétérinaire. Laboratoire de PharmacieToxicologie. Ann. Rech. Vet. 1981;12(1):93-97.

13. Mahmood N, Khan M U, U. L. Haq I, Afshan Jelani F, Tariq A. A case of dapsone induced methemoglobinemia. Journal of Pharmaceutical Policy and Practice 2019;12:22 DOI: 10.1186/s40545-019-0185-y

14. Bellik B, Derghaia K, Sancho J et al. A propos d'un cas de méthémoglobinémie grave suite à une intoxication volontaire par une teinture de chaussures. Annales de Toxicologie Analytique, vol. $\mathrm{XIH}_{\text {., }} \mathrm{n}^{\circ} 2$, 2001.

15. Ngo T-T, Truong J. Cas de méthémoglobinémie associée à la dapsone en oncologie pédiatrique. En direct de l'unité. Pharmactuel 2007; 40 (3) : 148-152.

16. Carrazza M Z N, Carrazza F R, Ogac S. Clinical and laboratory parameters in dapsone acute intoxication. Rev. Saúde Pública, 2000; 34 (4): 396-401. DOI : $10.1590 /$ S0034-89102000000400013

17. Wozel G. The story of sulfones in tropical medicine and dermatology. Int J Dermatol 1989;28:17-21.

18. Paccor A, Matsuda M, Capurso C e $t$ al. Metahemoglobinemia inducida por dapsona: presentación de un caso pediátrico. Arch Argent Pediatr 2018; 116(4):e612-e615.

19. Ghannoum M, Cormier M, Bernier-Jean A, et al. Extracorporeal treatments in a dapsone overdose: a case report, Clinical Toxicology. 2016; 54(9):886-889, DOI: $\underline{10.1080 / 15563650.2016 .1209769}$

20. Prasad, R., Singh, R., Mishra, O. P., Pandey, M. Dapsone induced methemoglobinemia: Intermittent VS continuous intravenous methylene blue therapy. The Indian Journal of Pediatrics, 2008; 75(3), 245-247. DOI: 10.1007/s12098-008-0053-2

21. Talarico J F, Metro D G. Presentation of dapsone-induced methemoglobinemia in a patient status post small bowel transplant. Journal of Clinical Anesthesia 2005; 17, 568-570

22. Skold, A, Klein, R. Symptomatic-Low Grade Methemoglobinemia Because of Dapsone. American Journal of Therapeutics, 2013; 20(6), e729-e732. DOI: $\underline{10.1097 / M J T .0 b 013 e 318217 a 5 a f}$

Cet article a été publié dans le « Batna Journal of Medical Sciences » BJMS, l'organe officiel de « l'association de la Recherche Pharmaceutique - Batna»

Le contenu de la Revue est ouvert « Open Access » et permet au lecteur de télécharger, d'utiliser le contenu dans un but personnel ou d'enseignement, sans demander l'autorisation de l'éditeur/auteur.

Avantages à publier dans BJMS :

- Open access : une fois publié, votre article est disponible gratuitement au téléchargement

- Soumission gratuite : pas de frais de soumission, contrairement à la plupart des revues « Open Access »

- Possibilité de publier dans 3 langues : français, anglais, arabe

- Qualité de la relecture : des relecteurs/reviewers indépendants géographiquement, respectant l'anonymat, pour garantir

la neutralité et la qualité des manuscrits.

Pour plus d'informations, contacter BatnaJMS@gmail.com ou connectez-vous sur le site de la revue : www.batnajms.net 


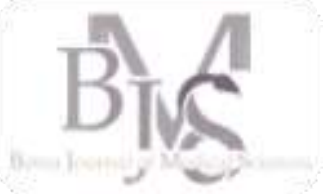

\title{
Certificat de décès en Algérie : intérêt, circuit, formulaire et directives de
} rédaction

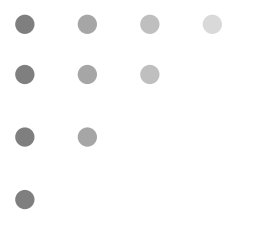

Service épidémiologie et médecine préventive (SEMEP) CHU de Sétif- Algérie

\section{Correspondance à :}

Meriem ABDOUN

drabdounmeriem19@gmail.com

DOI :https://doi.org/10.48087/ B]MSra.2020.7223

\section{Historique de l'article :}

Reçu le 16 septembre 2020

Accepté le 16 octobre 2020

Publié le 09 novembre 2020

Il s'agit d'un article en libre accès distribué selon les termes de la licence Creative Commons Attribution International License (CC BY 4.0), qui autorise une utilisation, une distribution et une reproduction sans restriction sur tout support ou format, à condition que l'auteur original et la revue soient dûment crédités.

\section{Pour citer l'article :}

Abdoun M. Certificat de décès en Algérie : intérêt, circuit, formulaire et directives de rédaction. Batna J Med Sci 2020;7(2):171-5.

\section{Death certificate in Algeria : interest, circuit, form and drafting instructions}

\author{
Meriem Abdoun
}

\section{RÉSUMÉ}

La comptabilisation annuelle des décès et la détermination des causes sont essentielles pour évaluer l'efficacité du système de santé d'un pays, que l'on estime aussi en mesurant l'impact des maladies et des traumatismes. Ces chiffres permettent aux autorités sanitaires de déterminer si elles prennent des mesures de santé publique adéquates. Les pays industrialisés disposent de systèmes pour déterminer les causes de décès dans la population. En revanche la plupart des pays en développement dont l'Algérie, n'en disposent pas. L'amélioration des statistiques des causes de décès passe par l'amélioration de la certification des décès, qui est encadrée par le format du certificat de décès et par le concept de la cause initiale du décès. Ainsi, le pivot de l'étude ces statistiques repose sur le certificat médical de décès, sa rédaction doit obéir aux règles et aux instructions édictées par l'organisation mondiale de la santé. Le modèle du certificat de décès doit être conforme à un modèle type préconisé par l'OMS, qui propose à tous les états membres un modèle international de certificat médical de la cause de décès, où figurent, suivant un ordre précis, une cause immédiate, une cause initiale et une ou plusieurs causes associées (intermédiaires) ayant contribués de manière indirecte au phénomène morbide. Ensuite, la codification des causes de décès s'appuie sur la classification internationale des maladies de l'organisation mondiale de la santé. Ce cadre garantit un certain niveau de qualité et de comparabilité nationale et internationale des données de mortalité.

Mots clés : certificat décès, cause initiale, causes mal définies.

\section{INTRODUCTION}

Les décideurs et planificateurs du secteur de la santé du monde entier font grandement usage des statistiques sur la mortalité. La qualité de ces statistiques dépend du degré de précision des certificats de décès que rédigent les médecins.

De bonnes statistiques ne sont possibles que si les diagnostics des causes médicales du décès et les circonstances sur le décès sont précis et complets. Malheureusement, dans de nombreux pays, les certificats de décès manquent considérablement d'exactitude. Cela réduit la qualité des statistiques nationales et internationales sur la mortalité et limite leur valeur pour la planification et l'élaboration de politiques de santé.

Le document de base de la production de ces statistiques est le certificat de décès dont la rédaction doit obéir aux règles et aux instructions édictées par l'organisation mondiale de la santé (OMS) [1].

\begin{abstract}
Annual accounting of deaths and determination of causes are essential for assessing the effectiveness of a country's health system, which is also estimated by measuring the impact of disease and injury. These figures enable health authorities to determine whether they are taking adequate public health measures. Industrialized countries have systems in place to determine the causes of death in the population. Most developing countries, including Algeria, do not. Improving cause-of-death statistics requires improved certification of death, which is framed by the format of the death certificate and the concept of the initial cause of death. Thus, the pivot of the study of these statistics is based on the medical certificate of death; its drafting must obey the rules and instructions laid down by the World Health Organisation. The model of the death certificate must conform to a standard model recommended by the WHO, which proposes to all member states an international model medical certificate of the cause of death, which includes, in a precise order, an immediate cause, an initial cause and one or more associated (intermediate) causes that have contributed indirectly to the morbid phenomenon. Secondly, the codification of causes of death is based on the World Health Organisation's International Classification of Diseases. This framework guarantees a certain level of quality and national and international comparability of mortality data.
\end{abstract}

Key words : death certificate, initial cause, ill-defined causes.

\section{INTERET DU CERTIFICAT DE DECES}

Le certificat médical de décès est un document officiel. Il doit être obligatoirement rempli par le médecin qui procède à l'examen de la personne décédée. Ce document présente plusieurs intérêts :

$\checkmark \quad$ Administratif : il permet d'établir l'acte de décès qui est un document d'état civil qui informe de la réalité du décès et la mise en place des opérations funéraires et qui est retardée en cas d'obstacle médicolégal (OML).

$\checkmark \quad$ Juridique : il permet aussi au médecin de s'interroger sur le type de décès, naturel et attendu en cas de pathologie connue, ou bien s'il s'agit d'une mort violente, ou de suspicion d'intervention d'un tiers et de mettre un obstacle médicolégal à l'inhumation, afin d'engager une procédure judiciaire pour déterminer la cause réelle du décès. 
$\checkmark$ Statistique : comptabilisation des décès par office nationale des statistique (ONS).

$\checkmark \quad$ Sanitaire : il permet de décrire les causes médicales de décès et la mise en place des programmes de santé d'un pays.

\section{LE CIRCUIT ADMINISTRATIF DU CERTIFICAT DE DECES}

Une fois le certificat de décès établit par le médecin, la déclaration auprès de l'officier de l'état civil est obligatoire (délais de 24 heures). L'officier de l'état civil garde la partie supérieure et la partie inferieure est collectée puis envoyée mensuellement au médecin chargé des causes de décès à la direction de la santé et la population (DSP) qui à son niveau la transmet à l'unité des causes de décès de l'institut national de santé public (INSP).

La collaboration des deux ministères Santé et Intérieur, par la diffusion de la circulaire interministérielle du 16 avril 2017, permet la centralisation et la transmission des certificats de décès des assemblées populaires communales (APC) vers la DSP. Cette dernière devrait améliorer les déclarations des causes de décès en Algérie.

\section{Le certificat de décès en vigueur en Algérie}

Tout décès survenu en Algérie doit faire l'objet d'un certificat médical de décès selon le modèle prévu à l'annexe du décret exécutif $n^{\circ} 16-80$ du 24 février 2016 fixant le modèle du certificat médical de décès [2] et de la circulaire interministérielle du 16 avril 2017 relative à la mise en application des dispositions du décret suscité [3]. Cette nouvelle version du certificat de décès est conforme au modèle international du certificat du décès recommandé par l'OMS [4].

Il comporte quelques adaptations pour tenir compte du contexte national. Ce certificat, révisé en l'année 2016 comprend deux volets :

\section{$\checkmark \quad$ Un volet administratif :}

Partie supérieure, nominative réservée à l'officier de l'état civil, dont copie est destinée, éventuellement, aux services concernés conformément à la législation et la réglementation en vigueur (Figure 1).

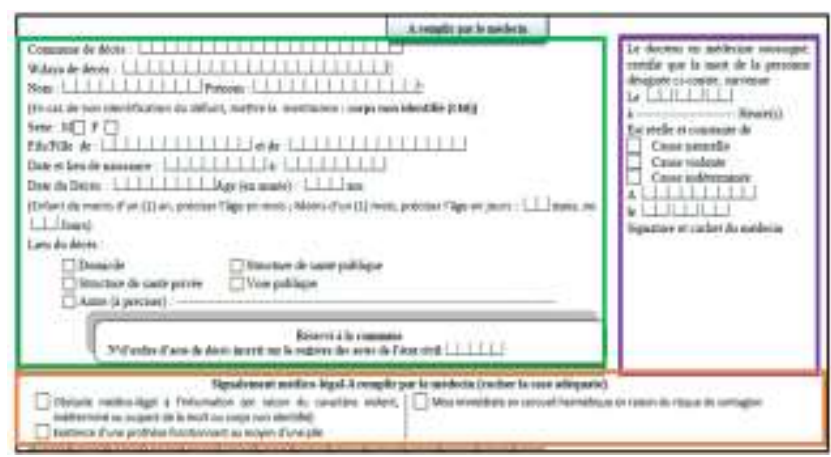

Figure 1 : Volet administratif

$\checkmark \quad$ Un volet médical :

Partie inférieure anonyme, réservée à l'usage des autorités sanitaires, où sont indiquées clairement les causes médicales de décès, destinée à l'unité des causes de décès (INSP) pour exploitation.

Elle doit être close par le médecin immédiatement après sa rédaction pour garantir la confidentialité des informations transcrites et ne pourra être ouverte que par l'autorité sanitaire habilitée à l'exploiter (Figure 2).

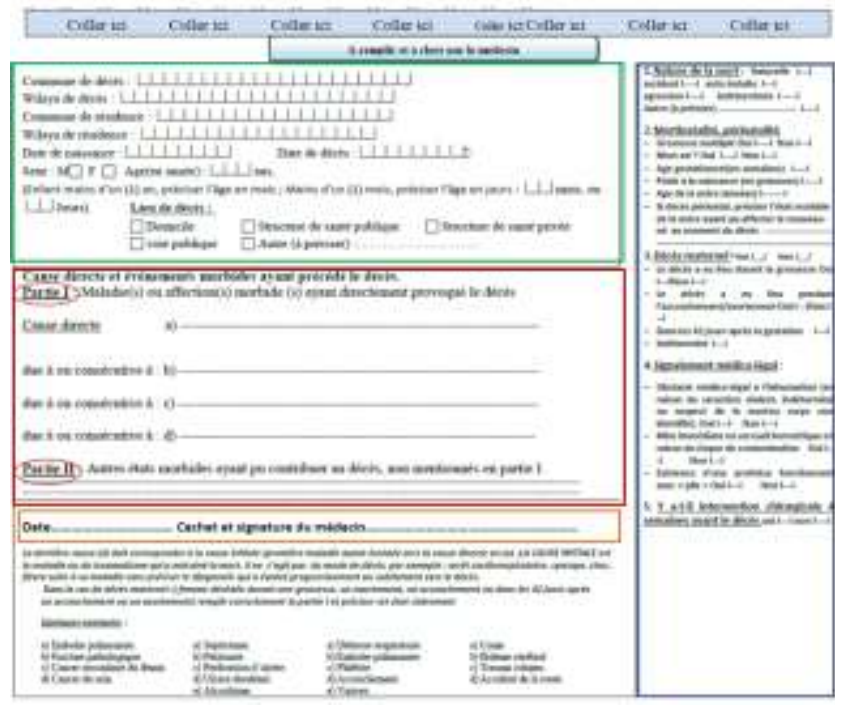

Figure 2 : Volet médical

\section{Codage des causes de décès}

Les informations médicales sur les causes de décès issues des certificats de décès sont codées selon la Classification Internationale des Maladies (CIM), dont l'appellation complète est la classification statistique internationale des maladies et des problèmes de santé connexes. C'est une classification médicale codifiée classifiant les maladies et une très vaste variété de signes, symptômes, lésions traumatiques, empoisonnements, circonstances sociales et causes externes de blessures ou de maladies. C'est une étape qui consiste à transposer les diagnostics de maladies ou causes de décès, en codes alphanumériques, ce qui facilite le stockage, la recherche et l'analyse des données [5].

\section{INSTRUCTIONS POUR LA REDACTION D'UN CERTIFICAT DE DECES}

Une fois le décès constaté, le médecin doit remplir les deux volets du certificat de décès. Toute information requise a son importance pour l'élaboration de tableaux de statistiques sur les causes de décès. La certification des causes médicales du décès n'a pas d'intérêt si le certificateur n'a pas fait mention du sexe, de l'âge et du lieu de la résidence du défunt par exemple. Pour les causes médicales du décès, il est demandé au certificateur de donner sa meilleure opinion quant à la séquence des événements ayant mené à la mort [6, 7].

\section{Rédaction du volet administratif du certificat de décès}

Sur cette partie du certificat de décès, destinée au bureau de l'état civil, le certificateur doit inscrire les informations suivantes selon les trois blocs (figure 1).

*Bloc 1

- La commune de décès et la wilaya de décès.

- Nom, prénom (si le défunt est non identifié mentionnez corps non identifié (CNI).

- $\quad$ Sexe, si le sexe est indéterminé écrivez le (exemples : cadavre putréfié ou malformation nouveau-né).

- Date et lieu de naissance.

- Date et heure de décès.

- $\quad$ Age du décès en années (si âge < à 1an en mois, si < 1 mois en jours).

- Cochez le lieu du décès (domicile, structure de santé publique, structure de santé privée, voie publique) si autres précisez (exemple lieu du travail, maison...).

- Ne pas remplir le numéro d'acte de décès, il sera rempli par l'agent de l'APC. 
*Bloc 2

$\checkmark \quad$ La date, le lieu et l'heure du décès (On note le jour, le mois et l'année ainsi que l'heure et la minute si on assiste au décès. Pour les arrivés décédés à l'hôpital ou les décès à domicile, il faut noter la date de la constatation du décès).

$\checkmark \quad$ Cochez la cause du décès :

- naturelle : résulte de l'évolution terminale d'un état pathologique endogène (exemple cancers, cardiopathies, diabète...).

- violente: elle est secondaire à une intervention extérieure. Il peut s'agir d'un suicide, d'un homicide ou d'un accident.

- indéterminée : la mort dont les circonstances de survenue ne peuvent être établies.

Signature et cachet : le certificateur doit signer et mettre son cachet à la partie réservée à cet effet.

*Bloc 3

$\checkmark$ Cochez obstacle médico-légal à l'inhumation si caractère violent, indéterminé ou suspect de la mort ou corps non identifié.

$\checkmark \quad$ Cochez mise immédiate dans un cercueil hermétique si risque de contagion.

$\checkmark$ Cochez existence de prothèses fonctionnant par piles si la notion existe.

\section{Rédaction du volet médical du certificat de décès}

Le volet médical du certificat de décès comprend plusieurs blocs :

*Bloc renseignement : Répondre les mêmes renseignements que le volet administratif sans le nom et prénom.

*Bloc causes de décès: Comporte deux parties partie I et partie II (Figure 2)

\section{Partie I}

- Comporte quatre lignes a, b, c, d qui permettent au médecin de décrire l'enchaînement causal des maladies qui ont directement conduit à la mort, de la cause immédiate rapportée sur la première ligne à la cause initiale mentionnée sur la dernière ligne remplie.

- S'il y a un enchaînement, la cause directe est notée en (a) et la cause première est notée à la dernière ligne ; toute cause intermédiaire sera notée à la ligne (b) ou aux lignes (b) et (c).

- L'affection enregistrée sur la dernière ligne de la partie I du certificat est la cause initiale de décès et sera codée. Elle sera utilisée pour présenter les statistiques des causes de décès, argumenter et à évaluer les politiques nationales de santé publique.

- Il faut rapporter la séquence des événements morbides ayant conduit à la mort, en partant de la cause immédiate (maladie terminale, traumatisme ou complication ayant directement entraîné la mort) jusqu'à la cause initiale du décès (maladie ou traumatisme étant à l'origine de la séquence des événements morbides ayant entraîné la mort). Un exemple est illustré dans la Figure 3.

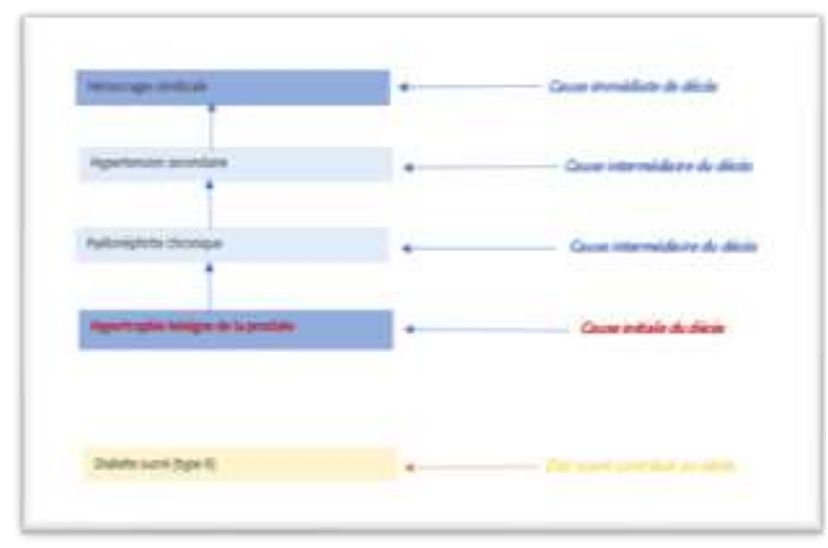

Figure 3 : Séquence/enchaînement des événements et autres états ayant provoqué la mort

\section{Partie II}

La partie II est réservée à tout autre état morbide important qui a contribué à l'issue fatale. Les causes contributives sont les autres maladies, affections ou lésions ayant contribué à la mort, mais sans appartenir à l'enchainement causal décrit en partie I :

- Affections chroniques (si elles ont contribué au décès) : insuffisance rénale chronique, diabète non insulinodépendant ...

- Intoxications chroniques : tabac, alcoolisme chronique, toxicomanie.

*Bloc renseignements complémentaires qui comprend :

1. Nature de la mort: Il faut cocher la case correspondante aux circonstances de la mort.

2. Mortinatalité, périnatalité : Pour une analyse approfondie de la mortalité périnatale, les données suivantes concernant tant la mère que l'enfant sont nécessaires :

$\checkmark \quad$ Pour l'enfant, il faut préciser s'il est né vivant ou mortné et le poids à la naissance en grammes.

$\checkmark$ Pour la grossesse actuelle, il faut préciser si elle est multiple ou non et aussi sa durée estimée en semaines.

$\checkmark \quad$ Pour la mère, il faut notifier son âge en années et la maladie ou l'affection maternelle ayant affecté le fœtus et le nouveau-né.

3. Décès maternel: S'il s'agit d'un décès maternel mentionner-le. Il faut préciser le moment du décès : pendant la grossesse, au moment de l'avortement ou 42 jours après l'accouchement ou indéterminé.

4. Signalement médicolégal :

$\checkmark \quad$ OML fait obstacle à l'inhumation et interdit l'ensemble des opérations funéraires. Si mort suspecte, mort subite, inattendue dont la cause est inconnue et de la mort violente : homicide, suicide ou accident., la loi fait obligation au praticien de s'orienter vers une procédure d'OML et éventuellement la procédure judiciaire.

$\checkmark \quad$ Si risque de contamination.

$\checkmark \quad$ Le retrait des prothèses à pile avant la mise en bière du défunt permet d'éviter, d'une part, la pollution des sols par les composants de la prothèse en cas d'inhumation. 
5. Intervention chirurgicale : Il est important pour des raisons de codage d'indiquer si une intervention chirurgicale a eu lieu dans les 4 semaines précédant le décès.

*Bloc signature et cachet :

Le certificateur doit écrire la date, signer et mettre son cachet à la partie réservée.

\section{Les recommandations de l'OMS pour la rédaction d'un certificat de décès}

Les médecins doivent effectuer la description la plus complète possible des états pathologiques ou les circonstances de décès pour faciliter le processus de classification et de codage de chaque certificat de décès.

\section{Cause initiale du décès}

Est " la maladie ou le traumatisme qui a déclenché l'évolution morbide conduisant directement au décès, ou les circonstances de l'accident ou de la violence qui ont entraîné le traumatisme morte». OMS, 1994 [8]. Les statistiques sur la mortalité reposent sur la cause initiale de décès, soit la maladie ou l'affection qui a déclenché la séquence/l'enchaînement des événements ayant provoqué directement le décès.

Par exemple, supposons qu'une personne meurt d'une hémorragie cérébrale des suites d'un accident de la circulation. L'hémorragie cérébrale est la cause directe (ou immédiate) du décès, tandis que la collision est la cause initiale de décès.

Le chirurgien s'intéresse au traitement de l'hémorragie cérébrale ; la santé publique s'intéresse aux moyens de prévenir les décès des suites de collisions de la route (la cause initiale de décès dans le présent cas).

\section{Causes de décès mal définies}

La consignation d'affections mal définies dans le certificat de décès n'a aucune utilité pour la santé publique. Il n'est pas acceptable de mentionner dans le certificat de décès comme cause de décès initiale :

$\checkmark \quad$ Le mode de décès: Les médecins ne devraient pas inscrire sur le certificat de décès le mode de décès comme « arrêt cardio-respiratoire » ou « mort cérébrale ».

$\checkmark \quad$ Les signes et symptômes : les termes douleurs à la poitrine, toux et fièvre...constituent également des états mal définis.

$\checkmark$ Défaillance d'un organe : il n'est pas acceptable comme cause initiale de décès une défaillance organique (exemple: insuffisance cardiaque ou rénale). Dans la mesure du possible, la maladie ou l'état à l'origine de la défaillance organique devrait être considérée comme la cause initiale du décès.

\section{Maladies cardiovasculaires :}

- Le processus de la maladie, la localisation.

- Le caractère éventuel aigu ou chronique.

- L'étiologie rhumatismale ou autre des cardiopathies valvulaires.

- Toute complication.

\section{Empoisonnement :}

- Si le décès est dû à un accident, un suicide, un homicide ou si le mode de décès n'a pas pu être déterminé.
- Le nom du produit, du médicament ou de la substance impliquée. S'ils vous sont inconnus, mentionnez-le également.

\section{Accident :}

- Le lieu où est survenu l'accident: s'il s'agit d'un accident de la circulation, de la voie publique, de travail, accident domestique.

- Faire une courte description de la façon dont le traumatisme est survenu en expliquant les circonstances ou la cause du traumatisme (chute d'une échelle).

- La localisation du traumatisme ainsi que la déficience associée à l'origine du décès (traumatisme crânien, polytraumatisme, ...)

Tumeurs :

- Le caractère malin ou bénin de la tumeur.

- Le site primitif aussi précisément que possible.

- Si le site primitif n'est pas connu, mentionnez-le.

Grossesse, accouchement et suites de couches :

- La nature de la complication.

- Le mode d'accouchement.

- Le moment du décès par rapport au stade de l'accouchement.

\section{Pneumonie ou une broncho-pneumonie :}

- La cause de tout antécédent pathologique à l'origine de la pneumonie.

- L'agent causal.

- Précisez s'il s'agit d'une affection primitive ou si elle est consécutive à l'inhalation d'aliments, de sang, ....

- Si la pneumonie a été causée par une dégradation de l'état général ou par une immobilité, veuillez mentionner l'affection qui a conduit à cette dégradation ou à, cette immobilité.

\section{Les infections :}

- Le nom de la maladie, l'agent infectieux lorsqu'il est connu, sa localisation.

- on ne devrait jamais entrer septicémie comme cause initiale si la source de l'infection (exemple: avortement septique ou pneumonie extrahospitalière) peut être identifiée.

- La septicémie est une affection mal définie et son inscription comme cause initiale est à proscrire.

Diabète :

- Insulinodépendant ou non ou autre.

- Natures des complications et leurs localisations.

\section{Vieillesse ou le grand âge :}

-Ne pas utiliser les termes "sénilité », " sénescence », « grand âge » comme cause de décès.

- En cas de poly pathologie avec causes très intriquées, il faut choisir la principale chaine causale et la rapporter en partie I et mentionner les autres pathologies en partie II. 


\section{ERREURS DE LA REDACTION DU CERTIFICAT DE DECES}

Voici une liste des erreurs rencontrées lors de l'évaluation et du codage des causes de décès que le certificateur doit éviter :

- L'écriture illisible : si l'écriture est illisible, elle entache l'exploitation du certificat de décès.

- Abréviations ambiguës : elles peuvent avoir plus d'une définition exemple: «IR» insuffisance rénale ou respiratoire.

- Données sociodémographiques incomplètes : lieu de résidence, âge, sexe, importantes dans l'analyse des données sur la cause de décès.

- Causes de décès mal définies : des termes comme l'hypoxie, l'arrêt cardiaque-respiratoire et l'insuffisance respiratoire.

- Enchainement causale incorrecte : si la séquence causale n'est pas logique, la sélection de la cause initiale reste difficile.

- Insuffisance des détails sur les circonstances des traumatismes (accident de la circulation, chute, accident de la voie publique...).

- Modèle du certificat utilisé : utilisation de plusieurs modèles du certificat de décès anciens et non conformes au modèle officiel du certificat le plus récent ou la certification des décès sur ordonnance.

- Signature et cachet non obtenus : le manque d'information sur le constateur et l'établissement où il exerce ne permet pas de le contacter en cas de besoin d'éclaircissement sur une information relative au certificat de décès.

- Les signatures et cachets cachent des informations importantes : quand les signatures et les cachets cachent des informations importantes, l'exploitation de ces données est difficile (exemple : cachet est sur la partie causes de décès).

\section{CONCLUSION}

Le certificat de décès est encore souvent considéré par les praticiens comme un document uniquement destiné à l'inhumation du sujet décédé et non pas comme un outil épidémiologique et de santé publique.

En Algérie, les déclarations de décès restent très limitées, autant sur le plan quantitatif (pourcentage de déclarations à l'INSP bas), que sur le plan qualitatif (informations médicales insuffisantes). L'amélioration de la qualité de la certification des causes de décès doit être une priorité.
C'est de l'exactitude et de la précision de la cause du décès que dépendra la qualité de la statistique nationale de mortalité qui aura un rôle à jouer dans le processus d'action et de décision en santé publique.

Déclaration d'intérêts: les auteurs ne déclarent aucun conflit d'intérêt en rapport avec cet article.

\section{RÉFÉRENCES}

1. Alain-Jacques Valleron. La mortalité et la morbidité mondiale, maintenant et demain : que connaît-on ? C. R. Biologies. 2008.331(12):991-1006. https://doi.org/10.1016/i.crvi.2008.09.002.

2. Journal officiel de la république Algérienne $N^{\circ} 12$. Décret exécutif $n^{\circ} 16$ 80 du 15 Joumada El Oula 1437 correspondant au 24 février 2016 fixant le modèle du certificat médical de décès. p.12.

3. Circulaire interministérielle du 16 avril 2017 relative à la mise en application des dispositions du décret exécutif n¹6-80 du 24 février 2016 fixant le modèle de certificat de décès.

4. Organisation mondiale de la santé. Le certificat médical de décès quatrième édition. Instruction à l'usage des médecins sur l'emploi du certificat médical de la cause de décès. Genève,1980.

5. Organisation mondiale de la Santé. Classification statistique internationale des maladies et des problèmes de santé connexes, dixième révision, vol. 2, 10e édition, Organisation mondiale de la Santé, Genève, 2016.

6. Manaouil C, Decourcelle M, Gignon M, Chatelain D, Jardé O. Le certificat de décès : comment le remplir et pourquoi ? Annales Françaises d'Anesthésie et de Réanimation. 2007;26(5):434-9.

7. Pavillon G, Laurent F. Certification et codification des causes médicales de décès. BEH 2003;30-31:134-8.

8. OMS, CIM-10. Classification statistique internationale des maladies et des problèmes de santé connexes : dixième révision, vol. 3, Genève, Organisation mondiale de la Santé,1994.

Cet article a été publié dans le « Batna Journal of Medical Sciences » BJMS, l'organe officiel de « l'association de la Recherche Pharmaceutique - Batna»

Le contenu de la Revue est ouvert « Open Access » et permet au lecteur de télécharger, d'utiliser le contenu dans un but personnel ou d'enseignement, sans demander l'autorisation de l'éditeur/auteur.

Avantages à publier dans BJMS :

- Open access : une fois publié, votre article est disponible gratuitement au téléchargement

- Soumission gratuite : pas de frais de soumission, contrairement à la plupart des revues « Open Access»

- Possibilité de publier dans 3 langues : français, anglais, arabe

- Qualité de la relecture : des relecteurs/reviewers indépendants géographiquement, respectant l'anonymat, pour garantir la neutralité et la qualité des manuscrits.

Pour plus d'informations, contacter BatnaJMS@gmail.com ou connectez-vous sur le site de la revue : www.batnajms.net 


\section{B Is}

- $\bigcirc$

- $\bigcirc$

P

○

1 Université BATNA 2, BatnaALGERIE

${ }^{2}$ Cabinet libéral, Batna-ALGERIE

Correspondance à :

Djohra HADEF

hdjohra@yahoo.fr

DOI : https://doi.org/10.48087/ BJMSra.2020.7224

\section{Historique de l'article :}

Reçu le 22 octobre 2020

Accepté le 4 novembre 2020

Publié le 09 novembre 2020

Il s'agit d'un article en libre accès distribué selon les termes de la licence Creative Commons Attribution International License (CC BY 4.0), qui autorise une utilisation, une distribution et une reproduction sans restriction sur tout support ou format, à condition que l'auteur original et la revue soient dûment crédités.

\section{Pour citer l'article :}

Hadef D, Slimani S.

Arthrite juvénile

idiopathique. Avancées et

défis. Batna J Med Sci

2020;7(2):176-81.

https://doi.org/10.48087/

B]MSra.2020.7224

\title{
Arthrite Juvénile Idiopathique. Avancées et défis
}

\author{
Juvenile Idiopathic Arthritis. Advances and Challenges
}

Djohra Hadef $^{1}$, Samy Slimani ${ }^{2}$

\section{RÉSUMÉ}

L'arthrite juvénile idiopathique (AJI) est la cause la plus fréquente du rhumatisme chez l'enfant. L'AJI est un terme générique qui regroupe toutes les formes d'arthrite chronique d'origine inconnue apparaissant avant l'âge de 16 ans. La classification des différentes entités de l'AJI était et reste toujours un sujet de discussion et de raffinement. La distinction de groupes plus homogènes permettra une thérapeutique plus ciblée. Le diagnostic de l'AJI est un diagnostic d'exclusion et il est purement clinique. Aucun test biologique n'est spécifique. Les explorations sont utiles dans le diagnostic différentiel et dans la définition de la sous-classification de la maladie. Établir le diagnostic d'une forme d'AJI est un défi qui nécessite parfois la demande d'avis d'expert pour une meilleure prise en charge diagnostique et thérapeutique. Il n'existe aucun traitement curatif pour l'AJI jusqu'aujourd'hui. Les avancées de connaissances de mécanismes physiopathologiques ont permis une meilleure approche thérapeutique de l'inflammation. L'avènement de la biothérapie ces dernières décennies a révolutionné la prise en charge de l'AJI et a nettement amélioré le pronostic.

Mots-clés : Arthrite juvénile idiopathique, Classification, Diagnostic, Biothérapie.

\section{INTRODUCTION}

L'arthrite juvénile idiopathique (AJI) n'est pas une maladie unique mais un ensemble d'affections hétérogènes qui ont en commun la présence d'atteinte articulaire inflammatoire chronique évoluant de plus de 6 semaines au niveau d'au moins une articulation, débutant avant l'âge de 16 ans et sans cause identifiable.

L'AJI est la maladie rhumatismale chronique la plus fréquente chez l'enfant [1]. Néanmoins elle est considérée comme pathologie rare mais non exceptionnelle [2]. Elle est l'une des principales causes d'invalidité à court, à moyen et à long terme [3].

L'étiopathogénie de la maladie n'est toujours pas claire. L'AJI est fort probablement la conséquence d'une réponse immunitaire aberrante à un facteur environnemental déclenchant chez une personne génétiquement prédisposée [4].

\section{ÉVOLUTION NOSOLOGIQUE}

La nomination de cette entité et sa classification ont toujours été un sujet de débat.

\section{ABSTRACT}

Juvenile Idiopathic Arthritis (JIA) is the most common cause of rheumatism in children. JIA is an umbrella term for all forms of chronic arthritis of unknown origin occurring before the age of 16 . The classification of the different entities of JIA was and is still a matter of discussion and refinement. Distinguishing more homogeneous groups will allow for more targeted therapy. The diagnosis of JIA is exclusionary and entirely clinical. No biological test is specific. Explorations are useful in the differential diagnosis and in defining the sub-classification of the disease. Establishing the diagnosis of a form of JIA is a challenge that sometimes requires expert advice for better diagnostic and therapeutical support. There is no curative treatment for JIA to date. Advances in knowledge of pathophysiological mechanisms have led to a better therapeutic approach to inflammation. The advent of biologics in recent decades has revolutionized the management of JIA and significantly improved the prognosis.

Keywords: Juvenile idiopathic arthritis, Classification, Diagnosis, Biologics.

Au début, elle était connue sous le nom de la maladie de Still, le nom de l'un des premiers médecins qui l'a décrit en 1897.

Aux années 70, les européens ont remplacé ce terme par ACJ (Arthrite Chronique Juvénile) ; alors que les américains ont adopté le concept PRJ (Polyarthrite Rhumatoïde Juvénile), avec deux différentes classifications pour les deux écoles [5].

Un consensus s'avérait nécessaire pour faciliter la progression des études multicentriques épidémiologiques et étiopathogéniques ainsi que les essais thérapeutiques.

Aux années 90, un comité international pédiatrique de l'ILAR (International Ligue of Associations of Rheumatology) a pu lever ce défi et il fut convenu de l'appeler AJI (Arthrite Juvénile Idiopathique) et de définir 7 sous-groupes classés selon des critères cliniques et biologiques présents durant les 6 premiers mois d'évolution (tableau 1) [6]. 
Tableau 1. Classification d'Edmonton 2001 des arthrites juvéniles idiopathiques [6].

\begin{tabular}{l}
\hline Forme systémique \\
\hline Forme Oligo articulaire \\
Persistante \\
Étendue \\
\hline Forme polyarticulaire \\
Avec facteur rhumatoïde négatif \\
Avec facteur rhumatoïde positif \\
\hline Arthrite en rapport avec une enthésite \\
\hline Arthrite psoriasique \\
\hline Arthrites indifférenciées
\end{tabular}

\section{DÉFI DIAGNOSTIQUE}

Le diagnostic de l'AJI est un diagnostic d'exclusion. Il est retenu lorsque des signes objectifs d'arthrite persistent chez un enfant pendant au moins 6 semaines en l'absence d'une autre cause identifiable. Le diagnostic est essentiellement clinique. Aucun marqueur biologique n'est spécifique à L'AJI, les tests de laboratoire sont utiles dans le diagnostic différentiel et dans la définition de la sous-classification de la maladie. L'imagerie articulaire est très difficile à interpréter car l'évaluation des articulations d'un enfant est difficile en raison des caractéristiques uniques du squelette en croissance. La radiographie conventionnelle reste la référence pour la démonstration des dommages structuraux aux articulations chez les patients atteints d'arthrite chronique [4]. L'IRM est le seul outil d'imagerie qui a la capacité de simultanément évaluer toutes les structures pertinentes dans les maladies articulaires inflammatoires [2]. L'échographie est bien adaptée au diagnostic et à l'évaluation des synovites et d'anomalies associées [4].

Forme systémique d'arthrite juvénile idiopathique (FSAJI)

La forme systémique d'arthrite juvénile idiopathique (FS-AJI), ou maladie de Still, est une forme particulière d'AJI et assez distincte des autres sous types. Elle est rattachée davantage au groupe des maladies auto-inflammatoires [10]. Elle est caractérisée par l'intensité des signes extra-articulaires qui dominent le tableau clinique. C'est la forme la plus aiguë et la plus grave représentant 10 à $15 \%$ des AJI [11]. C'est la seule forme d'AJI qui affecte également les garçons et les filles survenant entre 2 et 7 ans.

Selon l'ILAR, le diagnostic de l'arthrite systémique nécessite la présence d'arthrite accompagnée ou précédée d'une fièvre quotidienne d'au moins 2 semaines plus une ou plusieurs des manifestations suivantes : rash cutané caractéristique, hépatomégalie ou splénomégalie, adénopathies généralisées ou sérite.

Le plus souvent inaugurale, la fièvre est le symptôme majeur à la phase initiale. Elle doit évoluer sur une durée d'au moins 15 jours, et la courbe thermique typique est caractérisée par un pic fébrile par jour, à peu près toujours à la même heure, suivie d'une défervescence thermique en dessous de $37^{\circ} \mathrm{C}$.

L'analyse de la courbe thermique sur plusieurs jours avec prise de température toutes les 4 heures est donc primordiale pour étayer le diagnostic.
Au moment des pics fébriles, l'enfant a un aspect dit « toxique », avec visage pâle, état général altéré, myalgies et parfois arthralgies plus ou moins diffuses. Ces manifestations s'amendent avec la chute de la fièvre. Ce phénomène peut être utile pour distinguer l'arthrite systémique de la septicémie ou de la malignité dans laquelle l'évolution de la fièvre peut être similaire, mais où l'enfant garde un état altéré même en dehors des phases fébriles.

L'éruption cutanée est fugace, souvent maculeuse ou parfois urticarienne, siégeant souvent sur le tronc et la racine des membres et apparaît au moment de la fièvre ou peu après.

Elle peut se concentrer sur la peau recouvrant une articulation enflammée Les lésions cutanées peuvent être provoquées en frottant ou en grattant la peau non affectée (phénomène dit de Koebner), ou par un bain chaud ou un stress psychologique [12].

L'atteinte des séreuses est probablement très fréquente mais généralement infra clinique, elle peut intéresser le péricarde, la plèvre ou le péritoine. La plus fréquente est celle du péricarde objectivé à l'échographie cardiaque. Les douleurs abdominales que présentent souvent ces enfants correspondent probablement à la présence d'un épanchement péritonéal a minima.

Les adénopathies sont modestes au début puis deviennent plus importantes, elles sont symétriques et touchent plus fréquemment les régions cervicales, axillaire et inguinale. L'hépatomégalie est moins courante que la splénomégalie, mais si ces dernières sont importantes il faut penser à d'autres diagnostics.

Les manifestations articulaires peuvent se résumer à des arthralgies à la phase initiale mais la présence d'une authentique arthrite est nécessaire pour retenir le diagnostic d'AJI systémique, et elle peut ne survenir qu'après des semaines ou des mois après l'installation des signes extra articulaires ce qui rend le diagnostic difficile. Cette atteinte articulaire est variable, une mono-, oligo-, ou polyarthrite qui est la plus fréquente touchant les grosses et les petites articulations, de façon symétrique. Une atteinte diffuse ou atteinte de la hanche ou du rachis cervical ainsi que de l'articulation temporo-maxillaire semble être des facteurs de mauvais pronostic à long terme [13].

Sur le plan biologique elle est caractérisée par un syndrome inflammatoire majeure avec une vitesse de sédimentation (VS) voisine de $100 \mathrm{~mm}$ à la première heure et un taux de protéine réactive $\mathrm{C}$ (CRP) souvent supérieur à $100 \mathrm{mg} / \mathrm{l}$; une hyperleucocytose très marquée pouvant atteindre 20 à $30000 / \mathrm{mm}^{3}$ à polynucléaires neutrophiles, associée à une anémie inflammatoire souvent sévère, une thrombocytémie à $600000 / \mathrm{mm}^{3}$, voire plus mais moins fréquente, et une hypogammaglobulinémie [13]. Il n'existe aucun marqueur biologique spécifique de la maladie. La recherche de facteur antinucléaire et de FR est systématique : elle est négative dans cette forme.

Lors de la démarche diagnostique, il faut éliminer d'autres pathologies infectieuses (septicémie, endocardite bactérienne, brucellose, fièvre typhoïde, leishmaniose, infections virales), ou malignes (leucémie aiguë, neuroblastome métastatique), mais aussi des vascularites (la maladie de Kawasaki et la péri-artérite noueuse) [1].

Certaines maladies auto-immunes (lupus en particulier). Le rhumatisme articulaire aigu et certaines arthrites réactionnelles peuvent mimer une FS-AJI à la phase initiale.

L'élaboration de critères de diagnostic présomptif de FS-AJI est en cours par un groupe de travail de la Pediatric Rheumatology European Society (PReS), un diagnostic 
présomptif pourrait être porté après 2 à 3 semaines d'évolution, même en l'absence d'arthrite, devant un tableau clinique évocateur (en particulier une fièvre typique associée à des arthralgies et d'autres signes de la maladie), après avoir éliminé les principaux diagnostics différentiels [14].

Le syndrome d'activation des macrophages (SAM) est la complication aiguë la plus grave et potentiellement mortelle. L'arthrite de l'AJIS est souvent agressive et destructrice entrainant souvent une invalidité grave.

\section{Formes polyarticulaires}

L'atteinte polyarticulaire concerne $30 \%$ des AJI avec prédominance féminine nette [2]. Elle est définie par la présence d'une polyarthrite avec au moins cinq articulations atteintes au cours des 6 premiers mois d'évolution, les signes extra articulaires peuvent être observés mais moins importants que dans les formes systémiques tels qu'une légère fièvre, une perte de poids et une hépato splénomégalie. Elle comporte deux sous-groupes selon la positivité du facteur rhumatoïde (FR). Les diagnostics différentiels sont nombreux essentiellement la polyarthrite associée à d'autre type d'AJI (enthésite ou psoriasis), la polyarthrite associée à une pathologie générale (Lupus érythémateux systémique ou vascularite), une affection maligne le rhumatisme articulaire aigu et la maladie inflammatoire de l'intestin (MICI).

\section{Polyarthrites à facteur rhumatoïde positif}

Elles Constituent moins de $2 \%$ des AJI [15]. C'est une authentique polyarthrite rhumatoïde de l'adulte à début précoce touchant essentiellement l'adolescente et survenant sur un corps en croissance.

L'atteinte articulaire est en général bilatérale et symétrique à prédominance distale touchant les petites articulations (des mains et des pieds) et souvent aussi les grosses articulations (genoux et hanches). Le rachis cervical et les articulations temporo-mandibulaires sont fréquemment impliqués, tandis que le rachis thoracique et lombaire et les articulations sacroiliaques sont épargnés. Les arthrites sont le plus souvent fluxionnaires avec synovite et épanchement, mais parfois l'atteinte articulaire est peu ou pas hydarthrodiale avec enraidissement et rétraction musculotendineuse. Les nodules rhumatoïdes sont la caractéristique extra-articulaire la plus courante de la polyarthrite RF positive, rarement observés dans d'autres sous-groupes d'arthrite juvénile idiopathique, surviennent dans les sites classiques olécrane, point de pression, tendons fléchisseurs des doigts et tendon d'Achille chez environ un tiers des patients au cours de la première année de la maladie. Les autres atteintes extra articulaires sont exceptionnelles chez l'enfant pulmonaire, cardiaque, uvéite ( $<5 \%)$, etc.

Sur le plan biologique, on retrouve un syndrome inflammatoire d'importance variable, souvent intense avec une élévation de la VS, de la protéine réactive $\mathrm{C}$ mais pas d'hyperleucocytose, à la différence de la forme systémique. Une anémie inflammatoire modérée pourrait être retrouvée. Le FR est présent à deux examens successifs à 3 mois d'intervalle (critère diagnostique). Il faut savoir que le FR n'a aucune spécificité et peut être présent dans d'autres pathologies comme le LES ou d'autres formes d'AJI et même dans la population générale. La cause la plus fréquente d'une FR positive chez les enfants est une infection virale récente.

Les anticorps anti-CCP (peptides citrullinés cyclisés) sont présents dans $54 \%$ à $70 \%$ des cas, et pratiquement toujours négatifs dans les autres AJI [16]. Ils n'ont pas de valeur diagnostique confirmée ni de valeur pronostique propre. Les anticorps antinucléaires (AAN) sont parfois présents.

L'évolution se fait par poussées successives ou d'un seul tenant. Progressivement peut apparaître un enraidissement articulaire par rétraction capsulo-ligamentaire et/ou destruction ostéocartilagineuse. Le handicap est important dans 20 à $30 \%$ des cas, un retard staturo-pondéral peut survenir et aggraver le pronostic [13].

\section{Polyarthrites à facteur rhumatoïde négatif}

Cette catégorie est probablement la plus hétérogène de toutes les formes d'AJI. Il existe au moins trois sous types :

- D'authentiques polyarthrites de «type rhumatoïde ». Elle est similaire à la polyarthrite à FR positive sur le plan clinique, radiologique et évolutif, mais sans la présence de FR. Sur le plan biologique une VS accélérée et des ANCA négatifs. Début précoce de la maladie, touchant les deux sexes avec une évolution variable.

- Des oligoarthrites rapidement extensives au cours des premiers mois d'évolution ( $<6$ mois), elle se caractérise par une arthrite asymétrique, un début précoce, une prédominance féminine, des ANCA positifs, un risque accru d'uvéite et une association avec l'antigène leucocytaire humain (HLA) -DRB1* 0801 [1]. Ces formes ont un profil évolutif proche de celui des oligoarthrites extensives.

- Des polyarthrites "sèches ", forme plutôt masculine, peu hydarthrodiales, enraidissantes. La biologie reste le plus souvent normale sans auto anticorps. L'évolution se fait progressivement vers l'ankylose articulaire non douloureuse avec mauvaise réponse au traitement.

\section{Formes oligoarticulaires (oAJI)}

Les oAJI constituent une entité spécifiquement pédiatrique. C'est la plus fréquente des AJI (50\%), surviennent le plus souvent chez la fille avant l'âge de 5 ans [2]. Elles sont définies selon la classification d'ILAR par l'atteinte d'une à quatre articulations au cours des 6 premiers mois d'évolution en l'absence de signes systémiques, d'enthésiopathie, de psoriasis chez l'enfant lui-même ou un apparenté du premier degré ou de positivité du facteur rhumatoïde. Après les 6 premiers mois, l'évolution peut rester limitée (oligoarthrite persistante) ou s'étendre à plus de quatre articulations (oligoarthrite extensive) [6].

La plupart de ces enfants présentent un phénotype caractéristique, avec une arthrite asymétrique, une apparition précoce de la maladie $(<6$ ans), une prédilection féminine, une fréquence élevée d'ANA positifs et un risque élevé d'iridocyclite [17].

L'atteinte articulaire prédomine aux membres inférieurs ; elle est asymétrique et touche essentiellement les genoux et les chevilles. La hanche est rarement touchée ainsi que les petites articulations. L'atteinte temporomandibulaire doit être dépistée régulièrement pour éviter l'évolution vers le rétrognatisme. Le début est fréquemment monoarticulaire dans $70 \%$ des cas [13].

L'articulation touchée est enflée et souvent chaude, mais généralement pas très douloureuse ou sensible. Le motif de consultation est souvent l'augmentation de volume de l'articulation ou une boiterie indolore ou un flessum, un enraidissement articulaire, d'une amyotrophie ou asymétrie de longueur des membres inférieurs dans les formes vues tardivement. Les signes généraux sont inconstants avec un léger décalage thermique modéré et transitoire.

L'atteinte oculaire est péjorative, l'iridocyclite subaiguë à œil blanc asymptomatique uni- ou bilatérale peut précéder 
l'atteinte articulaire ou survenir au cours des 5 à 7 premières années suivant le début de l'arthrite [18]. Le risque est d'autant plus grand chez les filles, un âge $<$ à 7 ans et la présence de facteurs antinucléaires [19]. Au vu de la discordance entre l'évolutivité articulaire et ophtalmologique, l'examen ophtalmologique devra être répété régulièrement tous les trois mois pendant quatre ans puis tous les six mois entre quatre et sept ans puis tous les 12 mois [20].

La recherche d'un syndrome inflammatoire est systématique bien qu'il soit inconstant. Une élévation légère à modérée des taux de VS et de CRP avec anémie et leucocytose pendant la phase aiguë sont habituellement observées. La recherche de facteurs antinucléaires (de spécificité indéterminée) est impérative, ils sont présents dans 70 à $80 \%$ des cas et permettent d'affirmer le diagnostic, mais leur absence ne saurait exclure le diagnostic [21]. Le FR est absent et sa recherche est inutile.

En cas d'atteinte monoarticulaire, le premier diagnostic à envisager est celui d'une infection bactérienne si l'atteinte est récente, l'arthrite septique est une urgence et fréquente surtout avant deux ans. Les autres diagnostics possibles sont les suivants : un traumatisme, une malformation synoviale, une dystrophie synoviale, un angiome synovial, une tumeur synoviale, un ostéochondrome, un épanchement hémorragique secondaire à un trouble de l'hémostase, d'où nécessité de radiographie standard, d'échographie et parfois d'IRM et de ponction articulaire avec culture. La présence de manifestations cliniques atypiques comme la douleur intense, la perte de poids, la fièvre et la fatigue doivent toujours faire rechercher d'autres pathologies.

L'évolution se fait par poussées. Elle est de bon pronostic si elle reste oligoarticulaire avec risque d'asymétrie de la longueur des membres en cas d'arthrite persistante du genou et de pied plat valgus.

Le pronostic est plus sévère si elle s'étend devenant polyarticulaire avec risque de lésions ostéocartilagineuses et moins de rémission. Le risque d'extension et de destruction articulaire augmente si atteinte initiale de plus d'une articulation, atteinte du membre supérieur et augmentation de la VS [13].

Le pronostic ophtalmologique dépend de la précocité du traitement et de la gravité de l'iridocyclite avec risque de synéchies postérieures, de kératopathie en bande, d'œdème maculaire cystoïde, de glaucome et de cataracte voire de cécité définitive par amblyopie.

\section{Arthrite avec enthésite (ERA)}

L'ERA est le seul sous-type d'AJI le plus fréquent chez les garçons à l'âge de 10 ans; il représente 15 à $20 \%$ de toutes les AJI [2]. Par définition, il s'agit d'arthrite et d'enthésite, ou bien d'arthrite ou d'enthésite avec au moins l'un des critères suivants :

- Douleurs inflammatoires sacro-iliaques ou vertébrales ;

- Présence de l'antigène HLA B27, histoire familiale d'une pathologie associée au HLA B27 chez un parent du premier ou du deuxième degré ;

- Uvéite antérieure aiguë ;

- Début de l'arthrite chez un garçon âgé d'au moins 8 ans.

Sont exclus de ce groupe les patients avec antécédent personnel ou familial de psoriasis, la présence de signes systémiques et la présence du facteur rhumatoïde. La présence d'enthésite est la caractéristique la plus utile pour différencier l'ERA des autres formes d'AJI.

L'arthrite périphérique en ERA est souvent oligoarticulaire et affecte préférentiellement les membres inférieurs, souvent de manière asymétrique. Touchant le genou ou le pied le plus souvent ; la tarsite, gonflement diffus du dos du pied, est relativement unique à l'ERA.

Une autre atteinte particulière, évocatrice, des orteils réalisant une tuméfaction globale de l'orteil (orteil en saucisse), liée à l'atteinte des gaines tendineuses et des articulations interphalangiennes, évoquant au gros orteil un aspect pseudogoutteux. La hanche peut être atteinte de façon inaugurale contrairement aux autres sous-groupes d'AJI. La douleur est plus importante dans l'ERA que les autres soustypes d'AJI et répond aux AINS.

L'arthrite axiale des articulations sacro iliaques et des vertèbres est rare au stade initial et rarement isolée, elle peut s'installer après des années d'évolution de l'atteinte périphérique conduisant ainsi au tableau clinique de la spondylarthrite ankylosante (SA). Elle se manifeste par des rachialgies dorsales ou lombaires ou des douleurs fessières uni- ou bilatérales.

La présence d'enthésite (inflammation des insertions osseuses des tendons, des ligaments ou des fascias) est la caractéristique la plus utile pour différencier l'ERA des autres formes d'AJI. Les enthésites peuvent être inaugurales. Elles sont habituellement localisées au niveau du pied (insertion de l'aponévrose plantaire et du tendon d'Achille) et du genou (insertion du tendon rotulien sur la tubérosité tibiale antérieure). La douleur est provoquée ou reproduites à la pression de ces points.

Les manifestations extra-articulaires sont peu fréquentes. Une fièvre, une altération de l'état général avec asthénie et amaigrissement peuvent s'observer dans les formes à début aigu avec syndrome inflammatoire important.

L'uvéite dans l'ERA est aiguë et symptomatique (avec un œil rouge douloureux et photophobie), distincte de l'uvéite dans d'autres formes d'AJI et ne nécessite pas un examen ophtalmologique systématique. Généralement, elle est rapidement contrôlée par les traitements locaux avec un risque faible de séquelles.

Le syndrome inflammatoire est variable et inconstant. La présence de l'antigène HLA-B27 est un argument supplémentaire pour le diagnostic, mais son absence ne permet pas de l'exclure (car certains patients ERA sont B27 négatifs et de nombreux individus B27 positifs sont en bonne santé).

L'évolution se fait en règle générale par poussées. Le pronostic fonctionnel est dans l'ensemble assez bon ; risque de coxite destructrice uni- ou bilatérale (20 à $30 \%$ des cas) [13].

\section{Arthrite psoriasique}

Elle représente environ $10 \%$ des AJI. Selon les critères ILAR, la maladie est définie par l'arthrite associée à une éruption psoriasique ou à deux des éléments suivants : dactylite, piqueté unguéal ou onycholyse, psoriasis chez un parent du premier degré. Comme l'atteinte articulaire survient généralement quelques années avant le développement des manifestations cutanées, le diagnostic peut être difficile.

Il s'agit d'une entité hétérogène sur le plan clinique. Il apparaît ainsi deux catégories d'arthrite psoriasique : un sous-groupe à prédominance féminine, début précoce entre 2 et 5 ans, souvent associé à la présence de FAN et parfois compliqué d'uvéite à œil blanc (proche des oAJI) et un groupe à début plus tardif, prédominance masculine entre 12 et 13 ans, proche des arthrites avec enthésites.

L'atteinte articulaire varie de l'arthrite symétrique des petites articulations à l'arthrite asymétrique des grandes articulations des membres inférieurs selon le sous type, mais 
ce qui caractérise l'atteinte articulaire dans cette forme est l'atteinte de l'interphalangienne distale réalisant l'aspect classique orteil ou doigt en saucisse. Le tableau initial est oligoarticulaire touchant essentiellement le genou puis extension s'étendant respectant l'asymétrie.

Les manifestations cutanées sont souvent représentées par $\mathrm{du}$ psoriasis en plaques ou en gouttes habituellement au niveau cuir chevelu et les faces d'extensions des membres (coude, genou). Les ongles ponctués en dé à coudre sont très caractéristiques.

Il n'y a pas de manifestations générales en règle. Une uvéite à œil blanc est trouvée (par examen systématique à la lampe à fente) dans 6 à $20 \%$ des cas à début précoce et une uvéite aiguë (chez les patients HLA-B27 positifs) peuvent survenir.

Aucun test n'est spécifique. Le syndrome inflammatoire est inconstant. Le FR est absent (critère d'exclusion). Des AAN sont présents dans 20 à $60 \%$ des cas (habituellement dans les formes à début précoce de la fille avec possible uvéite) et l'antigène HLA-B27 est présent dans 11 à $28 \%$ des cas (particulièrement dans les formes plus tardives du garçon) [13].

L'évolution articulaire est indépendante de l'évolution cutanée. Elle se fait vers une atteinte polyarticulaire dans $60 \%$ des cas, mais le pronostic global est bon [13]. La présence d'AAN chez la petite fille augmente le risque d'uvéite et d'handicap fonctionnel.

\section{RÉVOLUTION THÉRAPEUTIQUE}

Bien que nous ne possédions toujours pas de médicaments capables de guérir de la maladie, le pronostic s'est considérablement amélioré, par rapport à il y a à peine dix ans, en raison l'avènement des biothérapies.

Les objectifs de la prise en charge sont la mise en rémission des manifestations articulaires aigues, l'adaptation du traitement en fonction de l'évolution, le dépistage et le traitement de l'atteinte oculaire et de l'atteinte fonctionnelle articulaire, la prévention et la prise en charge des effets secondaires des traitements, le maintien d'un bon développement psychosocial de l'enfant [22].

L'approche optimale de la prise en charge d'un enfant atteint d'AJI repose sur une équipe multidisciplinaire composée d'un rhumatologue pédiatrique, d'un ophtalmologiste, d'un chirurgien orthopédiste, d'une infirmière spécialisée, d'un physiothérapeute, d'un ergothérapeute et d'un psychologue [23].

Comme l'AJI n'est pas une maladie unique, les approches thérapeutiques doivent prendre en compte les différences entre les sous-types [4]. Le traitement de l'AJI doit être rapide et efficace. L'activité de la maladie doit être régulièrement évaluée (tous les 1 à 6 mois). Différents instruments sont utilisés dans l'évaluation de l'activité de la maladie et l'efficacité du traitement.

Les anti-inflammatoires non stéroïdiens (AINS) sont le principal traitement de cette maladie depuis des décennies [1]. Ils représentent le traitement médicamenteux de première intention dans toutes les formes d'AJI. Le naproxène et l'ibuprofène sont les plus utilisés à des doses de l'ordre de $30 \mathrm{mg} / \mathrm{kg} / \mathrm{j}$ en deux ou trois prises quotidiennes. Ils sont habituellement bien tolérés sans traitement gastroprotecteur systématique. Le délai minimal pour juger de l'efficacité est de deux à quatre semaines.

Les corticoïdes sont utilisés avec différentes modalités. Des injections intra-articulaires de stéroïdes, avec de l'hexacétonide de triamcinolone de préférence, sont fréquemment nécessaires au début de la maladie ou au cours de son évolution.

Les stéroïdes intra-articulaires sont suggérés comme traitements de première intention de l'arthrite juvénile idiopathique oligoarticulaire (oAJI) pour éviter les effets secondaires des médicaments systémiques et comme traitement d'appoint aux médicaments antirhumatismaux conventionnels. Bien qu'ils soient des anti-inflammatoires très efficaces, le taux de rechute est élevé.

La corticothérapie systémique à dose modérée ou élevée doit être réservée aux patients atteints d'arthrite juvénile idiopathique systémique avec des critères de gravité d'emblée ou en cas d'échec des AINS. Les bolus de la méthylprednisolone en intraveineux (10 à $30 \mathrm{mg} / \mathrm{kg} /$ jour jusqu'à un maximum de $1 \mathrm{~g} /$ jour pendant 1 à 3 jours consécutifs) est efficace, mais l'effet est souvent de courte durée. Par conséquent, une corticothérapie continue avec de la prednisone orale (1 à $2 \mathrm{mg} / \mathrm{kg}$ / jour jusqu'à un maximum de $60 \mathrm{mg} /$ jour en une seule prise ou en doses quotidiennes fractionnées) est fréquemment nécessaire. Une décroissance vers le 15e jour si l'état clinique le permet, en visant une dose inférieure à $0,3 \mathrm{mg} / \mathrm{kg} / \mathrm{j}$ à $3 \mathrm{mois}$ (ou $10 \mathrm{mg} / \mathrm{j}$ pour le grand enfant).

Dans les sous-types d'arthrite juvénile idiopathique autres que le sous-type systémique, les corticostéroïdes doivent être utilisés de manière très sélective vue les effets secondaires. Une courte cure de prednisone à faible dose (par exemple, 0,5 $\mathrm{mg} / \mathrm{kg}$ / jour) peut être envisagée pour soulager la douleur et la raideur chez les patients atteints de polyarthrite sévère réfractaire à d'autres thérapies ou en attendant le plein effet thérapeutique d'un traitement de fond.

Le méthotrexate (MTX) reste le médicament antirhumatismal conventionnel le plus largement utilisé dans la gestion de l'AJI en raison de son efficacité à contrôler la maladie et de ses effets toxiques acceptables [24]. La dose de 10 à $15 \mathrm{mg} / \mathrm{m}^{2} /$ semaine per os, sans dépasser la dose de 25 $\mathrm{mg} /$ semaine. En revanche, l'augmentation de la dose de méthotrexate parentéral à $30 \mathrm{mg} / \mathrm{m}^{2} /$ semaine n'apporte pas de bénéfice. Le MTX peut être administré par voie orale et sous-cutanée. Son efficacité se manifeste après 4 à 16 semaines de traitement.

Au cours des 15 dernières années, la prise en charge de l'AJI a été révolutionnée par l'introduction de modificateurs de la réponse biologique, qui ont fourni une option thérapeutique efficace pour le traitement des patients résistants aux médicaments antirhumatismaux conventionnels, à savoir le MTX ou la sulfasalazine. Ces médicaments ont été conçus pour cibler les cytokines et récepteurs clés impliquées dans la pathogenèse de la maladie.

Les médicaments biologiques sont souvent utilisés au début de l'évolution de la maladie de l'AJI et constituent un traitement efficace contre l'arthrite, les manifestations extraarticulaires de la maladie systémique et l'uvéite [4]. Les agents anti-TNF (Etanercept et Adalimumab) sont les produits biologiques de premier choix dans l'AJI non systémique, et l'anti interleukine-1 (Anakinra) et l'anti interleukine-6 (Tocilizumab) sont le premier choix dans l'AJI systémique [25].

Comme chez l'adulte et avant l'utilisation d'une biothérapie, la recherche d'une pathologie infectieuse et d'une tuberculose latente, avec vérification du carnet de vaccination et recherche d'un antécédent de varicelle, doit être systématique. Avant le traitement, la mise à jour des vaccins est donc nécessaire et la vaccination contre la varicelle est recommandée [14]. 


\section{Messages clés}

- L'arthrite juvénile idiopathique (AJI) est un diagnostic d'exclusion qui englobe toutes les formes d'arthrite chronique d'origine inconnue, commençant avant 16 ans.

- L'AJI n'est pas une maladie mais un groupe de six différentes pathologies définies par la classification ILAR (International League Against Rheumatism).

- L'AJI est un diagnostic clinique, aucun test biologique n'est spécifique.

- Le traitement médical est individualisé pour chaque enfant en fonction de la gravité et du nombre d'articulations touchées et de comorbidités.

- Le recours aux corticoïdes systémiques est limité. Les stéroïdes ne doivent pas être prescrits à un enfant présentant des douleurs articulaires ou un gonflement d'étiologie incertaine.

Déclaration d'intérêts: les auteurs ne déclarent aucun conflit d'intérêt en rapport avec cet article.

\section{RÉFÉRENCES}

1. Ravelli A, Martini A. Juvenile idiopathic arthritis. Lancet 2007; 369: 76778.

2. Prieur A.M, Quartier P, Bader-Meunier B, Glorion C. Maladies systémiques et articulaires en rhumatologie pédiatrique (2ème edition). Flammarion, 2009:37-84.

3. Job- Deslandres C. Arthrite juvénile idiopathique : critères de classification. Revue du Rhumatisme. 2010 ; 77 : 93-95.

4. Ravelli A. Handbook of Juvenile idiopathic arthritis. Springer, 2016: 1-10.

5. Duffy CM, Colbert RA, Laxer RR, et al. Nomenclature and classification in chronic childhood arthritis. Time for a change ? Arthritis Rheum 2005; 52:382-5.

6. Petty RE, Southwood TR, Manners P, et al. International League of Associations for rheumatology classification of juvenile idiopathic arthritis: second revision, Edmonton, 2001. J Rheumatol. 2004;31:390-2.

7. Job-Deslandre C. Juvenile idiopathic arthritis: definition and classification. Arch Pediatr. 2016;23:437-41.

8. Belot A. New classification for juvenile idiopathic arthritis: Is the Tower of Babel falling? Joint Bone Spine. 2018 Mar;85(2):139-141
9. Martini A. Are there new targets for juvenile idiopathic arthritis? Semin Arthritis Rheum. 2019 ; 49(3S):S11-S13.

10. Quartier P. Maladie de Still (forme systémique d'arthrite juvénile idiopathique). Arch Pediatr. $2008 ; 15: 865-866$.

11. Hofer MF, Mouy R, Prieur AM. Juvenile idiopathic arthritides evaluated prospectively in a single center according to the Durban criteria. Rheumatol 2001;28:1083-90.

12. De Benedetti F, Schneider R. Systemic juvenile idiopathic arthritis. In: Cassidy JT, Petty RE, Laxer RM, Lindsley CB, eds. Textbook of Pediatric Rheumatology. 6th edn. Philadelphia, PA: Elsevier Saunders; 2011;236248.

13. Job-Deslandre c. Arthrites juvéniles idiopathiques. EMC - Appareil locomoteur. 2007 ; 2(2):1-16.

14. Haute Autorité de Santé. Arthrites Juvéniles Idiopathiques. Saint-Denis La Plaine: HAS; 2017.

15. Quartier P. Arthrites juvéniles idiopathiques. EMC-Médecine. 2004; 1(6) :555-568.

16. Ferucci ED, Majka DS, Parrish LA, Moroldo MB, Ryan M, Passo M, et al. Antibodies against cyclic citrullinated peptide are associated with HLADR4 in simplex and multiplex polyarticular-onset juvenile rheumatoid arthritis. Arthritis Rheum 2005;52:239-46.

17. Giancane G, Consolaro A, Lanni S, Davì S, Schiappapietra B, Ravelli A. Juvenile Idiopathic Arthritis: Diagnosis and Treatment. Rheumatol Ther. 2016;3(2):187-207. doi:10.1007/s40744-016-0040-4.

18. Cassidy JT, Petty RE, Laxer RM, et al. Textbook of pediatric rheumatology. 5th edn. Philadelphia: Elsevier, 2005

19. Jari M, Shiari R, Salehpour O, Rahmani K. Epidemiological and advanced therapeutic approaches to treatment of uveitis in pediatric rheumatic diseases: a systematic review and meta-analysis. Orphanet J Rare Dis. 2020;15(1):41.

20. Foeldvari I. Ocular involvement in juvenile idiopathic arthritis: classification and treatment. Clin Rev Allerg Immunol 2014

21. Barut K, Adrovic A, Şahin S, Kasapçopur Ö. Juvenile Idiopathic Arthritis. Balkan Med J. 2017 Apr 5;34(2):90-101.

22. Bader-Meunier B, Wouters C, Job-Deslandre C, Cimaz R, Hofer M, Pillet $P$, Quartier P. Recommandations pour la prise en charge de la forme systémique l'arthrite juvénile idiopathique (maladie de Still). Arch Pediatr. $2010 ; 1639$ (7) : 997-1134.

23. Petty RE, Cassidy JT. Chronic arthritis in childhood. In: Cassidy JT, Petty RE, Laxer RM, Lindsley CB, eds. Textbook of Pediatric Rheumatology. 6th edn. Philadelphia, PA: Elsevier Saunders; 2011;211-235.

24. Gutierrez-Suarez R, Burgos-Vargas R. The use of methotrexate in children with rheumatic diseases. Clin Exp Rheumatol. 2010;28:S122-S127.

25. Blazina Š, Markelj G, Avramovič MZ, Toplak N, Avčin T. Management of Juvenile Idiopathic Arthritis:A Clinical Guide. Paediatr Drugs. 2016 Dec;18(6):397-412. doi: 10.1007/s40272-016-0186-0. PMID: 27484749.

Cet article a été publié dans le « Batna Journal of Medical Sciences » BJMS, l'organe officiel de « l'association de la Recherche Pharmaceutique - Batna»

Le contenu de la Revue est ouvert « Open Access » et permet au lecteur de télécharger, d'utiliser le contenu dans un but personnel ou d'enseignement, sans demander l'autorisation de l'éditeur/auteur.

Avantages à publier dans BJMS :

- Open access : une fois publié, votre article est disponible gratuitement au téléchargement

- Soumission gratuite : pas de frais de soumission, contrairement à la plupart des revues « Open Access »

- Possibilité de publier dans 3 langues : français, anglais, arabe

- Qualité de la relecture : des relecteurs/reviewers indépendants géographiquement, respectant l'anonymat, pour garantir la neutralité et la qualité des manuscrits.

Pour plus d'informations, contacter BatnaJMS@gmail.com ou connectez-vous sur le site de la revue : www.batnajms.net 

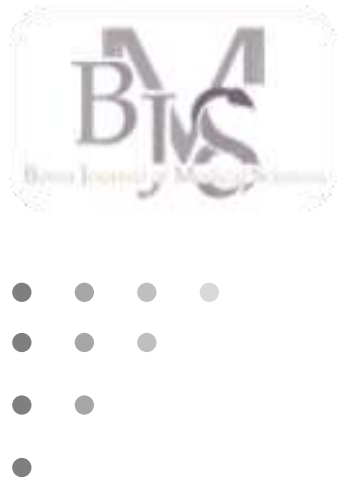

${ }^{1}$ Laboratoire LMCVGN, Faculté de Médecine, Université Sétif1,19000 Algérie

${ }^{2}$ Service de Pédiatrie, CHU Sétif, 19000 Algérie

\section{Correspondance à :}

Nada BOUTRID

n.boutrid@univ-setif.dz

DOI :https://doi.org/10.48087/ B]MSra.2020.7225

Historique de l'article :

Reçu le 10 juin 2020

Accepté le 28 septembre 2020

Publié le 09 novembre 2020

Il s'agit d'un article en libre accès distribué selon les termes de la licence Creative Commons Attribution International License (CC BY 4.0), qui autorise une utilisation, une distribution et une reproduction sans restriction sur tout support ou format, à condition que l'auteur original et la revue soient dûment crédités.

\section{Pour citer l'article :}

Boutrid N, Rahmoune H. La "Mesure de l'Homme":

Historique de l'anthropométrie et de l'auxologie. Batna J Med Sci 2020;7(2):182-5. https://doi.org/10.48087/BJM $\underline{\text { Sra.2020.7225 }}$

\title{
La "Mesure de l'Homme" : Historique de l'anthropométrie et de l'auxologie
}

\author{
The "Measure of Man": History of Anthropometry and Auxology
}

\author{
Nada Boutrid ${ }^{1,2}$ *, Hakim Rahmoune ${ }^{1,2}$
}

\section{RÉSUMÉ}

La croissance est un processus biologique correspondant à l'augmentation des dimensions, du poids ou du volume corporel objectivée par la science de la croissance soumise à des lois : l'auxologie, et toute altération de ce processus se reconnaît par l'anthropométrie : mesures corporelles pouvant détecter des anomalies telles que le retard statural ou l'acromégalie. Toutes ces notions, basiques pour les médecins d'aujourd'hui et notamment pour les pédiatres, ont été élaborées sur plus de 2 siècles de recherches cliniques, épidémiologiques et même radiologiques: la naissance de l'Anthropométrie ( «Science de la mesure de l'Homme ») et de l'Auxologie ("Étude de la croissance des êtres vivants») a connu bien des tumultes avant de donner vie à des concepts modernes et des modélisations statistiques et mathématiques dans le but ultime de distinguer les troubles staturaux pathologiques. Cet article brosse un tableau chronologique de la première courbe de croissance du Comte de Montbeillard au $18^{\mathrm{e}}$ siècle jusqu'aux dernières courbes de l'Organisation Mondiale de la Santé au début du millénaire.

Mots clés : Taille, Histoire, Anthropométrie, Auxologie, Âge osseux.

\section{INTRODUCTION}

La croissance est un processus biologique correspondant à une augmentation mesurable des dimensions corporelles, sa surveillance représente un temps essentiel dans le suivi d'un enfant et elle est régie par la science de la croissance soumise à des lois : l'auxologie [1-3].

Cette croissance requiert une mobilisation et une utilisation parfaite des apports énergétiques, coordonnées par de nombreux mécanismes notamment hormonaux et génétiques [4,5].

Une altération de ce processus se reconnaît par l'anthropométrie: des mesures pouvant détecter par exemple un retard statural qui correspond à un déficit de taille supérieur à moins deux dérivations standards (- 2 DS) pour l'âge [6-8].

Toutes ces notions, basiques pour les médecins d'aujourd'hui et notamment pour les pédiatres, ont été élaborées sur plus de 2 siècles de recherches cliniques, épidémiologiques et même radiologiques: la naissance de l'anthropométrie ("science de la mesure de l'Homme ») et de l'Auxologie («Étude de la croissance des êtres vivants ») a connu bien des

\begin{abstract}
Growth is a biological process corresponding to the increase in dimensions, weight or body volume as stated by the science of growth regulated through the biological laws of auxology, and any disorder during this process is recognized though anthropometry: the science of serial body measurements that can unveil abnormalities such as stature delay or acromegaly. All these concepts, basic for today's physicians and especially for pediatricians, were developed over more than two centuries of clinical, epidemiological and even radiological research: the birth of Anthropometry ("Science of obtaining systematic measurements of the human body) and Auxology ("Study of the growth of living beings") faced many disturbances before giving life to modern concepts and statistical and mathematical models with the ultimate goal of diagnosing pathological growth disorders. This article depicts a chronological view from the earliest growth curve of the Comte de Montbeillard in the $18^{\text {th }}$ century to the last growth charts of the World Health Organization at the turn of the millennium.
\end{abstract}

Keywords: Stature, History, Anthropometry, Auxology, Bone age.

tumultes avant de donner vie à des concepts modernes et des modélisations statistiques et mathématiques dans le but de distinguer les troubles staturaux pathologiques, souvent accessibles au traitement. [9].

Cet article brosse un tableau chronologique de la première courbe de croissance du Comte de Montbeillard au 18 siècle aux dernières courbes de l'Organisation Mondiale de la Santé au début du millénaire.

\section{Historique de "l'Anthropométrie" et de "l'Auxologie" de l'Antiquité à la Révolution Industrielle :}

Les Égyptiens et les Babyloniens furent les premiers à s'intéresser, en termes de mesures, à la croissance des enfants et à sa variabilité inter-ethnique [10].

Mais ce n'est qu'au 17ème siècle que les premières études rapportées sur la croissance furent réalisées à partir de données recueillies chez des hommes recrutés dans l'armée anglaise.

Ainsi, les mesures des recrues de la Marine Marchande et de la Marine Royale d'Angleterre depuis 17861786 reflètent les variations dites 
« séculaires » de la taille humaine ces derniers siècles [11].

\section{La « Mesure de l'Homme » : l'Anthropométrie}

Elsholtz (1623-1688) était le premier à utiliser le terme anthropométrie. Il lui donna la signification de " Mesure de l'homme ». Il s'inspira d'un instrument utilisé deux siècles auparavant par le sculpteur Léon Batista Alberti pour mesurer les proportions de ses statues afin de confectionner une sorte de toise qu'il appela « anthropometron » [10].

La première courbe de croissance: une histoire de noblesse !

Le 18e siècle voit l'éclosion des premiers travaux archivés relatant de l'accroissement de la taille humaine. Lambert est communément considéré comme pionnier par ses écrits de 1754 [10].

Cependant, la première étude longitudinale jamais réalisée n'est rapportée qu'en 1777 : Georges-Louis Leclerc de Buffon (1707-1788) dans la volumineuse « Histoire Naturelle » reprend le travail du Comte Philibert Guéneau de Montbeillard, qui mesura la taille de son fils tous les six mois depuis sa naissance.

Ainsi, la croissance d'un enfant, de la naissance jusqu'à la taille adulte, suivant un itinéraire représentant une « courbe » a été pour la première fois tracée à partir du 11 avril 1759 : le Comte de Montbeillard, curieux, pesa et mesura son fils, puis le fit de nouveau à intervalles réguliers, pendant dix-huit années successives. Puis, à partir de ce recueil de données, le Comte déduisit le rythme de croissance de son fils jusqu'à l'âge adulte [10-12].

Les valeurs numériques successives de la taille de cet enfant furent publiées dans les Additions à l'Histoire naturelle sous le titre : «Sur l'accroissement successif des enfants » (Figure 1).

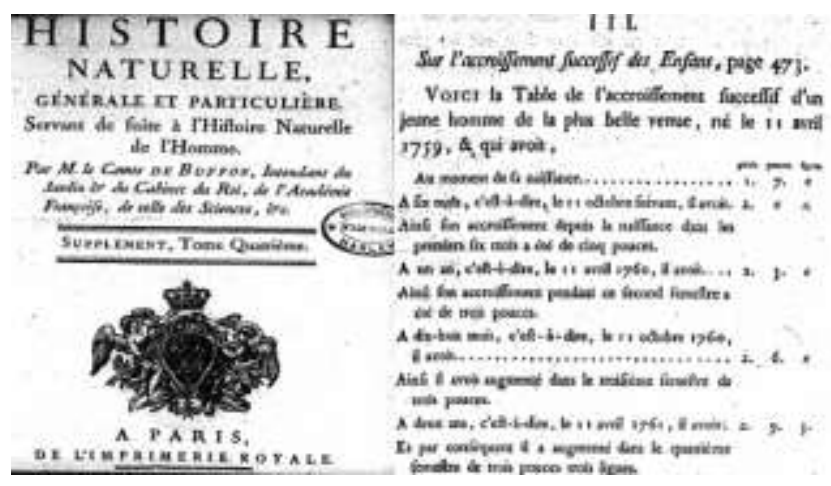

Figure 1 : « Histoire Naturelle, 1777 »

Les mesures de la première courbe de croissance d'un enfant ! [12]

Ces données numériques, initialement exprimées en anciennes unités, ont été ultérieurement reconverties en centimètres par Richard Scammon en 1927 [13]

Les équivalents des termes de mesures sont repris au Tableau1.

Tableau 1. Équivalents des termes de Mesure

\begin{tabular}{|c|c|c|c|}
\hline Toise & Pied & Pouce & Ligne \\
\hline $1,949 \mathrm{~m}$ & $0,325 \mathrm{~m}$ & $0,027 \mathrm{~m}$ & $0,002 \mathrm{~m}$ \\
& & & \\
\hline
\end{tabular}

Une fois convertis en centimètres, les mensurations régulières de l'enfant ont été reportée sur une courbe de "croissance » (Figure 2) qui montre que le petit : mesurait 51,4 cm à sa naissance

a entamé sa puberté vers 13 ans à une taille de $155 \mathrm{~cm}$. a achevé sa croissance à une taille a de $186 \mathrm{~cm}$.

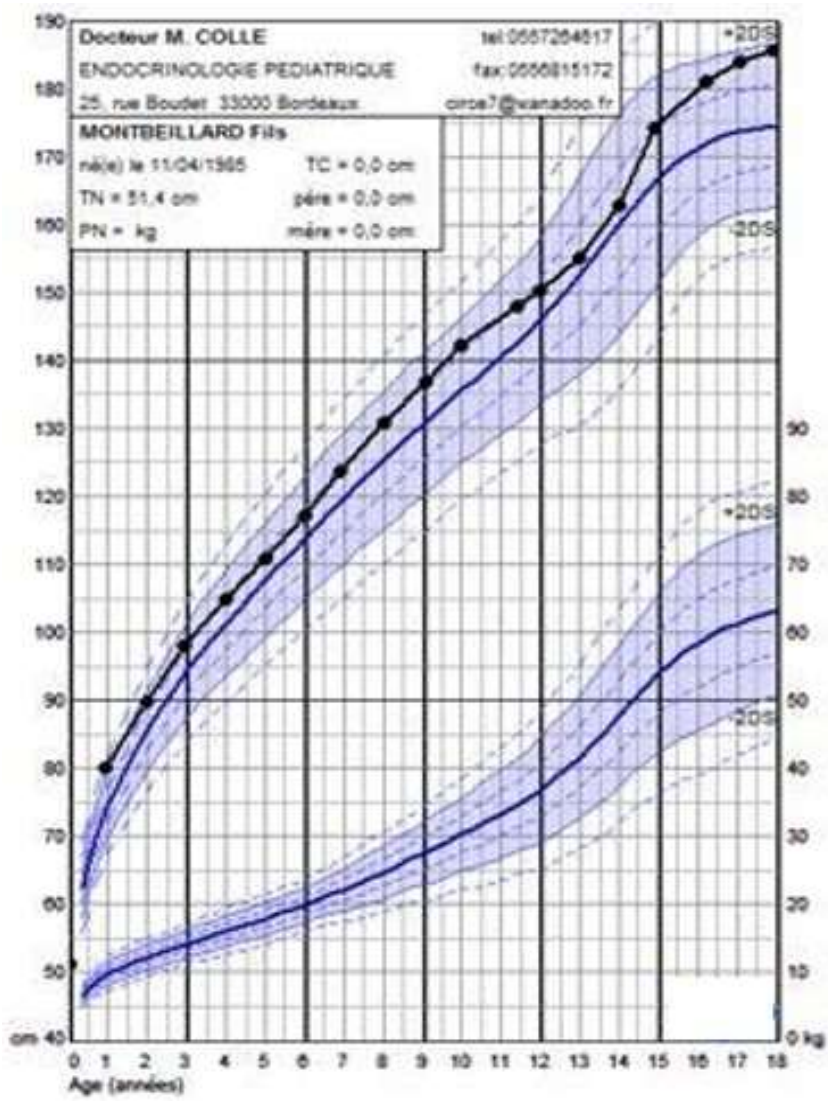

Figure 02. Première courbe de croissance [12]

Les principes de l'Auxologie - biométrie de la croissance- y sont alors clairement énoncés : le même enfant est mesuré à intervalles réguliers et dans des conditions aussi semblables que possible ("toujours pieds nus", "avec la même toise, la même équerre et par la même personne").

Quand Buffon a décidé d'en faire état dans son encyclopédique "Histoire Naturelle», il ne se doutait sûrement pas que cette courbe de croissance entrait dans l'Histoire humaine en présageant ce qui doit être fait consciencieusement pour chaque personne depuis son enfance : des mesures régulières et suivies de sa taille, établissant au final une courbe de croissance.

Historiquement, cette courbe est considérée comme la première à l'échelle individuelle !

Durant cette même période, à quelques encablures du Royaume du Lys, est suivie une cohorte d'élèves à l'école de Carlschule dans la ville de Stuttgart, en Allemagne (17721777), réalisant ainsi la première étude longitudinale de cohorte $[11,12]$.

\section{Le $19^{e}$ siècle et l'essor de la statistique}

\section{Quételet et son indice...}

La première étude statistique complète sur la croissance des enfants (poids et taille) a été publiée en 1835 par Lambert Adolphe Quételet, né à Gand en Belgique (1796-1874), Il était le premier à utiliser le concept de courbe normale actuellement appelée communément distribution normale ou courbe en cloche (courbe de Gauss) pour décrire la distribution des mesures de croissance. 
Il a aussi proposé en 1870 dans un document intitulé « Anthropométrie ou mesures des différentes facultés de l'homme » l'indice de Quételet: l'indice de corpulence poids/taille qui porte son nom à ce jour [11].

\section{Galton et ses percentiles...}

Sir Francis Galton (1822-1911), ténor de la statistique par ses analyses de corrélations et une myriade d'inventions, démontre " l'héritabilité » de la taille. Il est ainsi le premier scientifique à déterminer des percentiles afin d'étudier un individu par rapport à un groupe de personnes, avec son fameux schéma de la dispersion des tailles [11].

\section{Un nouveau venu : l'Auxologie :}

En 1903, Paul Godin dans un essai intitulé " Recherches anthropométriques sur la croissance des diverses parties du corps » a nommé "Auxologie" la science "métrique de la croissance et plus précisément l'étude de la croissance suivie chez les mêmes sujets pendant de nombreux semestres successifs par un grand nombre de mesures »[10,11]. Ce terme provient de la botanique (auxine: phytohormone de croissance végétale).

En réalité, dès la fin du $19 \mathrm{e}$ siècle, Franz Boas avait déjà noté que, tout au long de leur croissance, les patients pouvaient suivre des itinéraires très éloignés les uns des autres mais atteignant, en finalité, des tailles similaires. Il avait alors introduit le concept d'âge physiologique et proposé l'expression "tempo de la croissance" pour décrire les différents rythmes des sujets ayant une maturation lente ou rapide [11].

\section{Les grandes études longitudinales du $20^{\mathrm{e}}$ siècle}

On peut décrire deux périodes dans la réalisation des grandes études longitudinales : la première au début du 20ème siècle aux USA et la seconde après la seconde guerre mondiale en Europe.

Le Centre de Recherche du Bien-être de l'Enfant (dirigé par Baldwin et plus tard par Meredith) de l'Université de l'Iowa débute en 1917 une étude pionnière concernant les performances scolaires des enfants et leur relation avec la taille, le poids et la capacité vitale.

Les références dites de Harvard (élaborées par Stuart et Stevenson), dérivant de cette étude, sont alors rapportées en 1969 par Jellife [14-15].

Aux années 1950, la vague traverse l'Atlantique: le Centre International de l'Enfance (CIE) de Paris coordonne une étude internationale simultanément réalisée dans 7 pays européens différents (Suède, Finlande, Tchécoslovaquie, Pologne, Hongrie, Ecosse, France).

La partie française de cette étude, réalisée par l'équipe du Pr Sempé, a permis d'établir les courbes de référence françaises de la croissance qui figurent jusqu'à présent dans les carnets de santé des enfants français, et largement utilisées en Algérie jusqu'à la publication des dernières courbes de l'OMS en 2006 [16].

Dr. James Tanner, expert en croissance humaine de renommée mondiale, faisait partie de nombre de ces équipes de recherche et faisait référence dans l'aube de l'endocrinologie pédiatrique d'alors [17].

\section{Incorporation des données socio-démographiques}

À la fin des années 1970, une nouvelle école d'anthropométrie voit le jour avec l'émergence des historiens et des économistes, mettant en exergue les changements socio-économiques, politiques et les périodes de guerre. Elle
Elle permet de faire éclore un nouveau concept : le " gain séculaire » en taille, constaté notamment dans les pays industrialisés le long des deux derniers siècles ; initialement à la Marine Britannique [18, 19].

\section{Modernisations ... et Modélisations}

Depuis les années 1980-1990, une approche plus mathématique est adoptée via la méthode LMS ( $\mathrm{L}=$ Lambda pour le biais, $M=M u$ pour la médiane et $S=$ Sigma pour le coefficient de variation généralisé) ; une approche qui est fortement recommandée (particulièrement par les spécialistes en santé publique) pour l'élaboration des nouvelles tables de référence pour la croissance. Ces méthodes sont à l'origine de la plupart des courbes de référence nationales et internationales utilisées de par le monde $[20,21]$.

\section{L'Atlas de Croissance : Une histoire imagée !}

Pendant l'entre-deux-guerres, l'essor de la radiologie conventionnelle a permis d'étendre son usage en vue d'apprécier la maturation squelettique comme indice indirect de la croissance des os longs.

Leurs premières données ont été recueillies par Dr. William Walter Greulich et Dr. Sarah Idell Pyle de 1931 à 1942 à partir de radiographies prises à intervalles réguliers chez une cohorte d'enfants américains originaires d'Europe du Nord, à haut niveau socio-économique (par convention, toujours une radiographie de la main gauche).

Un livre dédié dénommé "atlas" est ainsi publié en 1959 par les deux médecins, il est dichotomisé selon l'âge et le sexe : chez la fille de 0 à 18 ans et chez le garçon de 0 à 19 ans $[22,23]$.

De nos jours, il existe plusieurs atlas d'âge osseux ; avec des versions adaptées à des populations particulières de patients ou à certaines pathologies chroniques, constitutionnelles ou génétiques [22].

\section{CONCLUSION}

Cet article dresse une brève chronologie des sciences de l'auxologie et de l'anthropométrie, depuis la courbe de croissance du Comte de Montbeillard au $18^{\mathrm{e}}$ siècle, véritable pionnière, aux dernières courbes de l'Organisation Mondiale de la Santé au début de ce millénaire dont l'application a permis d'asseoir une base solide pour la surveillance clinique de la population, essentiellement pédiatrique.

Déclaration d'intérêts: les auteurs ne déclarent aucun conflit d'intérêt en rapport avec cet article.

\section{RÉFÉRENCES}

1. Aknin J-J. Croissance générale de l'enfant. EMC - Médecine buccale 2008;3(1):1-11. [Article 28-800-C-10]. DOI : 10.1016/S12830860(08)49181-9.

2. Jouve J-L, Bollini G, Launay F, Glard Y, Craviari T, Guillaume J-M, et al. Cartilage de croissance et croissance en orthopédie. EMC - Appareil locomoteur. 2009;4(1):1-15. [Article 14-009-A-10]. DOI : 10.1016/S02460521(09)44798-3.

3. Thibault $\mathrm{H}$, Boulard S, Colle M, Rolland-Cachera M-F. Croissance normale staturo-pondérale. EMC - Pédiatrie. 2009:1-11. [Article 4-002-F-63]. DOI : 10.1016/S0246-0513(09)46315-6.

4. Ganong WF, Jobin M. Physiologie médicale. Bruxelles: De Boeck; 2012.

5. Bouvattier $\mathrm{C}$. Retard de croissance staturopondérale : diagnostic et prise en charge. EMC - Traité de médecine AKOS. 2006;1(4):1-6. [Article 30740]. DOI: 10.1016/S1634-6939(06)41292-8. 
6. Amin N, Mushtaq $\mathrm{T}$, Alvi S. Fifteen-minute consultation: The child with short stature. Arch Dis Child Educ Pract Ed. 2015;100(4):180-4.

7. Vidailhet M. Utilisation en pratique des données anthropométriques. Archives de Pédiatrie. 1999;6(7):787-93.

8. Salaün J-F, Brauner R, Gascoin-Lachambre G, Chalumeau M. Courbes de croissance : quels référentiels pour raccourcir les délais diagnostiques de quelles maladies ? Archives de Pédiatrie. 2011;18(5):H79-80.

9. Scherdel P, Botton J, Rolland-Cachera M-F, Léger J, Pelé F, Ancel PY, et al. Utilisation des courbes de l'Organisation mondiale de la santé pour la surveillance de la croissance des enfants en France. Archives de Pédiatrie. 2014;21(5):50-2.

10. Hermanussen $M$, Bogin B. Auxology - an editorial. Ital J Pediatr. 2014;40(1):8, 1824-7288-40-8.

11. Rolland-Cachera MF. Morphologie et alimentation de l'enfant : évolution au cours des dernières décennies. Cahiers de Nutrition et de Diététique. 2004;39(3):178-84.

12. "La première courbe de croissance d'un enfant". [En ligne]. [cité le 24 septembre 2020]. Disponible: http://courbedecroissance.com/fic/dossiers.php?c=13

13. Scammon RE. The first seriatim study of human growth. American Journal of Physical Anthropology. 1927;10(3):329-36.

14. Baldwin BT. Physical Growth and School Progress: A Study in Experimental Education Bulletin, United States Bureau of Education. Washington, DC: U.S. Government Printing Office; 1914.
15. Meredith HV. The rhythm of physical growth: a study of eighteen anthropometric measurements on lowa City white males ranging in age between birth and eighteen years University of lowa Studies in Child Welfare. 1935;11(3):7-128.

16. Badis N. Contribution à l'élaboration des courbes de croissance locales de la commune de Constantine [Mémoire en ligne]: Université de Constantine; $2014 . \quad$ Disponible: https://bu.umc.edu.dz/theses/agronomie/BAD6530.pdf az

17. Tanner JM. Growth at Adolescence. $2^{\text {nd }}$ ed. Oxford: Blackwell Scientific Publications; 1962.

18. van Wieringen JC. Secular Growth Changes. In: Falkner F, Tanner JM, editors. Human Growth. Boston, MA: Springer US; 1978

19. Rolland-Cachera MF, Deheeger M. Mesures anthropométriques chez I'enfant : Influence des facteurs nutritionnels. Cahiers d'anthropologie et biométrie humaine. 1996;14(1-2):381-8.

20. Cole TJ. The LMS method for constructing normalized growth standards. European journal of clinical nutrition. 1990 Jan;44(1):45-60.

21. WHO Multicentre Growth Reference Study Group. WHO Child Growth Standards based on length/height, weight and age. Acta Paediatr Suppl. 2006 Apr;450:76-85.

22. Adamsbaum C, André C, Merzoug V, Kalifa G. Âge osseux. Intérêt diagnostique et limites. EMC - Pédiatrie, 2005;2(1):1-1. [Article 4-005-A20]. DOI: 10.1016/S0246-0513(05)40372-7

23. Garn SM. Radiographic Atlas of Skeletal Development of the Hand and Wrist. Am J Hum Genet. 1959 Sep;11(3):282-3. PMCID: PMC1931997.

Cet article a été publié dans le « Batna Journal of Medical Sciences » BJMS, l'organe officiel de « l'association de la Recherche Pharmaceutique - Batna»

Le contenu de la Revue est ouvert « Open Access » et permet au lecteur de télécharger, d'utiliser le contenu dans un but personnel ou d'enseignement, sans demander l'autorisation de l'éditeur/auteur.

Avantages à publier dans BJMS :

- Open access : une fois publié, votre article est disponible gratuitement au téléchargement

- Soumission gratuite : pas de frais de soumission, contrairement à la plupart des revues « Open Access »

- Possibilité de publier dans 3 langues : français, anglais, arabe

- Qualité de la relecture : des relecteurs/reviewers indépendants géographiquement, respectant l'anonymat, pour garantir la neutralité et la qualité des manuscrits.

Pour plus d'informations, contacter BatnaJMS@gmail.com ou connectez-vous sur le site de la revue : www.batnajms.net 


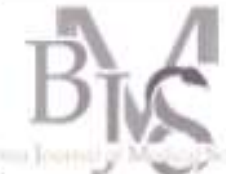

- $\bigcirc$

-

P

Service de Chirurgie Cardiaque, EHS Dr Djaghri Mokhtar,

Constantine - Algérie.

\section{Correspondance à :}

Redha LAKEHAL

lakehal.redha@gmail.com

DOI :https://doi.org/10.48087/ BJMScr.2020.7226

\section{Historique de l'article :}

Reçu le 4 octobre 2019

Accepté le 29 mai 2020

Publié le 09 novembre 2020

Il s'agit d'un article en libre accès distribué selon les termes de la licence Creative Commons Attribution International License (CC BY 4.0), qui autorise une utilisation, une distribution et une reproduction sans restriction sur tout support ou format, à condition que l'auteur original et la revue soient dûment crédités.

Pour citer l'article :

Lakehal R, Bendjaballah S, Aimer F, et al. Anévrisme de la crosse aortique distale englobant l'origine de l'artère sous Clavière gauche : à propos d'un cas. Batna J Med Sci 2020;7(2):186-8. https://doi.org/10.48087/ B]MScr.2020.7226

\title{
Anévrisme de la crosse aortique distale englobant l'origine de
} l'artère sous Clavière gauche : à propos d'un cas.

\author{
Aneurysm of the distal aortic arch including the origin of the left \\ subclavian artery: Case report.
}

\author{
Redha Lakehal, Soumaia Bendjaballah, Farid Aimer, Rabeh Bouharagua, Khacha \\ Khaled, Abdelmalek Bouzid
}

\section{RÉSUMÉ}

Introduction. Les anévrismes de la crosse aortique distale sont plus rares que ceux de l'aorte ascendante. Ils sont le plus souvent découverts à l'occasion d'une radiographie pulmonaire effectuée systématiquement ou pour une autre pathologie. Plus rarement, c'est une complication qui le révèle : soit compression, soit fissuration ou rupture. Le scanner et l'imagerie par résonance magnétique sont les examens de choix dans le diagnostic des anévrismes de la crosse aortique. Le traitement est chirurgical. Le but de ce travail est de monter un cas d'anévrisme de la crosse aortique distale englobant l'origine de l'artère sous clavière. Observation. Nous rapportons l'observation d'un patient âgé de 50 ans sans antécédents hospitalisé dans notre service à la suite de découvert fortuite lors d'un bilan d'une dysphonie d'un anévrisme de la crosse aortique. Préopératoire : Il était au stade II de la NYHA présentant une dysphonie depuis quelques mois. Radiographie pulmonaire: ICT à 0,60 . ECG : RRS. Échocardiographie: Anévrisme de la drosse distale, insuffisance aortique grade II à valve souple. Per opératoire : Volumineux anévrisme de la crosse aortique distale prenant l'origine de l'artère sous clavière gauche. Angio-TDM thoracique: Anévrisme de la crosse 82/72 $\mathrm{mm}$. Coronarographie: Fistule coronaro- auriculaire gauche. Le geste: Mise à plat de l'anévrisme, rétablissement de la continuité aortique par un tube en Dacron numéro 28 implanté en terminoterminale en hypothermie profonde et arrêt circulatoire et réimplantation de l'artère sous clavière dans la prothèse par l'intermédiaire d'un tube en dacron numéro 10. Les suites post opératoires étaient défavorables avec décès du patient en I 3 par défaillance circulatoire. Conclusion. Les anévrismes de la crosse aortique sont rares, d'étiologies diverses et posent des problèmes de technique chirurgicale très spécifiques. L'abord chirurgical de la crosse est très difficile.

Mots clefs: Anévrisme, crosse aortique, arrêt circulatoire, CEC

\section{ABSTRACT}

Introduction. Aneurysms of the distal aortic arch are rarer than those of the ascending aorta. They are most often discovered during a chest X-ray performed routinely or for another pathology. More rarely, it is a complication that reveals it: either compression, cracking or rupture. CT scan and magnetic resonance imaging are the tests of choice in the diagnosis of aortic arch aneurysms. The treatment is surgical. The goal of this work is to mount a case of a distal aortic arch aneurysm encompassing the origin of the subclavian artery. Observation. We report the observation of a 50-year-old patient with no history of aortic aneurysm who was hospitalized in our department following an incidental finding of a dysphonia of an aortic arch aneurysm. Preoperative: He had been in stage II NYHA with dysphonia for a few months. Chest X-ray: TBI at 0.60. ECG: RRS. Echocardiography: Aneurysm of the distal dross, grade II soft-valved aortic insufficiency. Intraoperative: Voluminous aneurysm of the distal aortic arch originating in the left subclavian artery. Thoracic CT angio: Aneurysm of the distal aortic arch $82 / 72 \mathrm{~mm}$. Coronary angiography: Left atrial coronary fistula. Procedure: Flattening of the aneurysm, restoration of aortic continuity with a number 28 Dacron tube implanted at the terminus in deep hypothermia and circulatory arrest and reimplantation of the subclavian artery in the prosthesis with a number 10 Dacron tube. The postoperative outcome was unfavorable with death of the patient on J 3 due to circulatory failure. Conclusion. Aortic arch aneurysms are rare, of various etiologies and pose very specific problems of surgical technique. The surgical approach of the aortic arch is very difficult.

Keywords: Aneurysm, aortic arch, circulatory arrest, cardiopulmonary bypass.

\section{INTRODUCTION}

Les anévrismes de la crosse aortique distale sont plus rares que ceux de l'aorte ascendante. [1]

Ils sont le plus souvent découverts à l'occasion d'une radiographie pulmonaire effectuée systématiquement ou pour une autre pathologie.

Plus rarement, c'est une complication qui le révèle : compression (trachée, œsophage, phrénique, récurrent), soit fissuration ou rupture avec hémomédiastin et hémothorax. [2]
L'angio-TDM et l'angio-IRM thoracique sont les modalités radiologiques préférées pour bilanter les anévrismes de l'aorte thoracique.

Le traitement chirurgical consiste également à remplacer la crosse par un tube prothétique en réimplantant les artères à destinée encéphalique dans ce tube.

Le but de ce travail est de rapporter un cas d'anévrisme de la crosse aortique distale englobant l'origine de l'artère sous clavière découvert fortuitement lors d'un bilan d'exploration pour dysphonie. 


\section{OBSERVATION}

Nous rapportons l'observation d'un patient âgé de 50 ans sans antécédents hospitalisé dans notre service à la suite de découvert fortuite lors d'un bilan d'une dysphonie d'un volumineux anévrisme de la crosse aortique distal.

En préopératoire :

Taille : Malade longiligne mesurant $177 \mathrm{~cm}$ de taille.

Classe fonctionnelle : Il était au stade II de la NYHA.

Il présentait une dysphonie depuis quelques mois.

Examen physique :

-Absence d'anomalies squelettiques et oculaires.

-Souffle diastolique 3/6 au foyer aortique.

Radiographie pulmonaire demandait dans le cadre d'exploration d'une dysphonie montrait un élargissement du médiastin supérieur avec un index cardiothoracique à 0,60.

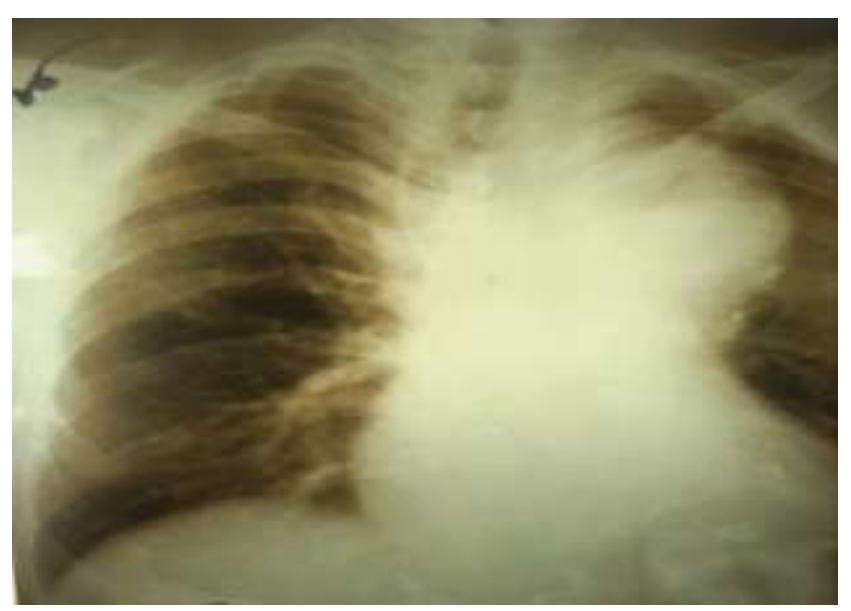

Figure 1. Élargissement du médiastin supérieur.

L'ECG montrait un rythme régulier sinusal. L'Échocardiographie montrait un anévrisme de la crosse aortique distale, insuffisance aortique grade II à valve souple, VG : 37/57 mm, FE : 64\%, PAPS : $37 \mathrm{~mm} \mathrm{hg}$.

L'Échodoppler cervical montrait une dilatation fusiforme du bulbe carotidien droit et du tronc artériel brachiocéphalique. L'Angio-TDM thoracique montrait un anévrisme de la crosse aortique mesurant $82 / 72 \mathrm{~mm}$.

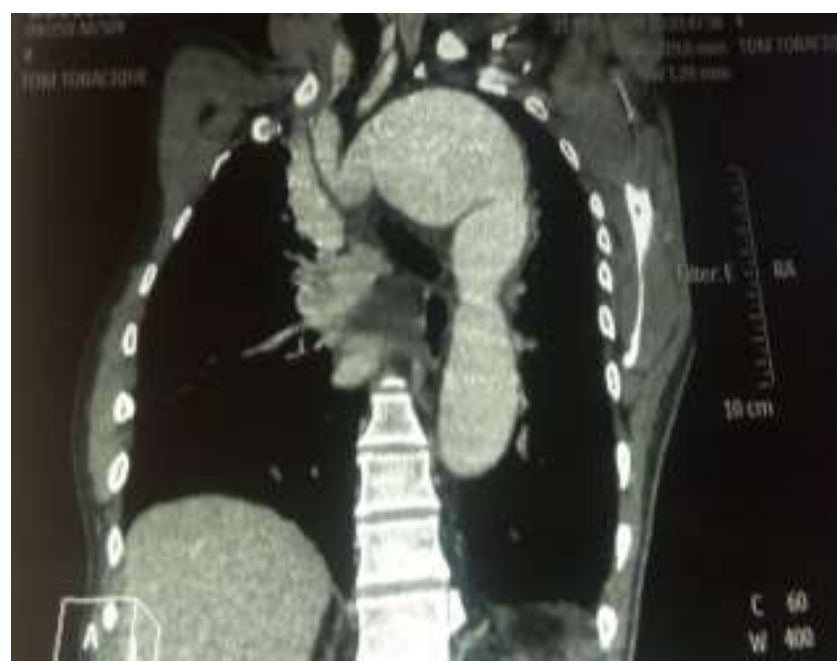

Figure 2. Anévrisme de la crosse aortique distal prenant l'origine de l'artère sous clavière gauche.
L'Angio-IRM thoracique n'a pas été demandé. La coronarographie faite dans le cadre d'un bilan préopératoire montrait une fistule coronaro auriculaire gauche alimenté par l'artère inter ventriculaire antérieure.

L'abord de la crosse aortique distale s'est fait par une thoracotomie postéro latérale gauche après installation d'une circulation extracorporelle (CEC) fémoro-fémorale.

L'exploration per opératoire montrait un volumineux anévrisme de la crosse aortique distale prenant l'origine de l'artère sous clavière gauche avec paroi aortique fragile sans signes de dissection ou de pré rupture.

Le geste consistait en la mise à plat de l'anévrisme après dissection et contrôle de ce dernier en amont et en aval, rétablissement de la continuité aortique par un tube en Dacron numéro 28 implanté en termino-terminale en hypothermie profonde et arrêt circulatoire sous CEC fémorofémorale et réimplantation de l'artère sous clavière dans la prothèse par l'intermédiaire d'un tube en dacron numéro 10.

Durée de la CEC : $253 \mathrm{mn}$, d'arrêt circulatoire : 39mn, de refroidissement : $63 \mathrm{mn}$ et de réchauffement : $190 \mathrm{mn}$.

Les suites post opératoires étaient défavorables avec décès du patient en 3 par défaillance circulatoire.

\section{DISCUSSION}

L'anévrisme de la crosse aortique peut s'exprimer par de complications mécaniques comme la dysphonie par compression du nerf récurrent [7].

L'indication opératoire pour ce patient était à la fois le diamètre de l'anévrisme et la complication par compression du nerf récurrent type dysphonie [4].

La chirurgie de la crosse aortique est complexe avec un risque péri-opératoire élevé [5]. Elle nécessite une planification minutieuse et une approche systématique afin de réduire la morbidité et la mortalité, ce qui permet d'opérer les patients avec un risque acceptable [5].

L'artère sous clavière gauche peut être ligaturé ou réimplanté directement ou par l'intermédiaire d'un tube prothétique sur la prothèse prothétique comme c'est le cas de notre patient.

La chirurgie de la crosse aortique sous CEC avec arrêt circulatoire n'est pas anodine de complications $[5,6]$.

L'insuffisance circulatoire apparait en post opératoire immédiat est une des complications de la CEC avec arrêt circulatoire et accroit la mortalité postopératoire [3].

L'avènement des techniques endovasculaires a changé radicalement la prise en charge des anévrismes de la crosse aortique distale [3].

\section{CONCLUSION}

Les anévrismes de la crosse aortiques sont rares et les étiologies sont diverses. Ils posent de problèmes de technique chirurgicale très spécifiques.

L'abord chirurgical de la crosse est très difficile car sur cette portion l'aorte quitte le médiastin antérieur pour le médiastin postérieur et passe de l'hémi thorax droit vers l'hémi thorax gauche.

L'avènement des techniques endovasculaires a changé le pronostic de ces patients (mortalité opératoire).

Déclaration d'intérêts: les auteurs ne déclarent aucun conflit d'intérêt en rapport avec cet article. 


\section{RÉFÉRENCES}

1. Buth J, Penn O, Tielbeek A, Mersman M. Combined approach to stentgraft treatment of an aortic arch aneurysm. J Endovasc Surg. 1998 Nov;5(4):329-32. doi: 10.1583/10746218(1998)005<0329:CATSGT>2.0.CO;2. PMID: 9867322

2. Kato M, Ohnishi K, Kaneko M, Ueda T, Kishi D, Mizushima T,et al. New graft-implanting method for thoracic aortic aneurysm or dissection with a stented graft. Circulation. 1996 Nov 1;94(9 Suppl):II188-93. PMID: 8901744.

3. Clouse WD, Hallett JW Jr, Schaff HV, Gayari MM, Ilstrup DM, Melton LJ 3rd. Improved prognosis of thoracic aortic aneurysms: a populationbased study. JAMA. 1998 9;280(22):1926-9. doi 10.1001/jama.280.22.1926. PMID: 9851478.

4. Coady MA, Rizzo JA, Hammond GL, Mandapati D, Darr U, Kopf GS, et al. What is the appropriate size criterion forresection of thoracic aortic aneurysms? J Thorac Cardiovasc Surg 1997; 113(3):476-91 [discussion 489-91].
5. Hiratzka LF, Bakris GL, Beckman JA, Bersin RM, Carr VF, Casey DE Jr, Eagle KA, Hermann LK, Isselbacher EM, Kazerooni EA, Kouchoukos NT, Lytle BW, Milewicz DM, Reich DL, Sen S, Shinn JA, Svensson LG, Williams DM; American College of Cardiology Foundation/American Heart Association Task Force on Practice Guidelines; American Association for Thoracic Surgery; American College of Radiology: American Stroke Association; Society of Cardiovascular Anesthesiologists; Society for Cardiovascular Angiography and Interventions; Society of Interventional Radiology; Society of Thoracic Surgeons; Society for Vascular Medicine. 2010 ACCF/AHA/AATS/ACR/ASA/SCA/SCAI/SIR/STS/SVM guidelines for the diagnosis and management of patients with Thoracic Aortic Disease: a report of the American College of Cardiology Foundation/American Heart Association Task Force on Practice Guidelines, American Association for Thoracic Surgery, American College of Radiology, American Stroke Association, Society of Cardiovascular Anesthesiologists, Society for Cardiovascular Angiography and Interventions, Society of Interventional Radiology, Society of Thoracic Surgeons, and Society for Vascular Medicine. Circulation. 2010 Apr 6;121(13):e266-369. doi: 10.1161/CIR.0b013e3181d4739e. Epub 2010 Mar 16. Erratum in: Circulation. 2010 Jul 27;122(4):e410. PMID: 20233780.

6. 6. Stecker MM, Cheung AT, Pochettino A, Kent GP, PattersonT, Weiss SJ, et al. Deep hypothermic circulatory arrest: I.Effects of cooling on electroencephalogram and evoked potentials. Ann ThoracSurg 2001;71(1):14-21.

7. Rizvi MM, Singh RB, Jain A, Sarkar A. Asymptomatic aortic aneurysm causing right vocal cord palsy and hoarseness: A rare presentation. Anesth Essays Res. 2014 Sep-Dec;8(3):397-400. doi: 10.4103/02591162.143157. PMID: 25886343; PMCID: PMC4258979.

Cet article a été publié dans le « Batna Journal of Medical Sciences » BJMS, l'organe officiel de « l'association de la Recherche Pharmaceutique - Batna»

Le contenu de la Revue est ouvert « Open Access » et permet au lecteur de télécharger, d'utiliser le contenu dans un but personnel ou d'enseignement, sans demander l'autorisation de l'éditeur/auteur.

Avantages à publier dans BJMS :

- Open access : une fois publié, votre article est disponible gratuitement au téléchargement

- Soumission gratuite : pas de frais de soumission, contrairement à la plupart des revues « Open Access »

- Possibilité de publier dans 3 langues : français, anglais, arabe

- Qualité de la relecture : des relecteurs/reviewers indépendants géographiquement, respectant l'anonymat, pour garantir la neutralité et la qualité des manuscrits.

Pour plus d'informations, contacter BatnaJMS@gmail.com ou connectez-vous sur le site de la revue : www.batnajms.net 


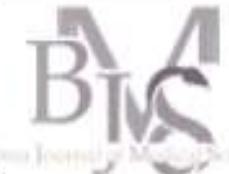

- $\bigcirc$

- $\bigcirc$

?

○

Chirurgie générale et

carcinologique, Établissement

Hospitalier Spécialisé Centre de Lutte Contre le Cancer Batna

Correspondance à :

Zohir BENABDELHAFIDH

lchirdems2016@gmail.com

DOI :https://doi.org/10.48087/B MScr.2020.7227

\section{Historique de l'article :}

Reçu le 17 décembre 2019

Accepté le 15 mai 2020

Publié le 09 novembre 2020

Il s'agit d'un article en libre accès distribué selon les termes de la licence Creative Commons Attribution International License (CC BY 4.0), qui autorise une utilisation, une distribution et une reproduction sans restriction sur tout support ou format, à condition que l'auteur original et la revue soient dûment crédités.

Pour citer l'article :

Benabdelhafidh Z. Tumeur neuroendocrinienne de l'ovaire, une localisation inhabituelle : A propos d'un cas et revue de la littérature. Batna J Med Sci 2020;7(2):189-91.

https://doi.org/10.48087/BJM Scr.2020.7227

Tumeur neuroendocrinienne de l'ovaire, une localisation inhabituelle : A propos d'un cas et revue de la littérature

\author{
Neuroendocrine tumor of the ovary, an unusual localization: About a case \\ and review of the literature
}

\title{
Zohir Benabdelhafid
}

\section{RÉSUMÉ}

Les tumeurs neuroendocrines (TNE) constituent un groupe des tumeurs susceptibles de naitre en tout point de l'organisme. Ces tumeurs sont rares. Bien que ceux-ci soient généralement associés au tractus gastro-intestinal, au pancréas et au poumon, des cas gynécologiques ont également été rapportés, Parmi celles-ci, les TNE ovariennes qui sont extrêmement rares. Seuls $15 \%$ d'entre eux existeraient sous forme pure, le reste présentant des composants tératomateux tels que des struma ovarii ou des kystes dermoïdes. Nous rapportons le cas d'une patiente âgée 36ans présentant un carcinome neuroendocrine primitif bilatérale de l'ovaire, confirmé par immuno- histochimiques, pour lequel une réduction chirurgicale optimale et une chimiothérapie a été réalisée. Nous insistons à travers cette observation et sous la lumière de la revue de la littérature sur les aspects épidémiologiques, morphologiques, immunohistochimiques et le défi diagnostic posé par ces tumeurs rares.

Mots-clés : Carcinome neuroendocrine, cancer de l'ovaire, chromogranine, immunohistochimie

\section{INTRODUCTION}

Les tumeurs neuroendocrines (TNE) constituent un groupe des tumeurs susceptibles de naitre en tout point de l'organisme. Ces tumeurs sont rares. Bien que ceux-ci soient généralement associés au tractus gastro-intestinal, au pancréas et au poumon, des cas gynécologiques ont également été rapportés, Parmi celles-ci, les TNE ovariennes qui sont extrêmement rares. Nous insistons à travers cette observation et sous la lumière de la revue de la littérature sur les aspects épidémiologiques, morphologiques, immunohistochimiques et le défi diagnostic posé par ces tumeurs rares.

\section{OBSERVATION}

Nous rapportons le cas d'une patiente âgée de 36 ans avec un BMI de 26,34 kg / m², G2P2. La patiente était suivie en endocrinologie pour une hypothyroïdie depuis 2011. La symptomatologie clinique évoluait depuis 11 mois par l'apparition des douleurs pelviennes.

L'examen clinique a trouvé une sensibilité latéro-utérine droite au toucher vaginal, et la perception d'une masse à travers le cul de sac postérieur. L'examen clinique a trouvé une sensibilité latéro-utérine droite au toucher vaginal, et la perception d'une masse à travers

\section{ABSTRACT}

Neuroendocrine tumors (NETs) are a group of tumors that could be born anywhere in the organism. Even Though these are generally associated with the gastrointestinal tract, pancreas and lung, gynecological cases have also been reported. Of these, ovarian NETs are extremely rare. Only $15 \%$ of them would exist in pure form, the rest presenting teratomatous components such as struma ovarii or dermoid cysts. We report the case of a 36-year-old patient with a bilateral primary ovarian neuroendocrine carcinoma, confirmed by immunohistochemistry, for whom optimal surgical reduction and chemotherapy were performed. We insist on this observation and in the light of the review of the literature on the epidemiological, morphological, immunohistochemical aspects and the diagnostic challenge posed by these rare tumors.

Keywords: Neuroendocrine carcinoma, ovarian cancer, chromogranin, immunohistochemistry

le cul de sac postérieur. Une échographie pelvienne a objectivé une image latéro-utérine solido-kystique droite sans épanchement péritonéal. Le dosage sérique du CA125 était de $13 \mathrm{UI} / \mathrm{ml}(\mathrm{VN}<35)$, ACE 0 ,47. Le complément IRM avait montré la présence de deux formations solido-kystiques ovariennes bilatérales mesurant $53 \mathrm{~mm}$ à droite et $27 \mathrm{~mm}$ à gauche suspectes, sans signes d'agressivité, ni extension loco-régionale (fig. 1).

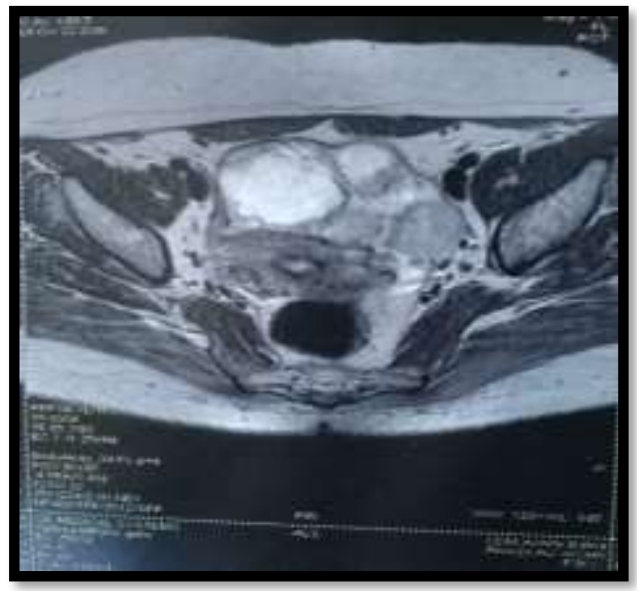

Figure 1. IRM pelvien montrant deux formations solido-kystiques ovariennes bilatérales mesurant 53 $\mathrm{mm}$ à droite et $27 \mathrm{~mm}$ à gauche. 
La patiente était opérée par cœlioscopie. L'exploration de la cavité péritonéale n'avait montré aucune lésion de carcinose ni ascite, une masse ovarienne de $7 \mathrm{~cm}$ prenant l'annexe droit et une autre masse ovarienne gauche de $5 \mathrm{~cm}$, d'où la réalisation d'une annexectomie droite avec biopsies multiples (Gouttières pariéto-coliques droite et gauche, l'espace vésico-utérin, grand épiploon, le douglas et l'ovaire gauche), dont le résultat anatomo-pathologique avec coloration immunohistochimique était une tumeur ovarienne carcinoïde bien différenciée avec expression diffuse des marqueurs neuroendocrine ( synaptophysine, chromogranine A) (fig. 2).

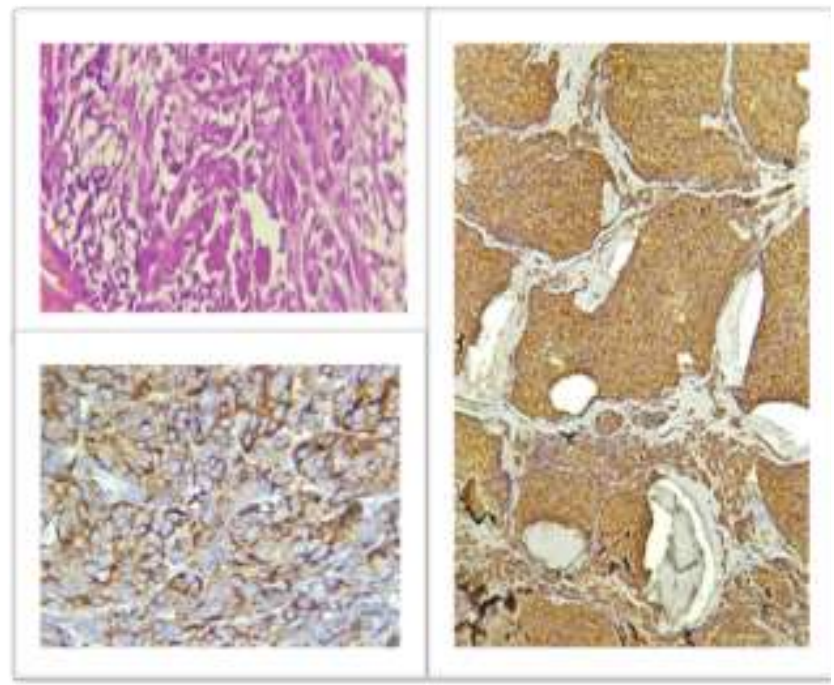

Figure 2. Images microscopiques montrant une positivité cytoplasmique diffuse et intense pour la chromogranine A et la synaptophysine dans les cellules tumorales (IHC).

Le bilan d'extension réalisé (TDM thoraco-abdominopelvien), a été normal, le dossier a été présenté au staff multidisciplinaire d'oncologie, il a été décidé 4 cures de chimiothérapie adjuvante à base de TAXOL+CARBO comprenant le carboplatine, et paclitaxel. Le geste chirurgical a été complété par une hystérectomie avec annexectomie gauche, omentectomie, appendicectomie et curage ganglionnaire pelvien (fig. 3 )

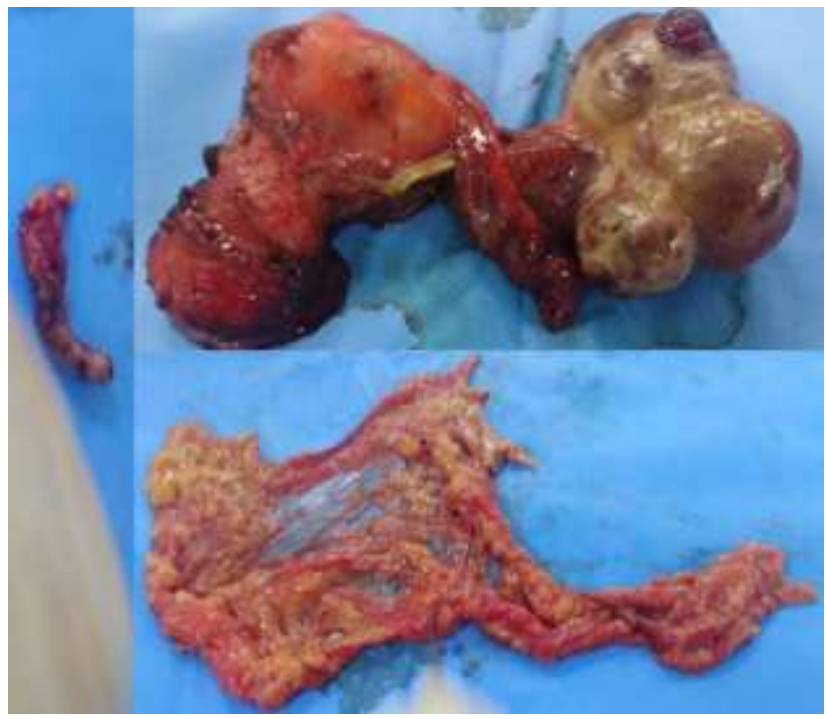

Figure 3. Pièce opératoire: hystérectomie avec annexectomie gauche, omentectomie, appendicectomie et curage ganglionnaire pelvien

\section{DISCUSSION}

Les tumeurs neuroendocrines (TNE) constituent un groupe des tumeurs susceptibles de naitre en tout point de l'organisme. Ces tumeurs sont rares. Bien que ceux-ci soient généralement associés au tractus gastro-intestinal, au pancréas et au poumon, des cas gynécologiques ont également été rapportés, Parmi celles-ci, les TNE ovariennes qui sont extrêmement rares [1]. La fourchette d'âge rapportée est comprise entre 22 et 76 ans, la manifestation clinique la plus courante étant une douleur abdominale suivie d'une distension ou un saignement généralisé et postménopausique [2].

Un tiers des patientes atteintes de TNE ovarienne présentent des symptômes neuroendocriniens cliniques tels que bouffées vasomotrices, diarrhée et crampes abdominales [3]. Seuls 15\% d'entre eux existeraient sous forme pure, le reste présentant des composants tératomateux tels que des struma ovarii ou des kystes dermoïdes [1]. Lorsque des TNE apparaissent dans l'ovaire, elles peuvent se présenter sous la forme de carcinomes à petites cellules, de variants de grandes cellules et de tumeurs carcinoïdes bien différenciées [4].

L'histogenèse des tumeurs neuroendocrines est inconnue et plusieurs hypothèses ont été émises. Premièrement, les cellules neuroendocrines sont présentes dans l'épithélium des tumeurs bénignes, borderline et malignes du tractus génital féminin. Deuxièmement, les cellules endocrines primitives peuvent se différencier en cellules endocrines et autres. Troisièmement, les tumeurs neuroendocrines ovariennes peuvent se développer à partir de cellules non neuroendocrines, qui activent des gènes favorisant la différenciation neuroendocrine. Ces cellules peuvent produire des amines et des peptides biologiquement actifs qui agissent comme des neurotransmetteurs, des hormones ou des régulateurs de paracrine [5].

Le diagnostic préopératoire de TNE est rarement possible. Le CA-125 est un antigène tumoral présent dans $75 \%$ à $83 \%$ des cancers épithéliaux de l'ovaire est rarement élevé dans TNE [6]. Les études radiologiques les plus couramment utilisées telles que la tomodensitométrie et l'imagerie par résonance magnétique (IRM) ne sont pas utiles pour le diagnostic différentiel avec d'autres tumeurs ovariennes et montrent généralement des résultats non spécifiques, où des études sur les radionucléides avec des isotopes spéciaux peuvent être plus utiles [7].

L'immunohistochimie est importante pour diagnostiquer le carcinome neuroendocrinien. Les marqueurs immunohistochimiques les plus couramment utilisés sont la chromogranine A, la synaptophysine, la cytokératine et le CD56. Pour diagnostiquer une tumeur neuroendocrine du tractus gynécologique féminin, il doit y avoir au moins deux marqueurs neuroendocriniens positifs.

La prise en charge thérapeutique des TNE de l'ovaire est similaire à celle des cancers épithéliaux et consiste en une résection chirurgicale complète de la tumeur suivie d'une chimiothérapie adjuvante [8],

Le principal point de controverse avec la chimiothérapie reste le fait que l'avantage de la chimiothérapie adjuvante après résection chirurgicale n'est pas prouvé en raison de la rareté de cette entité et du manque de données d'étude. Le pronostic des TNE de l'ovaire est difficile à déterminer en raison de la rareté de la maladie, du petit nombre de cas signalés et du manque d'études systématiques en population ou de données de registre. 


\section{CONCLUSION}

Les tumeurs neuroendocrines de l'ovaire constituent une entité tumorale très rare en oncologie gynécologique, La rareté de ces tumeurs rend difficile la formulation d'un consensus général sur le traitement standard. Cependant la chirurgie reste la pierre angulaire de la prise en charge et doit être pratiquée dans tous les cas. Des études multicentriques sont nécessaires pour apporter plus de lumière sur cette entité tumorale rare.

Déclaration d'intérêts: les auteurs ne déclarent aucun conflit d'intérêt en rapport avec cet article.

\section{RÉFÉRENCES}

7. Modlin IM, Sador A. Une analyse de 8305 cas de tumeurs carcinoïdes. Cancer. 1997;9: 813-829 2.

8. Collins RJ, Cheung A, Ngan HY, Wong LC, Chan SY, Ma HK. Carcinome primaire mixte neuroendocrinien et mucineux de l'ovaire. Arch Gynecol Obstet. 1991; 248:139-143.
1. Fisseler-Eckhoff A., Demes M. Tumeurs neuroendocrines du poumon. Cancers (Basel) 2012;4:777-798.

2. Reed NS, E. Gomez-Garcia, D. Gallardo-Rincon, B. Barrette, K Baumann, M. Friedlander, G. Kichenadasse, D. D. Lorusso, MR Mirza, InterGroupe Cancer Gynécologique. (GCIG) revue de consensus pour les tumeurs carcinoïdes de l'ovaire. Int. J. Gynecol. Cancer. 2014; 24 : S35-41.

3. Eichhorn $\mathrm{JH}$, jeune RH. Tumeurs neuroendocrines du tractus génital. Am J Clin Pathol. 2001;115 : S94-S112.

4. Bast RC Jr, Klug TL, E Schaetzl, P Lavin, JM Niloff, Greber TF, et al. Surveillance du carcinome ovarien humain avec une combinaison de CA 125 , CA 19-9 et d'antigène carcinoembryonnaire. Je suis J Obstet Gynecol. 1984;149:553-559.

5. Choi YD, Lee JS, Choi C, Park CS, Nam JH. Carcinome neuroendocrinien de l'ovaire. Gynecol Oncol. 2007;104: 747-752.

6. Rekhi B,Patil B,Deodhar KK : Spectre des carcinomes neuroendocrines du col utérin,. Ann Pathol Diagn 2013;17: 1-9.

Cet article a été publié dans le « Batna Journal of Medical Sciences » BJMS, l'organe officiel de « l'association de la Recherche Pharmaceutique - Batna»

Le contenu de la Revue est ouvert « Open Access » et permet au lecteur de télécharger, d'utiliser le contenu dans un but personnel ou d'enseignement, sans demander l'autorisation de l'éditeur/auteur.

Avantages à publier dans BJMS :

- Open access : une fois publié, votre article est disponible gratuitement au téléchargement

- Soumission gratuite : pas de frais de soumission, contrairement à la plupart des revues « Open Access »

- Possibilité de publier dans 3 langues : français, anglais, arabe

- Qualité de la relecture : des relecteurs/reviewers indépendants géographiquement, respectant l'anonymat, pour garantir la neutralité et la qualité des manuscrits.

Pour plus d'informations, contacter BatnaJMS@gmail.com ou connectez-vous sur le site de la revue : www.batnajms.net 


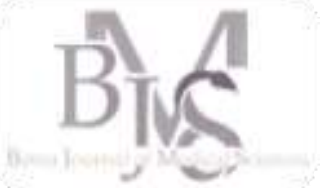

\section{Cas rare d'hémorragie digestive grave survenant sur une diverticulose jéjunale}

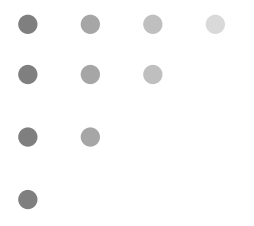

Service de chirurgie générale et oncologique, CHU Beni Messous, Alger - Algérie

\section{Correspondance à :}

Abdelkrim Chetib

karim.chetibi@yahoo.fr

DOI :https://doi.org/10.48087/ B]MScc.2020.7228

\section{Historique de l'article :}

Reçu le 24 octobre 2019

Accepté le 02 juillet 2020

Publié le 09 novembre 2020

Il s'agit d'un article en libre accès distribué selon les termes de la licence Creative Commons Attribution International License (CC BY 4.0), qui autorise une utilisation, une distribution et une reproduction sans restriction sur tout support ou format, à condition que l'auteur original et la revue soient dûment crédités.

\section{Pour citer l'article :}

Chetibi A, Saidani M. Cas rare d'hémorragie grave survenant sur diverticulose jéjunales.

Batna J Med Sci

2020;7(2):192-3.

https://doi.org/10.48087/BJ MScc.2020.7228

\section{A rare case of severe digestive hemorrhage occurring on jejunal diverticulosis}

Abdelkrim Chetibi, Mustapha Saidani

\section{RÉSUMÉ}

Les hémorragies digestives d'origine grêlique sont rares. Les diverticules jéjuno-iléaux sont rarement à l'origine d'une pathologie aiguë. La fréquence des diverticules acquis du jéjunum et de l'iléon varie de 0,06 à 2,3\% selon les séries. L'entéroscopie est l'examen de choix pour poser le diagnostic précocement. L'embolisation artérielle constitue un procédé efficace du traitement de l'hémorragie digestive associée à une diverticulose jéjunale. Nous rapportons l'observation d'une patiente qui s'est présentée aux urgences dans un tableau d'hémorragie digestive grave basse due à une diverticulose grêlique diagnostiquée suite à une laparotomie exploratrice en urgence et traitée efficacement par une résection jéjunale avec anastomose jéjuno-jéjunale T-T. Les suites post-opératoires étaient simples. Après 1 an de recul, la malade n'avait pas de récidive hémorragique.

Mots clés : Hémorragie, Diverticule, Entéroscopie, Résection, Anastomose.

\section{INTRODUCTION}

Les hémorragies digestives d'origine grêlique sont rares. Les diverticules jéjuno-iléaux sont rarement à l'origine d'une pathologie aiguë. Le scanner est l'examen de référence pour le diagnostic des diverticuloses du grêle. Ce sont les reformations coronales qui permettent de poser le diagnostic avec précision et certitude.

Une péritonite localisée dans le flanc gauche ou la région mésocolique en l'absence de pathologie antérieure et chez un sujet plus au moins âgé, doit faire suspecter dans un premier temps une diverticulose compliquée ou une perforation sur corps étranger.

La fréquence des diverticules acquis du jéjunum et de l'iléon varie de 0,06 à 2,3\% selon les séries. Ce sont des pseudo-diverticules, comme les diverticules sigmoïdo-coliques : hernie de la muqueuse et de la sous muqueuse à travers la musculeuse et la séreuse.

Les diverticules vrais comportent les 3 couches d'une paroi digestive normale. Surtout rencontrés au cours des 6ème ou 7ème décennie. Ils affectent les hommes plus que les femmes. Ils peuvent être uniques ou multiples (jusqu'à 100) avec un nombre moyen de 10-15. Leur taille est variable et peut dépasser $3 \mathrm{~cm}$. siégeant sur le bord mésentérique de l'intestin. Ils sont nombreux et plus larges sur le jéjunum proximal, plus rares et plus petits au niveau de l'iléon terminal.

\section{ABSTRACT}

Digestive hemorrhages of hailstone origin are rare. Jejuno-ileal diverticulas are rarely the cause of an acute pathology. The frequency of diverticula acquired from jejunum and ileon varies from 0.06 to $2.3 \%$ depending on the series. Enteroscopy is the examination of choice for early diagnosis. Arterial embolization is an effective treatment of digestive hemorrhage associated with jejunal diverticulosis. We report the observation of a patient who presented to the emergency room in a low severe digestive hemorrhage chart due to a veelic diverticulosis diagnosed following an emergency exploratory laparotomy and treated effectively with a jejunal resection with jejuno-jéjunal anastomosis T-T. The post-operative suites were simple. After a one-year setback, the patient had no hemorrhagic recurrence.

Keywords : Hemorrhage, Diverticulum, Enteroscopy, Resection, Anastomosis.

\section{OBSERVATION}

Nous rapportons l'observation d'une patiente qui s'est présentée aux urgences dans un tableau d'hémorragie digestive grave basse due à une diverticulose grêlique diagnostiquée suite à une laparotomie exploratrice en urgence et traitée efficacement par une résection jéjunale.

Il s'agit d'une Femme de 76 ans hospitalisée en urgence pour la prise en charge d'une hémorragie digestive grave suite à des mélénas abondants. On notait dans ses antécédents une hypertension artérielle. L'examen clinique était marqué par la présence d'une pâleur cutanéomuqueuse franche, une pression artérielle à 90 $/ 60 \mathrm{~mm} \mathrm{Hg}$ et une fréquence cardiaque de $100 / \mathrm{min}$. L'examen abdominal était normal et le toucher rectal ramenait du sang rouge.

L'hémoglobinémie était à 6,5 g/dl. Le bilan de coagulation était normal. La malade transfusait avec quatre culots globulaires en 48 heures. Après amélioration de son état général et hémodynamique, une fibroscopie oeso-gastroduodénale a été pratiquée révélant une hernie hiatale simple et une bulbite, sans traces de saignement local. La malade recevait 4 culots globulaires supplémentaires en 48 heures mais sans succès. Devant la persistance du saignement et l'aggravation de son état clinique, une laparotomie exploratrice en urgence a été décidée. 
L'exploration per-opératoire a révélé la présence de multiples diverticules intéressant la partie proximale du jéjunum à contenus sanguin traitée par une résection jéjunale avec anastomose jéjuno-jéjunale T-T (figures 1 et 2). Les suites post-opératoires étaient simples.

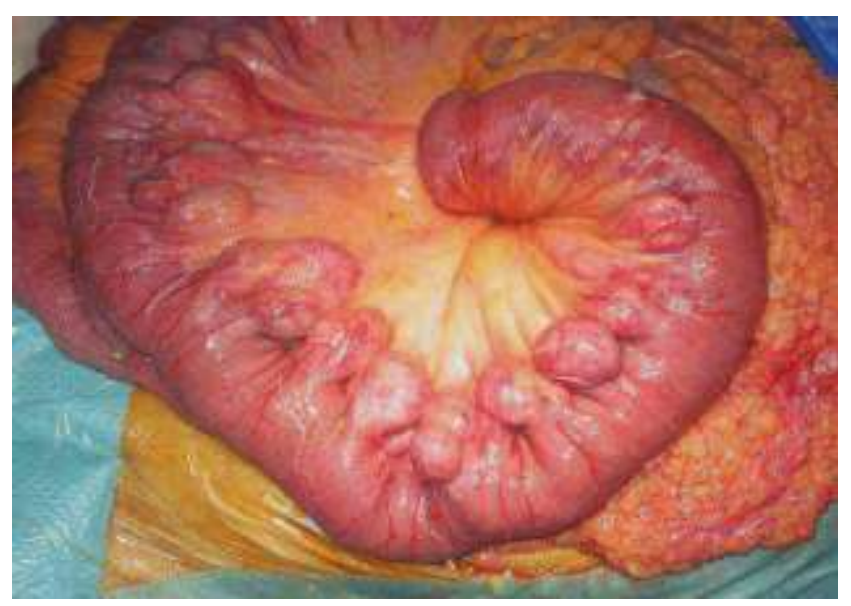

Figure 1. Diverticulose jéjunale

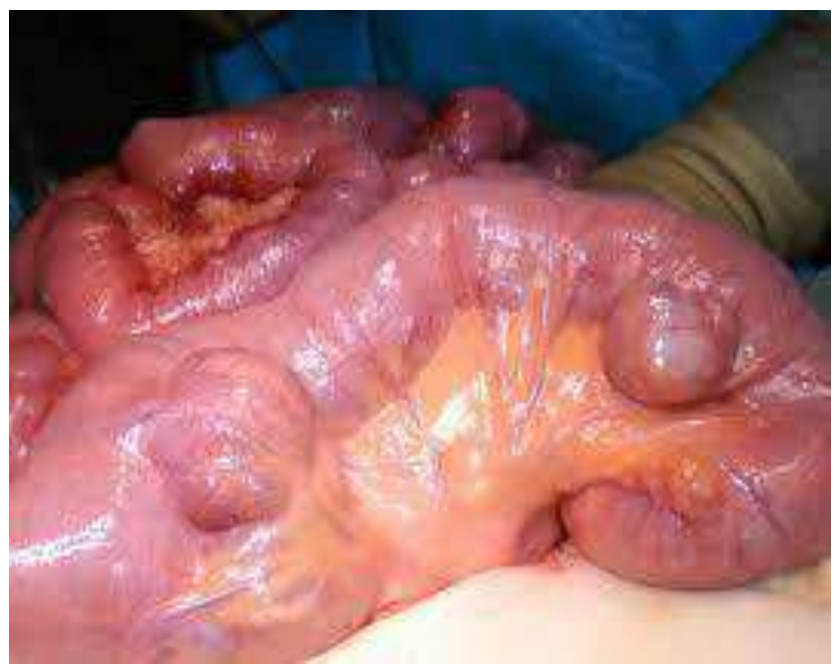

Figure 2. Pseudo-diverticules du jéjunum.

Après un an de recul, le malade n'avait pas de récidive hémorragique.

\section{DISCUSSION}

Il existe deux types de diverticules, les diverticules acquis localisés sur le bord mésentérique et les diverticules congénitaux siégeant sur le bord anti-mésentérique $(1,2)$.

Les diverticules acquis touchent les sujets âgés de plus de 60 ans. Ils sont plus fréquents au niveau de la partie proximale du jéjunum $(3,4)$. Les diverticules sont souvent associés à une diverticulose colique (5). Les complications surviennent dans environ $10 \%$ des cas. Il existe deux types de complications : les complications aigues représentées essentiellement par des sténoses grêliques, péritonites diverticulaires et très rarement les hémorragies). Les complications chroniques représentées par les douleurs abdominales, le syndrome pseudo-occlusif intestinal et l'anémie $(6,7)$.

Les hémorragies digestives d'origine diverticulaire représentent un vrai problème diagnostique et thérapeutique. L'entéroscopie est un excellent examen pour détecter cette lésion grêlique, mais sa pratique en urgence reste difficile à nos jour (7-9). L'artériographie pratiquée en période d'hémorragie associée à la vasopressine, ou par la réalisation d'une embolisation peut être une solution au traitement non opératoire (10-12).

\section{CONCLUSION}

A L'heure actuelle, Les hémorragies digestives d'origine diverticulaire représentent un véritable problème diagnostique et thérapeutique. L'entéroscopie est l'examen de choix pour poser le diagnostic précocement. L'embolisation artérielle constitue un procédé efficace du traitement de l'hémorragie digestive associée à une diverticulose jéjunale.

Déclaration d'intérêts: Les auteurs ne déclarent pas de conflits d'intérêt en rapport avec cet article.

\section{RÉFÉRENCES}

1. Miller, L. S., C. Barbarevech, and L. S. Friedman. Less frequent causes of lower gastrointestinal bleeding. Gastroenterology clinics of North America 23.1 (1994): 21.

2. Graupe, F., Rassek, D., Schwenk W., et al. Diverticulitis of the jejunum as a rare cause of acute gastrointestinal hemorrhage--diagnosis and therapy. Leber, Magen, Darm, 1995, vol. 25, no 6, p. 279-281.

3. Longo WE, Vernavaa AM. Clinical implications of jejunoileal diverticular disease. Diseases of the colon \& rectum, 1992, vol. 35, no 4, p. 381-388.

4. Ross CB, Richards WO, Sharp KW, et al. Diverticular disease of the jejunum and its complications. The American surgeon, 1990, vol. 56, no 5, p. 319.

5. Hochain P, Merle V, Capet C, et al. Upper digestive hemorrhage in patients over 80 years of age: incidence and prognostic factors. Gastroenterologie clinique et biologique, 1996, vol. 20, no 8-9, p. 638-644.

6. Agnifili, A, Gola, P, Gianfelice F, et al. Rare digestive hemorrhage caused by diverticular pathology of the small intestine. Minerva Chirurgica, 1990, vol. 45, no 10, p. 721-724.

7. Cellier C, Tkoub M, Gaudric M., et al. Comparaison de l'entéroscopie poussée et du transit baryté de l'intestin grêle dans les saignements digestifs et les anémies ferriprives inexpliqués. Gastroentérologie clinique et biologique, 1998, vol. 22, no 5, p. 491-494.

8. Schuster DM, Chapman WE., Ahl ET, et al. Jejunal diverticular hemorrhage localized by red blood cell scintigraphy. Clinical nuclear medicine, 2001, vol. 26, no 11, p. 936-937.

9. Okazaki M, Furui S, Higashihara $H$, et al. Emergent embolothérapy of small intestine hemorrhage. Gastrointestinal radiology, 1992, vol. 17, no 1, p. 223-228.

10. Bandi, R, Shetty PC., Sharma RP, et al. Superselective arteria embolization for the treatment of lower gastrointestinal hemorrhage. Journal of Vascular and Interventional Radiology, 2001, vol. 12 , no 12 , p. 1399-1405.

11. Krämer SC, Görich J, Rilinger N, Et al. Embolization for gastrointestinal hemorrhages. European radiology, 2000, vol. 10, no 5, p. 802-805.

12. Evangelista PT, Hallisey MJ. Transcatheter embolization for acute lower gastrointestinal hemorrhage. Journal of Vascular and Interventional Radiology, 2000, vol. 11, no 5, p. 601-606. 


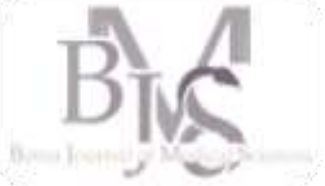

- $\bigcirc$

-

○

○

${ }^{1}$ Service d'ORL et de chirurgie cervico-faciale, CHU de Kamenge, Burundi.

${ }^{2}$ Service d'ORL et de chirurgie cervico-faciale, Hôpital Principe de Dakar, Sénégal.

Correspondance à :

Cheikh Ahmédou LAME

cheikhlame@gmail.com

DOI :https://doi.org/10.48087/ BJMScr.2020.7229

\section{Historique de l'article :}

Reçu le 15 juin 2019

Accepté le 04 septembre 2020

Publié le 09 novembre 2020

Il s'agit d'un article en libre accès distribué selon les termes de la licence Creative Commons Attribution International License (CC BY 4.0), qui autorise une utilisation, une distribution et une reproduction sans restriction sur tout support ou format, à condition que l'auteur original et la revue soient dûment crédités.

Pour citer l'article :

Seza KJC, Lame CA, Loum B, et al. Abcès cervical tardif sur plaie pénétrante par flèche : à propos d'une observation. Batna J Med Sci 2020;7(2):194-6. https://doi.org/10.48087/ BJMScr.2020.7229

\title{
Abcès cervical tardif sur plaie pénétrante par flèche : à propos d'une observation.
}

\author{
Delayed cervical abcess on penetrating neck injury by arrow: a case \\ report
}
Kandjoka Jean Claude Seza ${ }^{1}$, Cheikh Ahmédou Lame², Birame Loum², Leonard Bivahagumye $^{1}$, Gordien Ngendakuriyo ${ }^{1}$

\section{RÉSUMÉ}

Introduction. Les plaies pénétrantes du cou par flèche, quoique rares maintenant, peuvent être rencontrées dans certaines zones rurales de pays en développement, où l'accès aux soins hospitaliers demeure difficile. Observation. Nous rapportons le cas d'un patient congolais de 40 ans qui a consulté pour suppuration cervicale profonde, six mois après un traumatisme du cou par une flèche artisanale. L'exploration chirurgicale, après traitement antibiotique, a permis d'extraire le corps étranger vulnérant. L'évolution était favorable. Conclusion. Les plaies pénétrantes du cou par flèche artisanale nécessitent une prise en charge hospitalière précoce et adéquate par une équipe chirurgicale expérimentée afin d'éviter des suites compliquées voire désastreuses.

Mots Clés : Plaie pénétrante cervicale, abcès cervical, flèche.

\section{INTRODUCTION}

Les plaies pénétrantes du cou par flèche sont rares. Ces armes de basse vélocité restent cependant dangereuses par leur caractère tranchant et les complications infectieuses qu'elles peuvent entrainer sur cet espace anatomique réduit et renfermant des structures vitales. Les auteurs rapportent ici une observation de plaie pénétrante négligée du cou par flèche chez un cultivateur.

\section{OBSERVATION}

Un patient de 40 ans, cultivateur congolais, originaire d'une zone rurale forestière a consulté pour une tuméfaction cervicale douloureuse chronique.

L'interrogatoire rapportait une notion de traumatisme du cou par un projectile qui l'aurait atteint, six mois auparavant, dans un contexte de conflit interethnique.

L'examen, à l'admission, retrouvait un patient stable avec un empâtement cervical diffus associé à une plaie d'environ $2 \mathrm{~cm}$ à bords granulomateux, siégeant au niveau de la région latéro-cervicale gauche haute, et laissant soudre du pus (Figure 1).

\section{ABSTRACT}

Introduction. Penetrating neck injuries by arrow are a very uncommon entity. However, they can be encountered in some rural areas of developing countries, where access to medical care remains difficult. Case report. A 40 year old congolese man, farmer, from a rural forest area, presented with a deep cervical abcess, six months after neck trauma by an artisanal arrow. surgical exploration, after antibiotic treatment, was performed to extract the vulnating foreign body. Evolution was favorable without complication. Conclusion. Penetrating neck injuries by arrow require an early and adequate management by experimented surgical team to avoid complicated or even disastrous consequences.

Key words : Penetrating neck injury, cervical abscess, arrow.

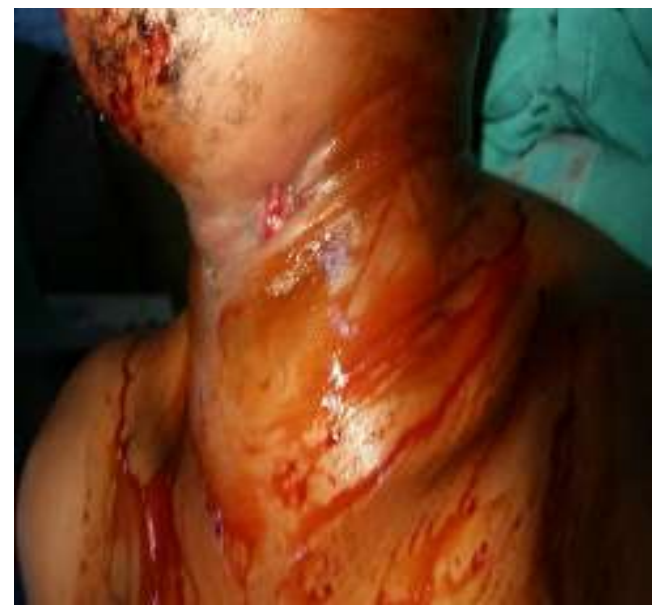

Figure 1. Tuméfaction cervicale antérieure avec plaie à bords granulomateux.

La radiographie standard du cou de face et de profil (Figure 2) mettait en évidence un corps étranger latéro-cervical gauche de tonalité métallique allant de la région sous maxillaire gauche à la région sus-claviculaire homolatérale.

Le scanner cervical confirmait la présence du corps étranger avec une infiltration diffuse des parties molles cervicales antérieure et latérale gauche (Figure 2). 


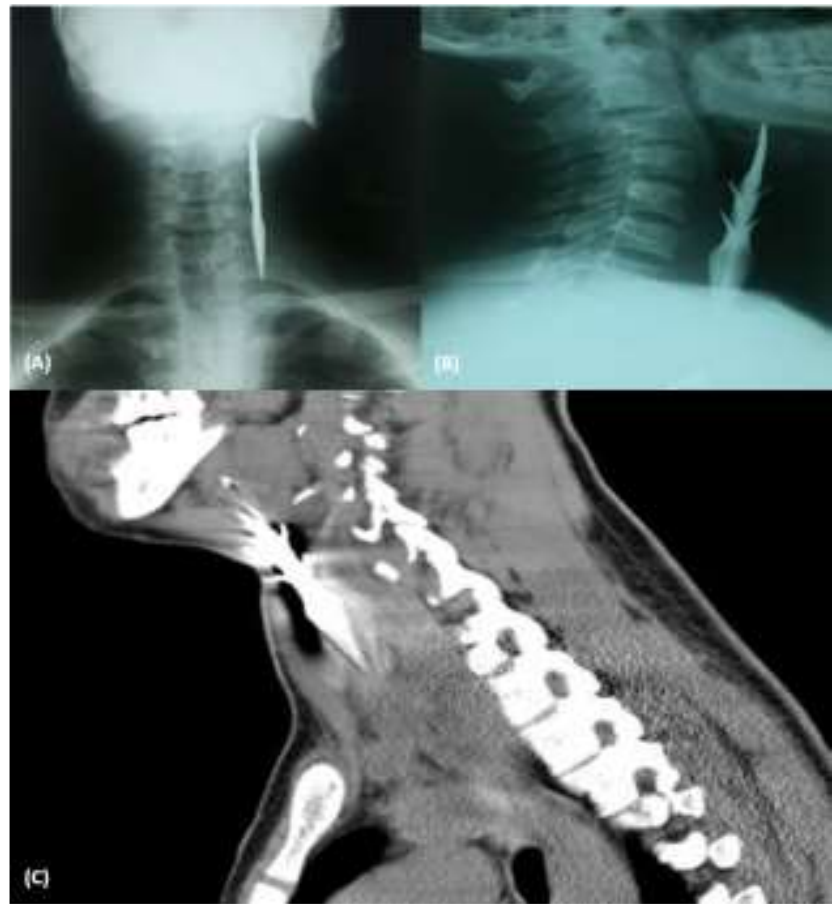

Figure 2. Radiographie montrant un corps étranger radio opaque. (A). Radiographie standard de face. (B). Radiographie standard de profil. (C). TDM cervicale en coupe sagittale montrant un corps étranger avec infiltration des parties molles cervicales.

La cervicotomie exploratrice (Figure 3), après une semaine d'antibiothérapie probabiliste, permettait d'extraire sans difficulté une flèche artisanale à bords dentelés (Figure 3) sans aucune lésion vasculaire ou viscérale associée. Les suites opératoires étaient simples.

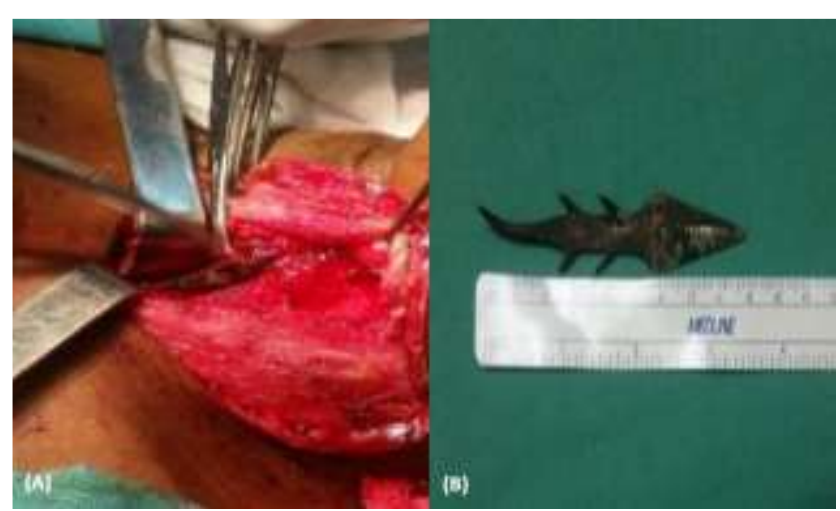

Figure 3. (A). Vue opératoire montrant la flèche figée en dedans du muscle sterno-cléido-mastoïdien. (B). Corps étranger extrait.

\section{DISCUSSION}

Toute plaie cervicale ayant franchi le muscle peaucier est définie comme une plaie pénétrante du cou (1-3). Ces plaies concernent une région anatomique complexe où sont concentrées plusieurs structures vitales dans un espace réduit $(1,4)$.

L'incidence des plaies pénétrantes du cou représente 5 à 10\% de tous les traumatismes $(1,2,5,6)$.

La nature de l'agent causal varie en fonction de l'aire géographique. Autrefois fréquentes, les plaies pénétrantes du cou par armes artisanales sont, de nos jours, rarement rencontrées $(6,7)$. Les principaux agents vulnérants sont les armes à feu, les armes blanches (couteaux, rasoirs, coupecoupe) $(1,3)$. Toutefois, les traumatismes cervicaux par armes artisanales type flèche ou lance peuvent être retrouvés dans les pays en développement notamment dans les sociétés tribales (5).

Sur le plan clinique, la présence de signes de gravité impose une prise en charge immédiate et une admission directe au bloc opératoire (1). Ces signes de gravité sont constitués par un saignement actif, un hématome expansif, l'abolition ou la diminution d'un pouls en aval de la lésion, l'existence d'un souffle à l'auscultation ou un état de choc circulatoire $(1,2)$. L'existence d'une détresse respiratoire aiguë ou la présence de bulles d'air extériorisées par la plaie cervicale sont des signes de gravité. Une lésion ignorée du tractus digestif constitue un risque septique majeur $(1,3)$.

La tomodensitométrie et l'Imagerie par résonnance magnétique lorsqu'elles sont disponibles, permettent de faire un bilan lésionnel vasculaire et viscéral qui aboutira à une bonne planification opératoire (3).

Les complications tardives des plaies pénétrantes cervicales par armes blanches ont été rarement reportées mais elles ne sont pas exceptionnelles en Papouasie, Nouvelle Guinée, au Nigeria et en Inde où les combats tribaux sévissent encore (5).

Ces complications tardives sont en général infectieuses $(5,8)$ comme chez notre patient qui a consulté pour un abcès cervical, six mois après le traumatisme.

Le traitement d'une plaie pénétrante du cou par flèche dépend de la localisation de la plaie, de la présence in situ de l'agent vulnérant, de la profondeur de la plaie et de l'état général du patient $(1,5)$.

Devant des signes de gravité, une exploration chirurgicale est indiquée $(1,3)$. La cervicotomie exploratrice permet de faire le bilan des lésions avant l'extraction minutieuse de la flèche. Cette extraction ne se fera jamais à l'aveugle. Elle est réalisée après contrôle premier des structures anatomiques nobles (6) .

Des pinces fortes (type Kocher ou Robert) permettent de désenclaver et d'extraire la flèche, dans le sens antérograde de la trajectoire du projectile pour éviter d'accrocher les gros vaisseaux et autres tissus importants $(5,6)$.

\section{CONCLUSION}

Les plaies pénétrantes du cou par flèche sont devenues rares. Elles peuvent engager le pronostic vital dans l'immédiat ou se manifester tardivement par une complication. Leur prise en charge requiert une évaluation clinique rigoureuse aidée de l'imagerie. La technique d'extraction, bien codifiée, doit être réalisée au bloc opératoire par un chirurgien expérimenté afin d'éviter une issue catastrophique.

Déclaration d'intérêts: les auteurs ne déclarent aucun conflit d'intérêt en rapport avec cet article.

\section{RÉFÉRENCES}

1. Nowicki J, Stew B, Ooi E. Penetrating neck injuries: a guide to evaluation and management. Ann R Coll Surg Engl. 2018;100(1):6-11.

2. Nepal A, Joshi R, Bhandary S, et al. Penetrating neck injury by an arrow: $A$ paradigm of age old assault. Nepal Med Coll J. 2010;12(1):58-60. 
3. Saito N, Hito R, Burke PA, Sakai O. Imaging of Penetrating Injuries of the Head and Neck:Current Practice at a Level I Trauma Center in the United States. Keio J Med. 2014; 63(2):23-33.

4. Van Waes OJ, Cheriex KCAL, Navsaria PH, et al. Management of penetrating neck injuries. Br J Surg. 2012;99(1):149-54.

5. Aremu SK, Dike B. Penetrated Arrow Shot Injury in Anterior Neck. Int J Biomed Sci. 2011;7(1):77-80.
6. Madhok BM, Roy DDD, Yeluri S. Penetrating arrow injuries in Western India. Injury. 2005;36(9):1045-50.

7. Shereen R, Oskouian RJ, Loukas M, Tubbs RS. Treatment of Arrow Wounds : A Review. Cureus. 2018;10(4):e2473.

8. Etoundi PO, Bob'oyono JM, Ela AA, Esiéné A. Plaie Pénétrante Thoracique par Jet de Lance. Health Sci Dis. 2014;15(4):1-3.

Cet article a été publié dans le « Batna Journal of Medical Sciences » BJMS, l'organe officiel de « l'association de la Recherche Pharmaceutique - Batna»

Le contenu de la Revue est ouvert « Open Access » et permet au lecteur de télécharger, d'utiliser le contenu dans un but personnel ou d'enseignement, sans demander l'autorisation de l'éditeur/auteur.

Avantages à publier dans BJMS :

- Open access : une fois publié, votre article est disponible gratuitement au téléchargement

- Soumission gratuite : pas de frais de soumission, contrairement à la plupart des revues « Open Access »

- Possibilité de publier dans 3 langues : français, anglais, arabe

- Qualité de la relecture : des relecteurs/reviewers indépendants géographiquement, respectant l'anonymat, pour garantir la neutralité et la qualité des manuscrits.

Pour plus d'informations, contacter BatnaJMS@gmail.com ou connectez-vous sur le site de la revue : www.batnajms.net 
- $\bigcirc$

P

${ }^{1}$ Département de médecine, Faculté de médecine d'Alger, Université d'Alger 1, Algérie.

2Département de Pharmacie, Faculté de médecine d'Alger, Université d'Alger 1, Algérie.

${ }^{3}$ Laboratoire de Biochimie, Centre Pierre et Marie Curie, Alger, Algérie.

\section{Correspondance à :}

Ammar CHIKOUCHE

chikouchea@gmail.com

DOI : https://doi.org/10.48087/ BJMScr.2020.7230

Reçu le 17 juin 2020

Accepté le 06 octobre 2020

Publié le 09 novembre 2020

Il s'agit d'un article en libre accès distribué selon les termes de la licence Creative Commons Attribution International License (CC BY 4.0), qui autorise une utilisation, une distribution et une reproduction sans restriction sur tout support ou format, à condition que

l'auteur original et la revue soient dûment crédités.
-

\section{Historique de l'article :}

Molecular analysis of an Algerian family of MEN2A at the CPMC, Algiers. Ammar Chikouche ${ }^{1,3}$, Nadia Ould Bessi ${ }^{2,3}$, Nawel Habak ${ }^{2,3}$

\section{RÉSUMÉ}

La NEM2A, qui se caractérise par l'association d'un cancer médullaire de la thyroïde (CMT) à un phéochromocytome et/ou une hyperparathyroïdie, fait partie avec la NEM2B et le CMTF des Néoplasies Endocriniennes Multiples de type 2 (ou NEM2). Ces NEM2 constituent des affections héréditaires rares, de transmission autosomique dominante dues à des mutations du proto-oncogène RET. La recherche de mutations du proto-oncogène RET chez un cas index NEM2A permet d'une part de conforter le diagnostic clinique et d'autre part d'identifier précocement grâce au dépistage génétique, parmi les apparentés du cas index, ceux qui sont porteurs de l'anomalie génétique, avant toute manifestation biologique ou clinique, pour une meilleure prise en charge. Les objectifs sont la caractérisation de la mutation ponctuelle du protooncogène RET chez le cas index et dépister chez les apparentés du cas index, les porteurs de la mutation germinale. Dans ce travail, nous avons mis au point les techniques de diagnostic génotypique par $\mathrm{PCR} /$ séquençage des 7 exons du proto-oncogène RET (8, $10,11,13,14,15,16)$ les plus fréquemment affectés. L'étude a été réalisée chez une femme avec CMT et phéochromocytome bilatéral (NEM2A). La mutation retrouvée chez le cas index, a été recherchée chez les et 07 enfants apparemment sains. La mutation identifiée chez le cas index (C634Y / exon 11) est retrouvée chez les 02 apparentés cliniquement atteints, ainsi que chez 04 des 07 enfants cliniquement sains. Cette mutation C634Y, commune au CMT et à la NEM2A, nous oblige à considérer les cas de CMT présentant cette mutation comme des NEM2A potentiels et de leur assurer une surveillance clinique et biologique identique. Une thyroïdectomie prophylactique sera proposée aux porteurs sains.

Mots clés : NEM2A, RET, Mutation.

\section{INTRODUCTION}

Les NEM2 ou néoplasies Endocrines multiples de type 2 (NEM 2) sont des affections familiales de transmission autosomique dominante du cancer médullaire de la thyroïde (1) qui se présentent en trois sous-types cliniques: NEM2A, NEM2B et CMTF (2-5). Le CMT des NEM2 est appelé CMT familial et représente 25 $\%$ (6). Dans $75 \%$ des cas le CMT est sporadique.

La NEM2A est caractérisée par la présence de cancer médullaire de la thyroïde (CMT) (dans $100 \%$ des cas), associé à un phéochromocytome (retrouvé $50-60 \%$ des cas) et à une hyperplasie des glandes parathyroïdes (présente dans $20-30 \%$ des cas). apparentés, 2 sœurs adultes présentant chacune un CMT

\section{ABSTRACT}

The MEN2A, which is characterized by the association of medullary thyroid cancer (MTC), pheochromocytoma and / or hyperparathyroidism, belongs with MEN2B and FMTC in Multiple Endocrine Neoplasia type 2 (or MEN 2). These MEN2 are rare hereditary diseases, transmitted as autosomal dominant and due to mutations in the RET proto-oncogene. The search for mutations in the RET proto-oncogene in an index case MEN2A allows one hand to confirm the clinical diagnosis and secondly to identify through genetic testing, including the related index case, those carriers the genetic abnormality early before any biological or clinical manifestation, for better management. The objectives are to characterize the point mutation in the RET proto-oncogene in the index case and detect in relatives of the index case, the carriers of germline mutation. In this studie, we developed genotypic diagnosis techniques by PCR / sequencing of the 7 exons of RET proto-oncogene $(8,10,11,13,14,15$, 16) whose most frequently affected. The study was performed in a woman with MTC and bilateral pheochromocytoma (MEN2A). The mutation found in the index case, was investigated in relatives, two adult sisters each having a MTC and 07 apparently healthy children. The mutation identified in the index case (C634Y / exon 11) was found in 02 related clinically affected and in 04 of 07 clinically healthy children. The C634Y mutation, common in FMTC and MEN2A, forces us to consider the case of MTC this mutation as potential MEN2A and provide them an identical clinical and laboratory monitoring. Prophylactic thyroidectomy will be offered to healthy carriers.

Keywords: MEN2A, RET, mutation.
Ammar Chikouche, Nadia Ould Bessi, Nawel Habak. famille algérienne de NEM2A au CPMC, Alger. Batna J Med Sci 2020;7(2):197-200. JMScr.2020.7230 Analyse moléculaire d'une https://doi.org/10.48087/B
La NEM2B où on retrouve chez les patients un carcinome médullaire de la thyroïde associé à un phéochromocytome, un syndrome marfanoïde et une neurogangliomatosis du tractus intestinal, mais il n'y a pas d'atteinte de la glande parathyroïdienne. Dans le carcinome médullaire de la thyroïde familial (CMTF), le carcinome médullaire de la thyroïde est la seule manifestation clinique de la maladie (5). Ces formes familiales, NEM2A, NEM2B et CMTF sont toutes dues à des mutations du protooncogène RET.

Le RET proto-oncogène localisé sur le chromosome 10 en 10q11.2. (13) code pour un récepteur membranaire à activité tyrosine kinase (14), exprimé dans des cellules dérivées de la crête neurale (15). 
Ce récepteur RET possède un domaine extracellulaire, un domaine transmembranaire et un domaine intracellulaire.

Un facteur neurotrophique nommé GDNF (glial derived neurotrophic facteur), lui-même lié un récepteur (GDNF-R) amarré à la membrane cytoplasmique par un intermédiaire glycosylphosphatidyl-inositol, se lie au récepteur RET. Ces trois composants forment un complexe qui entraine la transmission de signaux mitogènes $(16,17)$.

Nous rapportons la découverte, par des techniques de biologie moléculaire, d'une mutation du proto-oncogène RET chez un cas index de CMT confortant le diagnostic de forme familiale et permettant le dépistage génétique des apparentés du cas index porteurs de l'anomalie génétique cliniquement sains, avant toute manifestation biologique ou clinique, qui se verront proposer une thyroïdectomie prophylactique et d'assurer une surveillance clinico-biologique du cas index et de ses apparentés.

\section{OBSERVATION}

L'étude a été réalisée chez une femme de 50 ans avec CMT et phéochromocytome bilatéral (NEM2A). Cette patiente est la 3 ème d'une fratrie de 08. Dans les antécédents familiaux, la mère et la tante maternelle avaient un CMT associé à un phéochromocytome. Des prélèvements sanguins ont été réalisés sur tube EDTA après consentement éclairé de la patiente et suivi d'une demande d'analyse avec un résumé clinique.

La mutation retrouvée chez le cas index, a été recherchée chez des apparentés, 2 sœurs adultes (la 7ème et la 8ème de la fratrie) qui présentent chacune un CMT et un phéochromocytome.

Le dépistage génétique à la recherche de la mutation familiale a été réalisé chez 07 enfants apparemment sains. 01 enfant du cas index (le 3ème de la fratrie de 4), les 03 enfants d'une sœur et les 03 autres enfants d'une autre sœur après consentement éclairé. L'ADN génomique a été extrait à partir de globules blancs selon la technique salting Out ou méthode aux sels.

L'analyse génétique à la recherche de la mutation germinale, a porté sur l'exon 11 du proto-oncogène RET fréquemment mutés dans les cas de NEM2A et a été réalisée par séquençage direct (Big Dye Terminator 1-1) sur ABI Prism 3130 (Applied). Les amorces oligonucléotidiques utilisées pour amplifier l'exon du gène RET ont été conçues sur les séquences introniques flanquantes et sont : $11 \mathrm{~F} 5^{\prime}-3^{\prime}$ : CAGAGCATACGCAGCCTGTA et 11R 5'-3': ACACAGCGCCCTATGGAAAT. Les conditions PCR utilisées sont $4 \mu \mathrm{ld}$ 'ADN $(25 \mathrm{ng} / \mu \mathrm{I}), 2,5 \mu \mathrm{I} \mu \mathrm{I}$ de tampon PCR 10X; $1,25 \mu \mathrm{I}$ de dNTPs $(2 \mathrm{mM}, 1,5 \mu \mathrm{I}$ de $\mathrm{MgCl} 2$ à $25 \mathrm{mM}, 1,25 \mu \mathrm{I}$ de dNTP à $2 \mathrm{mM}, 1,25 \mu \mathrm{I}$ de solution d'amorces sens et antisens à $5 \mathrm{pmol} / \mu \mathrm{l}, 0,1 \mu \mathrm{l}$ Taq polymérase Roche $(5 \mathrm{U} / \mu \mathrm{I})$ et $13,15 \mu \mathrm{I}$ d'H2O.

L'amplification est programmée comme suit; une étape de dénaturation initiale de 5 minutes à $94^{\circ} \mathrm{C}$, puis 35 cycles d'amplification (comprenant dénaturation d'une minute à $94^{\circ} \mathrm{C}$ ; hybridation d'une minute à $60^{\circ} \mathrm{C}$ (pour l'exon 11) et élongation d'une minute à $72^{\circ} \mathrm{C}$ ) suivis d'une étape d'élongation finale de 10 minute à $72^{\circ} \mathrm{C}$. Le produit d'amplification était testé sur un gel d'agarose à $2 \%$ et les bandes visualisées par coloration au bromure d'éthidium. Les produits de PCR sont purifiés sur des plaques Millipore (Manu 30) et la réaction de PCR de séquence est réalisée avec 1,5 $\mu \mathrm{l}$ de produit PCR purifiée ; 0,8 $\mu \mathrm{l}$ de Big Dye terminator V1.1 (Applied Biosystem) ; 3,6 $\mu \mathrm{l}$ de tampon 5X (V1.1) et $2 \mu \mathrm{l}$ d'amorce sens ou antisens $(5 \mathrm{pmol} / \mu \mathrm{l})$.
Pour l'exon 11 : l'amplification est programmée avec 25 cycles comportant une étape de 30 secondes à $95^{\circ} \mathrm{C}$ suivi d'une étape de 4 minutes à $60^{\circ} \mathrm{C}$. Après purification par gel filtration au G50, les produits de PCR de séquence sont séparés par électrophorèse capillaire en utilisant la méthode de Sanger dans un séquenceur automatisé ABI Prism 3130 (Division Applied Biosystem). La présence de la mutation a été détectée par comparaison à la séquence de référence.

La patiente âgée de 50 ans présente une mutation due à une substitution de base une transition d'une guanine en une adénine en c.1901G>A à l'état hétérozygote, comme le montre la Figure1.
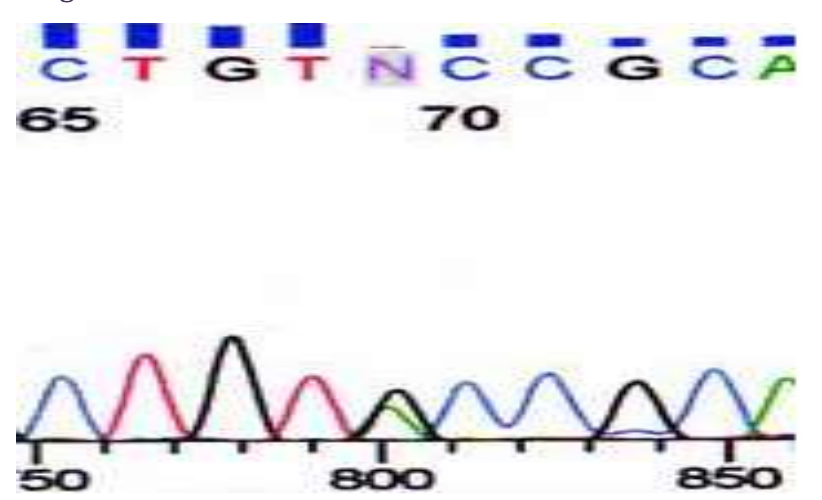

Figure 1. Mutation hétérozygote C634Y (TGC/TAC)

Ce remplacement de G par A conduit à la transformation du codon TGC en codon TAC et substitution d'une cystéine par une tyrosine au codon 634 au niveau de l'exon 11. Cette mutation germinale est notée C634Y. L'analyse génétique a été réalisée chez des apparentés (Figure 2).

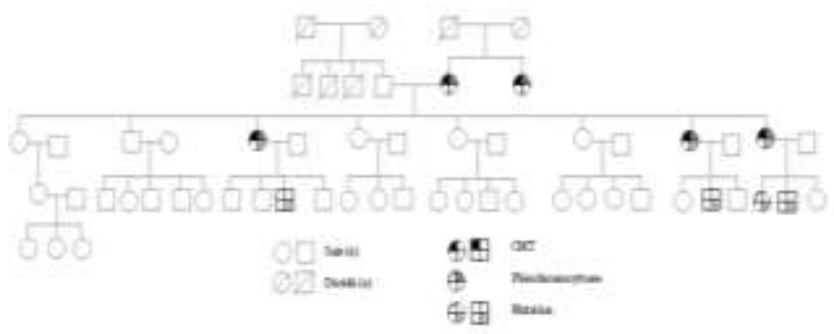

Figure 2. Arbre généalogique de la famille de NEM2A

La mutation identifiée chez le cas index est retrouvée chez les 02 sœurs cliniquement atteintes de NEM2A avec CMT et phéochromocytome, ainsi que chez 04 des 07 enfants cliniquement sains (Tableau 1).

Tableau 1. Résultats de l’analyse génotypique

$\begin{array}{lccl}\text { Diagnostic } & \text { Sexe } & \text { Age } & \text { Mutation } \\ \text { NEM2A } & \text { F } & 50 & \text { Présente } \\ \text { NEM2A } & \text { F } & 40 & \text { Présente } \\ \text { NEM2A } & \text { F } & 38 & \text { Présente } \\ \text { Enfant } & \text { M } & 23 & \text { Présente } \\ \text { Enfant } & \text { F } & 18 & \text { Absente } \\ \text { Enfant } & \text { M } & 10 & \text { Présente } \\ \text { Enfant } & \text { M } & 03 & \text { Absente } \\ \text { Enfant } & \mathrm{F} & 08 & \text { Présente } \\ \text { Enfant } & \mathrm{M} & 06 & \text { Présente } \\ \text { Enfant } & \mathrm{F} & 01 & \text { Absente }\end{array}$




\section{DISCUSSION}

La mutation retrouvée chez le cas index, une femme de 50 ans, et chez certains membres de sa famille au nombre de 06 est une mutation faux-sens, retrouvée au niveau du codon 634 (TGC/TAC) de l'exon 11. Cette mutation entraine le changement d'une cystéine de la partie extracellulaire de la protéine RET en une tyrosine, elle est dénommée Cys634Tyr ou C634Y (8). Cette mutation C634Y est commune aux cas de NEM2A (8) et du CMTF.

La plupart des mutations de RET retrouvées dans les NEM 2A (95\%) ont été trouvées dans les exons 10 et 11, qui code pour le domaine extracellulaire du récepteur. Ce sont des mutations faux-sens qui affectent l'un des codons correspondant à un résidu de cystéine positionnés dans la région juxta-membranaire riche en cystéine $(8 ; 9 ; 11 ; 18)$.

Les patients CMTF ont des mutations au niveau des mêmes codons que lors de l'atteinte sous forme de NEM2A mais avec d'autres substitutions d'acides aminés et au niveau de codons dans d'autres exons du gène RET.

La NEM2B est presque exclusivement associée à une mutation au niveau du codon 918 dans l'exon 16 (8; 9; 11; 18) et à une mutation la A883F au niveau de l'exon 15.

Il a été suggéré que les résidus cystéine du domaine extracellulaire du récepteur RET sauvage forment des ponts disulfures intramoléculaires.

Donc à chacun de ces syndromes, NEM2A, NEM2B et CMTF, des mutations spécifiques du proto-oncogène RET sont associés (7-11) (Tableau 2).

Le mécanisme pathogénique sous-entendu est que dans les NEM2A, les résidus cystéine non appariés du récepteur RET mutant forment des ponts disulfures intermoléculaires. Un changement d'une cystéine en un autre acide aminé, va automatiquement faire en sorte qu'une cystéine ne sera pas appariée. Cet état de fait fera que la cystéine non appariée d'un monomère RET va automatiquement s'apparier avec une cystéine d'en face d'un autre monomère RET.

Ces changements de conformation, suivies de la dimérisation du récepteur, entraineront l'activation du domaine Tyrosine Kinase (19). Cette dimérisation constitutive de RET entrainant l'activation de RET est indépendante du ligand (1 ; 20).

Tous les cas de NEM 2A rapportés à ce jour ont ce même mécanisme d'activation (1). Les mutations NEM 2A confèrent un potentiel de transformation dominant à l'allèle muté de RET $(1 ; 20)$.

La présence de la mutation la C634Y chez le cas index, qui présente un $\mathrm{CMT}$ et un phéochromocytome bilatéral confirme le diagnostic de NEM2A ainsi que pour ses 02 sœurs qui sont cliniquement affectées.

Les enfants cliniquement sains porteurs de la mutation familiale doivent être considérés comme étant des patients NEM2A. Ils doivent impérativement subir une thyroïdectomie prophylactique assez précoce avant que les signes du CMT n'apparaissent

Cela impose de rechercher un phéochromocytome, une hyperparathyroïdie et d'assurer une surveillance biologique et médicale aux patientes affectées et aux porteurs de la mutation.

\section{CONCLUSION}

Nous rapportons l'identification d'une famille de NEM2A avec cancer médullaire de la thyroïde et phéochromocytome, associée à une mutation du gène RET.

Chez cette famille, les patientes cliniquement affectées, au nombre de 03 , sont porteuses de la même mutation germinale $\mathrm{C} 634 \mathrm{Y}$, localisée au niveau de l'exon 11 du protooncogène RET.

Les sujets apparentés sains mais porteurs de cette mutation, au nombre de 04 , doivent être considérés comme étant des sujets qui présenteront une NEM2A. Ils doivent bénéficier d'une thyroïdectomie prophylactique et d'une surveillance adaptée au dépistage des différentes atteintes organiques potentielles (médullo-surrénale et parathyroïde).

Les apparentés non porteurs de la mutation sont rassurés et ne nécessitent aucune surveillance médicale particulière. L'étude génotypique d'autres apparentés est nécessaire.

Déclaration d'intérêts: les auteurs ne déclarent aucun conflit d'intérêt en rapport avec cet article.

Remerciements : Nous remercions Dr Daoud Chafia pour l'aide à l'élaboration de travail.

\section{RÉFÉRENCES}

1. Santoro M, Carlomagno F, Romano A, Bottaro DP, Dathan NA, Greco M, et al. Germline mutations of MEN2A and MEN2B activate RET as a dominant trasforming gene by different molecular mechanisms. Science1995; 267:381-383.

2. Modigliani E. Les néoplasies endocriniens de type 2. Presse Med 1998; 27:628-640.

3. Conte-Devolx B, Niccoli-Sire P. Néoplasies endocriniennes multiples de type 2. EMC, Endocrinologie-Nutrition1999; 10-036-A-08.

4. Murat A, Niccoli-Sire $P$. Le cancer médullaire de la thyroide. Mt endocrinologie 2000; $2: 430-437$.

5. Brandi ML, Gagel RF, Angeli A, Bilezikian JP, Beck-Peccoz P, Bordi C, et al. Guidelines for diagnosis and therapy of MEN type 1 and type 2 . J Clin Endocrinol Metab 2001; 86:5658-5671.

6. Schimke RN. Genetic aspects of multiple endocrine neoplasia. Annu Rev Med 1994; 35:25-31.

7. Block MA, Jackson CE, Greenawald KA, Yott JB, Tashjian AH. Clinical characteristics distinguishing hereditary from sporadic medullary thyroid carcinoma treatment implications. Arch Surg 1980; 115:142-148.

8. Eng C, Clayton D, Schuffenecker I, Lenoir G, Cote G, Gagel RF, et al. The relationship between specific ret proto-oncogene mutations and desease phenotype in multiple endocrine neoplasia type 2 : international ret mutation consortium analysis. JAMA1996 ; 276:1575-1579.

9. Mulligan LM, Kwok JBJ, Healey CS, Elsdon MJ, Eng C, Gardner E, et al. Germline mutations of the RET proto-oncogene in multiple endocrine neoplasia type 2A. Nature 1993; 363:458-460

10. Fagin JA. Molecular génetics of human thyroid neoplasms; Annu Rev Med 1994; 45:45-52.

11. Eng Charis. RET Proto-Oncogene in the Development of Human Cancer. Journal of Clinical Oncology1999; Vol 17, Issue 1: 380

12. Arighi E, Borrello MG, Sariola H ; 2005; RET tyrosine kinase signaling in development and cancer; Cytokine Growth Factor Rev 16:441-467. 
12. Ishizaka Y, Itoh F, Tahira T, Ikeda I, Sugimura T, Tucker J, et al. Human ret proto-oncogene mapped to chromosome 10q11.2. Oncogene 1989; 4:1519-1521.

13. Takahashi M, Buma $\mathrm{Y}$, Hiai H. Isolation of ret proto-oncogene cDNA with an amino-terminal signal sequence. Oncogene 1989; 4:805-806.

14. Takahashi M, Buma $\mathrm{Y}$, Iwamoto $\mathrm{T}$, Inaguma $\mathrm{Y}$, Ikeda H, Hiai H. Cloning and expression of the ret protooncogene encoding a tyrosine kinase with two potential transmembrane domain. Oncogene 1988; 3:571-578.

15. Durbec P, Marcos-Gutierrez CV, Kilkenny C, Grigoriou M, Wartiowaara K, Suvanto $\mathrm{P}$, et al. GDNF signalling through the Ret receptor tyrosine kinase. Nature 1996; 381:789-793.
17. Treanor JJ, Goodman L, de Sauvage F, Stone DM, Poulsen KT, Beck CD et al. Characterization of a multicomponent receptor for GDNF. Nature1996; 382:80-83.

18. Donis-Keller H, Dou S, Chi D, Carlson KM, Toshima K, Lairmore TC, et al. Mutations in the RET proto-oncogene are associated with MEN 2A and FMTC. Hum Mol Genet 1993; 2:851-856.

19. Borrello MG, Smith DP, Pasini B, Bongarzone I, Greco A, Lorenzo MJ, et al. RET activation by germline MEN2A and MEN2B mutations. Oncogene 1995; 11:2419-2427.

20. Asai N, Iwashita T, Matsuyama M, Takahashi M. Mechanism of activation of the ret proto-oncogene by multiple endocrine neoplasia $2 \mathrm{~A}$ mutations. Mol Cell Biol 1995; 15:1613-1619.

Cet article a été publié dans le « Batna Journal of Medical Sciences » BJMS, l'organe officiel de « l'association de la Recherche Pharmaceutique - Batna»

Le contenu de la Revue est ouvert « Open Access » et permet au lecteur de télécharger, d'utiliser le contenu dans un but personnel ou d'enseignement, sans demander l'autorisation de l'éditeur/auteur.

Avantages à publier dans BJMS :

- Open access : une fois publié, votre article est disponible gratuitement au téléchargement

- Soumission gratuite : pas de frais de soumission, contrairement à la plupart des revues « Open Access »

- Possibilité de publier dans 3 langues : français, anglais, arabe

- Qualité de la relecture : des relecteurs/reviewers indépendants géographiquement, respectant l'anonymat, pour garantir la neutralité et la qualité des manuscrits.

Pour plus d'informations, contacter BatnaJMS@gmail.com ou connectez-vous sur le site de la revue : www.batnajms.net 

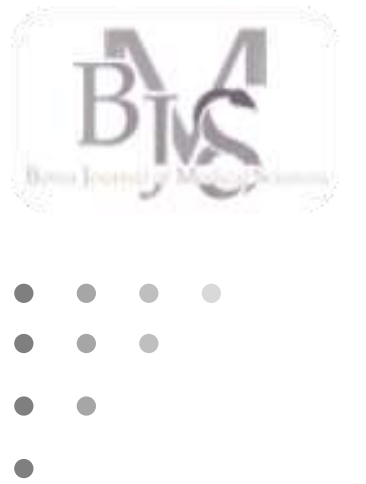

Service de réanimation polyvalente, CHU Tizi-OuzouAlgérie.

\section{Correspondance à :}

Yacine BENHOCINE

yacine001@yahoo.fr

DOI :https://doi.org/10.48087/ BJMScr.2020.7231

\section{Historique de l'article :}

Reçu le 10 septembre 2020

Accepté le 11 octobre 2020

Publié le 09 novembre 2020

Il s'agit d'un article en libre accès distribué selon les termes de la licence Creative Commons Attribution International License (CC BY 4.0), qui autorise une utilisation, une distribution et une reproduction sans restriction sur tout support ou format, à condition que l'auteur original et la revue soient dûment crédités.

\section{Pour citer l'article :}

Benhocine Y. Un cas d'embolie pulmonaire survenue chez un patient atteint de COVID-19. Batna J Med Sci 2020;7 (2):

201-3.

https://doi.org/10.48087/BJ MScr.2020.7231

\title{
Un cas d'embolie pulmonaire après guérison chez un patient atteint de COVID-19
}

\author{
A case of pulmonary embolism after recovery in a patient with COVID-19.
}

\author{
Yacine Benhocine
}

\section{RÉSUMÉ}

La maladie à Coronavirus (COVID-19) dans sa forme grave est souvent associée à une incidence élevée de coagulopathies et de thromboses, dans les dix premiers jours, qui justifie l'intérêt de prévenir les complications thromboemboliques notamment l'embolie pulmonaire (EP), en cours d'hospitalisation. Nous rapportons ici le cas d'un patient guéri d'une pneumonie à SARS-CoV-2 compliquée d'un syndrome de détresse respiratoire aigu ayant présenté une embolie pulmonaire (EP) à 11 jours de sa sortie de la réanimation du CHU Tizi-Ouzou. Cette rare observation pose le problème de la nécessité de la surveillance étroite des patients guéris, de la poursuite $\mathrm{du}$ traitement anticoagulant après la sortie d'hospitalisation.

Mots clés : embolie pulmonaire, COVID-19, coagulopathies.

\section{INTRODUCTION}

Selon la littérature, les patients admis dans les unités des soins pour des formes graves de COVID-19 présentent une incidence élevée de coagulopathie et de thrombose (1-4). Les recommandations des sociétés savantes ont été émises pour prévenir et minorer la survenue de ces complications, notamment en matière de thromboprophylaxie (5-6). Cependant, il n'est pas encore établi dans la littérature récente, si les risques thromboemboliques persistent chez des personnes guéries de COVID-19.

Nous rapportons ici l'observation clinique d'un patient admis le 05 avril 2020 dans le service de réanimation du CHU Tizi-Ouzou (Algérie), pour une forme sévère de pneumonie virale à SARS-CoV-2 compliquée de Syndrome de détresse respiratoire aigu de l'adulte (SDRA), qui a développé une embolie pulmonaire (EP) une dizaine de jours après sa sortie de réanimation, alors qu'il était cliniquement et biologiquement guéri, avec 2 tests PCR négatifs pour SARS-CoV-2.

\section{OBSERVATION}

Il s'agit d'un patient âgé de 62 ans, dont les antécédents sont constitués essentiellement d'un diabète type 2 et d'une cholécystectomie. Il était sous antidiabétiques oraux. Son histoire commence une semaine avant son admission par une fièvre et une toux sèche, puis s'installe au 6ème jour une dyspnée qui a motivé sa consultation à l'unité grippe du CHU Tizi-Ouzou où le test PCR SARS-Cov-2 se révèle positif.

\section{ABSTRACT}

Coronavirus disease (COVID-19) in its severe form is often associated with a high incidence of coagulopathies and thromboses, in the first ten days, which justifies the interest of preventing thromboembolic complications, in particular pulmonary embolism (PE), during hospitalization. We report here the case of a patient cured of SARS-CoV-2 pneumonia complicated by acute respiratory distress syndrome who presented with pulmonary embolism (PE) 11 days after leaving the intensive care unit of the Tizi-Ouzou University Hospital. This rare observation poses the problem of the need for close monitoring of cured patients and the continuation of anticoagulant treatment after discharge from hospital.

Keywords : pulmonary embolism, COVID-19, coagulopathies.

Il est admis le 04 avril 2020, initialement au service des maladies infectieuses, pour la prise en charge de cette atteinte respiratoire. Il sera admis en réanimation le lendemain de son hospitalisation, pour hypoxémie majeure. A l'examen clinique, il est lucide et cohérent, stable sur le plan hémodynamique. Il pèse 93 $\mathrm{kg}$ pour $169 \mathrm{~cm}$, IMC 32,63 kg/m².

Il présente une polypnée à 30 cycles par minute, l'auscultation pulmonaire révèle une diminution du murmure vésiculaire dans les bases.

La tomodensitométrie (TDM) thoracique montre des images hypodenses entourant les structures bronchiques avec des condensations des structures parenchymateuses para bronchiques ; et un comblement alvéolaire (figure 1).

Les gaz de sang réalisés sous 6 litres d'oxygène montrent une hypoxémie sévère : $\mathrm{PaO}_{2}: 38$ mmHg ; $\mathrm{PaCO}_{2}: 35$ mmHg ; pH : 7,49; $\mathrm{SaO}_{2}$ : $79 \%$ et Lactates : $1,5 \mathrm{mmol} / \mathrm{L}$. Devant l'aggravation de la dyspnée et la survenue d'une fatigue musculaire respiratoire, le patient est intubé, sédaté puis curarisé sous ventilation mécanique. Malgré l'optimisation des paramètres de ventilation protectrice, il demeure initialement hypoxique. Les gaz du sang réalisés avec $\mathrm{FIO}_{2} \quad 100 \%$ montrent une $\mathrm{PaO}_{2}$ à $90 \mathrm{mmHg}$; une $\mathrm{PaCO}_{2}$ à $36 \mathrm{mmHg}$, un Ratio $\mathrm{PaO}_{2} / \mathrm{FiO}_{2}$ à $100 \mathrm{mmHg}$. Il s'agit d'un SDRA sévère selon la classification de Berlin (8). 


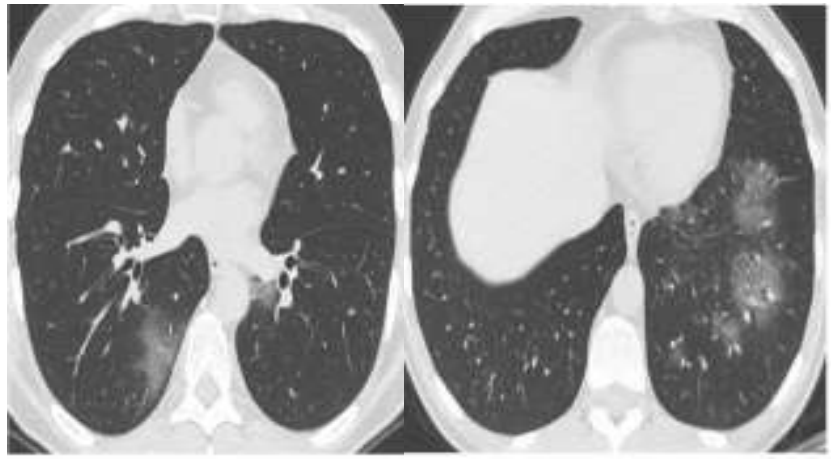

Figure 1. TDM à l'admission

Il est mis en décubitus ventral 6 heures après son admission avec une amélioration significative de l'hématose engendrant donc un début de la baisse de la $\mathrm{FiO}_{2}$ tout en maintenant une PEEP significative. Le patient bénéficiera au cours de son séjour des manœuvres de recrutement, entraînant une amélioration de l'hématose ; ce qui permettra l'arrêt des sédations, le sevrage ventilatoire et l'extubation le 20 avril, soit à J16. Le patient a reçu, au cours de son séjour, la chloroquine, l'azithromycine, une anticoagulation prophylactique par une Héparine à bas poids moléculaire (HBPM) et une antibiothérapie probabiliste par Amoxicilline / acide clavulanique et gentamycine, avec de la vitamine C.

Des examens complémentaires ont été réalisés, retrouvant une légère hyperglycémie à 1,58 $\mathrm{g} / \mathrm{l}$, une lymphopénie majeure à l'admission avec début de normalisation à J16, un taux de paquettes a $220000 \mathrm{el} / \mathrm{mm}^{3}$. Le taux de fibrinogène a été au maximum à 9,5 g/L à $\mathrm{J} 6$, avec un pic de D-Dimères à $1900 \mu \mathrm{g} / \mathrm{L} \quad$ (D'où l'anticoagulation curative). Le patient est porté sortant de la réanimation à J20, transférer dans son service d'origine (maladies infectieuses) sous oxygène aux lunettes nasales. Le sevrage à l'oxygène est réalisé huit jours après sa sortie de la réanimation. Devant la reprise complète de l'autonomie de toutes les activités de la vie quotidienne, l'anticoagulation est arrêtée, puis le patient est retourné à domicile après un séjour hospitalier total de 30 jours. Deux jours après son retour à domicile, il présente une angoisse, une dyspnée au moindre effort et une douleur basi-thoracique droite. A sa réadmission en soins intensifs, un infarctus du myocarde a été éliminé (ECG, troponine I et le BNP normaux). L'angioscanner thoracique confirme une embolie pulmonaire (EP) proximale, bilatérale avec des atteintes segmentaires et soussegmentaires (figure 2).

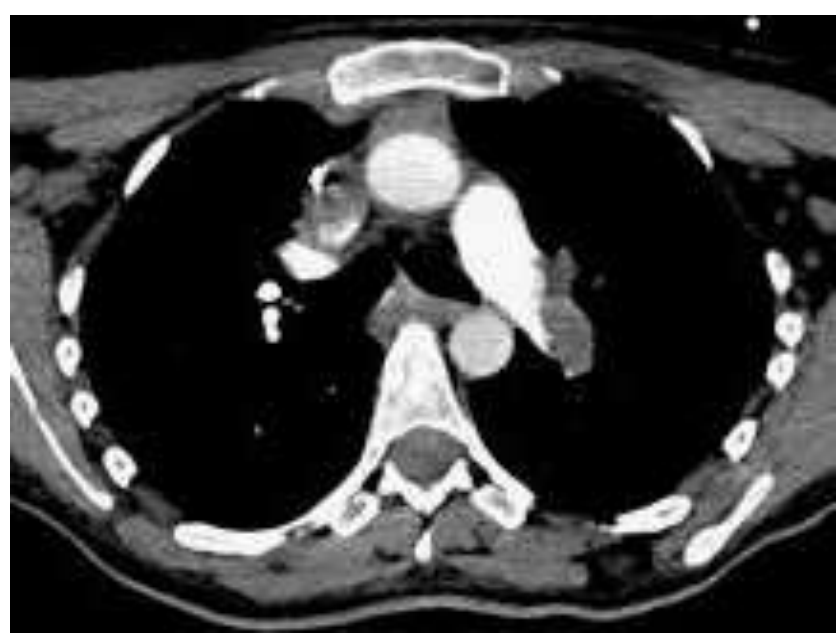

Figure 2. TDM révélant l’embolie pulmonaire
Il est mis sous HBPM à dose curative $(0,8 \mathrm{ml} / 12 \mathrm{~h} / \mathrm{s} / \mathrm{c})$ avec relais précoce par antivitamine $\mathrm{K}(\mathrm{AVK})$, pour une durée de 6 mois. L'évolution étant rapidement favorable, le patient sort de l'hôpital à J11 de sa réadmission.

L'échographie cardiaque est normale, sans signes droits, pas de thrombose veineuse profonde évolutive à l'échodoppler des membres inférieurs.

\section{DISCUSSION}

La majorité des publications qui accompagnent la pandémie de COVID-19 confirment que cette maladie s'exprime principalement par une pneumonie virale qui peut évoluer rapidement vers un SDRA (7-8). Il est également établi que l'évolution de la maladie peut se compliquer de défaillance multiviscérale qui serait favorisée par des troubles de la coagulation qui sont en partie attribuées à la dysfonction endothéliale (9), par l'orage cytokinique et par la dysrégulation immunitaire (10).

Dans ce contexte, il est décrit plusieurs cas d'embolie pulmonaire chez des patients admis en soins intensifs pour des formes gaves de COVID-19. L'EP apparait comme un facteur de gravité qu'il faut, par conséquent, rechercher chez les patients infectés au moindre signe d'aggravation (2- 5, 11). Les anomalies de coagulation rencontrées dans cette maladie ont conduit les experts internationaux à publier des recommandations qui visent à améliorer la prise en charge afin de réduire le risque de complication thromboembolique en instaurant une anticoagulation de préventive à curative en fonction du degré de risque thromboembolique (6-7).

Certaines équipes préconisent, sans en donner les modalités, le maintien d'un traitement anticoagulant après sortie de l'hôpital chez les patients avec des facteurs de risque (11). Si l'EP peut compliquer le parcours des patients avec la COVID19 lors de la phase aiguë de la maladie $(2-5,11)$, il n'existe pas à l'heure actuelle des recommandations quant au type d'anticoagulation à maintenir en dehors de la phase aiguë et surtout quand une guérison clinique et biologique est établie. Notre patient a présenté une EP alors qu'il était déclaré guéri et retourné à domicile. Il était redevenu autonome pour la marche et pour les activités quotidiennes, ce qui avait motivé l'arrêt du traitement anticoagulant. Le seul facteur de risque cardiovasculaire qu'il présentait, étant une obésité modérée avec IMC à 32,63, ne justifiant pas le maintien d'un traitement anticoagulant.

Qu'est ce qui pourrait donc expliquer la survenue de cette EP au moment où le risque de survenue semble le plus bas ? Estce la persistance des lésions endothéliales pour laquelle une équipe suisse (12) a émis deux hypothèses de survenue ? La première est une activation de l'endothélium par l'orage cytokinique ; la seconde est une atteinte directe des cellules endothéliales par le coronavirus ; en se fixant sur le récepteur de l'enzyme de conversion de l'angiotensine 2 (ACE2). Ce récepteur s'exprime dans différents organes : les poumons, le cœur, les reins, l'intestin mais aussi l'endothélium vasculaire. Or ce dernier est un organe à la fois paracrine, endocrine et autocrine indispensable à la régulation du tonus vasculaire et au maintien de l'homéostasie vasculaire. Son dysfonctionnement entraîne le risque de microthrombose et de vasoconstriction au niveau de ces différents organes cités ci-haut (13-14).

Une autre particularité est notée au niveau pulmonaire, où il est décrit un climat ultra inflammatoire observé et qui est considéré comme une atteinte pulmonaire spécifique baptisée « coagulopathie intravasculaire pulmonaire » pour la différencier de la coagulation intravasculaire disséminée (CIVD) qui, elle, touche de nombreux organes (4). 
Ce terrain hyper-inflammatoire persiste-t-il plus longtemps après la guérison et la négativation des tests RT-PCR pour le SARS-CoV-2 ? Cette hypothèse nous parait la plus plausible par le fait que notre patient a présenté cette EP bien à distance de la négativation des tests RT-PCR.

\section{CONCLUSION}

La maladie thromboembolique, notamment l'EP est une complication qui peut survenir chez les patients sévèrement atteints de la COVID -19. Il faut la prévenir et la rechercher activement en présence d'une aggravation respiratoire. Elle est rapportée dans la phase aigüe de la maladie. Sa survenue à distance de la prise en charge de réanimation et après guérison clinique et biologique doit-elle être rattachée au COVID-19 ? Quelle en serait l'hypothèse vraisemblable ? Est-il licite de poursuivre le traitement anticoagulant, bien au-delà de l'hospitalisation, mais pour quelle durée et à quelle dose ? Ce sont les interrogations que pose la présentation de cette observation rare.

Déclaration d'intérêts: les auteurs ne déclarent aucun conflit d'intérêt en rapport avec cet article.

\section{RÉFÉRENCES}

1. Poissy J, Goutay J, Caplan M, Parmentier E, et al. Pulmonary embolism in COVID-19 patients: awareness of an increased prevalence. 2020 https://doi.org/ 10.1161/circulationaha.120.047430.

2. El Boussadani B, Benajiba C, Aajal A, Ait Brik A, et al. Pandémie COVID19: impact sur le système cardiovasculaire. Ann cardiol Angeiol 2020 ; 69 :107-114.

3. Klok FA, Kruip MJHA, Van der Meer NJM, Arbous MS, et al. Incidence of thrombotic complications in critically ill ICU patients with COVID-19. https://doi.org/10.101016/j.thromres.2020.04.013

4. McGonagle D, O'Donnell JS, Sharif K, Emery P, et al. Immune mechanisms of pulmonary intravascular coagulopathy in COVID-19 pneumonia. https//doi.org/10.1016/ s2665-9913(20)30121-1.
5. Traitement anticoagulant pour la prévention du risque thromboembolique chez un patient hospitalisé avec COVID-19 et surveillance de l'hemostase. Available on http///sfar.org/ Traitementanticoagulation-et-surveillance-delhemosatase/consulted on March 2020

6. Behnood B, Madhavan MV, Jimenez D, Chuich T et al. COVID -19 and Thrombotic or Thromboembolic Disease: Implications for prevention, antithrombotic Therapy, and follow-up. JACC 2020. http://doi.org/10.1016/j.jacc.2020.04.031.

7. Wu Z, McGoogan JM. Characteristics of an important lesson from the coronavirus Disease 2019(COVID-19) outbreak in China: summary of report of 72314 cases from the Chinese Center for Disease control an prevention. JAMA 2020. Doi:10;1001/jama.2020.2648.

8. Qun Li, MED M, Guan X, Wu P, et al. Early transmission dynamics in Wuhan, China, of novel coronavirus-infected pneumonia. N Engl J Med 2020. https://Doi.10.1056/NEJMoa2001316.

9. Varga Z, Flammer AJ, Steiger P, Haberecker M, et al. Endothelial cell infection and endotheliitis in COVID-19. The Lancet 2020.https://doi.org/10.1016/s0140- 6736(20)30937-5.

10. Qin C, Zhou L, Hu Z, Zhang S, et al. Dysregulation of immune response in patients with coronavirus 2019(COVID-19) in Wuhan, China. Clin Infect Dis 2020 Mars 12. Pii: ciaa248.doi:10.1093/cid/ciaa248.

11. Grillet F, Behr J, Calame P, Aubry S, Delabrousse E. Acute pulmonary embolism associated with COVID-19 pneumonia detected by pulmonary CT angiography. Radiology 2020. Doi.10.1148/radiol.2020201544.

12. Catala Isabelle. Une dysfonction endothéliale à l'origine des complications vasculaires du COVID-19 ? https://francais.medscape.com/voir article/3605921, consulté le 15 mai 2020.

13. Moore HB, Barret CD, Moore EE, McIntyre RC, et al. Is there a role for tissue plasminogen activator as a novel treatment for refractory COVID-19 associated acute respiratory distress syndrome? J Trauma Acute Care Surg $2020 ; 88(6): 1-2$

14. Yuanling X, Xiang w, Pei Y, Shutong Z. COVID-19 complicated by Acute Pulmonary Embolism. Radiology cardiothoracic imaging 2020; 2 (2): e200067. https://doi.org/10.1148/ryct.2020200067.

\footnotetext{
Cet article a été publié dans le « Batna Journal of Medical Sciences » BJMS, l'organe officiel de « l'association de la Recherche Pharmaceutique - Batna»

Le contenu de la Revue est ouvert « Open Access » et permet au lecteur de télécharger, d'utiliser le contenu dans un but personnel ou d'enseignement, sans demander l'autorisation de l'éditeur/auteur.

Avantages à publier dans BJMS :

- Open access : une fois publié, votre article est disponible gratuitement au téléchargement

- Soumission gratuite : pas de frais de soumission, contrairement à la plupart des revues « Open Access »

- Possibilité de publier dans 3 langues : français, anglais, arabe

- Qualité de la relecture : des relecteurs/reviewers indépendants géographiquement, respectant l'anonymat, pour garantir

la neutralité et la qualité des manuscrits.

Pour plus d'informations, contacter BatnaJMS@gmail.com ou connectez-vous sur le site de la revue : www.batnajms.net
} 


\section{BIs}

- $\bigcirc$

- $\bigcirc$

-

${ }^{1}$ Faculté de Médecine Université BATNA 2, Batna - Algérie

2 Laboratoire de virologie EST, Université Claude Bernard Lyon A, Lyon - France

Correspondance à :

Nora RIGHI

noradoumandji@yahoo.fr

DOI :https://doi.org/10.48087/ BJMScr.2020.7232

\section{Historique de l'article :}

Reçu le 11 octobre 2020

Accepté le 5 novembre 2020

Publié le 09 novembre 2020

Il s'agit d'un article en libre accès distribué selon les termes de la licence Creative Commons Attribution International License (CC BY 4.0), qui autorise une utilisation, une distribution et une reproduction sans restriction sur tout support ou format, à condition que l'auteur original et la revue soient dûment crédités.

\title{
Premier cas d'un Zona exceptionnel du creux poplité
}

\author{
First case of an exceptional Zona of the popliteal fossa
}

\author{
Nora Righi ${ }^{1}$, Oum Kelthoum Mansouri ${ }^{1}$, Bruno Lina ${ }^{2}$
}

\section{RÉSUMÉ}

Le Zona est une infection virale aiguë, sporadique et douloureuse chez les personnes âgées, causée par la réactivation du virus de la varicelle-zona latent. C'est une affection dermatologique fréquente qui affecte jusqu'à $20 \%$ de la population, le plus souvent impliquant les dermatomes thoraciques et faciaux avec des lésions sacrées. L'herpès zoster affectant le creux poplité est une pathologie rare. La nature ambiguë de cette condition crée un dilemme diagnostique. Nous rapportons l'observation d'une patiente âgée ayant présenté une localisation exceptionnelle du zona : le creux poplité. C'est le premier cas décrit du zona du creux poplité confirmé par la PCR (Polymerase Chain Reaction) avec des caractéristiques distinctives secondaires chez une femme âgée.

Mots-clés: herpes zoster, dermatomes, creux poplité, Polymerase Chain Reaction.

\section{INTRODUCTION}

Le zona est une infection virale aiguë causée par la réactivation du virus de la varicelle-zona (VZV) latent [1]. Cette affection dermatologique est fréquente et touche entre 10 et $20 \%$ de la population générale [2]. Le zona est essentiellement une maladie de l'adulte de plus de 50 ans, mais peut survenir à n'importe quel âge [3]. Les personnes âgées sont des sujets à haut risque de récurrence du (VZV) et de survenue de ses complications [4].

Le zona est responsable d'une éruption cutanée douloureuse, classiquement métamérique [5]. Les dermatomes thoraciques, faciaux et cervicaux sont les plus touchés [5]. Des localisations atypiques ont été rapportées mais l'atteinte du creux poplité par l'herpès zoster n’a jamais été décrite.

Nous rapportons l'observation d'une patiente ayant présenté une localisation exceptionnelle du zona : le creux poplité. C'est le premier cas du zona du creux poplité confirmé par la PCR (Polymerase Chain Réaction) sur des lésions biopsies prêtant des caractéristiques distinctives secondaires chez une femme âgée.

\section{OBSERVATION}

C'est une femme âgée de 84 ans hypertendue depuis 10 ans, mise sous association Candésartan-diurétique thiazidique,Amlodipine et Acétylsalicylate de DL-Lysine. Admise au

\section{ABSTRACT}

Shingles is an acute, sporadic and painful viral infection in the elderly caused by the reactivation of the latent varicella-zoster virus. It is a common dermatological condition that affects up to $20 \%$ of the population, most often involving the thoracic and facial dermatomes with sacral lesions. Herpes zoster affecting the popliteal region is a rare pathology. The ambiguous nature of this condition creates a diagnostic dilemma. We report the observation of an elderly patient who presented with an exceptional localization of shingles: the popliteal fossa. This is the first described case of popliteal fossa zoster confirmed by PCR (Polymerase Chain Reaction) with secondary distinguishing features in an elderly woman.

Keywords: herpes zoster, dermatomes, popliteal fossa, Polymerase Chain Reaction

service des maladies infectieuses de Batna (Algérie) pour la prise en charge d'un zona surinfecté siégeant sur le creux poplité (métamère $\mathrm{S}_{2}$ ).

La symptomatologie a commencé par l'installation des douleurs type picotement et des paresthésies au niveau du creux poplité gauche irradiant vers la cuisse homolatérale entrainant une impotence fonctionnelle d'installation subaiguë exacerbée par la marche et calmée par le repos.

L'examen clinique a objectivé la présence d'un placard érythémateux au même siège des douleurs, centré par une éruption vésiculeuse à contenu trouble, associée à d'autres lésions vésiculeuses au niveau de la face antéro-interne de la cuisse gauche et à une adénopathie inguinale satellite, pas de déficit moteur. Le reste de l'examen clinique était sans particularité.

Des examens biologiques ont été demandés objectivant : un hémogramme normal, une glycémie postprandiale et à jeun normale, une hyperazotémie à $0,5 \mathrm{~g} / \mathrm{l}$; une créatinine sanguine normale, et un bilan lipidique normal. L'échocardiographie objectivait une insuffisance mitrale grade II. Une PCR VZV positive sur un fragment biopsie au niveau des lésions a permis la confirmation du diagnostic.

La patiente a été mise sous traitement antiviral

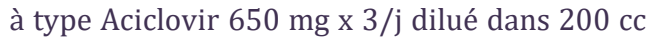
SSI (sérum salé isotonique) pendant 07 jours, 
associé au traitement antibiotique (amoxicilline acide clavulanique) et un traitement antiseptique local. La patiente a très bien évolué après 7 jours de traitement.

\section{DISCUSSION}

Le Zona n'est pas rare, et en général bénin. Le diagnostic de cette affection est essentiellement clinique basé sur la nature des lésions cutanées ainsi que leurs sièges, et la présence d'un syndrome neurologique. Le recours à la PCR s'impose en cas de doute ou de forme grave [6].

L'herpès zoster peut impliquer n'importe quel nerf avec l'éruption cutanée caractéristique dans la région de son innervation, et l'échec dans le système immunitaire qui mène la réactivation du VZV peut être provoquée par d'autres facteurs outre la maladie sous-jacente [7].

Le zona touche principalement le tronc jusqu'à 50\% - 60\% des cas, suivis de la région de la tête $\quad(10 \%-20 \%)$ avec des dermatomes sacrés impliqués dans seulement $5 \%$ des cas [7]. Des localisations atypiques ont été rapportées [8-12], mais l'atteinte du creux poplité n'a pas été décrite selon nos connaissances.

Cette observation illustre une localisation exceptionnelle d'un zona (creux poplité). Les symptômes initiaux pouvaient orienter vers une neuropathie sensitive d'installation subaiguë. Mais devant l'apparition des lésions vesiculopustuleuses typiques après un syndrome hyperalgique nous a inciter à pratiquer une PCR sur des lésions biopsies. Elle souligne également l'intérêt d'une bonne inspection cutanée quotidienne.

\section{CONCLUSION}

L'herpès zoster du creux poplité ,1er cas déclaré dans le monde ne doit pas être omis chez les patients présentant une éruption vésiculaire typique. Le diagnostic doit être évoqué devant toute neuropathie sensitive récente quelle qu'en soit la localisation chez une personne âgée. Par conséquent, une reconnaissance précoce et une prise en charge appropriée des patients atteints de zona sont importantes pour prévenir des issues potentiellement graves.
Déclaration d'intérêts: les auteurs ne déclarent aucun conflit d'intérêt en rapport avec cet article.

\section{RÉFÉRENCES}

1. Gnann JW Jr., Whitley RJ. Clinical practice. Herpes zoster. N Engl J Med 2002;347:340-6

2. D.W. Kimberlin, R.J. Whitley . Varicella-Zoster vaccine for the prevention of herpes zoster N Eng J Med, 356 (2007), pp. 1338-1343.

3. Lal H, Cunningham AL, Godeaux O, Chlibek R, Diez-Domingo J, Hwang SJ, et al. Efficacy of an adjuvanted herpes zoster subunit vaccine in older adults. N Engl J Med 2015;372:2087-96.

4. R.E. Hope-SimpsonPostherpetic neuralgia J R Coll Gen Pract, 25 (1975), pp. 571-575

5. P.O. Lang, J. Belmin, J.P. MichelLe zona du sujet âgé Presse Med, 38 (2009), pp. 571-583

6. R. Laurent. Varicella-Zoster., EMC-Médecine (2005), pp. 276-283.

7. Glynn C, Crockford G, Gavaghan D, et al. Epidemiology of shingles. J R Soc Med. 1991; 84:184.

8. Tatah F, Lakli S. A propos d'une localisation atypique d'un zona. Revue neurologique. $2015 ; 171$ (1) :144.

9. hopra A, Sivaraman K, Thomas BS. Herpes zoster of gingiva in an older woman: a rare case report. Gerodontology. 2017 Jun;34(2) 280-283.

10. Bjekic M, Markovic M, Sipetic S. Penile herpes zoster: an unusual location for a common disease. Braz J Infect Dis. 2011 NovDec;15(6):599-600.

11. Cukic V. The Uncommon Localization of Herpes Zoster. Med Arch. 2016;70(1):72-75.

12. Chung JY, Park JS, Kim YS. A Rare Cause of Acute Colonic Pseudoobstruction: Ogilvie's Syndrome Caused by Herpes Zoster. J Neurogastroenterol Motil. 2017;23(4):616-617.
Cet article a été publié dans le « Batna Journal of Medical Sciences » BJMS, l'organe officiel de « l'association de la Recherche Pharmaceutique - Batna»

Le contenu de la Revue est ouvert « Open Access » et permet au lecteur de télécharger, d'utiliser le contenu dans un but personnel ou d'enseignement, sans demander l'autorisation de l'éditeur/auteur.

Avantages à publier dans BJMS :

- Open access : une fois publié, votre article est disponible gratuitement au téléchargement

- Soumission gratuite : pas de frais de soumission, contrairement à la plupart des revues « Open Access »

- Possibilité de publier dans 3 langues : français, anglais, arabe

- Qualité de la relecture : des relecteurs/reviewers indépendants géographiquement, respectant l'anonymat, pour garantir la neutralité et la qualité des manuscrits.

Pour plus d'informations, contacter BatnaJMS@gmail.com ou connectez-vous sur le site de la revue : www.batnajms.net 


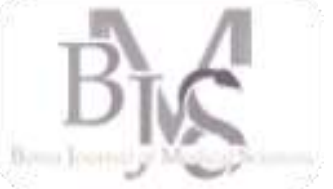

- $\bigcirc$

- 1

P

${ }^{1}$ L'équipe HAD Covid-19 Batna Algérie

${ }^{2}$ Laboratoire Sadelaoud, agréé COVID-19, Batna - Algérie

\section{Correspondance à :}

Nora RIGHI

noradoumandji@yahoo.fr

DOI :https://doi.org/10.48087/ B]MScr.2020.7233

\section{Historique de l'article :}

Reçu le 31 octobre 2020

Accepté le 6 novembre 2020

Publié le 09 novembre 2020

Il s'agit d'un article en libre accès distribué selon les termes de la licence Creative Commons Attribution International License (CC BY 4.0), qui autorise une utilisation, une distribution et une reproduction sans restriction sur tout support ou format, à condition que l'auteur original et la revue soient dûment crédités.

\section{Pour citer l'article :}

Righi N, Debbabi N, Sadelaoud M, et al. La COVID-19 atypique, à propos d'un cas. Batna J Med Sci 2020;7(2):206-7 https://doi.org/10.48087/ B]MScr.2020.7233

\title{
La Covid-19 atypique, à propos d'un cas
}

\author{
Atypical COVID-19. A case report.
}

\author{
Nora Righi ${ }^{1}$, Merim Debbabi ${ }^{1}$, Souad Hamoud ${ }^{1}$, Mounir Sadelaoud $^{2}$, Widad Toumi ${ }^{2}$, \\ Ferouz Kabache ${ }^{1}$, Mohamed Amine Benchadi ${ }^{1}$, Meloud Badla ${ }^{1}$, Allaoua Mahmay ${ }^{1}$, \\ Selma Faroudj ${ }^{1}$, Imen Hidoussi ${ }^{1}$, Rachid Derfouf ${ }^{1}$, Fatima Benaifa ${ }^{1}$
}

\section{RÉSUMÉ}

Le SRAS-CoV-2 du syndrome respiratoire aigu sévère, virus nouvellement émergeant, découvert en chine (Wuhan) a mené à une pandémie actuelle de maladie a coronavirus 2019 (Covid-19). Plus de 1.700 .000 de cas en Afrique avec 1.400 .000 de guérisons associés et 41000 de décès. 55,630 cas en Algérie avec 1,897 de décès (OMS 27 octobre 2020). Le SARS-Cov 2 peut infecter plusieurs systèmes y compris les voies urinaires ; cependant, la présence d'ARN viral n'était pas nécessairement prédictive des symptômes cliniques urinaires. L'objectif de cet article est de montrer que la Covid-19 peut être révélée par une symptomatologie urinaire haute, ce qui a été retrouvé pour la première fois chez un patient âgé de 56 ans, tabagique, ayant comme antécédents une anosmie et une agueusie remontant au mois de juin, consultait pour un tableau d'infection urinaire haute avec une leucocyturie aseptique, un bilan rénal perturbé. Devant la persistance de la fièvre et l'apparition des signes respiratoires, une sérologie Covid-19 lui a été pratiquée revenant négative, une PCR nasopharyngée positive et la PCR urinaire 3 jours après la mise en route du l'hydroxychloroquine était négative. Devant l'aggravation des lésions pulmonaires le malade est décédé dans un tableau d'insuffisance respiratoire aigüe. En conclusion, devant toute symptomatologie fébrile d'apparition aigue en cette période de pandémie, le praticien doit toujours avoir le réflexe de pratiquer une PCR nasopharyngée à la recherche du virus afin d'optimiser la prise en charge et d'éviter L'apparition des formes graves

Mots-clés: SARS-Cov 2, Covid-19, infection urinaire, insuffisance rénale, PCR.

\section{INTRODUCTION}

La récente pandémie de maladie à Coronavirus2019 (COVID-19) a posé de sérieux défis à la société humaine en Chine et dans le monde. Le COVID-19 a induit une pneumonie grave chez des hôtes humains et a entraîné une forte contagiosité interhumaine $(1,2)$. Les patients atteints peuvent présenter des symptômes sévères et certains d'entre eux peuvent même décéder de défaillances d'organes majeurs.

Le virus 2019-nCoV a démontré une capacité substantielle de transmissions interhumaines (2) et s'est rapidement répandu dans le monde (3)). Les patients infectés présentaient des symptômes considérablement variés et leurs résultats variaient de légers aux cas sévères (4). Il est nécessaire de mentionner que $61,5 \%$ des patients atteints de pneumonie COVID-19 présentant des symptômes critiques sont décédés dans les 28 jours suivant leur admission (3). La discrimination entre les patients gravement malades et ceux présentant des symptômes légers peut aider à comprendre les variations individualisées du pronostic.

\section{ABSTRACT}

The newly emerging virus SARS-CoV-2 of severe acute respiratory syndrome, discovered in China (Wuhan) has led to the current pandemic of coronavirus disease 2019 (Covid-19). There are over $1,700,000$ cases in Africa with $1,400,000$ associated healings and 41,000 deaths. 55,630 cases in Algeria with 1,897 deaths (WHO October 27, 2020). SARS-Cov 2 can infect many systems including the urinary tract; however, the presence of viral RNA was not necessarily predictive of clinical urinary symptoms. The aim of this article is to show that Covid-19 can be revealed by an upper urinary symptom, which was found for the first time in a 56-year-old smoker, with a history of anosmia and ageusia. dating back to the month of June, presenting with symptoms of high urinary tract infection with aseptic leukocyturia, a disturbed renal assessment. Faced with the persistence of fever and the onset of respiratory signs, he was tested for Covid-19 serology, which was negative, a positive nasopharyngeal PCR and urinary PCR 3 days after starting the hydroxychloroquine was negative. Faced with worsening pulmonary lesions, the patient died of acute respiratory failure. In conclusion, faced with any febrile symptomatology of acute onset during this pandemic period, the practitioner must always have the reflex to perform a nasopharyngeal PCR in search of the virus in order to optimize the management and avoid the appearance of severe forms

Keywords: SARS-Cov 2, Covid-19, urinary tract infection, renal failure, PCR.

L'objectif est de démontrer qu'il n'y a pas de spécificité de l'infection par le SRAS Cov 2 et que le malade peut présenter des tableaux cliniques variés au début de l'infection.

\section{OBSERVATION}

Il s'agit du patient A.T âgé de 58 ans tabagique, sans antécédents pathologique particulier mis à part une symptomatologie évoquant un Covid$19 \mathrm{au} 1^{\mathrm{er}}$ pic de la pandémie (juin 2020) notamment fièvre, syndrome grippal, perte d'odorat et du gout, la TDM était normale, la PCR non faite. Le malade a été mis sous azithromycine et l'évolution était favorable.

Le mois de septembre (21/09/2020), le patient consultait pour une fièvre évoluant depuis 2 jours associée à des frissons; l'interrogatoire retrouvait des signes d'infection urinaire type brulures mictionnelles et pollakiurie, une asthénie profonde avec des vomissements bileux. Pas de symptomatologie respiratoire; le patient a été mis sous Cefotaxime et gentamycine. Une sérologie Covid-19 revenait 
négative (IgM -, IgG -), et une échographie abdominopelvienne était en faveur d'une légère hypertrophie prostatique.

L'évolution a été marquée par la persistance de la fièvre et l'apparition d'une symptomatologie respiratoire à type de toux sèche, la saturation en oxygène était normale au début, La PCR positive sur un prélèvement nasopharyngé associée à une pneumopathie interstitielle à $50 \%$ sur la TDM thoracique.

Bilan biologique était comme suit: FNS : GB 5,4 .103 (une lymphopénie a $600 / \mathrm{mm}^{3}$ ) une anémie hypochrome normocytaire a 10,6 g/dl une thrombopénie a 87000/103, l'urée à $0,23 \mathrm{~g} / \mathrm{l}$ et une créatinine a $12.3 \mathrm{mg} / \mathrm{l}$. La troponine était normale, une hyponatrémie a $127,2 \mathrm{meq} / \mathrm{l}$, kaliémie 03,94 meq/l, chlorémie $98.70 \mathrm{meq} / \mathrm{l}, \mathrm{CRP}<06 \mathrm{mg} / \mathrm{L}$.l'ECBU été en faveur d'une leucocyturie aseptique à 110.000/ml, ECG normal sans trouble du rythme avec un Qtc a 403 ms.

Un traitement spécifique lui a été administré à base d'hydroxychloroquine à $200 \mathrm{mg} 3$ fois par jour associé à l'azithromycine $500 \mathrm{mg}$ le premier jour puis $250 \mathrm{mg}$ les 4 jours suivants, lovenox dose curative et une corticothérapie, vitamine C, Zinc Magnésium. Devant la persistance de La symptomatologie urinaire et l'installation d'une insuffisance rénale (urée : 0,45g/l, créatininémie : $28 \mathrm{mg} / \mathrm{l}$, clairance de créatinine à $30,56 \mathrm{ml} / \mathrm{mn}$ ), une recherche du virus dans les urines par PCR été faite revenait négative.

Devant l'aggravation du tableau clinique, Le malade a été orienté au service de réanimation, décédé $24 \mathrm{~h}$ plus tard.

\section{DISCUSSION}

Il y a encore beaucoup d'incertitudes concernant le nouveau coronavirus SARS-CoV-2, qui met actuellement en difficultés les systèmes sanitaires les plus solides, partout dans le monde. Cela comprend notamment quels sont les symptômes de la maladie et à quel point ils peuvent être variés.

Le diagnostic de la COVID-19 repose fortement sur les caractéristiques épidémiologiques, cliniques, les résultats d'imagerie et le dépistage des acides nucléiques (5). La délivrance du résultat du diagnostic par ces technologies prenait du temps et était sujette aux erreurs.

L'ARN viral a été détecté dans plusieurs organes chez des patients atteints de COVID-19 (4). Étant donné que le récepteur viral, la protéine angiotensine-convertase-2 (ACE2), est exprimé dans le rein, les testicules et la vessie, et de l'ARN viral a été détecté dans l'évier et les toilettes des patients atteints de COVID-19, le virus peut être sécrété par le système urinaire. Le SARS-CoV-2 infectieux a été isolé avec succès de l'urine d'un patient COVID-19 (6). Le virus isolé pouvait infecter de nouvelles cellules sensibles et a été reconnu par le sérum de ces propres patients. Des précautions appropriées doivent être prises pour éviter la transmission par l'urine. Le cas que nous décrivons s'est prêtait à un tableau typique d'infection urinaire haute avec une insuffisance rénale, la PCR SARS- Cov 2 dans les urines faites 3 jours après un traitement à base d'hydroxycloroquine azithromycine était négative. Cependant dans notre optique la Covid-19 peut prêter un tableau d'insuffisance rénale associé aux formes sévères très fréquentes, persistantes, et se présentent sous la forme d'une atteinte tubulaire ou tubulointerstitielle sans glycosurie (7).

\section{CONCLUSION}

À ce jour, il n'y a pas de liste de symptômes dont la spécificité ou la sensibilité à la COVID-19 est élevée. L'infection à Covid19 peut se manifester par un tableau d'infection urinaire: brulures mictionnelles, polyurie, pollakiurie associée à une fièvre. Toute symptomatologie fébrile dans ce contexte épidémiologique doit mener à penser à une infection à Covid19 et à pratiquer un prélèvement nasopharyngé à la recherche du virus.

Les patients doivent toujours être encouragés à consulter un médecin s'ils présentent de nouveaux symptômes ou si ceuxci s'aggravent. Ceci doit permettre d'optimiser l'orientation et la gestion des patients suspects de COVID-19. Le test de différents types d'échantillons peut être utile pour surveiller les changements de la progression de la maladie et pour établir un pronostic.

Déclaration d'intérêts: les auteurs ne déclarent aucun conflit d'intérêt en rapport avec cet article.

\section{RÉFÉRENCES}

1. Peng L, liu j, Xu W, et al.Sars-Cov-2 can be detected in urine, blood, anal swabs and oropharyngeal swabs specimens. J Med Virol. 2020; 92: 1676-1680.

2. Zhu N, Zhang $D$, Wang $W$ et al, A novel coronavirus from patients with pneumonia in China N Engl J Med 2020; 382:727-733

3. Yu IT, Li Y, Wong TW, et al. Evidence of airborne transmission of the severe acute respiratory syndrome virus. NEJM 2004 (350):1731-9.

4. Guan WJ, Ni ZY, Hu Y, et al. Clinical characteristics of coronavirus disease 2019 in China. N Engl J Med 2020; 382:1708-1720

5. http://www.chinacdc.cn/COVID19. Accessed on Oct 10th, 2020.

6. Jing Sun, Airu Zhu, Heying Li, Kui Zheng et al, Isolation of infectious SARSCoV-2 from urine of a COVID-19 patient. Emerg Microbes Infect. 2020;9(1):991-993.

7. Rubin S, Orieux A, Prevel R, Carré C, et al, Caractérisation de l'insuffisance rénale aiguë chez les patients de réanimation atteints du COVID-19. Néphrologie \& Thérapeutique 16 (2020) 247

Cet article a été publié dans le « Batna Journal of Medical Sciences » BJMS, l’organe officiel de « l'association de la Recherche Pharmaceutique - Batna»

Le contenu de la Revue est ouvert « Open Access » et permet au lecteur de télécharger, d'utiliser le contenu dans un but personnel ou d'enseignement, sans demander l'autorisation de l'éditeur/auteur.

Avantages à publier dans BJMS :

- Open access : une fois publié, votre article est disponible gratuitement au téléchargement

- Soumission gratuite : pas de frais de soumission, contrairement à la plupart des revues « Open Access »

Possibilité de publier dans 3 langues : français, anglais, arabe

- Qualité de la relecture : des relecteurs/reviewers indépendants géographiquement, respectant l'anonymat, pour garantir la neutralité et la qualité des manuscrits.

Pour plus d'informations, contacter BatnaJMS@gmail.com ou connectez-vous sur le site de la revue : www.batnajms.net 


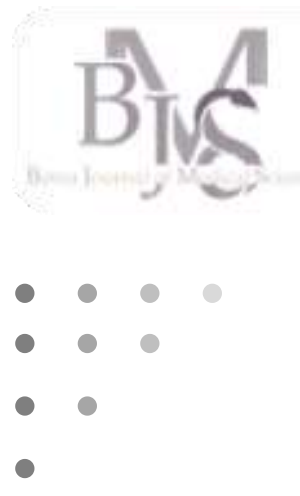

${ }^{1}$ Hematology and Cell Therapy; Etablissement Hospitalier Universitaire d'Oran.

${ }^{2}$ Faculty of medicine, University of Oran 1 Ahmed Ben Bella.

${ }^{3}$ Faculty of medicine, Abou Bekr Belkaid University Tlemcen.

\section{Correspondance à :}

Mohamed BRAHIMI

dr.brahimi.m@gmail.com

DOI :https://doi.org/10.48087/ B]MSle.2020.7234

Historique de l'article :

Reçu le 17 Mai 2020

Accepté le 16 Juin 2020

Publié le 09 novembre 2020

Il s'agit d'un article en libre accès distribué selon les termes de la licence Creative Commons Attribution International License (CC BY 4.0), qui autorise une utilisation, une distribution et une reproduction sans restriction sur tout support ou format, à condition que l'auteur original et la revue soient dûment crédités.

\section{Pour citer l'article :}

Brahimi M, Ramdani $\mathrm{H}$ Guenna M, et al. Coexistence of myeloblasts and B-CLL cells in peripheral blood. Batna J Med Sci 2020;7(2):208-9.

https://doi.org/10.48087/ BjMSle.2020.7234

\title{
Coexistence of myeloblasts and B-CLL cells in peripheral blood
}

\author{
Coexistence de myéloblastes et de cellules B-LLC dans le sang \\ périphérique
}

\author{
Mohamed Brahimi ${ }^{1,2}$, Hayet Ramdani ${ }^{1,3}$, Meriem Guenna ${ }^{1,2}$, Youcef Bouali ${ }^{1,2}$, \\ Mohamed Amine Bekadja ${ }^{1,2}$
}

B-chronic lymphoid leukemia (B-CLL) and acute myeloid leukemia (AML) are two separate diseases and they are clonally unrelated. [1] The coexistence of these two leukemias has been rarely described in the literature.[2] Most of these cases have been reported to occur after treatment of CLL with cytotoxic drugs suggesting that AML may be a secondary leukemia also knows as therapyrelated AML. [2]

This letter reports a case of a concomitant detection of myeloblasts and B-CLL cells in a patient and highlights the usefulness of cytomorphology and CD45/SSC dot plot in the recognition and discrimination of the 2 neoplasm cell populations.

An 87-year-old male with medical history of diabetes and high blood pressure presented to the department of hematology with complaints of fatigue, pallor and weakness for the investigation of a chronic lymphocytosis.

His blood tests showed hemoglobin $=5 \mathrm{~g} / \mathrm{dL}$; white blood cell $=18.6210^{9} / \mathrm{L}$; platelet count 70 $10^{9} / \mathrm{L}$ and slight lymphocytosis ranging from 6 to $1510^{9} / \mathrm{L}$ persisting for about 8 months.

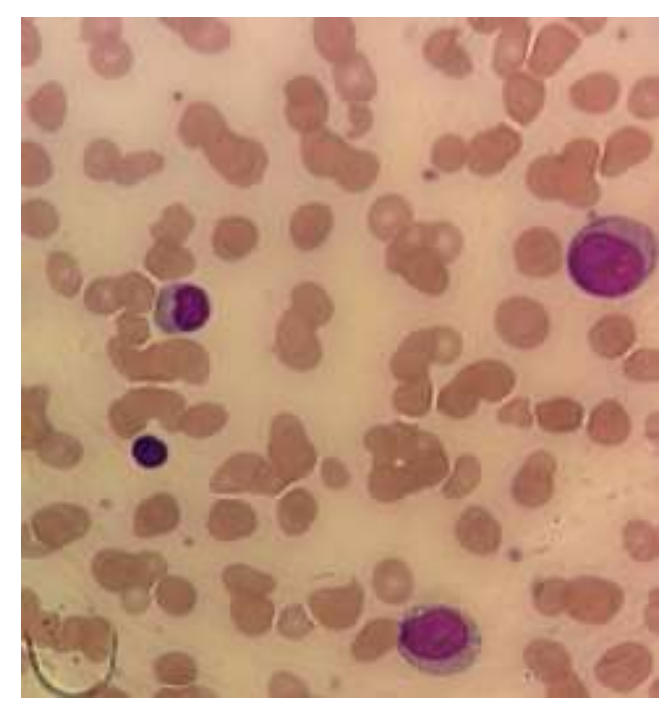

Figure 1._The peripheral bloods smear showing two circulating blast cells (on the right) with two small mature lymphocytes (on the left)
The peripheral blood (PB) smear showed small lymphocytes with scant cytoplasm and well condensed chromatin, and large blasts with high nuclear cytoplasmic ratio and fine nuclear chromatin, containing prominent nucleoli, however smudge cells were not seen (Figure 1).

The flow cytometric analysis of peripheral white blood cells was performed; and CD45 vs SSC Dot plot revealed two separate populations: large blasts with $\operatorname{dim} \mathrm{CD} 45$ expression located in the so-called "Bermude Area" [3] and small lymphocytes with high and heterogeneous CD45 expression (Figure 2).

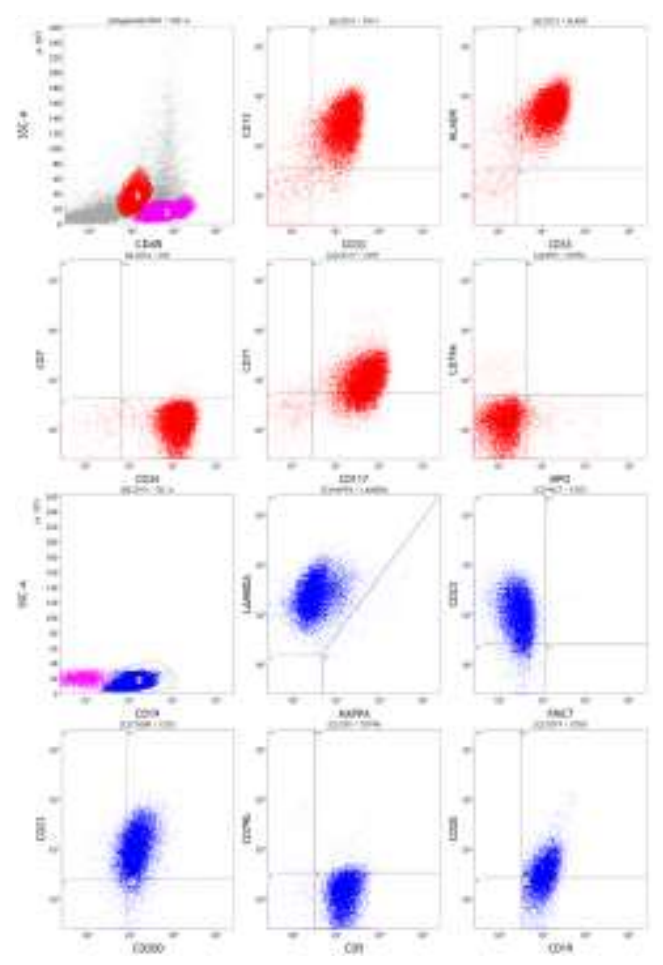

Figure 2:

Peripheral nucleated cells evaluated by flow cytometry. Myeloblasts (red color) was characterized by the co-expression of CD33, CD13, CD117 and HLA$\mathrm{DR}$, whereas B-lymphocytes (blue color) were identified by the heterogenous expression of CD45 and were characterized by typicall CLL immunophenotype: CD19+, CD5+, CD23+, CD20 + (dim), CD79a -, CD200+ and lambda light chain restriction (dim). 


\section{LETTRE À L'EDITEUR}

The European Research Initiative on CLL (ERIC) \& European Society for Clinical Cell Analysis (ESCCA) working group proposed that the application of CD45 must be left to the individual laboratory preference (i.e. "not recommended") because this marker is used

for identification of leukocyte subsets and provides a backbone to many gating strategies but is not essential to identify CLL.[4]

In our laboratory, the reason of adding CD45 to the lymphoproliferative disease antibody panel is that some acute lymphoblastic leukemia, so-called "ALL/L1 subtype" in the FAB classification, [5] can have small blastic cells with clomped chromatin. These cells are counted by a hematology counter as lymphocytes and can mislead to a diagnosis of CLL in the elderly, while the CD45 vs SSC dot plot allows us to separate the blasts from the mature lymphocytes.

In this case report, the blastic population expresses immaturity markers (CD34 and HLADR), and myeloid markers (CD117, CD13 and CD33) (figure 2), however, the myeloperoxidase (MPO) testing was negative by both cytochemistry and flow cytometry. Whereas the lymphocyte population was considered to be CLL cells by the expression CD19, CD20, CD5, CD23, CD200 and lambda light chain restriction and lack of expression of CD79a and FMC7 and CD34.

\section{RÉFÉRENCES}

1. Lu CM, Murata-Collins JL, Wang E et al. Concurrent acute myeloid leukemia with inv (16)(p13. 1q22) and chronic lymphocytic leukemia: molecular evidence of two separate diseases. American Journal of Hematology. 2006 ; 81 :963-968.

2. Lai R, Arber D A, Brynes R K, et al. Untreated chronic lymphocytic leukemia concurrent with or followed by acute myelogenous leukemia or myelodysplastic syndrome: a report of five cases and review of the literature. Am J Clin Pathol. 1999; 111: 373-378.

3. Brahimi M, Saidi D, Touhami H, Bekadja M A. The use of CD45/SSC Dot Plots in the Classification of acute leukemias. J Hematol Thromb Dis. 2014; 2: e107.

4. Rawstron AC, Kreuzer K-A, Soosapilla A et al. Reproducible Diagnosis of Chronic Lymphocytic Leukemia by Flow Cytometry: An European Research Initiative on CLL (ERIC) \& European Society for Clinical Cell Analysis (ESCCA) Harmonisation Project. Cytometry Part B 2018; 94B: 121-128.

5. Bennett J M, Catovsky D, Daniel MT et al. Proposals for the classification of the acute leukaemias French-American-British (FAB) co-operative group. British journal of haematology. $1976 ; 33(4)$ : 451-458

Cet article a été publié dans le « Batna Journal of Medical Sciences » BJMS, l'organe officiel de « l'association de la Recherche Pharmaceutique - Batna»

Le contenu de la Revue est ouvert « Open Access » et permet au lecteur de télécharger, d'utiliser le contenu dans un but personnel ou d'enseignement, sans demander l'autorisation de l'éditeur/auteur.

Avantages à publier dans BJMS :

- Open access : une fois publié, votre article est disponible gratuitement au téléchargement

- Soumission gratuite : pas de frais de soumission, contrairement à la plupart des revues « Open Access »

- Possibilité de publier dans 3 langues : français, anglais, arabe

- Qualité de la relecture : des relecteurs/reviewers indépendants géographiquement, respectant l'anonymat, pour garantir la neutralité et la qualité des manuscrits.

Pour plus d'informations, contacter BatnaJMS@gmail.com ou connectez-vous sur le site de la revue : www.batnajms.net 